









\section{ANNALES}

\section{SCWENES MTIREWES}

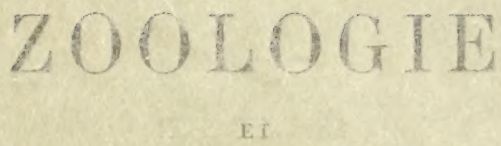

PALEONTOLOGHE

COMPREN ANT

1.ANATOMIE. IA PHYSGLOOLE, LA CLASSIFICATHON

ET L MISTOIRE NMTUIEILE DES ANIMATX

HeLLÉses SOIS L/. DXRECTTON DQ

M. EOMOND PERTIER

\section{EXTRAIT}

IIIBRARY

PARIS

DIvIsion of Crustacea

MASSON ET C, EDTTEURS

UA TAIRES DE Y ACADEMIE DE METEGTNE

120. Boulevard Satnใ-Germain $(6 \%)$

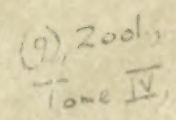

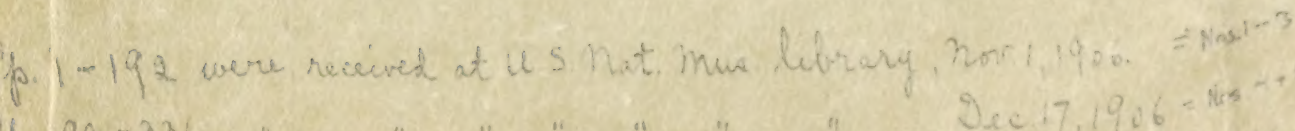


Conditions de la publication des Annales des sciences naturelles HUTTLĖME SÉrIE

\section{BOTANIQUE}

Publiée sous la direction de M. Pr. Van Twam. M.

L'abonncment est fait pour 2 volumes gr. in- $8^{\circ}$, chacun d'environ 400 pages, avec les planches correspondant aux mémoires.

Ces volumes puraissent en plusieurs fascicules dans l'intervalle d'une année.

\section{ZOOLOGIE}

Publiée sous la direction de M. Edmond Perrier.

L'abonnement est fait pour 2 volumes gr. in- $8^{\circ}$, chacun d'environ 400 pages, avec les planches correspondant aux mémoires.

Ces volımes paraissent en plusieurs fascicules dans l'intervalle d'une année.

Prix de l'abomement anmuel à chacune des parties, zoologie ou botanique

Paris : 30 francs. - Départements el Union postale : 32 francs.

\section{ANNALES DES SGIENGES GÉOLOGIQUES}

Dirigées, pour la parlie géologique, par M. HéberT, et pour la partie paléontologique, par M. A. MiLne-Edwands.

$$
\text { Tours I ì XXII (1879 a 1891). }
$$

Chaque volume............ $15 \mathrm{fr}$.

Celte publication est désormais confondue avec celle des Amnales des Sciences naturelles.

\section{Prix des collections:}

Premitre série (Zoologie el Bolanique réunies), 30 vol. (Kare). Deuxième série (1834-1843). Chaque partie, 20 vol. $200 \mathrm{fr}$. Troisième série (1844-1853). Chaque parlie, $20 \mathrm{vol}$. $250 \mathrm{fr}$. Quatrik̇esékie (18ă4-1863). Chaque partie, 20 vol. $250 \mathrm{fr}$. Cinouième sérte (1864-1873). Chaque partic, 20 vol. $200 \mathrm{fr}$. Sixik̇Me SÉrie (1874à 1885). Chaque parlie, 20 vol. $250 \mathrm{fr}$. SeptiÈme série (1885à 1894). Chaque partie, $20 \mathrm{vol}$. $250 \mathrm{lr}$. Géologite, 22 volumes.................... $330 \mathrm{fr}$. 


\section{DEVISIOD OR Crustacea}

\section{PAUNE CARCIYOLOGIQUE IIE LA MER ROUGri}

\section{DECAPOIES ET STOMATOPOIES}

Par le Dr G. NOBILI

(MUSEE DE ZOOLOGIE ET IXITOMIE COMPAREE, TUTIX)

\section{6}

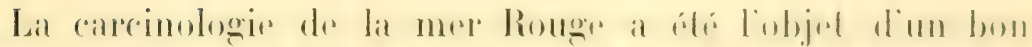
nomber de traratux. Je me bormerai a cons qui tratent direr-

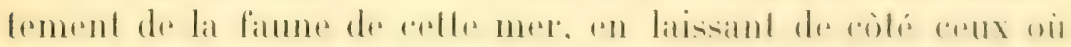

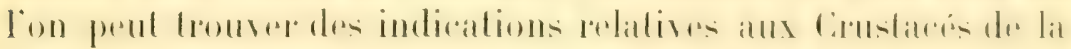

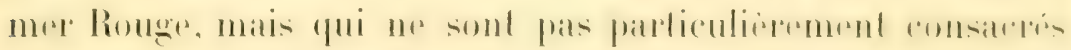
ì l'élude de cette faune.

Le premier travail, en ordre de date, trailant de la carci-

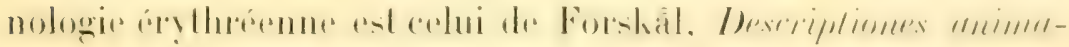
lium, elc., qux in ithere orientali observacit, etc., public is Copenhague en 1773 .

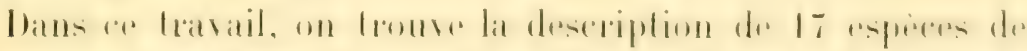

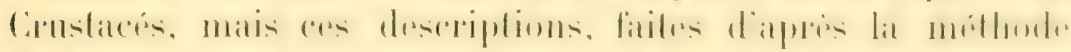

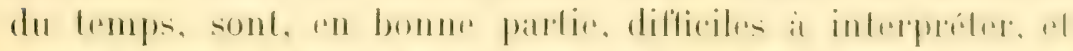

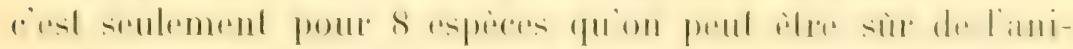
mal vu par Forskil.

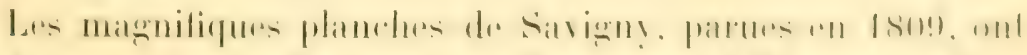

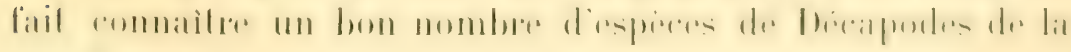
mer louge.

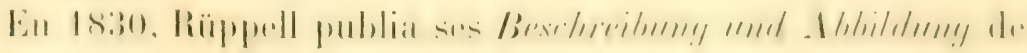
24 espèces de Brachyures de la mer Rouge. Les especes de

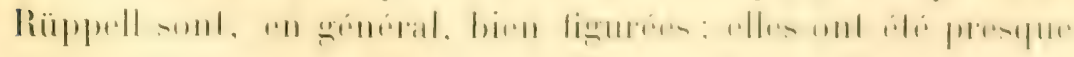

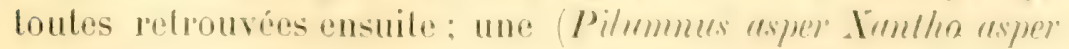
n'a plus élé revue; deux autres (Philyra cariergula el Ébuliu 
gremulatej sont dererites a nouvean et discutés par moi dans (e) meme travail, d'apres les types de Rïppel. One espece de la collection hüppretl restée incédite, a été publiée par De Man, en 1889, arre te nom manuscrit de Rüppell. Durrliteimire carinipes.

Dans la classique Histnire muturelle des Cmusterés de II. MilneEdwards (183\%-1839), de nombreuses especes sont reconnues det derrites, et daulres seront ajouténes a la faume de la mel Rouge dans les travaux suecessifs de eot autent mème el de M. Alphonse Milne-Edwards.

\& 8 Fu 1861 et 1862 , paraissent les importants Beiträge zun fient un nombe important d'especes nourelles et destypes inte-

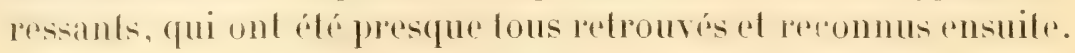
En appendice at son travail, Heller donnait une liste des esprers commues an 1869 de la mer Rouge. Ce nomber était

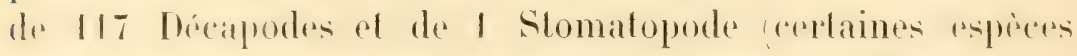

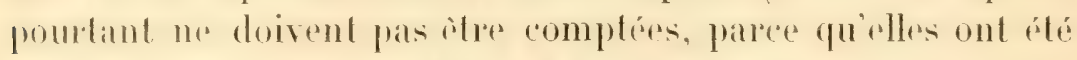

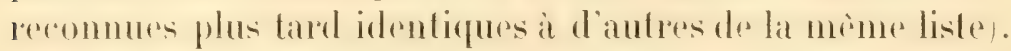

En 1866, von Martens décrit, parmi les Crustacés rapportés

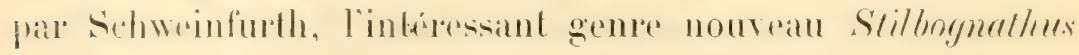
eryelliraeus.

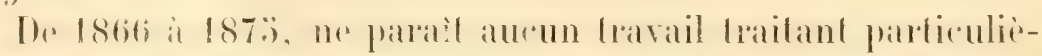
rement de la faume de la mer Rouge. Mais, en 1873, parail lo plus important travail pelatif a celle fiume, at le moins connu aussi : les Recherches sur les Crustrcés de lı mer Rouge,

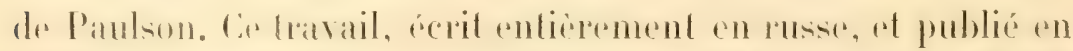
adilion a farle de cent exemplaires par l'Lniversite de Kieff,

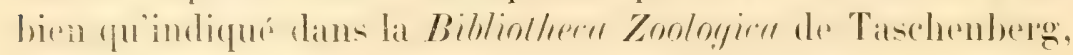

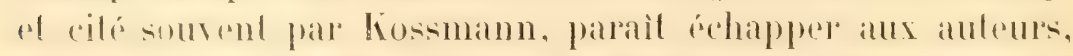

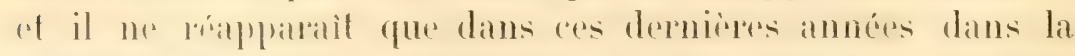

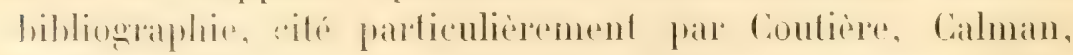

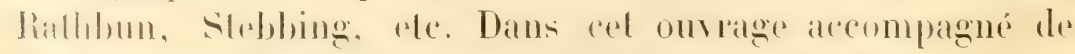

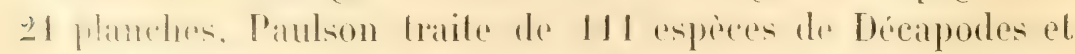

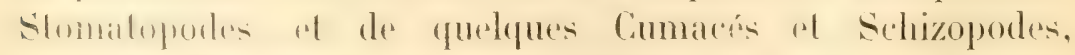

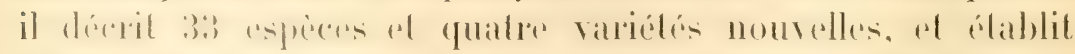

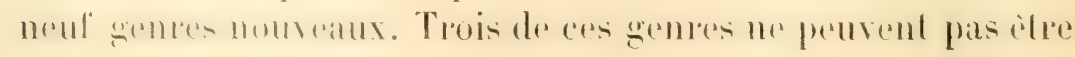
acceptés; deux autres sont des sous-genres de Lambrus. I la 


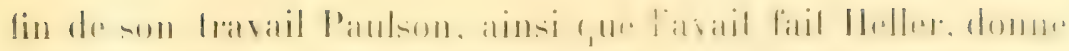

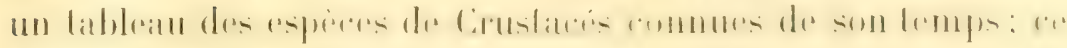
tableau comprend 170 Décapodes et 3 Stomatopodes.

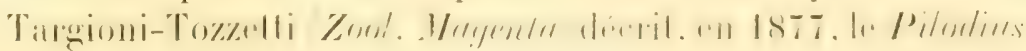
giranulalus.

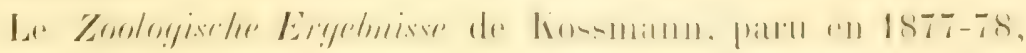
contient 9 especes nouvelles, dont 't seules peuvent itre maintenues.

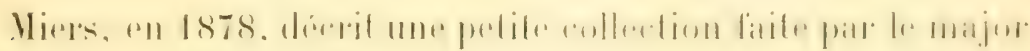
Burton dans le golle d'Akabah.

De Nan, en deux Lravaux publiés en 1880 el 1881 , traite

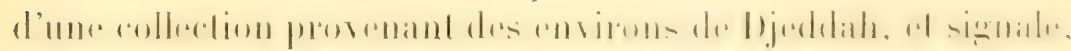

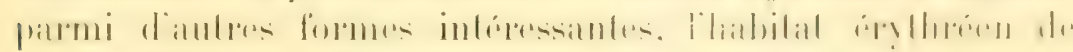
l'Arluer. Helleri 1. Edw. dont la localité álait inconnue.

Cano, en 1889, décrivil parmi les Cirustacés brachunes el Anomures recueillis pendant le voyage de la Tellor Pismi.

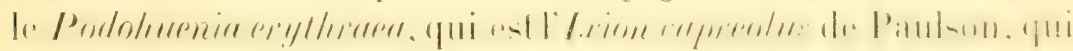
it son tour est identique avec le Cyplencercinus minulus $\mathrm{A}$. 11.

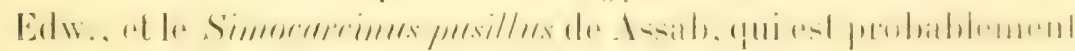
le jeune de S. simplex Dana.

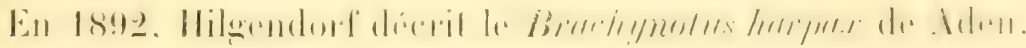

Un travail de A. Del Prato Crostarei della inllesinne erilren Bollegn. Alli Soc. Se. nal. 1896), part en 1896, n'at atucune vitleur pour la partic des Malacostracés.

En 1892 el 1897. paraissent deux mémodes tres interessants de M. Bouvier sur les l'aguriens de la mer liouge, arre: description do lrois formes nouvelles.

De 1896 a $190 \%$. 11. Couliere dans une série de notes at havatu bien connus, domme des contribulions tres preciensus

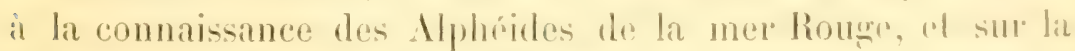
bionomie des Crustacés de Djibonti.

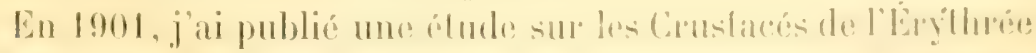

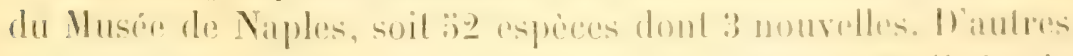

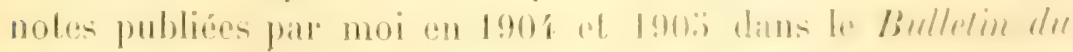

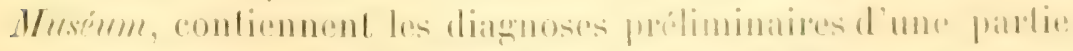

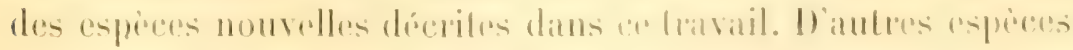

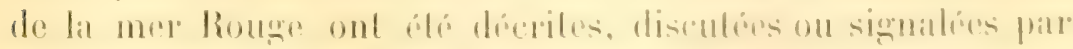

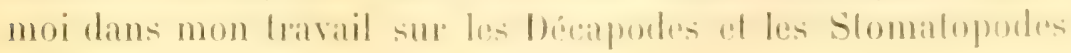


recueillis par MM. Bonnies et Pérez au cours de leur mission au golfe Persique Bulletin srientifique de la Fromere el de la Belgique, XL, 1906).

Dans cetle rapide revue de la bibliographie, je n'ai parlé (sauf pour quelques exceptions) que des travaux traitant expressément de la mer Rouge. On peut trouver des indications nombreuses d'especes de cette faune, dans les travaux de Hilgendorf, Miers, De Man. Henderson, Alcock, ete., que je ne fais qu’indiquer ici, mais qui sont cilés au cours du travail a leur place.

Les collections qui ont été mises à ma disposition pour la composition de ce travail étaient très importantes. Grâce a l'obligeance de II. le professeur E.-L. Bouvier que je remercie infiniment, les collections du Muséum (excepté lus Mlphéidés) m'ont été confiées, el ces collections ont formés de beaucoup la partie la plus grosse des matériaux éludiés (1). Ces récolles sont dues surtoul aux efforts de MM. L. Vaillant, R. Maindron, Ph. Jonsseaume, H. Coutièe, Ch. Gravier, abbi Cullierel, Du Bourg de Bozas, Clot Bey, Faurot, ete.

J'ai átudié ausio les collections assez riches du Musée de Turin ancienne collection, et récoltes récentes de MM. Clivio, Fatigati, Tellini, Magretti, Vinciguerra, ete.); du Musée de Gènes, failes par MM. Issel et Becearri; du Musée de Modène, faites par M. Ragazzi, et du Musée de Naples. A MM. les professeurs Camerano, de Turin; Gestro, de Gines: Rosa, de Modene; Honticelli, de Naples, je suis heureux d'exprimer ici mes meilleurs remerciements. Je dois aussi des remerciements particulier's it II. le Rér. Th. R. R. Stebbing, qui obligeamment me prèta el laissa longtemps à ma disposilion louvrage tres rare de Paulson, sams lequel ce travail aurait été impossible.

L'itude de cette sirie très large de Crustacés n’ayant permis d'augmenter de beaucoup le nombre des especes de Crustacés de la mer Rouge, et en étant relalivement pelit le nombre des especes signalées par d'autres auteurs qui me manquaient,

(l Puisque la plus grosse partie des matériaux éludiés appartient au Musémm, au cours du travail, guand il ny a pas pour les exemplaires lindicalion du Musée cela veut dire qu’ils sont conservés au Muséum. 
j’ai cru ulile d'indiquer dins mon travail aussi ces autres espèces non vues par moi, afin de donner un lableau aussi

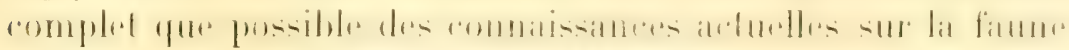

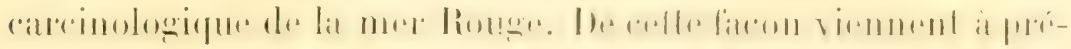

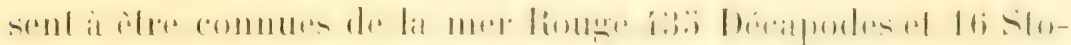

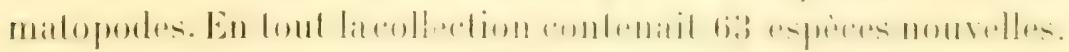

Les Cruslacés de la mer houge se partagent ainsi dans les différentes groupes :

\section{() ECIAP()Dis}

Natruntia.

Peniridir.

$\begin{array}{cc}\text { Genres. } & \text { Esprecs } \\ 1 & 13 \\ 2 & 3 \\ 1 & 1 \\ 1 & 1 \\ 10 & 41 \\ 3 & 4 \\ 4 & 7 \\ 9 & 22 \\ 1 & 1 \\ 4 & 6 \\ 2 & ! \\ 1 & 1 \\ 1 & 1 \\ 2 & 2\end{array}$

Reptantiu.

Palinurida....................

Scyllaride...................

Thalassinidite............... if

Paguride .................... 10

Gillitluidir..................... 1

Porcellanidie..................

Mippidra.................... :

Dromiidea ......................

Bruchyura.

Oxystomata................. 13 13

Oxyrhyncha ................ 16

Portunidie.................... 11

Xanthidie................... 39

Gonoplacide.................. 3

Grapside..................... 10 10 11

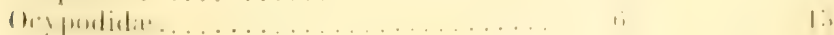

Pinnoleridie................ 3 , 11

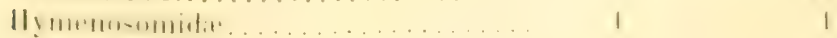

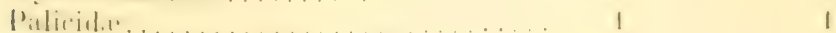

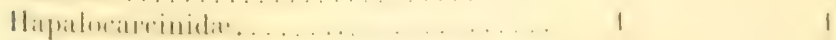

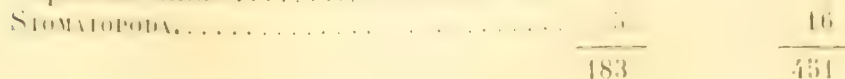




\section{DECAPODA}

\section{NATANTIA}

\section{PENAEIDEA}

\section{FAMILLE PENAID $E$}

A. Rostre denti en dessus et en dessous. Ine pleurobranchie sur la derniere paire de pattes (genre Penæus s. str.).

B. Rostre pourvu de $\frac{\tau-9}{4-6}$ dents ................. indicus Edw.

$B B$. Rostre pourvu de $\frac{8-10}{1}$ dents; deux sillons parallèles à la crète postrostrale continués jusqu'à l'extrémité de la carapace.

c. 'T'elson sans épines marginales. Les deux lames latérales du thelycum forment une espece de poche ouverte.................................

\section{P. canaliculatus}

OI.

cc. T'elson avec épines latérales; thelycum capsulaire... P. japonicus

Bate.

$B B B$. Rostre pourvu de $\frac{\tau-x}{3}$ dents; pas de sillons latéraux se continuant jusqu'au bord postérieur de la carapace.

d. Crête postrostrale distinctement sillonnée; crètes latérales au rostre s'arrêtant au delà de la première dent. P. ashiaka Kish.

$d d$. Crète postrostrale non ou très faiblement sillonnée; crêtes latérales au rostre s’arrêtant à l'extrémité de la

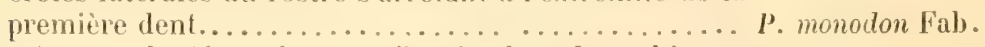

AA. Rostre non denté en dessous. Pas de pleurobranchie sur la dernière paire de pattes. Exopodites à toutes les pattes thoraciques, ou toutes sauf la dernière. Pas d'épipodites aux maxillipèdes externes et sur les deux dernieres paires de pattes. Carapace non fissurée $\ldots \ldots \ldots \ldots \ldots \ldots \ldots$ G. Metapenaeus.

B. Crète dorsale s'étendant jusqu'au bord postérieur de la carapace. Segments abdominaux $2-6$ carénés........... M. cognatus Nob. $B B$. Crête postrostrale ne s'étendant guère au delà de la moitié du dós de la carapace. Le deuxième segment abdominal n'est pas caréné.

b. Rostre plus court que les yeux ou à peine plus long.

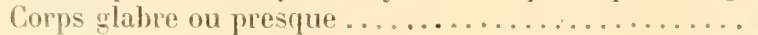

bu. Rostre long, ou, sil est court, le corps est très poilu. c. Petasma symétrique, arec deux pointes pour chaque verge, l'une verticale, l'autre transversale. Carapace et abdomen glabres ou presque. Mérus du g’e péréopode du mâle avec une échancrure et une dent............Stebbingi Nob. 
c. Pelasma asymétrique, l'une des verges prolongée en pointe ou dentée, l'autre rentlée, arrondie, plus courte et embrassant la premiere. Carapace et abdomen tres poilus (groupe du .I. velutimes).

d. La petite verge du petasma se prolonge en bec, non

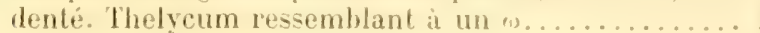

del. Petite verge du petasma denticulée à l'extrémilé. Thelycum arec deux plaques laissant entre elles une

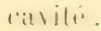

H. consobrinus

Xol.

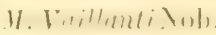

Penæus indicus lidw.

I1. Milne-Ldwards, H. n. Cr., II, 1837, p. 41:;.- Miers, Proc. Zool. Soc., 1878, p. 301-307. - Hilgendorf, M. B. Acad. Berlin, 1878, P. 8't. - Bale, Ann. Mar. Nat. Hist. (i), VIII, 1881, p. 177, pl. XII, fig. "; et Chullenger Warr. 1888, p. 249, pl. XXIII, fig. 2. - De Man, M. Weber's Zool. Ergebn., II, 189't, p. 511, pl. XXIX, fig. 53, et Zool. Jahrb. Syst., X, 1898, 1. 680. - Ortmann, Denkschr. Jena, VIII, 189', p.10. - P. merguiensis De IIan, Jou'n. Limn. Soc., XXII, 1888, p. 287, pl. XVlll, fig. 8.

". Obock (Jousseaume). Deux miles, lont l'un ì branches

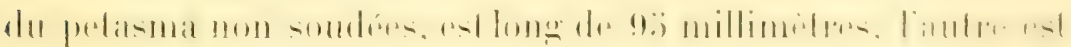
long de 103 millimelres. Ces exemplaires ont $\frac{7}{7}$ el $\frac{8}{7}$ denls atu rostre. Deux femelles, longues de 109 el 112 millimidres, arec $\frac{8}{3}$ el $\frac{9}{3}$ dents. Ces exempliares s'approchent de la variéti longriostris décrite par De Man, par leur rosloe non horizonlal, mais recourbe en haut el dépassiant ho scaphotérile d'un liers de sil longueur, depuis le bord antérierm de lis carapace. Dans la variéle de Cébebes, le rostro dópasse le scaphocerite de moilie de sa longuems. Comme dans celle

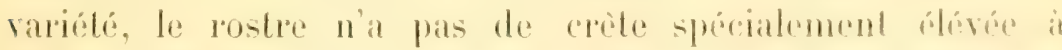
la base. Le liers qui depasse le seaphoceribe ast dípontron

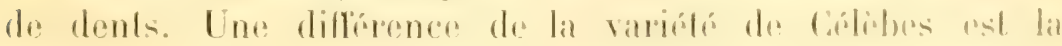

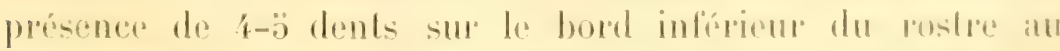
linello 8 .

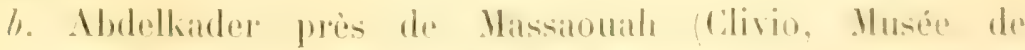

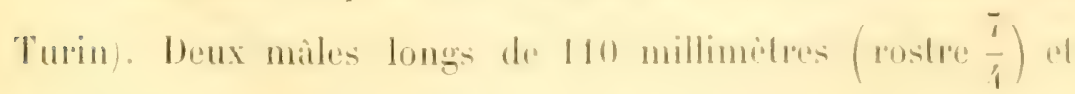

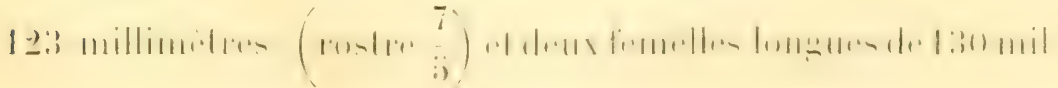


limelres $\left(\right.$ rostre $\left.\frac{8}{3}\right)$ el 13 r millimètres $\left(\right.$ rostre $\left.\frac{8}{6}\right)$. Ils ne ditfëerent pas sensiblement desexemplaires de Oboch par la forme du rostre, mais la partie dépassinnt le seaphocriplé est un peeu plus comte, ol la partie styliforme sans dents est, dans trois individus, peu plus longue.

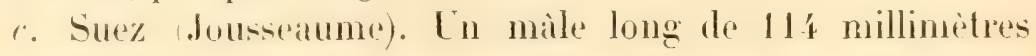
(rostre $\frac{8}{5}$ ) et deux femelles longues de 111 millimetres $\left(\right.$ rostre $\left.\frac{8}{i}\right)$ el 12 ' millimètres (rostre $\left.\frac{8}{6}\right)$. Le rostre a la mème forme que dans les individus de Obock of de Mbdelhater.

d. Baie de Djibouli (Gravier). Une femelle longue de io millimetres dont le rostre a la mime forme que dans les exemplaires qui précèdent, et a $\frac{8}{6}$ dents.

e. Ver Rouge (Y. Jousseaume). En individu dont le rostre est difierent, non recoube, mais dirige obliquement en haut depuis la base et pourvu de $\frac{10}{3}$ dents.

Jai examini nombreux aulpes exemplaires de cette espece de diflépentes localités indiennes el indo-nalaisiennes. Sur 39 exemplaires en tout, le rostre dans 37 exemplaires est gréle. élanré, non purliatièrement rehanssé en rarène ì la base, dé-

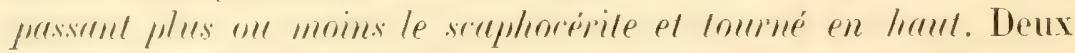
exemplaires seulement, l'un de Bombay, l'autre de Buntal (Sarawal), correspondent a la forme P. meryniensin De Man: leur rostre est soulevé en forte crête triangulaire à la base, il est

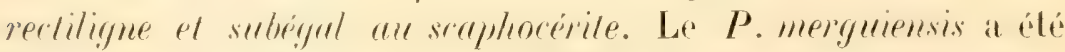
sigmalé par De Man, en plus qu à Mergui, dans la mer de Javat, par Lanchester ol par moi sur les cotes de Sarawak, of par moi a Bombay. Il est done bien probable quil s'agise d'une variété particulière à l'océan Indien.

Le thelycum ressemble beaucoup à celui de $P$. monodon;

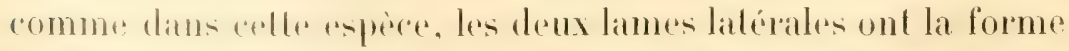
de deux valves semi-cioculaires, mappochés, avec les bords de conlatet refléphis al sonlevés, lasisant entre rux une fissure elliptique. La kame midiane est petile of se prolonge entre les 
deux latérales sous forme d'une mince crète droite, plus

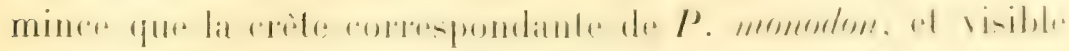
seulement en divaricant les lames externes. Le pelasma ou rundricum est aussi tres semblable it celui de conulicululus. monodon. ete.

Habilat. - Quelimane Hilgendorf); Dares-Galiam Ort-

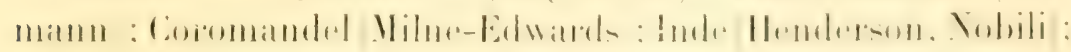

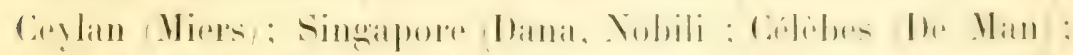

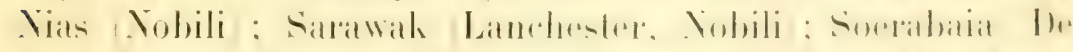

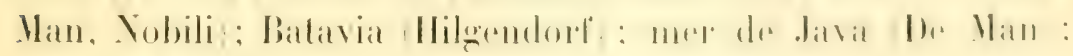
Amoy (Miers); Chefoo Miers); Philippines (Bate).

\section{Penæus canaliculatus Oliv. (1).}

Olivier, Encycl. méthod., p. 660. - Milne-Edwards, H. N. Cr., 2, p. \$1 . Bate, Ann. Mag. nat. Hist. (3), VIII, 1881, p. 17', et Challenger Hacr., P. 17. - Nobili, Boll. Wus. Torino, XY, 1901, no 397, p. 1, nee P. canuliculatus De llaan, Kishinouye.

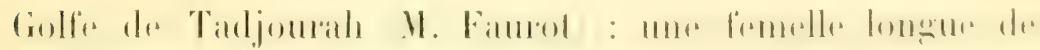

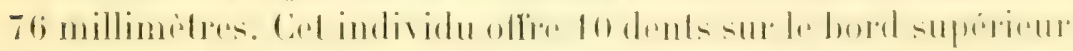

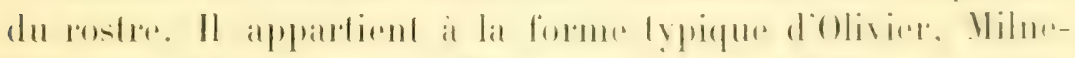

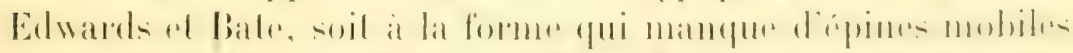

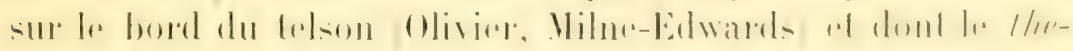

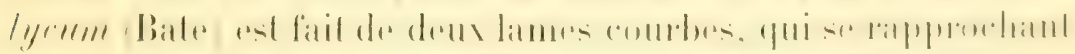
forment une poche ouverte de forme particuliere (Cil. ligure du "Challenger" ").

Un male de Buntal (Sarawak) a le pelasma it peu pres

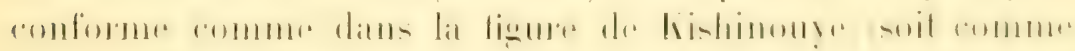
dans la forme joponicuss); mais la protuberance de la lame interne saillit libre en forme de crochet. lorsque dans jupnnicus elle est couchée sur le bord supérieur de la lame extertne.

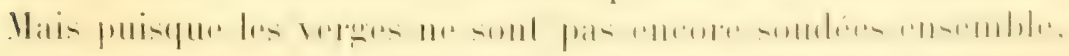
je ne crois pas pouvoir accorder une importance délinitive

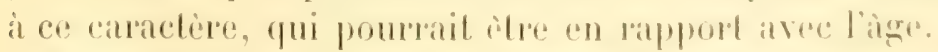

Lábule de la forme des appendices des spermatophores pourrait peut-ebe domner d'autres diffirences, ainsi qu'elle en

(1) Jai cité seulement la partie de la liltéralure qui se réfere, sans atucum doute, au viai $P$. cunuliculutus. 


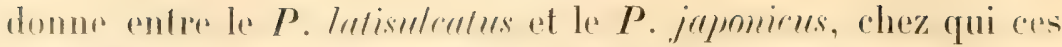
appendices sont connus. Mais, mème en laissant de côté ces

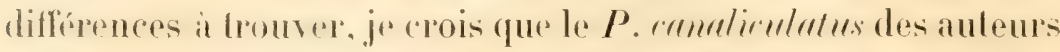
sat un groupe dre formes, dans lequel existent an moins quatre espèces, qu'on peut séparer ainsi :

A. Sillons longitudinaux latéraux de la carapace subégaux au sillon postrostral médian.

B. Telson sans épines marginales mobiles. Thelycum en forme de poche. Pas de lames accessoires entre la base de la $3^{\mathrm{e}}$ et de la $4^{\mathrm{e}}$ paire de péréopodes (Spermatophores?

P. canaliculutus Oliv.

$B B$. Telson avec épines marginales mobiles.

c. Thelyeum capsulaire. Pas de lames accessoires entre les péréopodes $3^{\mathrm{e}}$ et $4^{\mathrm{e}}$. Spermatophores avec un large appendice aliforme........... P.japonicus (Bate). cc. Thelycum formé par deux lames longiludinales (non réunies?). Une lame à la base des péréopodes $3^{e}$ et $4^{e} \ldots \ldots \ldots \ldots \ldots \ldots \ldots \ldots \ldots \ldots$.

AA. Sillons longitudinaux latéraux de la carapace plus larges que le sillon postrostral médian. Lame médiane du thelycum avec un prolongement bifurqué. Appendice des spermalophores en forme de bande fibreuse etroite. P. latisuleatus Kish.

\section{Penæus japonicus (Bate).}

Penæus canaliculatus (juponicus dans le texte) Bate, Ann. Mag. Nat. Hist. (5), VIII, 1881, p. 17\%

Penæus canaliculatus var. japonicus Bate, Challenger Macrura, 1888, p., 240, pl. XXXII, fig. 4. Penæus canaliculatus De Haan, F. Jap. Crust., p. 190. Niers, Proc. Zool. Soc., 1878, p. 298, 306 (pars). - Ortmann, Zool. Jahrb. Syst., V, 1890, p. 4'48, pl. XXXVI, fig. 2 (pars). - Kishinouye, Journ. Fish. Bureau Tokyo, VIII, 1900, p. 6,11, pl. I et VI, fig. 1.

Mer liouge M. Jousicatume, : 11 male bong de 137 millimètres, avec $\frac{9}{1}$ dents ou rostre, et une femelle longue de 1:3:) millimetres, arece 110 nombere gagl de dents. Dans cetle femelle le thelyrum porte les deux appendices raratelésidiques

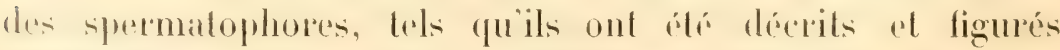

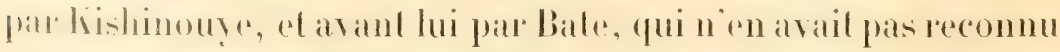
lit malurre, mais les avail considéés comme des parlies normales du thelyrum. Bate, dans son élude próliminaire sur les l'énéidés du "Challengere ", avait justenent recomnu que la forme japonaise décribe parr De Ilatan différait de celle décrite par Milne- 


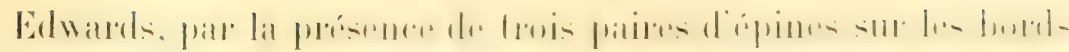
du telson, et avait proposé le nom de japonirus, dont il til plus lard une simple rariété du crmalimentus. La forme du thelycum, associé di la présence des épines, me paraissent

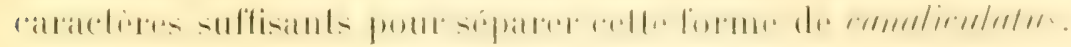

\section{Penæus monodon liab.}

Fabricius, Suppl. Ent. Syst., p. 408. - Bate, 1798, p. 408. A. M. X. H. (:3), VIll. 1881, p. 178, Chulleng. Macr., p. 2:30, pl. XXIT, fig. 1 (le or seulement). Ortmann, Denksch. Jena, VIII, 1894, P. 9, pl. II, fig. 1. -- De Man, Kon. Jahrb. Syst., X, 1898, D. 677. - Kishinouye, J. Fish. Bureau Tokyo, 1'll, 1900, p. 13̈, pl. Il, fig. 1, et pl. VII, fig. 3. - Vobili, Boll. Mus. Torino, Xllll, $\mathrm{n}^{0} 4: 2$, p. 1, et $\mathrm{n}^{\circ} 4.50$, p. 1, 1903 .

P. semisulcutus, var. exsulcatus Ililgendorf, H. B. Acad. Berlin, 1878.

Aucun exemplaire de la mer Rouge ne correspond it la

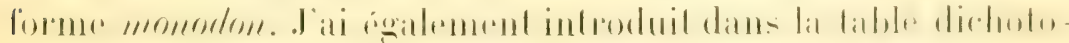

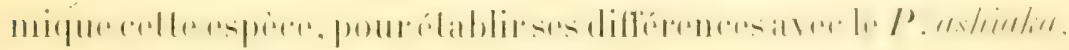
el pour ticher d'échaireir un peu la synonymie de ces formes.

On ne peut pas savoil exactement ce qu'ast he P. monndon

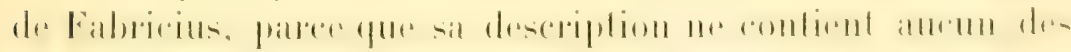
caractères employés aujourd'hui pour séparer colle esperex

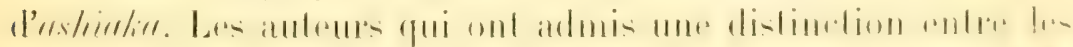

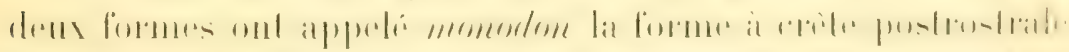

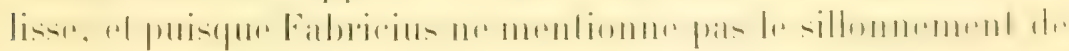
crete, il est bien probable que ses exemplaires correspondent vraiment it la forme délimitée plus tard par les auterurs.

Le P. monodon de Vilne-Elwards est, ansi que je l’ai établi dans mon travail sur les Cruslacés du goolte P'ersique, la forme it crite postrontale sillomme.

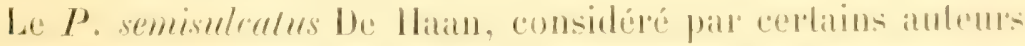
comme identique au P. monodom, al dont le nom a ble sombent

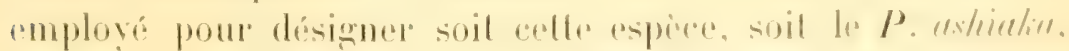
ful consideré par lishinouse, on 1900 , comme identique aree momodon, mais, an 1902. séparí pall misis lialhbun qui le

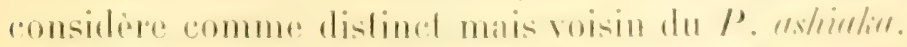

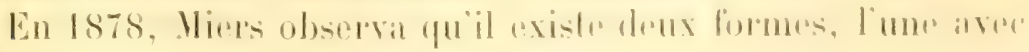

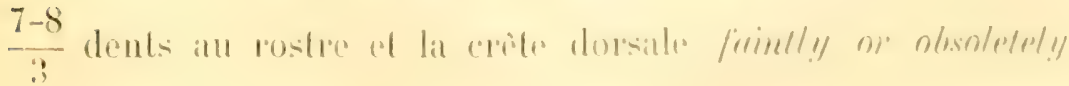


comulirululed, et avec le sillon gastro-hépatique faiblement défini, quil appelle semisulentus, l'autre avece crete non canaliculere of te sillon gastro-hépatique tris profond. Si pour crite dorsale faiblement canaliculée, on entend frublement par rapport it roucrlirululus of brosiliensis dont Vlers a parlé avant,

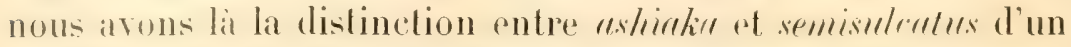
ròté si nous admettons que ces deux especes soient identiquest) et monodon de l'autre.

Hilgendorf, en 1878, décrit une forme de Zanzibar et du Mozambique sur la crète dorsale de laquelle ist.... nur eine

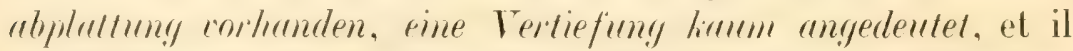
appelle cedle forme, ectr. exsuldalus du $P$. remisulcutus, tout en ayant le doute que celte forme soit le monodon.

Bate, en 1881, incline à considérer non seulement monodon et remixulerlus comme identiques, mais il a la menes opinion a propos d’indires. opinion qui ne peut pas itre soutenter et que Bate mème abandonne en 1888. Mais dans l’étude définitive des Crustacés du "Challengere", il sépare indirus, mais considere la forme monodon i crete non sillonnée comme le mile, et la forne semisulentus a crète sillonnée comme la femelle, d'une mime espece, ce qui est faux, puisqu'on trouve des mâles et des femelles des deux formes.

Ortmann, en 1891 (Zool. Jahrb., syst. V, p. 450), corrige lerreur de Bate at appelle semisuldalus la forme a crete sillomnere, et monodon la forme it crete lisse. En 189' te mime auteur conserve la mìme division, mais le caractere différentiel du thelyfum (sur lequel insista aussi be Van en 1898/ n'est qu'une différence d'ìge. Lne femelle de taille moyenne du P. ashichide provenant de Suez a le thelyrome a hames encore greiles et distantes comme celles du monodon de Ortmann, mais ces lames dans les femelles adultes se rapprochent ensuite "nl formant deux bords saillants le long dr len surfice de "onlaret, comme dans les adultes de deux "speces, el dans celles d'indicus.

Wr. Man. rn 1898, élablit bien les diffépences entre les deux formes an appelant semisulcales la forme a crite sillonnée.

hishinouye, "n 1900, fait de semisulerelus un synonymo de monodon, et appelle asticala la forme sillonnée. 


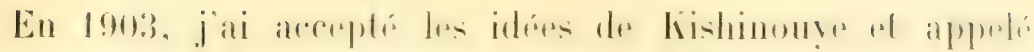

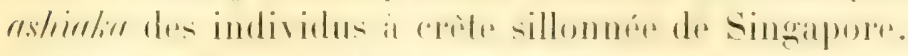

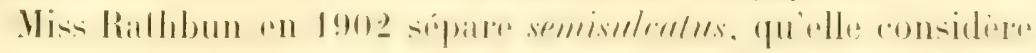
comme voisin de astrirlia el non de monodon.

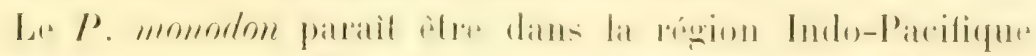

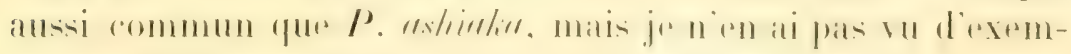
plaires de la mer Rouge.

\section{Penæus ashiaka Kish.}

Kishinouye, Journ. Fish. Bur. Tokyo, VIII, 1900, p. T, 14, pl. III et VIl, fig. 4. - Rathbun, Proc. Nat. Mus., XXVI, 1902, p. 38. - Nobili, Boll. Mus. Torino, XVIII, no 45̈, p. 2.-Bull. scient. Fr. Belg., XL, 190:3, p. 16.

P. semisulcatus Auct. (pars). - Yobili, Ann. Mus. Napoli, 1, 1901, n 3, p. 2.

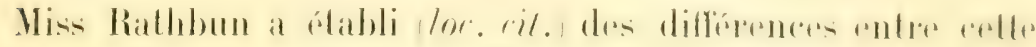

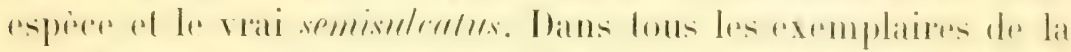

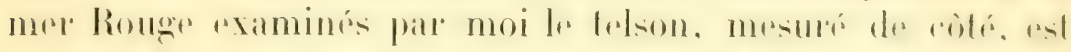

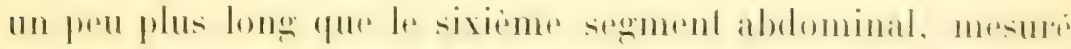

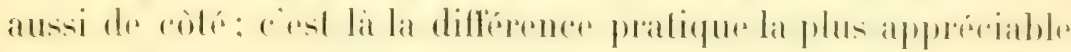
entre ashiatia el semisulealus; les autres, données par Miss

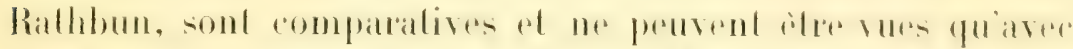

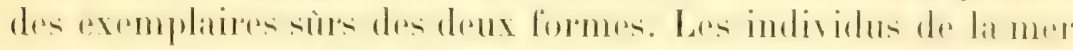

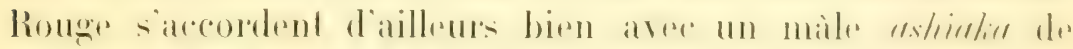
Nagasaki du Musée de Turin.

Her Rouge (Baudouin): un mâle ef une femelle. Her

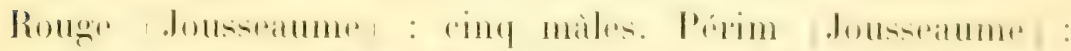

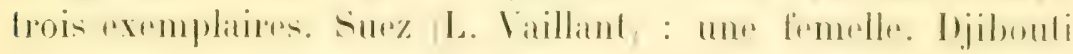
(Coulière) : nombreux individus jeunes

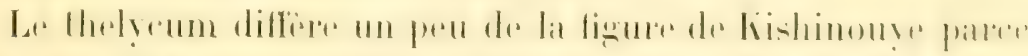

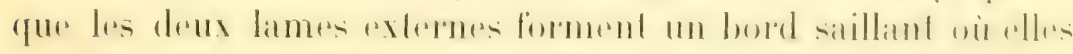

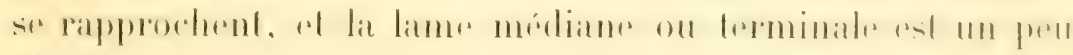
plus large.

La verge interne du petasma saillit un peu plus que dans

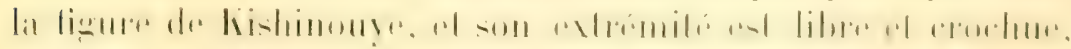

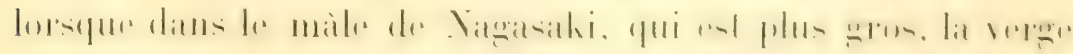
est raballue. 


\section{Genre METAPENAUUS Wood-Mason.}

Metapenæus brevicornis (Edw.).

Penæus brevicornis H. Milne-Edwards, H. N. Cr., II, p. \$17. - Nier's, Proc. Zool. Soc., 1878, p. 308. - Bate, Ann. Mag. Nat. Hist. (ö), VIII, 1881, p. 180, pl. XI, fig. 3. - Henderson, Trans. Linn. Soc. (2), V, 1893, p. 450.- De Man, Zool. Jahrb. Syst., X, p. 681, fig. í.

Une douzaine d'exemplaires tres jemes recueillis par M. Coutière, à Djibouti.

Le postre ces plus court que les yeux el pourvu de $6-8$ dents. Il ny a pas de crote sur la partir dorsale des la carapare. La carapace 11 at que quelques poils rares et courts, qui tres probablement disparaissent dans l'adulte.

M. arimslrix Dana, considéré par Bate et Henderson identique aree refte espece, est bien distinet par son rostre beaucoup plus loug ol prar une crebe sur lis carapare. CC. Nobili, Boll. Mus. Torino, XVIII, 1903, $\mathrm{n}^{\circ} 447$, p. 2.)

\section{Metapenæus cognatus Nob.}

(Pl. I, fig. 1.)

Nobili, Bull. du Mus., 1904, no 3̈, p. 229 (Diagn. prélim.).

Djibouti (M. Jousseaume) : une femelle.

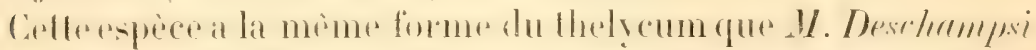
Nob., mais en differe pall rentans atrateleres importants.

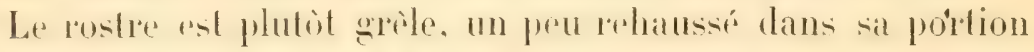

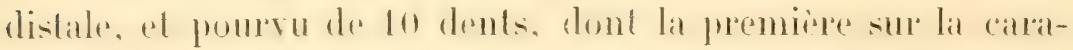

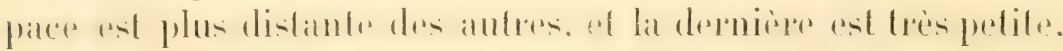
Il alleint lexhromilis de lavant-dernire antirle du pédoncule des antemmules. La crobr postrostrate sétemel jusquan bord postérieur de la carapace.

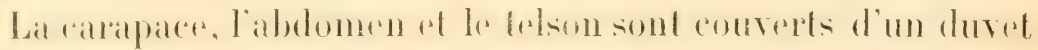

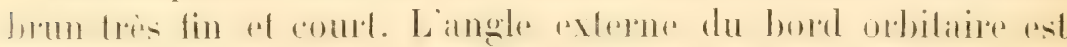
spiniforme; l'épine antennale et l'épine hépatique sont

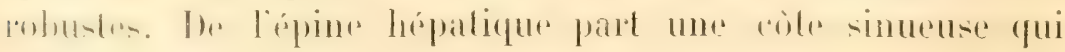
s'étend jusquau bord postérieur de la carapace.

Les yeux sont gros et réniformes; les pédoncules ont le

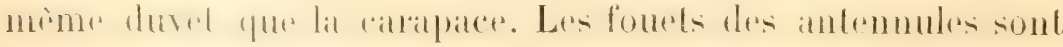
plus courts que le pédoncule. 


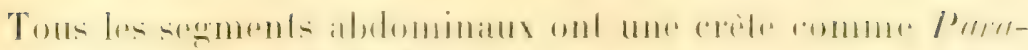

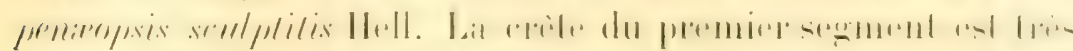

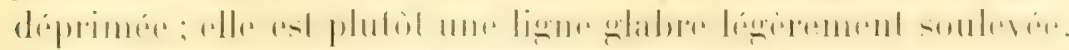

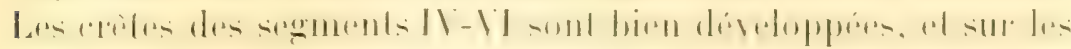
segments IV-V sont bifurquées à l'extrémité. Lues cotés des

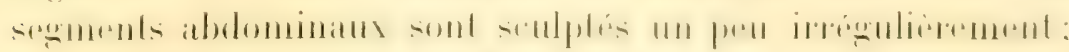
sur les cotés des segments 1 et VI il y a une crète distincte.

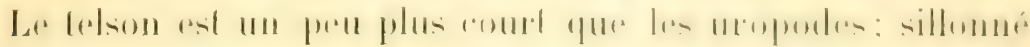

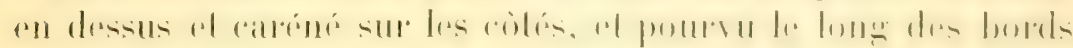
de nombreuses petiles spinules mobiles.

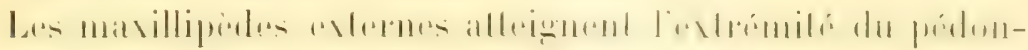

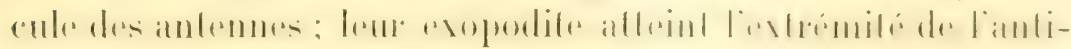

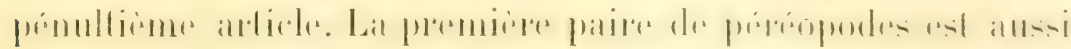

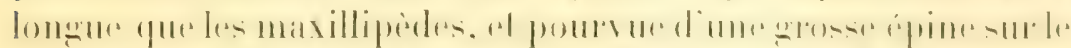

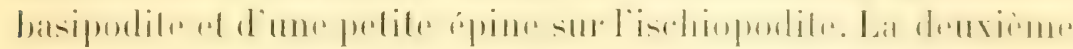
paire dépasse le pédoncule de toule la main; la troisième

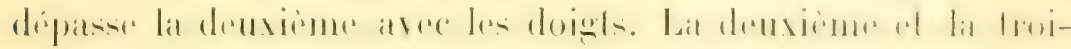

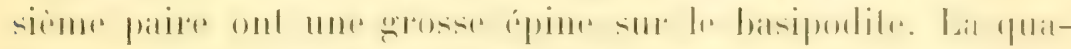
trieme paire a une pelile dilatation basale.

Le thelycum est formé comme dans 11 . Deschampsic (1); les

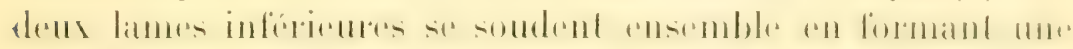

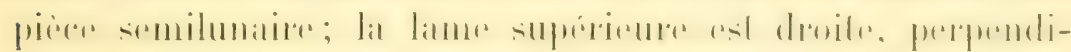
culaire, sillonnée (lisse dans Dexehampsi); les doux intermédiées sont interposées entre la médiane ef les cornes du croistant.

Cetle espece diftere de $M$. Deschrmpses par la pilosité, par

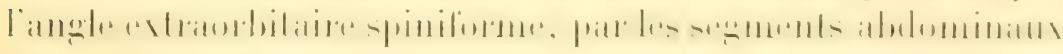
lous carénes et par son telson spinuleux.

Longueur, is millimelres.

\section{Metapenæus Stebbingi Nob.}

1.1. I. liz. :

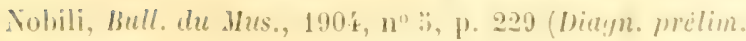

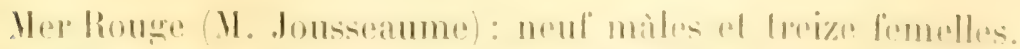

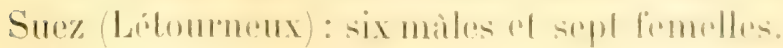

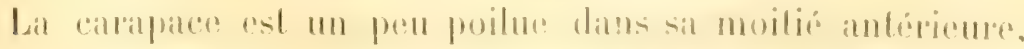

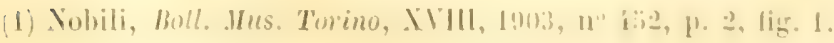


mais surtoul press des sillons. Les sillons: gastro-frontal, gastro-hrpalique, hépatiqur (partiellement), antennal, céphalique pour sés parties latrirales) sont distincts. L'épine hépalique of l'antemale sont bien formées. La crète postrostrale? scétend sulement par un petit trait en arriere de la derniere dent.

Le rostre "st plutòt grêle, légèrement recourbé en haut. denté jusqu’a l'extrémité; il alteint l'extrémité de l'avantdernier arlicle du pédoncule des antennules. et il est, conséquemment, plus court que le scaphocérite. Il a en dessus 8-10 dents, la premiere desquelles est placée près du tiers antirieur de la carapace et est distante de la deuxieme. Les fouets des antemmules sont aussi longs que les deux derniers articles du pédoncule el sont plutòt renflés. Le scaphocérite est sillonné le long du bord externe; le fourt des antennes est deux fois aussi long que le corps.

Les maxillipides externes alteignent l'extrémité du pédoncule des antennes externes; leur exopodite atteint l'extrémile de l'antépénultieme article. Les péréopodes de la première paire sont un peu plus courls que les maxillipedes externes; la deuxième paire dépasse arece les doighs le pédoncule? des antemnes; la troisieme paire atteint la moitié du scaphocérite. Les doigts de ces trois paires sont plus longs que la paume. Sur le basipodite des trois paires il y a une forte epine. La troisieme et la quatrieme paire a dans la femelle une dilatation laninaire au coxopodite. Le méropodite de la cinquieme parre a dans les mâles une incision suivie par une dent, comme dans $\boldsymbol{M}$. monoceros, affinis, mutatus, ete.

Dims la femelle, il ! a more cavité sternale, s'étendant entre la troisieme of la quatrieme paire de pattes, adossée aux deux lames du thelyeum, el couverte par les dilatations basales des prepopodes. Le thelyom est formé par deux lames quadran-

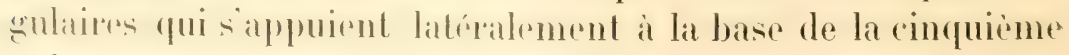
frime, antésientement a la dilatation du coxopodite de la

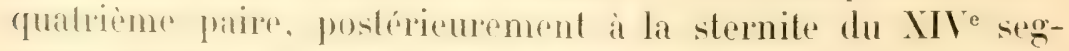
ment. Enlere lus denx lames il y a une troisieme pièce linéaire. qui es un prolongement de la sternite. Dans l'intérieur de la cavile proleswe par les deux dilialations, on peut voir les trois 


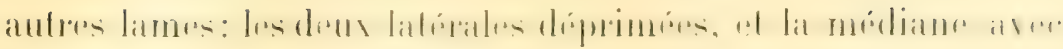
trois tubercules mamillaires.

Le pélasma est formó par une parlie colonnaire sillonnés

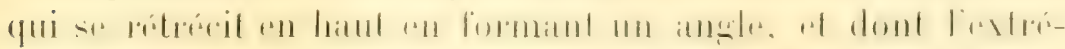

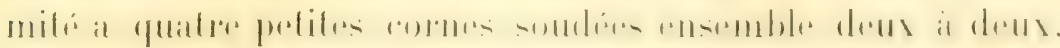

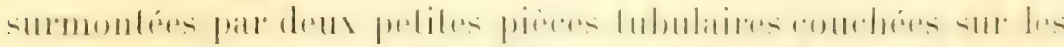

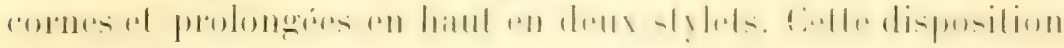
s'observe dans les màles adultes de "mer houge ", longs de

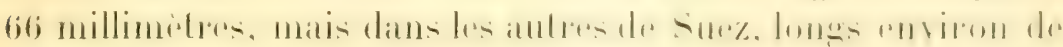

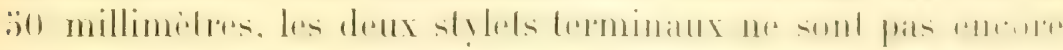

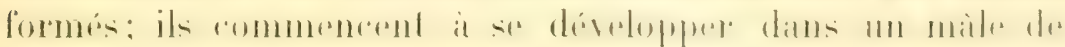
ว̋3 millimètres.

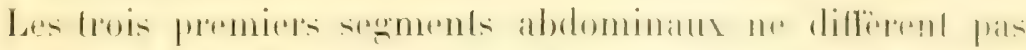

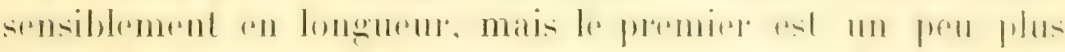

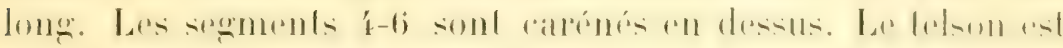
sillonné, plus court que les uropodes qui sont ítroits el

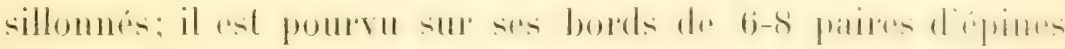
mobiles.

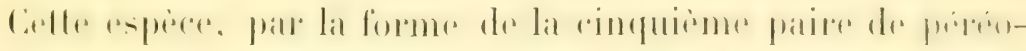

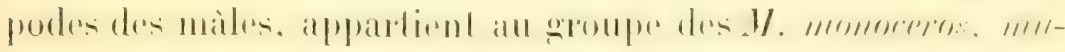
latus, affinis, incisipes, ele., mais elle dillere des especes dece

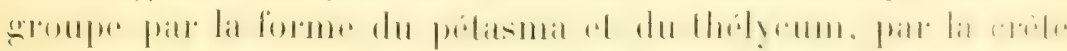
postrostrale très courte, par les épines marginales du tels(1). 1.t1\%

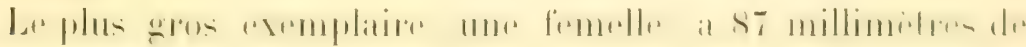
longueur.

\section{Metapenæus consobrinus Nob.}

(Pl. I, fig. 3.

Nobili, Bull. du Mus., 1904, no ï, p. 229 (Dia!n. meilim.).

Djibouli (II. Conlière), deux males et me lemelle.

Cette espice appartientau groupe du $1 \%$. relulimes, al, comme dans toutes les especes de ce groupe, le corps est courert de? duvet.

Le rostre dans te mile est courl, depassint it peine les yeux el alleignant presque l'extromilo du premier article du pédoncule des antemmules. Dans la lemelle il est plus long of 


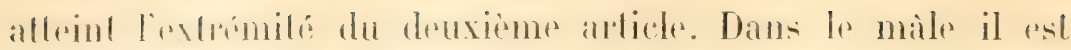
ansi lirige un pen plus en haut que dins la femenle. Le bord

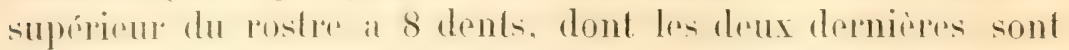

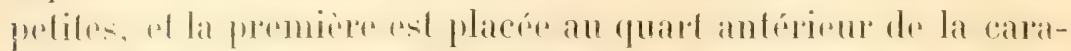
pace. Les yeux sont gros, les cornées réniformes.

Les maxillipedes axternes natteignent pas le boted anteriem

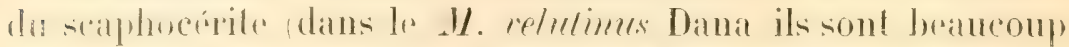

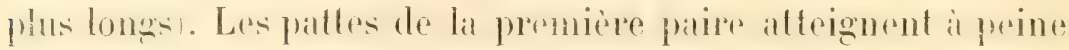

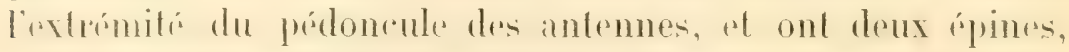
celles do la deuxième paire alloignent la moilio du scaphocérite el colles de la troisiene paire lextremite des maxillipedes. Elles ont une seule épine sur le hasiporlite. Il y a comme dans loules les formes du grouperetutimes, une pare d'ipines stermalus a la base des próropodes de la denxieme paire; ces épines sonl petites. Les palles de la quatrieme paire sont contes; celles de la cinquieme paire dépassent le pédoncule desanlennes, mais n’atleignent pas l'extrémité duseaphocépite.

Les segments abdominaux II-VT sont carénés, le segment II faiblement.

Le telson a trois paires dépines latérales fortes et deux paires d'rpinus dorsales. Sa pointe est trifurquée comme dans le $\boldsymbol{M}$. perlarum.

Le mile, qui a le pétasma completement développer, n’a qur 3:i millimedres dre longueur; cette espece est donc de petite faille. La pébasma est astmétrique. Loune des verges est grosse,

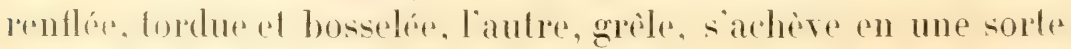
de bec. Les verges n'ont pas d'épines.

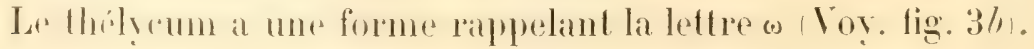

Metapenæus Vaillanti Nob.

(Pl. I, lig. 4.)

Nolili, Bull. du Mus., 190\%, nº̈, p. 229 (Diagn. prélim.j.

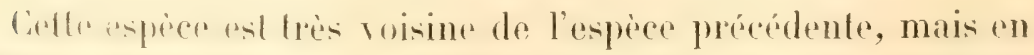
diffire neflement par la forme de son pétasma el surtout de son thily.um. Ello est représentée par quelques exemplaires

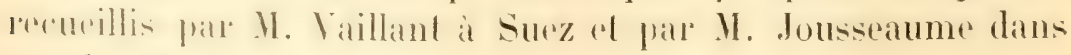
une localité non précisée de la mer Rouge. 
Le rostre dans le mile dépasse la moilié du deuxiène article du pédoncule des antennules; dins la fenelle, il est un peu plus long et atteint ou mème dripasse un peu l'extrémité de cel article. La forme est plutot rariable; le bord supérieur est pourvu de 7-9 dents, dont la premiere est placée près de la base du rostre sur la carapace, at une distanes médiocre des atutres. Le dos de la carapace n’est pas caréne.

Les maxillipedes externes alteignent les trois quats de lit longueur du scaphocérite. Les paltes de la premiere paries

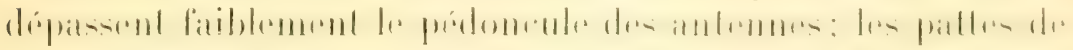

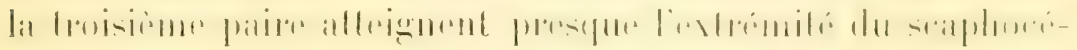

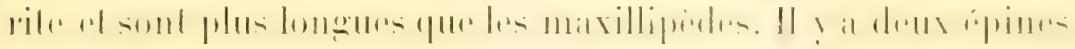
sur les paltes de la premiere paire, une seule sur les palles de

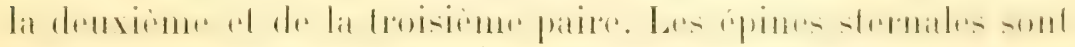
peliles.

Il y a une petite frace de crète sur le deuxieme segmenl

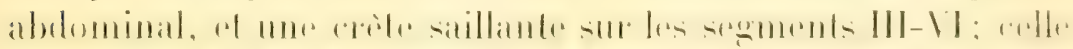

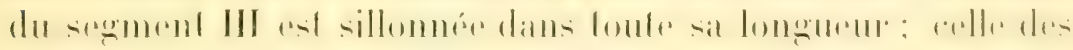

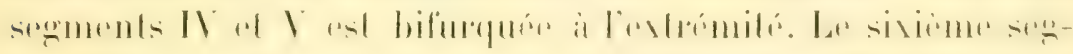
ment est long des trois quarls de la carapace. Le lelson est formé comme dans l'espèce précédente.

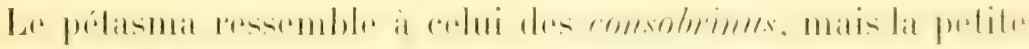

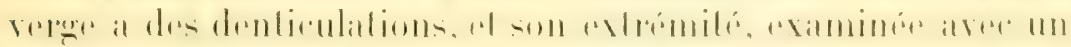

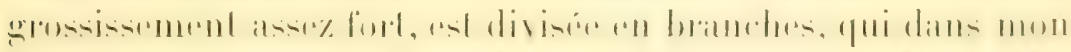

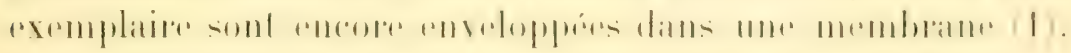
Le thélycum est voisin de celui de $\mathbf{M}$. perlarum. Lat latme supérieure est ample el angularire a la moitié de son hord supérieur; les deux lames inférieures sont soudées entre elles, les lames intermédiées sont petites; la cavité laissée entre ces lames est profonde.

Longueur, 46 millimétres.

Paulson décrit un Penduss celulinus. de ne crois pas que ce soit vraiment l'espece de Dana; mais sat deseription ne domne pas de délaits sur le pétasma of le thélyeum qui permedtent de reconnathe de guelle espere du spoupe elulimes il s'agisse.

(1) Dans la ligure, ces branches terminahes sont représentées plus grosses que ce que comporterait le grossissenent general de lorgane, qui est de 1.t diametres. 


\section{Metapenæus curvirostris (?) Stm.}

Trois frmelles de Massaouah M. Fatigati. Muse de Turin) me parassont appartenir prestue certainement a celle espece du dapon, de la mer de Chine el de la mered' Irafoura, mais leur thélyom est trop en maurais blat pour une déterminationsùe.

Le rostre dans deux exemplaires offre 8 dents, et il est peu rehausse, dans l'aute il a 9 dents et il est plus courbé. Toute la surface du corps est poilue. L'épine sus-orbitaire est distincte dans les trois individus. Il n'y a pas de fissures sur la carapace. Les maxillipredes externes alleignent la moilie du seaphocérite; la preminer pare de préropodes alleint presque l'extrémité du pédoncule dris anteinnes: les doigts sont environ hois fois ausi longe (fue la paume dans cette paire, lorsqu ils sont seulement un pert plus longs gue la paume sur la deuxieme paire, at ausi longs que la paume sur la lroisieme paire. L'épine sur le deuxième article des pattes de la première et de la denxieme paire st médiocrement developée. Les dactylopodites des palles ambulatoires sont notablement courls.

Il n'y a pas lrace de crete sur le deuxieme sogment abdominal: les segments III-VI ont une crote tris nette. Les bords posterieurs de lous les segments ont un petit sinus, plus fort sur les segments IV et $\mathrm{V}$. Le telson at profondément trisillommé. La sillon médian est rectiligne, les deux latéraux coment oblingerment of se rencontrent en formant un angle arece le sillon módian. Les epètes qui séparent ces sillons sont asserz fortes. Les frois parires de spinules des bords du telson sont distantes entre elles; les épines sont petites.

Les trois femelles ont respeclirement 63, iz at it millimètres de longueur.

Paulson décrit les formes suivantes que je n'ai pas vues:

\section{Penæus longipes (1).}

Paulson, p. 119, pl. XIX, fig. 1-1 a.

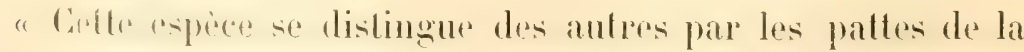

(1) Vu la rareté de l'ouvrage de Paulson, qui est devenu désormais introu- 


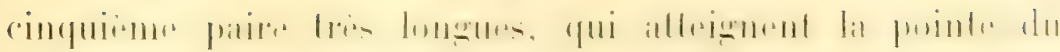

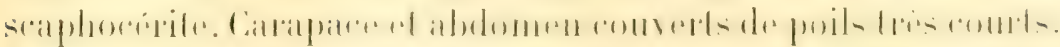
Rostre un peu tomrné an haul, is extrémité aignë; son bord supérieur avec 10 dents, la dixieme placée sur la carapace; bord inférieur sans dents el pourru de poils: les fouets des antennules ont de longs pédoncules, avec lesquels ils s'unissent (?). Pédoncules octilaires comrls. Partie antérieme de la carapace avec quatre épines: susorbitaire, antennale, hépalique el ptérygostomique. Deuxieme article des palles chéliformes armé d'une grosse épine, exo-

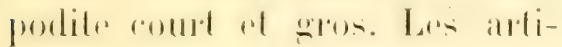
cles $3,4,5$ et 6 ont une crets

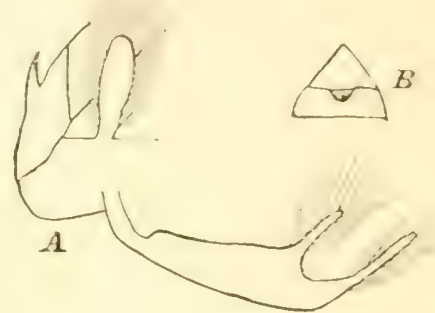

Fig. 1. - Penixus longipes Pauls.

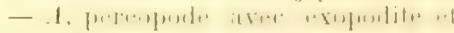
épipodite: $B$, thélycum (d'apres l'aulson). sur le bord; sur le bord posté-

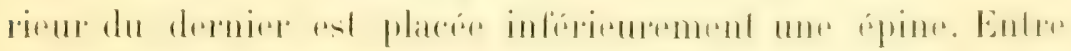
les pattes de la qualrième paire sur le sternum, on trouve

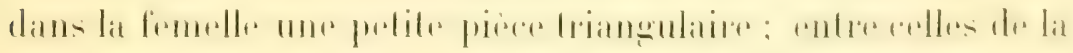

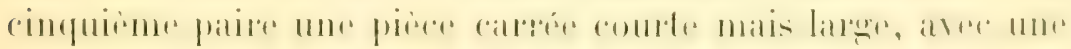

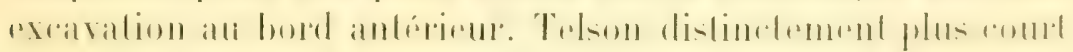

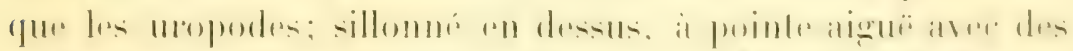

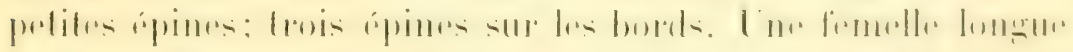
de 5 . millimetres. "

\section{Aphareus n. gen.}

Paulson, p. 117, pl. XVIII, fig. 3-3n.

"Mandibules simples. Deuxieme et troisiome paires de

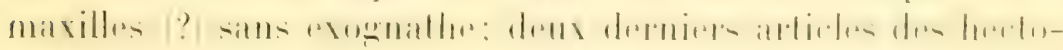

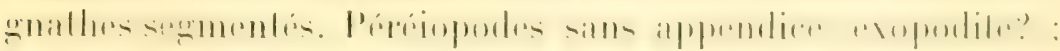

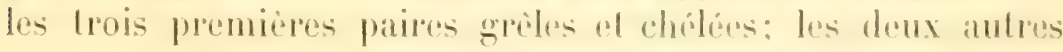
paires non annelees. Pleopodes it drux lames. Rostre cound, pédoncule oculaiere el pédoncule des anlennules fongrs."

vable, je crois ulile de donner une traduclion fonmise des diagnoses russes de ces especes nouvelles, dont jo nai pas va dexemplaires. Mans cette tratuc-

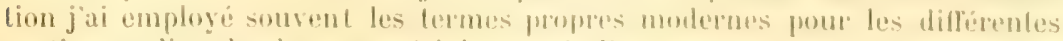
parties, au lieu des longrues priphorases de l'auleur. 


\section{A. inermis Pauls.}

"Pédoncule des antennes internes plus long que le seapho-

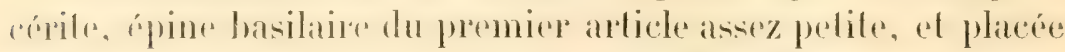
('n) haul; desurime et troisieme articles d'egale longueur, triangulanes: denxieme article plus lange que le troisiome. I la moilio de la partie antérieme de la carapare, il ! a unt crobtr denliforme, qui passe apres dans le eourt rostre. Sur les cotés

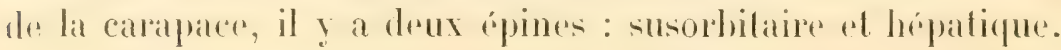
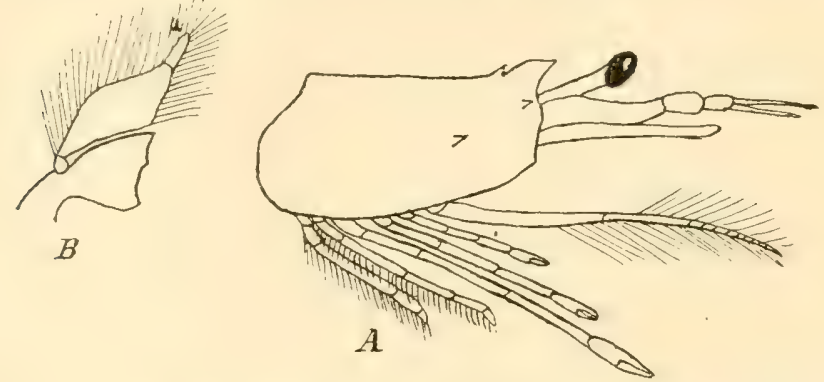

Fig. 2. - Aphareus inermis Pauls. - A, céphalothorax; $B$, mandibule (d'après Paulson).

"Pentognathe (xie) pédiforme. Ifectognathes plus longs que le pédoncule des antennes supérienes, lenrs extrémités samincissant fortrment: les deus derniers articles divisés en quatre segmonts. Paltes de la troisieme paire également longltes (1). Palles suivantes terminés par des dactylopodites aigus; les arlicles pourvus de longs poils. Sixième segment abdominal pouru d'une crote, et denx fois aussi long que le cinquieme. Theon lemine en pointe aree quatre épines de rhatque cote. Lropodes plus longs que le telson ; les internes

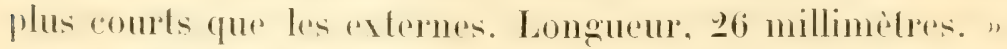

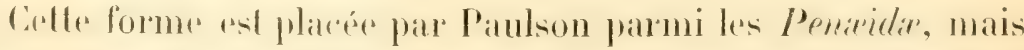
je crois que, par sa forme générale, par ses mavillipedes, par la forme des dernieres palles. des antemmules, de. elle appartient aux Sergestidix, bien que les pinces des trois premirens paries sobent plus déreloppres que ce qui advient d'habitude dans les Sergestidix.

Paulson signale encore dans la mer Rouge :

(1) Que les maxillipèdes? G. N 
Sycionia lancifer Oliv.

Paulson, p. 118, pl. 11111, lig. $2-26$.

\section{FAMILLE SERGESTID.E \\ Genne dCliles linw. \\ Acetes erythræus Nob.}

|'।. ! . |i ....

Nobili, Bull. Mus, 1905, no 6, p. 393.

Celle espèce paraîl être commune dans la mer Rouge. Le Musée de Turin en a recu des exemplaires tres nombreux de

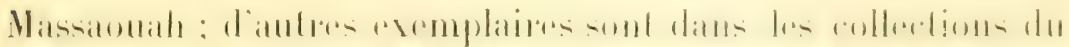

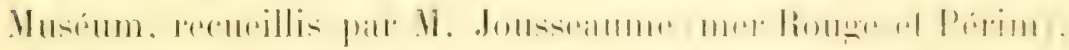
ainsi qu'un exemplaire pas encore mur, bien qu'ayant dégi

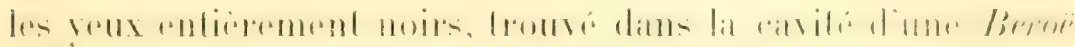
à Djibouli par H. Du Bourg de Bozas.

Je ne peux pas etre entierement sir que mon espece soit

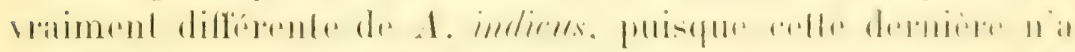

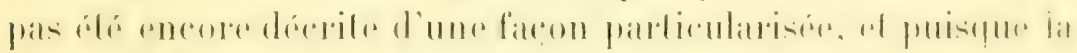

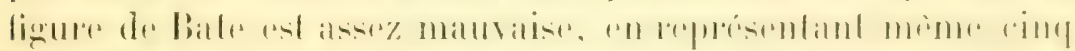

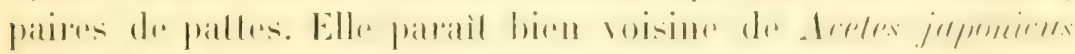

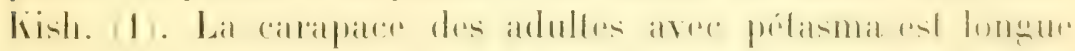
moins de $1 / 3$ de la longueur lotale du corps; elle est armée

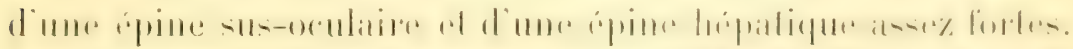

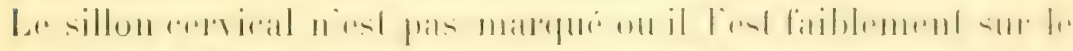

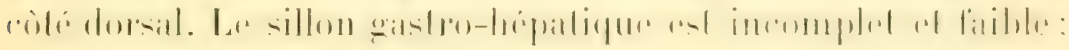
le branchiostégite offre en arriere une crete longiludinals flanquée par deux sillons. Le rostre est saillant, bidenté; un arriere du rostre il y a une aute dent sur la carapace.

Les yeux sont gros el pyriformes, les cornées ponflées al assez bien délachées du pédoncule qui stangil de la base it l'extrimité.

Le pédoneule des antennules as phe tong que leseraphoeririte, yu'il depasse d'environ moilie de son dernicer andicle. Le premier arlicle a une disposilion qui mesemble beatueoup it

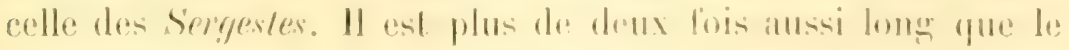

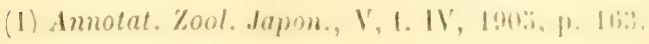


deuxieme, mesuré sur le bord interne; mais puisque son bord exterme savance au delit de l’insertion du deuxieme article, il est plus de deux fois ef demi aussi long si on le mesure sur ce borl. Le deuxieme article est court el un peu renfle par rapport au troisieme, ef deux fois ef demie aussi long que larese at l'extrémité. La portion du bord externe qui reste libre ent and du prolongement du premier article est a peine un pert plus longue que la moitir du bord interne. Le troisieme article est cylindrique, non renflé; il est phus d'une fois et demie aussi long que le bord interne do deuxieme arlicle, et Iroic fois aussi long que la portion libre du bord externe. Le premier ardicle est fortement excavé an desius pour la réceplion des yeux qui atteignent presque l'extrémité de cet article; du cote interne il a un fort rebord épais pour la protection de l'xil, qui ast ausi protigé partiellement par un rehaussement du coté externe. Lópine "xterne du premier article est placée un peu en arrière de la moitié, plus près de la base.

Dans la matr le gros four (lig. öd) est double parenviron 3 enticles; tous les exemplaires ont lextremite du fouet exterme cassée, et je ne peux pas en dire la longueur. Des deux alules fonels, l'un est séliforme, apparenment non segmentí, avec des denticulations sur lo segment qui le porte; lautre rst segmente, plus long, plus gros avec des appendices mobiles pendants. Le seaphocérite est étroit, plus de quatre fois ansi long que large; sa pointe est arrondie; la pelite épine apicale ne dépasse pas la pointe. Les antennes ont le fouet tres Long. dans le petit nombre d'exenplatres qui le conservent; il est conforme a peu pres comme dans $A$. jepmnirus, mais les poils sont beaucoup plus courts et souvent absents.

Les maxilliperdes extermes sont plus courts que la troisieme paire dre pattes. Ils sont pédiformes, sans exopodite, pt aucun de lemes articles nest élargi par rapport it reux des pattes. lls sont pourvus de longues soies, et leur sixiome article n'est pas segmente. Les trois premieres paires de palles croissent an longurur; cllesont, ainsique les maxillipedes, de longues soies; alles sonf formines par des pinces pudimentaires of microsco-

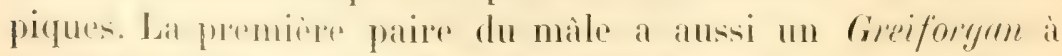
l'extremili do calpere a a la base du propodite, comme dans 
A. americanus Ortm. el dans d'auteres Sergestides, mais cet

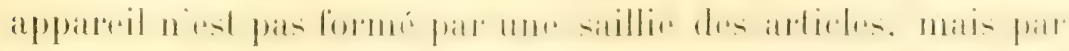

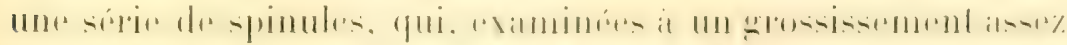

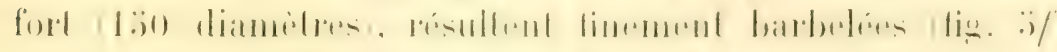
ainsi que paraîl être le cas aussi dans A. joponirns.

Les pattes 4 -ä manquent el c’est li un caractere générique.

Les branchies ont la même disposition que dans A. americanus (V. Ortmann, Decrop. Schizop. Planliton E.rpr., p. 39).

Le pétasma a une struclure curieuse, qu'on peut voir dans la figure ö e el qui est différente de celle de 1 . jopmnicus. Les pléopodes sont grêles et longs.

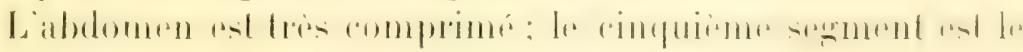
plus court, le sixieme est le plus long de tous.

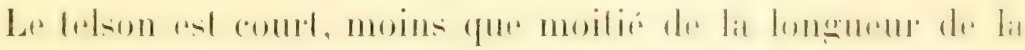

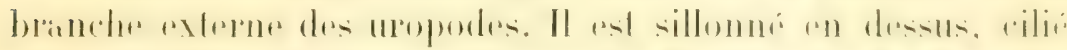

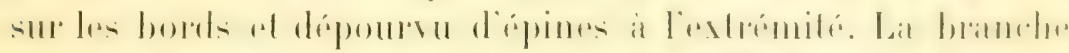

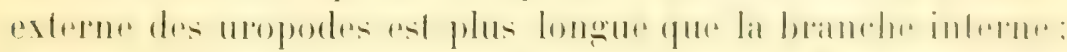

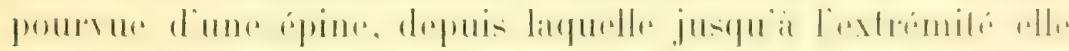

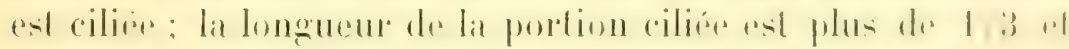

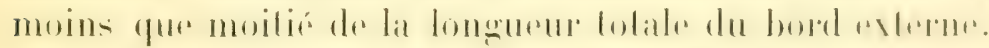

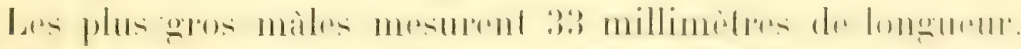

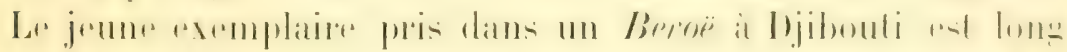

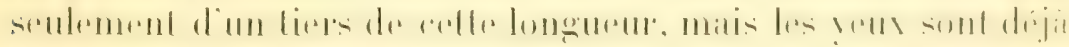
entierement noirs.

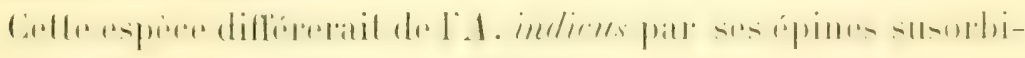
taires et par ses dimensions beaucoup plus grosses, al de l'A. japonicus par la forme de son pétasma.

\section{LUCIFERIN E}

Gente ladeifler 'T'momp.

Lucifer Hanseni Nol.

PI. II, lig. I.

Nobili, Bull. Mus, 190:3, no 6, P. 39! (Diugn. prélim.).

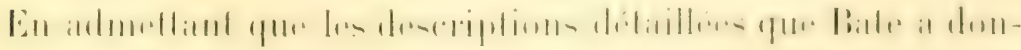

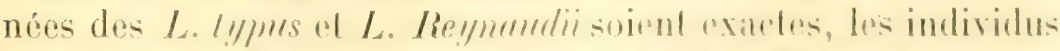

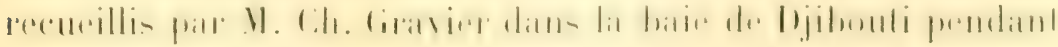




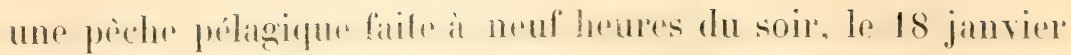
1904, appartiennent peut-ĉtre à une espèce nouvelle.

Lat portion éphlatique prolongée en atrant est deus fois

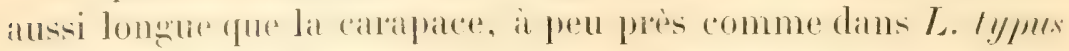
of heancoup plus longue que daus $l$. Reymundii; alle mesure

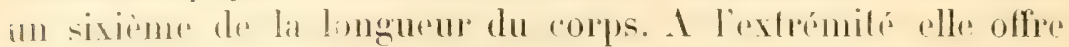
une pointe comme dans les deux espèces nommées et une

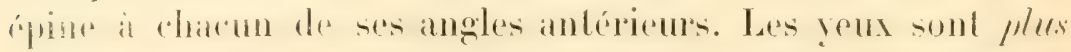

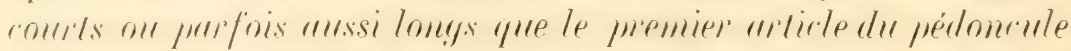
dex culemmlex / c'est le contraire dans les deux autres especest. Le premier article du pédoncule des antennules ast plus de deux fois ansid long que de denxiome: le troisieme est la moitié aussi long que le deuxième.

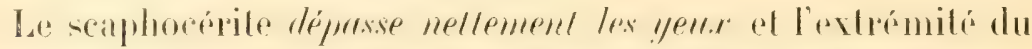
premier artiche du pédonoule. Le fouet des antemmules est asiez long; son premier article est presque aussi gros mais plus long que le troisieme article des antemules. Je ne vois pres de pheymorétile sur le cosocérile des antennes. Les palles thoraciques et les masillipedes ne me paraissent pas offrir de différences. La troisieme paire a un petit dactylopodite analogue ì celui de typus.

Les segments abdominaux (exrepté le sixième) n'ont pas

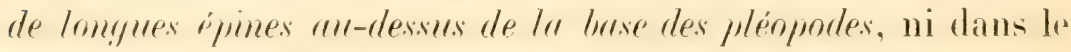
màle, ni dans la femelle. mais ils forment un petit angle spiniforme microsepique. En chla cotle wijece ressemble a L. Reynaudii, et paraît différer de lypues.

Les segments IV of I sont plus courts que les antres; les frois premiers sont subégaux.

Le siviente segment est plus cout que les segments IV et I

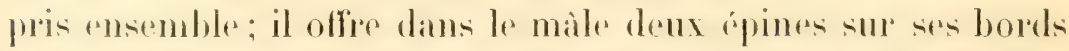
latéro-infórients, dont la dencieme, placén plus pros de l'exhémile st phus forte que la premiere. Iextrémile de son hord dorsal se prolonge en dent, asiez pelite. La forme des épines du màle ressemble à celle des épines de L. Iypus; dans la femelle it n'y a aucume épine.

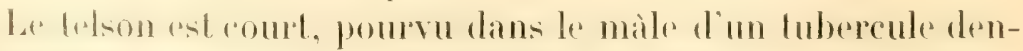
licule, qui mancpur dans la femelle. Il offere an dessus denx épines, y qualde pelites epines à son extrémite. Lat rame 


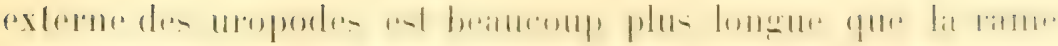
interne; alle est pourve sur son bork externe d'unc épinr placée pres de l'extrémité, aux :3/4 de lit longueur du borel externe. Lat portion ciliés de ce bord noecupe donc que 1/4 de la longuene du bord mime.

Le pétasma ressemble à celui dos doux aulles aspieces.

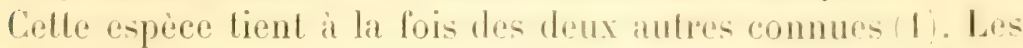

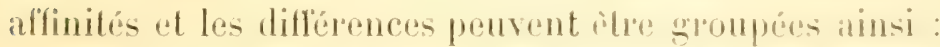

Lucifer typus,

1. Portion céphalique, $1 /$ 't de la longueur totale et plus de 2 fois la longueur de la carapace.

2. Premier article du pédoncule antennulaire, plus court que les yeux; un phymocérite; scaphocérite.

3. Bords des serments abdominaux arec une épine, plus forte dans la femelle que dans le mâle.

t. Sixieme segment ab)dominal avec deux épines dans le mâle et une dans la temelle.
I. Hanseni.

Portion céphalipue, $1 / 6$ de la longueur totale el 2 fois la longueur de la carapace.

Premier article du pédoncule des antenumles plus loner que les yeux; pas de phymocérite; scaphocérite plus long que les yeux.

Bords des segments abdominaux sans épine.

Sixième segrnent abdo. minal avec deux épines dans le male, sans épines dans la femelle.
I. Keynitudii.

Porlion réphalique, 1/:̈ de la longueur tolale el it peine de $1 / 3$ plus longue: yue la camapace.

Premier arlicle du júdoncule plus court que les yeux.

Bords des serments abdominaux sans épine.

Sixième segmentabdominal du male avec deux épines, dont l'une claviforme; dans la femelle une seule epine.

(1) Après la description donnée ci-dessus, j’ai eu l'occasion de voir de nombreux exemplaires d'une espèce que je cross le L. typus. Le L. Hanseni dillere des individus de typus (des iles Tuamotou) aussi par le sympode du premier

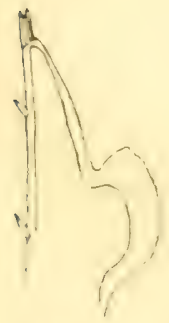

d

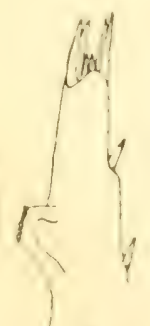

b.

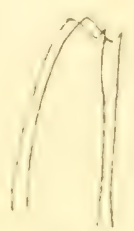

11

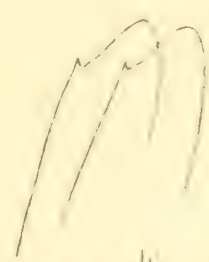

1,

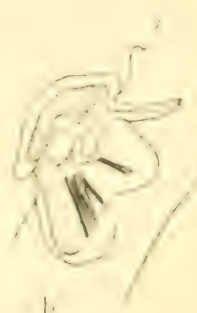

1.

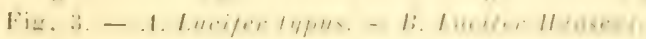

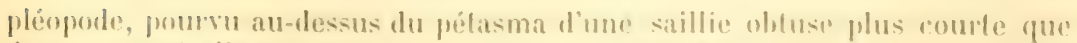
dans typus ou elle est prespue toujours spiniforme lo") : pat le luberente folsoni que du male plus court ib), plus étroit of de lorme difrérenle: par l'épine de l'exopode de l'uropode placre aux $3 /$ t de la lomaneme do bord externe (b'), at heaucoup plus éloignée de la prointe que dans typus ou dle est prespue terminale. 
Les plus gros exemplatires mesurent 12 millimetres de longuenr. Ils sont bion adultes. puisque les màles ont le pétasmat el le lubereult du lelson bien développés: un màle mème était en train d'émettre son spermatophore (1).

\section{Lucifer Reynaudii Edw.}

Paulson, loc. cit., p. 121, pl. XI, fig. 6.69.

Paulson a décrit des exemplaires de la mer Rouge de cette espece. Il est bien probable quelles' troure atsisi, parce qu'elle a étí houver dans toute la région Indo-Pacilique et dims l'océan Atlantique.

\section{EUGYPHIDEA}

\section{FAMILLE PASIPHAIDA}

Genre Leptochela Stmpson.

\section{Leptochela aculeocaudata Paulson.}

Paulson, loc. cit., P. 100, pl. XVl, fig. 1-1 s.

Djibouli M. Coulieper. Ln seul exemplate en matrais atat. Je prefere, dans les conditions de l'exemplaire donner, la lraduction de la deseription russe qui est assez détaillée, plutôt que deen dommer none nouvelle sur des materiaux insuffisints.

"Rostre pas plus long que l'ail, commençant en crête au milieu de la carapace. Pédoncules oculares courts et gros. Pédoncule des antennes supérieneses plus courts que le scaphocrerite; premier article plus long que les deux suivants, sa surface supérieure concire, arre rpine basale alteignant l'extrémite de l'arliche avec lequel elle est soudée pour plus de moitie de sa longueme, el quelque peu toumése sur son axe. Pédoncule des antemnes externes alussi long que celui des anlennes internes; bord externe du seaplocérite droit, bord inlerme convexe el pourva diune épine apicale; le premier arlicle a, ent plus de l'épine basile, une épine forte mais tres

(1) Bate a une figure analogue, mais d'une femelle de $L$. qui reçoit le spermatophore. 


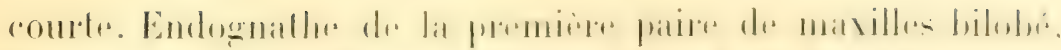

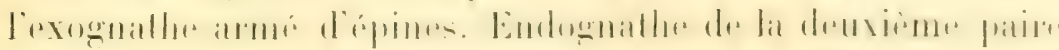

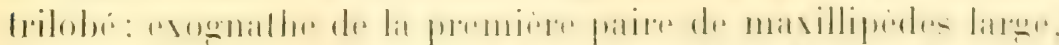

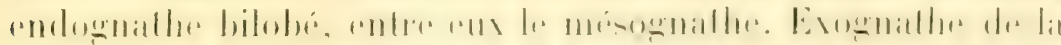

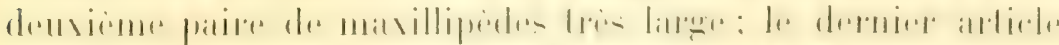

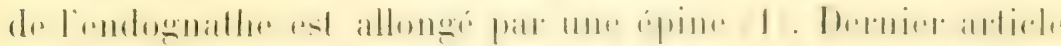

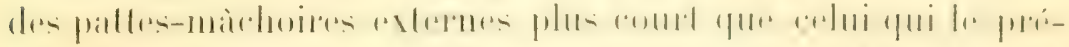
cède; son extrémité est dépourvue d'épines. I'remière el

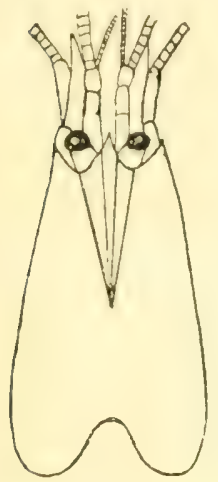

i
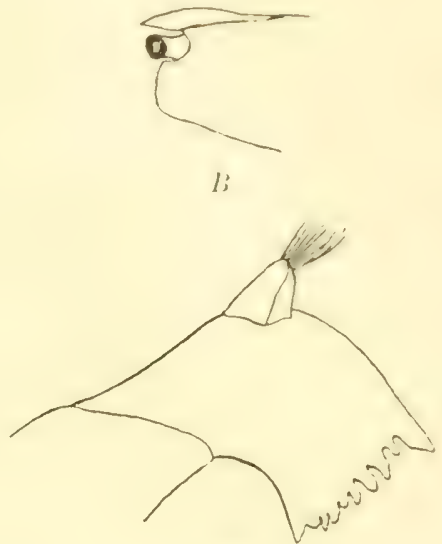

$1:$

Fig. 4. - Leptochela aculeocaudala Paulson. - A, carapace et antennes rues d'en haut; $B$, rostre vu de cite; $C$, mandibule.

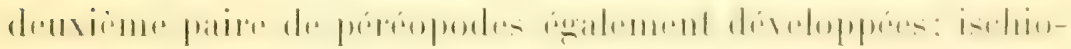
podite plus long que le mérus el le carpe; son bord interne armé de quatre ápines; sur le mérus el le carpe une série

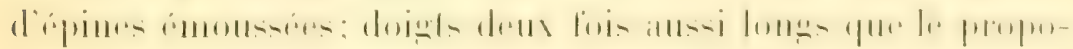
dite, avec le bord interne denté en seie. Troisieme paire de patles plus laibles que celles qui précedent ot plus courtes;

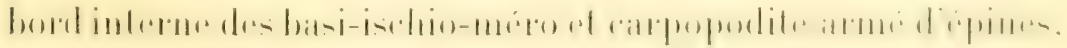

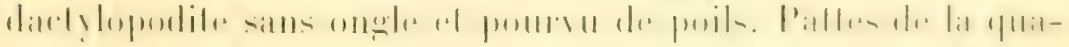
trieme el de la cincuieme paire igales, plus courtes mais plus grosses que celles de la froisieme paire; cinquieme pare plus courte que la quatrieme el dépourvere d'exopodte; ischiopo-

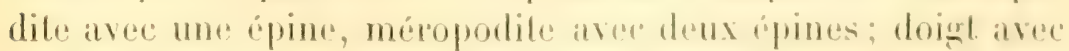

(1) Dans l'exemplaire de Djibouti il y a un groupe de quatre epones d'urale longueur. 
un ongle rudimentaire. Ablomen non replicen bas, comme dans

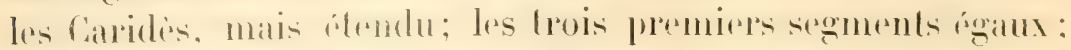

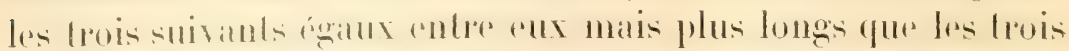

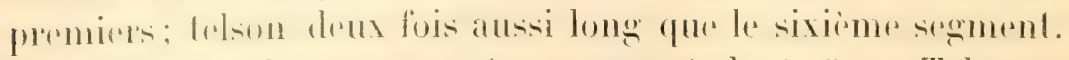

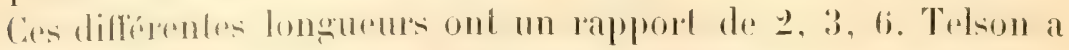

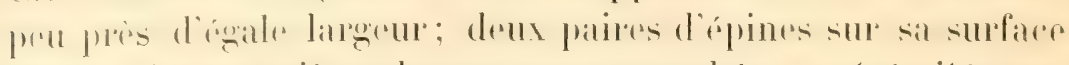

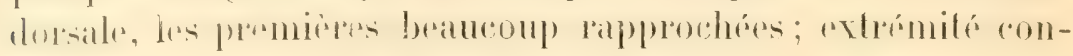
vexe, avec cinq paires d'épines, dont celles externes ont les bords lisses, et les internes sont dentées en scie. Uropodes "xternes plus courts que les internes; semberment la partie infé-

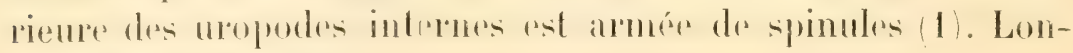
gueur, 11 millimètres. "

\section{FAMILLE ATYID E}

\section{Genre CARidina Edw.}

\section{Caridina nilotica Roux.}

Pelias niloticus Roux, Ann. Se. nat., XXVIII, 1833, p. 73, pl. VII, fig. 1.

Caridina longirostris Milne-Edwards, H. N. Cr., II, p. 363, 1887. - Heller, Sitzb. Akad. Wien, XL, p. 432.

Nec C. longirostris Lucas, Expl. scient. algér., 1849, p. 40, pl. 1V, fig. 1 (=Atyaëphyra Desmaresti).

C. nilotica De Ilan, I. Weber's Zool. Ergebn., II, p. 395, pl. XXIV, fig. 29l, m. mm. - Ortmann, Proc. Acad. Philad., 1894, 1). 403, 403 - Bouvier, Bull. scient. Fr. Belg., XXXIX, 1905, p. 72 et 78.

Nec C. nilotica Hilgendorf, M. B. Acad. Berlin, 1878, et Pfeffer, Mitth. Mus. Hamburg, VI, 1889 (三C. Wyckii Hicks.).

(gualpe individus recueillis par II. Jousseatume dans untr localité non précisée des côtes de la mer Rouge.

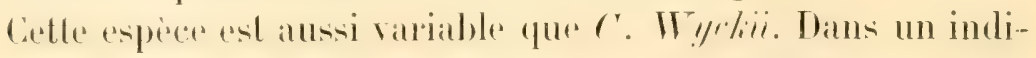
vidu long de 26 millimetres, le rostre offre $\frac{21+1}{17}$ dents; les trois premieres du bord superiem sont placres sur la carapace. La portion sans dents, antre la vingt-unieme dent af la dent aproale, est longue de presque les e/3 de la portion dentén libre; 1. rostro dépasse le scaphocérite d'un peu plus de 1/3 de sil longurur. Dans un autre exemplaire, il ! a $\frac{17+2}{11}$ dents ; deux

(1) Les rameaux externes sont denticulés ou spinuleux sur tout le bord externe. 
seulement sur la carapace: lo rostre dépasse de peu le scaphocérite el la portion sans dents est longue un peu moins que la moilic de la portion dentíc. Dans un autre exemplaire.

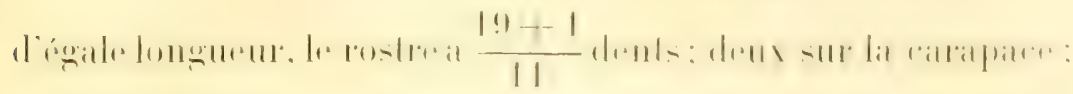
la portion sans dents est aussi longue que dans l'autre exemplaire, el la pointe dépasse de peu le scaphocérite.

Le carpe de la première parre est 1 fois 1/2 aussi long que sil largeur; le carpe de la denieme paire mesure it son extromilé 1/r de sa longueur.

Dans ces trois exemplaires (le quatrieme manque de carapace), nous avons les deux formes principales qur peut prendre le rostre de C. Wychili, soil : 1) lres long. dépassant beaucoup le seaphocérite et a portion sans denl assez longue, el 2) relativement court et it portion inerme raceourcie.

Cette espece habite le nord de l'Afrique et la valläs du Vil. De Man en a décrit en 1902 une variété de Cúlébes. La C. niln-

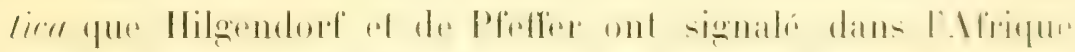
Orientale est, d'après Hilgendorf mème, C. Wyckili.

\section{FIMULE ALPHEID E \\ Genre ATIIANaS Iamari.}

Athanas Djiboutensis Cout.

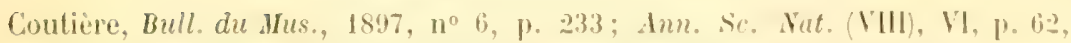
177; fig. 4, 207 (1899); Alph. maled. Laccad., 190', P. 8.66, figr. 129.

Ner Rouge (II. Jousseaume). Un exemplaire mulite.

\section{Gaxte SYNALPHEUS Bate.}

\section{Synalpheus triunguiculatus Paulson.}

Paulson, loc. cil., p. 103, pl. XIV, fier. 1-1!); (1/pheus); Nohili, Imn. Mnus. . Yapoli, I, n" 3, 1901, P. 2; Bull. scient. Fr. Bely., XI. 1906, 1. 2.'.

Massanouh Musere de Gines). Lim dizanne d'exemplaters. Massaonah (Musce de Naples). 


\section{Synalpheus biunguiculatus Stimpson.}

Mastantuh (Musie de Gènes). Une dizaine d'exemplaires. Massaouah (Musée de Naples). Un mâle.

\section{Synalpheus tricuspidatus Hell.}

Alphous tr. Heller, S. B. Akad. Wien, XLIT, p. 267, pl. III, fig. 1:.

Massaouah (Musée Naples). Quatre màles.

Genre AlpheUs Fab.

Alpheus ventrosus Edw.

Alphens ventrosus II. Milne-Edwards, H. n. Cr., t. II, p. 3\%2 (1837). - Coutière, Alph. maled. Laccad., p. 882. - Nobili, Boll. Hus. Torino, 1905, n 506, p. 2. Alpheus lxvis Randall et Auct.

Nombreux "xemplaires de la mer Rouge el de Massaouah (Musées de Turin, Gènes et Naples).

\section{Alpheus bucephalus Cout.}

Coutière, Alph. maled. Lıccad., 1903, p. 890, pl. LXVIII, fig. 29.

Un exrmplaire mutilé de Massaouah (Musée de Turin).

\section{Alpheus insignis Heller.}

Heller, S. B. Acad. Wien, XLIV, p. 269, pl. III, fig. 17, 18. - Nobili, Ann. Mus. Napoli, 1, 3, p. 2. - De llan, Abh. Senckenb. Ges., XXV, 1902, p. 864; pl. XXVI, fig. 60. - Coutière, Ann. Sc. nat., loc. cit., p. 89, fig. 55, et Alph. maled. Laccad., p. 899.

Érythrée (Musée Naples). Un màle.

\section{Alpheus pacificus Dana.}

Dana, U. S. Expl. Exp., p. s42, pl. 34, fig. J.-Coutière, Alph. maled. Laccad., p. 909, pl. LXXXV, LXXXVI, fig. 47.

Un mxemplaire mutilé, recueilli par VI. Jousseanme, est attribué avec quelques doutes à celte espèce. 


\section{Alpheus rapax liab.}

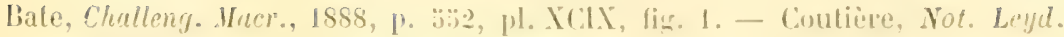
Ilus., XIX, 1897, p. 293, et Ann. Sc. nal., loc. cil., p. 233, fis. 28:, el Alph.

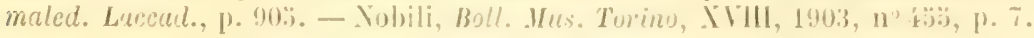

Jassaomah Musée de 'Turin). Uin miln.

\section{Alpheus Audouini Coul.}

(Alpheus Edvardsii Auct., pars.)

Coutiere, Alph. maled. Laccad., p. 911, pl. LXXXYII, lig. :3:.

Her Rouge Mus. de Turin). Une fencelle ot un male.

\section{Alpheus strenuus Dana.}

Dana, U. S. Expl. Exp., p. 343, pl. XXXIY, fig. t. - Cioutière, Fol. Leyd. IILs, XIX, p. 199, et Alph. maled. Laccad., p. 913, pl. LXXXYll, fig. :33.

Her Rouge (II. Jousseaume). Guelques exemplaires. Mndelkader, près de Massaonah (Musée de Trurin). Un màle (1).

\section{FIIIILLE H/PPOL,TT/J}

\section{Genre VIRBIUS Stimpson.}

Virbius orientalis Heller.

IIippolyte orientalis Ileller', S. B. Akad. Wien, XLII, 1861, 1). 277.

Virbius proteus P'atulson, lor. cit., p. 10!1, pl. XVIII, ligr. 1, et pl. X, lig. 2-";.

Djibouli (1) Couliere). Trente-sept exemplaires.

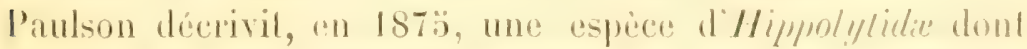

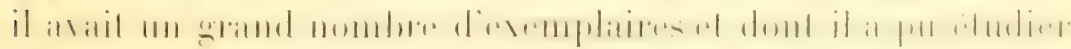
la variabilité. Les varialions présentés par colle esperes sonl

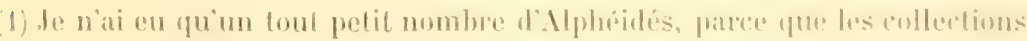

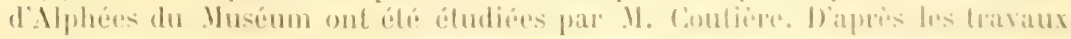

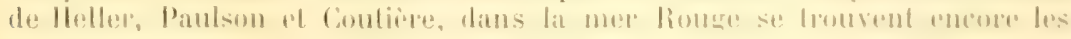
espreces suivantes :

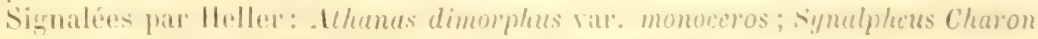

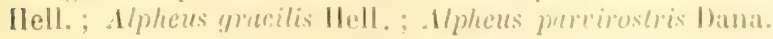

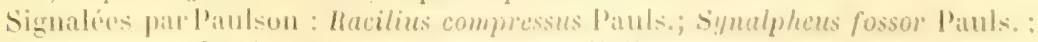
s. tumidumenus Pauls.; H/pheus cressimumes Ilell.

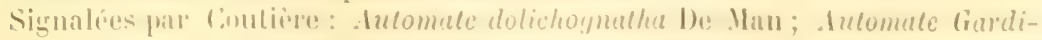

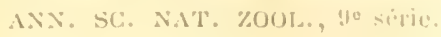


Lrès nombreuses; je crois done mieux donner la version entiere

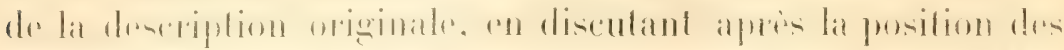

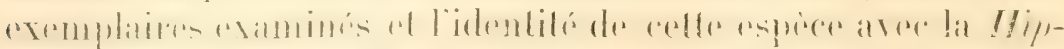
polyle orientalis de Heller.

Paulson groupe les formes de celle espece en deux catégories I et B, qu'il partage ensuite en grompes:

" 1. - Comprenant les formes à rostre bas, également haul dans toute sa longueur, avec pédoncules antennulaires attei-

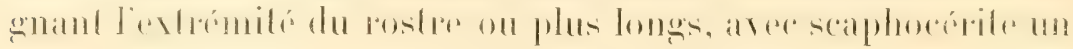
peu plus long que le pédoncule des antennules.

Premier groupe. - Le pédoncule des antennules alteint

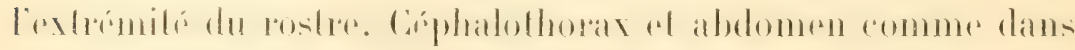
le genre Virbius, partie dorsale du cinquième el du sixième el

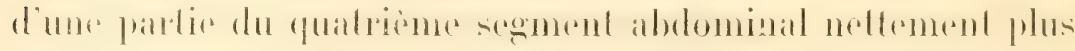
étroite que la partie basale, avee un sillon sur les côtés. Cara-

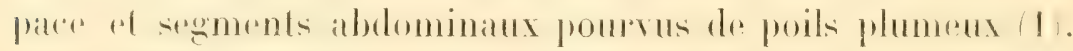

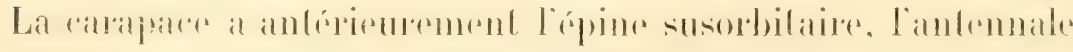
et la branchiostégale. Rostre bifide a la pointe, ses deux bords non dentés. Epine basale des antennes externes un

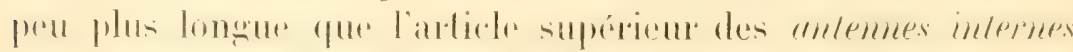

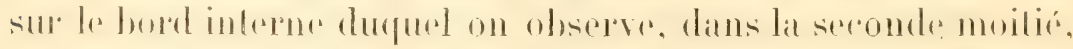
une autre petite épine; le gros fouet a huit articles. Maxilli-

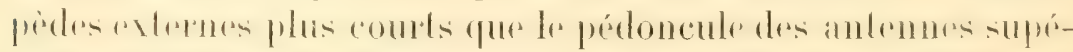

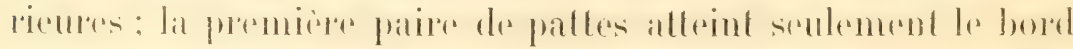

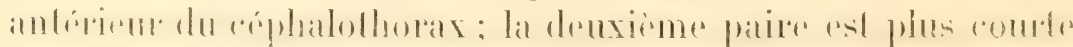

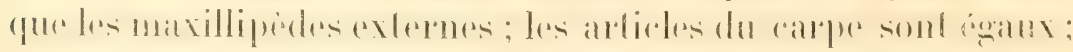
la main est plus longue que l'avant-dernier article. Les trois autres paires de paltes ont la mème longueur el dépassent

neri Cout.; Athanas dimorphus (Ortm.; Athanopsis platyrhynchus Cout.; Arcte indicus Cout.; Jousseumea serratidigita Coul.; Jouss. latirostris Cout.; J. cristatu Cout.; Amphibetxus Jousseaumei Coul.; Alpheopsis xqualis Cont.; Synalpheus neomeris De Ian; S. Gravieri Cout.; S. paraneomeris Cout.; S. biunguiculatus var. pachymeris Cout.; Alpheus laviusculus Dana; A. Bastardi Cout., el sa var. Hululensis Cout.; A. hippothoè De Man; A. Maindroni Cout.; A. euchirus Dana; denteropus Ililgd; 1. collumianus Stm.; A. paragracilis Cout.; A.microstylus Bate; A. aculeipes Cout.; A. spongiarum Cout.; A. gracilipes Stm.; A. paratrinitus Iliers; 1. alpheopsides Cout.; A. barbatus Cout.; 1. splendidus Cout.; A. pachychirus stm.

A ces espèces il faut ajouter le typique A. Edwardsi Aud., délimité par Couliim

(1) Tris rares. G. N. 


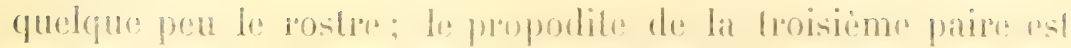

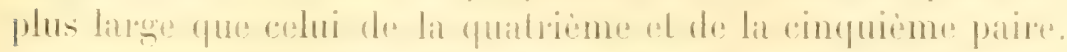

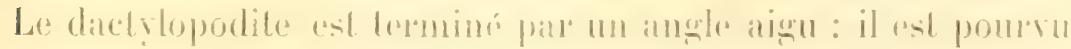

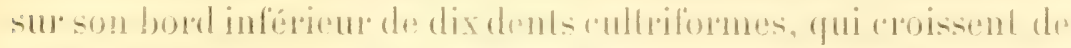

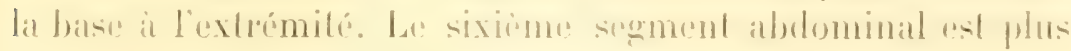

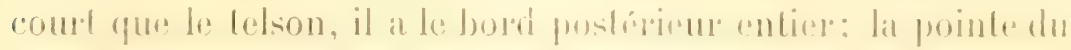

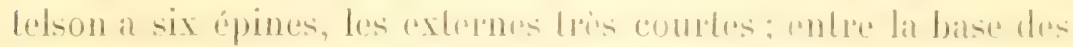
cpines il y a un poil.

Le rostre peot aroile:

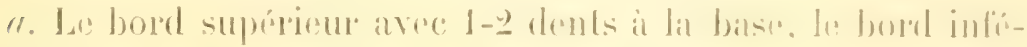

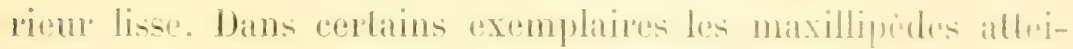
gnent lexténétí du pédonculo des antennules.

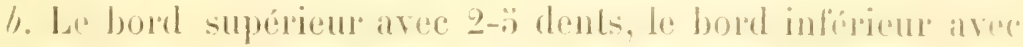

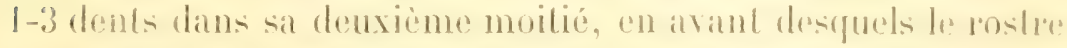

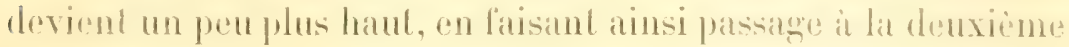
cialégonse.

r. Le bord supérieur arre ane dent press de la loase, linfir-

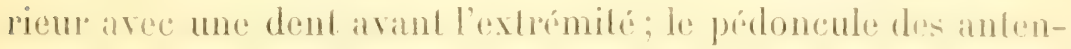

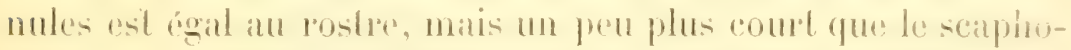
cerile. Ces formes conslibent ainsi nu pastage di la denxiome

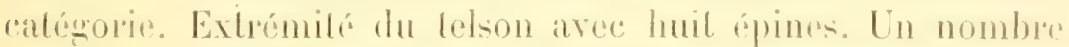
rigal d'épines sobserve dans de deuxieme groupe of diens lis

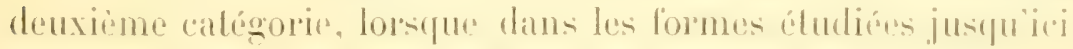
il y a Loujours six ṕpinm, sauld daus un individu.

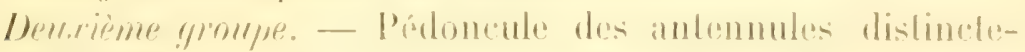

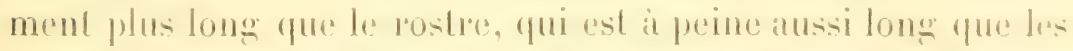
yeux out mome plus could. Le rostre peut aroile des formes

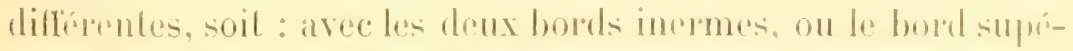

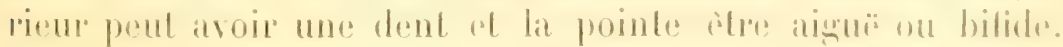

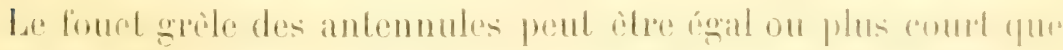

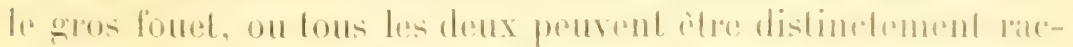

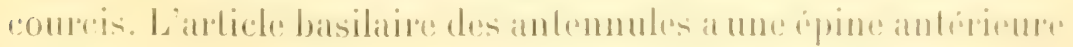

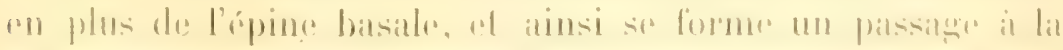

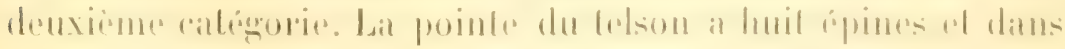

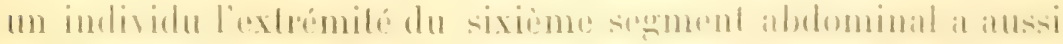
11110 (i)

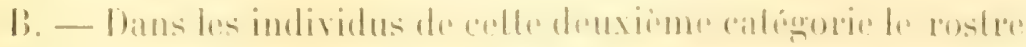

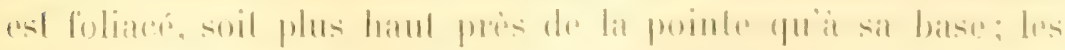




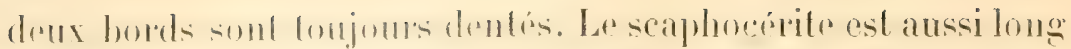

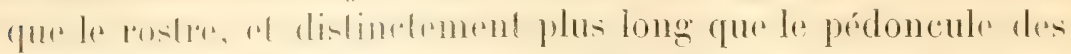

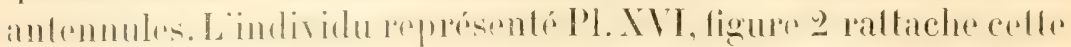

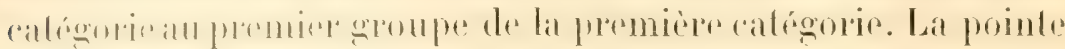

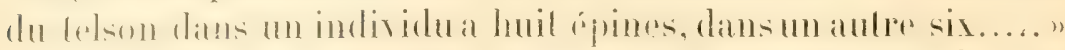

J'ai donné in extenso la traduction de la description de

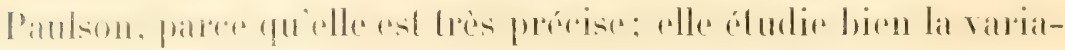
tion de cette forme, et c'est sur elle qu'il faut se fonder pour

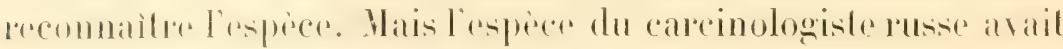
élé déjà vue en partie par Heller.

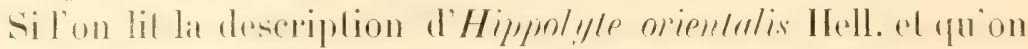

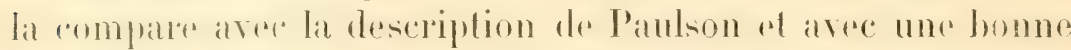

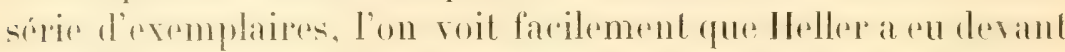
lui. un exemplaire appartenant à la catégorie B de Virbius

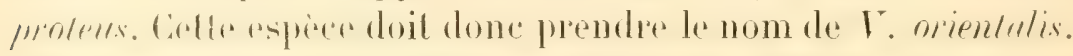

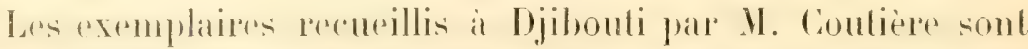
fres variables. Ancun individu ne correspond a la categorie I

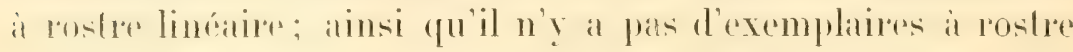

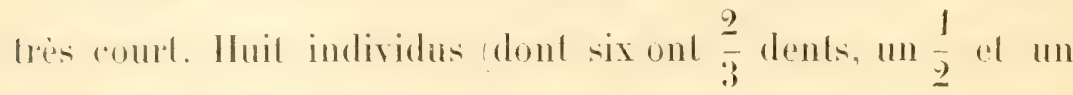

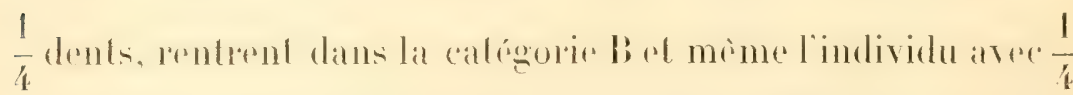
coprespondant is la ligure 3 de la planche XVI. Les aubers

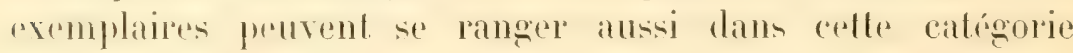
ou dans les formes de passiger entre colle ratégorie of la 1 , dans lespuelles le rostre porte un nomber variable de dents et son bord antérieur s'élargit un peu dans la parlie anté-

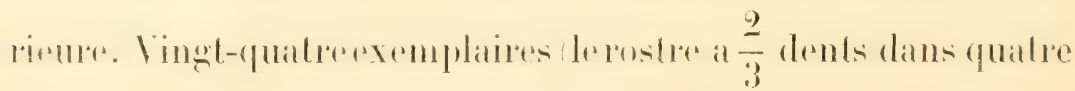
individus, $\frac{2}{1}$ dans quatre, $\frac{2}{2}$ dans dix, $4 \frac{3}{1}, 1 \frac{1}{2}$ dents dans les autres) appartiennent ì ces formes de passage.

Thes vardables alussi sont les longueders proportionmelles du

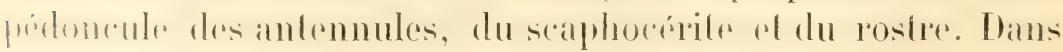

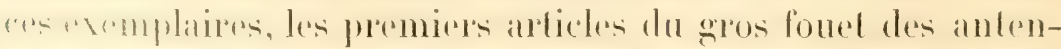

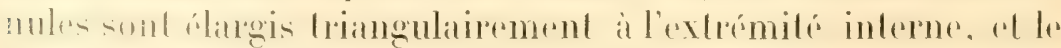
fouct apparait denté; et, en cela, ils s'aceordent avec la description de $H$. orientalis. 
bon nombre de ces exemplaires offrent parteci, par-li sur la carapace des poils plumenx latres of tres curieus, qui ne sonl pas du lout particuliers it lat caldigorie 1, mais quis'observenl aussi dans la catégorie lj, meme dans une grosise fomelle de 20 millimetres de longueur, qui correspond exactement it bis ligue 3 de la P'lanche 11 I de l'antion.

Un exemplaire de la ner houge (1. Jousicatume) appartient aussi it la calégorie B, el son rostre a $\frac{2}{\underline{y}}$ dents.

Virbius (?) jactans Nob.

PI. II, lig. 2.)

Nobili, Bull. du .Ius., 190', no \%, p. 239 (Diagn. prélim.).

Celle espece nouvelle n'est représentée que par uno peliter femello ovigere recueillie a Djibouti, par 11 . Coutiere. Ceel exemplaire n’est pas en bon chat, fo jo ne peux end donner que

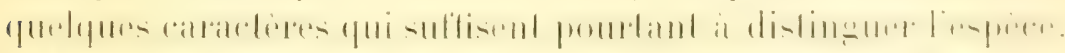

Le rostre est surélevé, oblique en haul, conlinué en une carene courte sur le dos de la carapace; pourvu de ä dents sur le bord supérieur, dont 3 placées sur la carapace, a sans dents sur le bord inféricur. Le rostre est tres court. Les

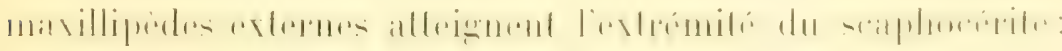
ils ont quelques épones à l'extrémité.

les palles de la deuxieme pare n’alleignent pas l'oxhrimilé du scéphocérite; les trois arlicles du carpe sont equax. Les palles III, IV, I ont une ṕpine ì l'extrémité du móropodite. Le propodite a une forme canteléristique : il s'élargil un pen vers son extrémité ou il est tronqué obliquement. Le dictylopodite est simple, coutbe et peut s'atapter contre

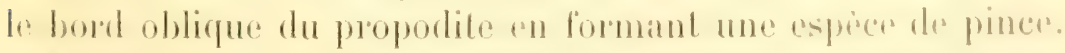

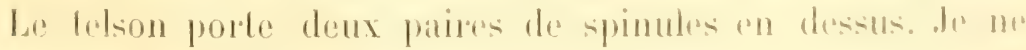
prous rion dire de sa poinle.

\section{Genre Paschocaris Nob.}

Nobili, Bull. du Mus., 190\%, no 6, 11.3!1.

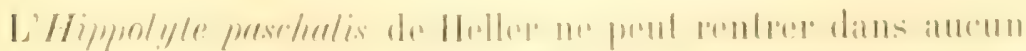

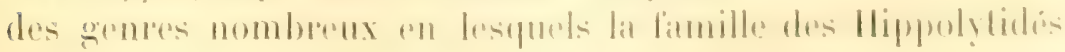


a été divisée. de crois done qu'il faut placer provisoirement cette forme dans un nouveau genre qu'on peut caractériser ainsi :

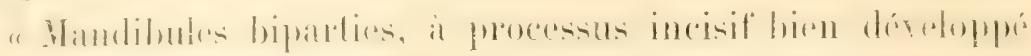

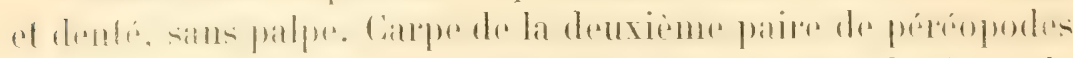
divisé en six articles. Branchies disposées selon la formule suivante :

\begin{tabular}{|c|c|c|c|c|}
\hline . & Podobranchies. & $\begin{array}{l}\text { Arthroliranchies } \\
\text { antérieures. }\end{array}$ & $\begin{array}{l}\text { Arthrobranchies } \\
\text { postérieures. }\end{array}$ & I'leuroliranchies. \\
\hline \multirow[t]{2}{*}{ 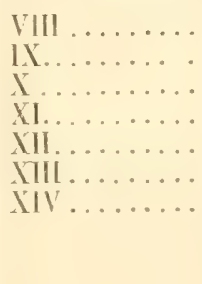 } & $\begin{array}{l}1 \\
1 \\
0 \\
0 \\
0 \\
0 \\
0\end{array}$ & $\begin{array}{l}0 \\
0 \\
0 \\
0 \\
0 \\
0 \\
0\end{array}$ & $\begin{array}{l}0 \\
0 \\
0 \\
0 \\
0 \\
0 \\
0\end{array}$ & $\begin{array}{l}0 \\
0 \\
1 \\
1 \\
1 \\
1 \\
1\end{array}$ \\
\hline & 2 & 0 & 0 & $\because$ \\
\hline
\end{tabular}

Pas d'épipodites ni d'exopodites aux pattes thoraciques.

Paschocaris paschalis Heller.

(Pl. 1II, fig. 1.)

Hippolyte paschalis Heller, S. B. Akad. Wien, vol. XLIN, 1. 276, pl. III, tig. 2f. - De Man, Arch.f. Nat., 1887, p. 3̈34.

Sept individus recueillis par H. Coulière à Djibouli.

Le rostre est typique dans six exemplaires, c'est-it-dire pourvu de quatre dents, dont deux placées sur la carapace, et sa pointe est distinctement bilide. Il atteint l'extrémité des cornées. La carapace a seulement l'épine antennale.

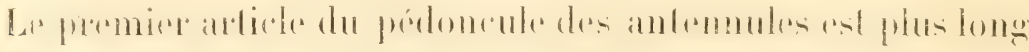
que les deux suivants pris ensemble; le slylocérite alteint l'extrémité du deuxiène arlicle, son bord externe est droit, l'interne convere, l'extrémité pointue el longue. Le deuxième article a une petite spinule a l'extrémilé de son bord exlerne. Le troisieme article n'a pas d'épines, mais il a it son extrémité. en dessus, une lamelle triangulate mobile. Le gros fonet est formé d'environ dix arlicless. Le scaphocérite, armé d'une 


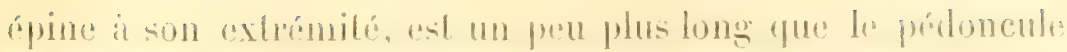

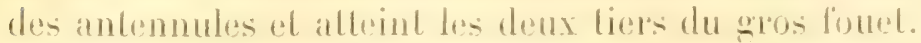

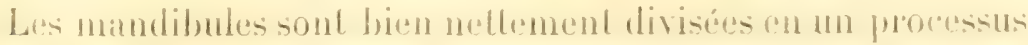

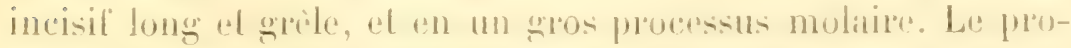

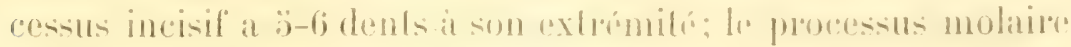
est formé de deux patres, un huberoule molaire comprat arves

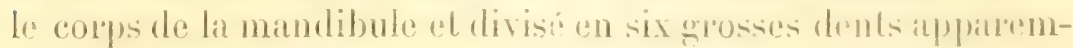

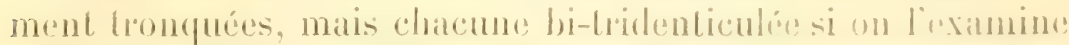

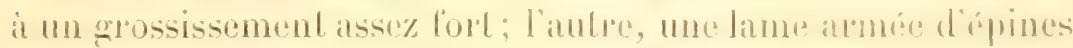

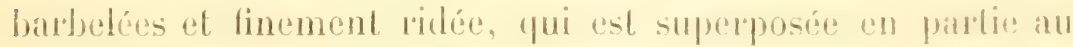
tubereule molaire. Le palpe mitnegue.

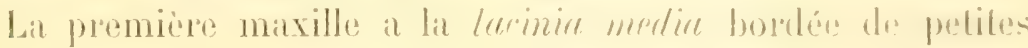

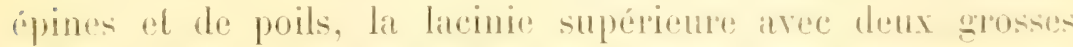

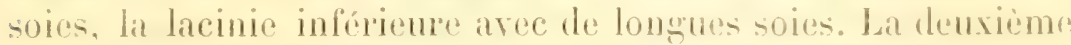
maxille a le lobe interne lribobé el pourro de poils. lo lobe externe gros el arondi aux deux cxlormites, la pelilu piece

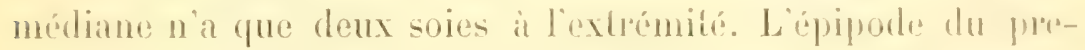
mier maxilliperde est bilobé; l'exopode ast flagallilorme cet

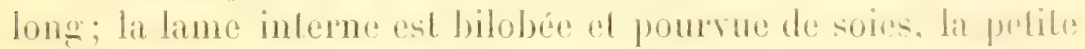

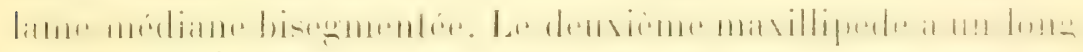

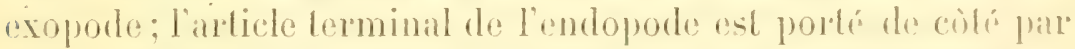
le pronultiene arlicle. Les froisiènes maxillipedes sont lones,

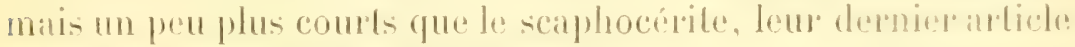
porte des poliles spinules bromes.

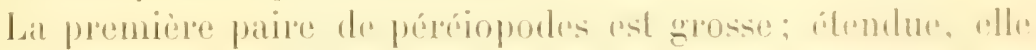

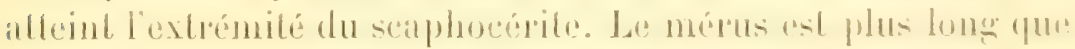

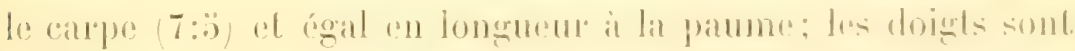

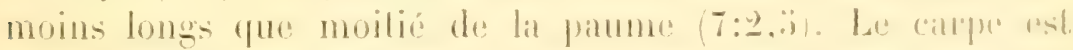

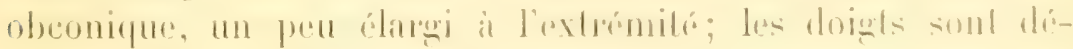

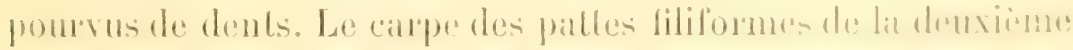

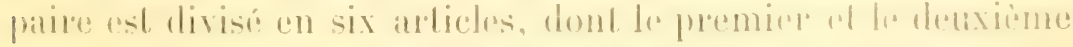

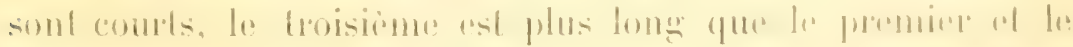

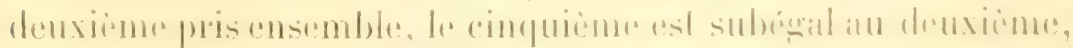

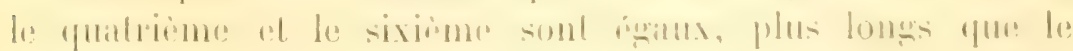

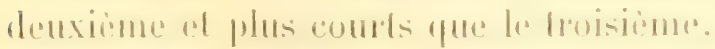

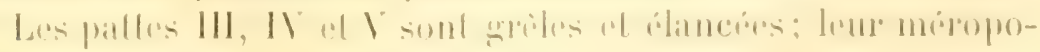

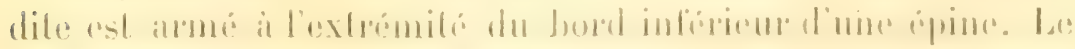

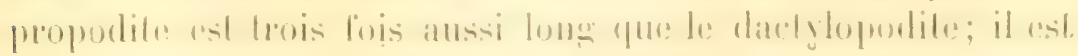




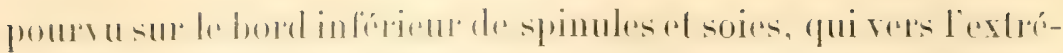

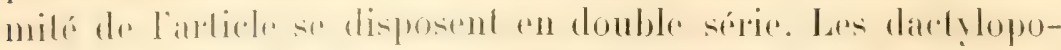
diles sont fermines par deux onguicules bruns de fongueur

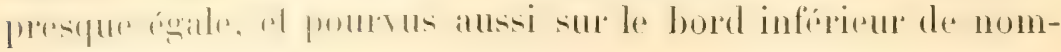
breuses spinules.

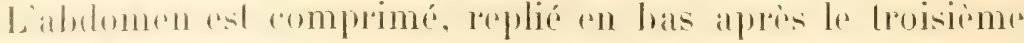
smmite. Les plutrons des segments I a l sont angulaires, les autres arrondis. Le telson porte sur son dos trois paires de spinules: son apex as tres courl. Alanque par sis épines, les deux externes très courtes, les deux médianes un peu plus courtes que les deux intermédiées qui sont assez robustes.

six exemplaters appordiemment a la forme lypique a rostre

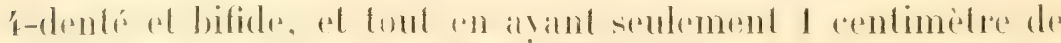
longurur ont déjit des arufs. Mars il y a ausio un septieme exentplaire. lons de 19 millinderes, qui correspond a la forme

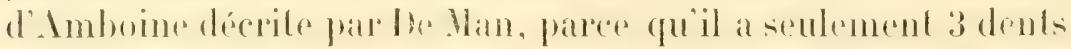
et la pointe du rostes simple. La pédoncule des antemnules est conformé romme datns les autres exemplateres: mais lo gros fout a dix-sept articles au lieu de dix.

\section{Genre SARON Thallw,}

\section{Saron gibberosus Edw.}

Hippolyte gibberosus H. M.-Edwards, H. N. Cr., II, 183i, ヤ. 3is; Atl.Cuv., pl. LIII, fig. 4.

Ilippolyle gibberosa De Man, Irch. f. Nat., 1887, p. 533, et Zool. Jahrb. Syst., IX, 1897, p. 761, pl. XXXVI, fig. 68 (pars).

Hippolyte marmoratu Ortmann, Zool. Juhrb. Syst., V, 1890, p. 497.

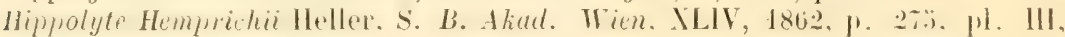
fig. 23. - De Man, Not. Leyd. Mus., III, p. 10\%.

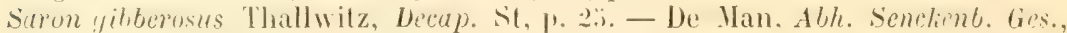
XXV, 1902, p. 852, pl. XXV1, lig. 57. - Nobili, Bull. scient. Fr. Belg., XL, 1906, p. 35.

Saron marmoratus Borradaile, P. Z. S., 1898, p. 1009 (pars). - Nobili, Ann. Mus. Zool. Napoli, p. 3.

Mer Rouge (I. Jousseamue). Une femelle.

lles Musha (H. Gravier). Dragage Iö mètres, sable. Cinq mâtes, quatre femelles.

Djibouli (M. Gravier). Un male. "Vivant sur les polypiers.

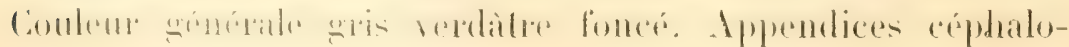


thoraciques it bandes bhenes el bhanches allernees. Tarehes oculiformes an tulson."

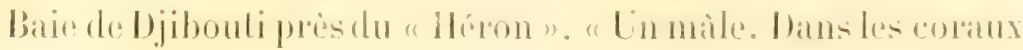
virants. Tatehes grises avee des ponchations blenes on blanches. Appendices du cóphabotholax it bandes lranswersales

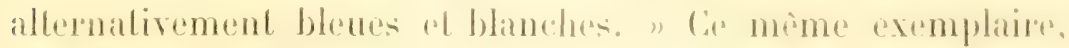
dont 11. Gravier a si soigneusenent nolé er rion la coloration, a en alcool des bandes rougeatres, ce qui proure que collo coloralion, observée par moi aussi dans des exemplateres du golle Persique, est due a l'action de l'alcool.

Erythréc Nus. Naples). Une femelle.

\section{FIIIILE LATRELTIDE}

GenRe LA'TrEUT'ES S'TAI.

\section{Latreutes pygmæus Nob.}

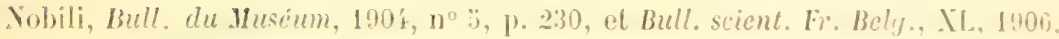
1. 3i, , l. III, lis. itill.

Djibouti (I. Coutiere). Neul individus de longueur vatriant entre 13-17 millimetres; quelques-uns ovigeres.

\section{Latreutes Gravieri Nol.}

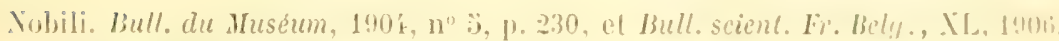
1. 3!), pl. III, lig. :j-:ii a.

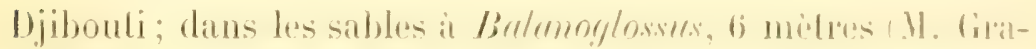
vicr). Un jenne individu decril al ligure par noi, lor. ril.

\section{Latreutes mucronatus var. multidens nov.}

(Pl. 11, lier. 3.)

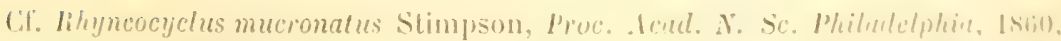

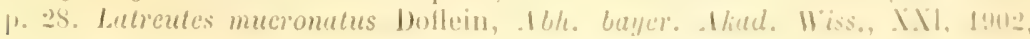
1). 6:38. pl. Y, lig. 6.

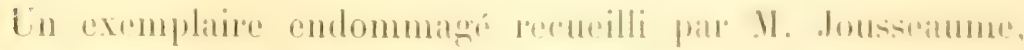

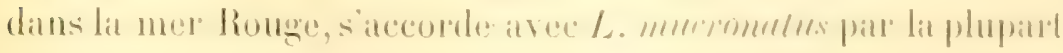

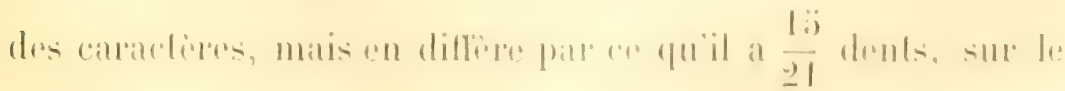




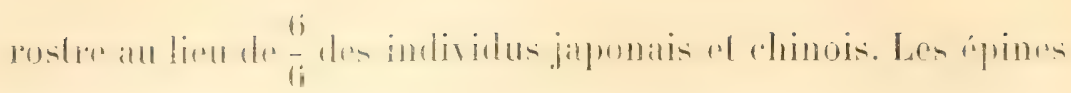
près et en dessus de la base des antennes sont bien distinctes. Entre lia base de l'oxil et l'insertion de l'antenne, il y a une épine aiguï.

Dans les collections du Musée zoologique de Thurin, il y a quatre individus recueillis a Java pendant le voyage de la "Wagenta " qui concordent avec cette variété, mais montrent

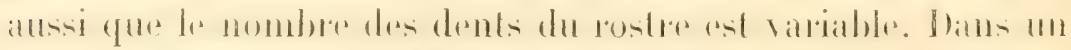

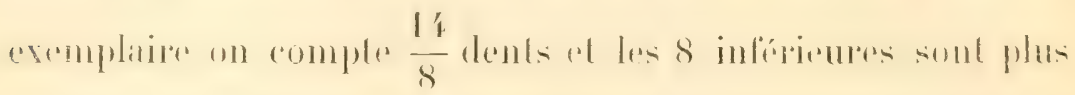

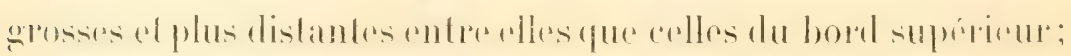
dans les trois autres exemplaires on a : $\frac{12}{10}, \frac{12}{8}, \frac{12}{9}$. D'apres ces

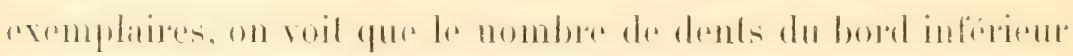

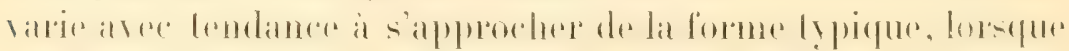
ceux du bord supérieur restent toujours en nombre au moins double de celui de la forme typique.

Dans les exemplaires de Java, le rudiment de la $2^{\circ}$ dent dorsale sur la carapace est bien distinct, plus que dans celui de la ner Rouge.

La forme typique n'est connue que de Hong-liong el de la baie de Sagami.

GENRE ANGASIA BATE.

(Tozeuma Stimpson.)

A. Rostre très long. Pas de dent dorsale à la base, pas d'épines susorbitaires. Bord inférieur avee 27 dents....... A. armata Pauls.

A A. Rostre un peu plus long que le scaphocérite. Une dent dorsale, des épines susorbitaires. Bord inférieur arec

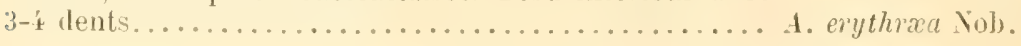

\section{Angasia armata Paulson.}

Tozeunate armatum Paulson, loc. cit., p. 99, ph. XV, fig. 2-20.

Angusia stimpsoni llenderson, Trans. Linn. Soc. (2), I, 1893, p. 43\%. pl. XL, lix. $18-20$.

Mer Rouge (11. Jousseaume). Une femelle ovigère.

Lianimal est long de 73 millimetres, dont 26 sont oceupés par le rostre. Le rostre s'étend pour un long trail sur le mème 
plan que la cartpace el les frois premiers segments abdominaux, puis il se dirige quelque peu en haul. I la base, va

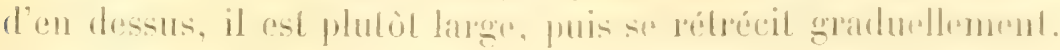

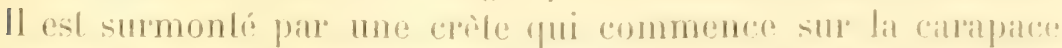
In peu avant le commencemont du rostre el qui sie prolonger

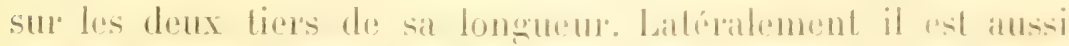

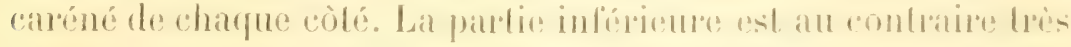

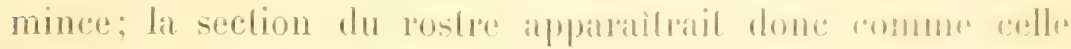

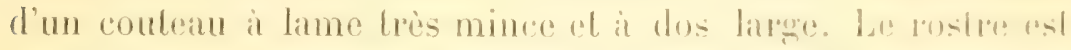
lisse en dessus ot pourvu en desious de 27 dents. P'pis des max

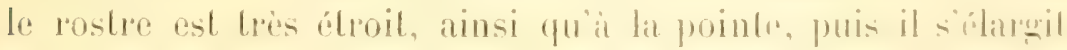
brusquement et va ensuite se rélrécissant graduellement jusqu'it la pointe. Les veux sont lies couts.

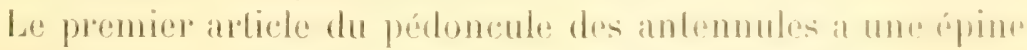
basile; il est presque aussi long que les deux andms pros ensemble. Lépine basale dépasse l'extrémile du premione

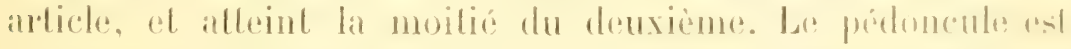
distinctement plus court que la moilie du scaphoserite al meme le gros fouct alteint it peine la moilie du seaphocépitr.

Le premier article du pédoncule des antennes ist amé inforienement d'une épine. Le scaphocérite est longe alpoil. linéare, lancéolé, et profondément sillonné en desis.

Lat carapace est un peu plus atroite antorientement yere

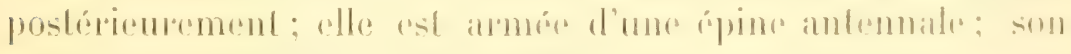
angte antero-intéricur "sint sintorme.

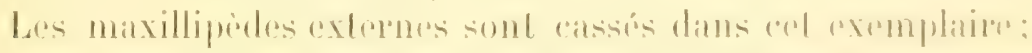

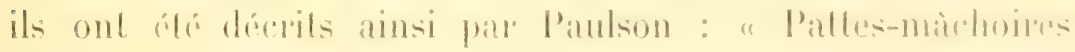

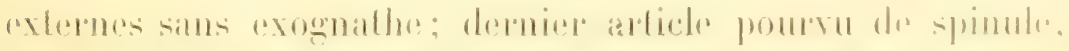

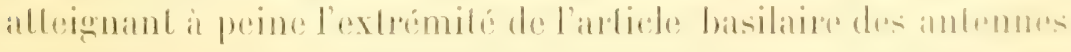

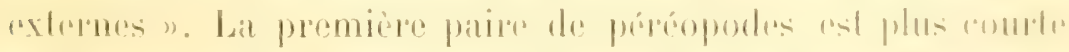

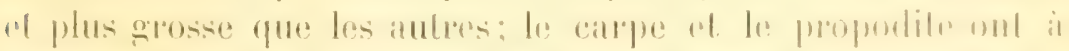

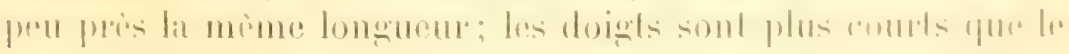

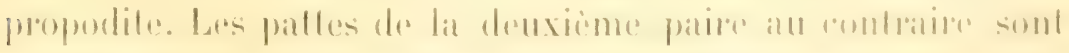

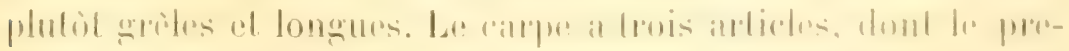

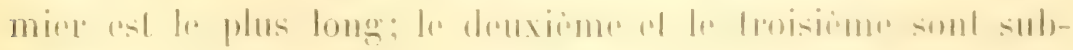
inillils.

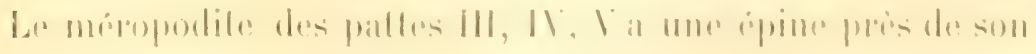

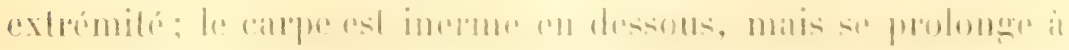




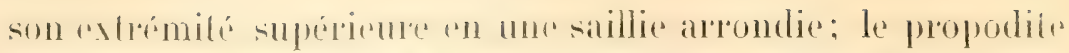

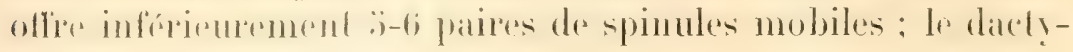
lopodite a $3-4$ spinules.

Lablumen at bes comprimé latéralement: les plenons sonl atromblis ol amples. Les segments $3-3$ sonl oblusement

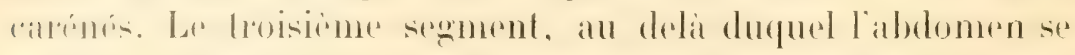
reploie en bas, a à son extrémité une saillie crochue; le qua-

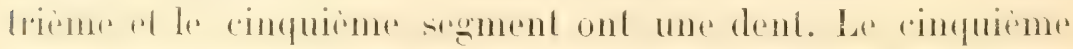

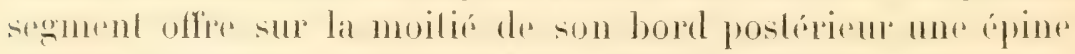
dirigan obliquement an bats. Le sixieme segment at long al

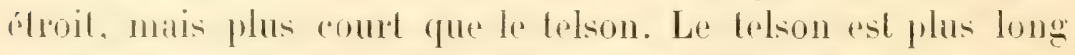
que les uropodes, tris élroil, linéare, bifurqué à l'extrémité

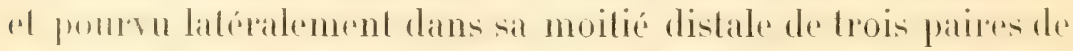
sprimules. Lexopode des mopodes a deux spimules prosis de la sulure avec la pièce terminale.

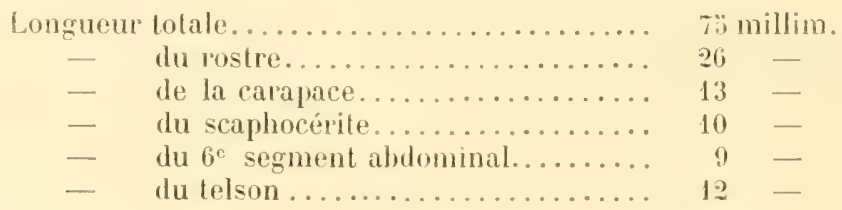

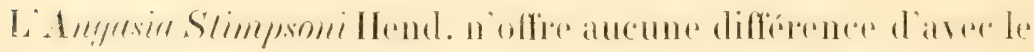
$T$. armatum. Le rostre est bien un peuplus recourbé en haut,

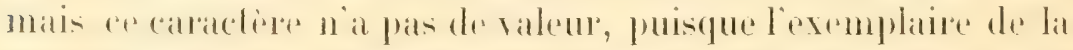

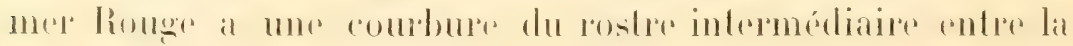
ligure de Paulson et celle de Henderson.

\section{Angasia erythræa Nob.}

Tozeuma erythr. Nobili, Bull. 1hus., 1904, no 3, p. 231 (Diugn. prélim.).

Mer Rouge (Musée de Turin). Quatre individus.

Cetle espèce se distingue tres lacilement de $A$. armata l'anls. Le rostre a la mème forme droile et à couteau dans les deux espèces, mais pendant que dans a'mula il est très long, dans erylhrae il dépasse de peu le scaphocérite. Iu d'en dessus il est plutôt large à sa base, et fortement caréné dans

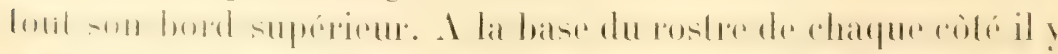

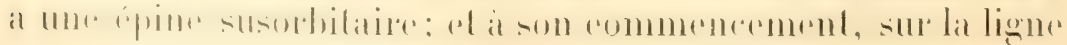


médiane-il y a une épine. Le bord inférien du rostre as lrós mince ret lamellaire, el n'a yue 3-4 dents dont lune sous lat pointe. Dans A. "rmale le rostre n'a pas de dent dorsile at ia base: les épines susorbilates manquent, al les dents du bord inférieur sont tres nombreuses. Lescaphorárite at un pelu plus long que la carapace, et arme d'une ipine robuste. late pédon-

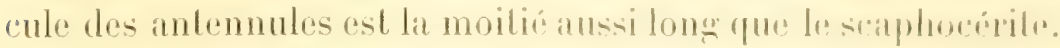
Son premier andicle est phus long que las deux suivants pris ensemble; le stylocérite styliforme dípasse quelpue peu lindré-

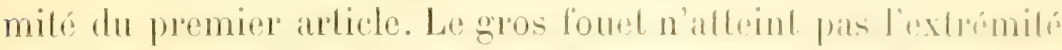
du scaphocérite; les yeux altagnont la moitió du promion

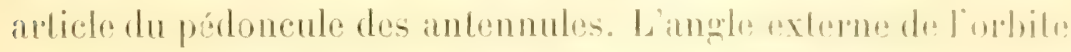
ast dentiforme el presque aussi long que l'apino antomnaire. Langle plérygostomien est aigu; en arriere el plus haul que cet angle est placée une épine qui n’alleint pas le bork antio rieur de la carapace.

Les plemons des segments abdominaux $1-33$ sont aromdis. cenx du quatrieme segment angulaire, ceux du cinquirmo ont une pelite dent pres de l'extrémilé. Le troisieme secrment lait une saillie postérieurement, pas si grosse que colle de armera, les segments a el ä nonl pas la saillie dentiforme de

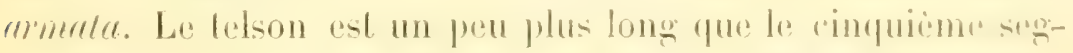
ment, subrgal aux mopodes; sur son dos il y a deux pairus d'opines mobiles.

La troisieme paire de maxillipides alleint lextrémiti du

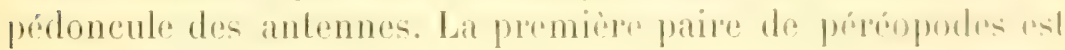
courte el grosse, el dépasse il peine la base du seaphorérite. Le mérus el le carpe y sont subegaux, ou birn le carpe as un

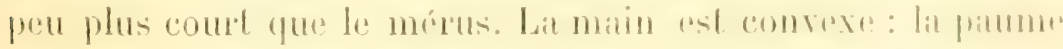
ast aussi longue que les doights.

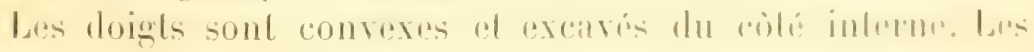

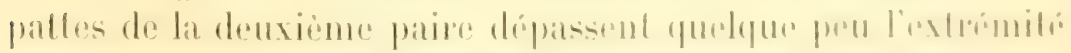

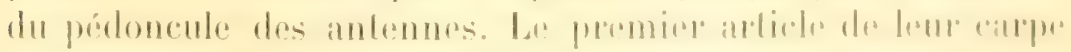

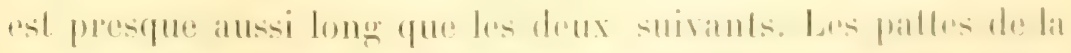

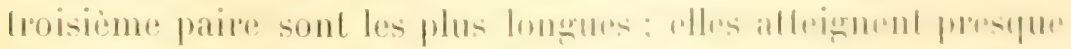

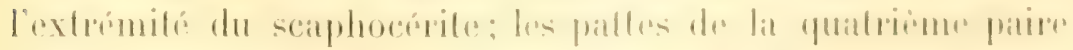

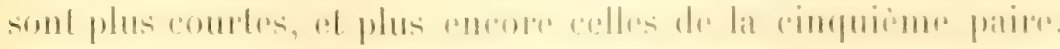

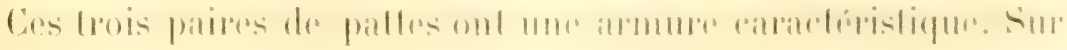




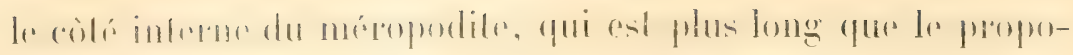
dile, on observe une série de cinq épines coniques, relativement prosies el implantées dans un alréole, ce qui les rend mobiles. Sur le carpe il y a aussi une épine du mème genre. Le bord inférieur du propodite a de nombreuses spinules

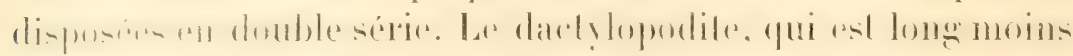
que moilié du propodite, est combé el pourvu en dessous de

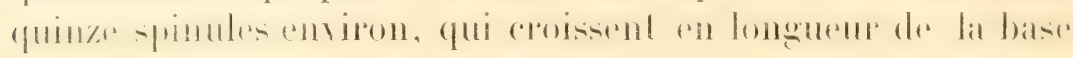
ì l'extrémilé.

Le plus gros individu est long de 32 millimètres.

Celte espece ressemble un peu ì l'Angasia paroninu Bate, mais ell differe nettement par ses épines susoculaires, par l'épine basale du rostre, par la présence d'une épine bran-

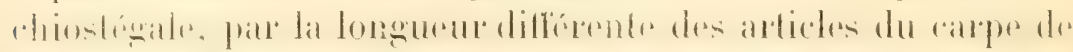

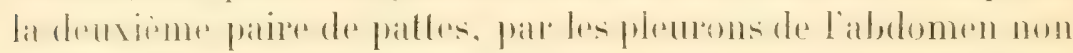
prolongés en arrière, ete. Cff. Bate, Proc. Zool. Soc., 1863, p. 498, '1. XI, tig. I).

\section{Genre HipPOLYSMAT'A Stinipson.}

\section{Hippolysmata vittata Stimpson.}

(Pl. II, lig. 4.)

Stimpson, Proceed. Acad. N. Sc. Philadelphia, 1860, p. 26.

? H. tiltutu var. De Man, Areh. f. Naturg., 1887, p. 494 (Amboinensis).

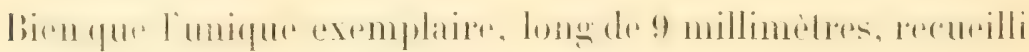
par II. Couliere a Djibouli, soil mulilé, je crois pouvon reconnaitre en lui la vaie $H$. villate Stm., qui serait en ce

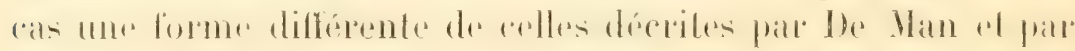
Thallwilz. Ce qui me porte it altribuer mon exemplaire à la

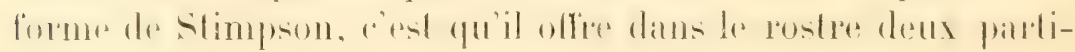
cularités, bien décrites par Stimpson.

Le rostre est grèle et porte en dessus six dents à peu près

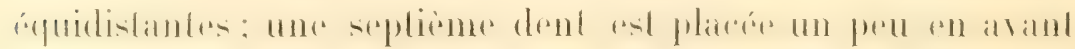
de la moilié de la carapace, el la distance entre celle denl

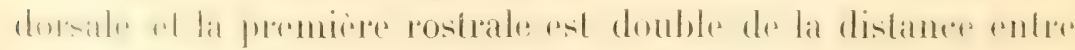

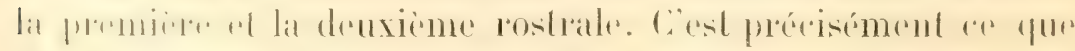
dit Stimpson : dente posteriore rel primo parce ante medium crminumcis sito el dente secundo intervallo duplo remolo. 


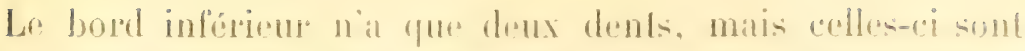

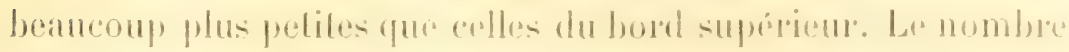

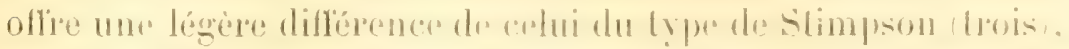

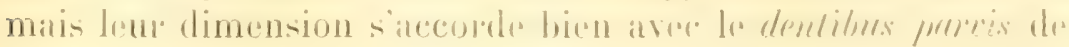
-

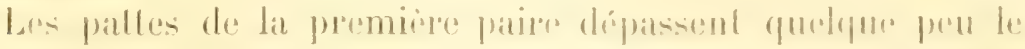

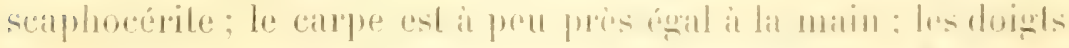

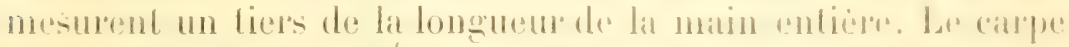

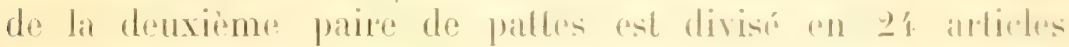
environ; le márus a des lraces smperlicielles de division. Lue atules palles manquent.

\section{Hippolysmata multiscissa Nol.}

(Pl. II, lig. : b.)

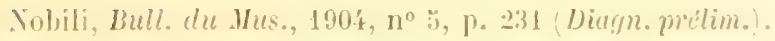

Djibouli (M. Couliere). Yeuf individus.

La disposilion des dents sar le rostee est variable, mais il? a toujours plus de dents que dans II. cillula. Les dents sonl

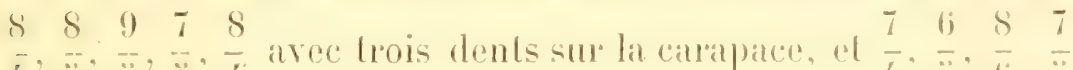

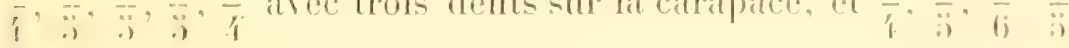
arec deux dents sur la carapace.

Ouand il y a

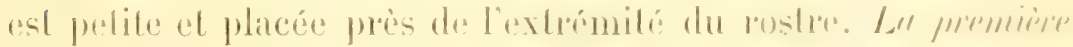

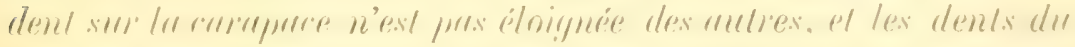

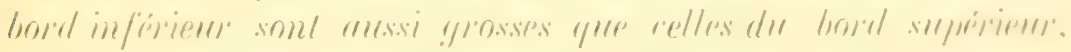

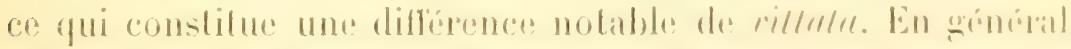

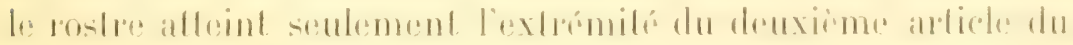
pódoncule des antemnules, mais il pend aussi an allumdere la

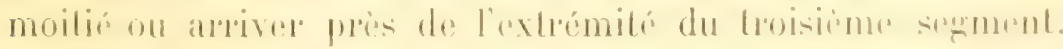

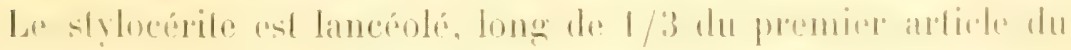

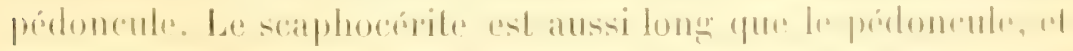

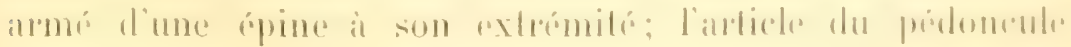

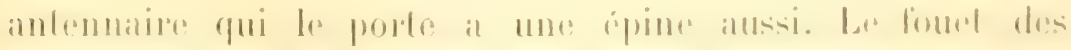

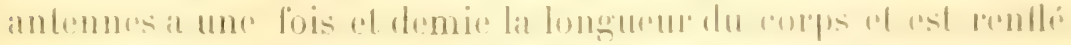

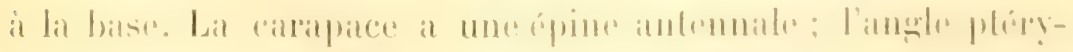
gastomian est spiniforme.

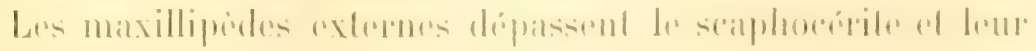


dernier article est spinuleux. La première paire de patles

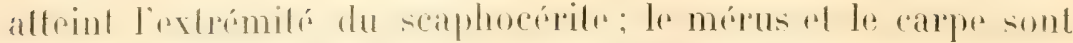
subégaux: la paume est longue plus d'une fois et demie le

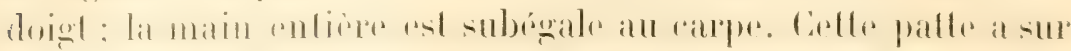

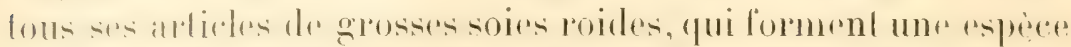

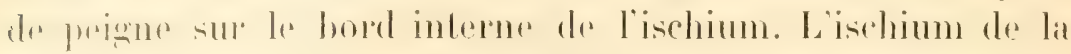
deuxième paire de pattes est divisé à son extrémité en 4 articles; le mérus est divisé sur toute sa longueur en

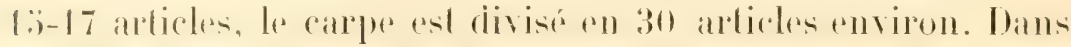
viltala le mérus seul a des traces de division, el le carpe est

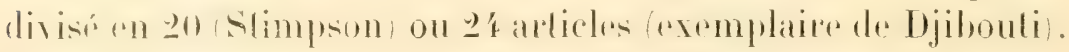
Dans la forme voisine de Amboine. De Man compla 17-19 alficles, et Thallwitz 17 dans sa var. sublilis de Cebu. Dans des

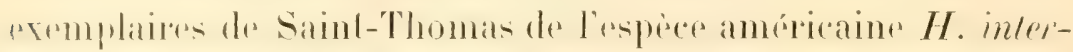
medter ligst. examinés par moi, le mépos es aussi pluriarliculé

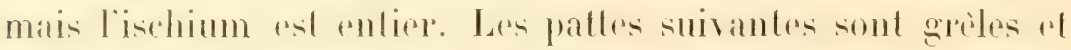
longurs: lo merus est atrmé inferieurement de lrois épines appliqueses conter la surfice, qui furent décertes allsis par

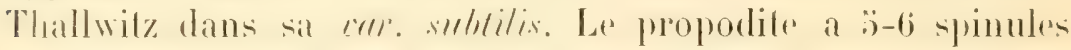

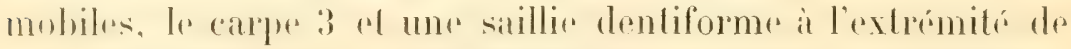
son bord superient la dactylopodite a double onguicule of 2-3 autres spinules.

Les branchies sont disposées ainsi :

\begin{tabular}{|c|c|c|c|c|c|}
\hline VII............ & $\begin{array}{l}\text { Podob. } \\
0 \text { ep. }\end{array}$ & $\begin{array}{l}\text { Art. ant. } \\
0\end{array}$ & $\begin{array}{c}\text { Art. post. } \\
0\end{array}$ & $\begin{array}{c}\text { Pleturob. } \\
0\end{array}$ & $=0 \mathrm{ep}$. \\
\hline V111........... & 0 ер. & 0 & 0 & 0 & $=1 \mathrm{ep}$. \\
\hline $1 \times \ldots \ldots \ldots \ldots$ & $r+\mathrm{ep}$. & 0 & 0 & 1 & $=1+r+e p$ \\
\hline$X \ldots \ldots \ldots \ldots$ & 0 ер. & 0 & 0 & 1 & $=1 \mathrm{ep}$. \\
\hline XI........... & 0 ер. & 0 & 0 & 1 & $=1 \mathrm{ep}$. \\
\hline XIl............. & $0 \mathrm{ep}$. & 0 & 0 & 1 & $=1 \mathrm{ep}$. \\
\hline XIII........... & 0 ер. & 0 & 0 & 1 & $=1 \mathrm{ep}$ \\
\hline \multirow[t]{2}{*}{ XIV.......... } & 0 ep. & 0 & 0 & 1 & $=1 \mathrm{ep}$. \\
\hline & $1+r-$ & $\overline{0}$ & $\overline{0}$ & 6 & $\equiv 7+r+$ ep \\
\hline
\end{tabular}

Le telson a deux paires de spinules en dessus.

Le plus gros exemplaire est long environ de 30 millinietres. 


\section{FAIILLE DISE PONTON/IISAE \\ Gavize PliRICIJIIENTES Costa. \\ (Anchistiu l)ana.}

\section{Periclimenes Petitthouarsii (Iurl.).}

Palemon Petithouarsii Autouin, Expl. pl. Savigny, p. 91. - Savigny, Descr. Elypte Coust., pl. X, fig. 3.

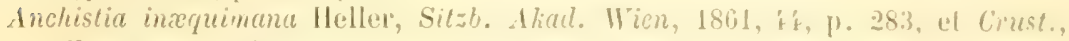
Novara "), p. 109.

Anchistia Petitthoursii Paulson, loc. cit., [r. 11'. - Knssmann, loc. cit.. II,

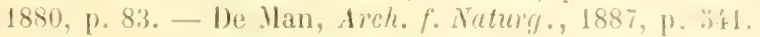

Periclimenes Petilthouarsi Bormalaile. Ann. Ha!. К. H. (7), vol. II, |x!s, p.381. - Nobili, Lmmario Mus. Zool. Napoli, 1, 1901, n. 3, 1). 6. - De Jan, Lble. Senckenb. Ges., XXV, 1902, p. 82't (rar. spinifern). - Yobili, Bull. scient. Fr. et Bely., 1906, p. 1 .

Massaouah (MM. Antinori, Issel, Beccari. Musín dro (rênes). 10 exemplanes; Djibouli (11. Couliere), 21 exemplanist:

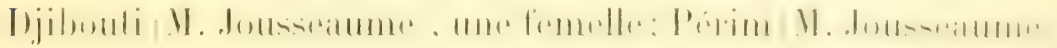
2 individus; Erylhrée (Nusée de Naples), a males; Ervlhrée Musée de Turin), ö mites.

Laxamen de ces nombreux exemplaires démontre que la

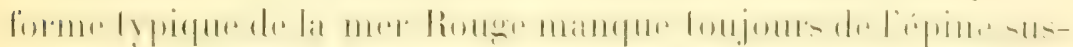
oculaire, qu'on observe au contraire chez los exemplares malais el polynésiens, que II. De Ilan a séparés avec le nom de illi. syilityptel.

\section{Periclimenes ensifrons (Dana).}

Anchistia ensifrons I)ana, U. S. Expl. Exp., I, 1852, p. 380. Lab. XXXIII, fig. 1. - De IIan, Wreh. f. Naturg., 1887, p. sł.i. - Müller, Vert. Yat. lies., Basel, VIII, 1887, p. 471. - Ortmann, Denkschr. med. Xat. Gies. Jenu, 1'll, $189 \%$, p. 16 .

Periclimenes ensifrons Borradaile, loc. cil., p. 382. - Nobili, Amm. Mus. Ciu. sl. Yut. Genoea, XL. 1899, 1).23\%. - De Man, Alh. Senckent. (ics., XXI, 19(1)2, ए). $8: 0 ;$.

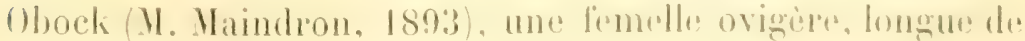

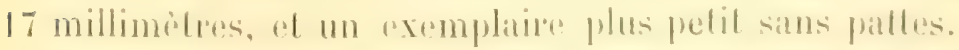

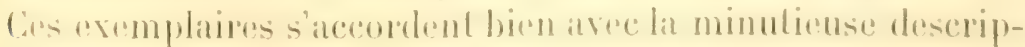

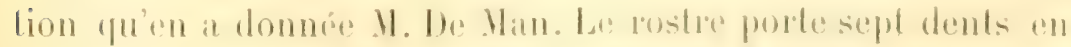

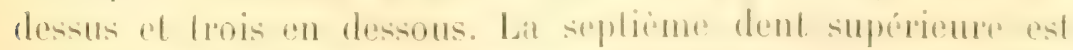

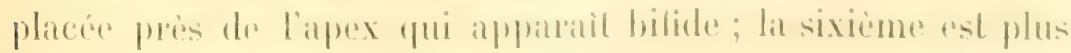
ANN. SC. NAT. ZoOL.., ge sibie. 


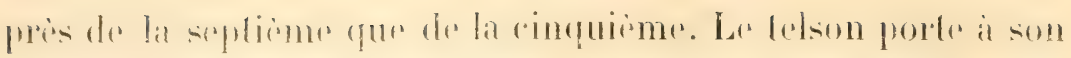

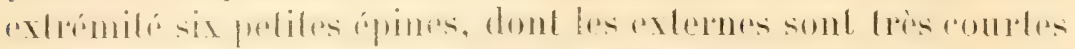

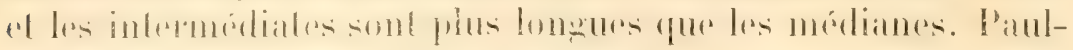

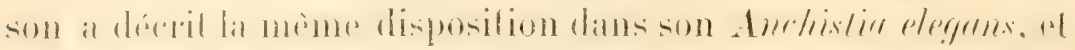
insiste mème sur ce caraclère, en écrivant : la "caractéristique pour celle espière est le bord postérieur du telson qui dénole une purenté acer les Palrmon ". Cette disposilion n'est pas du lout particulière à celte espece. de l'ai notéc chez toutes les Periclimenes décrites dans ce travail, et il est étrange que

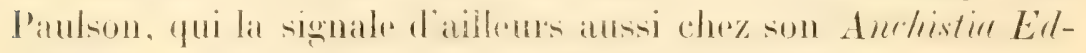
urudsii, ne l'ait pas vue chez la P. Petithourmsii!

H. De Han considè identique à cetle espèce la $P$. viliensis. Borr.

En 1899, je considérais ces deux espèces comme distincles,

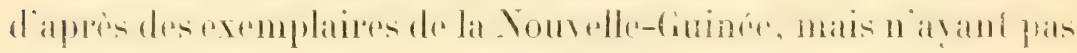

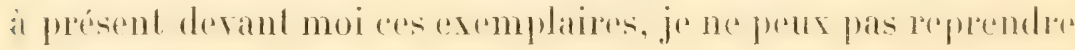
la question.

Cette espèce est nouvelle pour la faune de la mer houge.

НАв. : Dar es Salaam (Ortmann); Trincomali (Müller);

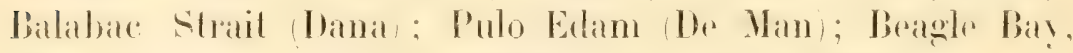
Nouvelle-Guinée anglaise (Nobili); Ternate (De Man).

\section{Periclimenes soror Nob.}

(Pl. Il, fig. 6.)

Nobili, Bull. du Mlus., 190ł, n5 5, p. 231 (Diagn. prélim.).

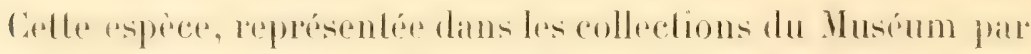

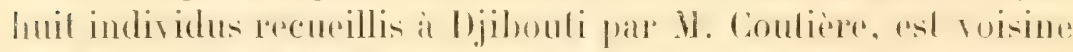

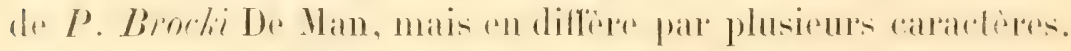

Le rostre est plus long que le pédoncule des antennes

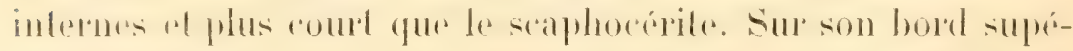
rieur convexe, il porte de 11 ì 13 dents, toutes rapprochées

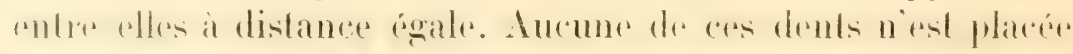

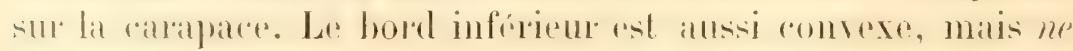
porte pas de dents.

La carapace porte une épine antemnale; direclement en

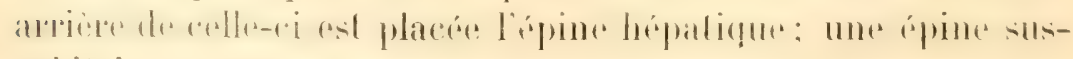
orbitaire manque. 


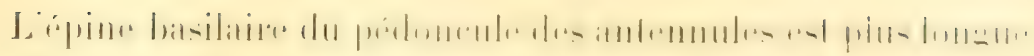
que la moitie du premier article: l'épine apdacte du premier arlicle est courte.

Le telson porte en desins deux paiders de spinules mobiles:

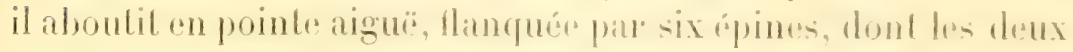

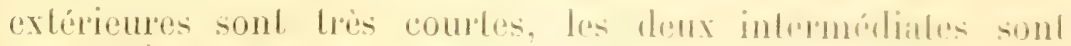
longues, el les deux médianes sont plus courtes fun celles-ci.

Les pattes de la premiere pare arrivent a peime il loxtrimilé du scaphocérite, el sont grrides. Le mépoditr at un peu plus long que le carpopodite. Le carpopodite schargil un pen ¿ l'extrémilé, ou il mesure en largeur le quat de sit longurme. La main est courte, Iongue à peine des deux fiers du carpe. Sus doigts, qui sont un peu plus longs qute lit portion palmaire.

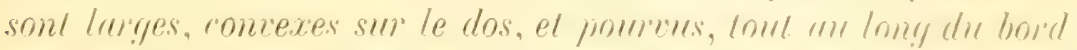
extérieur. de dentimules mennes el nombrenses, Loul commo chez P. Pelillenumisi.

Les patles de ladeuxieme paire sont courtes, grodes, inermes. el dépassent le seaphocérite de la moilié de la paume. Le mérus, qui est subégal ou ì peine plus long que lischium. est complètement inerme. Le carpe est tres courl, obconique.

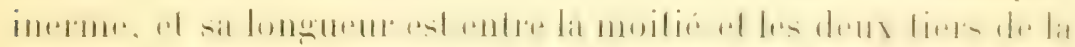

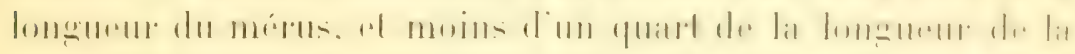
main entiere, et un peu plus courte que les doigh. La main ast linéaire, allongée; les doigh sont tries couls, longs it peine d'un quart de la tongueur de la main entiere, el un liers de. la longueur de lá portion palmaire.

Sur les palles suivantes le carpe est environ la moilio aussi long que le propodite. Les dachlopodites sont counts. courbés, plutòt larges à la base, et pourves pres de lindrémitio

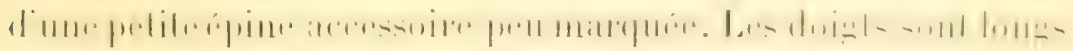
i peu pres un neurieme de: la longueur du propodile.

Cette especes se distingue facilement de $P$. Bmoliti par les caracteres suivants :

$$
\text { P. Brockii Me Man. }
$$

P. snror Vuliili.

1. Rostre plus long que le seaphocé- | 1. hostre plus courl que hr scaphocivile, pourvu de $\frac{9-10}{1}$ dents.

2. Epine antérieure du premier artich. 2. Epine, ete, tries courle. du pédoncule des antenuules longue. 


\section{P. Broctii De Man.}

\section{P. soror Nobili.}

3. Doigts de la première paire de pattes 3. Doigts finement dentés comme chez non dentés.

P. Petilthouarsi.

4. Doigts de la deuxieme paire longs la 4 . Doigts de la deuxieme paire longs moilié de la paume.

\%. Dactylopodites sans épine acces- 5. Dactylopodites pourvus d'une épine soire. accessoire.

Le caractère le plus intéressant de cette espèce est donné par

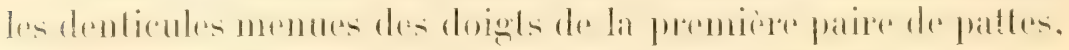

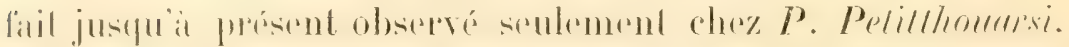

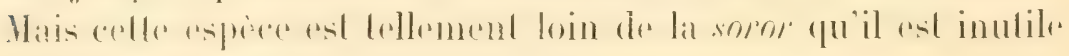
de dommer les diflérences entre elles.

II. Paulson a décrit un genre nouveau el deux autres nouvelles espéces de ce groupe que jo n'ai pas vues. Je donne ici la traduction des descriptions du carcinologiste russe.

\section{Anchistia elegans Pauls.}

Anchistic elegans Paulson, p. 113, pl. XVII, fig. 1.

"Rostre un peu élévé supérieurement, aussi long que le

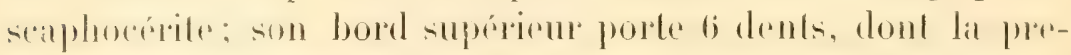

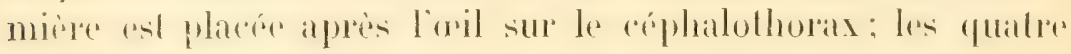

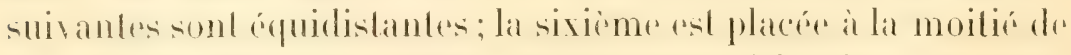
la partie restante du rostre; l'apex est bifide. Sur la partie antéreme du bord inférieme il y a 3 dents, dont la premiere

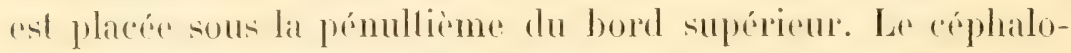

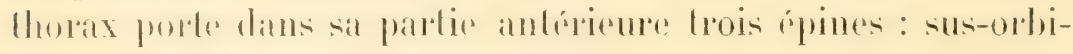
laire, antennaire et branchiostégale (1). Le pédoncule des

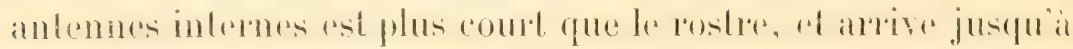

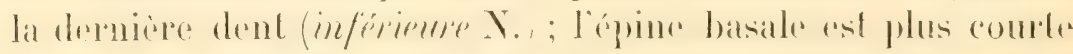

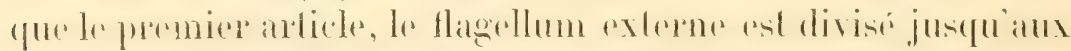

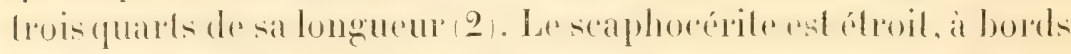

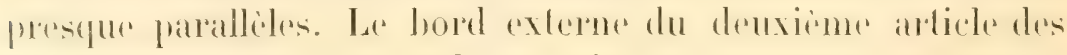
heclognathes est pourvu d'une épine, et est aciculé à l'extrémité. Pattes antérieures plus longues que le rostre, arrivant jusqu'au propodite de la deuxième paire, el ayant la mème

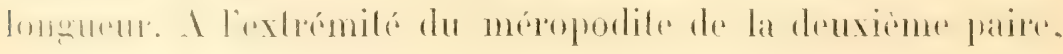

(1) J'après la figure, cette épine est l'hépatique, non la branchiostégale.

(2) Le denier quart, d'apres la figure. 


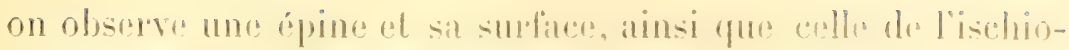

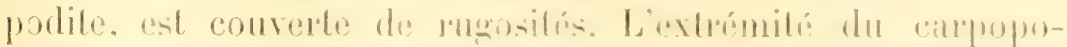

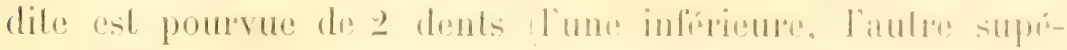
rieure $\mathrm{X}$.).

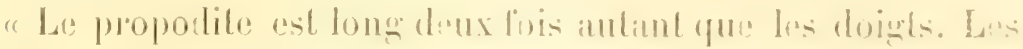

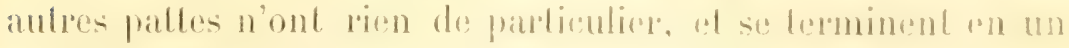
ongle simple.

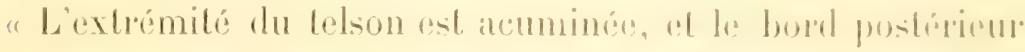
porte six petites épines, dont celles de la demxione patio sunt plus longues; les internas sont plus combes que la moilio de celles qui precedent, at sont convertes do poils sur lomes bords.

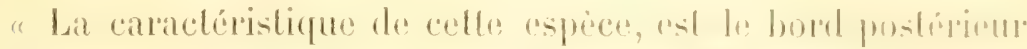
du telson qui indique une parenté atree les Polimmon.

Celle espece me parail roisin: de la l’. ensifinns.

\section{Anchistia Edwardsi.}

Paulson, p. 11.', pl. XYll, lig. 2.

"Iostre prespue horizontal, aussi longe que le seaphocurilu:

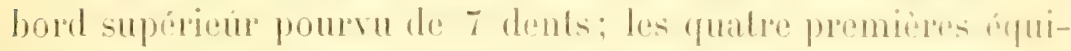

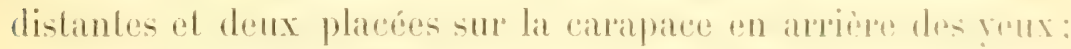

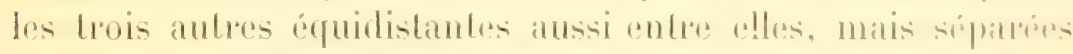
de celles qui les précedent par un intervalle; pointe liblide:

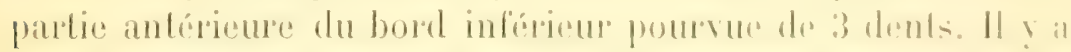

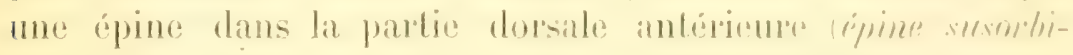
Irive X.), comme chez A. elergmes. Le pédoncente des antemme-

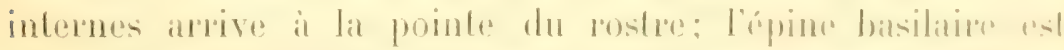

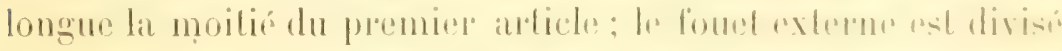

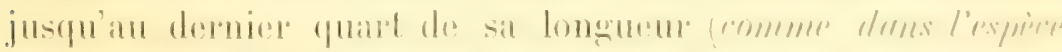

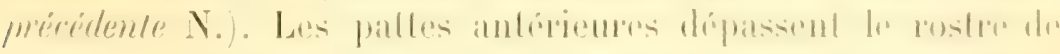

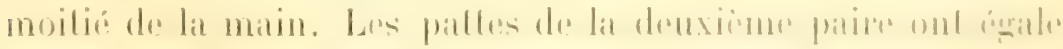

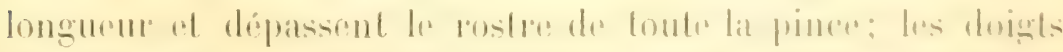

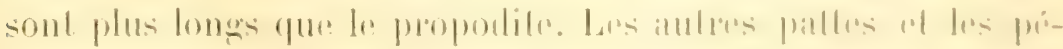

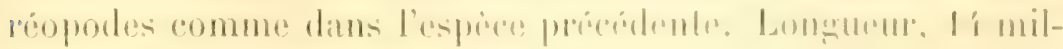
limelers, yeompris le rostre.

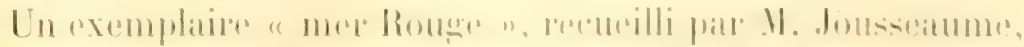

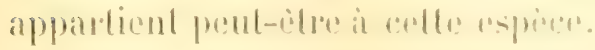


Anchistioides n. gen. (Paulson).

" Corps comprimé. Pédoncule des antennes supérieures court; fonet externe très gros, et divisé jusqu’à la moitié

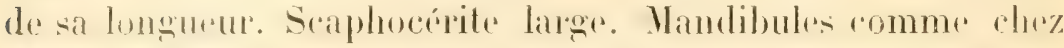

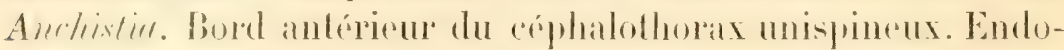

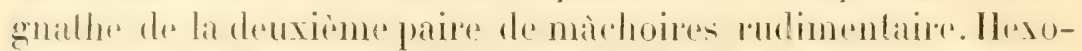
whather de la premiere paire de maxillipedes foliace. Maxilli-

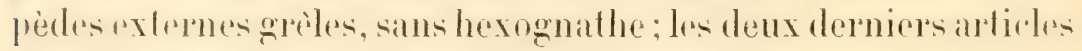
distinctement plus courts que le deuxieme. Les deux premiires paires de propopodes pourvates de pinces: la deuxieme paire plus direloppere que la premiere. Ramean extrieur de la premiere paire de paltes aboloninales du male courl el biparti; fartie rolerne crochue, sareordant avec les Cardés par la patte acessone suivante. Deuxieme paire comme chez Alsrlistie el Prlamon. Extrémité du telson droite, sans pointe."

\section{Anchistoides compressus.}

Paulson, p. 113.pl. XIX, fig. : "..

"Rostre tong, foliace, pourve d'une cote latriate, commenrant is la moitié du rephalothorax; borel supérieur pourvu de lo dente grosses, pointe aigü. P'édoneule des antennes supri-

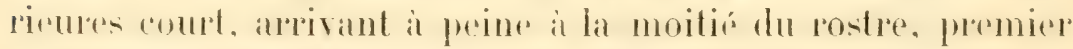
article assoz court, mais blargi; an plus de l'ópine basale coutverte par l'organe auditif, il y a une ćpine ferminale; deuxieme

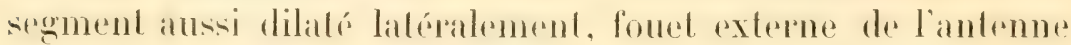

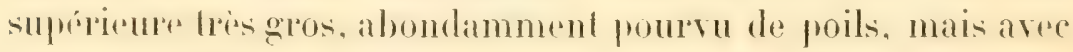
sa face supérieure glabre.

Mrliele basilaire de l'antenne externe sans épine: scapho-

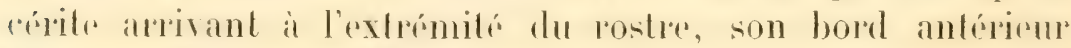
frompur obliquement. Il y a, sur lo bord anterpirur de la carapace,

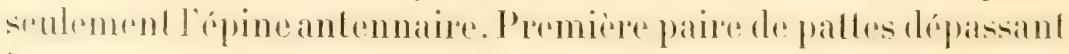
la restre de la longueur de la pince. Deuxieme paire de palles ratsone matis, it en jugere par les articles basilaters, plus fortes

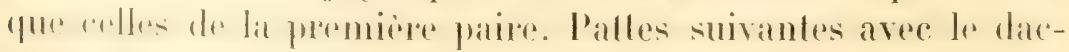

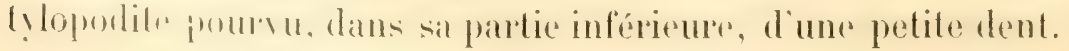




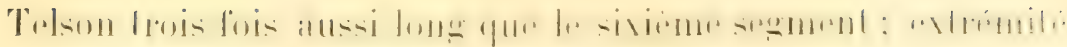
droite el armée de six ípines, dont les externes tres pulites.

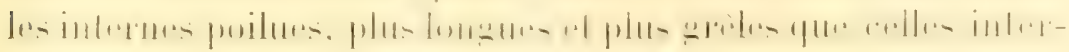
médies. Entre les intermediósel les internes, il y a de chatgur

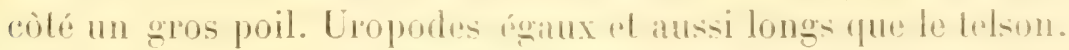
Un male long de lä millimidres.,

Paulson ne parle pas, dans celle description, du bork inlii-

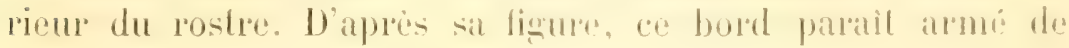
1 dents peu marequécs el irrónulivers.

\section{Genre CORALloGithls Stripsox. (OEdipus Dana.)}

Les huit especes de Comallinaris commues justpüit presunl dans la mer Rouge peuvent se diflérencive ainsi:

A. Protubérance basale des dactylopodites des prattes III-Y bien formée, sans: épine accessoire.

B. Rostre denté.

C. Rostre denté sur le bord supérieur seulement.

D. Rostre pourvu d'une seule dent. Doigts movables dans un plan presque horizontal.... C. mucrophthalmu lidw.

DD. Rostre pourru de f-ï dents. Doigts morables dans un plan vertical............. C. hecale Yob.

CC. Rostre denté en dessus el en dessons.

$E$. Mains des pattes de la deuxieme paire égales de dimensions el de forme ............. ('. superhit J)ama.

$E E$. Vains très inégales et de forme tris diflérente, lune de Corulliocuris, à doigts tombs: l'autre presque normalement cardienne... C. lucinu Vob.

BR. Rostre non denté.................... Ludinastris Hell.

1. Protuberance basale des dactylopodiles réduite ou nulle; il y a une épine accessoire; partois aussi des denticules (sous-renre Onycocaris lob).

F. Bostre non denté, mérus inerme, mains non granu-

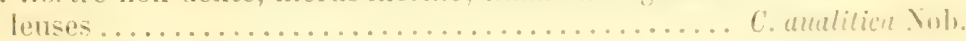

$F F$. Rostre avee $\frac{\tau-9}{0-1}$ dents: ischium et mérus denticu-

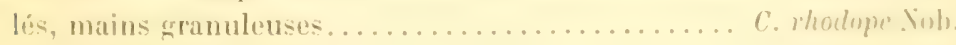

\section{Coralliocaris superba (1)illit .}

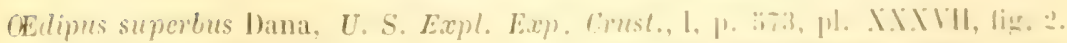

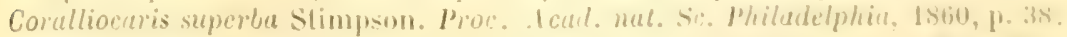

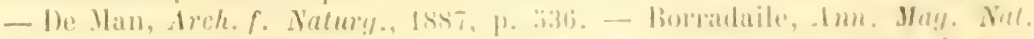

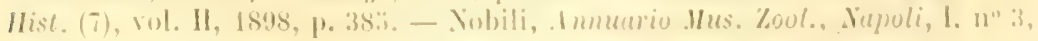
$1901, \mathrm{p} .3$.

OEdipus dentirostris P'aulson, p, 11:2, pl, XII, lig. 7-7 d. 
Djibouli M. Maindron. Is93 drux miles el deux femelles; Djibouli (M. Coulière), un individu anomal.

Le poster des exemplaires recurillis par II. Maindron porte. chez les deux màles $\frac{\ddot{a}}{\underline{a}}$ dents, chez les deux femelles $\frac{\ddot{a}}{\underline{a}}$ et 7

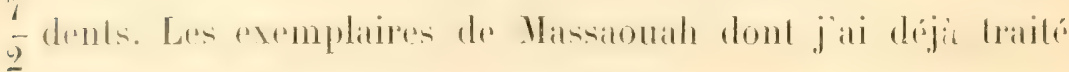

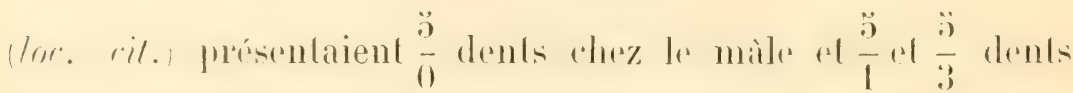
chez les femelles. L'exemplaire recueilli par M. Couliere présente une curieuse anomalie : le rostre ne porte qu'une dent

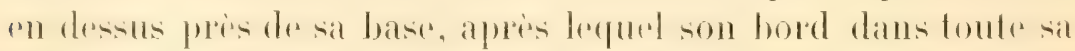
longueur est inerme et courbe; en dessous, il porte 3 dents. J'ai représenté ce rostre à la planche II, figure 7 .

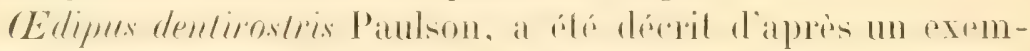
plaiere qui manquait de palles de la deusieme paire. Le rostere

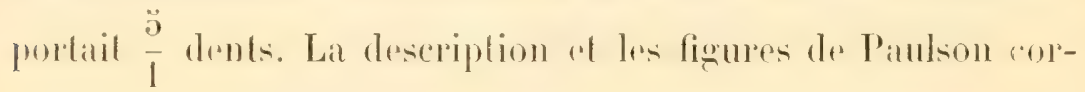
respondent parfaitement, pour ce qui est du rostre, des antromes of de la prolubérance hasale des dactylopodiles, a C. superber.

Har. : Massanuah, bate de Rahavia, Tongatabou, Tahiti.

\section{Coralliocaris macrophthalma (Edw.j.}

Pontonia macrophthalma II. Milne-Edwards, H. N. Cr., L. II, 1837, p. 350, et All. Cuvier R. Anim. Crust., pl. LII, fig. 3.

Coralliocaris macrophthalma Borradaile, Amn. Mag. Nat. Hist.(T), vol. II, 1888, p. 385. - Nobili, Annuario Mrıs. Zool. Napoli, I, 1901, no 3, 1) 3.

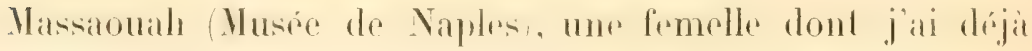
donné une description détaillée dans le travail cité.

\section{Coralliocaris nudirostris (Heller).}

OEdipus nudirostris Heller, Sitzb. Acad. Wicn, vol. XLIV, p. 279, 11. III, lig. 2.5.

Coralliocaris mudirostris Borradaile, loc. cit., 1. 383. - Nobili, Ann. Mus. civ. St. Yat. Genova, XL, 1899, p. 235.

Cielte espèce a été décrite d'après des individus de la mer 
Rouge. de n'ai malheneusement pas vu d'exemplaines de cedte

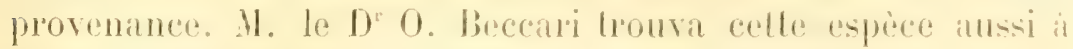
Mafor, dans la Yourelle-Ciluiniue.

\section{Goralliocaris lucina Nob.}

Yobili, Innuario Mus. Zool. Napuli, 1, 1901, n०3, 1). :3.

Coralliocaris lamellirostris De Man, Abh. Sunclenb. Gesells., IIT, 1!102. 11. 8i2, pl. XXVI, ligr. $3 i$.

?Coralliocaris lamellirostris Stimpson, Proc. Acul. Y. Si. Philurlelphir. Ision. P. 38 .

Ervthée (Mus. Naples), 30 individus; Massaonah MII. Inlimori, lssel, Beccari, Mus. de Geines), 20 individus; Djibonti (11. Couliere), 16 individus; mer Rouge (11. Jousseatume). 14. individus; Djibouli, dans les Madrepora virantes. 8, I. 1909 (Ch. Gravier).

En 1901, je décrivais une Corallinearis de la colonie Erythrón. que je considérais nonvelle. L'année suivante, le D) I.-fi. Dr Ian décrivil el figura, aree la précision el le soin qui lui sonl habiluels, la mème espèce, d'après une femelle do Tornate. Excepté quelques diflérences dans les détails, lícilement explicables et de peu d'importance, la forme que II. De Man

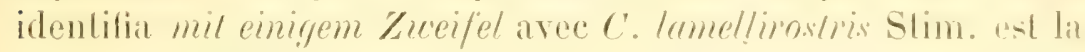
mème que ma C. lucina. Il x'y a pas de doute que pour ce qui est du rostre ef de la grosse pince, mon especes saccorde fris bien aree l'espece des iles hioi-liiu. de crois poutant quil s'agit la de deux especes differentes. Liesperee de la mex

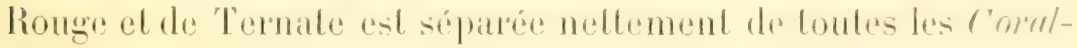
lincaris par la forme des palles de la deuxieme paire qui sonl très differentes entre clles: l'une est grosse, a doigh tordu al at. complexirement, la conshuction lypique de Comenlliomerix:

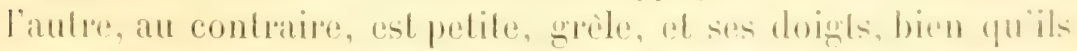
soient langes, convexes en desisus el concaves dans lat pallio?

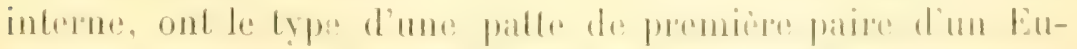

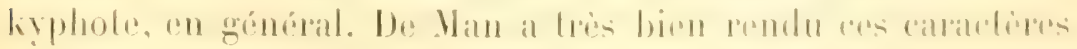

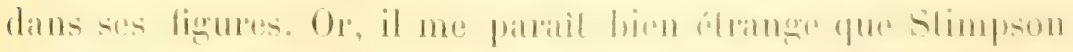
(auleur concis mais diligent, lant que l’on a pu identifire lat

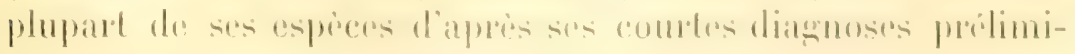

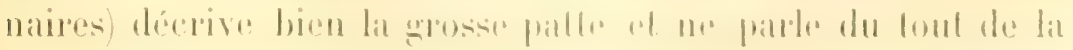


pelite, qui purmant "xistait chez son exemplaire, puisquil scrit: pedes secumdi insequales. Cela me fait supposer, et, je

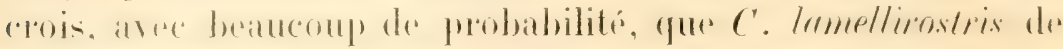

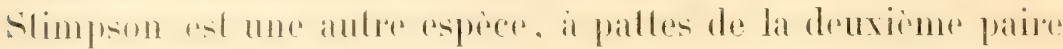
inégales de dimensions, mais non de forme très différente, ainsi yor nous observons, par "ximple, chez la $C$. inaryulix () Imann.

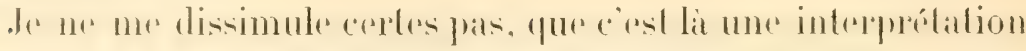
purement personmelle, ef quine description insuffisante est foujour posible, mime de la prat de stimpson. Ses ly pes

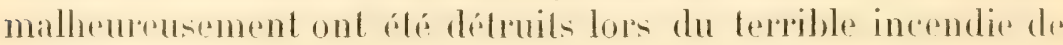
Chicago.

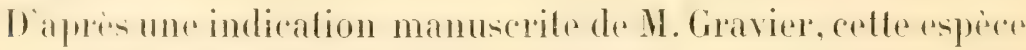
est de couleur rose pille.

\section{Coralliocaris hecate Nob.}

(Pl. III, lig. 2.)

Nobili, Bull. Mus. Paris, 1904, no క, p. 2:2 (Diagn. prélim.).

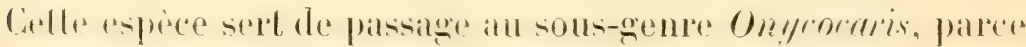
que la probubérance basale des dactylopodites est plus réduite

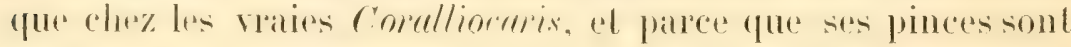
disposées verticalement.

Elle ent représentén dans les collections du Musiom par un

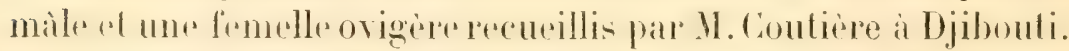

Le rostre est petit, grèle, et porte en dessus 4 dents aiguës spiniformes; sa pointe est aiguë; son bord inférieur est dépouru de dents, ef il n'est pats diargi il hase. Il est plus

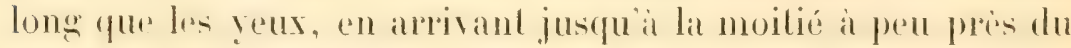
deuxime article du proloncule des antennes internes. Les venx sont rylindriques of les pédoncules sont plutot longs. Le stat-

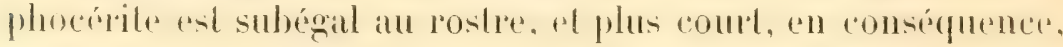
fure le pédoncule des antrnnules; il porte une petite epine i son "xtrémité antérieure. Le premier article du pédoncule des anlemmules est un pea plus long que l'aril, el presque ansi

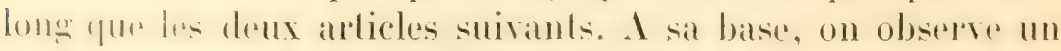

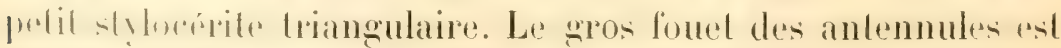
brièrement dirisé après le huitième article. 
Le fouel des antennes externes at i pen pros ansi long que lat carapace.

Les pattes de la premiere paree sont labbles; mérus et carpe sont subégaux et plus longs que lat main, dont les doights at lat paume sont aussi subégaux.

Les palles de la deuxiene paire sont tres incogales: at landis

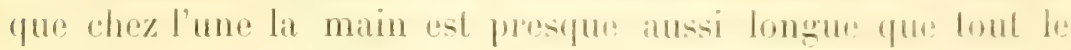
corps, chez l'autre la main esl a peine aussi longue que las carapace. Lat grosse pince, chez l'unique exemplates pui ail lese deux paltes, est celle de gauche. Le mexus ast un peu plus lones que l'ischium. Son bord supérieur est convexe, son hord inférienr concare et completement inerme. Le arpu as obconique el court. La grosse main est longue, presffue autant qur la distance entre la pointe du rostre jusquan dornier anlicte

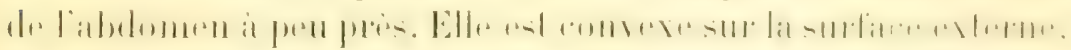
et un peu moins sur la surlace interne, large it la base of un peu rétrécie à l'extrémité. Les doights sont un pen plus longs qu'un liers de la longuenr de la paume. Ils sont un peu recourbés vers la patie interne; le doigt mobile wht haut. tres comprimé, el porte une grosse dent prés de sil bitso: le doigt fixe, comprimé aussi, porte e dents; les poinfes des deux doigts sont aigues. Laumbe main est beatueoup plus pelite, moins haute, longue a peine de la moitie al sus doigls onl

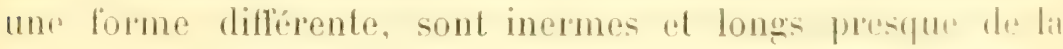
moilie de la paume. Les deux patles ne sont pats disposines plus ou mons horizontalement comme dans la pluparl des

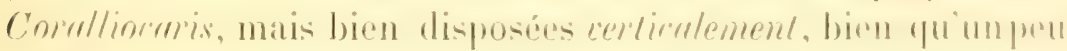
obliques.

Les trois paires suivantes de prevopodes sont mólioneres: le

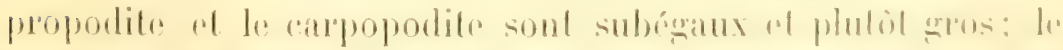

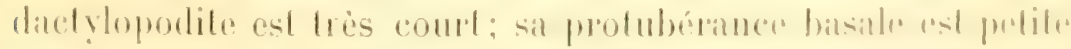

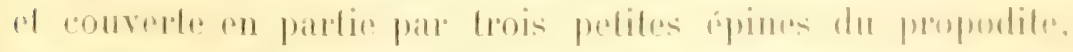

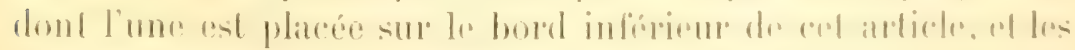

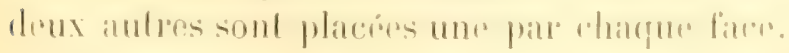

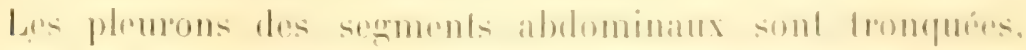

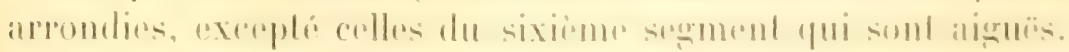

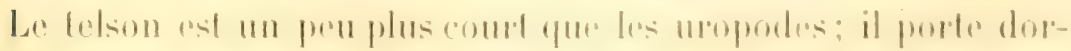
salement dens paires despimules. ol it sil pointe six spimules. 
les latérales comples, les quatre autres longues, mais les deux mitoyennes un peu phus courtes que les intermédiées.

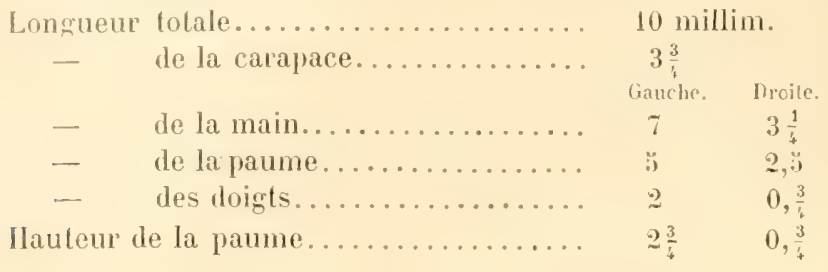

\section{Onycocaris Nob.}

Nobili, Bull. Irus. Paris, 1904, no 3̈, p. 252.

Ge nouveau sous-genre de Coralliocaris a élé établi par moi

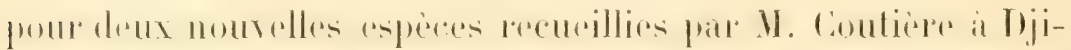
houli, qui offrent les patticularités suirantes. La prolubérance

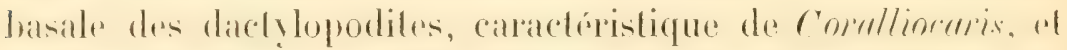
très réduite dans l'une des espèces $(C$. aualitica) et absente chez l'autre $(C$. rhodopo). Chez les deux espèces, l'on observe

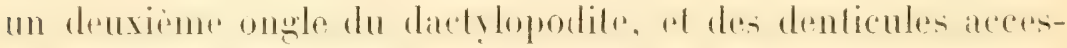
soires. Les chélipèdes sont disposés verticalement comme

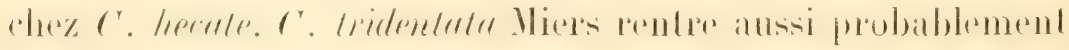
dans ce sous-gente.

\section{Goralliocaris (Onycocaris) aualitica (1) Nob.}

(Pl. III, fig. 3.)

Nobili, loc. cit., p. 252 (Diagn. prélim.).

Une femelle ovigère longue de 9 millimètres, et un petil mâle recueillis par $\mathbf{H}$. Coutière a Djibouti.

La carapace est courte, large et assez convexe chez la femelle, moins large el moins convexe chez le màle. Le rostre,

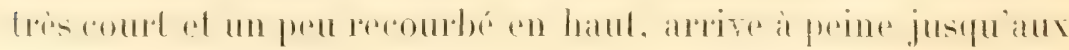
cornées des yeux. Les yeux sont gros, renflés el courls. L'angle

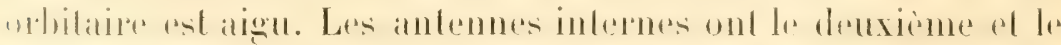

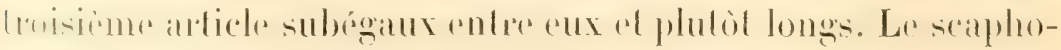
cérite est court, ovalaire, étroit, et manque complètement

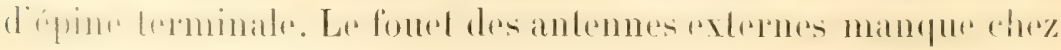

(1) \نxخ:-rs, localité près de Zeila, probablement le golfe de Tadjourah. 
la femelle: chez l'aule individu, il arrive anx deux liers environ de la longueur de la caraparer.

Le merus et le carpe des palles de lit premiere patresont subégaux.

Les palles de la denxime paires sont rates chez la femelle, at légerement inçates chez lo male. Eilles sont comprimós

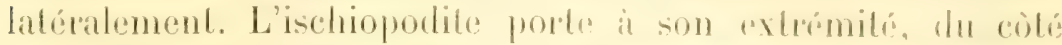
interne, une saillie dentilorme. Le mérepodite a les deux bords plutôt converes, el est relativement cont. La "arpe qui ast

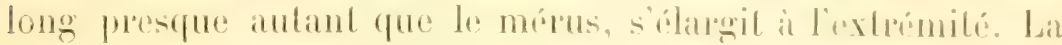
main est comprimée, large, courte, arece las doights un peu plus courts que la paume, al a uir aspect alpheöle, qui rappelle celle de liarilius rompressus íands. Lus doigh ne joignent pas exactement. Le doigh mobile porte uno dent, it laquelle correspondent deux petites dents sur le doigt fixe. Examiné a fort grossissement, son bord tranchant, apres lit dent, est linement denticulé jusqua a la pointe.

Les paltes suivantes portent cing spimules sur bord infirrieur du propodite. Les daclylopodites courts ont une épino aceessoire denticulée à l'extrémilé, et une petile probubéraner basale denticulée.

Le telson porte it son extrémité quatre spinules subigates, et est plus court que les uropodes, dont les bords sont finement denticulés.

\section{Coralliocaris (Onycocaris) rhodope Nol,.}

Pl. II, fir. s.)

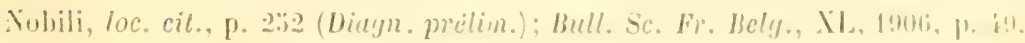

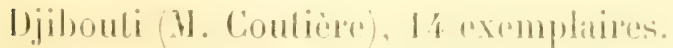

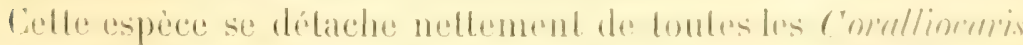

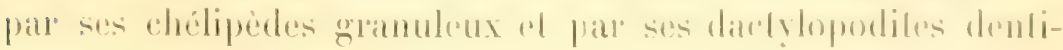

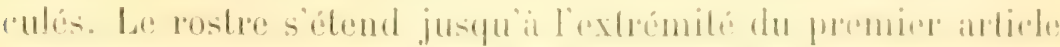

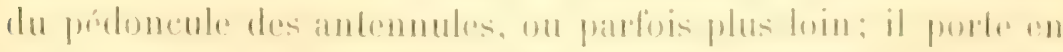

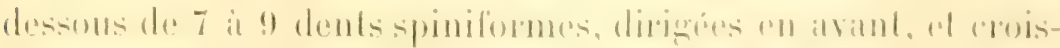

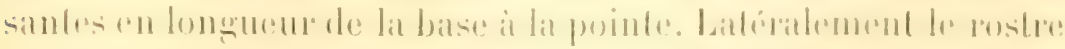

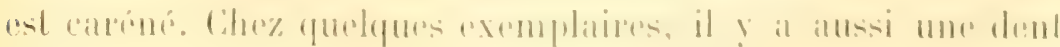

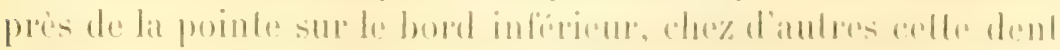




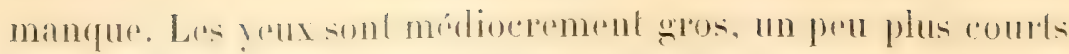
que le rostre ou igalement longs.

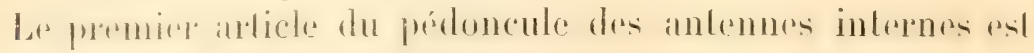

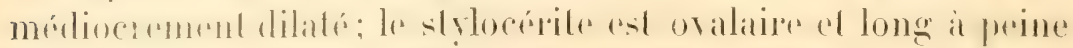

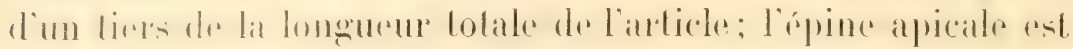

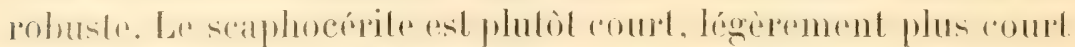

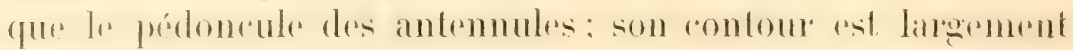

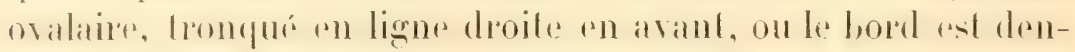
liculé, et son épine externe apicale est longue el forte el dépasse notablement le bord antérieur du scaphocérite.

Sur la carapace, on observe une épine antennale forte; manque l'épine hépatique et la sus-orbitaire.

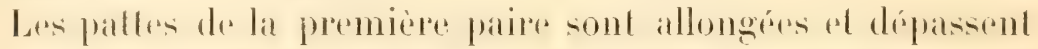

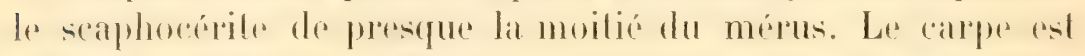
subégal au mérus ou légirement plus long; les deus arlicles sont allongés. La main an contraire est plus conte que la moritir du carpese at ses doights sont phus couts que hat paume.

Les pattes de la deuxième paire sont inégales, el, comme

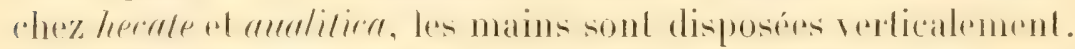

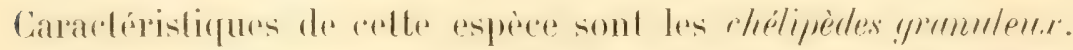

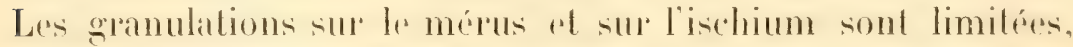
particulierement an bord supripeur, ou quelques-unes, en derenant plus grosises, donnent à ce bord une apparence denti-

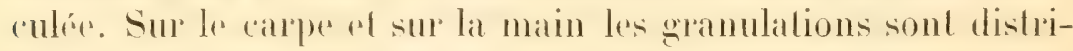
buens sur les deux fares, mais elles sont plus nombereuses of serrés du rotio interne; celles des bords sont aussi plus grosises el domment anc bords un aspecel denticule. Sur lar grosse pine

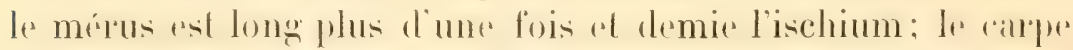
est court, obconique, la main grosse, convexe sur les deux

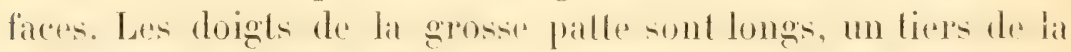
longueur de la main entiere, cenx de la pelite patle sont plus longrs. Lat paume de la grosse palle est aussi longue que deux fois les doigts. Elle est aussi haute un prel plus que moilio de

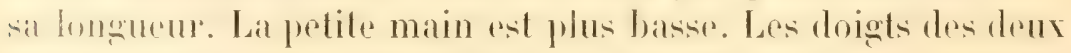

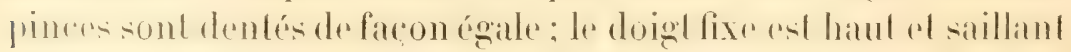

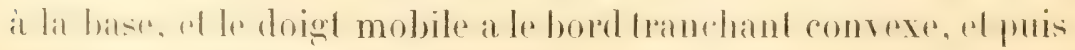
rétréci en pointe courbe.

Les palles suivantes sont gredes. La mépopolite porte sur le 


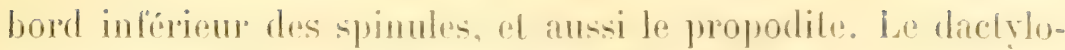
podite a une deuxiome cipine. phutot petile; sa probuberance basale est prespue nulle. Lat protubérance rudinentaire el lo trait entre l'ongle tominal el laceessoile sont denticulés.

Le telson est presque aussi longe que les uropodes; il porte en

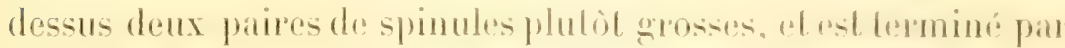
six épines disposées comme chez les autres Conallinerris.

Le plus gros individu, une lemelle ovigere, est long de 10̈ millimetres.

\section{GENTE HARPIIIUS DANA.}

\section{Harpilius Beaupresii (Aud.}

Palamon Beaupresii Audouin, Expl. pl. San., 182:, p. 91. - Savigny, Descr. Egypte, Crust., pl. I, lig. 4.

IIarpilius Beaupresii Ileller, Silzb. Acad. Wien, XLIV, 1862, 1).280. - Paulson, loc. cit., [). 113. - De Man, Lech. f. Yoturg., 1887, 1. 339. - Locradaile. Ann. Mag. Nat. Hist. (7), vol. II, 1898, P. 386. - Yobili, Imn. IJus. Zool. Napoli, vol. 1, 190', $11^{\circ} 3$, 1. 3.

Massaouah (11. Lsel el Beccari, Mus., Gènes), 36 exem-

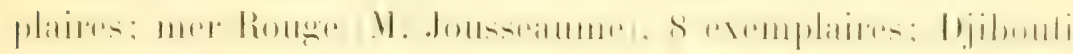
(II. Coutiere), 30 exemplaires; Djibouli (II. Jousseatume). I exemplate: Périm (II. Jousseaume), une frmelle; Erythrou (Mus. Naples), 30 exemplaires.

Le rostre porte en genéral $\frac{3}{3}$ dents, at at plus court que le scaphocérite, mais la longnem at le nombre des dents paraissent dre bien variables.

Has: Her Rouge, Malaisie.

\section{Harpilius lutescens Dana.}

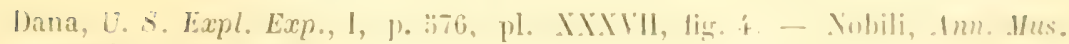
Iapoli, 1, n" 3, 1., 3.

Eryllnee (Mus. Nipples), un male.

\section{GENRE ANCIIST'US BOUIR.}

Anchistus Miersi (I)e Min).

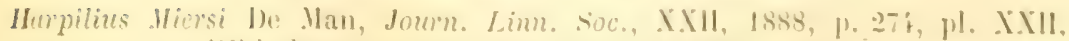
lig. 6-10. - Whitulegge, Austr. Mus. Mem., III, 18!7, p. Iis. 
Anchistus Miersi Borradaile, Amm. Mug. Nat. Hist. (7), II, 1898, p. 38\%, et Willey's, Zool. Res., IV, 1899, p. 408. - Nobili, Bull. Sc. Fr. Belg.; XL, 1906, p. is.

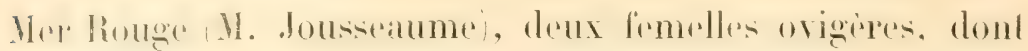

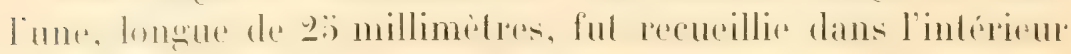
d'une Pinna.

Le rostre, dans ces deux exemplaires, alleint l'extrémité du deuxieme article du pédoncule des antennules, et n’a pas la

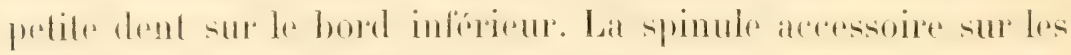

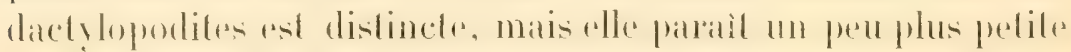

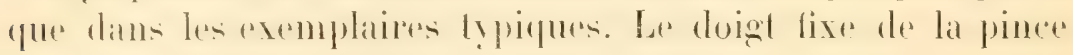
dans l'exemplaire plus jeune a 4 dents, l'autre 8. Le lelson a six épines mobiles à son extrémité, les deux externes très

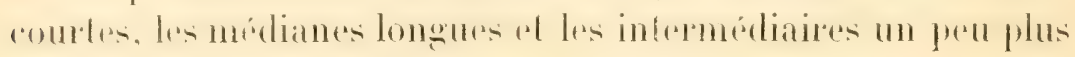
longues que les médianes.

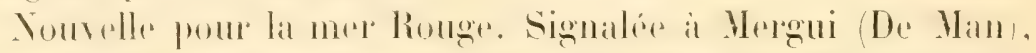

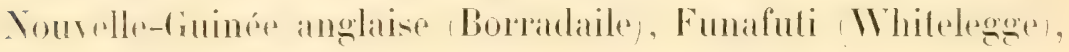
golfe Persique (Nobili).

\section{Genre ANGylocaris Schenkel.}

\section{Ancylocaris aberrans Nob.}

Palemonella aberrans Nobili, Bull. du Mus., 1904, no 5, p. 233.

Ancylocaris aberruns Nobili, Bull. scient. Fr. Belg., XL, 1906, p. 52, pl. IV, fig. $9-9 b$.

Djibouli (M. Coutière), un mâle (type de Palamonella aberrons).

Djibouti (M. Coutière), " nageant en dessus et dans la "sphère d'influence " du disque de Discosoma giganteum ". Deux males et une femelle.

Je n'avais eu d'abord qu'un mâle en mauvais élat et j'avais cru que cette espèce fül une Palamonella; après jen trouvais une grosse femelle, très bien conservée, dans les collec-

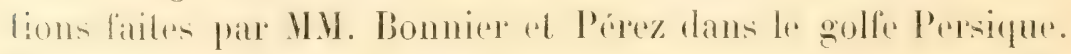

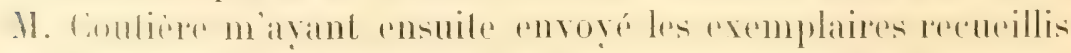
sur le disque de Discosoma, je pus' reconnaître que ma pré-

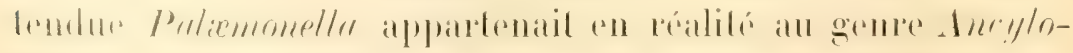
caris, fondé par Sehenkel pour une espèce de Célèbes, qui 


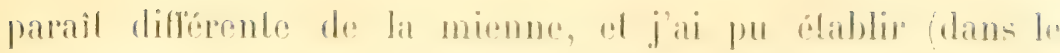
deuxieme des mémoires cilés l'átrange ditrérence antre les

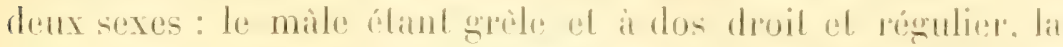

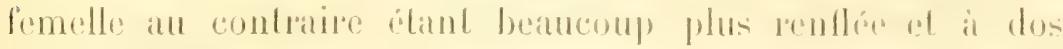

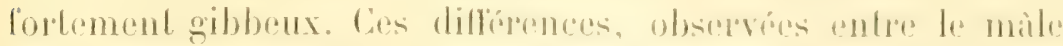

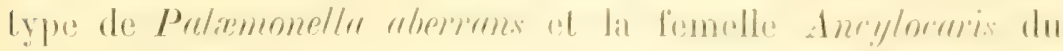
golle l'ersique, sont confirmés pall lese exemplatires pris

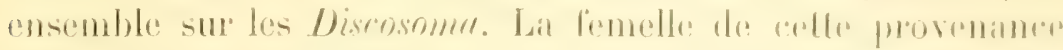
ast tres nettement gibbeuse sur toute la surfince dorsale de sil carapace, bien qu'elle le soil un peu moins que lanule femollo. du golle Persique, ee qui vient de ses dimensions plus pelites.

Le lostre dans les trois exemplanies de Disenomer est quel-

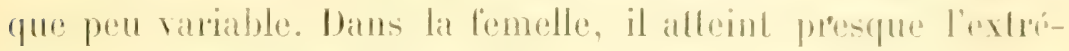
milé du dernier ardicle du pédoncule des antemmules, ot il as armé de $\frac{6}{1}$ dents; il est conlormé de meme dans l'un des

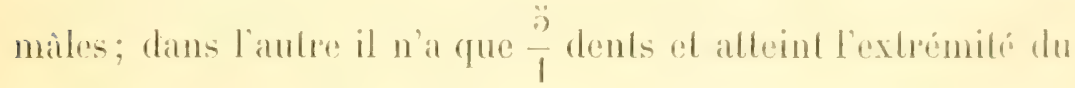
pédoncule des antennules.

Jai dégit dit (Bull. Sr. Fr. Bely. XI, p. ö3) que les ligumen lo

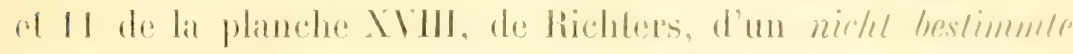

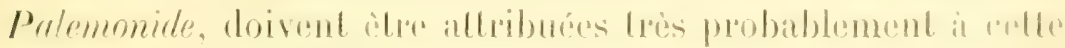
espece.

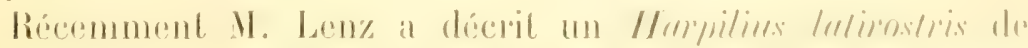
Zanzibar qui patail bien voisin de celle esperes.

GENRE PONTONIA LAT. (Borralaile restr.)

Pontonia pinnæe Urtu.

$\because$ Cuncer custos forskal, Descr. Inim., 177\%, p. ().

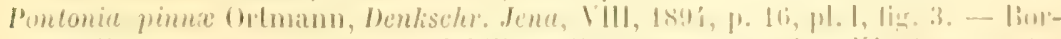

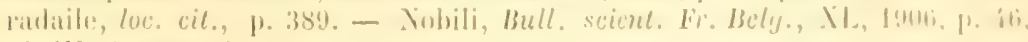
pl. 11, lis.11-116.

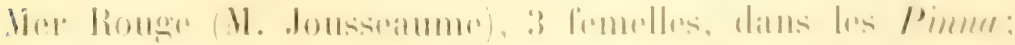

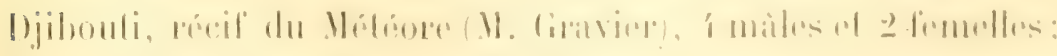

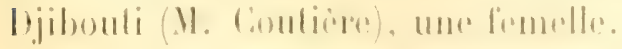

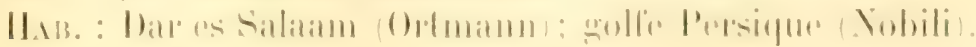




\section{Genre CONGHODYTES Peters.}

A. Doigts des pinces lisses en dessus.

$B$. Rostre plus long que le scaphocérite.

C. tridacnx Pet.

$B B$. Rostre plus court que le scaphocérite......... C. meleagrinx Det. 1. Doigts des pinces avec une grosse saillie quadrangulaire sur leur bord externe.

\section{C. biunguiculata} Pauls.

\section{Conchodytes meleagrinæe Peters.}

(Pl. III, fig. ए.)

Peters, Gesellsch. Nuturf. Fr. Berlin, 1851 (fide Ililgendorf); M. B. Acad. Bertin, 1852, p. 594; Areh.f. Naturg., 1852, p. 594. - Ililgendorf, H. B. Lliad. Berlin, 1878, p. 836. - Borradale, loc. cit., P. 390 ; Proc. Zool. Soc., 1898, p. 1007; Willey's Zool. Res., pt. IV, p. 409.- Nobili, Inn. Mus. Civ. Genova, XL, 1899, p. 235.

Pontonia meleagrinx Bate, Challeng. Macr., p. 707, pl. CXXIY, fig. 1-2.

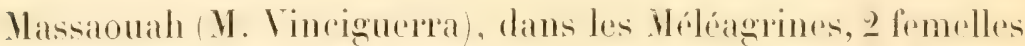

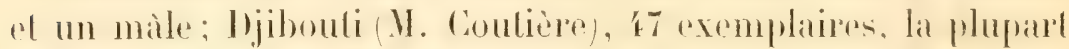
femelles; Tjibouli (II. Jousseamme), i frmelles; more Rouge, dans les Pinna (M. Jousseaume), כ̈ femelles el 3 màles.

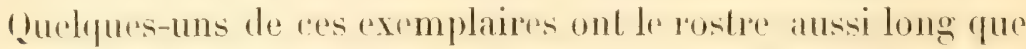
le pridoncule des antemnules el mime plus long. mais loujoms

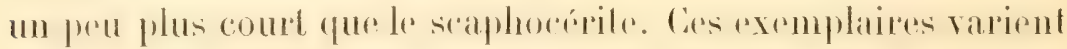
dans le sens de $C$. Iridacne, d'autant plus que le carpe des

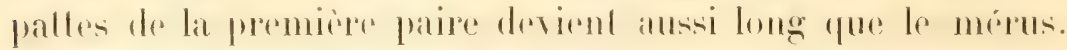

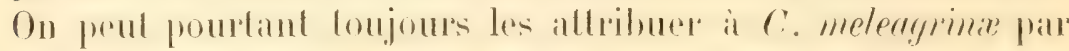

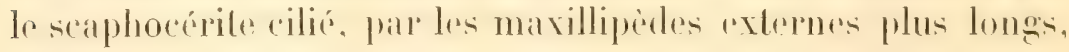
et par le doigh fixe des pinces armé de deux dents, parmi lespuelles vient se placer la dent do doigh moloilr. J" nolerai a ce propos que la deuxième dent du doigt lixe devient dans les gros axemplaires un lobe oblique laibloment dentelé.

Tronvere ansid a Mogadiscio-Benadie, fall M. Taramasso (Musée de Turin).

Conchodytes tridacnæ Peter's.

V. Hilgendorf, M. B. Acad. Berlin, 1878, p: 83:i. 
Conchodytes biunguiculata (

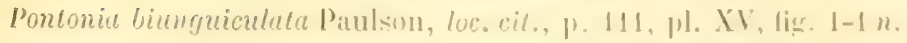

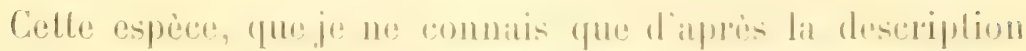

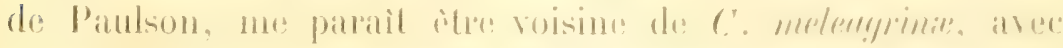

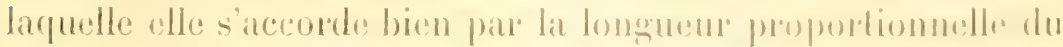

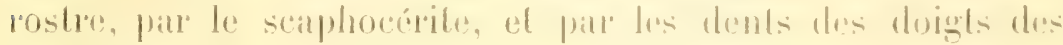

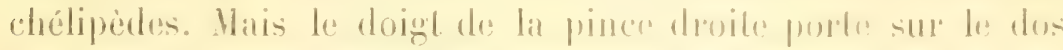

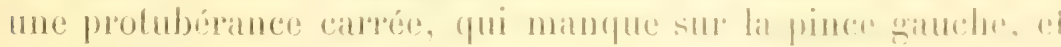

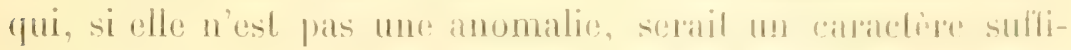

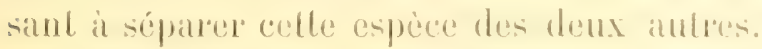

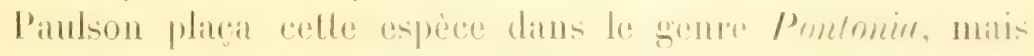
d'apres ses ligures, surtoul celle dos datelylopodiles. on wail que c'est bien une Cionchodyles.

Gexin TYPTON liostu.

\section{Typton Bouvieri Noh.}

(l. III, fig. 4).

Nobili, Bull. du Hus., 190:, 11" "i, 1.. 23:3 (Diann. pritim.).

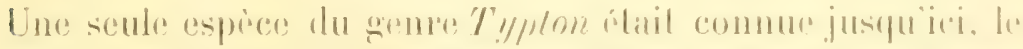

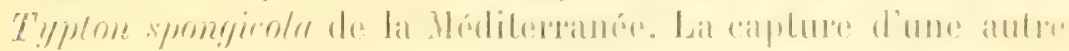

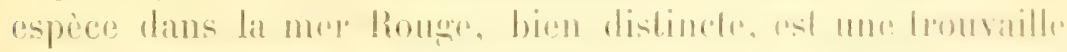
assoe lematle(pual)le.

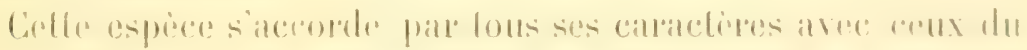

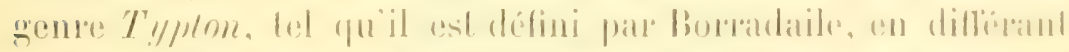

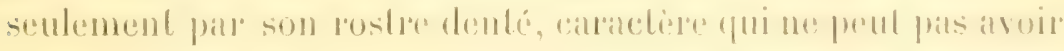

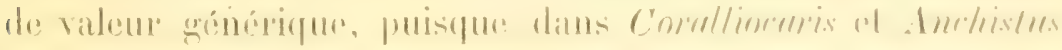

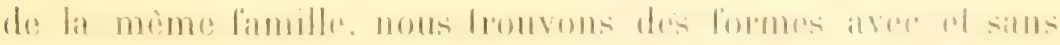
douls all bostor.

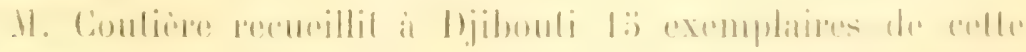
appice.

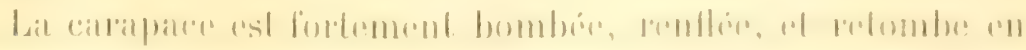

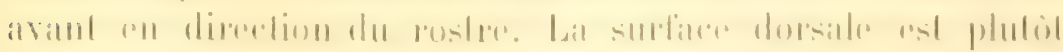

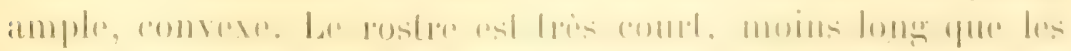

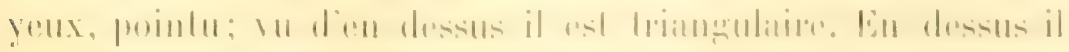

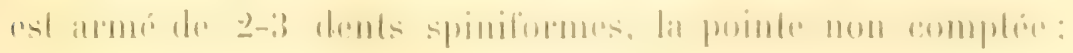




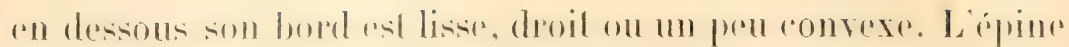

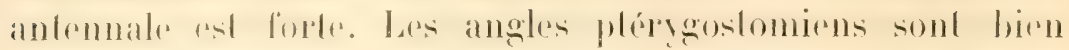

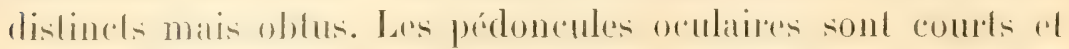
gros, coniques, élargis à la base; les cormées sont peliles et

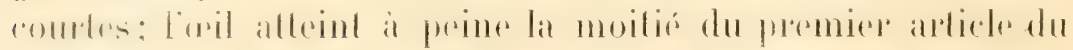
pédoneule antennulaire.

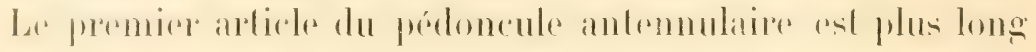

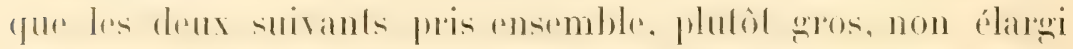

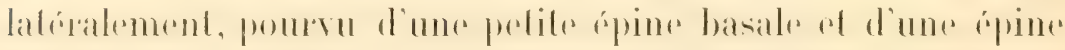
apicale très menue. Le deuxième et le troisième arlicle sont

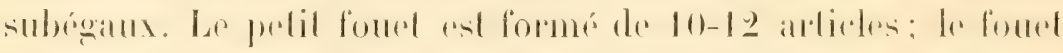
plus gros, qui n'est pas renflé ni bifide, a le mème nombre d'articles, le premier desquels est assez long. Les antemnes n'onl pas un vali scaphlocerpite mats sentemenl un rudiment:

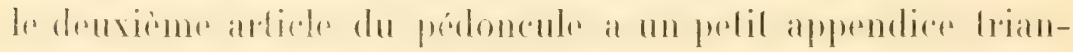

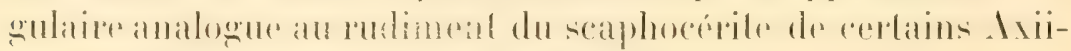
dés. Le fouet des antennes est court.

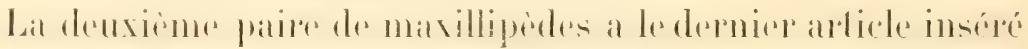

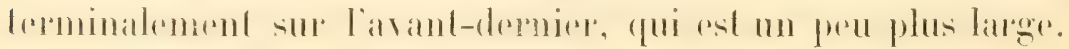

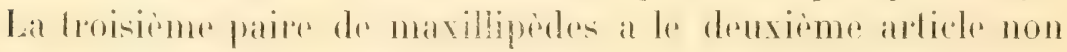
ćlargi el subégal ou un peu plus court que les deux articles suivants pris ensemble, qui sont subégaux entre eux; l'exopode dépasse un peu le deuxième article.

La première paire de péréopodes a le carpe un peu plus court que le mérus. Les mains sont bien conformées, el les

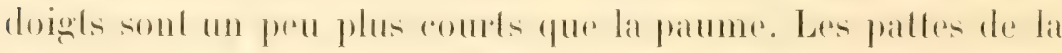
demieme paire sond un pen incigales, matis ont la méme forme. Sur la grosise palle, Tisehimm de le merus sont subégaux, le carpe est très court el conique. La main entière est six fois

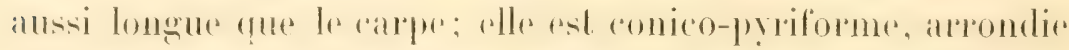

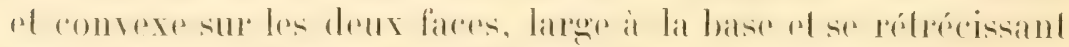

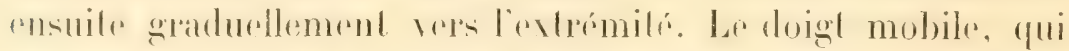

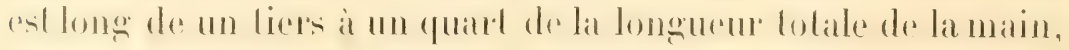
est haut, comprimé, arqué, sans dents et lerminé par une courte pointe courbée. Le doigl fixe est plus grèle el a une

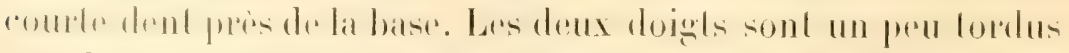
vers le còté interne. La pelite main ne diffère de l'autre que pour etre un peu plus basse el un peu plus courte. Les 


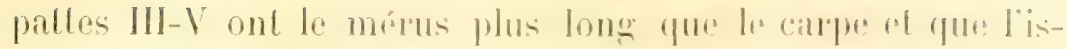

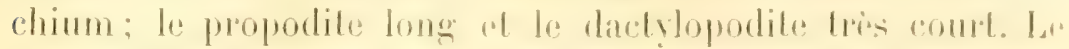

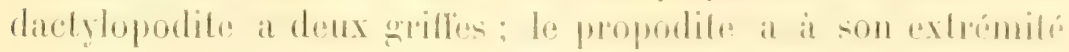

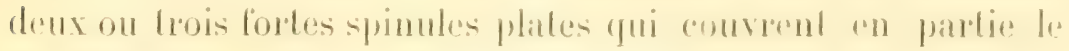
diactylopodite.

Les pleurons des cing premiers sexments abdominatux sonl arondis, ceux du sixieme segment sonl angulaters. Le Lelson

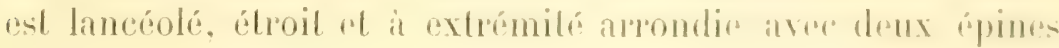
petites el quatre longues.

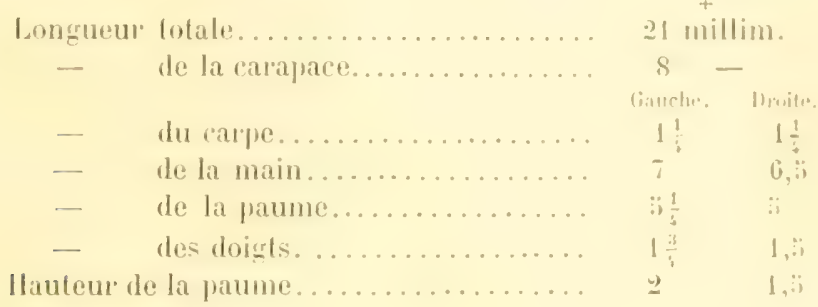

\section{FIMILLE HYHEVORER/I).E}

GeNRE HYMENOCHRA Lat.

Hymenocera elegans IIell.

Heller, Fitzb. Acad. Wien, XLIX, 1861, p. 26', pl. II, fig. 9-1't. - Hitgendorl, M. B. Acad. Berlin, 1878, 1). 828. - Ortmann, Zool. dahb. Syst., Y, Is!n,

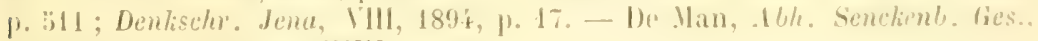
XXY, 1902, p. 82.2, pl. XXY, lig. :2.

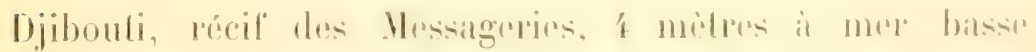
(II. Cravier), mo limelle avee arufs, longum í millimithm.

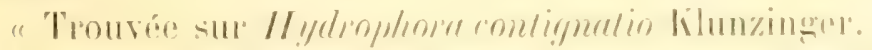

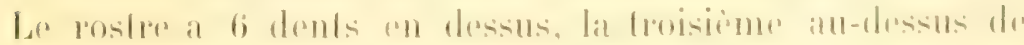

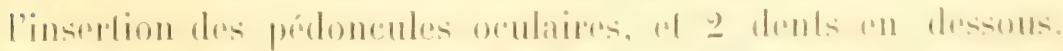

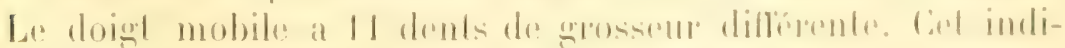

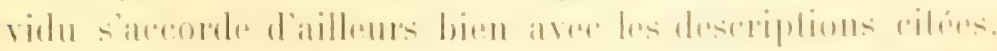

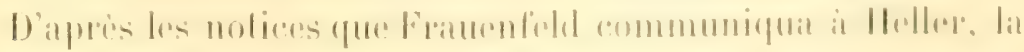

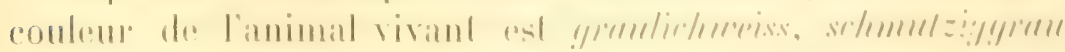

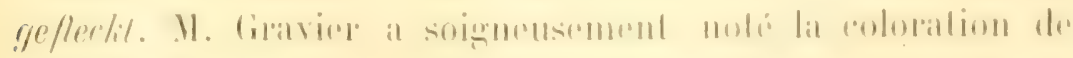
celle liacon

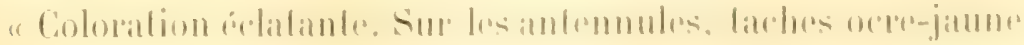




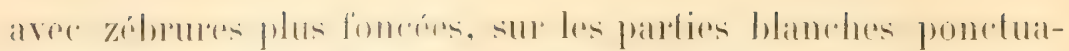
lions ocre. Haxillipèdes : Arlicle basilaire, aire centrale claire entourée par une zone plus sombre, blene; $2^{e}$ article, ponctualions couleur samon, en avant taches oculiformes

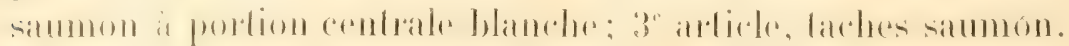

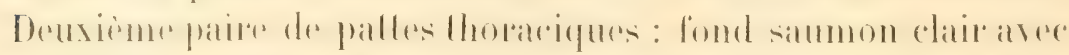

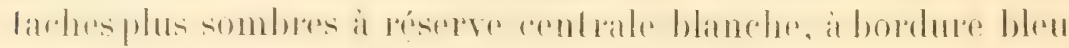

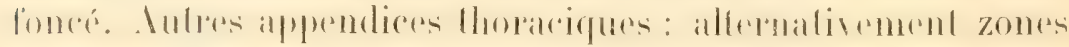

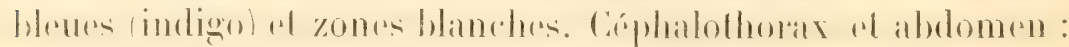
sur le fond blane laches ocre brun foncé avec un liséré bleu foncé fondu vers le centre de la lache. Taches bleues sur le telson. ")

Heller, à propos des pléopodes, a écril : "Die Banchfüsse sind normal gebildet n. Cela sera cerhamement vrai pour le

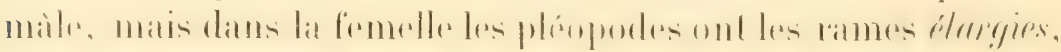

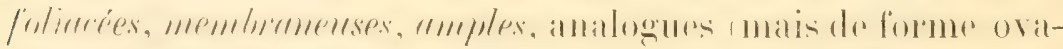

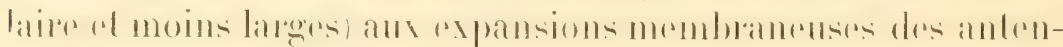

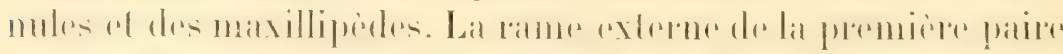
de pléopodes est foliacée, l'interne très réduite el coute. I es

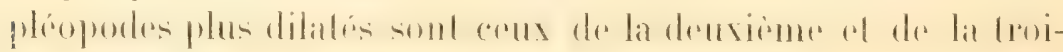
sième paire.

Hals. : Tor, dans les coraux (Heller); Mozanibique (Ilil-

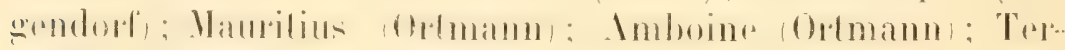
nale (De Man).

FANILLE PALAEONIDA.

Genre PALAmonelda Dana.

Palæmonella tenuipes var. (ann. sp.?).

Cf. Palemonella tenuipes IJana, loc. cit., p. 582, pl. XXXVIII, lig. 3. - De Man, Arch. f. Naturg., 1887, p. 5i:1, j]. XXIl a, lig. 4. - Ormann, Zool. Jahrb. Syst., $\mathrm{V}, 1890, \mathrm{p}, \mathrm{g} 2 \mathrm{\tau}$.

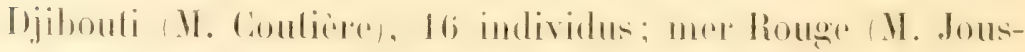
seaume), une femelle; Éylhrée (Mus. Naples), un mâle.

J'ai quelque doute que ces exemplaires soient vraiment la Palamonellu renuipes, parce que, s'ils s'accordent pour la plupart des caractères avec les trois descriptions cilées, ils 
ofleent mene dibrence importante dans les palles de la deuxieme patio.

Le rostre dans lous les exemplation a $\frac{8}{2}$ dents. Les deux pore-

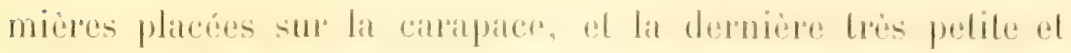
placée lout pies de la pointe. Les palles de la premiere pario ont les menes proportions dans les andicles que dans lat description de lo Man. Sourent lo brats n'altrint pats l'extrémilé du rostre. Les palles de la deuxiene paires sont subrogales al

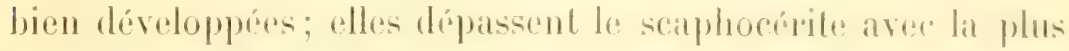

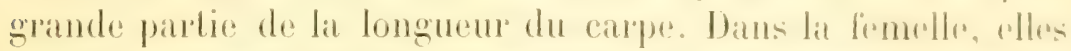
sont plus courles que le corps. Dins une palle mosurien de

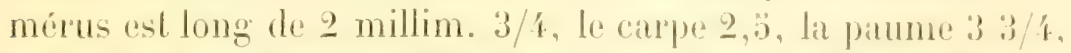
les doigls 2 millimelres. Dans un aulre exemplatio, la paume est longue de - millimidres, le doigh 3). Cues dimensions statecordent donc assez bien, saut pour les doights, avececlles de la ligure originale, el aussi avee la descriplion de Oremann

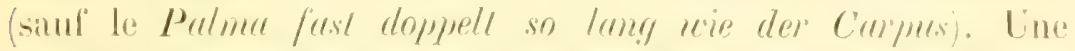
dillúrence notable s’observe dans le mérus, qui n est pas suprol infraque arulo, mais qui a seulement une éprine ì l'erlóémilé de son horel inférienr. Le carpe est armé d'une épine it son exlrémité supero-interne. Les doigls sont dentes; il y a 3 denls rolitive-

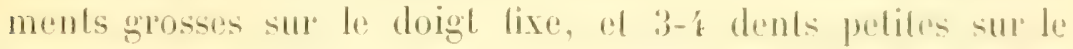
doigl mobile.

\section{Palæmonella biunguiculata Nob.}

(P). III, lig. (3.)

Vobili, Bull. du Ifus, 190't, n" "3, p. 2333 (Miugn. prélim.).

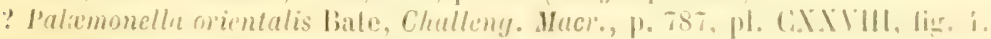

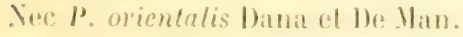

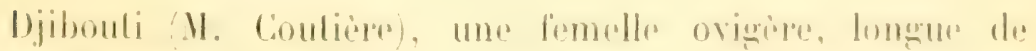
11 millimilnes.

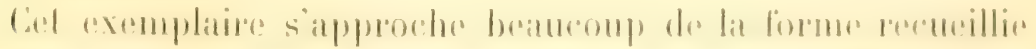

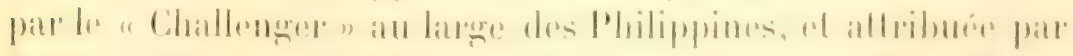

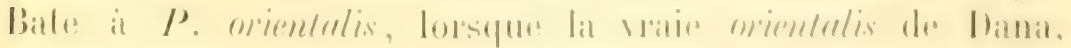

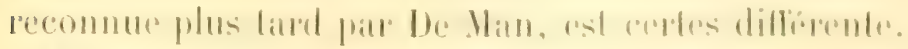

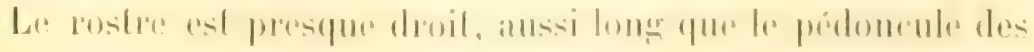

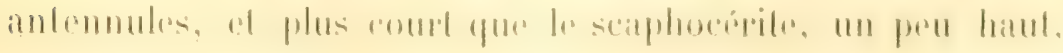


et pourvu en dessus de 8 dents, dont les deux premières sonl placées sur la carapace; il n'y a pas de petite dent sub-apicale, et il y a une seule dent sur le bord inférieur. Le fouel plus court des antennules est soudé avec le fouel voisin par

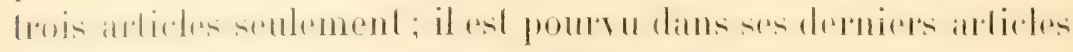

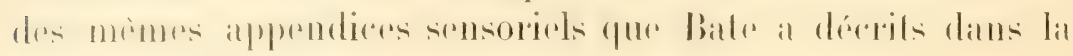
forme des Philippines.

La carapace a une épine antennale el une épine hépatique

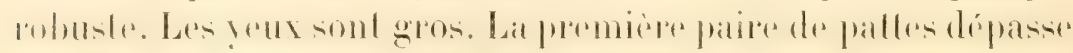
avec les doigts l'extrémité du scaphocérite; le carpe est subégal au mérus, et un peu plus long que la main; paume el doigls sont subégaux, ou les doigts un peu plus courts. Les

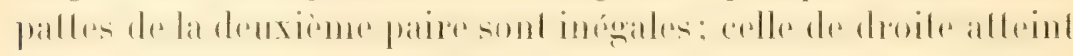
presque avec le mérus l'extrémilé du scaphocérite; celle de

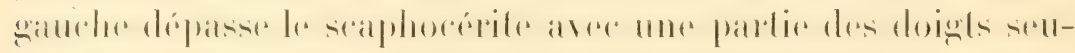
lement. Le mérus est notablement plus long que le carpe; le

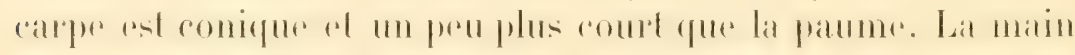

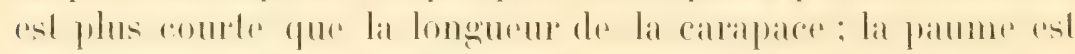

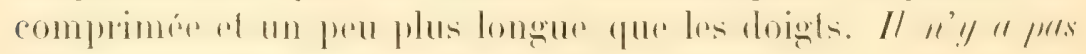
d'éprines ni sur le mérus ni sur le corpe.

Les pattes III-I sont grèles et longues et atteignent 0:1

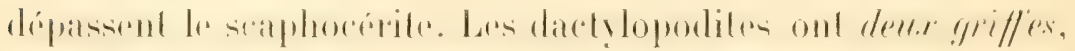

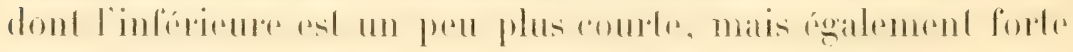
que l'auble.

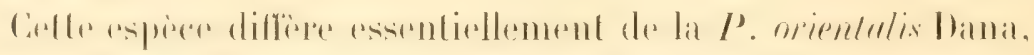

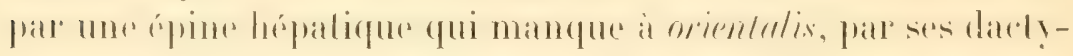

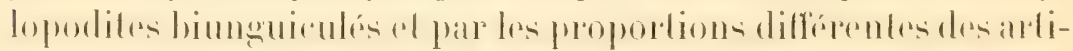

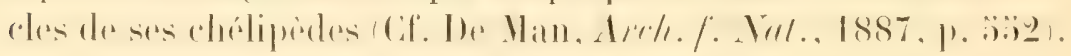

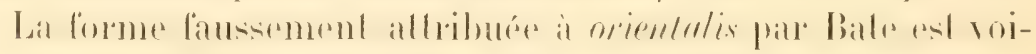
sine de celle-ci, mais Bate ne dit rien de l’épine hépalique.

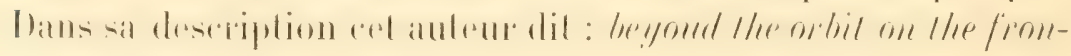
lat margin is a first antennal Ionth and according to Dana, ano-

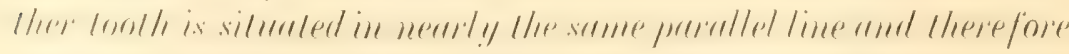

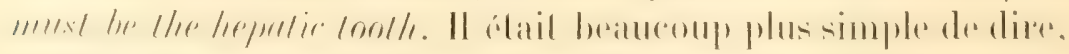

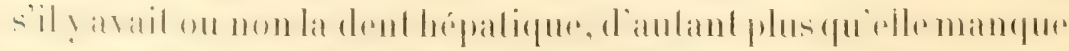

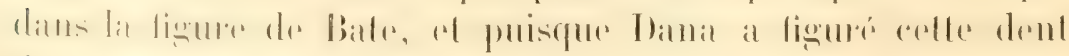

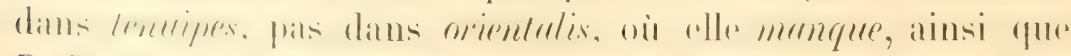
De Man l'a confirmé! 
Le dactrlopodite de la forme de bate, terminales in luen ameyurl umryes; dans la figure, le deuxieme ongle n'est pas lerminal ef robuste, comme dans mon espece, mais bloigne et pelil.

\section{Palæmonella gracilis Piuls.}

Paulson, p. 117, pl. X'II, lige 6-6f.

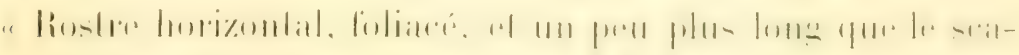

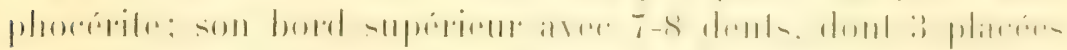

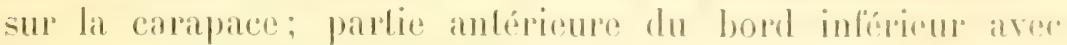
3 dents; pointe bilide. Pédonenle des antemnules ansi long que? le scaphocérile; épine basale courle, épine supéreme acuminée; seuls les quatre derniers articles du fonel exlerme sont libres. Les maxillipedes externes atteignent sentement le deuxieme article du pédoncule des antennes supérieures: lo deuxième article est plus court que les deux derniers: le dernier est armé de spinules. Première paire de paltes fres grebles.

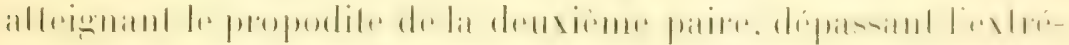
milé du rostre avee la moilié de la longueur de la main.

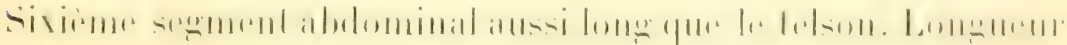
du phus gros des six exemplaires, 93 millimiles. "

Celle description ne donne aucun dibitil des patlas de la deuxieme paire. D’apres la figure, l'épine hépalique mancue. el il y a une épine branchiostégate.

\section{GENRE LEANDEH S'TM.}

\section{Leander pacificus Stimpson.}

Stimpson, Proc. 1cal. N. Ic. Philadelphia, 1860, p. 40. - De Man, Nö. Leyd.

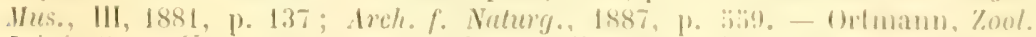

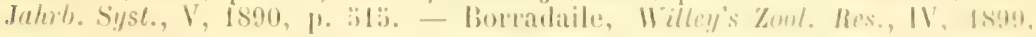

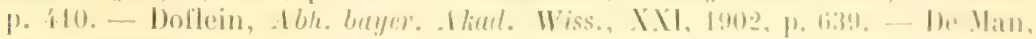
Abh. Senclienb. Ges., XXI, 1902, p. sot.

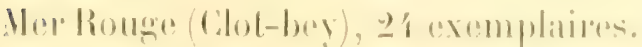

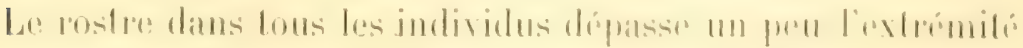

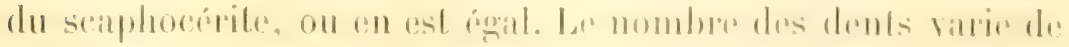

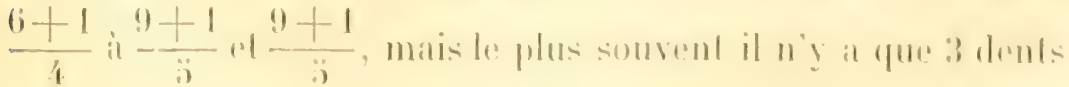


en dessous. Dans un exemplaire anormal il y a $\frac{10+0}{\%}$ dents :

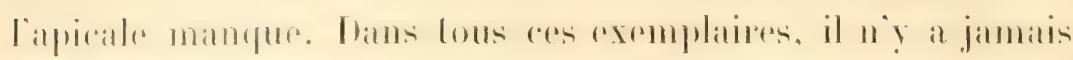

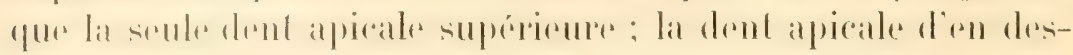

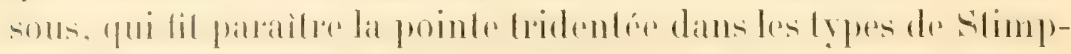
soll, manque toujours.

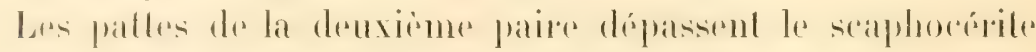
de plus d'un liers de la longueur du carpe.

Celle espèce est connue des mers de Chine, du Japon, de la

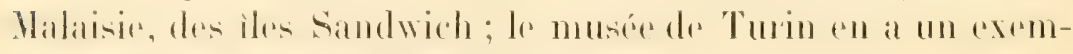
plaire du eap de Bonne-Espérance.

\section{Leander natator Edw.}

Patiomon natator II. Milne-Edwards, H. N. Cr., II, p. 393. - Dana, loc. cit, p. 588, pl. XXXVIIl, fig. 11. - Heller, Crust. sudl. Eur., 1863, p. 268, pl. IX, fig. 11-12. - Bate, Challeng. Hacr., p. 784, pl. CXXVIII, lig. 6-7.

Palæmon latirostris De Haan, F. Jap. Crust., p. 170, pl. XLV, fig. 12.

Leander natator Stimpson, Proc. Acad. N. Ac. Philad., 1860, p. 109. - De Man, Not. Leyd. Mus., III, p. 143; Arch. f. Jaturg., 1887, p. 563.-Ortmann, Zool. Jah b. Syst., V, p. 525. - Nobili, Ann. Mus. Napoli, 1, n 3, 1901, p. 6. Palemon torensis Paulson, loc. cit., p. 116, pl. XVII, fig. 3-3 f, 4, 5.

Périm (I. Jousseaume), 13 exemplaires; Aden (M. Jous-

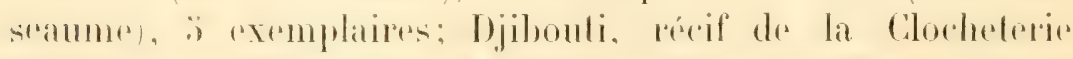

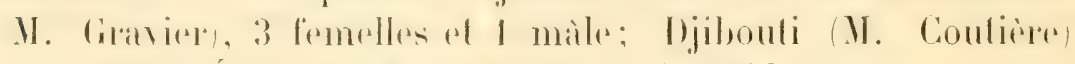
2 femelles; Éplhrée (Mus. Naples), 8 individus.

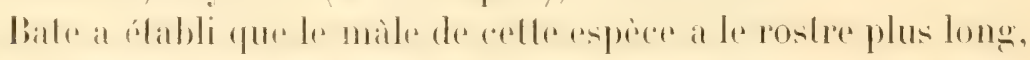

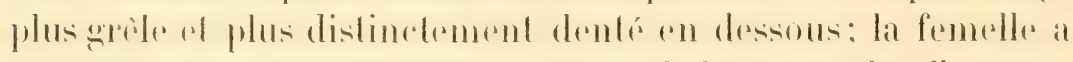

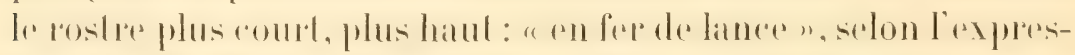
sion de II. Milne-Elwards. Ces differences sobservent ansis dans ces rexmplateres, mais les dents du borel inférieme du rostre sont tantôt plus, tantôt moins marquées.

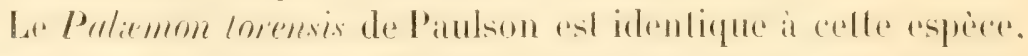

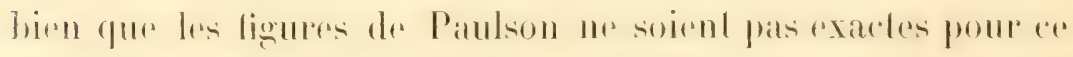

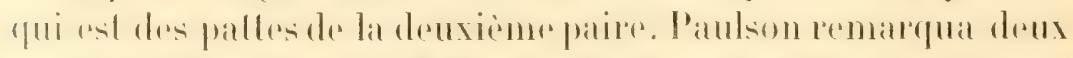
variélés: l'une, variété $\Lambda$, qui est représentée dans mes maté-

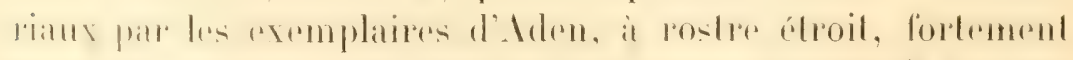

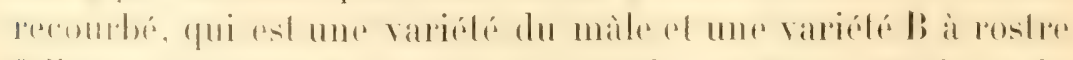

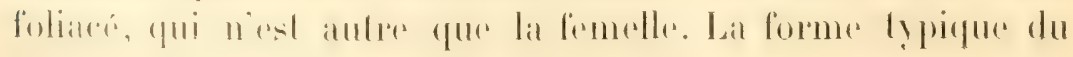
P. Corensis ast la forme normale du mâle. 


\section{Palæmon Audouini Hell.}

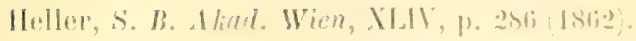

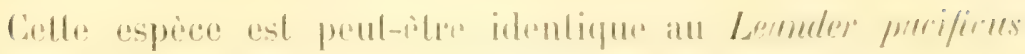
Slimpson, 1860.

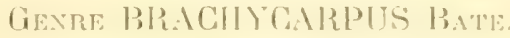 \\ Brachyearpus advena Noh. \\ (Pl. IV, lig. 1.)}

Sobili, Bull. Mus., 190:3, n" 6, p. 39:3.

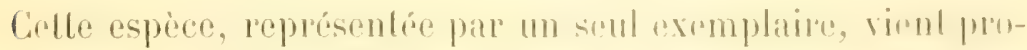
bablement de la mer liouge; mais si provenance n'est pas

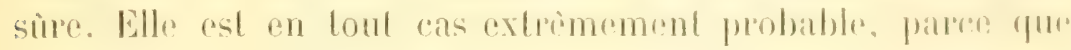

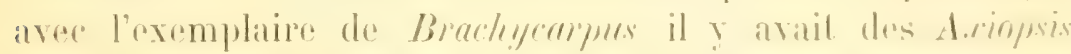

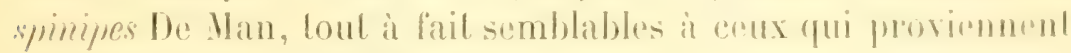
sirement de la mer Rouge.

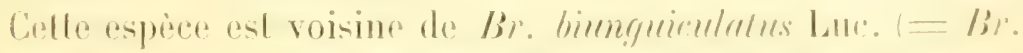

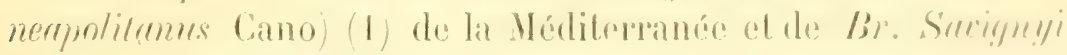
Piale de la Nourolle-Zélande.

Limique exemplaire at long de 3:3) millimelres de la poinde

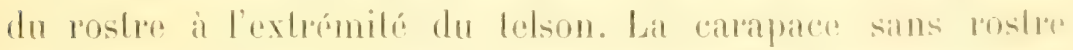

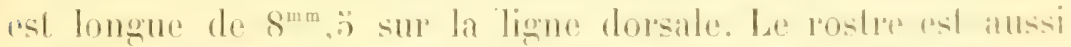

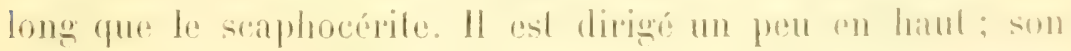

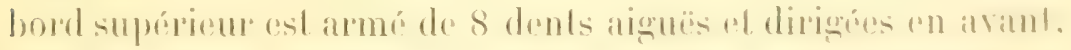

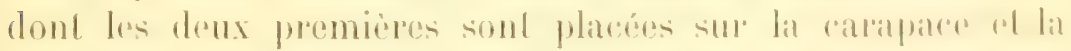

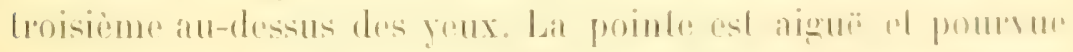

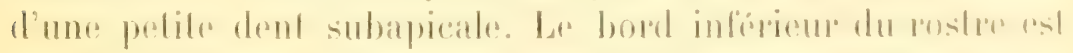

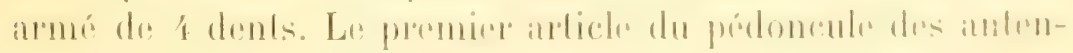

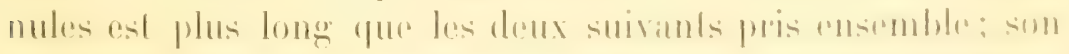

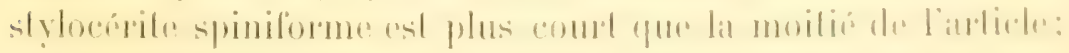

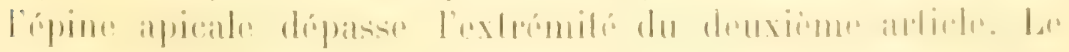

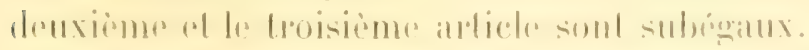

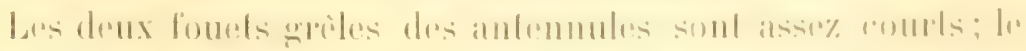

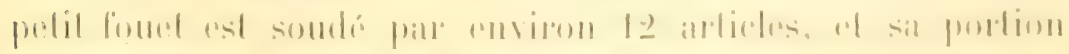

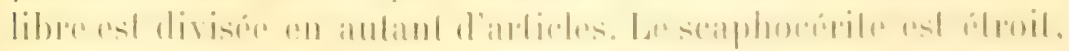

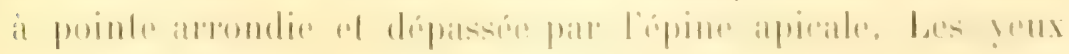

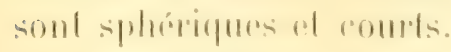

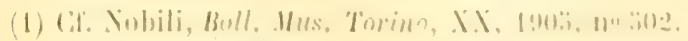


L'épine antennale est forle, l'épine hépatique aiguä, mais plus pelite.

Les maxillipèdes externes dépassent de plus de moitié de lenr avant-dernier article le pédoncule des antennes.

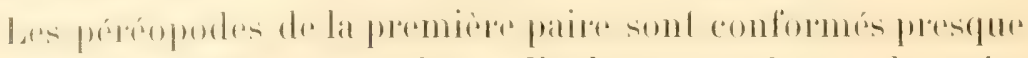

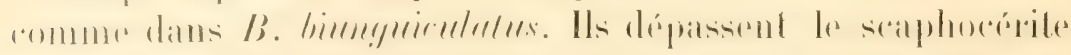
d'une partie de leur carpe; leur mérus est plus long que le

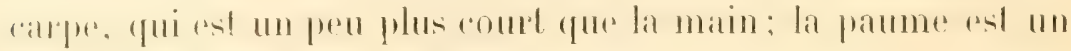
peu plus courte que les doigls.

Des paltes de la deuxième paire il n'y a que celle de droile,

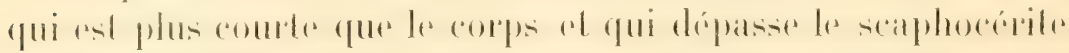
de plus de moilié de son carpe. Le mérus est cylindrique, un peu comprimé el armé à l'extrémité antérieure de son bord

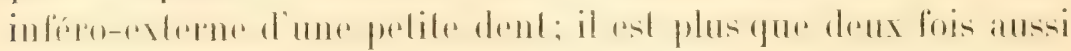
long que le carpe. Le carpe est obconique, à bord antérieur

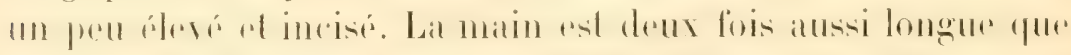

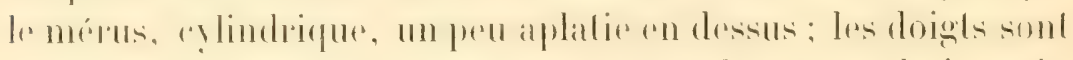
un pen plus routs que lat paume. Le bord interne de lis main est un peu concave, parce que les doights sont un peu tournés an dedians. Les doigts joignent bien, onl des petits poils du còté interne, et sont armés comme ceux de biumguiculalus. Les palles ambulatoires sont conformées comme celles de 1). limengluirulullux.

Les plempons des segments abdominams I-III sont arrondis. ceux des segments IV-VI angulaires et aigus. Le telson ne diflère pas de celui de biunguiculatus.

Celle espece est bien voisine du $B$. biunguiculatus dont elle

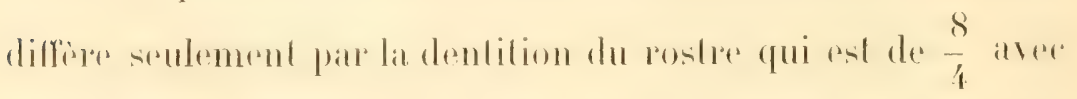

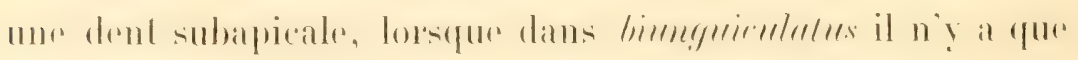
$\frac{7}{3}$ dents el pas de dent subapicale, par ses maxillipèdes

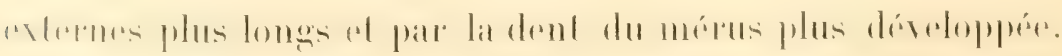
Les quatre espèces de Brachyrarpus comnues jusqu'ici peuvent être reconnues ainsi:

A. Daclylopodites avec un seul onguicule. Rostre avec $\frac{9}{b}$ dents..

B. Audouini Bale.

(Nouv.-Lélande). 
A. Dactylopodites avec deux onguicules.

B. Rostre avec $\frac{7}{3}$ dents. Main de la premiere paire de pattes plus courte que le carpe. Doigh de la grosse pince bâllants. Doight lixe avee deux grosses dents.

B. Sivignyi bate licemuda).

BB. Rostre avec $\frac{-}{3}$ dents. Mains de la première paire un peu plus longues que le carpe. Inoigts de la gronsice pince joignant bien et sans grosses dents. Mitrillipèdes externes dépassint le pédoncule des antennes avec le dernier article seulement............... B. biun!mirululus Lue (Nererie, sicile, golfe de Naples).

BBB. Rostre avee $\frac{8}{4}$ dents. Mains des denx premieres paires comme dans l'espece précédente. Maxillipirdes externes dépassant l'extrémité du pérloncule de plus de moilié de leur avant-rlernier article......... B. adrenre Vobs.

FAMILLE GNATHOPUYLIOLE Gantze GNATIOPIIYLLUM Lat. Gnathophyllum americanum Guirin.

Gnathophyllum anericanum Guérin, R. de la Sagre Hist. Cuba, III, p. xx, All. VIII, pl. II, fig. 1.1. - Rabhbun, Bull. U. S. Fish. Coumn., $1900(191) 1$, n" 2, P. 126.

Guuthophyllum fasciolatum Stimpson, Proc. Ac. Sc. Philud., 1860, 1). 28. - Ilas:well, Cat. Lustr. Crust., 1882, p. 181. - De .lan, 1ruh. f. Nat., 1887, p. isti, 16h. Senctient). (Ges., XXY, 1902, 1). 762.

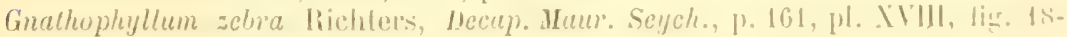
20 .

? Gnathophyllum pallidum Ontmann, \%. Jaheb., 1, 1890, 1. \$3.3.

Jjibouli (H. Couliere), 2 individus; iles Mushar (II. Ciritvier), un individu. Dans l'un des individus de l)jihouti l.

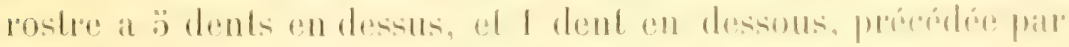

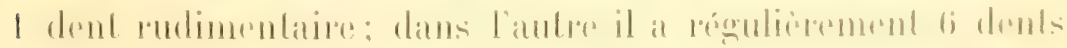

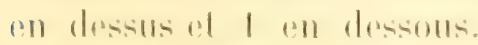

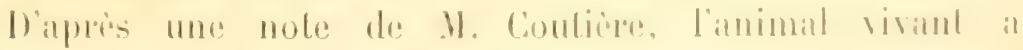

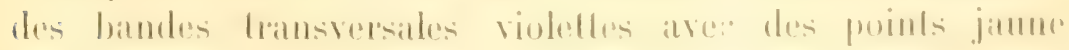
l'or:

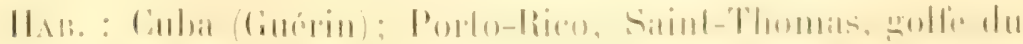

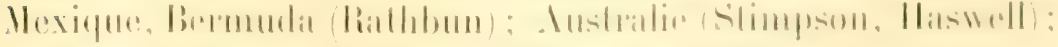

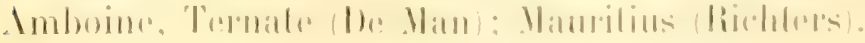




\title{
FAMILLE PROCESSIDA
}

\author{
Genre PROCESSA Leach. \\ (Nika Risso.)
}

\section{Processa Coutierei Nob.}

(I. I), liw. 3.)

Nobili, Bull. du Mus., 190', nº :, p. 234 (Diugn. prilim.).

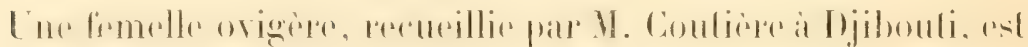
en si mauvais étal que je ne peux donnel qu'une partie des caractères. Cette espèce paraît voisine de $P$. ranaliculrula, mais toulefois elle en est différente.

Le rostre dépasse les yeux; vu de côté il est mince, presque droil el retroussé à la pointe; vu d'en haut il est triangulare, caréné en dessus et sur les côtés, cilié sur le bord et plus large

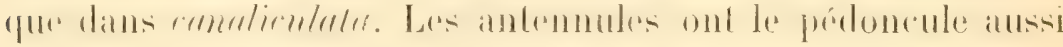

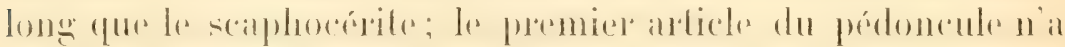
pas d'appendice; il est plus coml que les deux suivants pris ensemble, qui sont subégaux. Le gros fout est divisé en

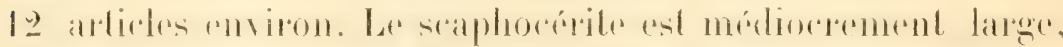

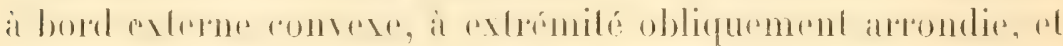

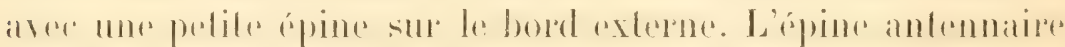
manque, mais l'angle entre l'orbite et la base des antennes est aigu.

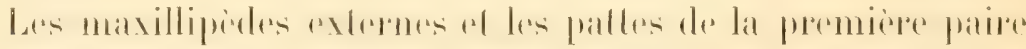
manquent. Il n'y a qu'une palte de la deuxième paire; son carpe est divisé en six articles.

Dans les palles HII a II. Ie propodile est plus rount que le carpe el dépourvu d'épines sur le bord inférieur; dans les

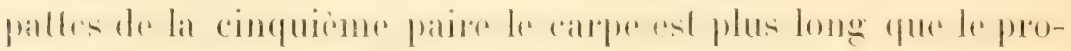

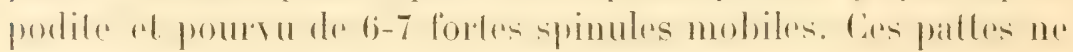
dépassent pas sensiblement le scaphocérite.

Le telson a une courte pointe, flanquée de deux épines de chaque côté, les externes coutes, les internes très longues; entre les hases des épines médianes, il y a de longues soies

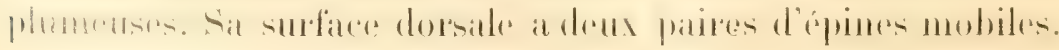

Longuenr, 15 millimètres.

Il y a encore dans les collections du Muséum deux pelits 


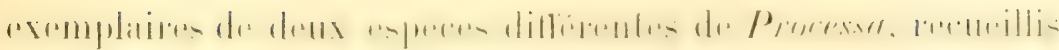
a Obock par M. Jousseaume, mais ils sont en frop mauvais chal pour une álude probitabla.

\section{Processa æquimana Pials.}

Nika xquimana Paulson, loc. cit., p. 97, pl. Xil, lizr. bi-tiu.

"Bord frontal quelque peu saillanl en avant. of pourvu

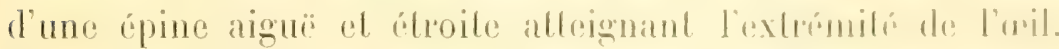
L'arlicte basilaire des antennules a, pres de la base, un appen-

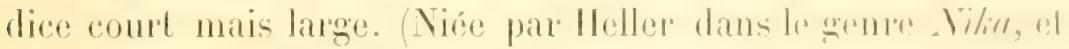
par consépuent absente dans $N$. pdulix.) Palfes-matehoires exlermes dépassant le pédoncule des antemules avec une patlie du pénultieme article. Sur le bord antérieur du criphatolhorax il y a une ipime antennale. Paltes de la deuxieme parion

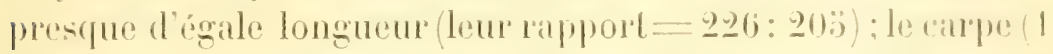
est divise dans le mime nombre d'articles que dans $N$. fremriensis, mais les quatre premiers ne sont pas lusionnic ensemble. Une femelle.

Longueur, 6 millimètres; longueur de lir carapace , $^{m,}, 0$ :

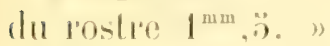

D’apres la figure de Paulson, le rostre sorigine dlune siallie semicirenlatere du bord frontal.

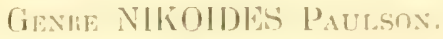

Nikoides Danæ Pauls.

$$
\text { II. ., Ii-.. 1.1 }
$$

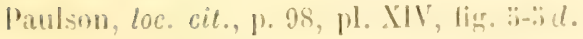

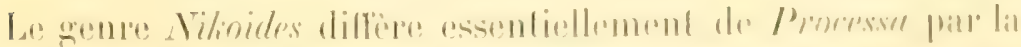

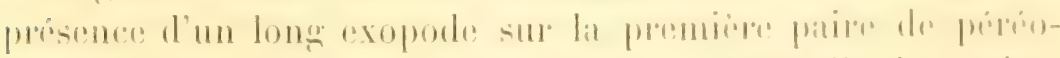

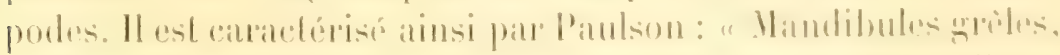

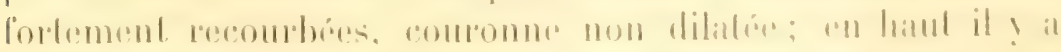

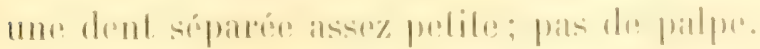

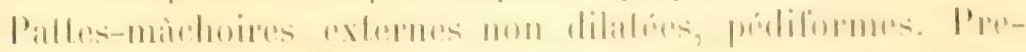

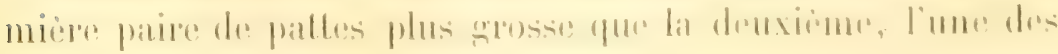

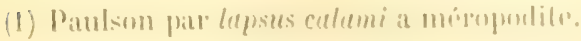




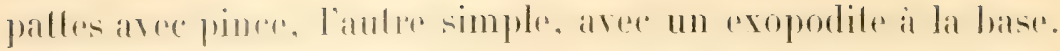

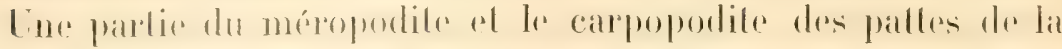

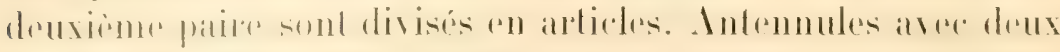

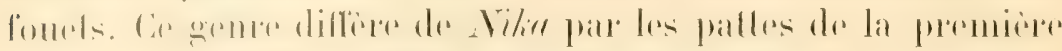

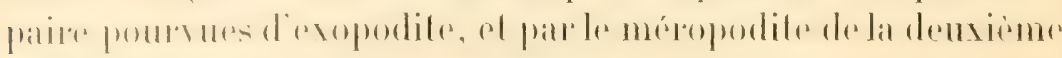
paire segmenté » (1).

J'en ai ru hrois exemplaires : une grosse femelle ovigire

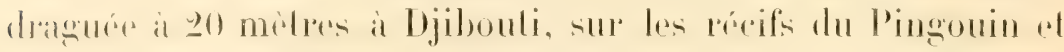
du Météore, par M. Ch. Gravier; une petite lemelle ovigère

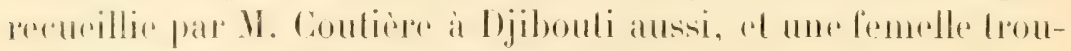
vée à Périm par M. Jousseaume.

Le rostre est caréné en dessus, recliligne, ou un peu tourné en bas, aussi long que les yeux (dans la figure de P'aulson un

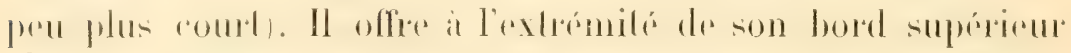
une dent, dirigée en avant en ligne droite el séparée de la

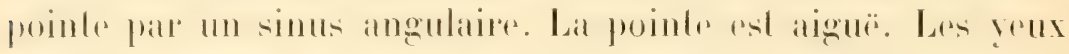

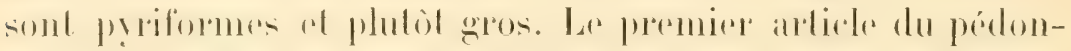
cule des antennules est plus long que le deuxième, excavé en dessus, cilié à l'extrémité, el pourvu à sa base du côté externe d'un appendice slylocérilique large, court et tronqué en aranl, mais pourvo d'une dent à l'angle latéro-exterme.

Le deuxième article est environ deux fois aussi long que le Lroisieme.

Le fonet externe est long moins de moitié de l'externe: celui-ci est long plus de moilié du corps. L'angle externe de

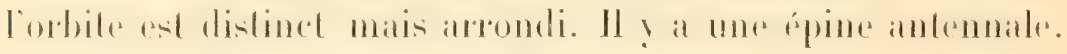

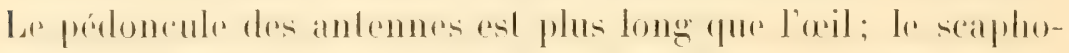

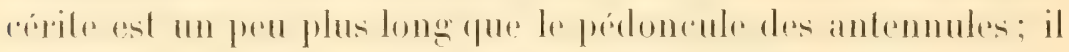

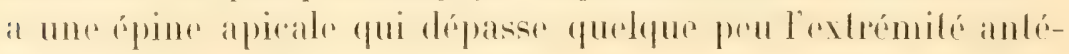
lieure.

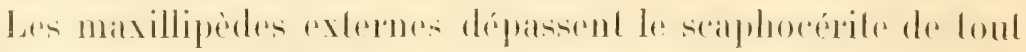

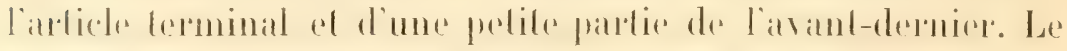

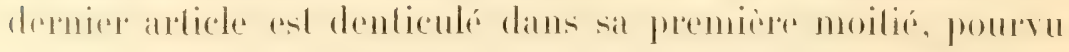
de 2-3 spinules et cilié. Les palles de la première paire sont un pou inégales, mais pas beaucoup.

(1) Line autre espèce de ce genre habite la mer Noire : ciest la $\boldsymbol{N}$. pontica Sowinsky (Voy. Sowinsky, K’faunie ralioolnuznich Cernavo Moriu (Mém. Soc. Scc. Kieff', 1882). 
Las doux pattes ont un exopode assez long. qui alloint he

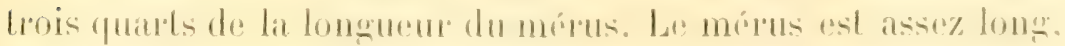

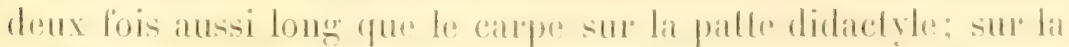

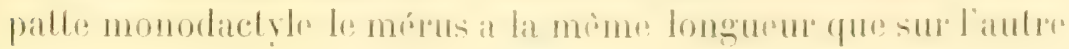

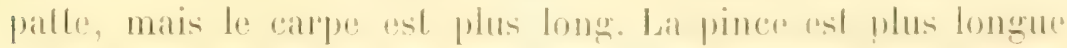

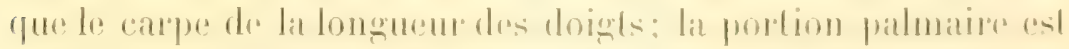

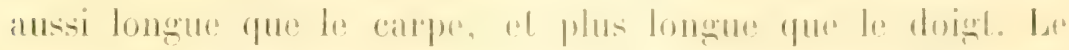
propodite de la palle monodactyle as notablement allongé.

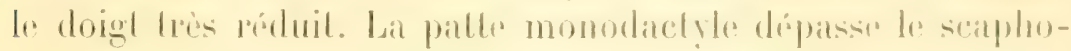
cérite de la longuene du doigh. Les patter filitormes de lit deuxieme paire sont tres inégales; celle de gatuche at plus courte que celle de droile: dle dépetsise le seaphocérile de presque toul le carpe, lorsque celle de droibre depasse le scaphlo-

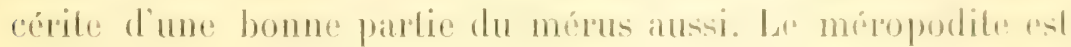
plus ou moins distinctement segmenté; d'apris la figure de

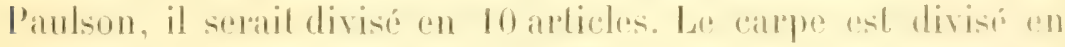

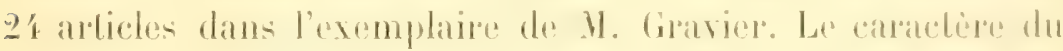
mérus segmenté n’a pas de valeur générique, paree que jai observé les mène fail dans des individus de Poncessen romelimelela Leach (- Vilie edulis Risso) provenant de Speziat.

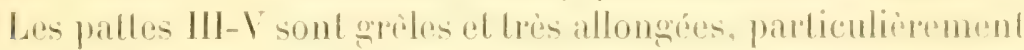
celles de la quatrieme paire qui dépassent le scaphocéribe de plus de la moilie de la bongueur du propodite. Les palles de lit

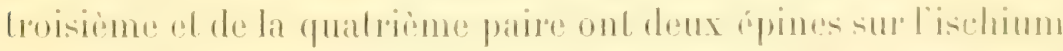

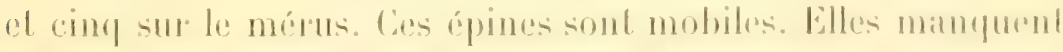

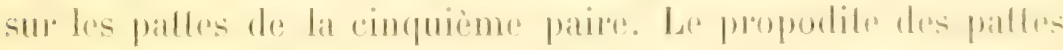

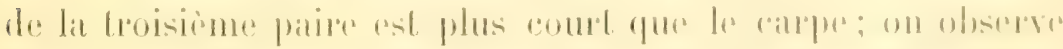

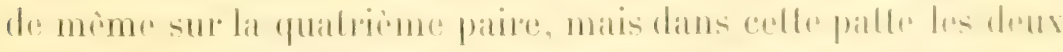
arlicles sont lodes longer le propodite des palles de la cin-

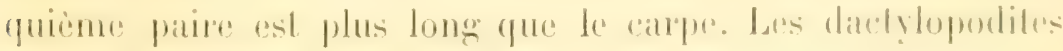
sont atsore courls al simples.

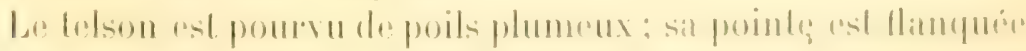

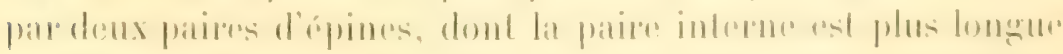
que lat parme axterme.

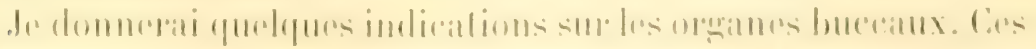

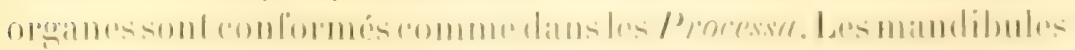

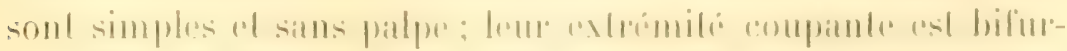

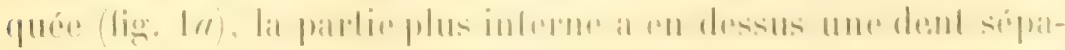

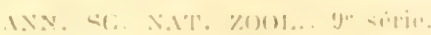




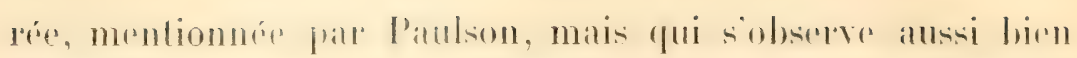

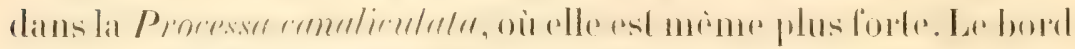

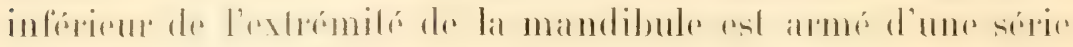

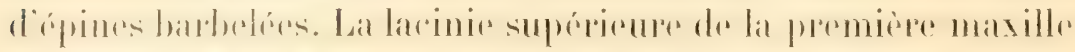

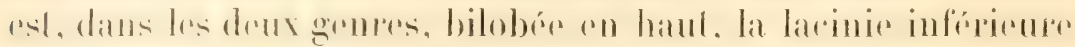

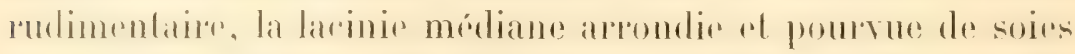

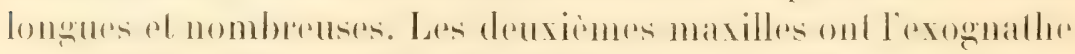

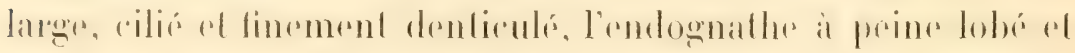

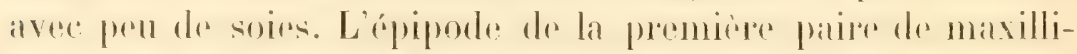

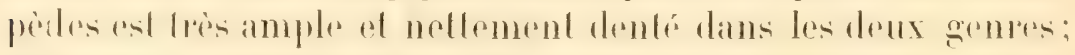
levognathe Hagedliforme a des leaces de division dans sal pautie

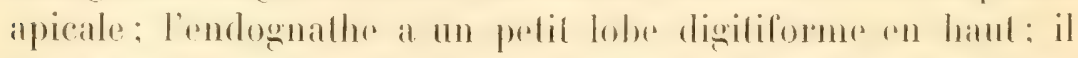
11 est plus divises ensule, mais former un larege lobe aree des

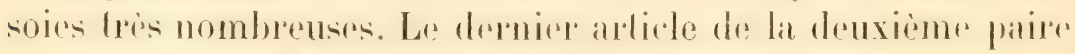

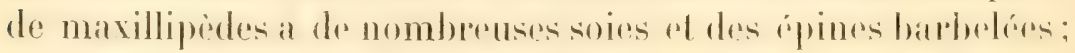

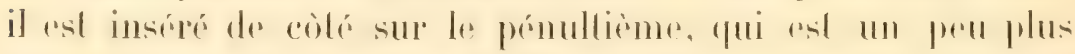

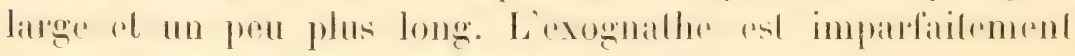
divisé en arlicles dans sa partie lerminale.

Ce genre au fond ne diffère du genre Processu que par les palles de la premiere paire pourvues d'exopodiles.

Cetle espèce atteint la haille de la Proressu cranulirulata.

\section{FAMILLE CRANGON/DA}

\section{Genre CORALLIOCRANGON Nobil.}

\section{Coralliocrangon Perrieri Nob.}

(Pl. IV, fig. 2.)

Nobili, Bull. du Mus., 1904, no :, p. 23.4 (Diagn. prélim.).

Les caracteres du genre peuvent etre élablis ainsi :

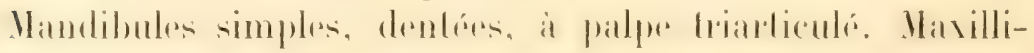

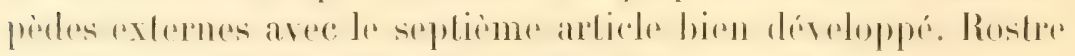

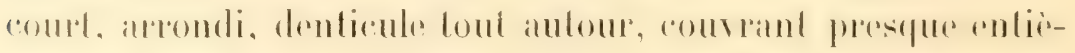

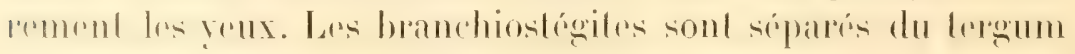

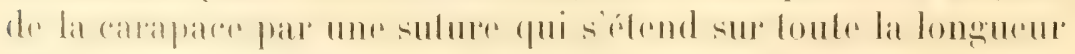

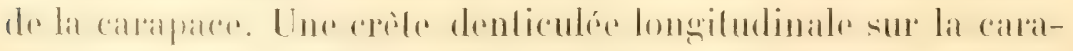

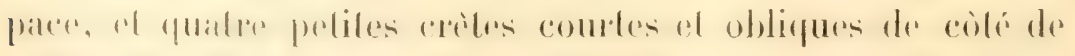

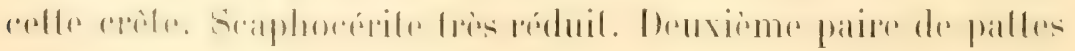




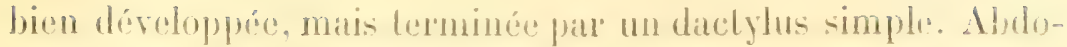
men lrès peu seuplé el sans apine ventate. Le premier segment est dépourvu de préropodes (1).

11. Coutière recureillit à Djibouli deux exemplatires, malleu-

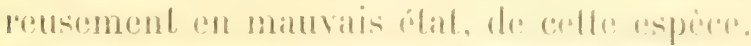

Lat calrapace est plutol aplatie al nollement divisie pall

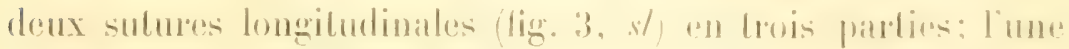
médiane dorsale el deux branchiostégaldes latérales. I air partie médiane dorsales de la carapace esl parenarue longilublinale-

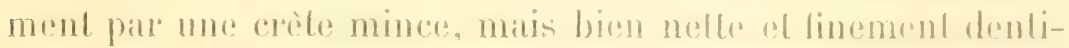

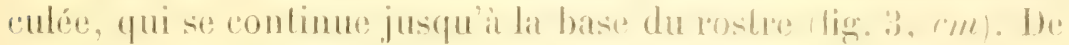

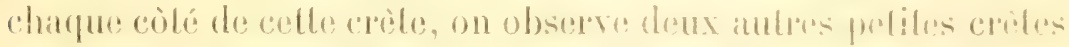

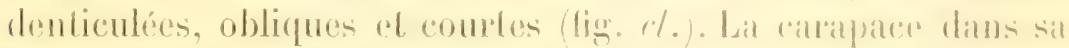
partie antérieure a des aspérités microseopiques. It rostrer sallit en avant sous forme d'une lame carrere mats formanl

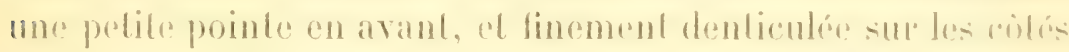

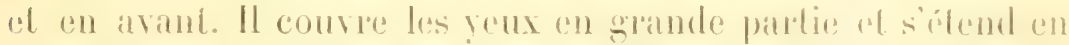

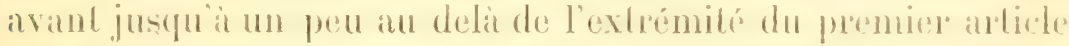
du pedoncule des antemules. Le bort orbitane est ansi denliculé el son angle externe est saillant el dentilopme. Les vens

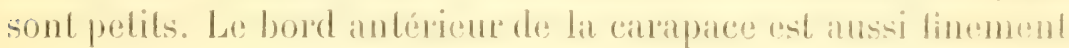

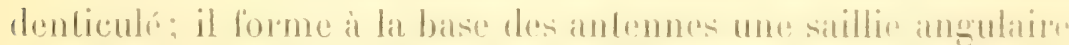

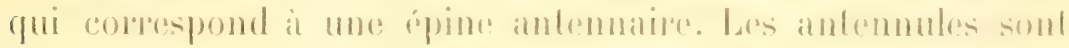

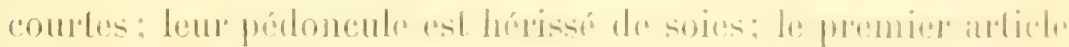

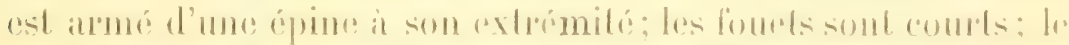

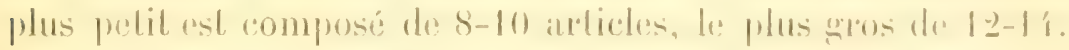

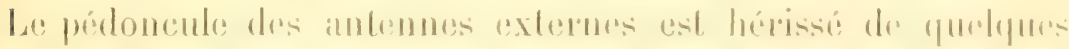

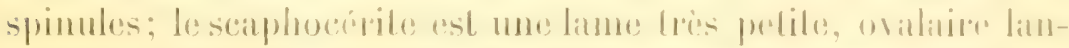

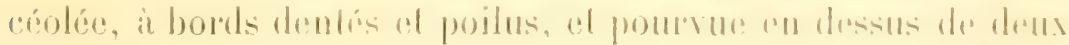
lignese d'aspérités.

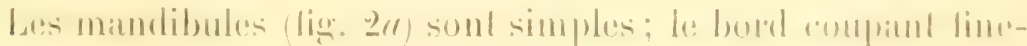

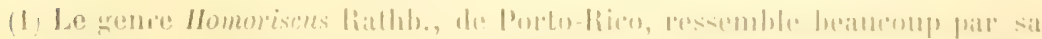

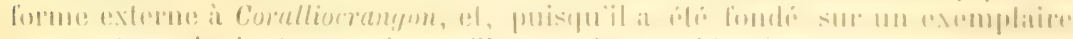

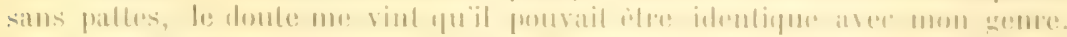

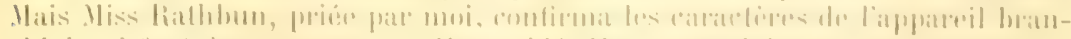

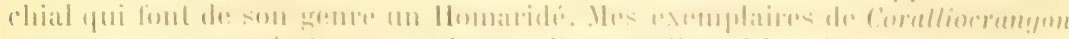

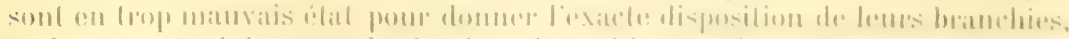

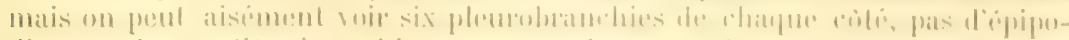

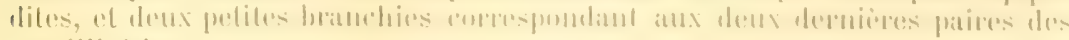
masillipiedes. 


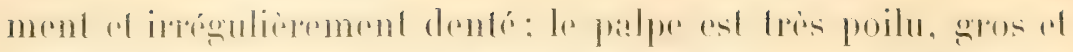

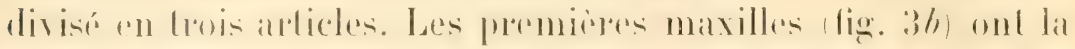

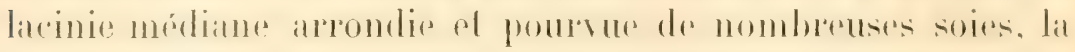

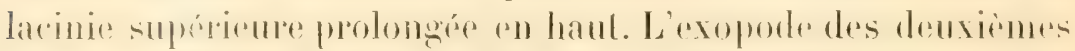

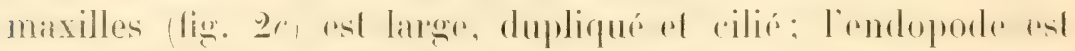

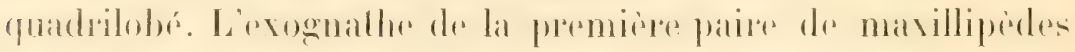

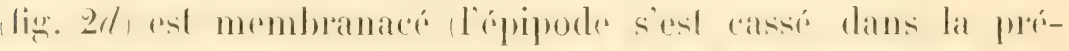

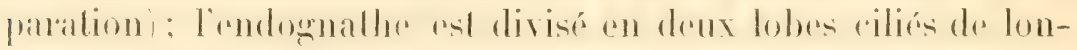

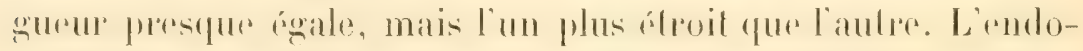

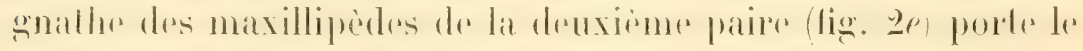

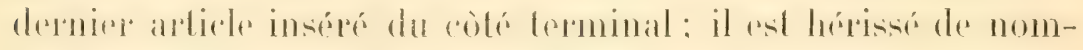

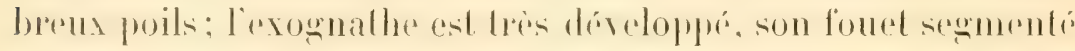

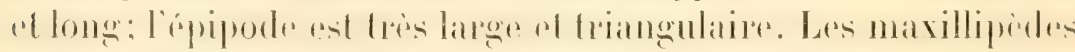

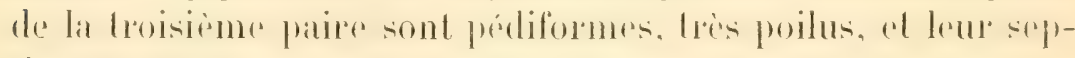
lième article est bien dévcloppé el ovalaire (fig. 2f').

Les palles de la première paire (fig. 2g) ont le mérus triangulaire, un peu denticulé à son extrémité; le carpe court el

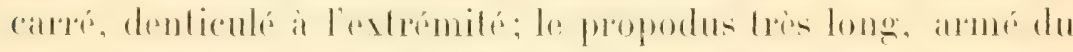

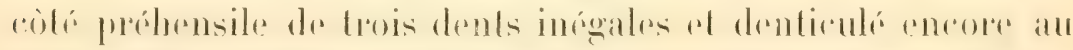

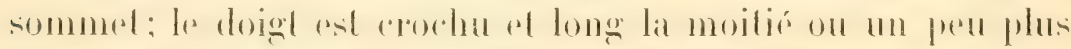

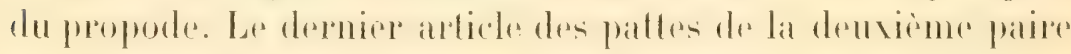

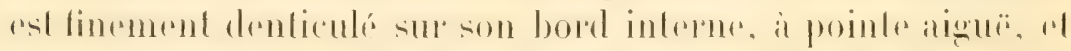
couvert en dessus d'une forte loulfe de poils (tig. 2h).

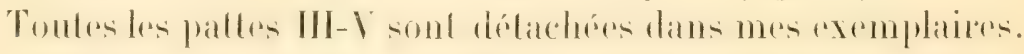

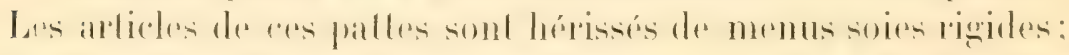

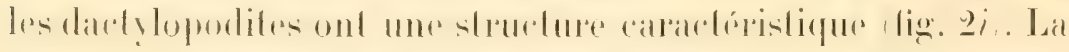

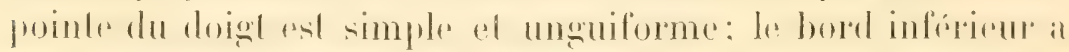
une rangée d'environ une trentaine de spinules menues el

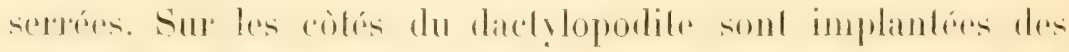
spinules mobiles.

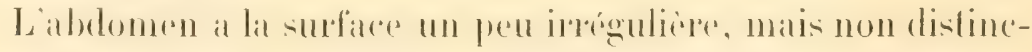

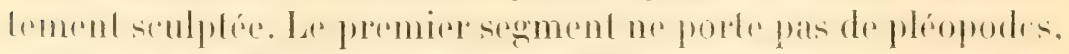

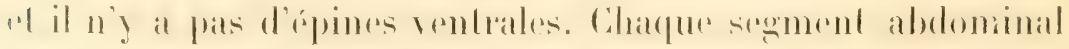

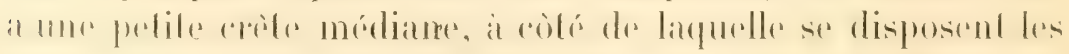

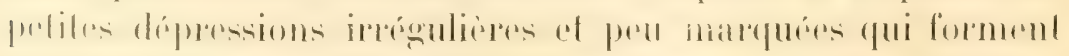

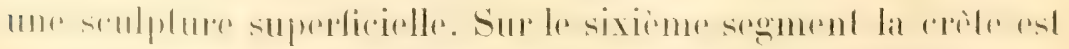

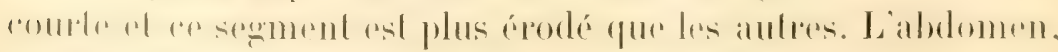

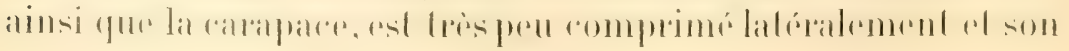


aspect, par la combure plus ample des arceaux, ast plubit homarien quencyphidien Las plemons sonl lous atrondis an arriere et tronguós sur la bord.

Le telson est arrondi it la pointe of lange, aver deux pelits

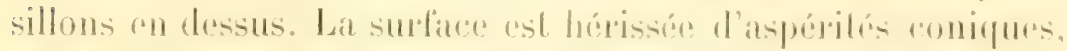
microscopiques. Les uropodes, arrondisct d'igale longueur, sont séparés par une sulure mobile en deux patdes: le long de lat sulure, il y a une ligne de spinules. Sur lo dos de lexopode

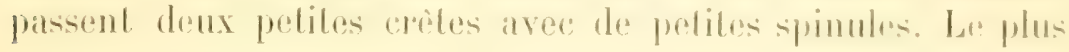
gros exemplaire est long do 16 millimblest

\section{STENOPIDEA}

FIMILLE STENOPIIISE

CHENRE S'TINOPJS IAT.

\section{Stenopus hispidus (Oliv.).}

Milne-litwards, II. X. Cr., 2, p. 407, pl. XXV, lig. 1; Cuv. R. Anim. C'rust.. pl. L, fig. 2. - Adams et White, "Samarang " Crust., 1830, p. 61, pl. 111. lig. 6. - Dana, loc. cit., p. 607, pl. XI. fig. 8. - Bate, Challeny. Macr., p. 211. pl. XXX. - De Man, dreh. f. Nat., los̃, p. \$66. - Rathlun. Bull. U. s. Fish Commission, 1900, vol. II (1901), p. 99.

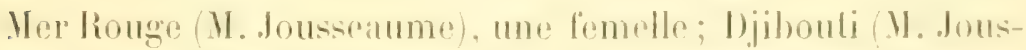

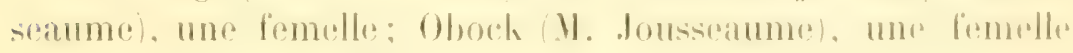
ovigere; Ijibouli (1). Conlieme), un individu jenme.

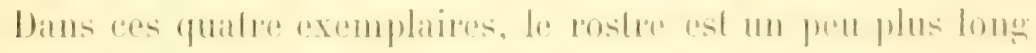

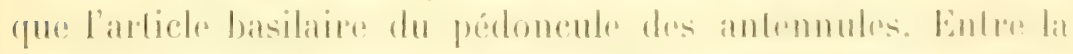

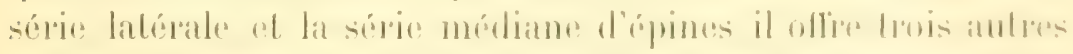

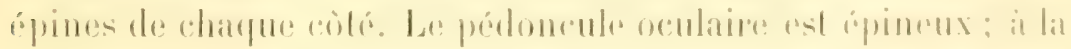

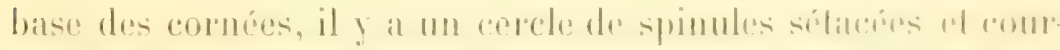
beres qui enclosent l'ail ab le prolignent.

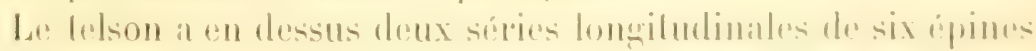
chiteime.

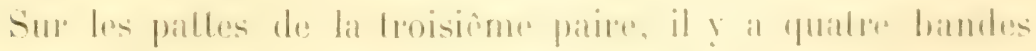

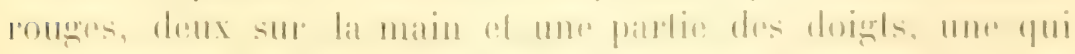

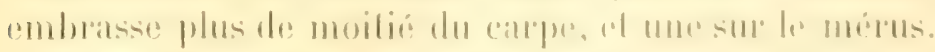

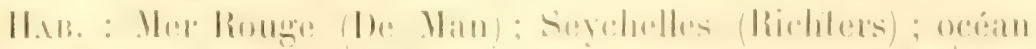

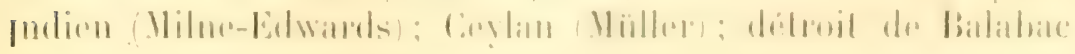




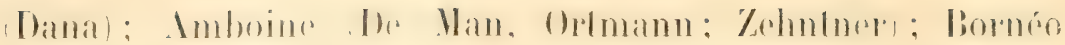

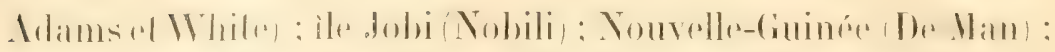

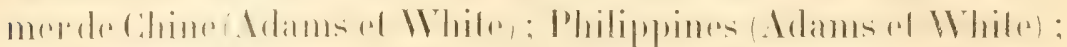

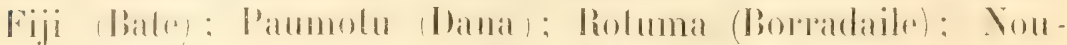

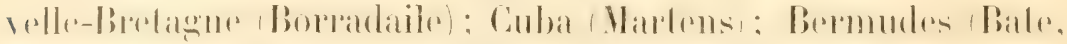
Herrick); Porto-Rico, Floride, Jamaïque, Santa Lucia (Rathbun).

\section{Genre S'I'ENOPUSCUI,US Richters.}

\section{Stenopusculus crassimanus Richters.}

Richters, Decap. Maurit. Seych., 1880, p. 168, pl. XVIII, fig. 27-29. - De Man, Areh. F. Wht., 188i, 1. itiii.

Djibouli (M. Coulière), 4 individus.

Les individus de Djibouli s'accordent mieux avec les exem

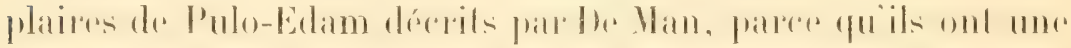

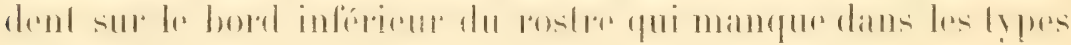
de Maurilius.

Le rostre a 4-ä dents sur le bord supérieur el une portion distale sans dents phus ou moins longue. Les deux épines audessus el au-dessous de l'insertion des antennes sont les plus

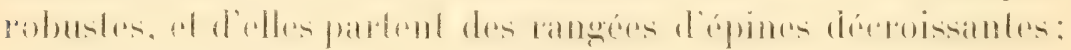
celle qui part de l'épine inférieure décourt en série rectiligne

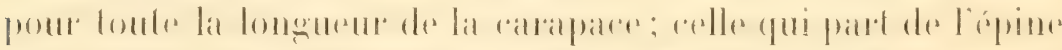

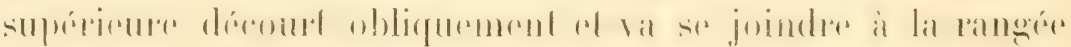
inférieme. La surface de la carapace est en plus hérissée de spinules tris petiles.

Le carpe des chélipèdes offre une face supérieure lisse délimilée par deux crêtes denticulées. Le reste de sa surface est lıérissé de petils granules spiniformes. La main a en dessus

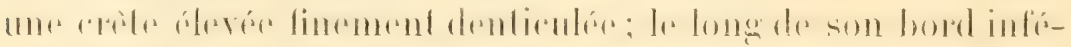
férieur, il y a deux rangées de denticules, rapprochées el parallieles.

L'exopode des uropodes a 10-12 denticules marginales,

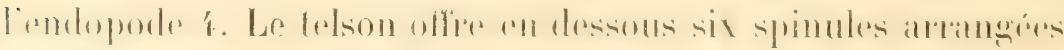
en deux séries parallèles de trois épines chacune.

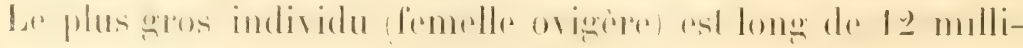
mètres.

Cetle espèce n'est connue que de Maurilius el Pulo-Edam. 


\section{REPTANTIA}

LORICATA

\section{FAIILLE SCYLLA RIII) E}

GENRE SCYYIARUS FAm.

(Aretus Jana.

\section{Scyllarus pumilus Nol.}

Tobili, Bull. Mas., 190:, n" 6, p. 39:.

Un exemplaire conservé it sec du Muséc le 'Torin, provenanl

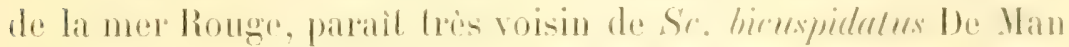

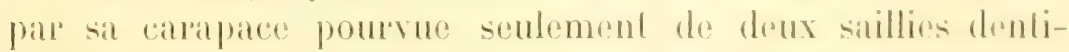

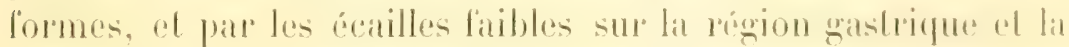
cordiale. Il en differe par l'extremilé anteriener du sternum

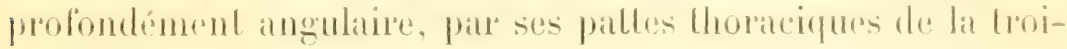
sieme el de la quatrieme paire non sillonnées; par les dents du dernior arlicle de l’antenne (en nombre de $(j)$, séparées pitr des pelifes incisions, landis que les incisions sonl plus pro-

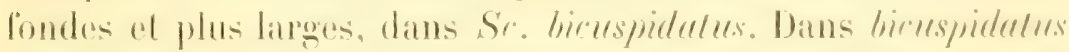
il y a en arovere de la deuxieme saille, entre celle-ci el lo sillon corvical. 2 pelites dents Do Man in lill.) plarene l'ums

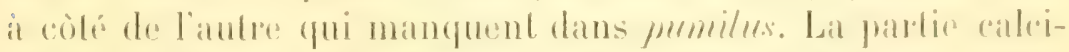

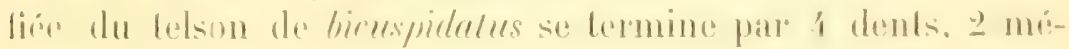

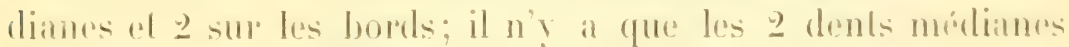
(lians S'. jum milus.

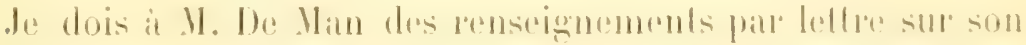

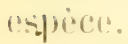

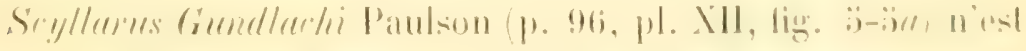

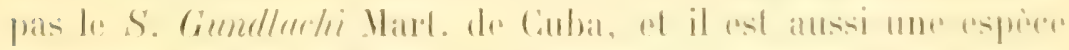

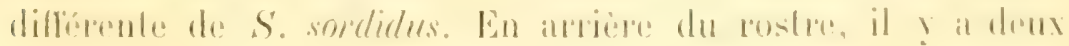

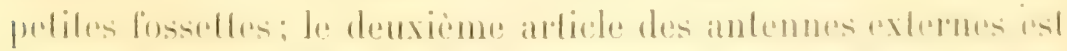

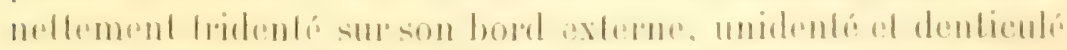

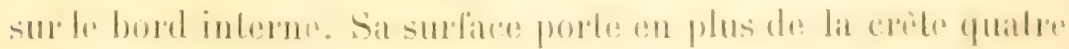

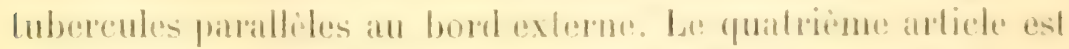

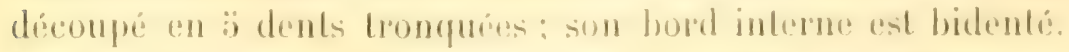

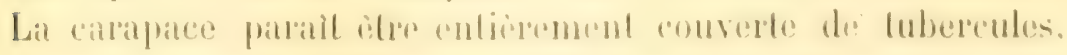

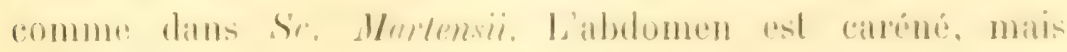




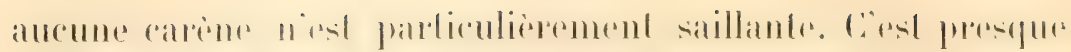

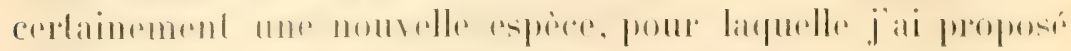
le nom de Scyllarus Paulsoni (l. cil., p. 393 ).

\section{GENRE THENUS.}

Thenus orientalis Rumph.

II. Milne-Edwards, II. N. Cr., t. II, p. 286; Atl. Cuv. R. Anim. Crust., pl. XLI, lig. 2; Ilaswell, Cat. Austr. Crust., p. 170. - De Man, Areh. f. Xat., 188i, p. 485. - Bate, Challeng. Macr., 1888, 1).66. - Ortmann, Zool. Juhrb. Syst., VI, 1891, 1. 46. - Henderson, Trans. Linn. Soc. (2), V, 1893, p. 433.

Massaouah (M. Faligali, Musée de Turin), une dizaine d'exemplaires.

\section{FAIILLE PALINURIDAE.}

\section{Genre PANulirus Gray.}

Panulirus penicillatus (Oliv.).

Pulinurus penicillatus Olivier, Enc. Wéth. VIII, 1811, p. 674. - Milne-Ldwards, II. N. Cr., II, p. 299. - Stimpson, Proc. Acad. Philad., 1860, p. 28. - De Man, Not. Leyd. Mus., II, 1880, p. 18\%. - Ilaswell, Cat. Iustr. Crust., 1882, p. 172. - Richters, Decap. Maur. Seych., 1880, p. 161.

Palinum Ehrenbergi Ileller, S. B. Acad. Wien, XLIV, 1862, p. 260, pl. II, fig. 8; Novara Crust., 1868, p. 9\%. - Iloflmann, Crust. Echinod. Iadagascar, p. 30, pl. VIII, fig. 6.

Palinurus (Senex) penicillatus Pfelfer, Verh. Nat. Ver. IJamburg, 1881, p. 3i. Senex penicillatus Ortmann, Zool. Jaherb. Syst., II, 1891, 1. 28.

Panulirus penicillatus (Oliv.) Bate, Challeng. Wacr., 1888, p. 82, pl. XII, p. 2.

- Borradaile, Proc. Zool. Soc., 1898, P. 1014; Willey's Zool. Res., IV, 1899, p.419. - Henderson, loc. cit., p. 433.

Ner Rouge (Musée de Turin), un gros mâle.

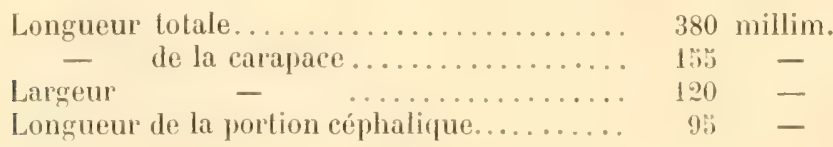

La carapace est très hérissée d'épines, mais celles-ci ne sont pas entourées de poils; il n'y a pas de poils sur la carapace. L'anneau antennal a quatre épines fortes, inclinées

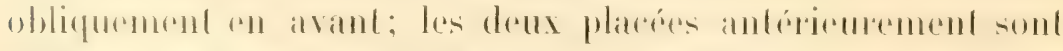

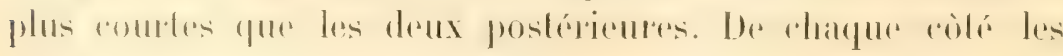
épines sout soudées à la base; les quatre épines forment 
ainsi deux groupes sépares par un espatee libre. L'angle antó-

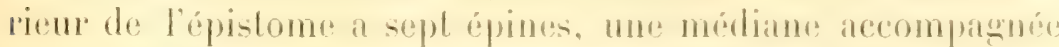
par deux latérales presque soudés avee elle par la basi: drux grosses aux extremités at une de chargue cotri plus pelile entre les latérales el le groupe médian.

Les maxillipedes de la troisieme pare ont un exopode combl qui alteint à peine la moilié de l'ischium: les maxillipardes de la deuxième paire ont un exopode poursu d'un long louel divise en 14 arlicles.

Le pédoncule des antennules dépassese celui das antennos de tout le dernier article el d'une partie du pémulliemo. Lu basipodite de la cinquième paire de palles a unu ápine probuste du còté axterme.

Les sillons des segments abdominaux ne sont pas inturrompus; le bord antérieur de chaque sillon n'est pas erénelí.

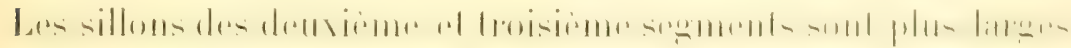
el plus profonds, ceux des segments 4 el $\ddot{j}$ sonl plus átroils u plus superficiels; celui du sixieme segmenl est le plus large de

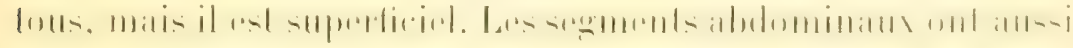
des grosses ponchalions.

Les cornes épimériennes du deuxieme segment ont i danls str le bord antérien el 2 sur le lobe; celles du troisieme sogmentrespectivement 2 et 1 ; celles du quatrieme of du cinquieme segment 1 el 1; celles du sixieme segment nonl pas de denls.

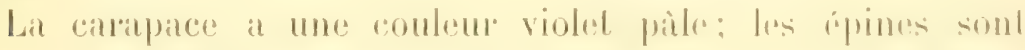

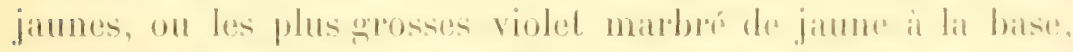

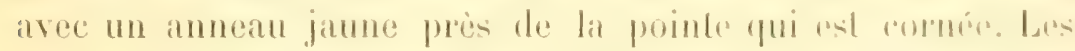

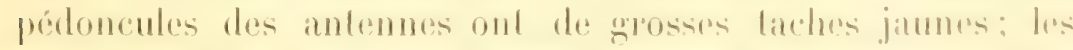
pédoncules des antenmules sont rayés longiludinalemonl de jature sul fond violet. Les palles ont lat mòme disposilion de

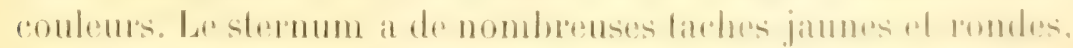

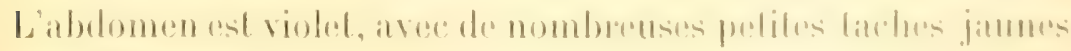

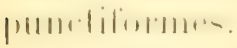

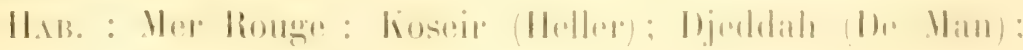
Nkabah (Miorsi).

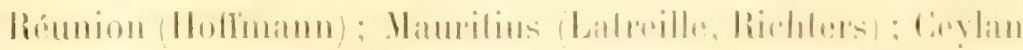

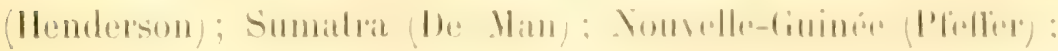




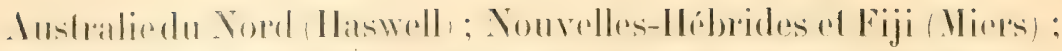
Tahili stimpson, Bale); Roluma, iles de la Loyaulé (Borrat(laile); mer du Sud (Ortmann).

\section{Panulirus homarus (Herbst).}

Pfefler, Mitth. Nat. Mus. Hamburg, 1896 (1897), p. 255-263 (ubi syn.).

Cancer homarus Herbst, Nat. Krabb. Krebs., 1796, p. 86, pl. XXXI, lig. 1.

Palimurus omatus Fabricius, Suppl. Ent. Syst., 1798, p. 400. - Milne-Edwards,

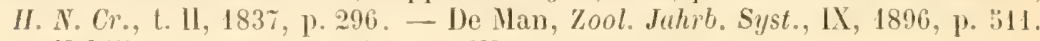

- Nobili, Inn. Mus. civ. Genova, XL, 1899, p. 243.

Obock (M. Jousseaume), 2 jeunes mâles, longs de 83 el

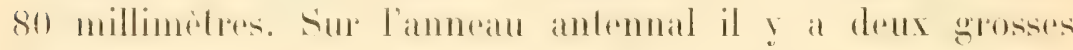

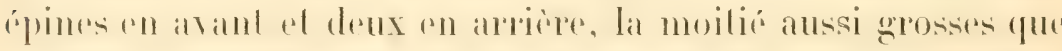
les premières; entre ces quatre épines, il y en a deux autres très petiles. Les épimères abdominaux ont 3 -4 dents.

Celle espèce est répandue de Zanzibar ì la NouvelleGuinée.

\section{Panulirus sp.}

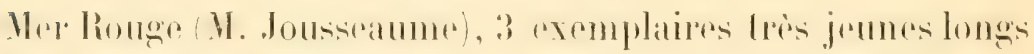
de 18,19 et 20 millimètres).

les indirdus sont indelerminables, soil paree quils sont fres jeunnes, soil aussi falee quils sont roisins de plusirurs espèces, sans concorder entièrement avec aucune.

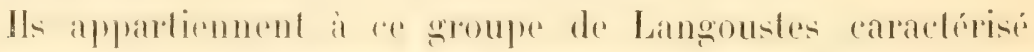
par l'absence de palpe atux maxillipedes exlernes, el de foued

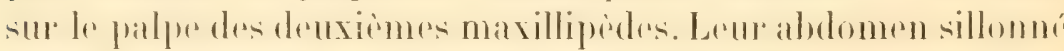

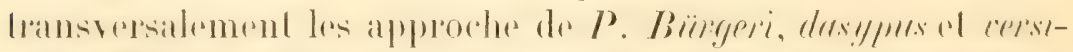
rolor. Vais les sillons non inlerempom les séparent des deux dernieresesperese pendant que le nombre el la disposilion des

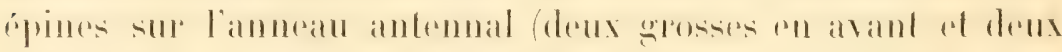

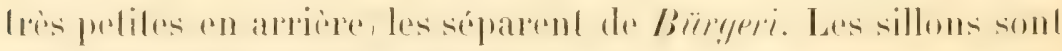
cénelés sur leur bord antérieur.

\section{Genre puerulus Orta. \\ (Puer Ortm.)}

\section{Puerulus spiniger Ortm.}

Ineer spiniyer Ortmann, Denkschr. Mel. Nat. Ver. Jena, VIII, 1894, p. 19, pl. II, liv.:. :. 


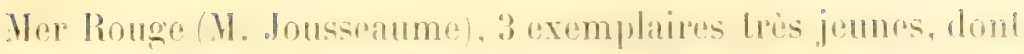
le plus gros mesure 1!) millimitres de lomemome.

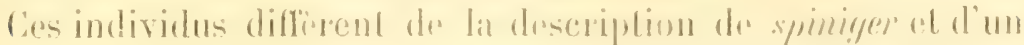

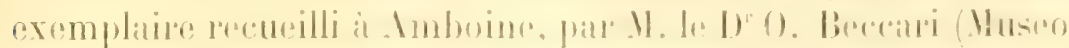

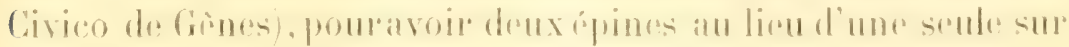
la crote latépale de la carapace.

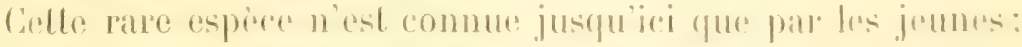
olle a été aussi signaléesenlement it Amboime.

THALASSINIDEA (I).

\section{FIIIILLE AYIIT) A}

GENHE A.TOPSIS BORTADAIL:

\section{Tableau des Ariopsis de la mer liouge:}

4. Portion dorsale antérieure de la carapace aplatie. Serapho- al slylocripilu asiez développés. Wains longues.

li. Extrémité des segments sternaux des deux dernières paires de patles spiniformes. Éailles it la base des

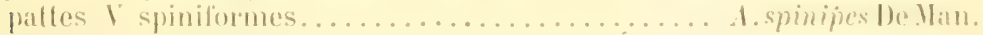

BR. Extrumilé dus segments sternaux obtuse. Eeailles

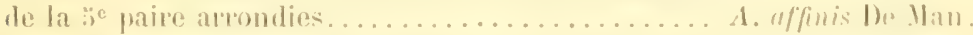
1. Carapace sans aine aplatie. Scapho- et stylocérile trís

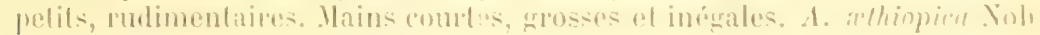

\section{Axiopsis spinipes (1) Mall.}

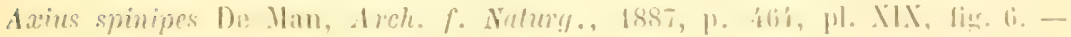

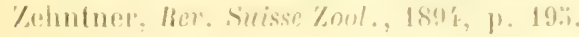

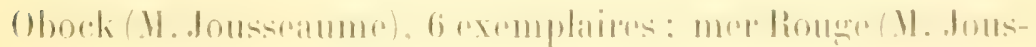

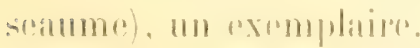

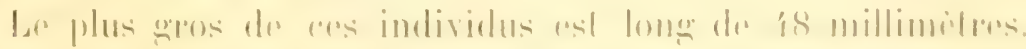

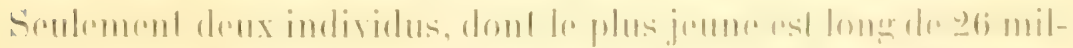

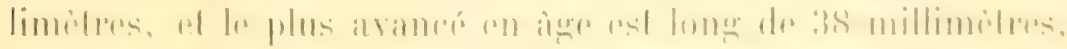

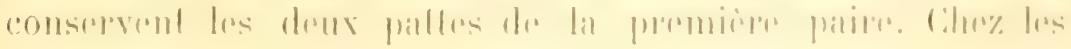

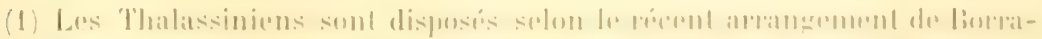

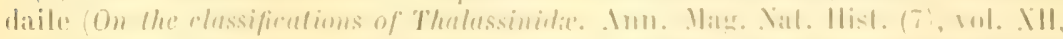

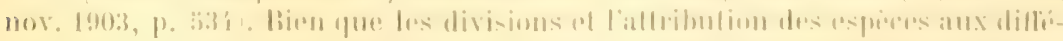

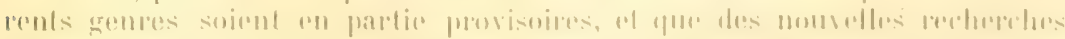

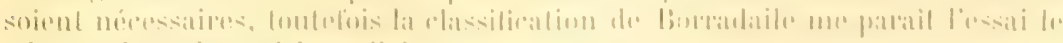
plus rationatel tonte justuriati. 


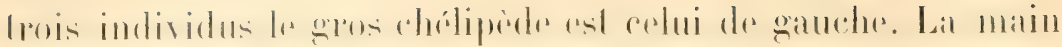

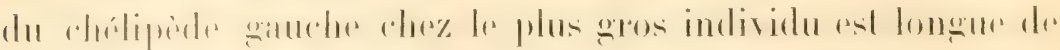

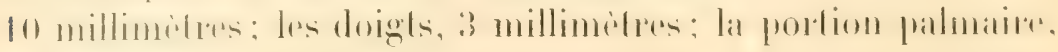
7 millimètres.

La hauteur de la portion palmaire est de 3 millimètres; la

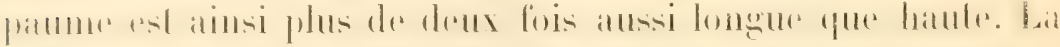

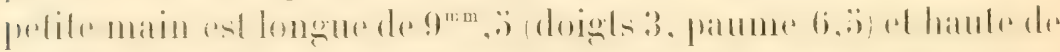

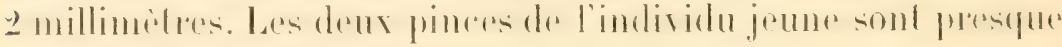
égales de dimensions.

Celte espèce est un peu variable. La petite dent sur le bort

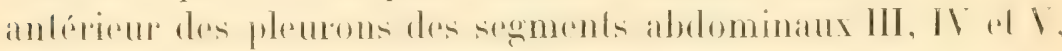
manque chez trois exemplaires, mais est présente chez les

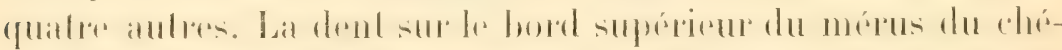

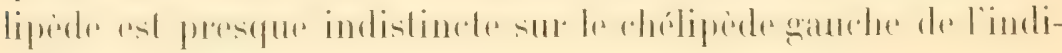
vidu plus pelil.

Celte espèce n'avait jamais élé trourée dans la mer Rouge. Elle a été signalée à l'ìle Noordwachter et à Amboine.

\section{Axiopsis affinis De Man.}

Axius affinis De Ilan, Areh. f. Naturg., 1887, p. 469, pl. XX, fig. 1.

Obock (11. Jousseaume), b̆ exemplaires.

Les différences entre celte espèce et celle qui précède sonl ássez menues, mais constantes, et je crois ainsi que les deux formes peuvent bien ìtre séparées spécifiquement.

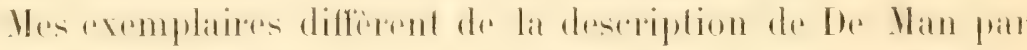

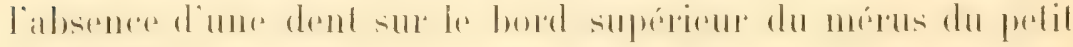
chélipède.

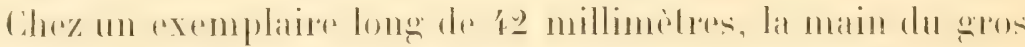

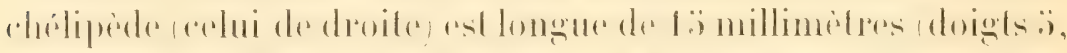

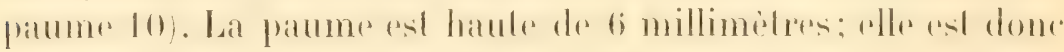

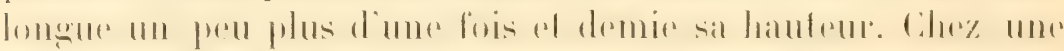

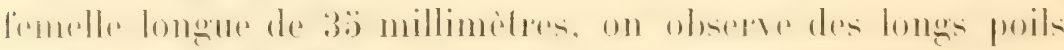

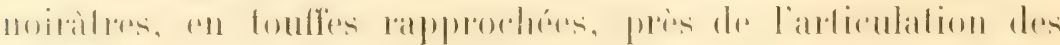

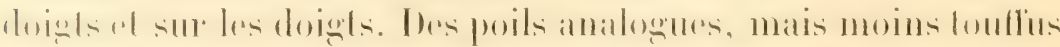
et plus clairs, s'observent aussi plus ou moins bien chez les autres exemplaires.

Les mains de lous les individus sont carénées sur le hord 


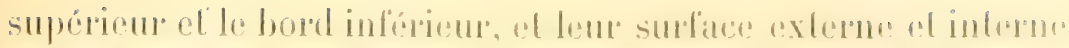
est converte de granulations déprimres el squamitormes.

Nouvalle pour la mer Rouge. Inabile Imboine.

\title{
Axiopsis (Paraxiopsis) æthiopica Nul.
}

\author{
(I). VI, lig. 1.
}

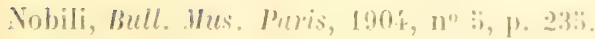

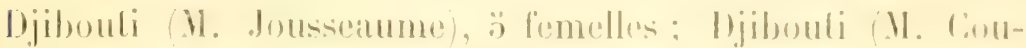

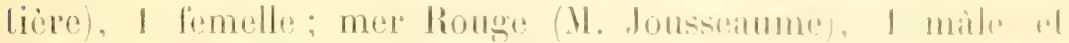

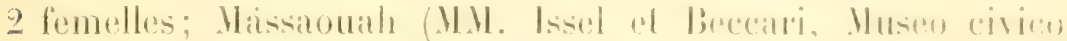
de Genes), I lemelle.

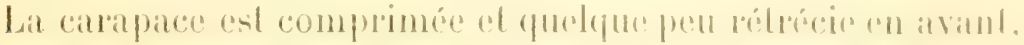

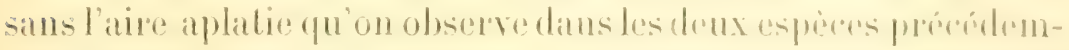

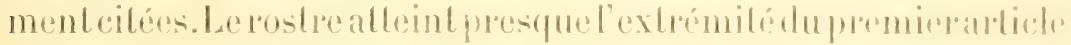
du pédoncule des antenmules; il asl done plus long qua les yox.

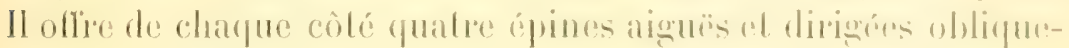

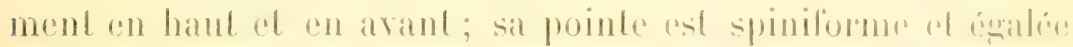

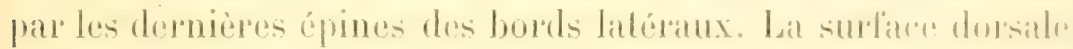
du rostre, an pen en arriere de la pointe, porte une sirie dre cinc épines aigues, robustes el dirigées en avant, qui śatend

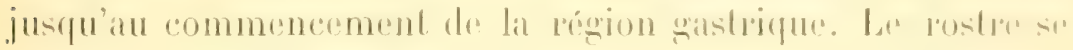

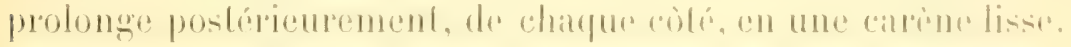

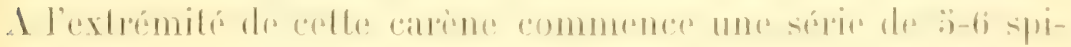

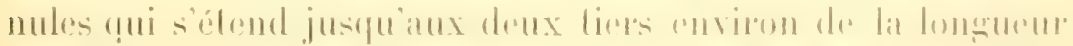

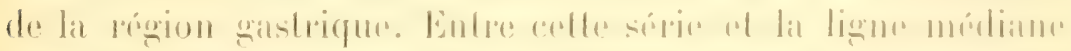

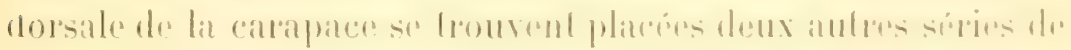

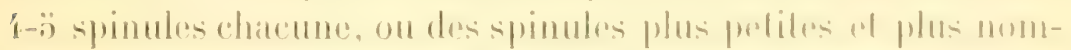

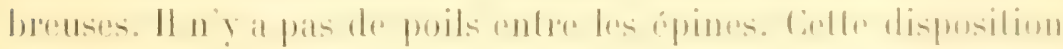

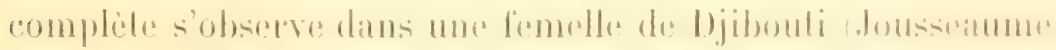

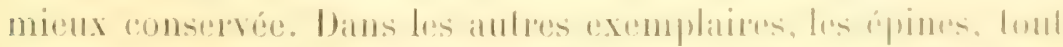

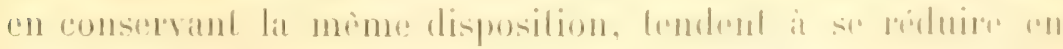

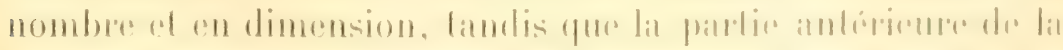

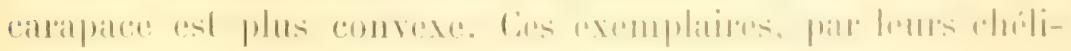

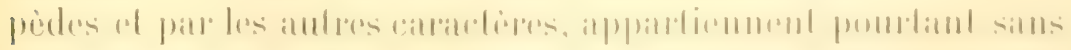

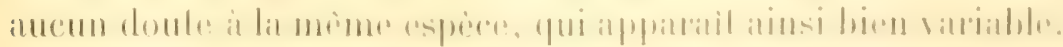

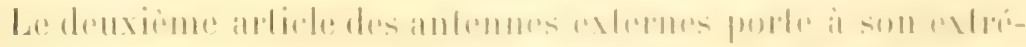

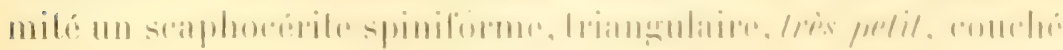


sur la surface supéricure du tro isièno artiele, et saillant en dent

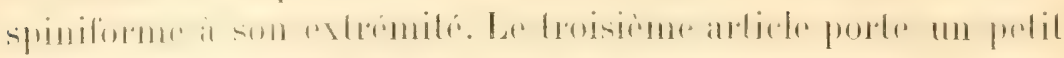
slylocérite à l'oslrémité supérieure, inmédiatement en avant de In dent scaphocéritique, el cel article apparail ainsi, au

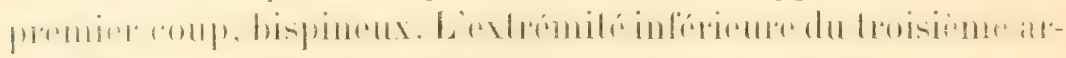
licle porte aussi une dent spiniforme, plus pelite que le stylocérile. Scapho-etslylocérite sont benuenup) plus pelits que dans les deux espèces précédentes (lig. la).

Les fouets des antennules mesurent en longueur les deux liers de la longueur de la carapace.

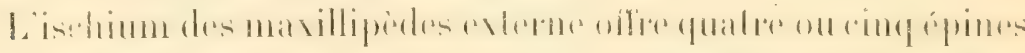
qui croissent en longueur de la base à l'extrémilé de l'arlicle.

Les pattes de la premiere pare sont fortement inégales, lant par leur forme que par leurs dimensions. Le coxopodite offre une pelite saillie conique ì son extrémité antérieure, mais non une véribable épine. Le basipodile est entierement inerme.

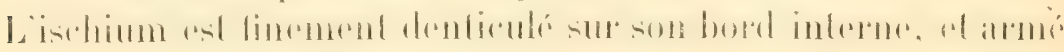
d'une pelite épine obtuse it son extrémité. Le mérus est long un peu moins que deux fois sa largeur; il est convexe sur sil lace externe. Son bord inférieur est denticulé, les dents sont

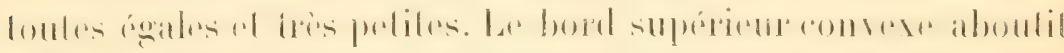
cn une pointe obtuse el courle. Le bord latéro-antérieur se

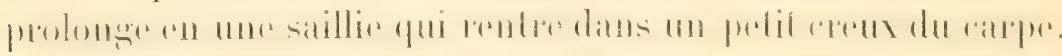
Le carpe est long les trois quats de sa plus grande hauleur, laiblement caréné sur son bord supériem el pourvu d'une dent obluse sur le bord inférieur. La main est bien concexe extérieurement. La paume est plus longue que les doigls main,

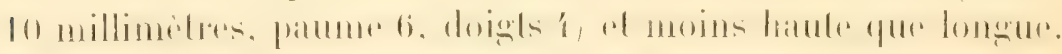
Elle est presque entierement lisse sur sa surface externe.

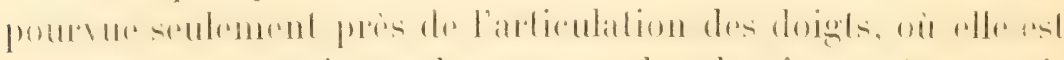
un peu concave, de quelques granules déprimés el squami-

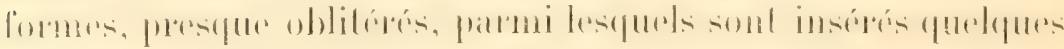
poils jaunatres. Il y a aussi de rares poils sur le restant de la surface. La face interne est aussi convexe el lisse, el offere aussi des granules pres de l'arliculation des doigls, mais moins dislincts que ceux de la fice externe. Les doigts sont sillonnés longitudinalement; le doigt lixe porte $2-3$ dents. le doigl mobile est peu denté. Le mérus de la pelite palte esl complè- 
convexe. A l'extrémilé du bord externe, on remarque deux on

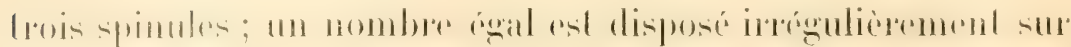

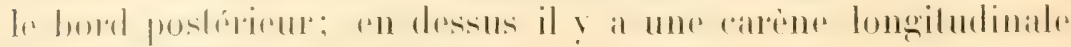
ill'mée de $2-3$ spinules.

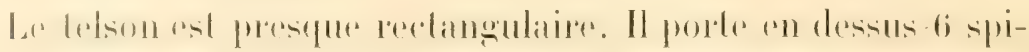
nules, distribuées en fer à cheval. La surface entre ces épines est concave. Les bords latéraux ont une épine à la base el deux

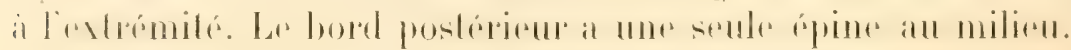

Longueur tolale...................

- de la carapace............

l.mpnent du mérus................

Largeur $\quad-\quad \ldots \ldots \ldots \ldots \ldots \ldots$

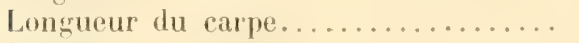

Largeur _ $\quad \ldots \ldots \ldots \ldots \ldots \ldots . . . .$.

Longueur de la main...............

- de la paume............

llauteur de la paume............. 1 .
१(Djihouli).

26 millim.

\begin{tabular}{cc}
\multicolumn{1}{c}{11} & - \\
Droile. & Gauche. \\
3,3 & 1 \\
2,5 & 3,3 \\
3 & 3 \\
2 & 4 \\
7 & 10 \\
4 & 6 \\
3 & 4 \\
1. & 3
\end{tabular}

Je crois que cette espèce doit rentrer dans te sous-genre Parraxiopsis, récemment fondé par De Man.

\section{FAIILLE CALLIANASSIDE.}

Gente UpOglebIa Leach.

Tableau des $U$ poggebia de la mer Rouge.

A. Doigt fixe de la pince beaucoup plus court que le doigt mobile (pince imparfaite).

B. (Pas de dents du côté interne de la paume.) Paume proportionnellement haute................. osiridis Nob.

BB. Pas de dents du côté interne de la paume.) Paume

très grèle, proportionnellement à la longueur....U. U. hirtifrons Wh.

A. Doigt lixe aussi long que le doigt mobile, formant une pince parfaite (Calliadne $=$ Gebiopsis).

C. Pédoncule des antennules subégal ou un peu plus court que celui des antennes. Carpe des chélipèdes avec une épine en-dessous el en dessus.

D. Bord frontal avec quatre épines. Mérus des chélipèdes spinuleux sur le bord inférieur. Bord postérieur du sixième segment abdominal lisse. U. Danwini Miers.

DD. Bord frontral avec 8 épines. Mérus des chéli$=(G$. intermedia De Ilan $)$. pèdes non spinuleux. Bord postérieur du sixieme segment abdominal finement den-

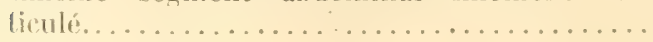

U. octoceras Nol). 
CC. Pédoncule des antemnules heaucoup) julus courl que celui des antennes. Carpe sims épines ou avec un tuberenle sur le bord inliurimur.

E. Rostre relativement courl, arec : $\dot{x}-\ddot{3}$ granules sur les bonds. Carpe avec tubercule inléricur nul ou tris peu développé. Hain lisse inférienrement......................... E. Sarignji stahl.

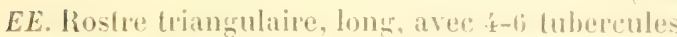
=(isodactigla l)itm.). spiniformes sur les bords. Carpe arec tubercule inlérlen. Bord inférien de la main dente.... U. mulhremes Fon.

\section{Upogebia Osiridis Nob.}

Nobili, Bull. Mus, 190', no :), p. 235 (Diatyn. prèlim., et Bull. scient. Fr. Bełs., XL, 1906, 1). 62, p. I. II, fig. 1't.

Mer Rouge (I. Jousseame), 2 màles el 'temolles; lojibouli

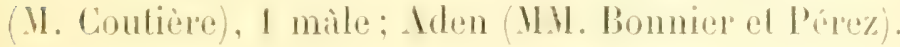

\section{? Upogebia hirtifrons (Wh.).}

CI. Xobili, Bull. scient. F). Belg., XL, 1906, p. (il, pl. 11, fig. 13 (uti lit.).

Denx exemplaires de Djibouti, en mavais état, appartionnenl probablement it celte espece.

\section{Upogebia CGalliadne (1) Darwini (Mier's!}

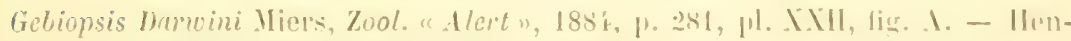
derson, 'Trans. Limn. Soc. (-2), 1, 1893, 1). 132.

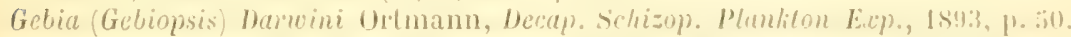

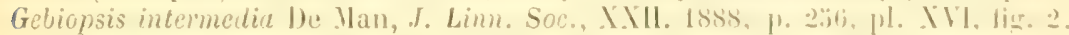

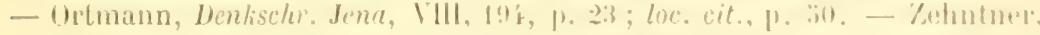

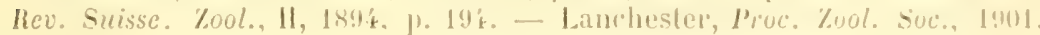
P. :3:i:

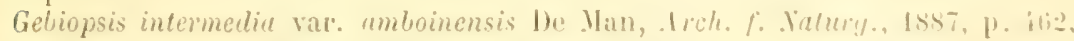
el .16h. Senckenb Ges., XXI, 1902, 1. 7i:9.

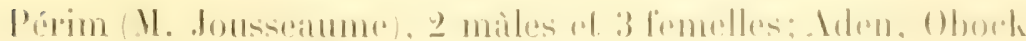

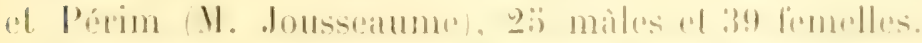

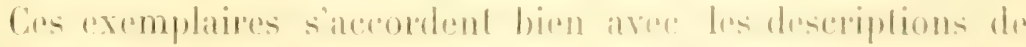

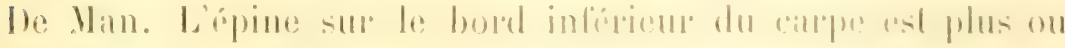

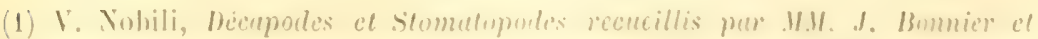

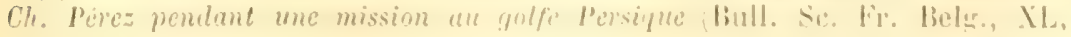
1906, p. 61 . 


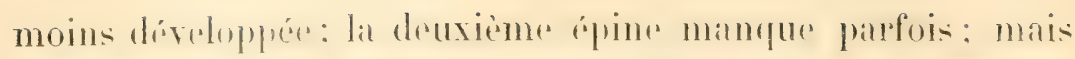

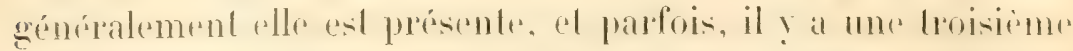
spinule plus petite placée en arrière el en dessus des deux autres. Lets spindes du mérus sonl moins nombrenses que dant l'exemplaire de Mergui, décril par De Man.

\section{Upogebia (Galliadne) octoceras Nob.}

Nobili, Bull. Mus., 1904, nº 5, p. 236 (Diagn. prelim.).

Périm (II. Jousseatume), I mile: Mlen, Obock et Pórim (M. Jonsseaume), 10 mâles, 11 femelles ; mer Rouge (M. Jousseaume), 1 femelle.

Cielle espece est roisine de $L$. Damini, dont elle differe par:

$1^{\circ}$ Le front pourvu de huit épines recourbées en arrière;

2 Par le mérus des chélipèdes dépourvu de spinules;

$3{ }^{\circ}$ Par le sixieme segment ablominal linement mais dislinctement denticulé au long de tout son bord postérieur.

Ces caractères sont constamment associés diuns tous ces exemplaires, el je n'ai jamais trouvé aucun d'eux dans les soixante-neuf exemplaires examinés de U. Darwini.

\section{Upogebia (Calliadne) Savignyi (Strahl).}

Savigny, Descr. Egypte, j)l. X, fig. 3 (sans nom).

Gebia stellata Audouin, Expl. Pl. Savigmy, p. 80.

Calliadne Savignyi Strahl, 1I. B. Akad. Berlin, 1861, p. 1064.

Gebia isodactyla Ortmann, Zool. Jarhb. Syst., I1, 1891, p. 3̈5, pl. I, fig: 9.

Gebia (Gebiopsis isodactyla Ortmamn, Schizop. Plankton Exp., Descap. 18!93. p. 50 .

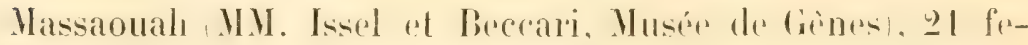
melles; Djibouli M. Coutires. '4 miles. 16 femelles: Southim. dans les éponges (M. Jousseaume), 12 femelles.

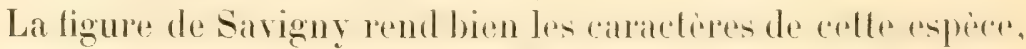

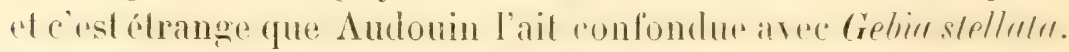
En lsti, Strahl en donna une dreceriplion lirée de la figure.

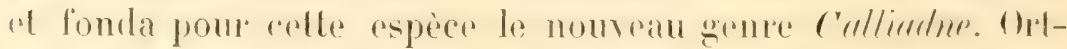
mann, en 1891, décrivit celte espèce sous le nom de Gebiu

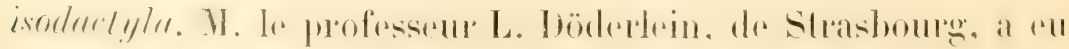

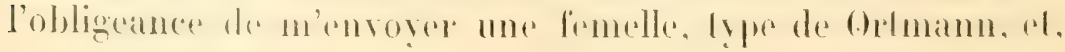


d'apres la comparaison arec mes axemplatres, je n'at ateun doute que l'espece dontmann me soil identipue a celle de Savignt.

Le rostre est triangulaire, obtus atu bout ef plus court que les pédoncules oculares. Il es rourat en dessus de petils

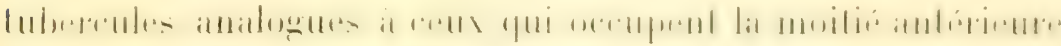
de la rógion gastrique. Les bords du rostre sont atriés de 1-ö granules, pas plus gros que ceux de la surfaco dorsile. Les cretes qui bordent les cotés de la rógion gastrique sont sonlevées, prolongées en arant en une dent distincte, el finement

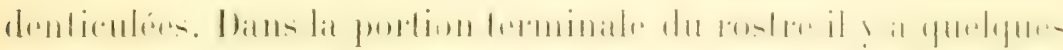
Louffes de poils, qui ne forment pas une masse tomentueuse comme dans $U$. Darmini et $L$. neloceras. Les cretes ont des longs poils. Les pédoneules oculaires sont gros it la base el st rétrécissent à l'extrémité, ou les cornées sont implantées obliquement; la plus grande extension de la cornée est du coté externe.

Les maxillipedes externes, qui portent des longes poils, nonl

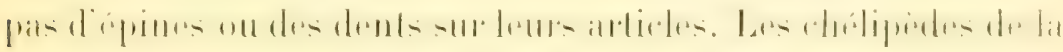
premiere paire sont ́ganx. Les articles basikires n'ont pas d'épines. Le mérus, qui est presque frois fois ausi long que large, a sur son bord inforieur les longues soies des aulpes especes. Le bord inféricur n’a pas une série de spinules comme C. Darmini; mais, en faisanl passer parmi les soles une

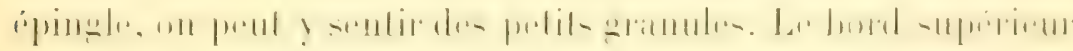
près de l'extrémité a un Luberente pulimentaire, qui sonvent peut manquer. Le carpe est lres coml, al lid lémite de som bord inférieur pent se trourer un lubereule petil, qui peut anssi manquer. Lat main est convexe sur les deux surfaces, poilue, matis les poils ne forment pas des lignes obliques comme dins / Ju'exini, mais sont distribués plutot uniformément. Lal portion

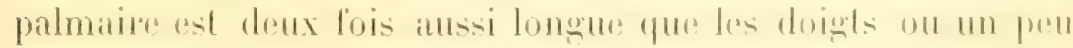

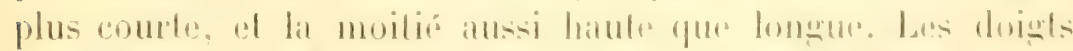
sont pointus; le doight tixe est ansisi longe qua le doigr mobile. Les autres palles nont rien de particulier.

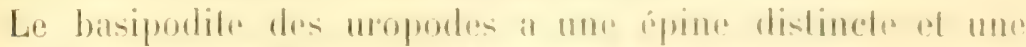
autre it la base de lexopode. Laxopode a denx cretes longilu-

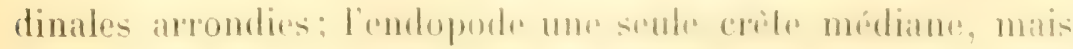




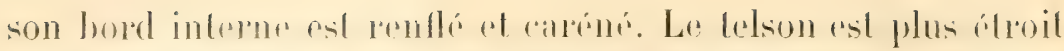

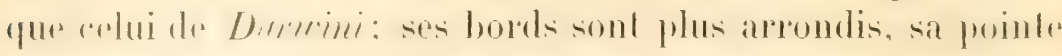

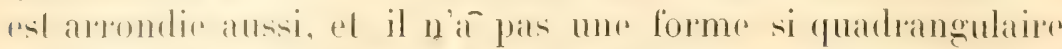
que dans Darwini. Sur sa surface dorsale, on observe deux

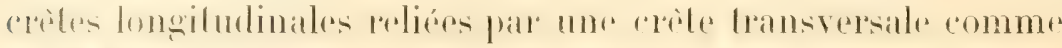

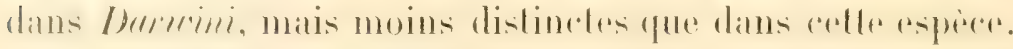

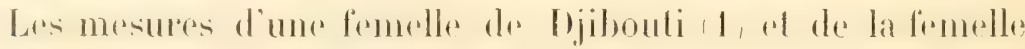
type de Gebia isodactyla (2) sont:

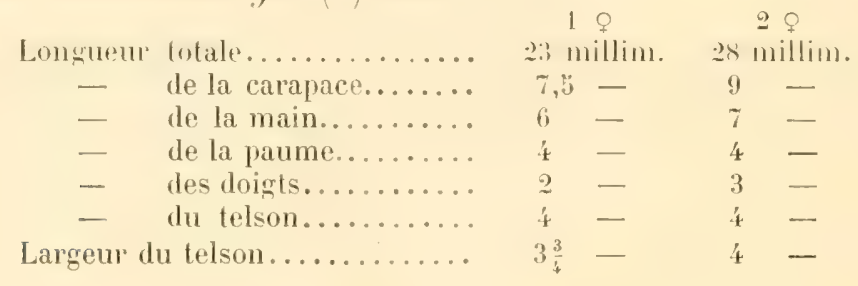

\section{Upogebia (Galliadne) rhadames Nob.}

Upogetia (Gebiopsis) rhadames Nobili, Bull. du Mus., 1904, n5, P. 235 (Diagn. prelim.).

Souakim, dans les éponges (I. Jousseaume), 9 mâles et

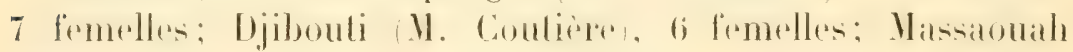

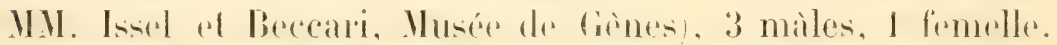

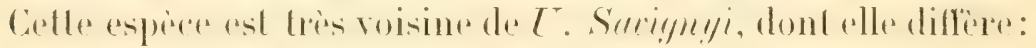

$1^{\circ}$ Le rostre est beaucoup plus long, triangulaire, arec ö-6 dents spinilormes de chatque coté, divergentes en dehors udaus Sarigmyi, les dents des bords du rostere ne sont pats plus développées que celles de la surface dorsale);

$2^{\circ}$ Le bord inférieur de la paume est distinctement denticulé ;

30 Le carpe offre inferienerment nu lubercule dentiforme bien développé.

\section{Genre CALIIANASSA Leach.}

A. Telson presque aussi long ou aussi long que l'endopode des uropodes.

B. Maxillipèdes externes étroits. Pas d'épine rostrale. Pas de saillic dentiforme ou spiniforme sur le mérus des pattes de la $1^{\text {re }}$ paire (Cheramus).

c. Ischium du gros chélipède denticulé sur le bord inférieur et sur le bord supérieur, près de la base. Carpe plus long que la paume............ C. Calmuni Nob. 
c. Ischium seulment granule sur le bord inlérieur, lisse en dessus. Carpe plus court rque la

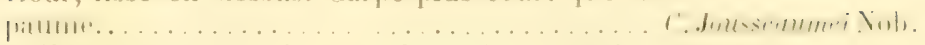

BB. Naxillipedes externes larges. Lne épine rostrale. Pas de saille spiniforme on de lobe distinct it la base du mérus du gros chélipède (Trypea).

c. Carpe du gros chélipède plus court que la paume. Telson arec denx crètes longitudinales. C. Bourieri Cob.

co. Carpe du gros chélidede aussi long que la main. Telson sans crètes................ C. Gravieri Toh.

A. Telson plus court que l'endopode des uropodes Callichirus).

B. Ln lobe a forme de crochet il la base du mérus du gros chélipède. Joigts de la grosse pince non dentés. C. Rosa Xob.

BB. Pas de lobe à la base du mérus.

c. Ischium des chélipèdes pourvu d'envipon dix épines aiguës. Basipodite des uropodes arec 1-2 spinules. Rostre trispineux............. Coutierei Nol.

cc. Ischium à peine denticulé à l'extrémilé. Basipodite des uropodes sans spinules. Rostre uni-

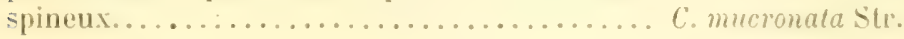

\section{Callianassa Cheramus) Jousseaumei Nob.}

(Pl. VI, figr. 2.)

Nobili, Bull. du Mus., 190k, no:, p. 236 (Diagn. prélim.).

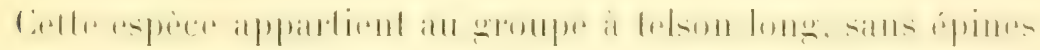
rostrales el avec le mérus des chélipedes dépourvu de prolon-

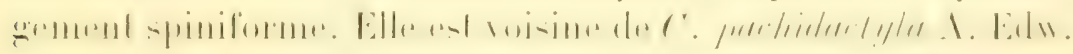
du cap Vert el de C. rombninensix. Elle ast représuntée par de nombreux axemplates : Jjibouti el Périm (11. Jousseatme), 10 males, 9 fomelles; mer Rouge (II. Jousseaume), 2 fenelles; Djibouli (11. Couliere), \& males, 3 fomelles; golle de Tadjourah (II. Famol), I mile el I lemello.

Le bord antérieur de la carrapace forme pulpe la base des youx un cout prolongement triangulane; il n'y a pas d'épines fostrales. Les sallies laterales enter les yeux al los antennes

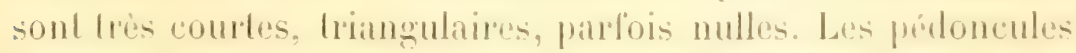
ocularies sont déprimós el triangulaters, it poinles subarigne el a bord externe obligue, anomdi, les veux sont pelits, convexes

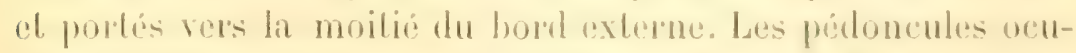
labes alteignent l'extrémilé de premier andicle du pédoncule des anfenmules. Le troisieme andicle de pédoncule des antennules est un peu plus long quo lo deuxieme article (comme ö 
¿̇ 20); non plus court ainsi que j’ai dit par erreur dans la diatsnose; il n’alteint pas la moilié du dernier article du pédoncule des antennes. Le fonel supérienr est un peu rentlé i l'extrémite: l’inférieur a des cils nombreux du còté inférieur.

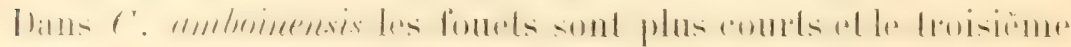

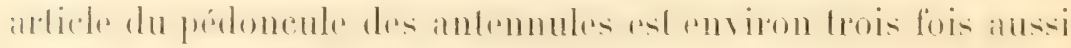
long que le deuxième.

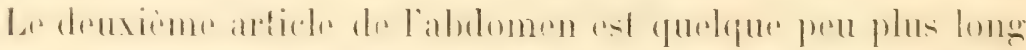

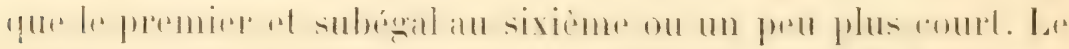

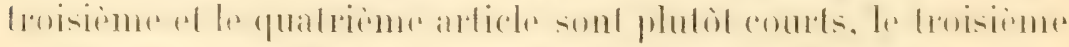

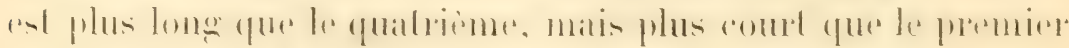
et le deuxieme. Le sixieme segment est le plus long, il mesure environ 1 fois $1 / 2$ la longuen du lebon. Le lelson est presque aussi long que large, ou un peu plus large que long; il a les bords latéraux ef le bord terminal arrondis. Le bord terminal a souvent une pelite échancrure arrondie. Sur sa stirface il oflre une proéminence arrondie el peu saillante dans sa pro-

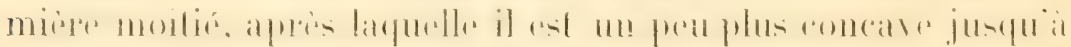

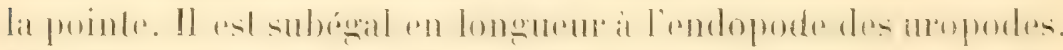

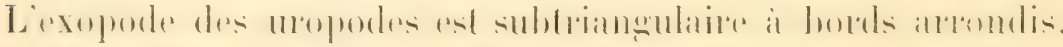

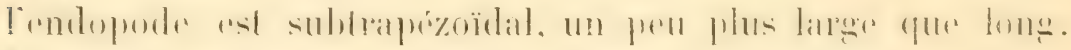
Liexopode porte une crète lisse, l'endopode une aussi.

Les maxillipèdes externes sont médiocrement élargis. Les chélipèdes sont fortement inégaux; le plus gros pent-idre

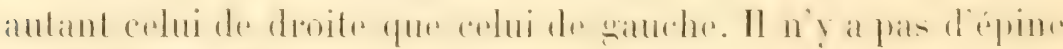
sur le basipodite.

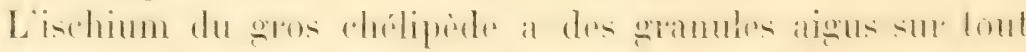
son bord interne; le bord supérieur est lisse. Le bord supérieur du mérus est lisse. le bord inférieur est distinctement

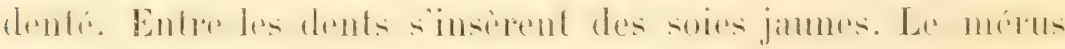
est large des $3 /$ ' de sa longueur.

La carpe est aussi haul que la paume, mais sa longueur va de $1 / 2$ aux $2 / 3$ de celle de la paume; il est bien convere; son bord supérieur est aigu, son hord inférieur est pourru

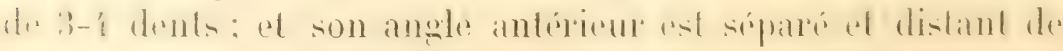
la main. Le bord inférieur a des longs cils. La main est haute, assez convexe, et la paume est plus longue que los

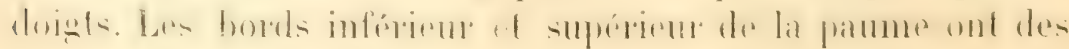


loulles de soies: mais stre la surface externe, il n'y a gure quelques louffes de poils pres de l'articulation des doights.

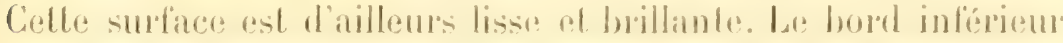
de la main est denticulé; le bord superienr lisse. Les denticules du bord inferreur de la main sont repliés un peu vers la còté interne, el pen visibles exterieuremenl; ils s'aretent it la base du doigl. Le lobe de la paume qui se trouve entre las bases des doigts est denticule; il y a allssi un pelit lolue dentiforme au-dessus de la base du doigh lixe. Le doigh mobile est assez gros, fres courbé, se crobistut ares le doigt lixe an dedans at en grande partie cachr par celui-ci; il est ponrou de deux grosses dents. Le doigt fixe est unilormément dente. mais ces dents ne se voient qu'en ouvrant la pince. Il y a des loultes de poils sur la surface de conbact des doights.

Sur le petil chélipède, l’ischium, le mérus el he bord inféricur du carpe el de la main ne sont pas dentés: le mérus n'est pas élargi, ni divisé par une còte en deux portions, mais uniformément convexe : une fois et demie aussi long que latge. Le carpe est un peu plus court que le mérus el que la paume. Les doights nont pas de grosies dents.

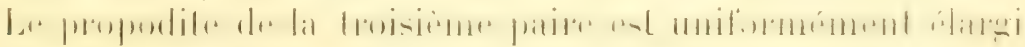
du colri inférieur.

Les mesures d'une grosse lemelle sont:

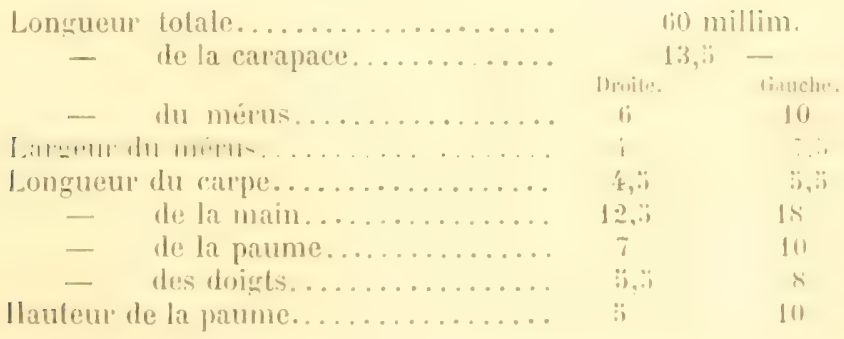

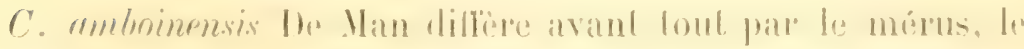

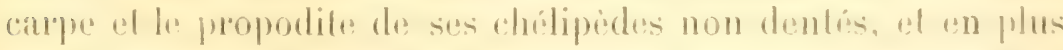

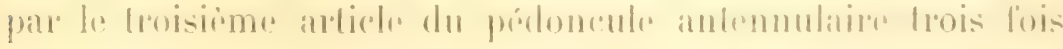

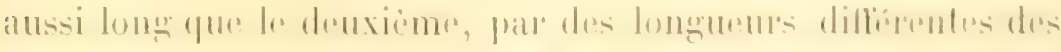
arlicles de la premiere parios de paltes, of des andicles abdominaux, el par la forme de son folson.

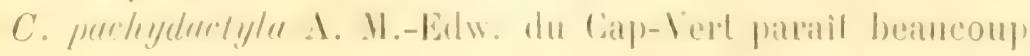


plus voisine. It n'en ai pas vu d'exemplaires, a je dois jugere

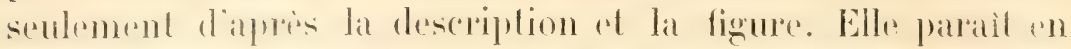

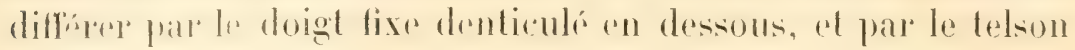
incisé-sinué postérieurement.

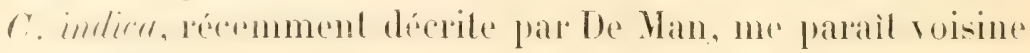
de celte espèce.

\section{Callianassa (Cheramus) Calmani Nob. \\ (Pl. I, fig. 2.)}

Nobili, Bull. du Hus., 1904, no :3, p. 226 (Diagn. prélim.).

Obock (I. Jousseaume/, un seul exemplanie long do ho millimètres.

Le bord frontal de la carapace forme entre la base des veux une petile sallie friangulaire comme dans $C$. amelonimensis of C. Jonssemmei. Le troisieme article du pédoncule des antenmules as 10 pen plus long que le deuxieme. Les antemnes manquent à l'unique exemplaire.

Sur la premiere paire de pépropodes l’ischimm du gros chí-

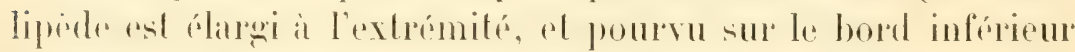

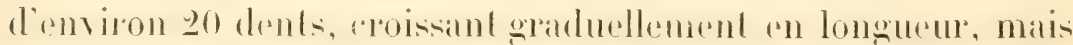
ne derenanl jamais ausi gros ni spiniformes eonme ceux de

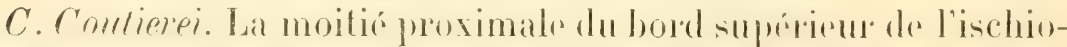
porlite est ausi denticulér. Le mépopolite lessemble it celui

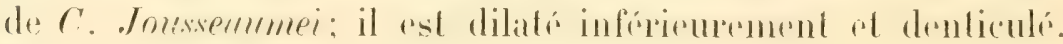
Le carpe est plus long que la paume el que le mérus, caréné sur les deux bords qui sont un peu tomenes end dedans: le bord infrieur est finement denticulí. Lat patmme est un pell plus

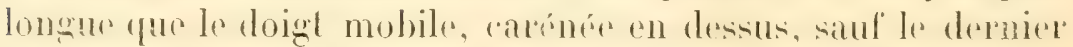

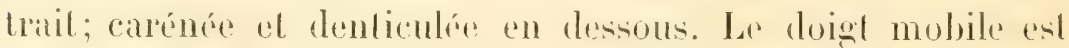

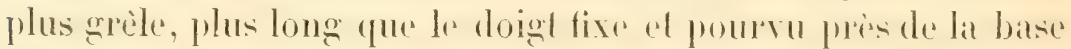
d'un gros tuberente bidenté, puis lisse al sins dents. Le doigh fixe est plutôt court, gros, denté sur le bord inférieur en continuation du bord palmatere, dépourve de dents sul le bord (odpant. Entre le doigh dixe et lo doigt mobile la paume fail

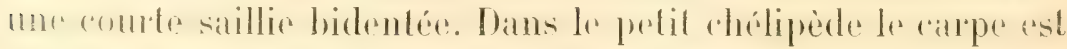

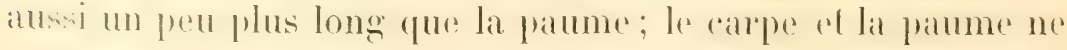

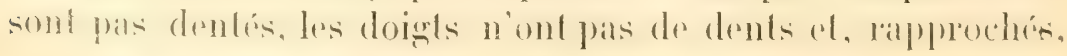


laissent un large aspace vide: ils sont plus longs que la paume.

Lat hoosieme parre de paltus manque dans l'unique exemplaire. Le belson of les mopodes resiemblent a ceux de

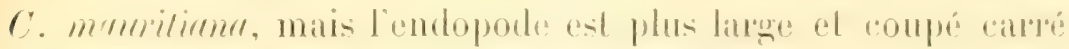
¿̀ l'extrémité.

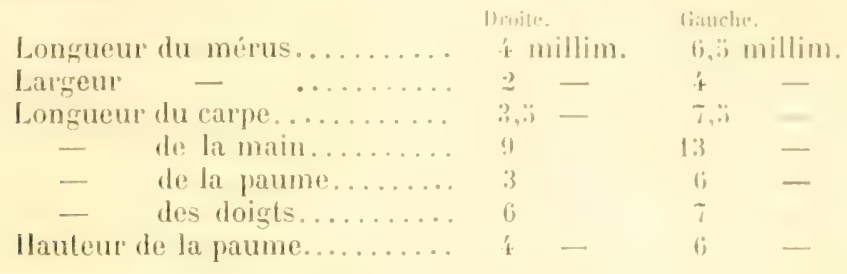

On peut distinguer facilement celle espece des $C$. . Inoussermmei el $C$. amboinensis, par la forme des mains, par la longueur du carpe, par l’ischium denté sur le bord supérieur, ate.

\section{Callianassa (Trypæa) Bouvieri Nol,}

(PI. II, fig. 3.)

Nobili, Bull. Mrus., 190', no :3. p. 236 (Diugn. prélim.).

Djibouti (11. Coutière), un seul exemplaire.

Le front se prolonge antérieurement en une ápine rostrales longue, dépassant les cornées, el atteignant les deux liors de

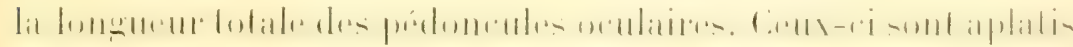
dorsalement el les cormées arrondies sont placées an milinu des leur surface. Le troisieme atsicle du pédoncule des antermules est plus long que ceux qui le précedent; le pédoncule des

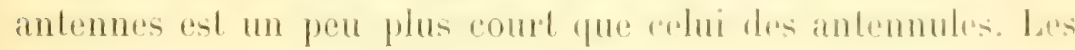
maxillipedes externes sont lries larges.

Le gros chélipede (a droite) a l'ischiam non dilaté a l'extro-

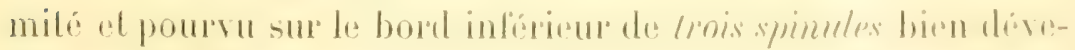

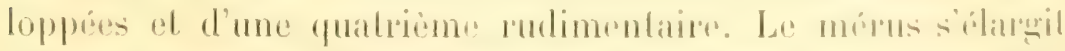

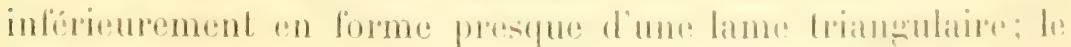
coté postériem de ce trianghe ast fablement denticule; le coble

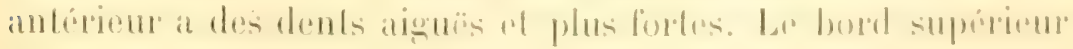

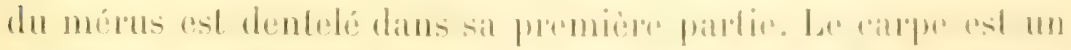

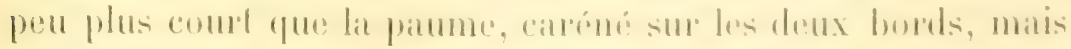

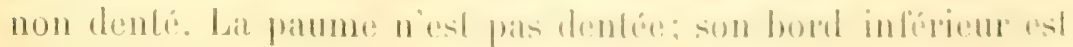
an ligne droile aree le doigh lixe; alle est un pere plus longue 


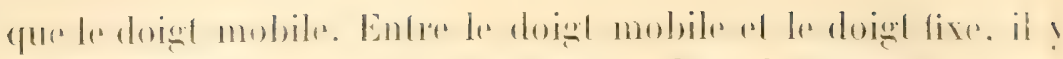

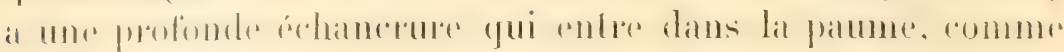
dans C. mantiliana, californiensis el uncinalu. Le doigt fixe esl

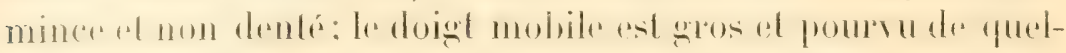

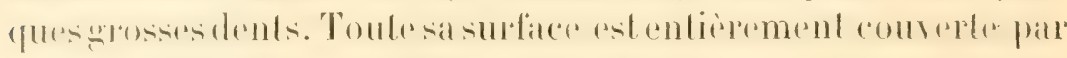

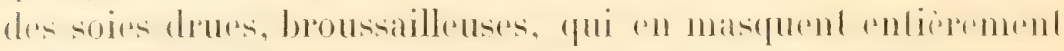

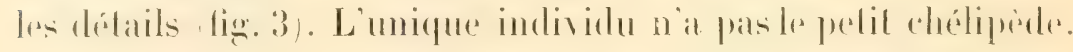

Le propodite de la troisième paire de palles est médiocrement élargi, et ne fait pas de saillie spéciale à son extrémité

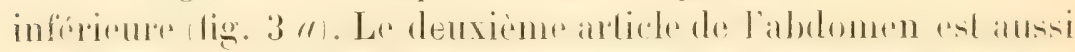
long que le troisième et le quatrième pris ensemble, et plus long que le sixième.

Le telson est aussi long que l'endopode des uropodes, un

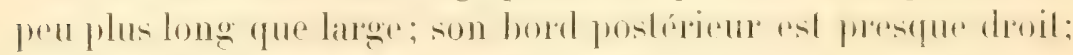

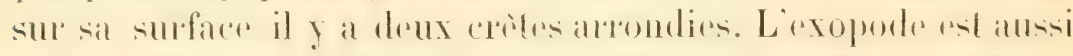

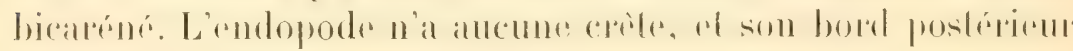
est presque droit.

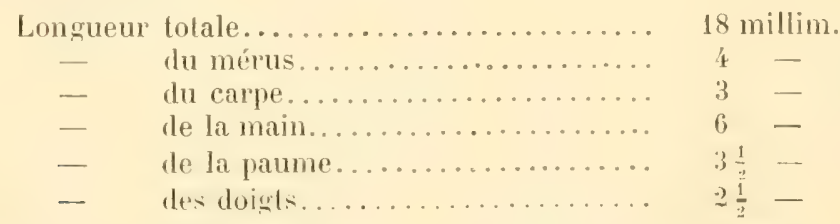

Cette espèce par la paume de son chélipède fendue s'ap-

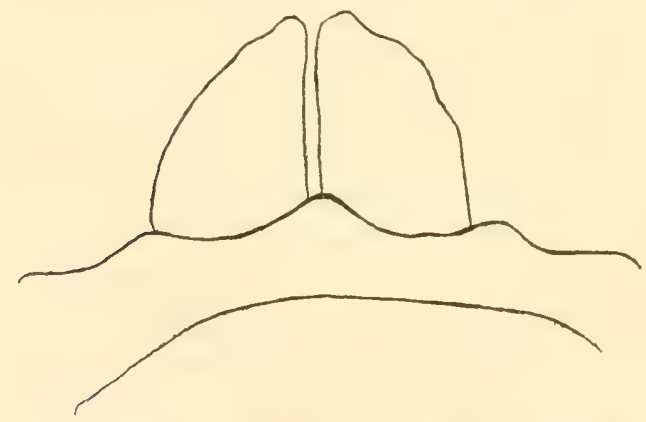

Fin. :3. - Rostre et yeux de Callianasse munitiana Miers (d'après le type du British Nuseum, figure du Dr W. T. Calman).

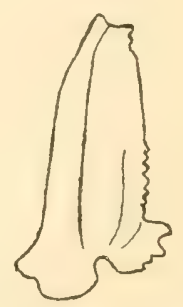

Fiğ. 6. - Mérus de $C$. manritiana (d'apres le type du british Museum figure itu 1) ${ }^{\mathrm{r}}$ W. T. Calman).

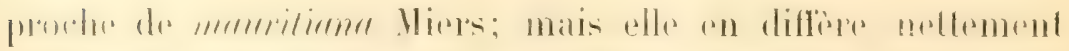
par la forme de son rostre qui est une épine, par son mérus 


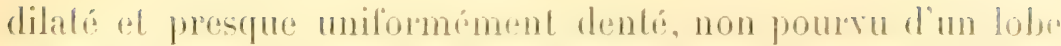

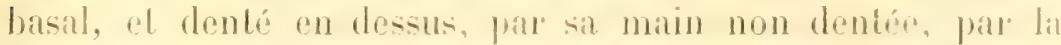
forme dillúrente du belson.

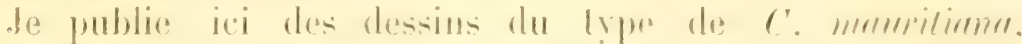
que je dois a lobligeance du J) II. T'. Calman, du brilish Huserma

\section{Gallianassa (Trypæa) Gravieri సiol.} (PI. II, lig. 't.

Nobili, Bull. du .Mus., 1903, nº 6, p. 39:3 (Diagn. prélim.

Celle espece, représentée par un seul exemplater pocurilli

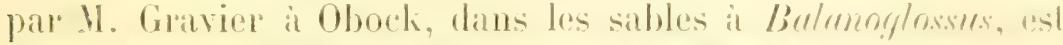

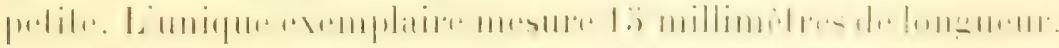

Le rostre est spiniforme ol se prolonge justgu'i moilié des cornées qui sont placées au milien des pédoncules. Ises pódoncules des antennules sonf plus longs rue ceux des anlennes: leur moisiome aticle est poesque deux lois aussi long qur lo deuxieme.

Les palles de la premiere paire sont peu inégales. Lat plus grosse a l’ischium lres finement demliculé an dessous; le mún

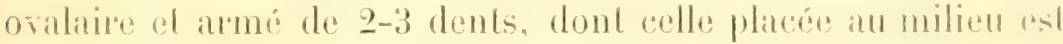
tres sailante. Le carpe est subégal en longuent it la main entière. lisse, non denté. Lat main est aussi lisse sur les deux boods; les doigls sont un pen boillants el le doigl lixe est fine-

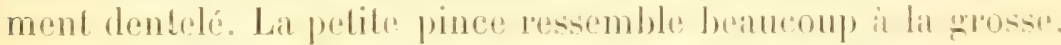
mais son merus n'a qu'une seule grosso dent un peu avant lit moitio de son borel inferiens. Le cinpe y est dislinctrment plus long que la main al lisse ansit.

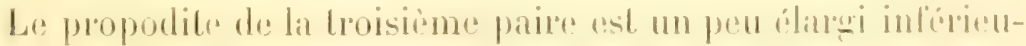
moment, mats alrondi et non saillant en lobe?.

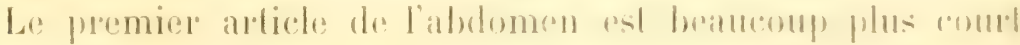

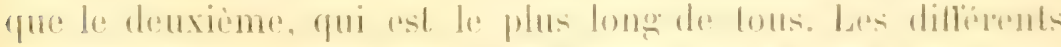

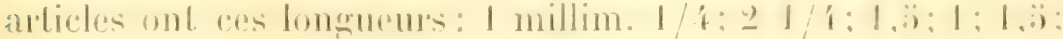

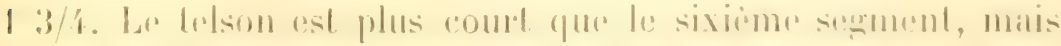

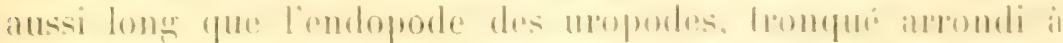

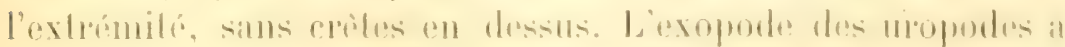

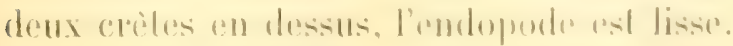




\section{Callianassa (Callichirus) mucronata Strahl.}

Callianassa macronata Strahl, H. B. Akarl. Berlin, 1861, p. 1056. - A. MilneEdwards, Nouv. Arch. Mus., V1, p. 9.. - De Ilan, Arch. f. Nat., 1887, p. 484, pl. XXI, tig. 2.

Callianassa Novæ-Guinex, Thallwitz, Decap. St., p. 31, pl. I, fig. 9. - De Man, A bh. Senck. Ges., XXV, 1902, p. 757. - Ortmann, Zool. Jahrb. Syst., VI, 1891, 1). 57; Denkschr. Jena, VIII, 1894, 1.23.

Djibouli el Perim, 37 individus. Djibouti II. Coutire); mer Rouge (M. Jousseaume), 2 femelles; Périm (II. Jousseaume), I male: Djibouli, dans les polypiers (M. Cravier), une dizaine d'exemplaires.

Ces individus s'accordent très bien avec les descriptions cilées. Une femelle a les dimensions suivantes :

Longueur totale.

$\overline{-}$ de la cara $\ldots \ldots \ldots \ldots \ldots \ldots$

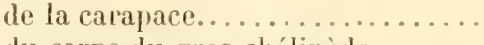

du carpe du gros chélipède.......

de la main (doigt étendu)........

de la paume................

du doigt mobile.............
47 millim.

$10-$

$4-$

14

$8-$

Il.13. : Philippimes Strahl; Imboine De Man, Ortmann): Nouvelle-Guinée (Thallwilz); Maldives (Ortmann).

\section{Callianassa (Callichirus) Rosæ Nob.}

(Pl. VIl, fig. 2.)

Nobili, Bull. Mus., 190', no $̈$, p. 23\%.

Lne femelle recurillie par N. le Dr Ragazzi dans une localité non elablie de la mere Rouge, el appartemant all Vlusere de Modène.

Ler rostre est une longar épine, dépassiant les pédoneules orulaires at atusi longure que le premier article du pédonente des antennules. En dehor's du rostre le bord frontal de la "atrapare fail deux pelites sallies angulaires. Les pédoncules des anlinmules sont tres longs, plus longs que ceux des antemmes

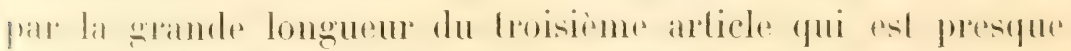

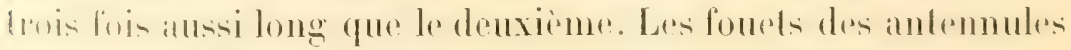

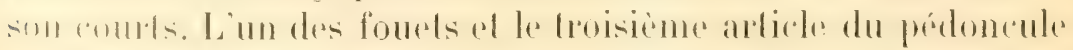

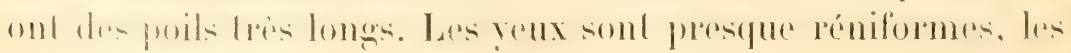


cornés occupent une grande partie de la surlace du pédoncule.

Les maxillipedes externes no sont pas particulierement élargis. Les palles de la premieres paire sont inógales le chélipede plus gros est celui de gatuche. Sur celle palle, on observe des pelits tubercules aigus sur le bord inferient de lischimm. Le mérus est distinctement divisé par une còte en une portion superieure convexe ol un inferinte un peu concare: a son extrémité proximale le mépus sallit en un fort lobespiniforme.

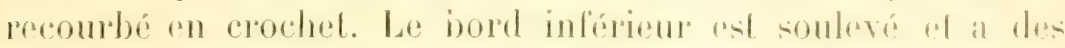
dents menues mais dislincles. Le mérus est plus de deux for aussi long que large. Le earpe est aussi hat que la main. arec les deux bords aigus, mais sans dents ni épines. Il est un peu plus court (1) que la portion palmaire de la main. Lat main as convexe, lisse el luisante, ainsi que le carpe, sins dents ni epines. La paume est aussi longue que le doigh mobile blendu. Le bord tranchant du doigt mobile est courbe en S, par un gros lobe qui occupe une grosse parlie du bord, mais il nest pas denté; bien que, examiné a la loupe, on y voil de lrés finces cémulations. Le doigh fixe n'a pas non plus de dents: il offre un petil lobe subtriangulaire près de sa base.

Le pelil chélipède est absolument inerme. Le mérus n’est pas pareouru par une cote; il est un peu moins de deux fois atusi long que large, el dépourvu d'épines. Le carpe est un peu plus cout que la portion palmatre de la main, qui est un peen plus courte que les doigls. Ausi bien la main que le carpe ont les deux bords aigus, mais non dentés. Les doights sont un peu baillants; le doigh mobile est caréné en desists al pourva de

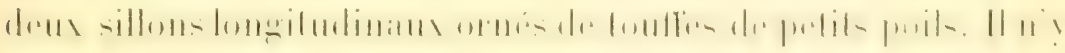
a pas de dents sur les deux doights le doigh lixe rel fortement poilu sur son bord tranchant. Le propodile de la troisiome patim

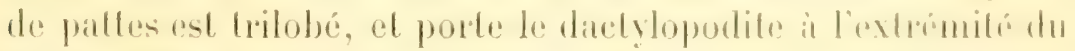
lober médian.

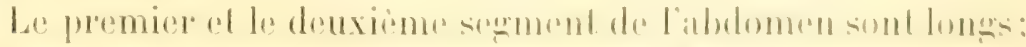

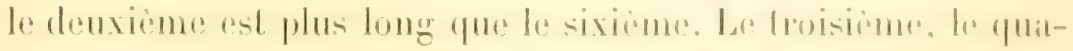

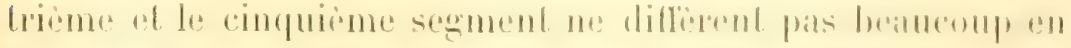

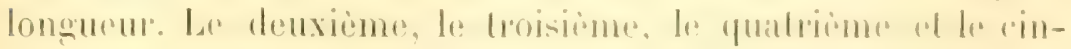

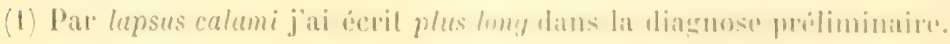




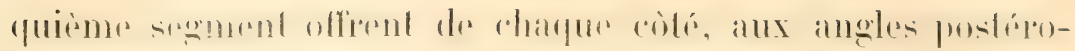
latéraux, une fossette circulaire membranacée.

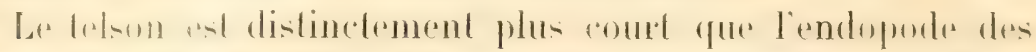

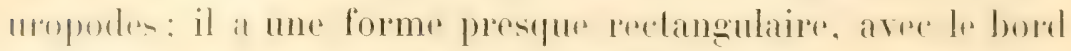

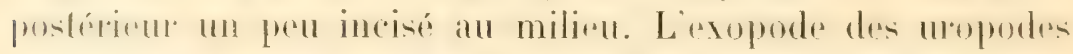

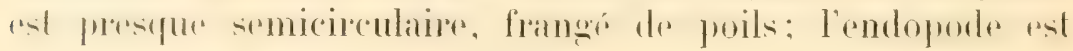
étroil et lancéolé.

Longueur totale..................

- de la carapace.............

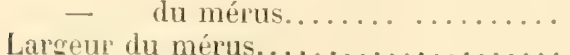

Longueur du carpe.................

- de la main...............

- de la paume..............

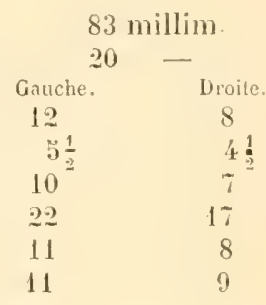

Par la hrivelé du telson el par lit présence diune ípine rostrale, celle espèce a quelques affinités avec $C$. breicondala

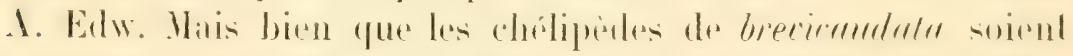

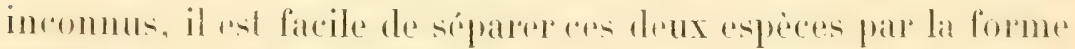

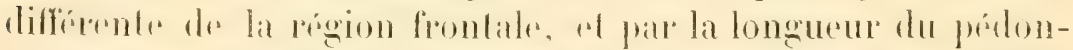

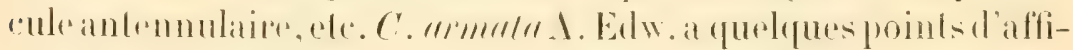
nilé, mais elle ditfere ausi par un gramel nomble de caracteres.

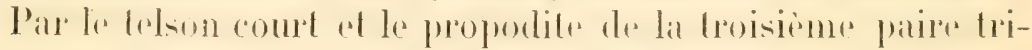

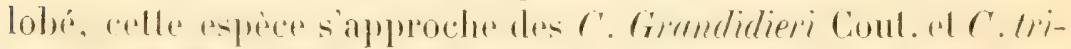

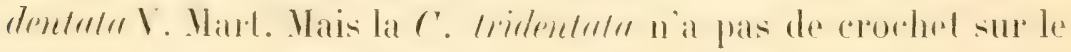
mérus, le $C$. Giandidieri a les mains épineuses et les deux espèces ont le front tridenté.

\section{Callianassa (Callichirus) Coutierei Nob.}

(PI. VII, fig. 1.)

Nobili, Bull. du Mus., 1904, no 3 , p. 237 (Diagn. prélim.). Bull. scient. Fr. Belg., XL, 1906, p. 60 .

C. Wartensi Coutière, Bull. Wus., 1899, no 6, p. 287 (nec C. Wortensi Miers).

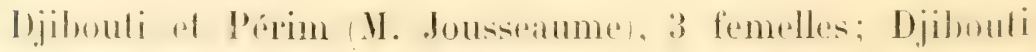
(11. Coulière), 2 grosses femelles.

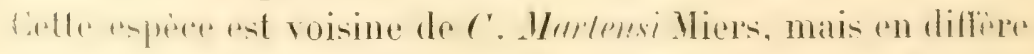

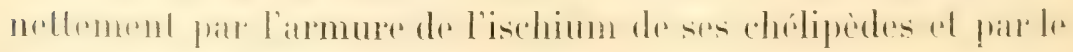
basipodite de ses uropodes. 
Lefront se prolonge antiriemenent en une pointe rostrale

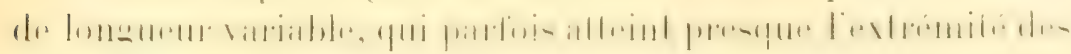
pédoncules oculares, parfois en alloint seulement la moilié. De chaque cote de l'épine, il y en a deux aulers phos pelites al plus courtes. Les pédoneules oculaires ont la surlited dorsale convexe, presque cylindrique: les cormées sont terminales. et embrassent presque loute la portion terminale du profoncule. qui införieurement se prolonge an dedir des cornées en un lubercule. La surface inférieure da pédoncule ast divisue par ane crute médiane en deux còtés. Le deuxième artich du piedoncule des antennules est plus cout que le troisieme: l'extrimití

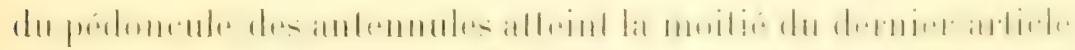
du pédoncule des antemnes. Les fouets des antennes dépassent l'extrémité du deuxième segment de l'abdomen.

Le sillon cervical est net; la partic antérieme de la portion céphalique usl faiblement rugueuse. Les maxillipedes externes sont módiocrement élargis.

Les chélipedes sont incógaux. Le basipodite n'a pas d'ópines (il y en a une dans Mnrensi d'apres De Man). Lisehimm des deux chélipedes a une rangée denviron dix épines qui croissent en longueur de la base it l'uxtremité, et dont relles distales sont recelilignes.

Jans C. Marlensi. il n'y a que des lubercules oblus el petils ainsi que lon peut volu d'aptris le desin du lyper que jedois al l'obligeance de .I. Le I) IV. T'. Calman.

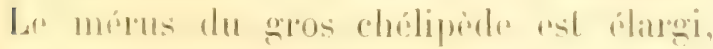

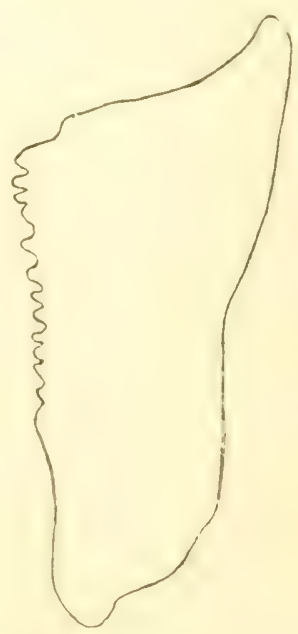

Fig, ¿. Is-hium d, ílliunussun Horlensi

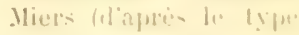
du litilish Musemu.

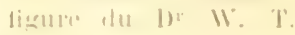
Calmatur.

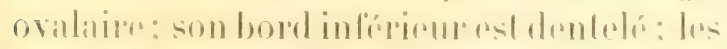

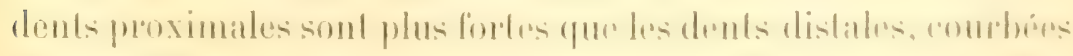

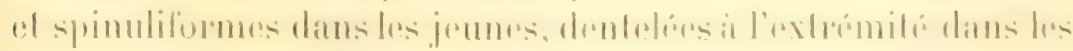

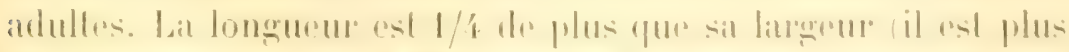

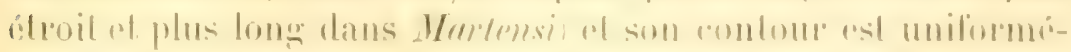

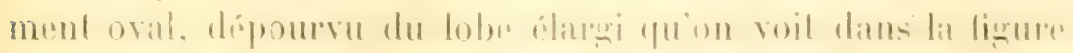

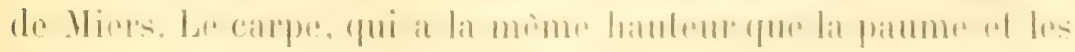




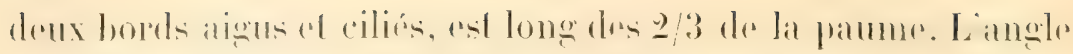

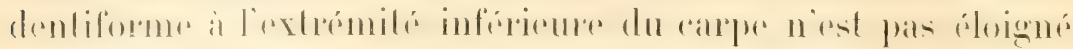
de la main comme dans la figure de Warlensi, mais il en est

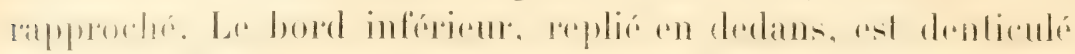

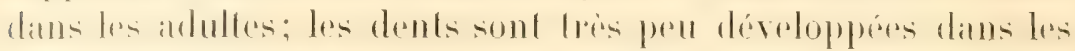

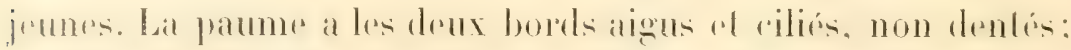

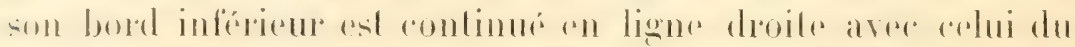

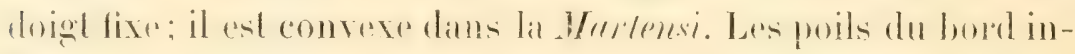

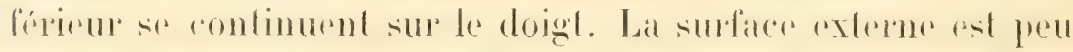
convere, et a de rares touffes de soies. Les doigts sont un peu

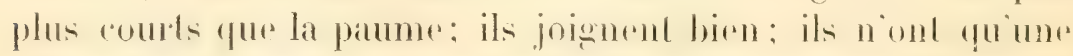
grosse dent chacun, et des touffes de soies jaunes. Dans les virux remplaires le coble introme de la grosse pince a des grat nules petils et nombreux à l'extrémité du carpe el de la paume.

Le petit chélipède a l'ischium également armé; le mérus

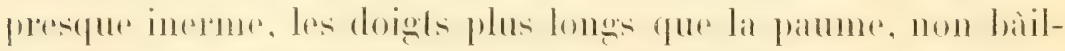
lants. Les doigts de la denxieme paime de pattes sont plus contes que la patume. Le propodile de la troisimme paire de patles a it peu près la même forme que dans la Murtensi, mais il est moins élargi inférieurement.

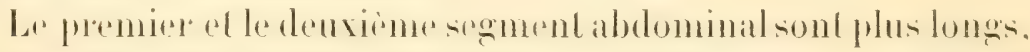
le denxieme at plus long que le premier. Le sixieme segment at aussi long que le cinquieme of les $2 / 3$ ausi longe que le

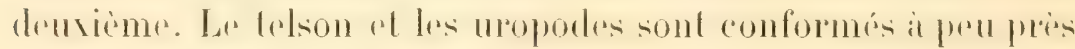
commere cens de la C. Marlensi. matis le basipodite des mopodes

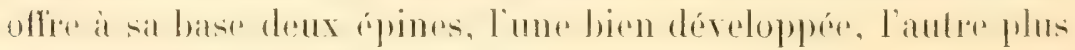
petile el parfois rudimentaire.

Les grosses femelles de Djibouti, recuesilies par M. Couliere. atteignent une longueur de 80 millimètres.

J'ai envoyé un exemplaire de celle espèce à M. le $D^{\text {r }}$ W. T.

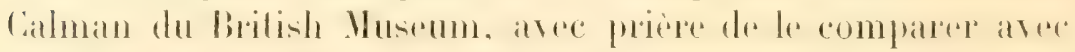

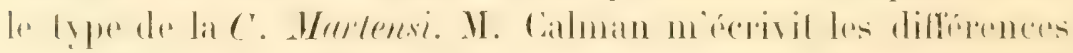
suivantes: "Le rostre est distinctement plus long chez la $C$.

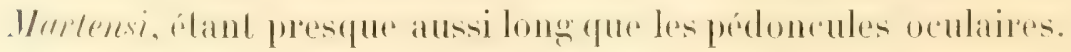

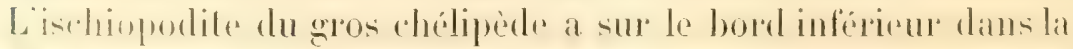

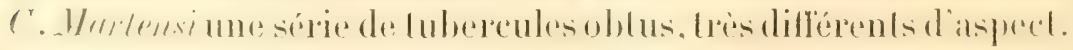
L'exopode des uropodes n'a pas d'épine là où elle se trouve 
dans ros exemplaires. Il y a ausi une légèe diflérence dans le contour, puisque l'exopode est un peu plus abroit at plus aigu. "

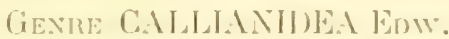

\section{Callianidea typa Esw:}

Callianidea typa II. Milne-Eiwards, H. n. Cr., 2, 18:37, P. 320, pl. XI bis, lig. 8-1\%. - Borradaile, Proc. Zool. Soc., 189k, p. $101 \%$; Willey's Res., IV,

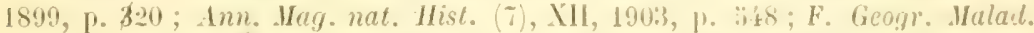
Laccad., II, p. 7f2. - De Man, Ibh. Senckenb. Gies., XVI, 1902, 11. Tiil. Callianidea mueronuta Kossmann, Zool. Ergebn. roth. Heer., 11. 1880, 1. 84).

Djibouti (I. Jousseanme), ̈̈ individus; Obock (11. Jousseanme), un individu; Djibouli (I. Coulieve) 17 exemplaires: Djibouli dans les polypiers (II. Ciravier'), un individu; iles

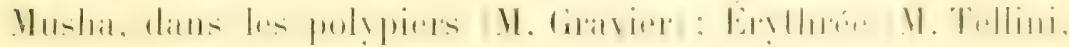
Musée Turin), „2 exemplaires.

De Van a donné une nourelle deseription étendue de cette espece. Son indiridu manquait du gros chélipede. de vais donc donner guelque notice de celle palte, pour compláter lix descriplion de De Van.

La grose pince est tantò à ganche, tantòt à droite. L'is-

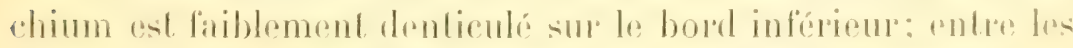
dents, il y a des loulles de soies. Le mérus est convexe sur sit sulface axterne, mais divisé en drux moiliés, qui font angle an milieu. Ses deux bords, superiene of infriteur, sont fortement convexes: to contour de landicle vient ainsi it etre ollipsoülal.

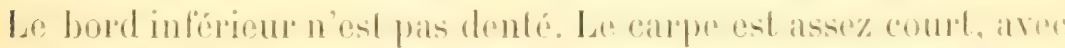

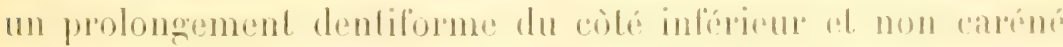

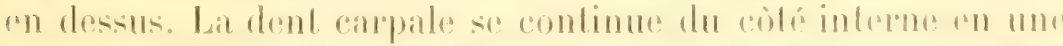
pedile crote armée de z-3 dents. La main, qui est convexe rell

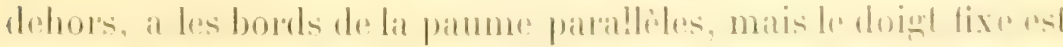
dirige un pen en bas. Le bord inféreu "st distinctement

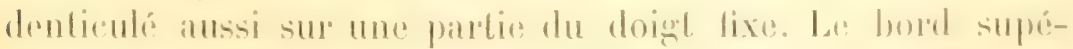

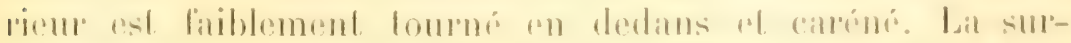

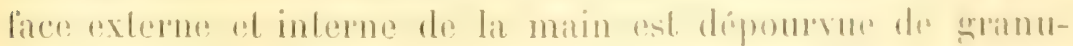
lations; elle offere des toutfes de solus jaumatres. l'atralliblement. au bord probensile du doigh lixe, il y at, du cote interme of du coté rexterne, une rangere de erramulations, plus ou moins AN. SC. NAT. \%OOL., !y sirip. 


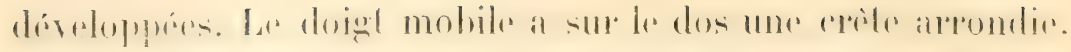

liossmann décrivit en 1880 une nouvelle espèce de Callirnider de la mer houge, suiil appela $C$. muronala. Celle espece serait caractérisée, d'après son auteur, par une petite denl frontale aigué, et parce que le fonel supérieur des antennules est renflé non à la pointe, mais au-devant de celle-ci, le plus gros article átant le quatorzième. Pour ce qui est du premier caraclère, il n'a ancune valeur, parce que la pointe ros-

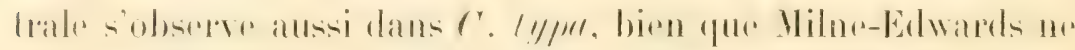
l'ail pas décrite. Le caractere décrit des antennules est assez rariable, et il ne peul pas non plus avoir de valeur. De Man dans les typa de Ternate observa que le plus gros article est

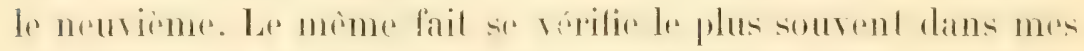

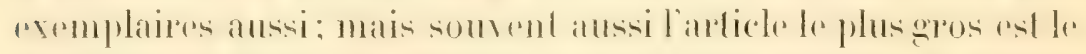

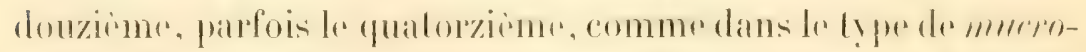
nula, et mème dans deux eas, dans l'une des antennules les

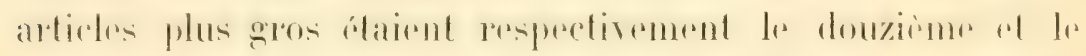
treizieme, dans l'autre le sixième ef le huitieme. Ces varia-

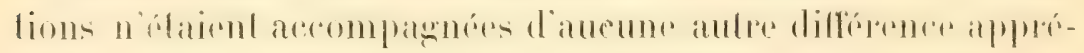
ciable. La $C$. mueromate est done identique à la $C$. Iypar.

\section{PAGURIDEA}

\section{FAMILLE PAGURIDA}

\section{GFNRE PAGURISTIES}

\section{Paguristes Jousseaumei Bouv.}

Bouvier, Bull. Soc. Philom. Paris (VIII), no 4, 1891-92, 1. :2.

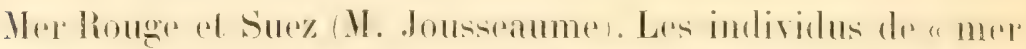

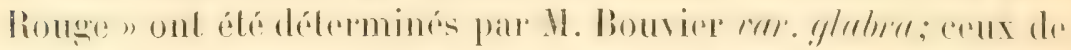

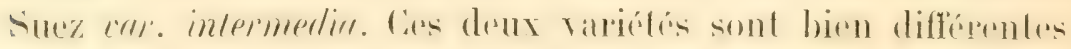
entre elles. La ian. glabra a les paltes très peu poilues (et à

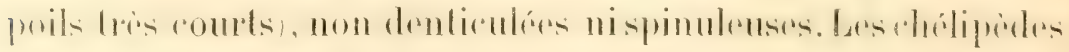

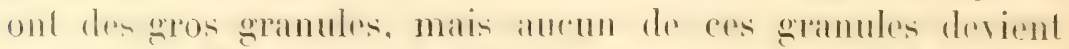

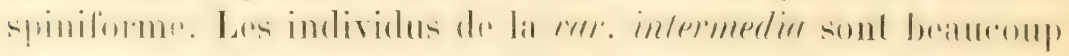
plus poilus; les poils sont plus longs, les propodites des

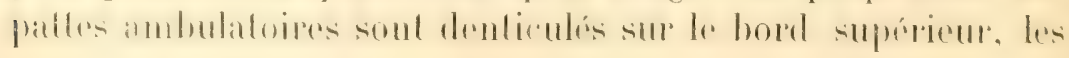


carpopodiles sont spinuleax, at les Lubereules sur le bord

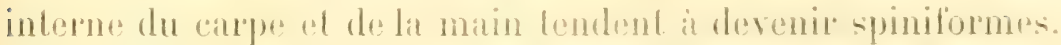

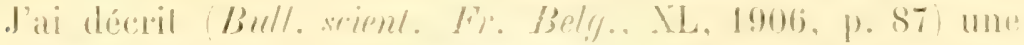

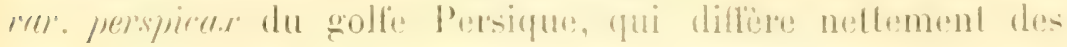
formes de la mer liouge par ses reux constamment plus hongs que les pédoncules antennulates. Dans celle forme. on observa atssi, quant aux palles, la menno variabilité que dans les of oms-

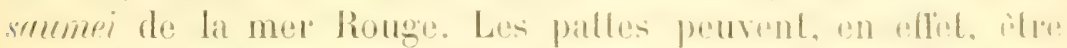

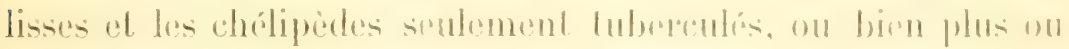

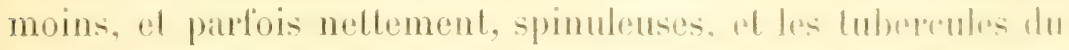
bord interne des chélipedes deviennent des dents spinilonmes.

\section{GENHE CLIBANARIUS DANA.}

Tablean des especes de la mer hionge.

1. Dactylopodites de la 3" paire distinctement plus long: que les propodites.

B. Céphahothorax vert vlivitre. Paltes de lat 20" et de lia $3^{\circ}$ paire avec des bandes longitudinales hleues. marginées de rouge..................... Congitarsus I) Haan.

BB. Céphalothorax taché de rouge. Paltes avee des bandes longitudinales rouges........................ strolatus l)ana.

A.1. Daclylopodites de la 3" paire plus courts que les propodites.

a. Couleur rouge avec de nombreux points blanes. Dactylopodite el propodite de la troisieme patte de

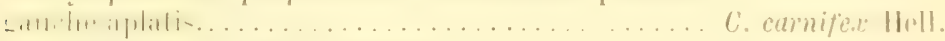

na. Coloralon disposée par hambes, ou phus ou moins uniforme, mais asee des anneaux sur les dactylupodites. Propodile el dactylopodite de la torsieme: palte gauche non aplatis.

b. Couleur fondamentale jaunalre, aver handes longitudinales longers. Pas d'anneaux eolurés sur les dactylopodites. .................... simnutus Hull.

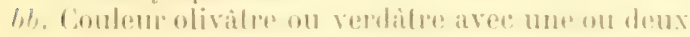

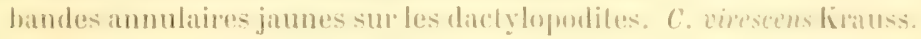

\section{Clibanarius striolatus | Illit.}

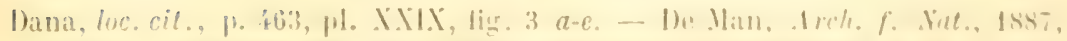

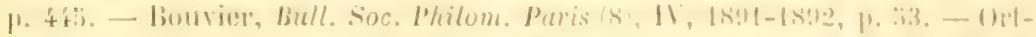

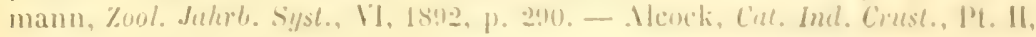
1. Ali, pl. II, lig. 7 .

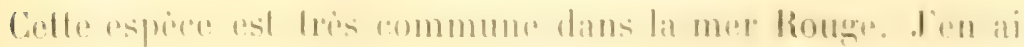




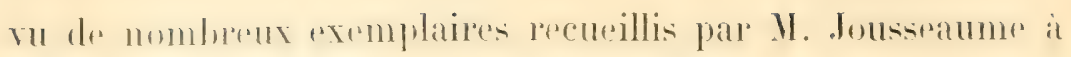
Périm, Aden, Obock, ele.

\section{Glibanarius longitarsus (De Haan).}

Pagurus longitarsus De Haan, F. Jap. Crust., p. 211, pl. L, lig. 3.

Clibanarius longitarsus Hilgendorf, Dechen's Reise Ost. Afr., P. 96. - De Man, Arch.f. Nat., 1887, p.41. - Nobili, Ann. Hus. Civ. Genova, XL, 1900, p. 492 ; Bull.scient. Fr. Bely., XL, 1906, p. 83. - Alcock, Cat. Ind. Decap., 11, 1905, P. 158 (ubi lit.).

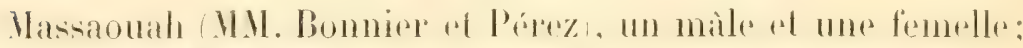
Massaonah M. Magrelli, Musée de Turin/, Im geros male rn

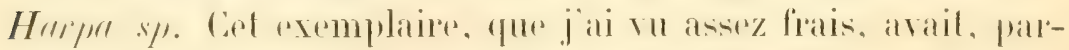
dessus les lignes rongeatres, me coloralion blen métallique dilluse.

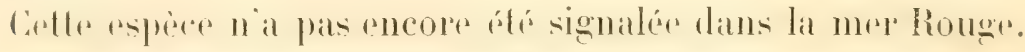

\section{Clibanarius infraspinatus Hilgd.}

Hlilgendorf, loc. cit., p. 97 (note). - De Man, J. Limn. Soc., XXII, 1888, 1. 237. - Ortmann, loc. cit., P. 290. - Henderson, Trans. Linn. Soc. (2), V, 1893, p. 423. - Nobili, Boll. II tos. Torino, XVIII, n ${ }^{\circ} 55,1903$, p. 19. - Alcock, loc. cit., p. 4 .

Signalé dans la mer Rouge par Ortmann.

\section{Glibanarius carnifex Hell.}

Heller, S. B. Akad. Wien, 千́4, 1862, p. 250. - Bouvier, loc. cit., p. 53 . - Nobili, Bull. scient. Fr. Belg., XL, 1906, 1. 87.

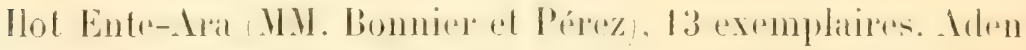
(II. Jousseaume), 2 individus.

\section{Clibanarius signatus Hell.}

Heller, ine cit., p. 20ั2. - Paulson, loc. cit., p. 92. - Bouvier, loc. cit., p. ๖3. - Nobili, loc. cit., p. 83.

Nombreux exemplaires de l'ìlot des Frères ou Brothers

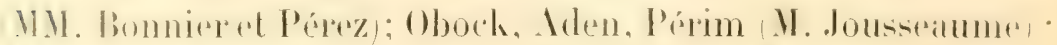

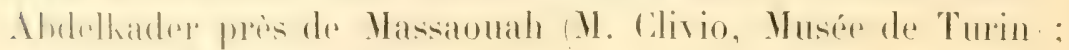
Obock (1I. Maindron). 


\section{Clibanarius virescens líauss.}

Patfurus virescens lirauss, Sudufi. Crust., 184.3, p. :36, pl. 11, fig. 3.

Clibanarius virescens Dana, loc. cit., 1. 29, fig. 6. - Ililgendorf, loc. cit., 1. 9.; : 11. B. Akad. Berlin, 1878, p. 821, pl. I11, lig. 11. - 1)e Man, Journ. Lim. Soc., XXII, 1888, p. 2'ĩ. - Bouvier, loc. cit., p. 3̈. - Nobili, loc. cit., p. 86.

Combreux exemplaires d'Obock, P'rim, Aren, Djibouti (11. Jousseaume) el Iten (III. Bonnier el P'érez). Les exemplaires qui ont séjoumé plus longtemps daus laaleool sont devenus rougeatres, mais l'anneau des dachlopodites est toujours clairement visible.

\section{(iNNRE CALGINLS I)ANA.}

I. Main du petil chélipède pourvue en dessus d'unc haule crète divisée en cing dents. Couleur de l'animal frais vert et jaune, en alcool rougeitre, mais toujours avec un anneau rouge sur les daclylopodites............ C. latens Rand.

II. Main du pretit chélipède avec dés tubercules peu ou médiocrement saillants, sur son bord supérieur. Couleur rouge passant au violet foncé, avec des tubercules blancs. 'as d'anneau sur les dactylopodites......... C. rosaceus Hell.

\section{Calcinus latens Rand.}

I'agumus latens Randall, Journ. Acad. Philaul., VIII, 1839, D. 133.

Calcimes latens Dana, loc. cit., p. 459, pl. XXYII, fig. 11. - Heller, Crust. Horara, 186\%, p. 88. - Ililgendorf, M. B. Acuel. Berlin, H. 823.- (1)tmann. loc. cit., [. 293. - Bouvier, loc. cil., ]. :3. - Yobili, loc. cit., p. 83, pl. 1, fig. 20. - Alcock, loc. cit., p. is, pl. l, ficr. :3.

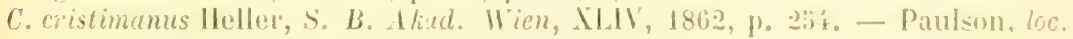
cit., p. 9:2.

C. intermedius I)e Man, Yol. Leyd. Hus., 1II, I, 102.

Nombreux exemplaires de Djibouli (II. Coulière et II. .lousseaume): Obock (II. Jousseaume): Périm, Aden (.I. .lousseaume); mer Rouge (M. Jousseatume).

Signatée atussi it Djeddah.

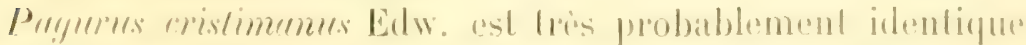
avece 6 . latens. Il est en tout cats extremement probable yue les

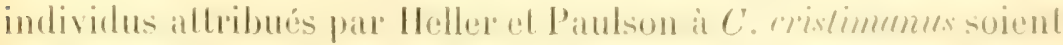
des lulens.

\section{Calcinus rosaceus $\|\mathrm{c}\|$.}

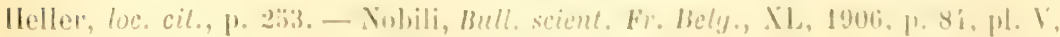
lig. 21 . 
Iles Musha (II. Gravier), me femelle s'accordant parfaitement avec l'exemplaire des còtes d'Irabie, décrit par moi, loc. cil.

\section{GeNRE DIOGENES DANA.}

(Rostre en pointe simple.)

A. Pédoncules oculaires plus longs que les pédoncules antennaires; un peu plus longs ou aussi longs que le pédoncule des antennules. Carpe des pattes deuxiemes avec deux épines; carpe des pattes troisièmes avec une seule épine. Pince gauche fortement poilue......... D. senex llell.

14. Pédoncules oculaires plus courts que le pédoncule des antennes. Carpe des pattes $\stackrel{-}{-} 3$ spinuleux. Pince peu ou point poilue.

B. P'édoncules oculaires atteignant l'extrémité du pédoncule des antennules. Cinq rangées de granules aigus ou de denticules sur le propodite des pattes

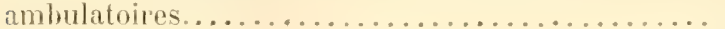

BB. Pédoncules oculaires n'atteignant pas l'extrémité D. denticulatus Chevr. et Bour. du pédoncule des antennules. Propodites des pattes ambulatoires avec une seule série de spinules ou presijue lisse.

a. Bords latéro-antérieurs de la carapace non dentés. Pince non épineuse à doigt fixe fortement

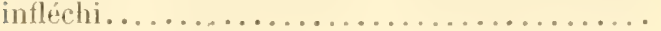

aa. Bords latéro-antérieurs de la carapace dentés. b. Pince à granulations peu ou point saillantes; à crète médiane plus ou moins développée et granuleuse, mais non épineuse. Bord inférieur de la main formant un angle plus ou moins marqué arec le doigt fixe (au moins dans les adultes).............

bb. Granulations plus aiguës. Crête médiane de la main armée de $3-6$ spinules. Bord inférieur de la main et du doight droit ou convexe.............................
D. pugilator var. avarus llell.

D. pugilator Roux.

D. pugilator var. brevirostris.

\section{Diogenes senex Hell.}

Heller, Crust. Novara, 1863 , p. 83̈, pl. VII, fig. 3. - Bouvier, loc. cit., p. 53.Tobili, loc. cit., p. 78 .

Pagurus (Diogenes) senex Ililgendorf, M. B. Akad. Berlin, 1878, P. 83\%.

Suez (II. Jousseaume), un exemplaire, déjà signalé par MI. Bouvier; Djibouli (H. Jousseamme), ö individus.

Le pédoncule des yeux dans ces exemplaires alleint quel-

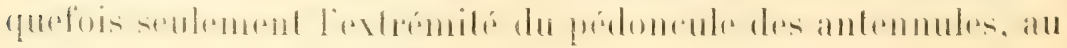




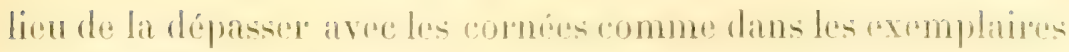
du golle l'ersigute.

\section{Diogenes denticulatus Chevr. of Bour.}

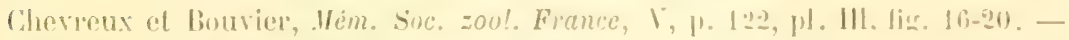
Bouvier, loc. cit., p. ii.

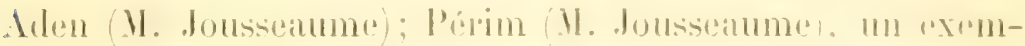
plaire de chargue localité, qui sont les memes déjit signalés par II. Bouvier.

\section{Diogenes pugilator Roux.}

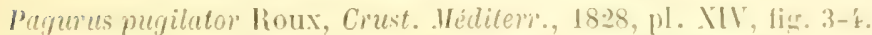

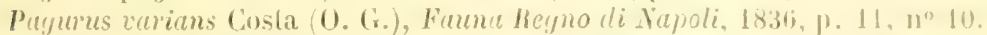

Diogenes variuns Ileller, Crust. Sull. Eur., 1863, 1. 170, p1. 1, lier. 1:3-11.

Diogenes pugilator Bouvier, Mém. Soc. zool. Fr., II, 18!1, J. 394i uhi s!nn., al

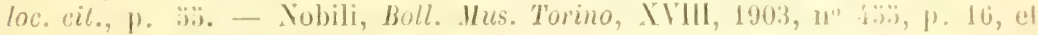
Bull. scient. Fr. Bely., XL, 1906, p. 76.

D. uvarus Alcock, Cat. Ind. Conest. 11, 1903, p. 68, pl. I1, fier. 6.

Les tres nombreux exemplaires recueillis par 11 . dousiratume it Suez, Aden, P'rim, Djibouli, ete. prourent que cetle especes est tres repandue dans la mer Rouge. Elle est ausis communt? daus le golfe l'ersigue. Lat plupart des exemplates araiont dija

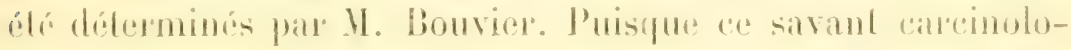
giste a pu examiner une immense serve d'exemplateres de lat

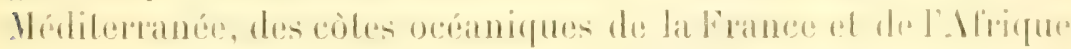
occidentale, je n'ai aucun doute de lidentile de cers axmplates avec l'espece lois varbable de l'kurope.

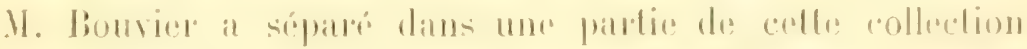

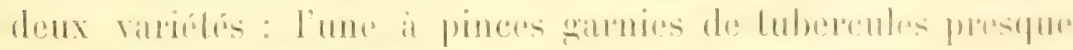

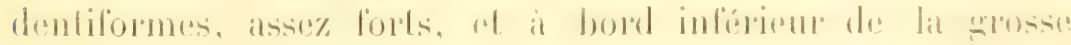

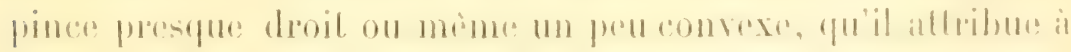

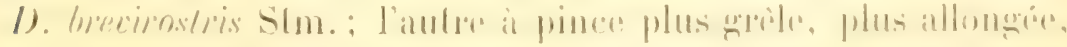

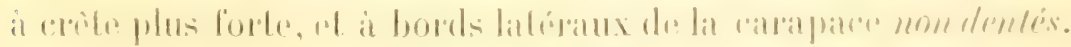

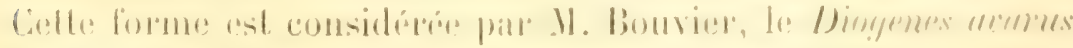

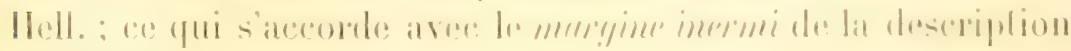

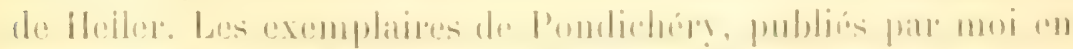

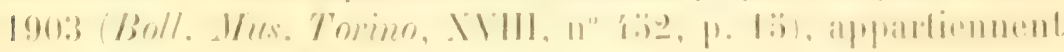

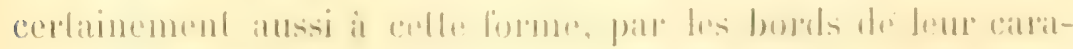
pace non dintion. 
Le D. acomes décrit par Alcock a les bords latéraux dentés.

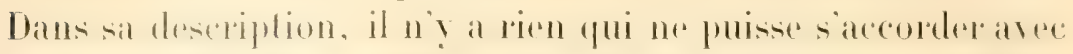

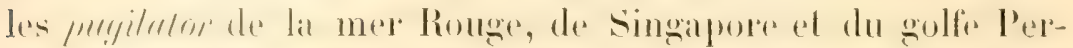

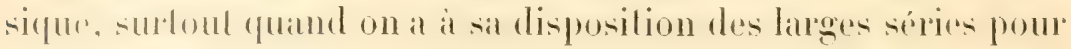
observer les très nombreuses variations de cette espèce. Je crois done démontré que le $D$. acarus des auteurs est iden-

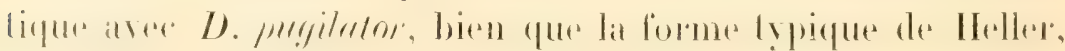
¿a borts non dentés, en soit une variété.

\section{Genre TrOGLOPAGURUS.}

Troglopagurus Jousseaumei Bouv.

Bouvier, Bull. du I/s., 1897, p. 231-232, fig. 6. - Alcock, loc. cil., p. 75, pl. I. lim. 6 .

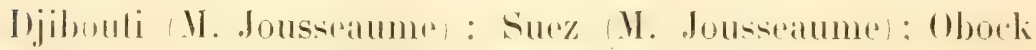
(II. Jousseaume); mer Rouge (II. Jousseaume).

Ciefle espece habite la mer Rouge, le golfe Persique el les côles occirlentales de l'Inde.

\section{Gexre PAGURLS Auct.}

A. Chélipèdes armés de tubercules ou d'épines, pas de lignes écailleuses imbriquées.

$B$. Pédoncules oculaires longs, à peu près comme le pédoncule des antennules ou mème plus. Cornées occupant pas plus d'un tiers de la longueur du pédoncule. Pattes très poilues.

C. Carapace et pattes ocellées. Chélipède gauche beaucoup plus large et plus long que le droit. Articles de la seconde moitié du fouet antennal non rentlés........................

P. punctulatus

Olivier.

CC. Carapace el pattes non ocellées. Une bande rouge sur le mérus et le carpe des pattes ambulatoires.) Chélipède gauche plus large mais de peu plus long que le droit. Articles de la seconde moitié du fouet antennal rentlés...... P. euopsis Dana.

BB. Pédoncules oculaires gros et courts, n'atteignant pas l'extrémité du pédoncule antennulaire; cornées grosses, atteignant un tiers ou même plus de la longueur du pédoncule.

D. Bord supéro-interne du doigt mobile du chélipède gauche en crète tranchante.......... P. deformis Edw.

D7). Bord supéro-interne du doigt mobile du chélipède gauche pourvu de rangées longitudinales de granulations................... tinctior (Forsk.). 
A. Chélipèdes ornés de lignes squamiformes imbriquées et ciliées. Pédoncules oculaires sros et subéraux aux pédoncules antennulaires. P'itles ambulaloires ornées partiellement de lignes ciliés................ Prrosor (Ilerbst).

\section{Pagurus arrosor (Herlist).}

Cancer (trosur Ilerbst, II, J. 170, pl. XLIII, lim. 1, 17!96.

Payums striatus Latreille, Hist. nat. Cr. Ins., 11, 1). 163, 1802. - lioux, Coust.

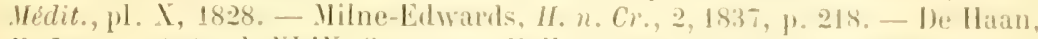
F. Jap., p. 206, pl. XLIX, fig. 1. -- Heller, Crust. Sudl. Eur., 1815i, p. 17't. - Ilenderson, Challeng. Anom., 1888, p. 3... - Ottmann, loe. cit., p. 283. Whitelegge, Wem. Austr. Ifus., IV, 1900, I. 166. - Savigny, Deser. Eg!/f)te, pl. IX, lig. 1.

Petrochims arrosor Rathbun, Proc. U. S. Mat. Mas.

Pagurus arrosor A. Wilne-Lirards et Bouvier. Crust. Trarailleur et Tulisman. p. 178. - Alcock, loc. cil., p. 168 (uli sym.).

Suez (II. Letommenx), un maile.

Lonerueur de la carapace............. 23 millim.

- de la portion céphaligue........ 12 -

- du bord antérieur............. 10 -

- des pédoncules oculaires....... 9. -

d'ai comparé avee altention cel individu avee d'aulese do la Méditerranée el avec un gros individu du Japon, el je n'ai lrouvó aturune dillérence appóciable.

Celle espece, en plus que dans la Mródilerrance el dans

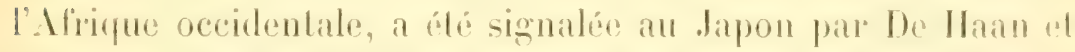

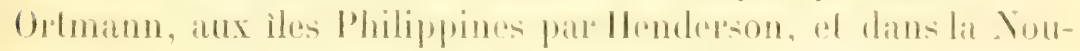

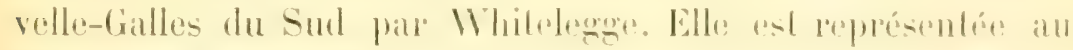
liresil par des variótís.

\section{Pagurus punctulatus Oliv.}

(it. Alenck, loe. cit., 1).81, pl. 1111, lig. 1 (ubi lit.).

Signatio dams la mor Roune par Hilgendort.

\section{Pagurus euopsis I Mnil.}

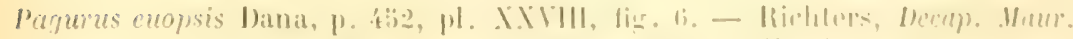

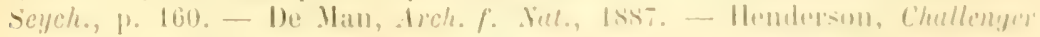

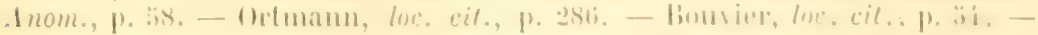

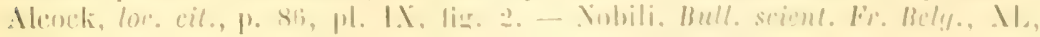
$1906,1 \%, 82$. 
P. depressus lleller, S. H. Akad. Hien, XLIV, 1862, p. 248. - Kossmann, p. 76. - Hilgendort, M. B. Alad. Berlin, 1878, 1. 814. - De Man, loc. cit, p. 431 .

Dardanus Ilellerii Paulson, loc. cit., p.90, pl. Xll, fig. 't-'c.

Périm, Obock el Alen (M. Jousseame), nombreux exemplaires; mer houge (Musée de Modène), un gros màle dont la carapace est longue de 29 millimetres.

\section{Pagurus tinctor (Forsk.).}

Cancer tinctor Forskaol, Deser. I nim., P. 93.

Pagurus tinctor Nobili, Bull. scient. Fr. Belg., XL, 1906, p. 81; Boll. Nus. Torino, $190 \%, \mathrm{XX}, \mathrm{n}^{\circ} 306, \mathrm{p}$. 4.

Pagurus varipes Heller, loc. cit., p. 244, pl. I, lig. 1, et pl. 11, fig. 2-3. - De Man, Not. Leyd. ILus., II, 1. 18; III, 1. 129; Irch.f. Yat., 1887, p. 436.Bouvier, loc. cit., p. "3t. - Alcock, loc. cit., p.90, pl. IX, lig. 7.

Nombreux exemplaires de la mer houge (Jousseaume); Obock dans Ranella spmosa (M. Culliéred); Massaomah (MV. Bonnier et Pérez), el d'autres de la mème localité

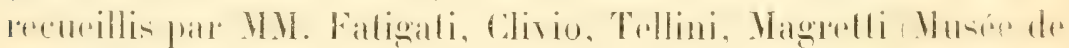
Turin); Assab, Moka (Musée de Modène).

La plupart des exemplaires de Massaouah ont la coquille couverte de nombreuses Actinies du genre Adamsir.

\section{Pagurus deformis Edw.}

11. Milne-Edwards, Lnn. Sc. Nat. (2), VI, 1836, p. 272, pl. XIT, lig. 2. Mliers, Zool. Erebus Terror Crust., p. 3, pl. II, lig. 3. - De Man, Iroh. f. Naturg., 1887, 1. 435.-Ortmann, loc. cit., 1.288. - Alcock, loc. cit., p. 88, pl. IX, fig. 4 .

Un exemplaire dans les collections du Muséum, dont la provenance de la mer Rouge n'est pas toul it fait sume, bien qu'elle

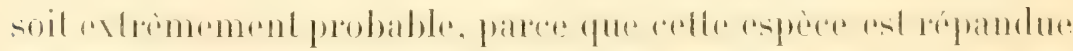
dans loute la région Indo-Pacifique.

\section{(ifNRE ANIGULUS I) ANA.}

\section{Aniculus strigatus (Herbst)}

Cancer strigatus Herlost, III, IV, 180x, p. 23, pl. I.XI, fig. 3.

Pagurus strigatus Oliv. - Ililgendorf, H. B. Ikad. Berlin, 1878, J. 820, pl. II, lig. 8. - Ortmann, loc. cit., 1. 28\%. - Bouvier, loc. cit., 1. 5x. - Borradaile, Willey's Zool. Res., IV, 1899, p. 23. - Nobili, Boil. Mus. Torino, XVIII, 1903, no 432, 1). 1:i. 
Aniculus strigalus Henderson, Tians. Limn. Sive. 2). 1, 1898, 1), +2.2. - 11-

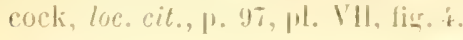

Périm (I. Jousseaume), un exemplater. Les annoilux squatmilormes qui exelent lespatles sont rouges, les poils jaumes:

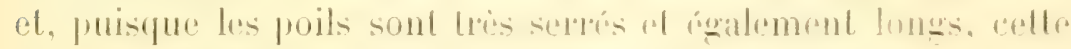
espece apparail annelée allemalivement de rouge al do jatue.

signalé anssi à Men, par M. lionviur.

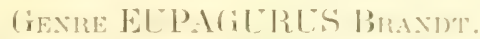

Eupagurus cavicarpus P'uls.

Paulson, p. s1, p. XII, fir. 3-3

Cedte espece m’est inconnue. Elle a dés décrite ainsi part Paulson.

" Patr la forme de ses chálipedes celle esposer rappelle

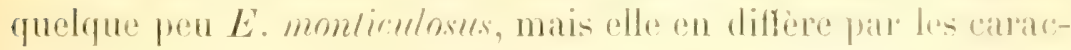
leres suivants : pédoncules oculaires distinctement plus longs et plus gros; pédoncules des antennes externes ansi longque les peux; l'acicle arrive dans l’un jusqu'il la cormón

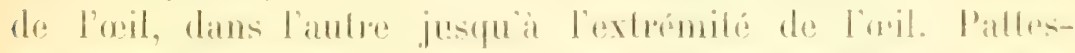

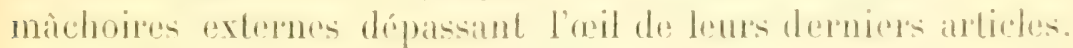

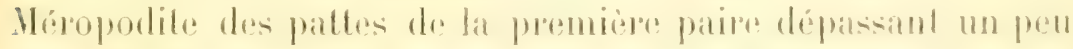

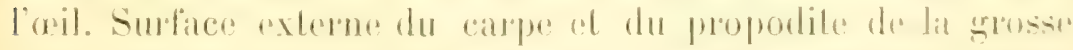

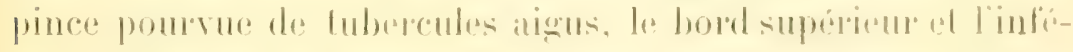
risur du propodite canaliculés; pattie módiane aonsexe, dams

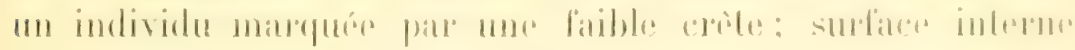

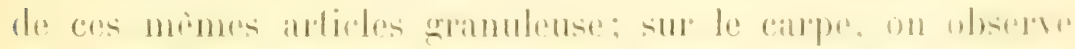

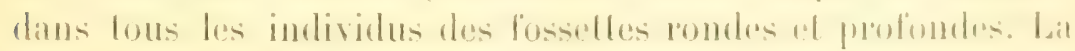

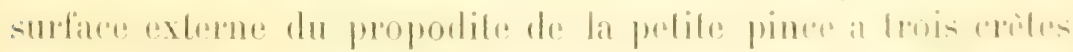

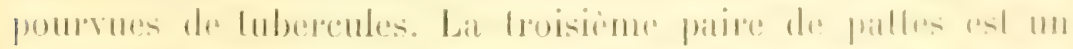

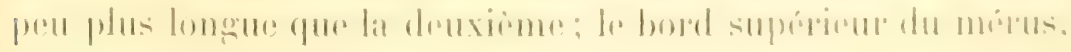

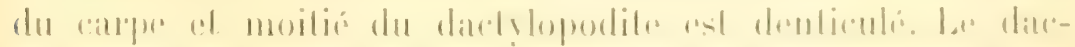

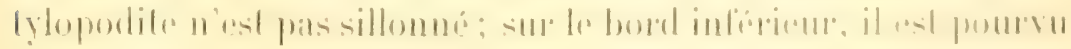

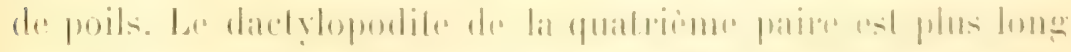

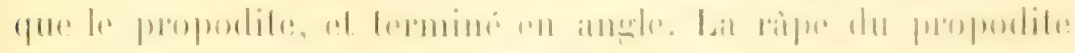

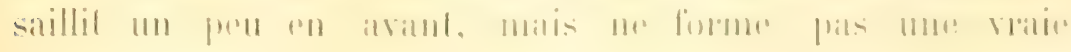
pince. " 


\title{
Genre Cestopagurus Bouvier. \\ Cestopagurus Coutierei Bouv.
}

Bouvier, Bull. du Nus., 1897, nº 6, p. 229, tig. 1-马.

T)jibonbi M. Coutiere, un exemplaire lype de lesperes.

\section{FAMILLE CQENOBITIDAE}

Gœnobita rugosus var. Jousseaumei Bour.

Bouvier, Bull. Soc. Philom. Paris (8), II, 1889-90, p. 146.

Tres nombreux exemplaires de Obock (11. Jousseaume); Djibuti MV. Jousseaume, Couliere, Gravier); P'érim el Aden Il. Jousseaume): mer Rouge, Assab, Buia, Périm (I. Ragazzi, Musée de Modene); Massaouah (MII. Clivio. Magretli, Mus. Turin, golfe d'Mabah M. Ireonali, Mus. Turin).

\section{GALATHEIDEA}

\section{FAMILLE GALATHEID}

\author{
Genre GALATHEA.
}

A. Rostre étroit, pourvu de trois épines seulement de chaque côté. P’as d'épine sur la carapace ou à la base du

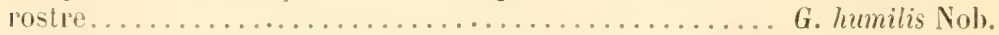

1A. Rostre pourvu de quatre épines de chaque côté. Des épines à la base du rostre ou sur la carapace.

B. Pattes de la première paire très allongées; le mérus à lui seul est deux fois et demi aussi long que la

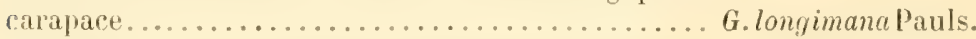

$B B$. Pattes de la première paire de longueur normale ou courtes.

C. Pattes de la première paire plus de deux fois aussi longues rue la carapace. Main aussi longue que la carapace avec le rostre............. G. segyptiaca Pauls.

CC. Pattes de la première paire à peine d'un quart plus longues que la carapace; main plus courte que moitié de la carapace................ Grevimana Pauls.

\section{Galathea humilis Nob.}

(Pl. VIII, fig. 1.)

Nołili, Bull. Mus., 1903, no 6, p. 396.

Lietle petite espèce est représentée par sept exemplaires 
sans paltes el par Lrois pinces détachérs, hourées par II. Conlière à Djibouti.

La carapace des trois exemplaires, de la pointe du rostre au bord posterieur, mesure 3, 3 it í millimetres de longueur. Les femelles ont des auts.

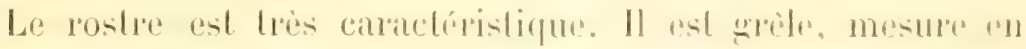

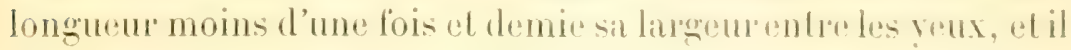
est armé sur les cotés de 3 dents sentement. dont l'une placéce pres des yeux, la deuxième est assoz doignés de colle-ci at la troisieme est plus rapprochée de la pointe. Lal deuxieme dent est la plus longue. Sa surfite est concave at 141 peu poilur. Lai longueur du rostre est entre un tiers at la moilio de lat longurur (le lia carapace.

L'angle externe de l'orbitre est aigu. Sur les bords lateraux de la carapace, il y a ö-6 pelites épines. Le dos de: la cirripatere est traversé par un petit nombre de lignes poilues, dont celles en avant du sillon cervical, qui n'est pas plus plofond que hes

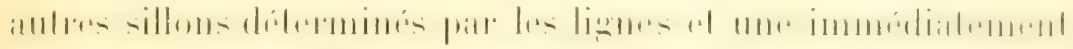
en arriere de ce sillon, sont interrompues. Il n'y a aucune ápine sur les lignes du dos de la carapace.

Les maxillipedes externes sont courts of tres poilus. Le mé-

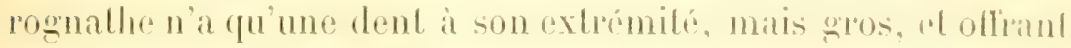
ansi une entaille dentilorme sur son hord.

II n’y a que des chélipèdes débachés, qui sont it pou prés deux lois aussi longs que les camapaces des autres exem-

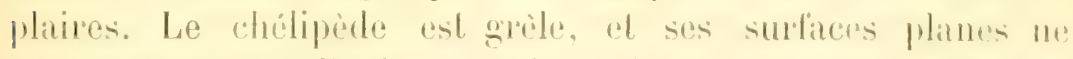
sont pas armées d'épines, mais seulement àples. Les ípines des bords sont aussi peu nomberuses. L ischium as laiblement

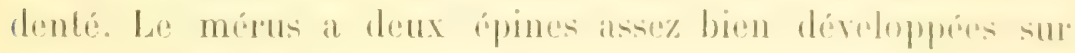

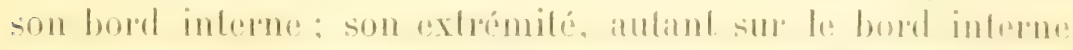

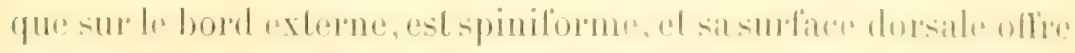

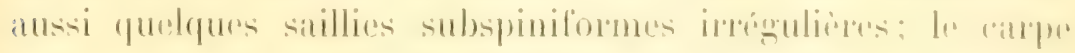

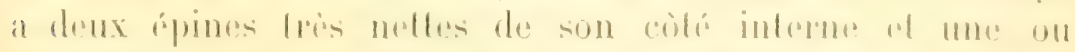

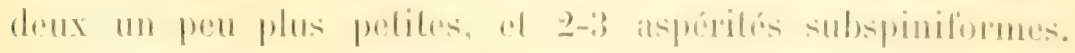

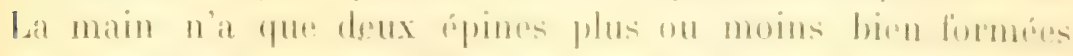

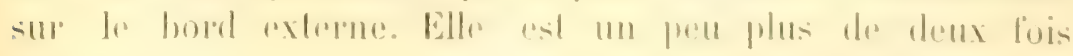

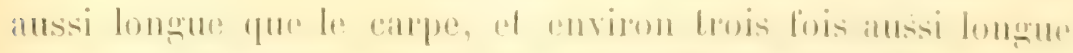

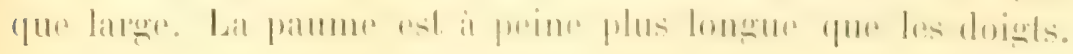


Ceux-ci sont baillants, à pointe excavéc el dentée, pourvus de longs poils; le doigt mobile a deux grosses dents émoussées: le doigt fixe une seule.

Les pattes ambulatoires manquent entièrement.

Celle espèce, bien que j'aie dù la décrire avec trop peu de

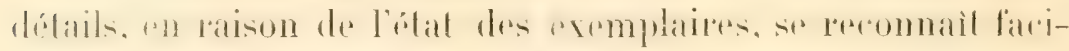

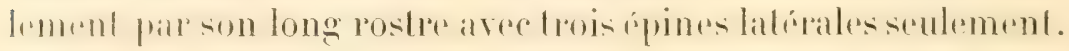

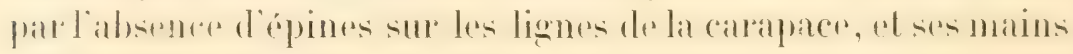
it surface plane non épineuse.

Galathea æg:yptiaca Pauls.

(Pl. VIi, fig. 3.)

Paulson, loc. cit., p. 94, pl. XII, fig. 1-16.

(i). spinosi rostris Dana. Nobili, Am. Hus. Yupoli, I, no 3, 1901, p. 6.

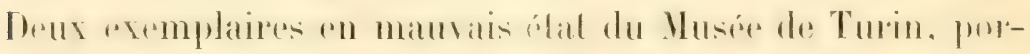

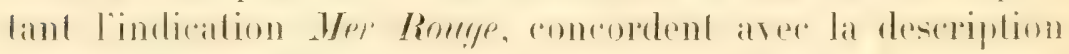
russe el la figure.

La carapace de l'individu plus gros est longue de $3^{\text {mam }}, 6$ de

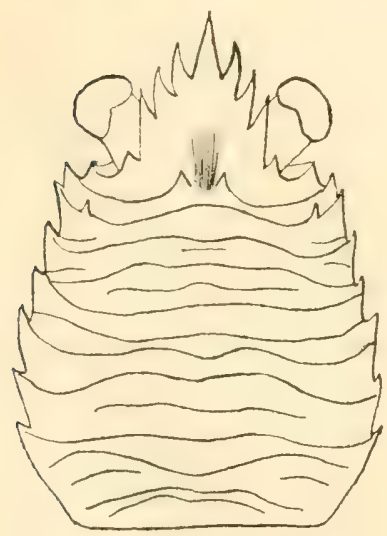

Fig. 8. - Galathen regyptiaca (d'apres P'aulson). l'extrémité du rostre au bord postérieur. Son rostre est pourvu de chaque côté de quatre épines, l'une placée près des orbites et petite (c'est cette épine qui manque ì $G$. humilis), les autres épines longues, a bord externe presque droit; la pointe dépasse nettement l'extrémité de la dernière paire d'épines. La largeur du rostre à sa base est les $4 / 3$ de sa longueur. Le rostre est concave en dessus; en arrière du rostre sur la premiere ligne de la carapace, il y a deux petiles épines. L'angle orbitare externe est aigu; les bords latéraux de la carapace sont convexes el armés, en plus de l'épine extraorbitaire, de 6-7 épines. La surlace de la carapace est parcourue, ainsi que de règle, par des lignes eiliées; sur la deuxième de ces lignes, lout près des bords latéraux, il y a 
une épine de chanure colr. Il n’y a pas de sillon cervical sur lat calatace.

Les palles-machoires axtromes, étendues. dépassent de

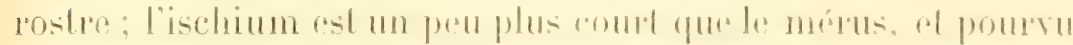
a lextremite de son bord interme de denx pelites dents: les

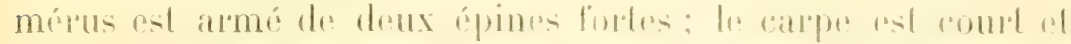

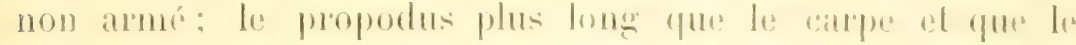
dactrlus.

Les chélipedes sont plus de deux lois ausi longes que la carapace. Le mépus a deux fipines forten ed une pelite sur son

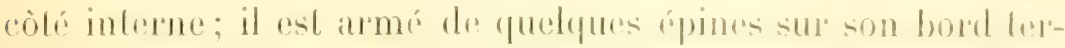
minal. Sur sa surface dorsitre il y at deux rangeres de spinules petites. Le carpe est armé d'une épine forte sur son bord interne, of d'une autre moins pobuste it son cxtromite of de deux rangés d'épiness sursilsurlace dorsale. La main os un peu plus de drux fois aussi fongue que le carpe; la paume est un

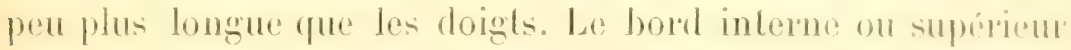
de la main n'a que 2-3 spimules, le bord inférien ou exterme est armé de T-S dents spiniformes, qui se conlinuent, an diminuant, sur le bord infórieur du doigh fixe. Lat sufface de lat main est parcourue par dens lignes de spinules lres pelibes. Les doigh sont baillants. Le doigt mobile a une dent tubereculilorme a sa moilié le doigl fixe a une dent a son extrimiti.

Les paltes ambulatoires ont le bord supérien du mérus armé de spinules aiguis: le hord inferien a allsid de pelites

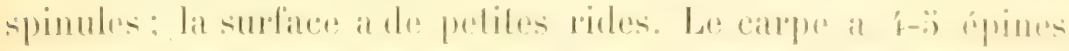

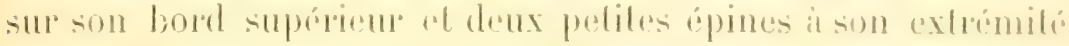

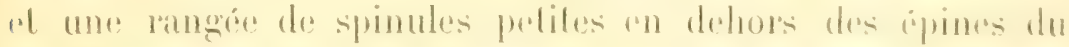
bord suprexeme. Le propodite "st ame de spinules pelites en

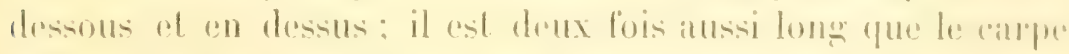

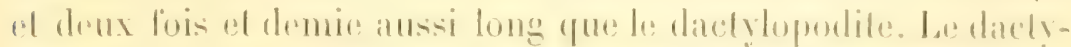

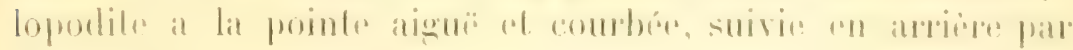
:-6 spimules.

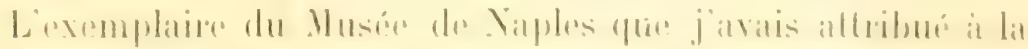

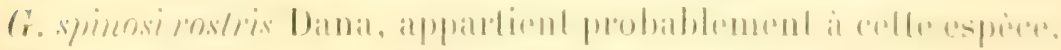

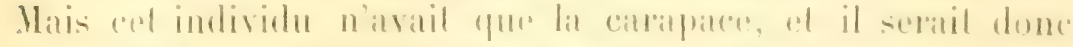

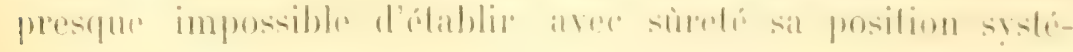
maliveur. 


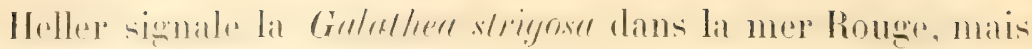
eelte espèce doit être réexaminée.

\section{Galathea brevimana Pauls.}

Paulson, loc. cit., p. 9:.

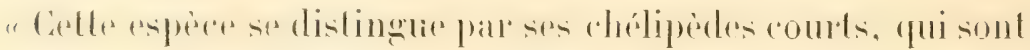

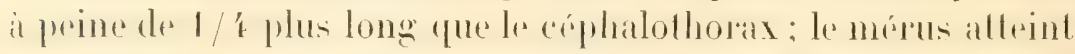

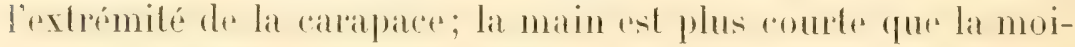

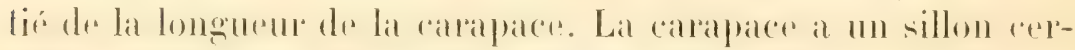
vical el des lignes transversales. Sur le bord hépatique, il y a 2 denticules; sur le bord branchial ö; une dent ast placée

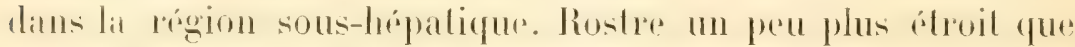

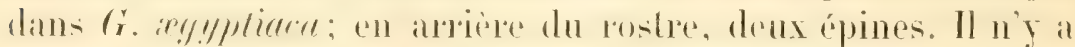

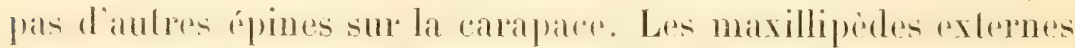

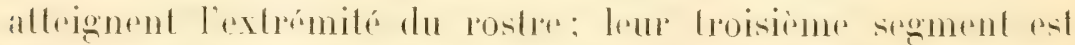

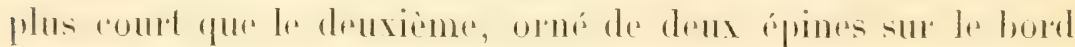

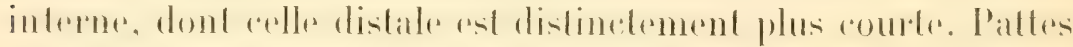
ambulatoires comme dans $G$. ixg!ypliaca. w

\section{Galathea longimana Pauls.}

Paulson, loc. cit., p. 94, pl. XII, fig. 2-2a.

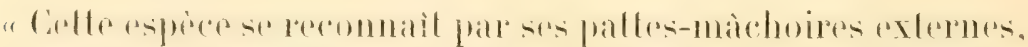
par son rostre étroit, par la disposition des spinules sur la carapace, el par ses longs chélipèdes, donl le mérus est deux

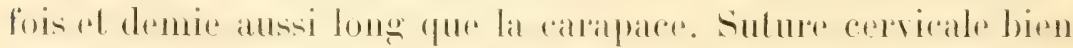

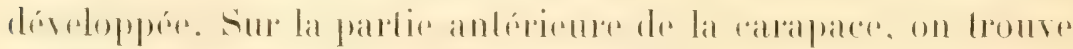
une ligne squamiforme, transversale en arière, pourvue

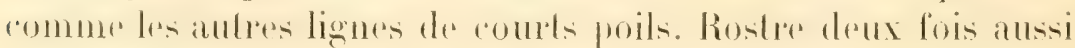

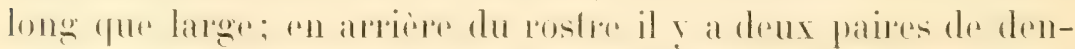

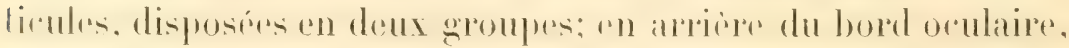

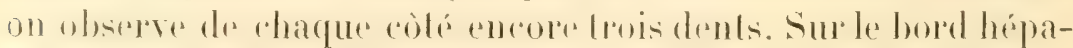

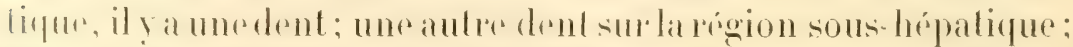

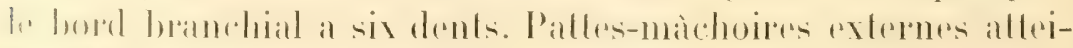

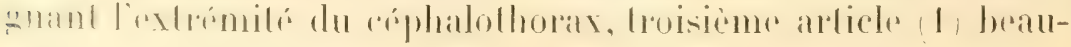


coup plus court que le deuxieme; son bord interne arme de deux dents, l'externe d'une; quatrieme of sinquisme articles également longs.

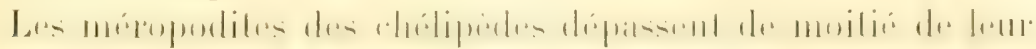
longueur le rostre. Le propodite arec les doigls est plus long que la carapace et le rostre. La surlace aterne ot les bords du

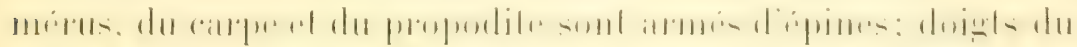

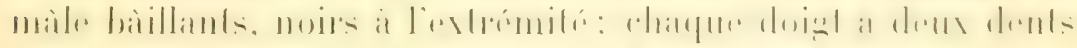

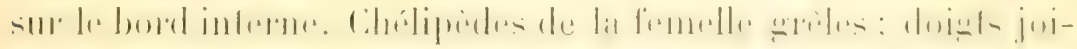
gnant bien, at bord interne denticulé.

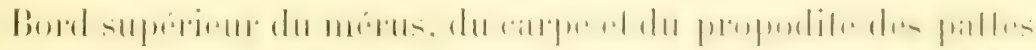

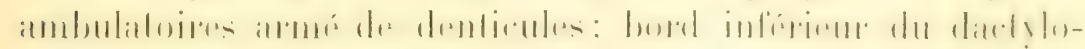
porlite pourvu de spinules."

\section{FINILLE PORCELLANTISE}

\section{GENTE PETROIASTHES STM.}

A. Bord externe de la paume non denté, ni tuberculé.

B. Surlace de la carapace pourvue de lignes poilues

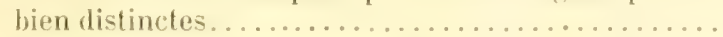

BB. Surface de la carapace sans lignes poilues (ou avec lignes microscopiques) el sans grosses granulations. l’as d'épine épibranchiale.

C. Carpe relativement large (1,6 aussi long que large) avec $3-;$ dents bien formées.......... I'. rufescens (IIell.).

$C C$. Carpe allongé, de deux it trois fois aussi long que large, avee $2-3$ dents peu marquies.......P. leptocheles (Ilelleri.

A. Bord externe de la paumedentí, ou pourvu de tubercules.

B. Pas d'épine supra-orbitaire.

C. Carapace avec des gros tubercules arrondis. Pas dépine épibranchiale. Bord de la paume tuberculé. Peu de poils sur la carapace. Méropodites tuberculés................. omatus Pituls. $=\left(\boldsymbol{I}^{\prime}\right.$. mossambicus llilged.

CC. Carapace fortement poilue, sans tubercules. Lne épine épibranchale très petite. Bord externe de la paume denticulé el trís poilu. Méroporlites lisses et poilus................. J'. viryutus l'auls.

(= tririryutus (1)tm.).

BB. Lue épine épibranchiale et pas d'épine sus-orbilaire. Méropodites denticulés en dessus el aver une

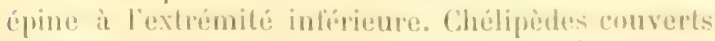
de tignes saillantes poilues. Front denticule....... P. tomentosus Dana.

BBB. Ine éprine sus-aculaire el une épine épibranchiale. Front lisse................... I'. carinipes Ilell. 


\section{Petrolisthes Bosci (Aud.).}

Savigny, pl. VII, fig. 2.

Porcellana Bosci Audouin, Expl., p. 88. - Heller, loc. cit., p. 2:36. - De Man, J. Limn. Soc., XXII, p. 217; Not. Leyd. Mus., III, 1881, p. 10'.

Petrolisthes Bosci Stimpson. Paulson, loc. cit., 1. 8\%. - Kossmann, Zool. Ergebn. rolh. Heer., p. 74. - Ortmann, Z. Jahrb. Syst., X, p. 283-284. Nobili, Inn. Mus. Napoli, 1, 1901, n³ 3. p. 7; Bull. scient. Fr. Belg., XL, 1906, P. 66.

Porcellana rugosa II. Milne-Edwards, H. n. C 2,2, p. 282.

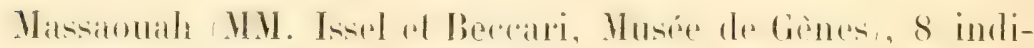

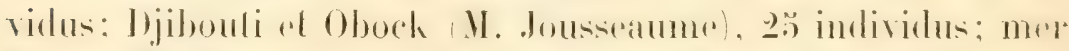

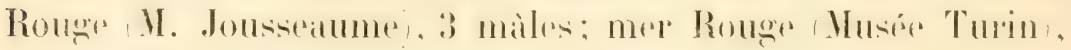
4 individus; ille Daret (Musée de Naples), 1 femelle.

\section{Petrolisthes rufescens Hell.}

Porcelluna rufescens Heller, loc. cit., p. 25\%, pl. II, fig. 4.

Petrolisthes mufescens Paulson, loc. tit., p. 88. - Hilgendorf, M. B. Akad. Ber-

lin, 1878, p. 825, pl. II, fig. 7. - Nobili, Ann. Mus. Napoli, 1, 1901, no 3, p. 7;

Bull. scient. Fr. Belg., XL, 1906, P. 66.

Petrolisthes Lamarchi Ortmann, Denkschr. Jena, IIII, 189x, P. 26 (pars).

P. Lamarcki var. rufescens Borradaile, Proc. Zool. Soc., 1898, 1) 66.

?. P. rufescens Kossmann, loc. cit., p. 73.

P. clentatus Haswell, Cat. Iustr. Crust., 1882, p. 146. - Ortmann, Zool. Jahrb. syst., VI, 18\$2, 1. 262.

Mer Rouge, Aden et Obock (I. Jousseaume), 60 exem-

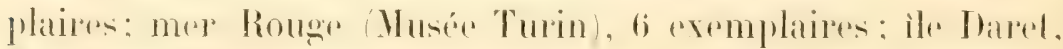

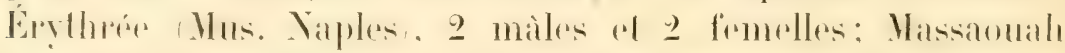
(Mil. Issel et Beccari, Irus. Gènes), 12 exemplaires; Aden (M. Chaper), 1 màle et 1 femelle; Suez (II. Létourneux), 12 exmplaires: Obock M. Maindronf, I mate: I jibouli (M. Maindron), 1 mâle.

Dans mon chude sur les Crostacés du golfe Persique, jai

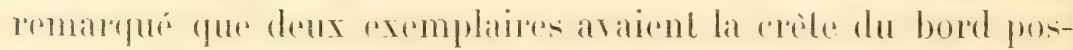

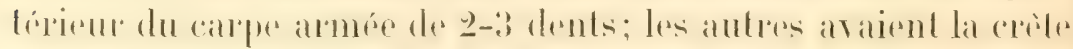

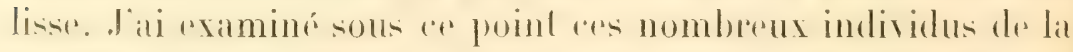
mer Rouge, al je vois que, foul en restant constants les callatelères de l'espèce, on peut y séparer deux formes:

l" Lime typique correspondint au lype de Iteller salns dents sur le bord postérieur du carpe et qui par ce caractère s'éloigne le plus de P. dentatus ou Lamarclii. 
Petrolisthes leptocheles Ortmann, Denlischr. Jena, VIII, 1894, p. 23. - De Man, Not. Leyd. II Lus., XV, 1893, p. 292.

? Cancer antennatus Forskal, p. 89.

Djibouti ef (Obock (I. Jousseame), nombreux exemplaires: Massaouah (MM. Issel et Beccari), כ้ exemplaires.

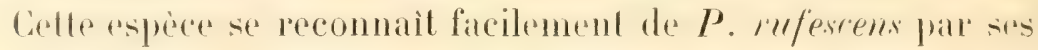
chripedes fort allongés dans les adultes, par ses carpes phus grêles, et avec une disposition dillérente des dents.

La carapace n’a pas d'épine épilnanchiale ni d'épine supratoculaire). Elle est un peu plus larger que longure; sil surface 11 a rien de différent des autres Petrolisthes de ce groupe : elle ast glabre at ponctuée, el pourve de quelques rides sur les parties laterales el doune petile crete an arriere du front. Le front a le sillon habituel en dessous: son lobe mídian est amomeli el savance bien au-devant des latéraux qui sont un peu obliques. Les bords latéraux de la carapace sont carénés.

Les chélipedes sont trè longs ol grobles dans les atulter. moins longs dans les jeumes. Dans un male adulte de Djibouti, les chélipedessont plux de trois forix answi longx que la carapace: dams les individus plus jemes, ils ne mesment qu'un peu plus de deux fois la longueur de la carapace. Le mórus forme it son Polrénite anterieme un lobe subatigu; le bord de ce lobe est denticule. Il n'y a pas de dents sur la face inférieure de rent anticle. Dans les miles adultes le carpe est um peu plus long que la carapace: sa longueur dans l'adulte déjic nommé wet de trois fois sa largeur a la base (y compris la dent); dans les jeunes celle longuerur descend mime a deux fois sentement. Le bord antéreur a loujours une dent bien nelle a la base: puis le bord mince devient irrégulier, el forme prestgue loujours un lobe vers la moilié, el un lobe ress l'extremile, qui, foul en pourant dans les jeumes itue plus ou moins aigus, sonl loujours fulits ol pen saillants el me ressemblent an rien a la figure de Iletler, latpelle, si mon identilicalion de l'esperee est exarde. n'est pas bonne. Le bord postérieur du carpe n'a qu'une sentr dent a son extrémite. Les mains sont allongées, de mime

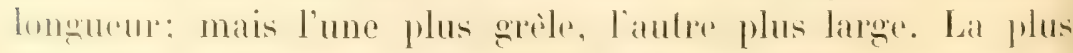

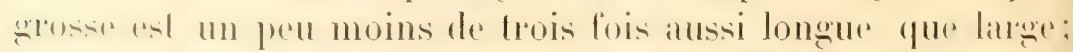

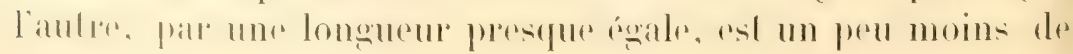


quabe fois aussi longue que laren. Il n'y a pas de vraies lignes

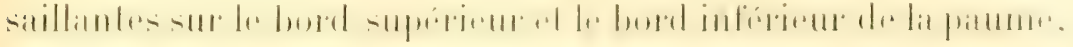
mais ces deux bolds sont un pen comprimés et minces. Le bord externe de la main est droil sur ha pince plus petite. un peu convexe par suite de la coubure du doigt fixe sur l'aulpes pince. Les doigts de la petite pince joignent bien, ceux de la

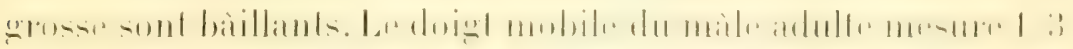
de la longueur tolale de la main; sur la grosse pince, il a un pelit lubereule dentiforme aigu, oblique it la base: sur la petite en Lubercule manque. Les deux doigts sur les denx mains sont bien poilus du coté interne; les poils sont courts.

Les pattes ambulatoires n'ont pas d'épines; mais l'uxtré-

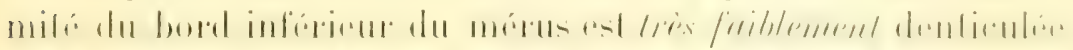

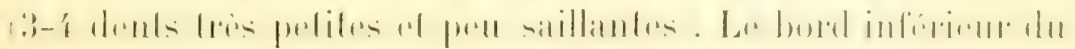
propodite finit en une pelite épine.

Le Concer antemalues que Forskal a trouvé à Suez in /oraminihus Spongixe offirinalis violarex est certainement (par ses antenne selacex thorace tripjolo longiores: rarus in brachymis charmeter), un P'orcellanien el par ses compri introrsum bidentali se ritpproche de celle espece.

Les exemplaires mieux conservés sont sourent lavés de blumbe comme ceux de P. rufexcen.

\section{Petrolisthes ornatus Pauls.}

Paulson, loc. cit., 18\%i, p. 86, p1. XI, fig. 3.

Porcellana (Petrolisthes) mossambica Hilgendorf, II. B. Akat. Berlin, 157- 1).82:i, fil. 11, lin. 1i.

Djibouli, Obock el P'erim (I. Jousseaume), I0 mailes el If femelles; mer Rouge (Mus. Turin), 3 exemplairs.

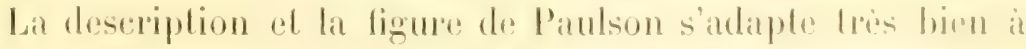

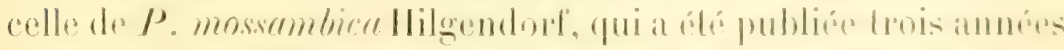
plus lard. La dillérence principale ast seubement dans le lolue médian du front qui est plus court of plus arrondi dius la

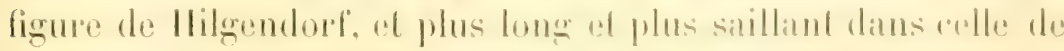
Paulson. Cedte difference pourtant n'a pas de valeur patree que l'on trouve les pasiages entre les deux lommes. 


\section{Petrolisthes virgatus Pauls.}

Paulson, toc. cit., p. 8\%, pl. XI, fig. 4.

Petrolisthes trivirgatus Ortmann, Denkschr. Jena, VIII, 1897, 1). 27, 28; Zool. Jakt?. Syst., X, 1897, p. 287, 288.

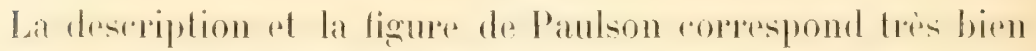

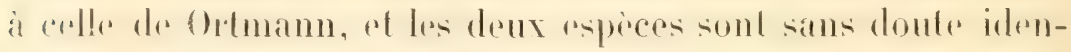
liques. Je molerai que Paulson signale la présence diume pedite

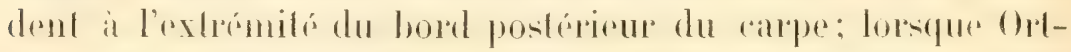

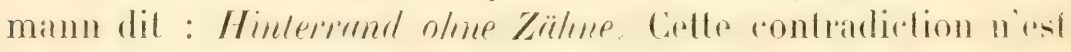
poutant qu'apparente. paree que dans la figure des deux

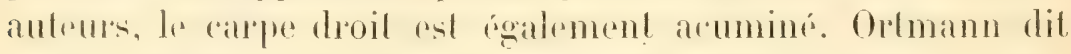

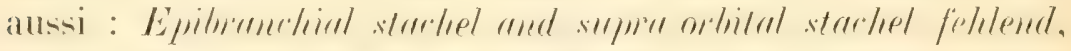
lorsque Patulson déril une dent laterate. mais celle dent asl pelite mime a un grosisisement de vingl fois : alle pent done: bien avoir échappé à Ortmann.

La coloration est du mème type : Dünliel vinletl... mit drei

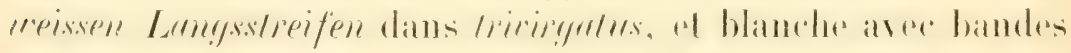

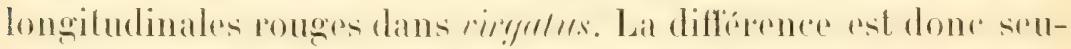

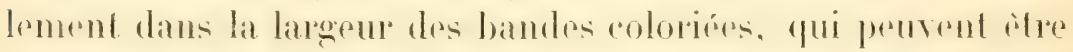

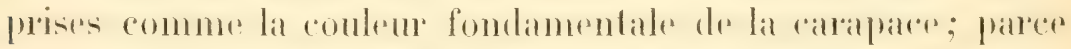

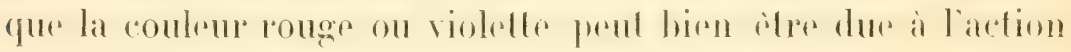
de l'alcool. Sourent d'ailleurs on troure des animaux rouges el d'autres violels dans une même espèce (1).

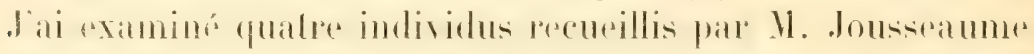
à Obock. Ils s'accordent très bien avec les deux descriptions. La bordure de poils sur le bord externe de la main rsl longere et touffue; parmi les poils se trouvent cinq ou six petites epines. Les palles ambulaboires sont aussi poilues que les ché-

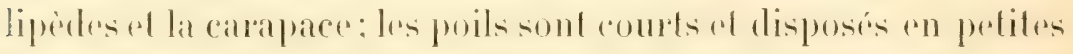

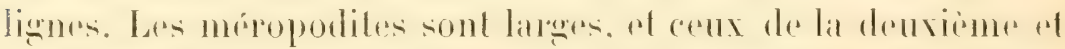

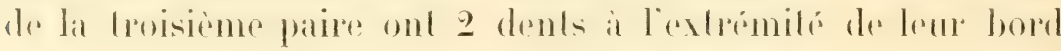

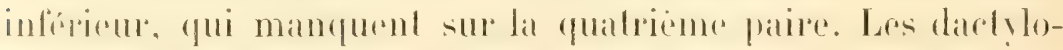
podites ont deux ou trois petites épines cornées.

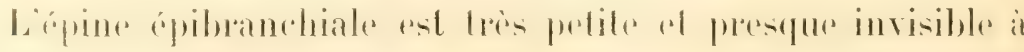
l'oil nu.

(1) Jai observé ce fait, par exemple, dans Homalaspis plana et dans Platyxanthus crenulutus. 
Longueur de la carapace...... 10 millim. a millim.

Lonerueur du carpe............

- de la main......... $12-11-$

- des doigts......... i; - ij -

Canlle espece nost connue que de la mer Rouge cl de Kanzibar : Dar es Salaam.

\section{Petrolisthes carinipes Hell.}

Porcellana carinipes Ileller', loc. cit., p. 2:57, pl. II, fig.::- - De Man, Hol. Leyd. IIus., III, 1881, p. 10't.

Petrolisthes curinipes De Man, Not. Leyd. Mus., XY, 1893, p. 299, pl. I'Il, tig. ̈̈. Ortmann, Zool. Jahth. Syst., X, 1897, p. 287, $28 \%$.

Mer Rouge (Mus. Turin), '́ individus; Djibouli (M. Jousseaume), 2 femelles.

Ces exemplaires s'aceordent bien avec les descriptions de Heller et de De Man, si ce n'est par le lront qui me parail plus large et a lobe moren moins saillant. Dans tous les exemplaires, la carapace el les pattes sont ornées de petites lignes ou tubercules squamiformes poilus, ì peu près comme dans P. Bosci, mats moins forts. Les carpes de denx exemplaires du Musée de Turin ont 3 dents it lem bord antirixur. ot. i. épines, dont l'apicale est double, a leur bord postérieur: un exemplaire de Djibouli a ö dents it droile of ta ganches sur le borl anterieur.

Cielle espece n'ost connue rue do la mer houge.

\section{Petrolisthes tomentosus Dinal.}

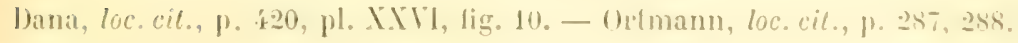

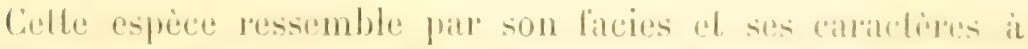

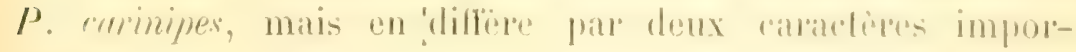
lanls:

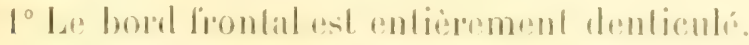

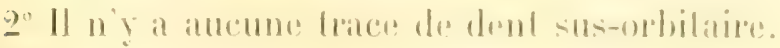

Elle est représenté par dema exemplanires do Obock M. Jousseatli11( ). 


\section{Genre PAGHYGHELES Stmipson.}

\section{Pachycheles sculptus (Edw.).}

Porcellanc sculpta II. Milne-Ldwards, H. n. Cr., 2, p. 253.

porcellanu (Pisisoma) sculpta De Man, Arch. f. Nat., 1887, p. 413 ; Journ.Linn. Soc., XXII, p. 218.

Pisisona sculptum Ortmann, Zool. Jarhb. Syst., VI, 1892, p. 263. - De Man, ibid., IX, p. 378.

Pachycheles sculptus Ortmann, Denkschr. Jena, VIll, P. 29; Zool. Jahrb. Syst., X, 1897, p. 292, 294. - De Ilan, Abh. Senckenb. Ges., XXV, 1902, p. 701. Nobili, Bull. scient. Fr. Belg., XL, 1906, p. 67.

Pisosoma natalensis Krauss. Paulson, loc. cit., p. 88, pl. XI, fig. 5.

Massaouah My. Issel el Beccalli, Mus. Gènes), un jeune individu; mere Rouge Mus. Turin; 6 molividus; Oboch et Djibouti (M. Jousseaume), 3 mîles et ว̆ femelles.

Tous ces exemplaires, excepté une fermelle en mue, ont un pinceau de poils sur la fite interne de la main, a l'articulation du doigt.

\section{Genre POLYONYX Stimpson.}

A. Pinces à peu près d'égale forme; la petite pince non pourvue d'épines ou de soies rudes sur sa surface. Bords de la carapace non dentés.

B. Front tridenté à lobes infléchis. Une petite crête sur la face interne de la main. Méropodites ambulatoires

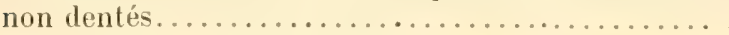

$B B$. Front presque droit. Pas de crête sur la face inP. triunguiculatus Zehntn.

terne de la main. Héropodites ambulatoires dentés. 1 . pedulis Nob.

AA. Pinces de forme inégale, l'une grosse et lisse, l'autre petite, épineuse ou poilue. Bords de la carapace avec des dents spiniformes.

B. Petite pince épineuse.................. P. pugitaton Nob.

BB. Petite pince poilue mais non épineuse........P. denticulatus Pauls.

\section{Polyonyx triunguiculatus Zehntn.}

Polyonyx triunguiculatus Miers, Alert, p. 539. - De Man, Arch.f. Nat., 1887, p. 421. - Nobili, Ann. Mus. Napoli, 1, 1901, n 3, p. 7 (nec Dana).

Polyony acutifrons De Man, Zool. Juhrb. Syst., IX, p. 384, fig. 49; Abh. Senckenb. Ges., XXY, 1902, p. 709.

Polyonyx triunguiculutus Zehntner, Rev. Suisse Zool., II, 1894, p. 185. - Nobili, Boll. Mus. Torino, XX, $1905, \mathrm{n}^{\circ} 506$, p. 3.

()hock (II. Jousieaume), un individu: Djihouli (II. Gravier'),

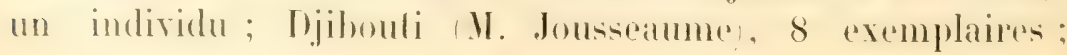


Érylhée (Jus. Naples), un exemplaire; mer Rougu Mus. Turin), un exemplaire.

Ces exemplaires appartiennent bion tous it P. Iriungrumblutus par la forme du front et par la pelite crète sur la face interne de la main. Je note pourlant que la crète du bord infériente de main est, sur la potite pince, finement denliculée, caractire que je ne trouve dans aucune des descriptions, et que les points et les fosseltes imprimés sur la face externe de la petile main sont sourent profonds el si serrés que la surface de la main el du doigt mobile en apparail tuberculée. Je noterai ausi gum les doigts sont la plupart des fois batillants, of la saillie a la

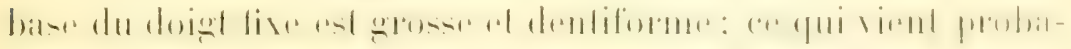
blement de ce que les exemplaires sont males.

Signalé anssi a Snez, par Miers.

\section{Polyonyx pedalis Nol,}

(Pl. YIII, fig. 3.

Nobili, Bull. Mus., 190:3, no 3, p. 396.

Colle espece a quelques affinilés arec P. comeler Miallier (- P. enphroxyne De Man). Elle est représentée par deux

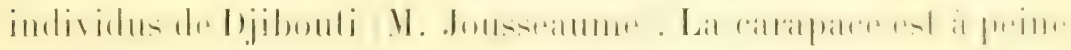
phus longue que large, à bords latéraux presque parabledes. dropourvus de coèle el de dent épibranchiale. Lat surfice n a rien

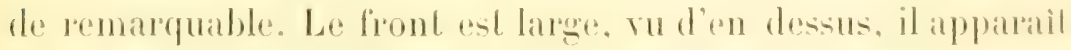
droil: vu d'en bas, il ofle un fres pelit lobe au milieu, qui rst it peine plus long que les lobes latéaux, gui sont formés pale

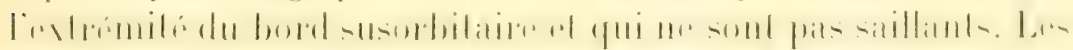
orbiles sont petiles, leur angle externe n'est pas saillant.

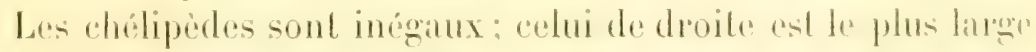

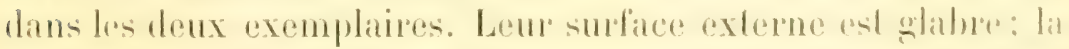

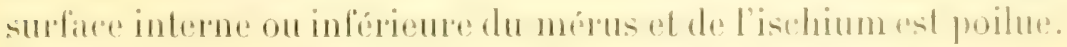

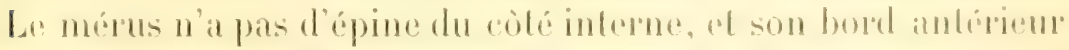

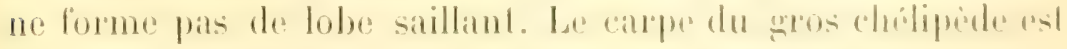

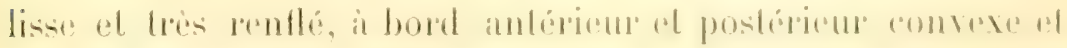

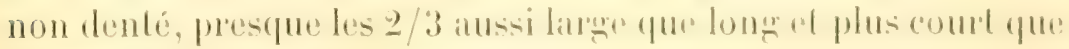
lit milin.

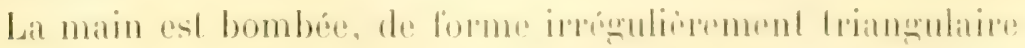




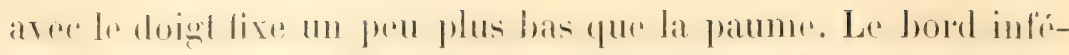

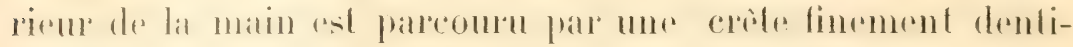
culce ef pourvue de courts poils, qui remontent aussi sur le doigl fise. Le doigh mobile est forl, arochu, salls dents. plus

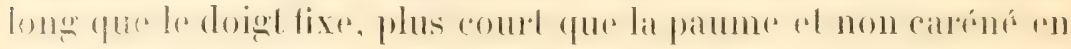

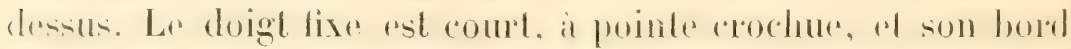
lamehant est presque entierement ocruper par la satlie basi-

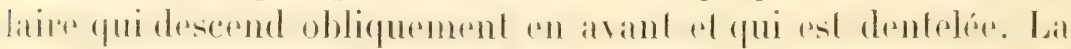
fater interme de la main est glabre. celle du carpe poilue. La

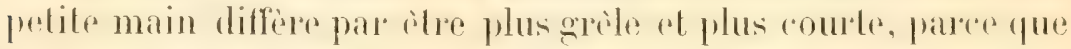

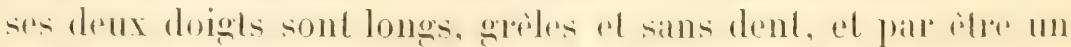
peu plus poilue.

Les paltes ambulatoines sont contes al prilues. Le meropo-

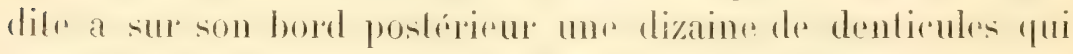
sonl bien visibles lorsquion entiere les poils: le propodite as

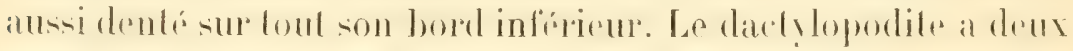

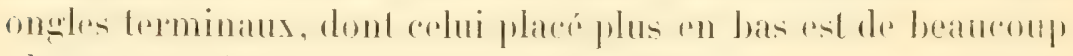
plus gros, suivis par deux pelites spinules.

Longueur de la carapace...............

Largeul

Longueur du carpe.

Largeur

Longueur de la main.

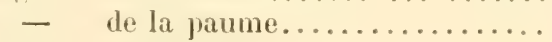

Hauleur de la paume................

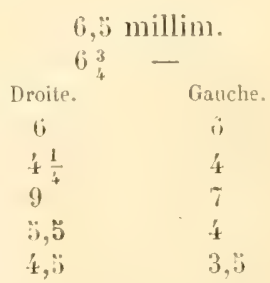

Cetle espèce se distingue facilement du $P$. cometes par sa

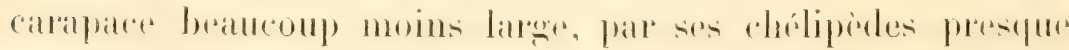

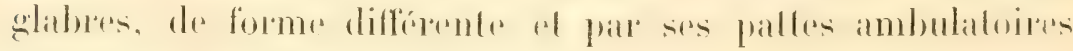
denticulées en dessous.

\section{Polyonyx denticulatus Paulson.}

Paulson, loc. cit., p. 89, pl. XI, fig. 6.

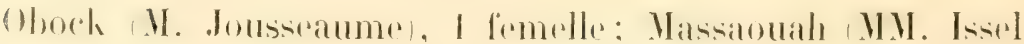
el Beccari, Mus. Gènes), 3 exemplaires.

La carapace est un peu convexe, à peine plus large que

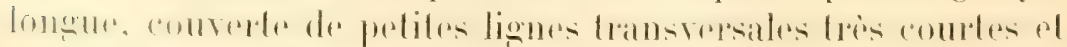

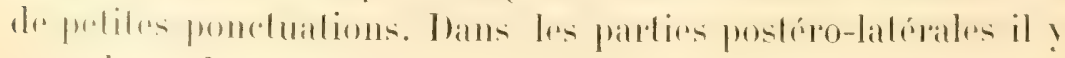
a quelques longs poils épars. La suface de la carapace est à 
peu pres unie, sans régions: sul le sillon cervical est not. mais superficiel. Des deux robés de la region cordiale des lignes obliques brunes forment un dessin an forme dr D. Le front est tris large, nellement tridenté, mais inflechi en bas; et puisque le lobe mádian est lros concave sul sit surtace. vu d'en dessus il apparail droit avee une concavite au milieu. Les lobes externes sont, a leur bord antireour presque droits, peu ou point arancés el inflechis; le lobe médian at long. infléchi verticalement et finement denticula sur ses bords.

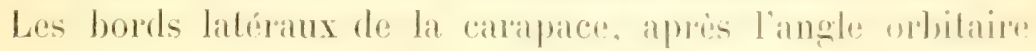
externe, décoment droits pour 1/'t emviron de la longueur de la carapace, puis, apres un élranglement. Les bords bran-

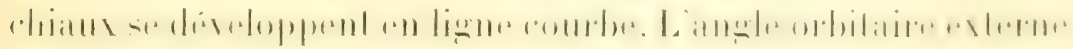
est aigu, spiniforme, quelquefois double (poul-èler par anomalie). Sur le premier trait rectiligne des bords lateraux de la carapace, il y a 2 dents spiniformes. En haut de la constridion latérale, sur te dos de la carapace il y a mo épinr ancére. Sur le bort convexe ou branchial il y a quatre epines. dont la premiere ast pelite ef les autres croissent an longurar. Las parties postéro-latérales ont des petites pides lansversedes.

Lus chélipedes sont inégaux, le plus gros est placé it droite. Le gros chéliperde est glabre, laubre poilu.

Lr merus du gros chélipide a sur sa surlace infirienre

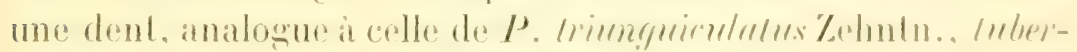

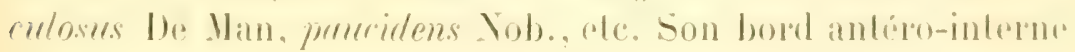

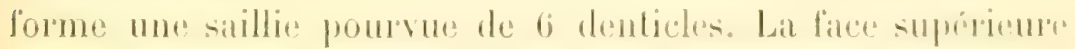
est haversee par des lignes ruguruses; son bord postáneme a

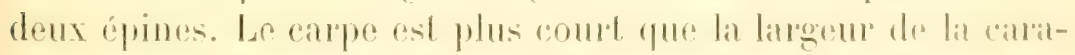

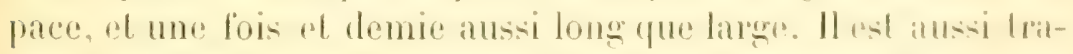
verse par des lignes roguenses; son bord anterium laminaire a 3 dents friangulaires dirigeses en avant et une qualrieme denl

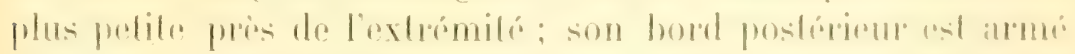

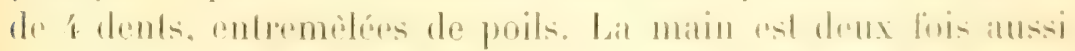

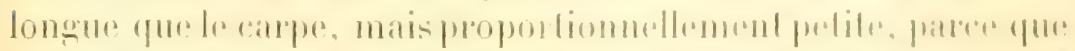

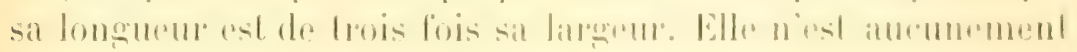

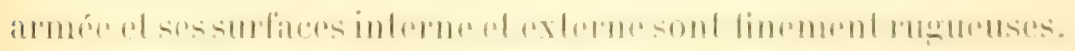

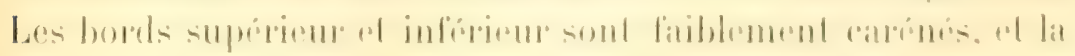

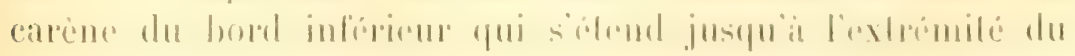




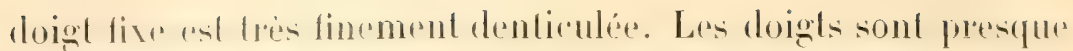
anssi longes que da paume, of un peu biallants. Le doigh mobile est caréné en dessus, et cette crête est presque imperceptiblement denticulée. Il ny a pas de grosises dents sur le coté labuchant. mais sedement une petite saille sur chargur doight, après laquelle le bord est finement denticulé.

Le petit chélipède est très hérissé de poils. Le mérus est

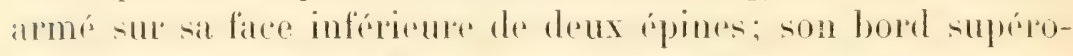
inferm Laminaire a 4 dents spiniformes. Le carpe est proporliomnellement plus comt que sur la grosse pince; il est armé

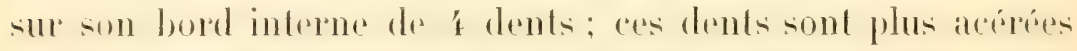
que tedles de lautre pince el conformés differemment, chant faites doune partie basale large et deroupere daus le bord, surmontée par une arête grêle en forme d'épine. La face supé-

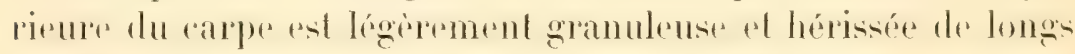

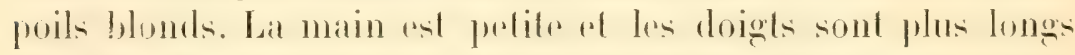

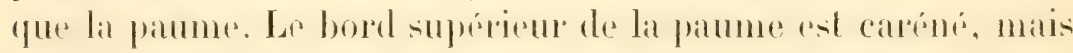
non drule; le bord superirur du doigh mobile est catrene attsisi et la crite est dentée. Le long du bord inférieur de la patume

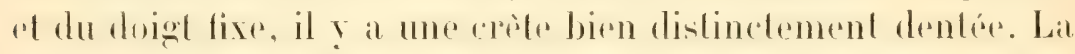
strfiere de la main na pas de granulalions remarquables, ni

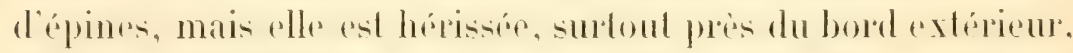
dr. longe poils blonds, comme ceux du carpe. Cess poils sont nombreax entre les dents du bord inféreur. Les doigh joiganent bien, noul pas de grosies dents, mais sont linement

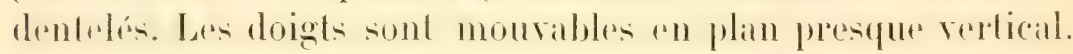

Les palles ambulatoires sont queleque peu ruguenses el ont des longs poils. Le méropoditr n'offre pas d'épine stre aurum bord. Le dactylopoditr est armé de deux ongles, dont celui placé plus bas est un peu plus gros. Le propodite a 3-4 épines mobiles.

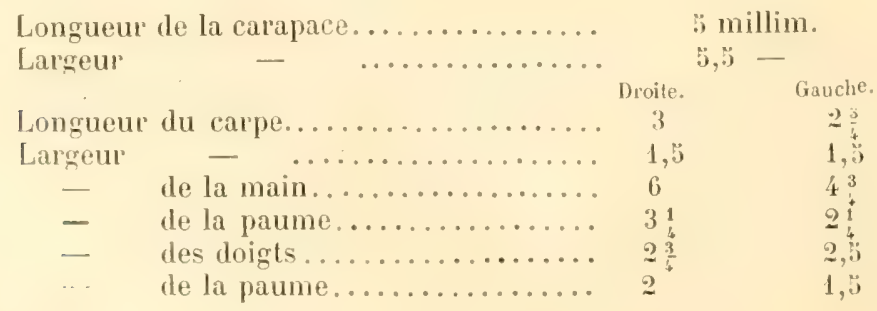




\section{Polyonyx pugilator Nol.}

Tobili, Bull. Hus., 190\%, no 3, ]. 161; Bull. scient. Fr. Belg., XL, 1906, J. 71,

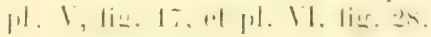

Massanah (MII. Issel of Beccari. Musere de Gines). I Pemelle; Djibouti, récils du Métere (I. Gravier), I male jeune; Jjibouli (II. Jousseame), I mites et 2 femelles: mer Rouge (11. Jousseaume), I màle.

Ces exemplaires s'aceordent bien avec les types du grolle: Persique, mais ils oflrent quelques varialions. Ainsi dans l'un des exemplaires de Massatouah, il y a ö dents sur le borel anterieur du carpe du petit chélipede. Les épines de la main du mème chélipède sont émoussées el moins régulièrement arlangées en séries. Le jeune male de Djibouli n’a pas de dents sur le bord postérieur du carpe du gros chálipede. comme les adultes qui en manquent aussi, ce qui prouve que l’absence de ces dents est constante a tous les ìges. Le male

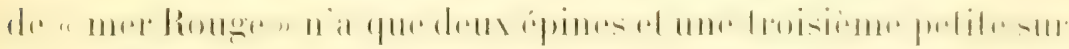
le bord antérieur du carpe du petil chélipède, et ses ípines

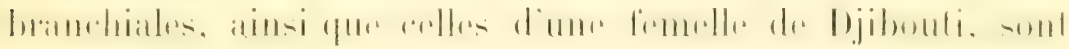
aiguës.

\section{GENRE PORGELLANA.}

\section{Porcellana inæqualis Hell.}

Ileller, S. B. Akad. IVien, XLIV, 1862, 1. 2.i9, pi. II, Kig. 7. - Nobili, Bull. scient. Fr. Belg., XL, 1906, p. 7', pl. Y, figr. 18.

Djibouti (I. Couliere), quelques axemplaires; mer liouge al Dijoouli (I. Jousseaumo), S exemplates.

Celle espece est voisine de P. semolifonns, mais on prul lat recomaitre par diverses particularibs. L'extromile du hord interne da carpe de la grosise pince forme un gros lobe arrondi gui manque dans sferenlifinms: la grosse main est beaucoup

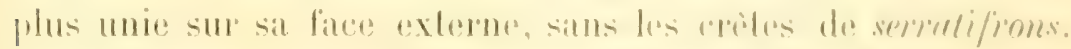
La petile main a une forme tric diflurente: les doights y sont

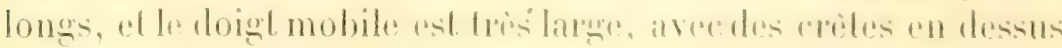
el concave en dedans; la concanter as remplie de proils. Lai

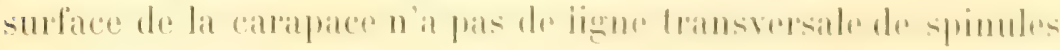




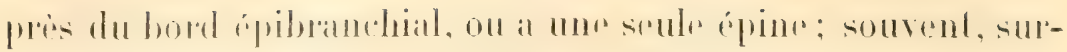
tout dans les femelles, on observe le mème fail dans serralifirnies aussi.

\title{
Porcellana serratifrons Stimpson.
}

Stimpson. Proc. Acud. Nat. Sc. Philadelph., 18:38, p. 242. - De Man, Arch. f. Naturg., 188i, p. 417. - Nobili, Bull. scient. Fr. Bely., XL, 1906, j. 75.

Mer Rouge el Djibouli (M. Jousseaume), 6 femelles.

\section{HIPPIDEA}

\section{FAUILLE ALBUNEID E}

\author{
Gente AlBUNha Fab.
}

1. Doigt de la troisième paire de péréopodes pourvu près de sa base d'un tobe linéaire saillant................. symmysta (Lin.). 14. Doigt de la troisieme paire de péréopodes sans lobe saillant ............................ Thurstoni Ilend.

Albunea symmysta (Linn.).

Albunea symmysta II. Milne-Edwards, H. $n$. Cr., t. II, 1837, p. 203, et $\mathbf{A} t$. Cuvier R. Anim. Crust., pl. XLIl, fig. 3. - Lucas, Rev. Hag. Zool. (2), V, 18:33, pl. I, fig. 8. - Hiers, Journ. Linn. Soc., XIV, 1878, p. 326. - Henderson, Trans. Lim. Soc. (2), V, 1893, p. 409.

Albunea symmysta Ortmann, Zool. Jaherb. Syst., IX, 1896, p. 223, 224 (ubi syu.).

Aden (M. Jousseaume), 2 exemplaires; Obock, dans les

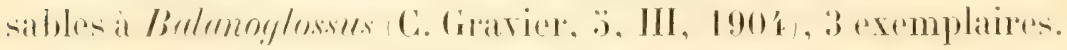

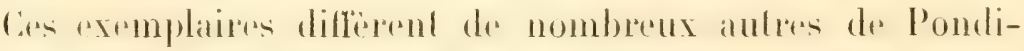
chéry du Musée de Turin par les caractères suivants:

$1{ }^{\circ}$ La longuem des pédoncules oculaires dépasse deux fois la largeur de leur base; la courbure des bords extérieurs est prestue nulle, atu lien que rhez les individus de Pondichery la

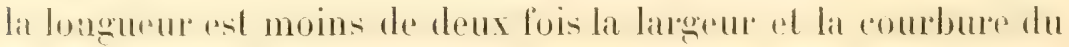
bord est plus forte.

yo Le telson est moins large (q), sa pointe est plus obluse. of sit lorme est plus miforménent ovale. Il est pareouru par dens prominemers longitudinales, qui sont bertucoup moins marquées dans les exemplaires indiens. 
Hab.: Beiloul (Cano); Mascareignes (Ilenderson); Inde Lueas. Miers, Henderson, elc.): Ceylan (Vüller); Vicobares Heller:

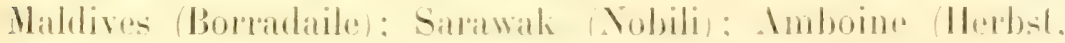

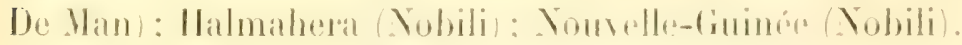

\section{A. Thurstoni Henl.}

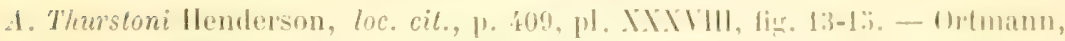
loc. cit., p. 223, 224.

de rapporte a celle rare spece de l'Tule huil individus de Beiloul (Mus. Turin) qui different de A. symmenstr, surtoul par labsence du lobe sure dachlopodite de la troisieme pare de paltes. Its staceordent assez bien arec la description de: Henderson. La longueur des yeux est un peu phus de deux fois leur largen it la base. Le telson porte deux faibles crotes arrondies, comme dans les symmystre de Aden. Chez la femelle ce segment est ovalaire, chez les miles lancéolé, par suile d'une dilatation qu'on observe sur les cotés.

Le plus gros individu est long de 12 millimetres of large

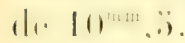

Un exemplatire trouvé a Djibouli par 11 . Jousseatume me parail anssi appartenir it celle espere: mais, malheureusement. il manque les paltes de la hosieme parte.

Ils.s. : lheval-1'ar Inde:

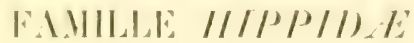

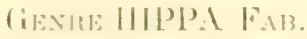

\section{Hippa asiatica Elw.}

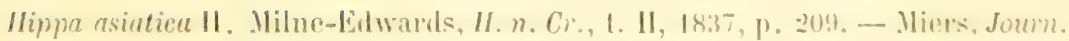
Limn. Soc., XI1, 1. 32., pl. 1, tig. 11. - (1)tmann, loc. cil., 1. 231, 233. Sobili, Boll. Mus. Torino, XVIII, no 4.2, , 1. 16.

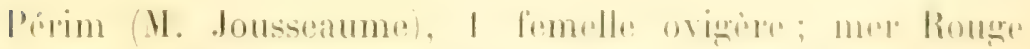
(11. Jouscatume), 3 jemes exemplates.

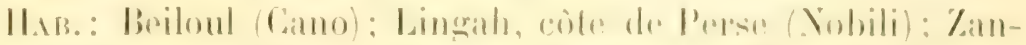

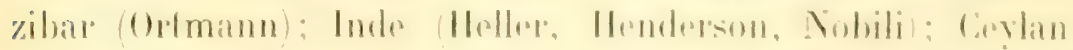

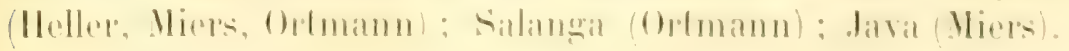




\title{
GENRE REMIPES LATR.
}

\section{Remipes pictus Heller.}

Remipes pictus Ileller, Sitzb. Lkad. Wien, 4́, 1861, p. 243. - Kossmann, loc. cit., II, 1880, p. 71. - Cano, Boll. Soc. Nat. Napoli, III, 1889, p. 262. - De

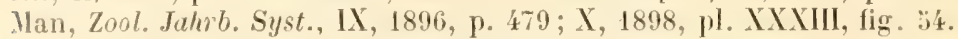
Remipes testudinarius var. pictus Paulson, loc. cit., p. 8t, pl. XI, fig. 2-2f.

Inp Rouge (Clot-Bey), 13 individus; Beiloul (Musere de Rome. Exemplaire de Cano?), 1 femelle.

Tous aes remplaires stacordent assez bien avee lat descrip-

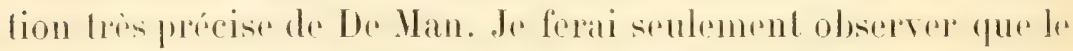
nomber des articles du fouet plus court des antemnes internes est sujet à varier. De Man a compté 10 et 11 articles chez le màle et 8 chez la femelle, Heller 11 chez la femelle, Paulson et Kossmann 10 chez le mâle et 8 chez la femelle. Dans hois femelles du Vusiom qui ont les antemnes entieres.

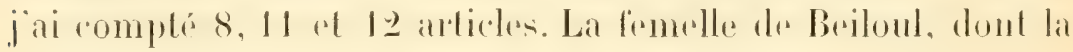
caripace est longue de $11^{\mathrm{mm}}, \mathrm{g}$, a 10 articles.

\section{BRAGHYURA PRIMIGENIA DROMIAGEA.}

\author{
Genre Dromia Fabr.
}

\section{Dromia Rumphii.}

Dromic Rumphii H. Nilne-Edwards, H. n. Cr.. I. II, p. 174. - De Haan, F. Jap.Crust., p. 107, pl. XXXII. - Alcock, J. As. Soc. Bengal, LXVIII, 1899, p. 137, et Descr. Cat. Ind. Decap. Crust., I, 1901, p. 4t, pl. II, fig. 4. - De Man, Abh. Senckenb. Naturf. Ges., XXV, 1902, p. 687. - Borradaile, F. Geogr. Maled. Laccad. Archip. (Marine Crust., pt. IX), 1903, p. 576.

Obock (I. Jousseaume), un gros mâle dont la carapace

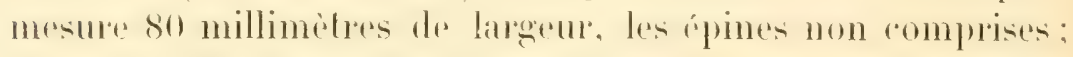

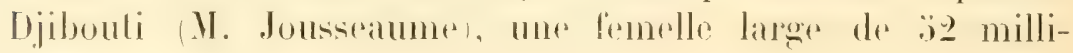
mètres.

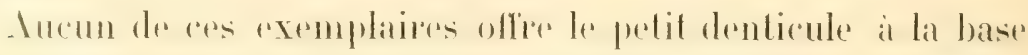

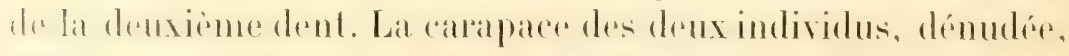
offre un grand nombre de points violets, qui tendent à se grouper irrégulièrement. 
(iEXTE DROMIH)I A S'TM.

\section{Dromidia unidentata Riipl.}

Dromiu unidentata Rüppell, Beschr. 2.4 Krabb. Roth. Heer., 1830, p. 16, MI. IV, fig. 2 ; pl. 11, fig. 9. - II. Minne-Ldwards, H. n. Cr., L. II, p. 17s. - Ileock, loc. cit., p. 139, et loc. cit., p. 47, pl. 11, lis. 2.

Dromidia unidentuta Kossmann, p. 6\%. - De Man, J. Linn. Soc., XXII, P. 207,

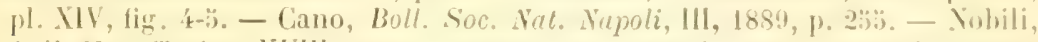
Boll. Mus. Torino, XYIII, 1903, no $43: 3$, p. 23, et Bull. scient. Fi. Bely., 1906, p. 92.

Assab (Dr. Ragazzi, Mus. Modine), I lemelle de $30 \times 33$ millimètres; mer Rouge, Aden et Obock (M. Jousseaume). I male el 3 femelles; Djibonti (II. Gravier), I femelle; mer Rouge (1l. Jousseaume), 2 màles et 4 femelles: Djibouti (II. Jousseaume), I femelle; mer Rouge (Musée Turin). 2 femelles.

Guclques-uns de ces exemplaires correspondent, par la forme du front et par la courbure des bords latéraux, at la variété des iles Mergui décrite par De Man, d'autres sont intermédiaires entre cette variété el la forme typique. Cela prouve quilil n' a pas de différence entre la forme de la mer Rouge el celle de l'Océan Indien, mais seulement des varialions qui peurent ctre comblées par des points intermédiaires.

\section{Gexte CRYPTODROMIIA STM.}

\section{Cryptodromia canaliculata Stm.}

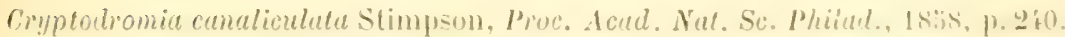

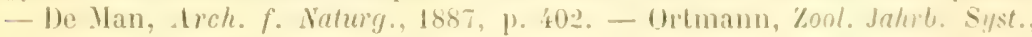
11, 1892, p. \$3\%. - Meock, loc. cil., p. 1.2, et loe. cil.. 1). 31). pl. II, lig. 8 .

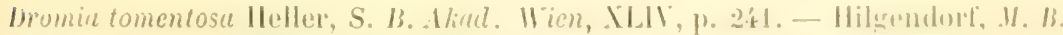

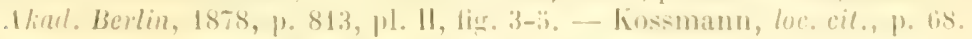

Criptodromia tomentosa paulson, luc. cit., p. 83.

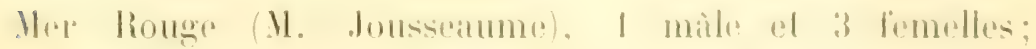

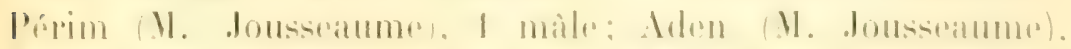

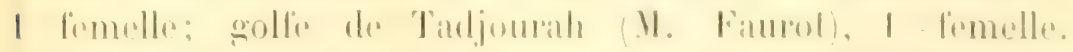

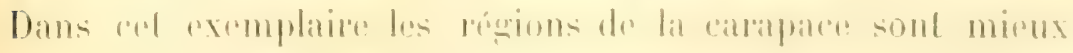

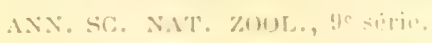


mareques que dams les aubes. Chez lous les exemplaims la

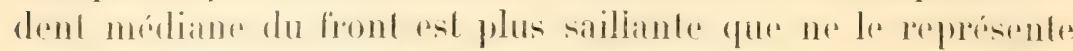
la figure de Ililgendorf.

\section{Cryptodromia pentagonalis (Hilgendorf):}

Dromia pentagonalis Ililgendorf, M. B. Akad. Berlin, 1878, p. 814, pl. II, lig. 1-2.

Cryptodromia pentagonalis Henderson, Trans. Linn. Soc. (2), V, 1893, p. 406.

Aden (M. Jousseaume), une femelle.

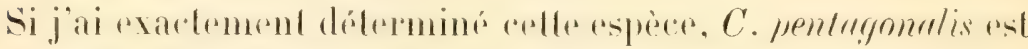

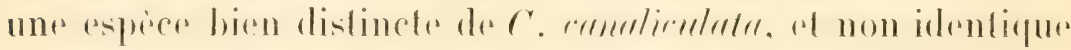

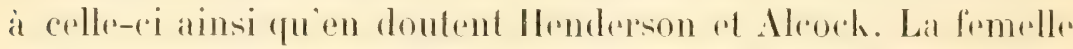

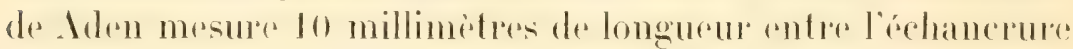

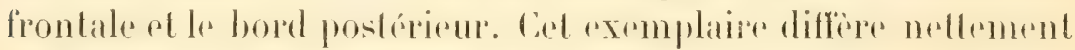
des autres $C$. comoliculata par sa carapace plus convexe el tomie, sans délimitation de régions, autant unie que celle de C. Hilgendorfi, patr son fronl beaucoup plus saillanl, of par ses bords latero-antérieurs armés dime sedule dent pen saillante.

Nouralle pour la mer Rouge. Habile Mozambique Uligenendorf), Maurilius (Henderson) et l'Inde (Henderson).

\section{Gryptodromia Hilgendorfi De Man.}

Cryptodromia Hilgendorfi De Man, Arch. f. Naturg., 1887, p. 404, pl. XVIII, fig. 3. - Alcock, loc. cit., p. 145, et loc. cit., p. 52, pl. III, fig. 11. - Nobili, Ann. Mus. Civ. St. Nat. Genova, XL, 1899, 1. 249, et Bull. scient. Fr. Belg., 1906, p. 93.

Dromides Hilgendorfi Borradaile, A. H. N. H. (7), XI, 1903, p. 299, et Faunu Geogr. Maled. (Mar. Crust., IX), p. 577.

T)jibouti M. Jousseaume), une femelle qui mesure $7^{\mathrm{mm}}$, , d de largere sur 7 millimetres de longueme, et un male ave son

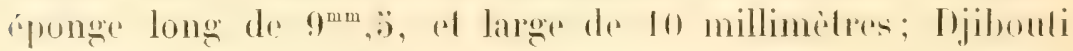
(1). Coutibere, un màle habilant dans mone éponge conique : la

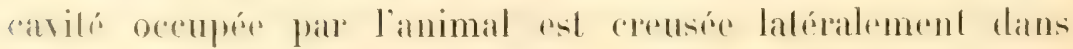
l'éponge, qui est ainsi couchée transversalement sur la Dromie. 


\section{BRACHYURA}

\section{OXYSTOMA}

\section{FANILLE CAJAPPIDA}

\section{CALAPPINAE}

Genre CALAPPA Fabr.

Calappa hepatica Linn.

V. Aleock, J. As. Soc. Beng., LXV, p. 141, 143 (ubi syn.).

Mle Rouge Mus. Turin!, imblivilus; Beiloul, \& metres (C)sini, Mus. Turin), un màle et une femelle; Djibouti (M. Couliere), 6 imdividus: golfe de Tadjourah (I. Famol, 2 femelles:

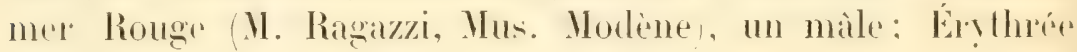
(11. Tellini), une femelle.

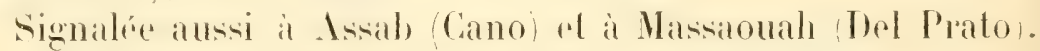

\section{Calappa philargius (Linn.).}

Calappa cristata Fab. fl. Milne-Edwards, H. n. Cr., t. II, p. 105, pl. XX, lig. 2. - Ortmann, Zool. Jahrb. Syst., V1, 1892, p. 563.

Calappa philargius (Linn.), De Ilaan, F. Jap. Crust., 1) 71, pl. XIX, fig. 1. Alcock, loc. cit., p. 140, 140 (ubi syn.).

Mer Rouge M. Jousieatume), 111 jelume mile qui mesture

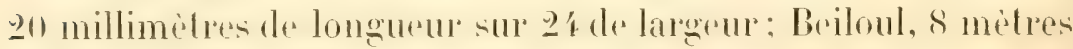
(M. Orsini, Mus. Turin), un mâle très jeune.

celte espere, repandue depuis le golfe Persique jusqualu Japon, n’a pas encore été signalée dans la mer Rouge.

\section{Calappa gallus (Herbst).}

Calappa gallus (Herbst), II. Milne-Edwards, H. n. Cr., t. II, P. 10\%. - Brito Capello, Jorn. Sc. Lisboa, III, 1870-71, p. 133, pl. 11, fig. 4, 14. - Alcock, loc. cit., p. 146. -.. Rathbun, Amn. Inst. Jamaica, 1, 1897, p. 36.

Calap ra galloides Stimpson, Ann. Lyc. New-York, YII, 1859, p. 71.

Assab (M. Ragazzi, Mus. Modene), un mâle el 2 f'emelles. 
Les exemplaires de Assab mesurent:

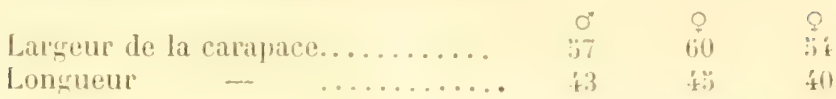

La forme du front dans ces exemplaipes et dans d'autres de provenances diverses que j'ai examinés est très variable; tantob il est plus, tantòt moins avancé au milieu, lantót entier, lantol légèrement émarginé.

Cielle espèce est répandue dans loule la région indo-pacifique et aussi dans l'océan Mllantique, depuis l'Afrique oecidentale ef le cap Vert jusqu'a la Floride of aux Intilles.

\section{MATUTIN AE}

Geste Matéti Fab.

\section{Matuta Banksii.}

V. Alcock, loc.cit., p. $1: 38$ (uti syn.).

Obock (II. Jousseaume), 3 femelles; mel Rouge et dilen (II. Jousseaume), un male el 2 femelles.

\section{Matuta victor $F_{i}$ b.}

V. Alcock, loc. cil., J. 161 (ubi s!yn.).

Ner houge el Aden (II. Jousseaume), 3̈ males et 3 fomelles: Obock (11. Jousseaume), un mite et ö lemelles; golfe de Tadjourah (11. Faurot), un male; Obock, a mer basie (II. Cirat-

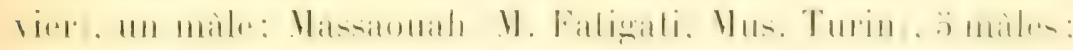
Assab (I. Ragazzi, Mus. Modène) un maile.

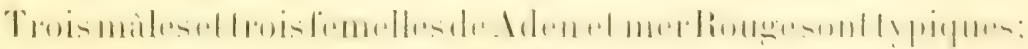

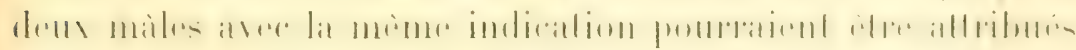

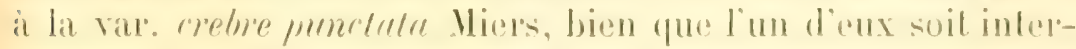
médiaire entre eetle variété a laches lapprochés el formant des taches composes it centre chare da forme typique it faches

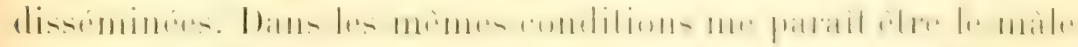
du golfe de Tadjourah el celui de Oboch recueilli par M. Ciravier. Les individus d'obock recucillis par .11. dousseatume sonl des crebre punclule qui ofrent des latits de passige it la forme 
typique, partere que les petiles laches, lout en formant des

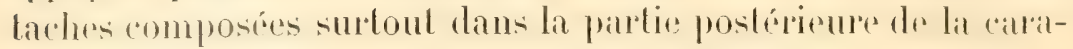
pace, sont généralement moins rapprochées.

\section{LEUGOSIINAE}

\section{FAMILLE IEUCOSIIDAE}

\section{Genre OREOPHORUS Ruppelt.}

\section{Oreophorus horridus Rïppell.}

Oreophorus horridus Rüppell, loc. cit., p. 19, pl. IV, lig. 5. - II. Milne-Edwards, H. n. Cr., II, p. 131. - Bell, Trans.Linn. Soc., vol. XXI, p. 306.- A. MilneEdwards, Ann. Soc. ent. France, 1863, p. 151.

Obock et Aden (I. Jousseaume), 3 males et 2 f'emelles; Djibouti (M. Jousseaume), une femelle.

La figure de Rüppell ne rend pas bien l'aspeet de l'animal. La forme générale de la caraprace est, ainsi que Bell a éril, subtrirngulala, et a une certaine analogie avec celle d'une

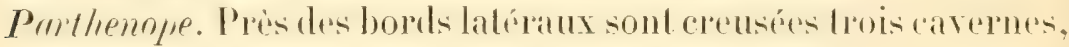
qu'on ubserve aussi dans 0 . relirululus .d. Wh. Parfois une? pelite galerie sous lo lest fail communiquere la premiene aree la deuxieme carerne. Le vrai bord postérieur el la région intestimale sont séparés des antres pardies de la carapace par un sillon

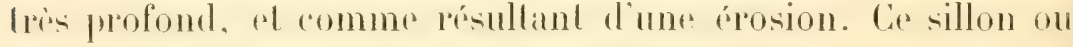

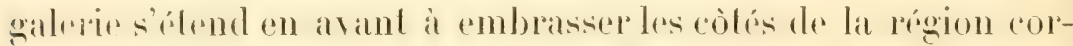
diale, dont le bord postérieur "sl siparé par un sillon analogue de lat région intestinale. Les creites sur les régions branchiales sont forles. Les régions sont ponelués al creusées de glosses fosictles, ainsi que le stermum at liabdomen. Liabdomen du male est encaissé profondément dans le sternum eoncave. Les doigts des pincessont incegaus it la paume ou un peen plus longs.

IIAв : Tor (Rüppell).

Genre NURSiA Leach.

Tableau des Nursia de la mer Rouge.

A. Carapace sans aucune crête sur le dos............. mififera Müll. ג. Carapace pourvue de crètes épibranchiales et d'une partie de la crète longitudinale...................... Jousseaumei Nob. 


\section{Nursia Jousseaumei Nob. (Diugn.plélim.).}

(Pl. I.I, lig. 'x.)

Nobili, Bull. Mus., 190\%, n 6, p. 397.

Her Rouge el Périm (M. Joussoaume), 10 miles el 9 fomelles: Obock (II. Jousseaume), un mile.

Par l’absence de crobes hépaliques at de la croble lransversale parallele el voisine du bord postérieur do: la caldapace. celle sepece vient se placer dans le groupe de l. persirn Ile. el de Y. rbbreciralı Bell.

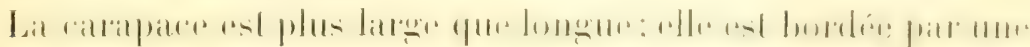

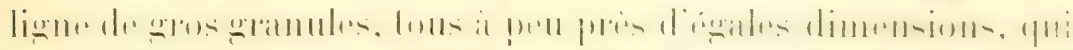
suit loule sa marge, excepté le front. Le front est concave en dessus; son bord antériemr ext faiblememt tridenté, le lobs. on dent médiane, est trés peu saillant. Les régions sous-hépa-

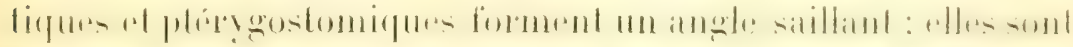

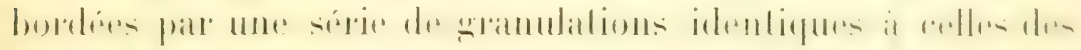

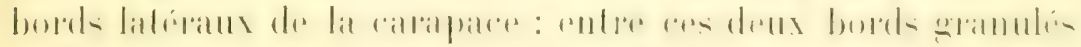
se forme ainsi une furelle tres nelle. Au dela de celle fatcelle

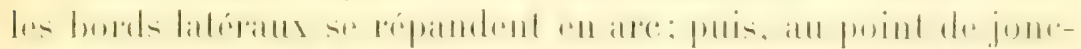

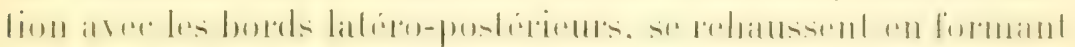

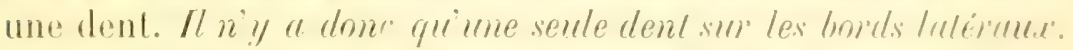
Les bords latéro-postérieurs converogent obliquement vors le

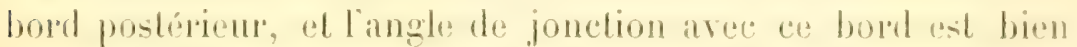
prononcé. Le bork postéricur ost sinurux; il offre an milieu un

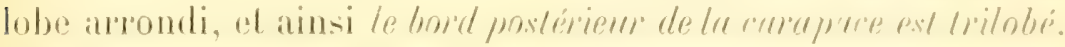

De lat dent décribe il la jonction des bords lintéro-intériens

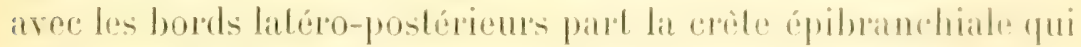

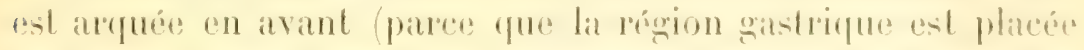
un peu en arant d’une ligmo imaginaire unissant los dents des

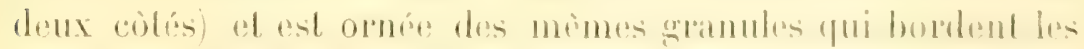

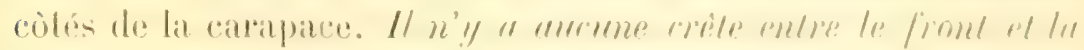

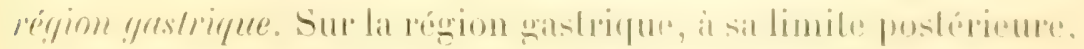

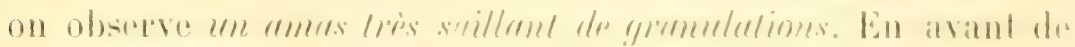

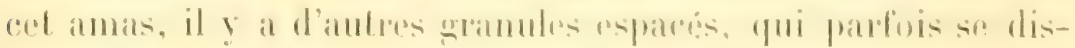

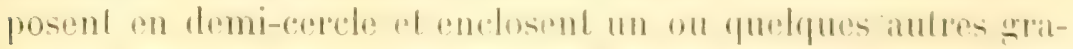

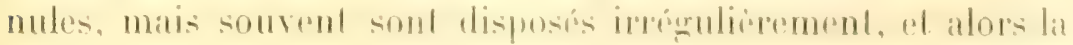




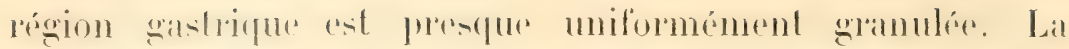

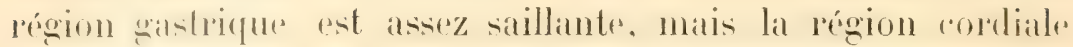
l'est encore plus, bien que par le repliement en bas de la carapace, elle se trouve placée à un niveau inférieur. Elle saillit sous la forme d'un gros luberente, souvent lurriformes

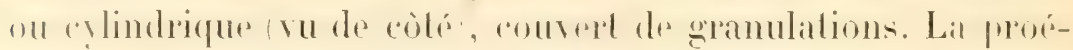

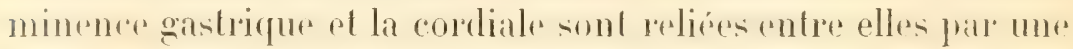
ligne de gramulations, qui reprósente les vestiges de la rrote

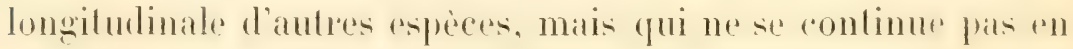
arriere de lat saillie cordiale. Ainsi chez cette espece la repte

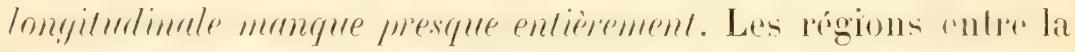
weite ípibranchiale. Jes sallies decrites ot les bords lateraux sont entièrement lisses.

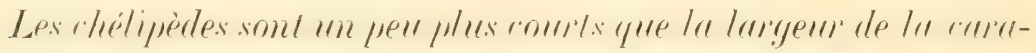
pure. Le bras est finiment gramulenx, plus en desious ef en avant quien dessus. Le earpe porte me petite ligne de grantulations pres de son hord inferienr. Lat main est faiblement "arénée "n dehors et pourvure du coté interne el tout pres du borel inférexur d'une petite ligne de seanulations plus saillantes, qui sobend jusquan doigl tixe. Les doigts sont phos courts que la paume, sillonnés; ils ne joignent pas.

Les pattes ambulatoires somi courtes of faiblement geranuleuses.

Lavant-deruies article de l'abdomen du mile porte a son extrémite une forte dent laminare, dont la pointe stecourbe en arrière.

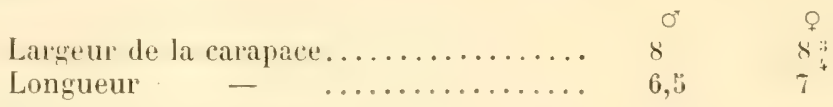

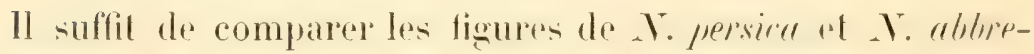
vialu pour reconnaître tout de suite les différences.

Nursia Jousseaumei var. cornigera Nob.

Nobili, loc. cit., p. 397.

(!uatwe exemplaires de mer Rouge M. Jousseaume) different par les proportions plus petites, par les bords latéro-intérieurs non répandus en arc, mais passant presque droits 
dans les angles latéraux qui sont très forts, comifomes recourbés en homl. el bien dótachés des bords de la carapace. L’amas de granulation sur la partio postérienre de lia carapace est tres fort el suspendu an haut par un promenle Ione. La"

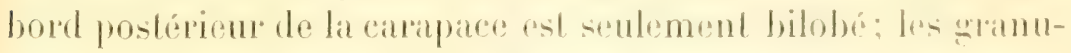
lations du sternum et de l'abdomen sont beaucoup plus lortex que dans les femelles typiques.

\section{Nursia rubifera Mïll.}

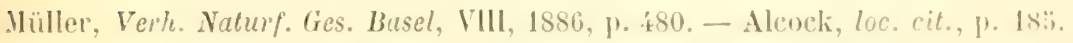

Mer Rouge (11. Jousseaume), g̈ màles el 7 lemelles.

Celle espèce a un facies de Cryplornemus, el resiemble mìme quelque peu ì Cryplocn. Grandidieri 1 . Edw.

La carapace est élargie latéralement, el a un contour octogonal, dù à la présence de deux bords latéro-postériems les

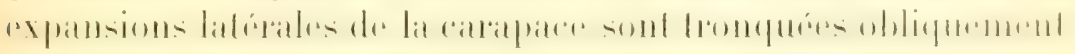
en arrière; non conlinuées avec le vrai bort postérieur ef loorment ainsi deux bords latéro-postérieurs). Les cótés de cel

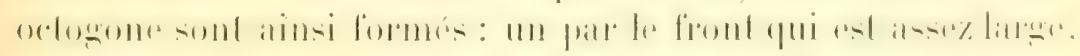

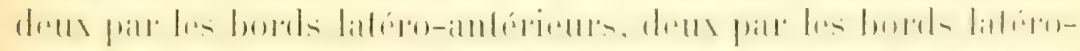

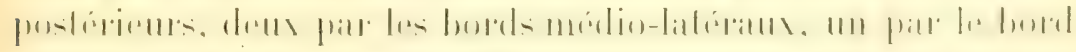

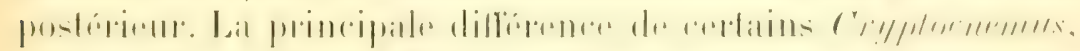

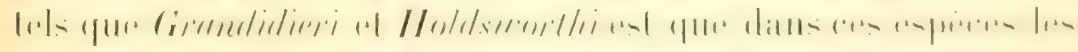
expansions latérales de la carapace se continnent avece lo bord postéreur en ligne presque droite. Le front est large, saillant, bilobé. Les parties antérieures de la carapace sont déclises, la partie postérieure est concave, par suite du rehatusconent des

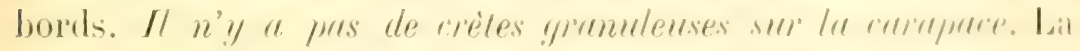

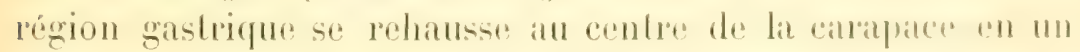

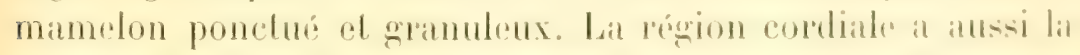
forme d'un gros lubereule rond. Les borels de la ratrafrare

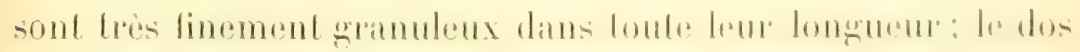
de la carapace al grossierement ponctue mais sills gramlations, saul toul pres des bords lialiro-postibreurs.

Lexognalle est lange it pen pris comme l'ischiognalhite: son bord anteriene et son bord postipiene forment une courber 


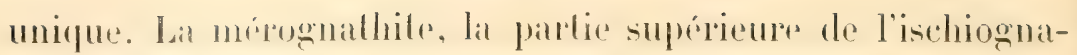
thite el lexognathe sonl gramuleux. Le stermum es concares, ses bords lationax portent de prosies gramulations grompés ensemble. Les articles II-VI de l'abdomen du màle sont soudes ellsemble, sans sulures de séparation, mais les bords externes sont sinués et montrent ainsi trace de la division primilire des articles. A l'extrémité antérieure de l'article VI, foul pres de la base du septieme, il y a $u$ fort lubereule conigue.

Laabdomen de la femelle n'a gur trois articles; mais l'arlicle plus long. formé par Ia soudure de quatere articles, oflre pris de sa bate une suture bien nette. Lat surface de cet article est grossièrement ponctuée.

Les chilipedes ont une longueur inférieure à une fois et demie celle de la carapace. Le bras est triangulaire, et charun de ses bords porte me sirice continue de granulations : la fiere

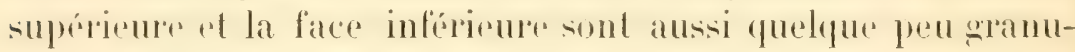
louses. La carpe porte des granulations mentes mais sorreses. Lat main at les doigls sont aussi gramulens ; la main porte meme

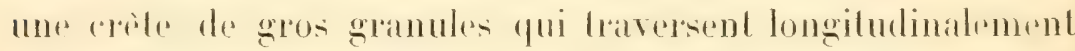
sa face supéricure (interne). Son bord externe (supérieur) s'amincil, mais il ny a pas une vraie crete. Les doigts sont atusi longes que la portion palmatre el pourvus de crates longi-

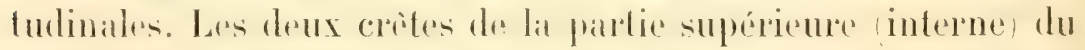
doigl tixe se continuent en deux crites de gramules sul le bord inférieur de la main.

Les pattes ambulatoires depassent le borel de la carapace

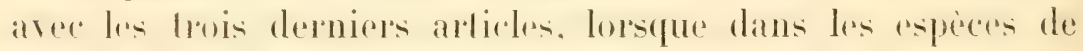
Cryplocnemus (excepté Obolus Ortm.), les doigts seuls dépassent le bord de la carapatre. Les articles sont gramuleux. Le

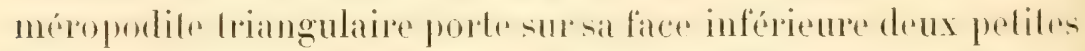

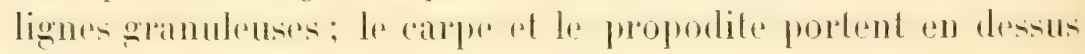
deux petiles crètes parallèles.

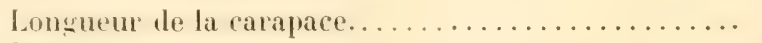

Largeur

$\tau,: i$

Longueur des chélipèdes.........................

II. Ir [) Jean Roux, Cuslos du Musée de Bitle, a eu la bonté de complarex pour noi un milk de la mer Rouge aree les ly pes 
de Trincomali, el de m'écribe que cet exemplaire "concorde parfaitement bien a arec le lype.

Cette espece n'est connue que de Trincomali.

\section{GiFire EBAIJA LeACH.}

A. Bord postérieur de la carapace convere, ou avec les angles non ou peu prononcés.

B. Région hépalique non concave.

C. Bord postérieur de la carapace convexe, mais avec les angles marqués. Propodite de la première paire de pattes non caréné. Pénultième article de l'abdomen du male plus long que large............................. gramulata liüp.

CC. Bord postérieur convexe sans angles marqués. Propodite de la première paire de pattes avec une crete mince et saillanle sur le bord inférieur. Pénultième article de l'ablomen du mâle plus large que long................... E. abdominalis Vob.

BB. Région hépatique profondément concave........ E. orientulis liossm. A. Bord postérieur de la carapace rectiligne à angles mar-

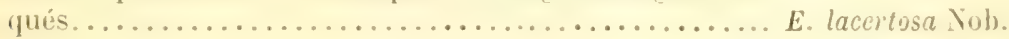

\section{Ebalia granulata (Rüpp.).}

Pl. IX, fig. I.

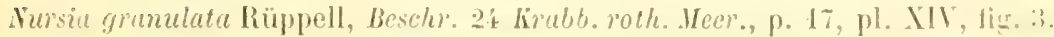
? Yee Nusia grunulata Paulson, loc. cit., p. 79, p!. X, lig. 2-2 f.

Nec Ebalia grumulate Viers, "Alert" Crust., p. "3:9.

II. Le professeur F. Richters, de Francfort-sur-Mein, a eu l'obligeance de m'envoyer le type de Sursia granulall Rüpp. dont je donne ici une pholographie. de vais décrire à noureau cette espece, d'apros le type mème. Mes meilleus remereiments sont dus it 11 . le professeur Richters.

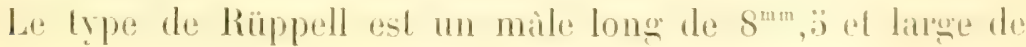
8 mill. 1/4. La surface en est presque enlierement pranulense: to front seulement ef les orbiles sont lisses. Les entanulations sont fines el tres petiles sur la parlie anterieure de lat carapated soil sur la premiere moilié de la régron patstrigue el sur la région hépalique, alles deviennenl phus grosies sur les rigions branchiales, sur la partic módieme ol postirieure de la région

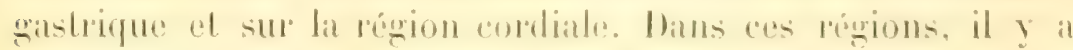

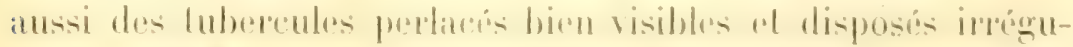

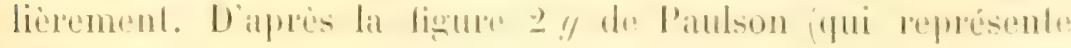




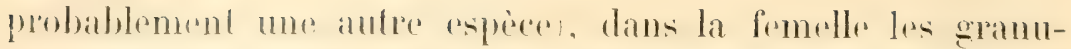
lations seraident plus petites. Aurune des régions n'est particulierement saillante. Ixes sillons longitudinaux de la raparaer sont bien marqués, mais médiocrement profonds.

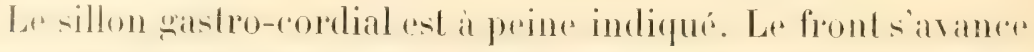
an delit du bord épistomien, il rol mellement bilobépar le sillon en dessus; chaque lobe est triangulaire. Dans l'espèce de Pandson lópistome dípasise lo front. Lal région hépatique est

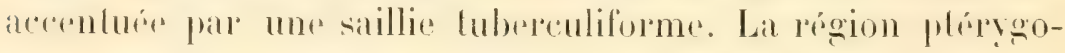
stomiegue forme anssi un lubereule sallant; le bord hépalique

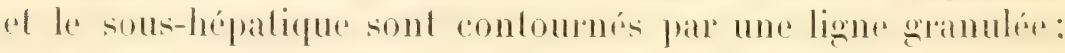
il y a ainsi une facette latérale. Les bords latéraux ont une ligne de granulations dont quelques-unes deviennent plus

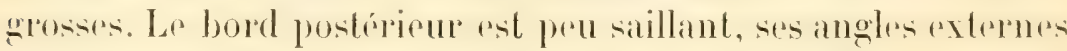
sont aceuses, il y a aussi une pelite saille an milieu; le loail entre les angles externes et la saillie médiane est un peu obligue. La tigure de Paulson a les angles of la saillie beatucoup plus marpues, ot la portion intermédiaire du bord ast concave.

Le sternum est ponctué. L'avant-dernier article de l'abdomen du mole est allongé, plus d'une fois of demie ansi long que larene a sa base. ol deux fois ausi long que large is

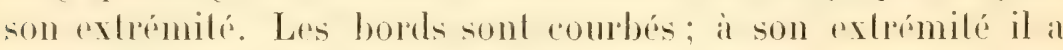
un tubercule rond et peu saillant.

Liexognathe des maxillipides est phos rourt que l'endognathe. etroil: son bord externe fail me courbe unique are le borl antérixur. Le mérognathe est i peu près anssi long qur l’ischiognathe; sa surface n'est pas granulée.

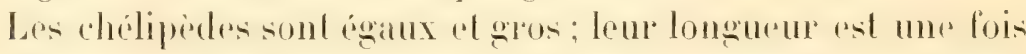
el demie celle de la carapace. Le mérus est subtriangulater. plus dilaté a la base qu'a lextrimite. La surface superperure a les rangéses de granulations a la base et pres des bords; antre-

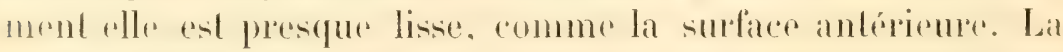
suldae inférieme est lisse. Le carpe a une ligne de granulations tris faible pres de son bord interne. La main est grosse, aussi longue que la largene de la carapace ; sa portion palmaire est un peu plus courte que les doigts; la hauleur de la portion palmaire us les trois quarls de la longueur. Ia surface est 


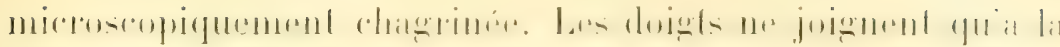

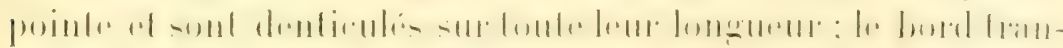
chant fait une petite saille it la base. Ils sont finement sil-

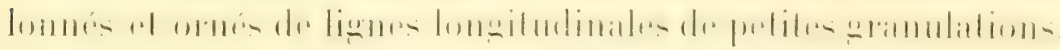
Une ligne granulare assez fortr sur lo doigl fixe se continue

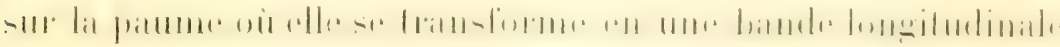
formée par 3-4 séries de petits granules.

Les paltes ambulatoires ont les propodites tris fiablement catrénés

La Nursin gromulata P'auls. a les hrois lobers du bord postílérieur de la carapace très accentués. Cost pent-idre une autre espece.

LiEbalia gramulata Mier's de P'rovidence-Istand n'est certainement pas celle espece. Sa carapace et ses patles sont converte de granulations tres fines qui deviennent plus grosses sur l’abdomen el sur les maxillipedes. Sa forme est aussi circulaire. Celte espece d'ailleurs, d'apres Miers, est roisine de Ebratia miliaris A. M.-Edw. qui est une espèce tres diflérente.

\section{Ebalia abdominalis Nol.}

['. IX, fig. 2.

Nobili, loc. cit., p. 399.

Her Rouge, Aden et Périm (IV. Jousseaume), 34 miles a

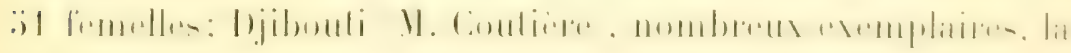
plupart jeunes.

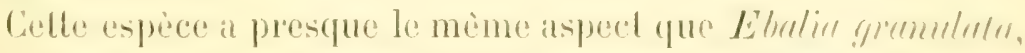
mais on peut facilement l'en séparer par des dibremens constantes.

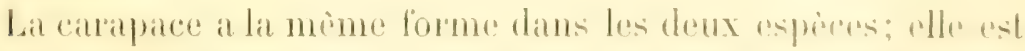

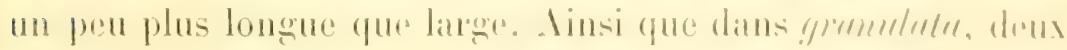
sillons longitudinatux séparent les régions módianes de lat catrat-

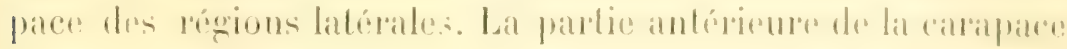

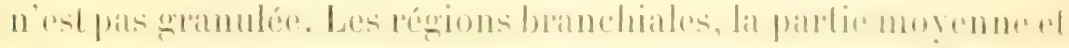

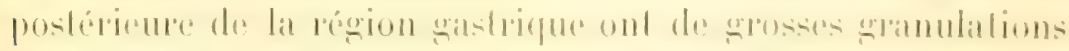
vésiculenses, dont quelques-unes sur la ligne módiane longilu-

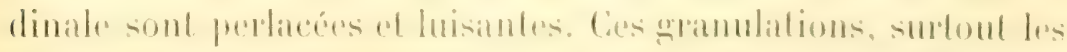

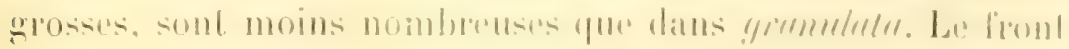


a lit mème forme que dans gremulale : dans les femelles of les males jeunes, il est plus rombl que le bord épistomien; lans les adulles. il dépasie ce bord. Le bord susorbitaire offre dro nombereux petils sillons, courts al lígers ot noiriters, qui forment sur ce bord, en plus des fissures, un plisire lres fin. Lat région hépatique est peu prononcée, la sous-hépatique el la plérgostomienne sont peu saillantes el nont pas le gros lubercule de E. gramulalu. Les bords lithaux sont finement gratnulés. Le bord postérieur de la carapace est amondi mniformément, non saillant, ni aux angles cxtermes, ni au milien.

L'avantederniere atlete le labblomen du male a une forme caractéristique: icest article est laroge, quadrangulaire, dépassant en dehors les autres allickes, ol plus large gur long. Il porte en dessus un gros lubercule lriangulaire et dentiforme. Les chélipides du male adulte ont deux fois la longueur du corps; dins la femelle el le male jeune ils sont plus courts. Le bras est plus gros pres de la base, a par la disposition de ses

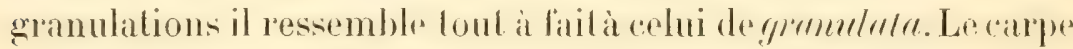
at une serve de granulations tris faibhes, pres du bord interne. La main du mile est rativement grosse. La portion palmaire est plus courtr que les doigls; sa hauteur est un peu plus des $2 / 3$ de sa longuenr. La main est tres finement granulée. mais elle n'a pas la bande de granulations arrangéses en série que l'on voit dans grommlatu. Les doigts sont sillomnés longitudinalement et granules. Le doigt fire porte sur sa face superieure une rangée de granulations quistarrede prestue a moitié de sat longueur oì elle se lermine en un gros granule perlacé ul blane, mais ne se contimue pas sur la main. Les doigts dans la moitié proximale bioillent largement, dans la partie distale joignent et sont finement denticulés.

Less propodites des pattes ambulatoires noul pas de rraies cretes dans les mâles jeunes el dans les femelles; dans les màles artultes, au contraire, le bord inférieur du propodite de la premiere paire porte une crète hanchante el bien saillanle, qui s'arrète avant l'extrémité de l'article.

Las fomelles jeunes de Djibouli (M. Couliere) s'accordent entierement arec les femelles lypiques reeueillies par M. Jousseaume. Mais deux miles de Djibouli lout "n s'accordant avec les jeums 


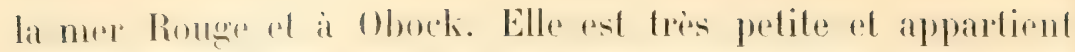
au groupe de algivica, Wood-Dasoni, diadumena, ete., soit à ces espèces qui ont les régions saillantes, les latérales sifratres des milogennes par des sillons profonds, w le bord

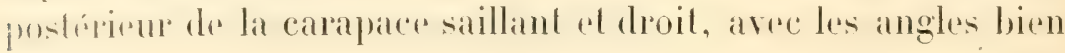
nets.

La carapace est un peu plus large que longue, de forme presqur hexagonale. La surface not finement gramulée, avec des gramules plus gros sur les rógions médianes el sur une partie des régions branchiales.

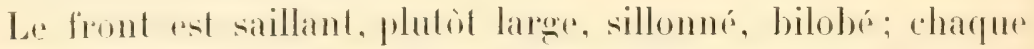

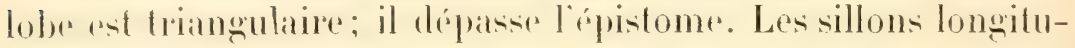
dinatus de ha carapares sont hres profonds; les régions hépalipues sont un peu concaltes. La région gatstrique ef la cordiale qui est unire aree l’intestinale sont saillantes; la cardio-intesti-

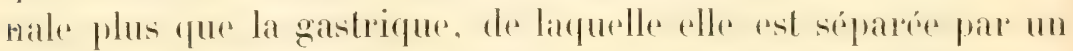
sillon. Les régions branchiales sont renflées de côté et en desus. Il n1 a pas de ficedle hépatique, mais langle dr la région phergostomique est prononcé. Les bords de la ralraparesont margines par une ligne de granulations. Le bord pos-

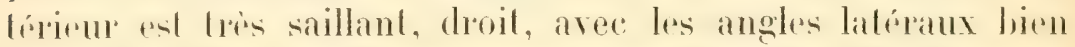

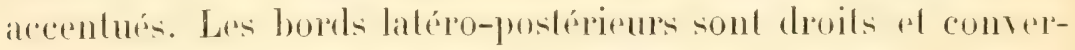
gents, margines par une forte ligne de granulations. Le sternum of l'abdomen sonl granulís: l'avant-dernier article de lablulomen est plus bong que large et poursu d'un petil lubercule arrondi.

Les chélipèdes sont plus de deux fois aussi longs que la carapace. Ils sont relativement gros. Le mérus est très peu Elanule, exrepté pres de la base el des bords; le carpe a me

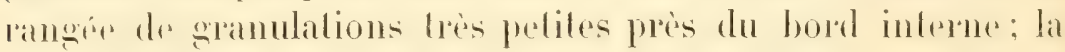
main est lisse, et son bord inférieur est sinneux. Le doigt

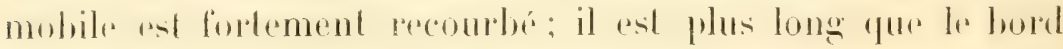
suprepere de la paume. Les doights ne joignent et ne sont dentes quit lit prointe. Les palles ambulatoires nont rion de rematlquable.

Longueur de la carapace............... 4 millim. 


\section{Ebalia orientalis liossm.}

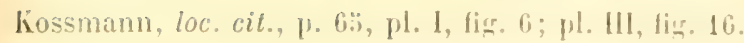

do n’a pas vu d'exemplaires de celle espece. Elle parail

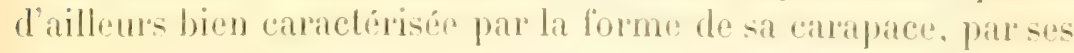
régions hépaliques profondément déprimés el concares, el par les régions médianes de la carapace leeliées an front par un pont ílloit.

\section{Gevire NUGIA IANA.}

A. Carapace presque globulaire. Thubercules maryinan: laibles.

\section{N. tuberculosin}

A. M.-Eilw.

A. Carapace a contour irrégulier, avec de gros tubercules marginaux.

B. Deux sillons longitudinaux obliques enclosent en un triangle les régions médianes longitudinales de la

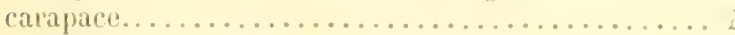

BB. Pas de sillons longitudinaux obliques. Carapace tres

N. pulchellu X. M.Edw.

bosselée............................... Pfefferi De Man.

Nucia tuberculosa A. Milne-Liwards.

(PI. IX, lim, 6 .

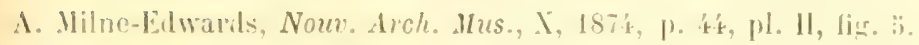

Aden (II. Jousseaume). 2 femelles.

Ces deux individus sont beaucoup plus gros que le lype, qui

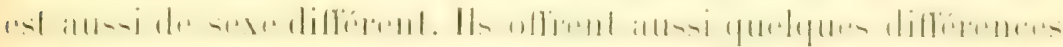
qui ne permettent pas d'itre absolument sur de la déprminalion. J'ai envoyé ees individus à V. Bouvier, qui a eu l'obligeance de les comparer au lype, et qui mª écrit al ce sujel: "de crois qu'on doit identilier les deux Nurien d'Aelen avee la N. luberrenlose X.- II. Edw. I mon avis les differencessont purement de nature sexuelle; ros deux exemplates abut des les-

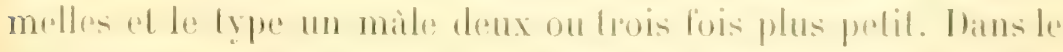

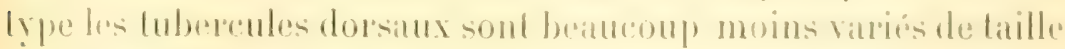
gue renx d'Alen; its ont le plus sourent lat forme suivante $n$

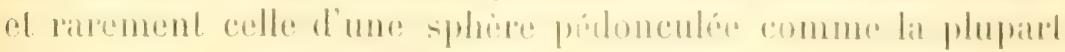

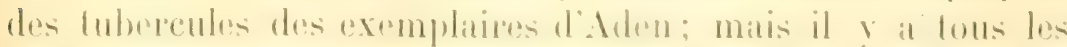

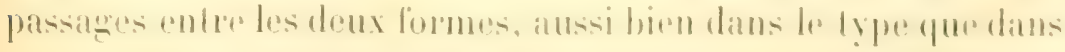

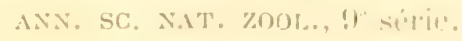

$1 \mathrm{~V}, 11$ 


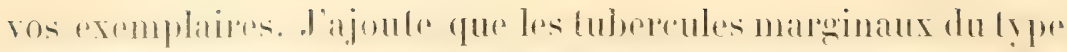

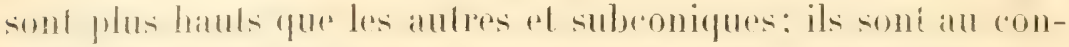

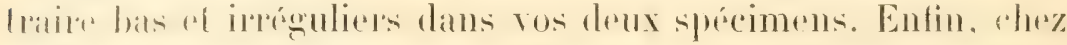

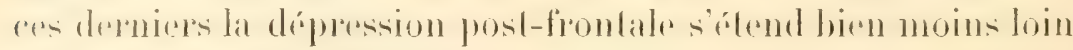
en arrière que dans le type."

Les dimensions des deux femelles sont:

Longueur de la carapace.

Largeur

8,5

8
ร

$6 \frac{3}{4}$

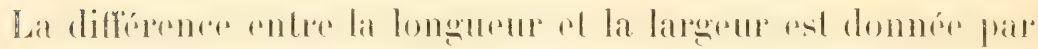

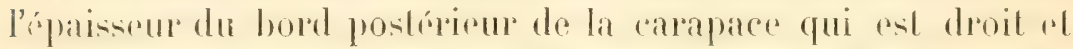
mellement stopré par un sillon. Il n'y a d'autres sillons sur ha

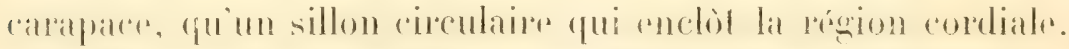

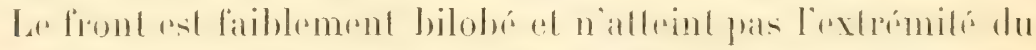

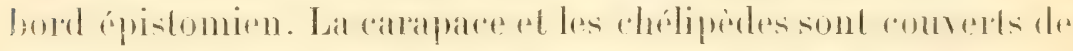

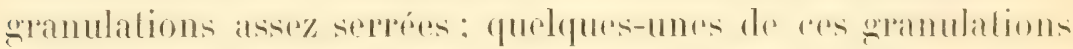
deviennent plus grosses et rarient de forme, les unes élanl coniques, on en forme de $\cap$, les autres étant sphériques el

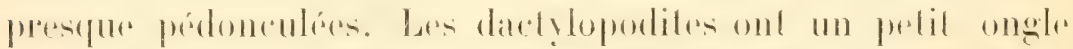

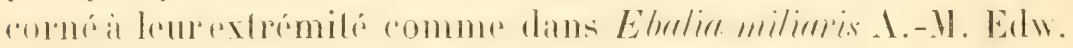

Je suis d'accord avec M. Bouvier que les différences remar-

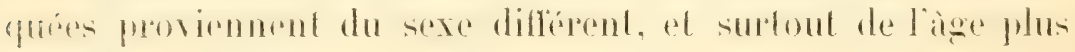
avancé.

\section{Nucia Pfefferi (De Man)}

Ebalia Pfefferi De Man, Arch. f. Nat., 1887, p. 390, p!. XYII, fig. 4. - Henderson, Trans. Limn. Soc. (2), I, 1893, p. 402.

Nucia Pfefferi Alcock, loc. cit., p. 191. - De Man, Abh. Senckenb. Ges., XXI, 1902, p. 684.

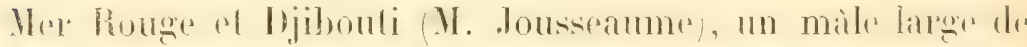

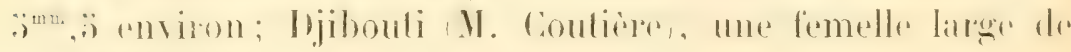
$3^{\mathrm{mm}}, \mathrm{s}$ environ.

Ces exemplaires diflèrent par quelques points des descrip-

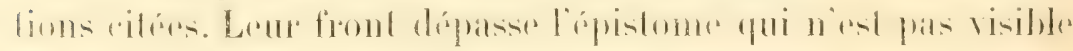

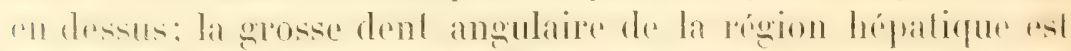

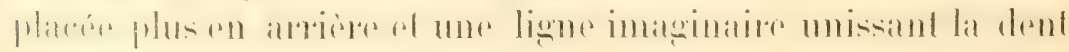

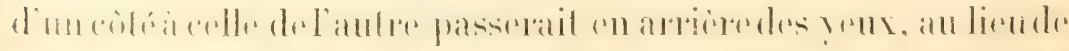
passer sur leur bord antérieur. Les bosselures de la région 
branchiale sont moins marpueses et variables. La dent plér Ygostomique est it peine marquie. Le bord postérient de lit campace est droit. La région cordiate as délimiléts seulement

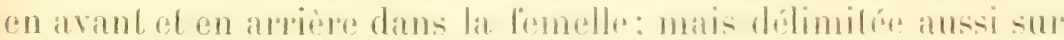
les cotés par un sillon circulaire dans le mile.

II.n. : Amboine, Ternate (De Man/. Inde. Yaturilius Hen$(1+1)+(1))$.

Nucia pulchella (1. .1.-Edw.

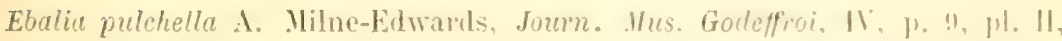
lig. 2.

Her Rouge (II. Jousseaume), un jeune mile qui mesure $3^{\mathrm{mm}}, \ddot{\partial}$ de largeur, soit un peu plus de la moitio de la largerur du type. Le front est aussi avancé que dans les deux individus de l'espece précédente. Les bords latérax de la carapace onl leois gros lobes saillants, lriangulaires el denticulés. Le premier lobe surtout, en correspondance de la région hépalique. est très saillant en dehor's. En arriere des trois gros bobes. il y a encore f-ö petils lobules dentiformes. Cus dents sont nota-

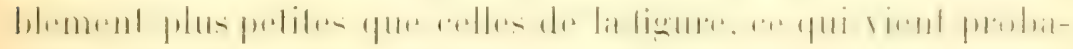
blement de l'itge plus jeune.

La disposition des régions sur la carapace est haubement coractéristique. Deux sillons se départent en arriere du front et se portent obliquement jusqu'au bord postéreur de lat caratpace, en délimilant aninsi une longzue rógionn médiane triangulaire qui est formés par la gastrique, la coldiate el l’inteslinale.

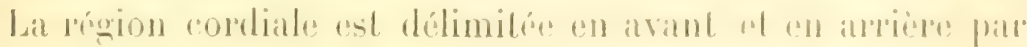

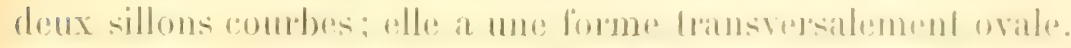

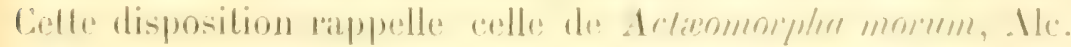

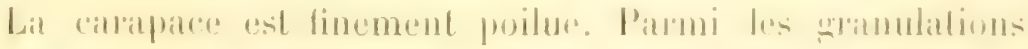

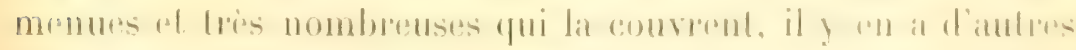
plus grosses el asise\% nombreusers.

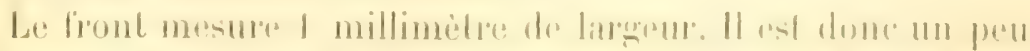

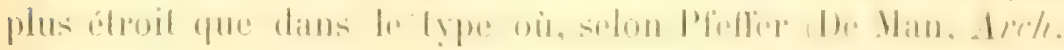

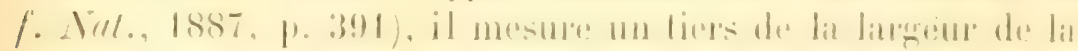
carapatce. 


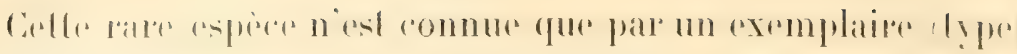
des îles Vili.

\section{Gente MYRA Leach.}

1. Facette hépatique distincle; bords latéraux de la carapace marrfués d'unc: ligne granulée ; épines postérieures aiguës ou coniques.

B. Epines du bord postérieur de la carapace aigrǘs ef longues; chélipèdes très longs (presque trois fois la longueur de la carapace dans l'adulte); carapace très linement granulée (dans les jeunes, la carapace a cing épines marginales et des épines au bord postérieur, et est distinctement granulée)........... fugax (Fab.).

$B B$. Epines du bord postérieur de la carapace plus courtes; celle du milieu longue, les autres dentiformes ou coniques; carapace avec des granules dislincts; dans les vieux individus, chélipèdes de une lois et demie à un peu plus que deux fois, la longueur de la carapace........................ offinis Bell.

A. Facette hépatique non distincte dans les adultes; bords latéraux de la carapace non marginés par une ligne de granulations; bord postérieur armé de trois tubercules aplatis et pétaloïdes............... Kessleri (Pauls.) _

\section{Myra fugax (Fab).}

H. Milne-Edwards, H. n. Cr., II, 1. 126; Cuvier R. Anim. Crust., pl. XXV, fig. 3. -De llaan, F. Jap., p. 134, pl. XXXIII, tig. 1.-Bell, Trans.Limn. Soc., XXI. 183\%, p. 296. - Alcock, loc. cit., p. 202 (ubi syn.).

Myra carinatu Bell, loc. cit., p. 297, pl. XXXII, lig. 3.

Myra coalita Ililgendorf, M. B. Akad. Berlin, 1878, p. 812, pl. 1, lig.6-7. Myru ventacantha Alcock, loc. cit., p. 204.

Obock (M. Jousseaume), 3 mâles jeunes.

Je suis entierement d'accord avec Neock que $\boldsymbol{M}$. coulita M. cominata ne sont que des stades non adultes de $M$. fungax, mais je crois aussi que $M$. pentarantha Alcock n'est que le stade tout jeune de la mème espèce. M. Mleock, d'ailleurs, doutait déja fortement de ce fait. Les trois exemplaires re-

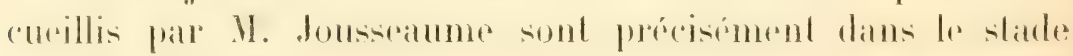
pentacanlha; ils ont aussi les mêmes dimensions que cette:

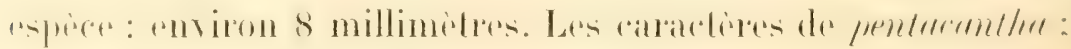

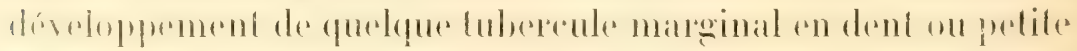

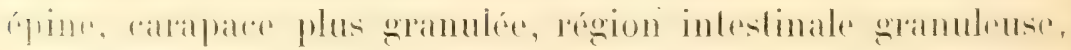
chélipèdes courls, ete., sont bien les mèmes caracteres de lous les jeunes des Lencosiens et des Myra en particulier. Les 
ppines du bort postériem sont bien celles de Myre fugar. Cette espece a élé signalée ansi à lisilonl par Ciano.

\section{Myra affinis Bell.}

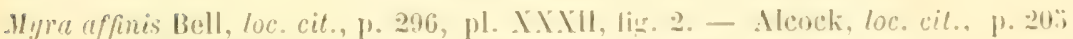

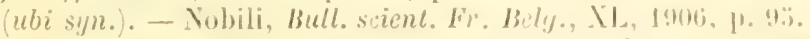

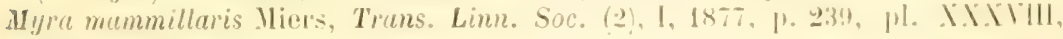
lim. $2:-27$.

Myju subgranulata Kossmann, loc. cit., p. $6: 3$, pl. 1, ligr. 7.

Jer Rouge (II. Jousseatume), un jeune mate qui correspond

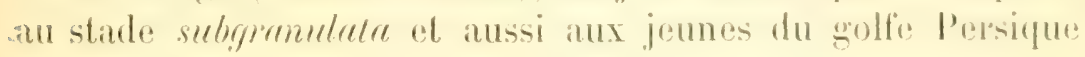
récrits patr moi, loc. ril.

\section{Myra Kesslerii (['auls.).}

- Cullidactylus Kesslerii P'aulson, loc. cit., 1). 80, pl. Xl, lig. 1-1 c.

Myra darnleyensis Haswell, Proc. Linn. Soc. N. S. IV., IV, 1879, p. 32, MI. W, lig. \&, Cat. Austr. Crust., 1882, p.122. - Mier's, Challenger Brach. 1886, 1. 316. - Alcock, loc. cit., p. 207. - Borradaile, F. Geogr. Mald. Lrecead., II, 1). 438 .

La descriplion el la figure de P'aulson, comparócs attenti-

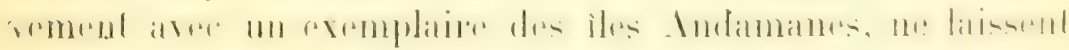
aucun doute que le Callidarlylus Kesslerii de Paulson est lir

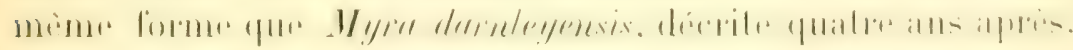

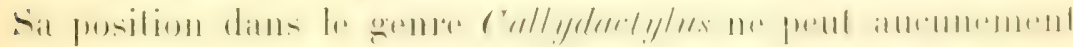

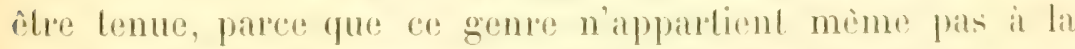

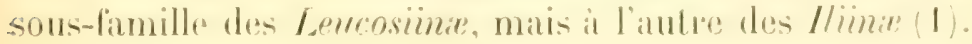

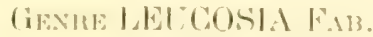

1. Carapace nettement phus longue que large, sans pubescence; bord épimeiral non visible en dessus dans toute son étendue; sinus thoracigue délini, aver des tuberules perlacés. Méropodites des palles ambulatoires non writnules.

(1) A propos de symonymie d'oxystomes, je molite de leaceasion pour faime

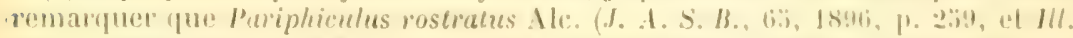
\%ool. Incestigator, pt. XXX, lig. i) est certainement identipue aves: llia mu-

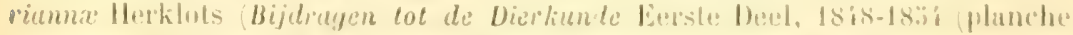

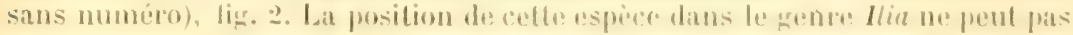

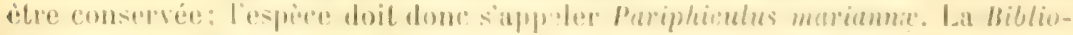

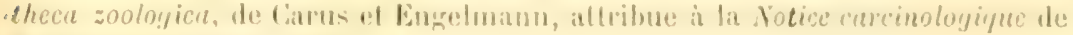
dlerkitoto la date do 18:-2. 
$B$. Bord cxterne de la main caréné ; bord postérieur de la carapace droit.

C. Angles du bord postérieur de la carapace arrondis; vrais bords postéro-latéraux de la carapace granulés jusq'au-dessus de l'avant-dernière paire de pattes; front très peu concave en dessus. (Une ligne brune repliée en 11 traverse la carapace.)

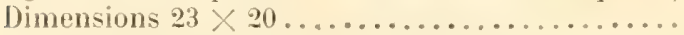

L. signata l’auls. CC. Angles postérieurs prononcés; bords postérolatéraux granulés jusqu'à la première paire de pattes; front convexe. Dimensions : 10-11 $\times 8-9$.

$A A$. Carapace aussi large que longue. Méropodites des paltes L. corallicola AIcock. ambulatoires avec deux lignes de granulations en dessus et en dessous.

$B$. Une masse de tomentum noir sur l'angle épibranchial. Sinus thoracique délini en avant........... L. elata A.M.-E. $B B$. Pas de tomentum sur l'angle épibranchial. Sinus thoracique entièrement indélini en avant......... L. hilaris Nob.

\section{Leucosia signata (Pauls.).}

Leucosia urania var. signata Pauls., loc. cit., p. 76, pl. X, fig. 1-1 c. Leucosia fusco-maculata Miers, loc. cit., p. 236, pl. XXXVIII, fig. 1. Lencosia signata Nobili, Boll. Mus. Torino, XX, 1905, no 506, p. 4 .

Beiloul (11. Orsini, Mus. Turin), un male al e lemelles:

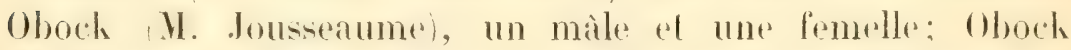
(1I. Cravier), dragage daus le sable du recif de la Clochederie, une femelle: Djibouti II. Coutière), une femelle: Djibouti (H. Jousseatume). une femelle. Localité inconnue: : 2 femelles. Ces individus sout plus gros que ceux de Zanzibar décrits par moi, loc. cil.

\begin{tabular}{|c|c|c|c|c|}
\hline & \multicolumn{2}{|c|}{ Beiloul. } & \multicolumn{2}{|c|}{ Alcock. } \\
\hline & $\sigma^{\prime \prime}$ & Q & రో & \& \\
\hline Longueur de la carapace........ & 23 & 22 & $20, ;$ & 20 \\
\hline$\ldots \ldots \ldots$ & 20 & 19 & 17 & 16,5 \\
\hline
\end{tabular}

La figme, an forme de 11 , w plus ou moins nefle daus tous les exemplaires. Les tubereules pétaloüles du sinus thoracique valient de 3 ì 4 .

Haв. : Her Rouge (Paulson); golfe de Suez (Miers); Zanzibar (Nobili).

Leucosia corallicola Alc. (var.?).

Aleock, loc. cil., p. 224, fig. 4, lllustr. zool. Invest. Crust., pl. XXX, fig. 2.

Denx exemplates de l'órim at un mile de "mer Ronge ". Lous recurillis par II. Jausseatume. Le fronl de ces exemplatres 


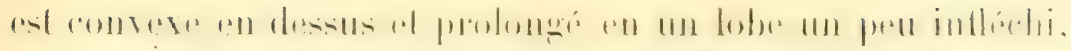

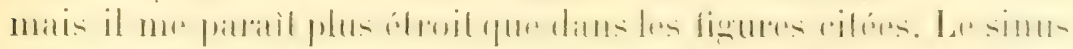
Horacique a ö granulations au lieu de 3-4. La carapace est distinctement poncluée il la loupe. La couleur générale est

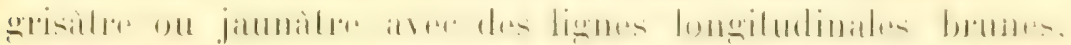

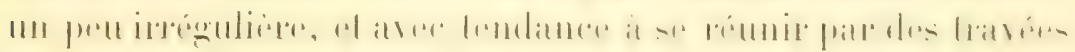
latérales. Ces lignes sont peu marpuées, peu nombreuses el assez distantes entre elles.

Les deux branches antérieures de l'Y du simus thoracique sont presque igales. Les bords latéro-postérieurs s'arreblent au-dessus de la premiere pare de paltes ambulatoires.

Le bord inférieur de la main, ainsi que cetui- du carpe, esl

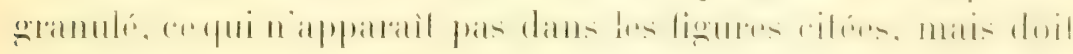
se trouver aussi dans les types, puisque Aleock dit que les mains correspondent ì celles de L. pallida.

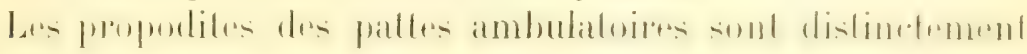
carénés.

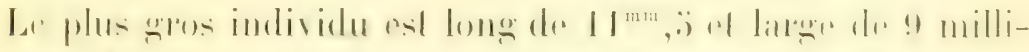

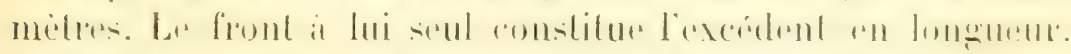

Cette espece n'est connue que des còtes du Malabar 29 bras).

Leucosia elata A. M.-Ldw.

A. Milne-Edwats, Wouv. Arch. Mus., X, 187', P. H, pl. II, lig. 2. - Alcock, loc. cit., p. 2208.

Obock (II. Jousseaume), 2 màles el une liemelle.

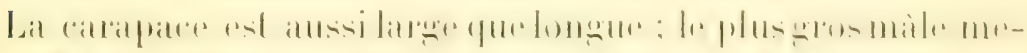

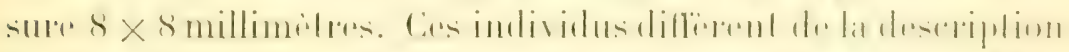

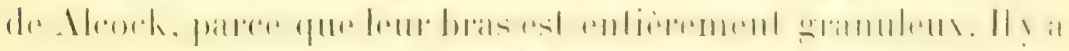

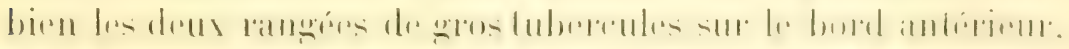

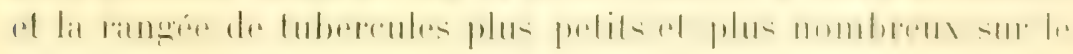
bord postárieur el les gros lubereules do la base: mais en phus de ces tubercules la partie distate du bras est entierement couverte de granules déprimés el de forme irreguliere.

Le méropodite des paltes ambulatoires est bicitrone en dessus el en dessous, les pelites carines sont gramuleuses. Ises propodites sont distinctement carromes sur les deax bords. 
La coloration sipproche plus de colle des exemplaires de la Nouvelle-Caládonie que de celle des exemplaires de l'Inde. La coulen orangén, qui dans los exemplaires types ne formen que des laches, envahit iei presque toutr la rarapace, en lacisant seulement de petites ares verditres. Les chélipedes el les palles sont orangés. Les plargues de fomentum sont prescue noires.

Haz. : Nouvelle-Calédonie (1. Nihne-Edwards): Lpolu, Ceylan, golfe Persique (Alcock).

\section{Leucosia hilaris Nob.}

Nobili, Bull. Mus., $1903, n^{\circ}$ 5, p. 162 ; Bull. Scient. Fr. Belg., XL, 1906, P. 100, pl. V', fier. 9.

Mer Rouge aj Djibouti (M. Jousseame), un màle, qui a déjà été décrit par moi, loc. cit. (1).

\section{Genre PHILYRA Leach.}

A. Carapace subcirculaire. Chélipedes trés longs. Bord postérieur de la carapace arrondi.

B. Épistome et région sous-hépatique saillant en avant du front comme la mâchoire d'un bulldog. Carapace aver. quelques granulations visibles à l'ail nu. Ph. scabriuscula

(liab.).

$B B$. Épistome médiocrement saillant au delà du front.

Carapace lisse ou presque, à neil nu......... Ph. variegata

$\boldsymbol{A} A$. Carapace allongée, subhexagonale. Chélipèdes ne dé(Rüpp.). passant pas une fois et demie la longueur de la carapace. Surface de la carapace inégale et grossement ponctuée; bord postérieur saillant et droit.

Ph. rectangularis

lliers.

\section{Philyra scabriuscula (Fab.).}

11. Nilne-Edwards, H. n. Cr., II; p. 132, pI. XX, fig. 9-10. - Bell, Trans. Linn. Soc., XXI, 1853, p. 299. - De Man, Not. Leyd. Mus., III, 1881, p. 126.

- Henderson, loc. cit., p. 399. - Alcock, loc. cit., p. 239 (ubi syn.).

Obock (M. Jousseaume), 2 mâles.

Le plus gros rexplatire a une coloration brune uniforme,

1) Hilgendorf cite, d'apres Ehrenberg, la $L$. urania et la Philyra porcellana, matis il fiudrait revoir les remplaires, parce que les anciens auteurs ont souvent donné ces noms à des espèces bien différentes. 
le plus jeune a une coloration jaunatre rayée el tachelée de

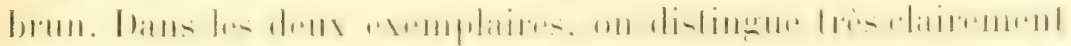

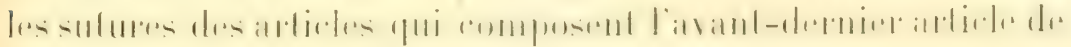
l'abdomen du mile.

\section{Philyra variegata (Rïjग\%.}

Myra variegata Rüppel, loc. cit.. p. 17, pl. IV, lig. t.

Philyra variegala Miers, Challeni. Brach., 1886.

Philyra platycheira P'aulson, loc. cit., p. 2':2, pl. X, firr. 3, 3 c.

Philyra platychira Alcock, loc. cit., p. 222 pors (les seuls exemplaires du coulle

Persique).

Djibouli (11. Couliere), 7 mìles, 3 lemelles; Djibouli, sur

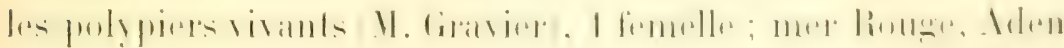
et Périm (11. Jousseaume), 6 femelles; Obock (11. Jous-

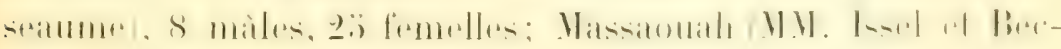
cari, Mus. Gènes), I femelle.

de dois a l'obligeance de 11 . le professeur Dr F. Richters,

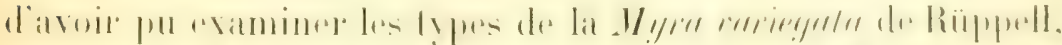

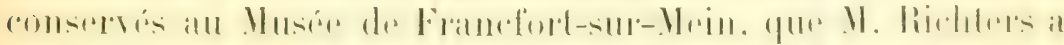

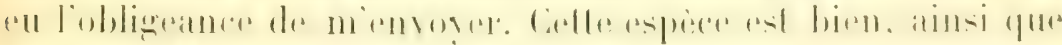

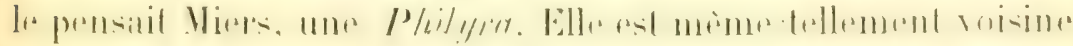

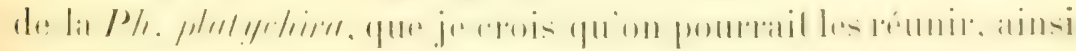
que P'aulson a fail. En ce cas, pourtant, il faudrait donner it l'espece le nom de irmiergatr.

Les petites différences entre les deux formes ont ith dejir vues par M. Aleock, qui pourtant ne comnaissail pas la P/. ret rieguld. Lu savant carcinologiste de Calcultat, dans son travail

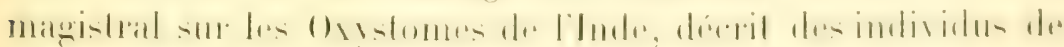

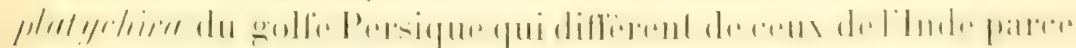
qu'ils ont the don'sul surfare murh mollled with green and hrourn, and the immobile finger denticulute heyond the line of hruirs. Cas

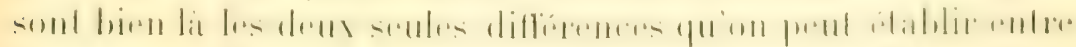
les lypes de Riuppell et mes exemplatires de la mor Rouge d'un

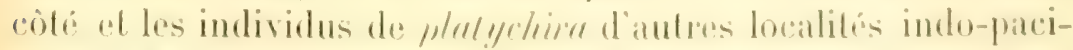
fiques. Lat coloration des individus inde-paciliques est, ainsi

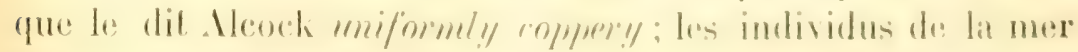

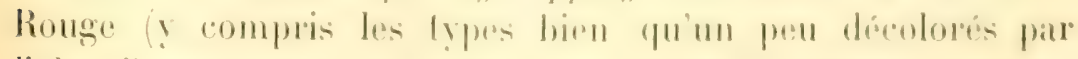

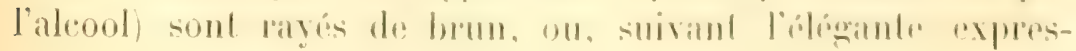




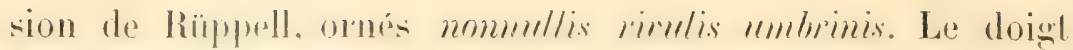
fire offre romstamment quelques denticules saillants, apres lit ligne dre poils dans les exemplities de la mer houge: il sel entier, a cet endroil, dans los individus d'auleres provenances.

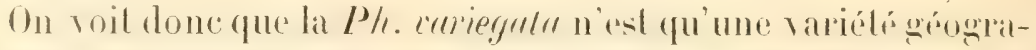

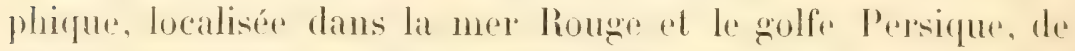
la Ph. plrelyrliren largement répandue dans la région indopacifique.

\section{Philyra rectangularis Miers.}

Mier's, Alert. Crust., p. 546, pl. XLIX, fig. A.

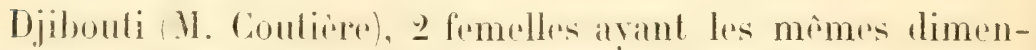
sions que le type, presque 6 millimètres de longueur.

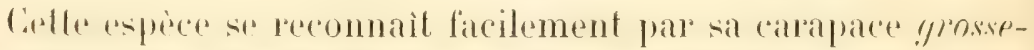
ment ponctués, par la portion antépiene déprimée. la portion intestinale saillante, of son bord postérieur saillant ef droit. Les angles. de ce bord sont droits, mais non séparés de la carapace par une exhancerure comme dans la ligure de Miers. Les régions branchiales sont ponctues ot anssi granuleuses.

Celle espeece niest connue que par une seule femelle trourée aux îles Seychelles par l' "Nlert ".

\section{ILIIN FE}

Gen rePHiCulus Adams et White.

Iphiculus spongiosus Ad, et Wh.

Adams et White, "Samarang" Crust., p. รั , pl. XIII, fig. วั. - Stimpson, Proc. Acad. Philad., 1858, p. 161. - Miers, "Alert " Crust., p. 2马3. Alcock, loc. cit., p. 256. - Lanchester, Proc. Zool. Soc., 1900, p. 766. Nobili, Boll. Mus. Torino, XVIII, 1903, n⿳455, p. 24.

Her Rouge el Djibouti (H. Jousseaume), InI mitle et une femelle.

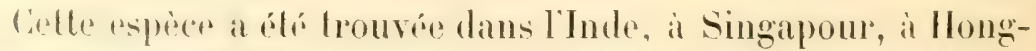
hong, aux iles Philippines el dans la mer d'Mafoura. 
GENRE ARCLINIA JEACH.

Arcania septemspinosa (Fal.).

(if. Alcock, loc. cil., p). 26i:.

Obock (11. Jousseaume), 2 miles; mer Rouge a lojibouti (11. dousseaume), 2 màles; Massaouah (I. Fatigati. Mhuse dr 'Turin), I mate mesurant 20 millimetres de longueur sur ?f) de largeur.

L'individu de Massaonah difrere de deux antres de Sandheads (Inde), du Husée de 'Thuin, par ses régions hŕpatiques moins convexes, les doigts un peu plus courts que la paume

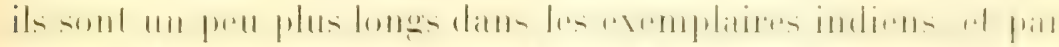

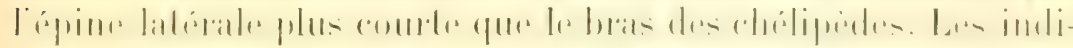

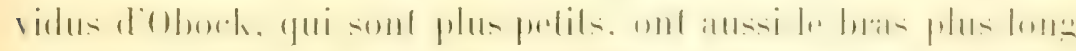

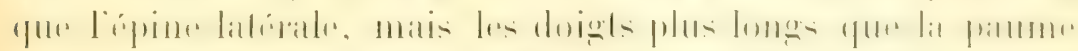
comme dans les individus indiens.

Celle espece a été houvée dans le golfe Persique, dans l'Inde, dans la Malaisie el en Chine.

\section{GENTE IX\ IAACH.}

\section{Ixa inermis l.outh.}

(if. Nleock, loc. cit., p. 272.

Dans l'incertitude qui existe encore an sujet de cette aspoce.

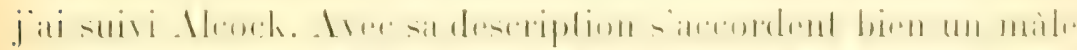
at une femelle de Massaouah (M. Fatigati, Musée de Turinj.

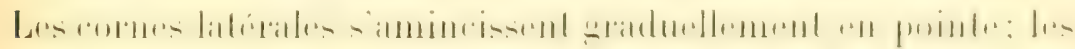
sillons du dos de la carapace sont superficiels ot olabere les

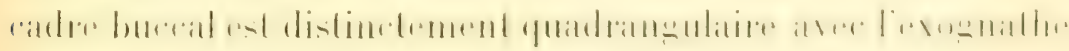
convexe al granulé. Un antre exemplatire, qui n'est lopresentis que par tune carapace dessechese of molommagés se trouve

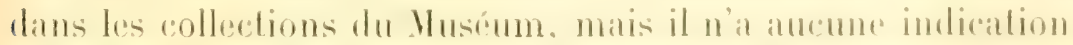
de localite. Coet exemplaire est bien interessant paree que toul

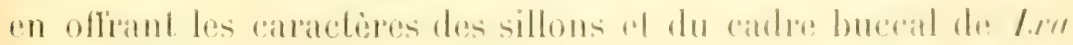

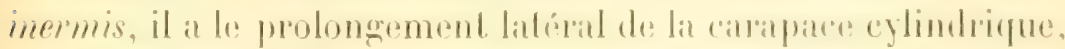

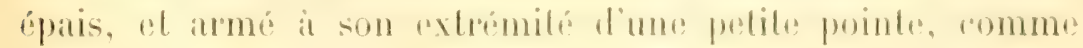
dims I. cylindres. ("est probablement une anomalie due it lat régération de la corne rassón. 
Ixa. Eduardsi Lucas (Ann. Soc. Enı. fi., 183̈8, p. 181, pl. IY, fig. $3 ;$ A. Nilne-Edwards, Ibid., 1859, p. 1506, pl. VI, fig. 1),

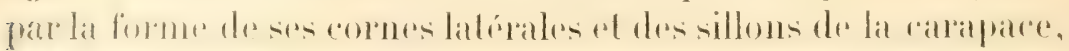

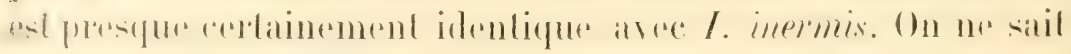
pourtant rien de la forme de son carle buccal.

Cette espèce, bien que décrite depuis un siècle, paraît ètre sare. Leach n'en a pas signale ha localibe. Les caphores sumes

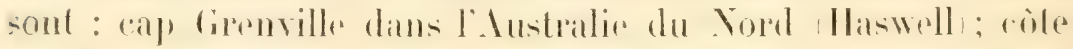

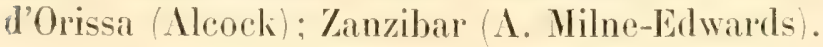

\section{FAMILLE DORIPPIDA}

GENRE DORIPPE FAB.

\section{Dorippe dorsipes (Linn.).}

lif. Alcock, loc. cil., p. 277 (ubi syn.).

Obock (I. Jousseaume), une femelle.

\section{OXYRHYNGHA}

\section{FAMILLE MAMAIDA \\ Genre CaMpOSGia latis. \\ Camposcia retusa Latr.}

Guérin, Iconogr. R. Anim. Crust., pl. IX, fig. 1. - H. Milne-Edwards, H. $n$. Cr. I, p. 283, pl . XV, fig. 15-16, et CuvierR. Anim. Crust., pl. XXXII, lig. 1.— Ortmann, Zool. Jahrb. Syst., V11, 1893, p. 35.-- Alcock, J. As. Soc. Beng., 64,1895, p. 184 (ubi syn.).

Mer Rouge (Musée de 'Turin), un mâle.

$$
\text { Genre ACANTHONYX LATR. }
$$

Acanthonyx elongatus Miers.

Miers, P. \%. S., 1877, p. 673, pl. LXXIX, fig. 1.

Signalé dans la mer Rouge par Miers.

Acanthonyx consobrinus A. M.-Edw.

Paulson, loc. cit., p. 7, pl. III, fig. 1.

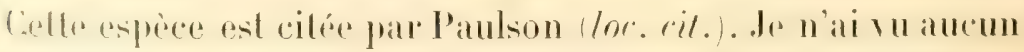

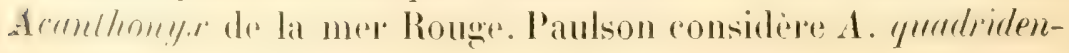


talus linasus identique aree colle aspece. Puisque je ne connais

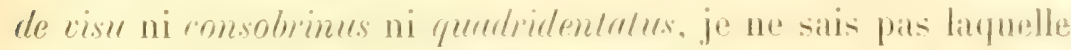

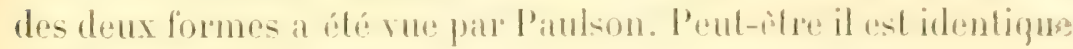
it elongralls.

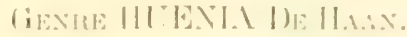

\section{Huenia proteus I) llian.}

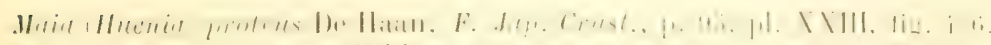

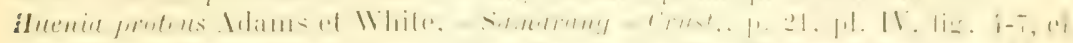

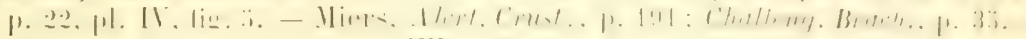

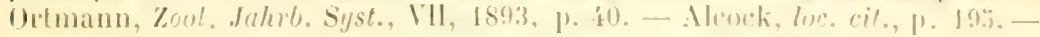

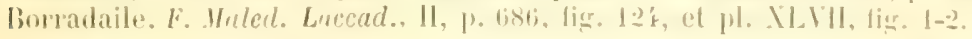

Her houge (I. Jousseamme), une femelle; Djibouli (II. Cintlieres), un jeune màle.

La femelle a le lobe apibranchial carre ol phes aboil que le lobe hépatique. La region gastrique porte hois luberendes biens nets, et la région cardiale un seul, dans la limelle, le maile n’a pas de lubereules distinels sur la région gaslrique.

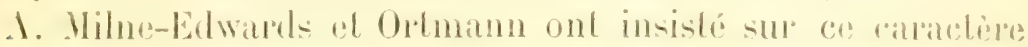

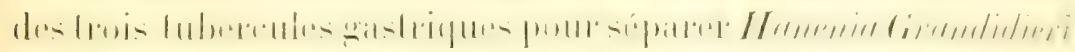
de H. protens; mais je ferali observer que les ligures de tee Ilatu, soil des lypesmèmesdir molens, ont bien frois lubereules. Vu la grande variabilité de $/ /$. moteus, je doute fort yue II. Gromelidieri ne soil identique avere celle espece.

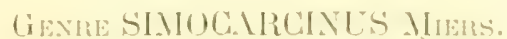

\section{Simocarcinus simplex (I)ana).}

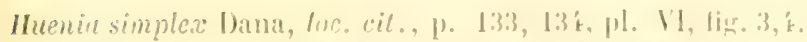

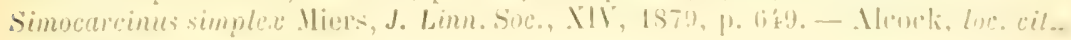
1). 1916.

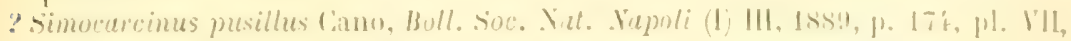
lig. 3- 3 .

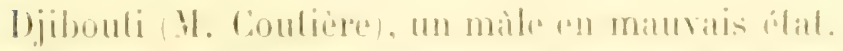

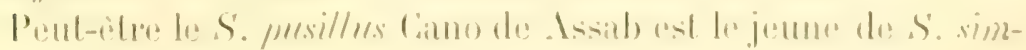
pher.

\section{Simocarcinus pyramidatus.}

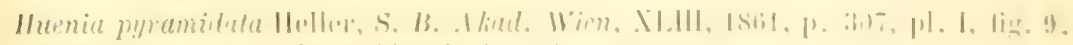

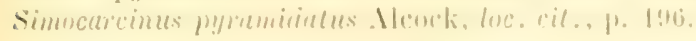

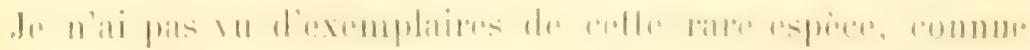

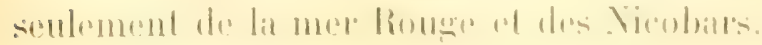




\section{Simocarcinus Helleri (Pauls.).}

Ifuenia Helleri Paulson, loc. cit., p. 8, pl. III, fig. 2.

"Voisine de $\boldsymbol{H}$. pyramiduta, mais distincte par son rostre dont la surface supérieure se rétrécil, l’infériemre est un peu concave, mais élargie vers la pointe; surface latérale

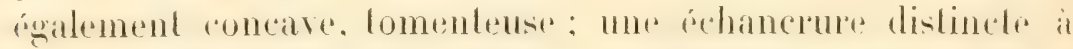

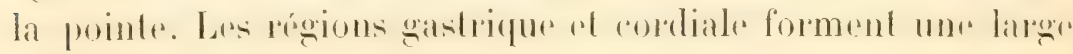
saillie.

Doigl des chélipides arece de pelits denticules, joignant bien.

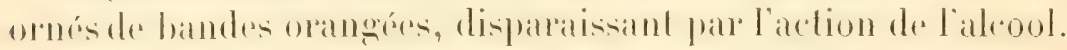

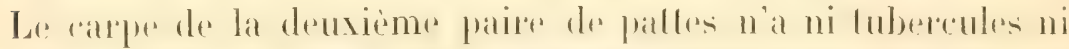
sillons: le propodus est sitns dents: le doigh "st denlirulé.

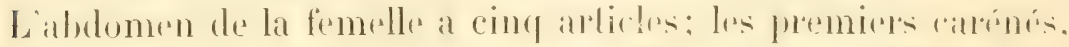

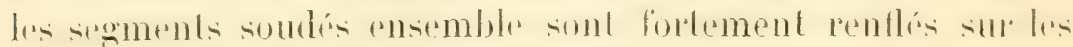
còlés, el pourvus d'une goutlière au milieu.

\section{Genre MENOE'THJUS EDM.}

Menothius monoceros (Lat.).

Cf. A. Milne-Ldwards, Nouv. Arch. Mus., IV, 1868, p. 70, et VIII, 18\%2, p. 2:22, 253. - Alcock, loc. cit., p. 197 (ubi syn.). - Paulson, loc. cit., p. 6, pl. H, fig. $2,3 a, 3 b$.

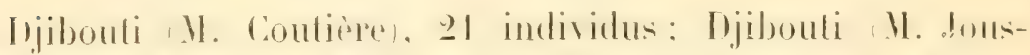

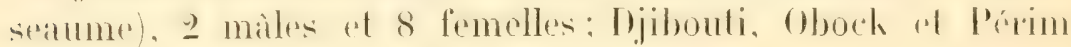
(I. Jousseaume), 8 màles, 4 femelles.

\section{GENRE HYASTENUS $\mathrm{W}_{\mathrm{H}}$.}

Hyastenus spinosus A. Edw.

A. Milne-Edwards, Nouv. Areh. Mus, V111, 18:2, 1. 250. - Alcock, lue cil.. P. 211. - Nobili, Boll. Mus. Torino, XVIll, 1903, n 4:3̈, p. 27. H. diacanthus Cano, loc. cit., p. 178 (nec H. diacenthus De Ilaan). 11. diacanthus bituberculatus Lanchester', Proc. Lool. Soc., 1900, 1' i2.3. Chorinus aries Bianconi, Spec. Zool. Hossambicana, 1851, p. 75.

Djihouti (M. Coulière), un mâle jeune el mulilé, chez qui

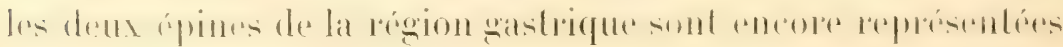
seulement par des tubereules. 
Tous an individus appardioment is la forme representure par les figures $1 d$ et $1 /$, de l'ouvrage cilé de $\Lambda$. Mihne-Edwards, mais les cornes propres du rostre paraissent un peu plus

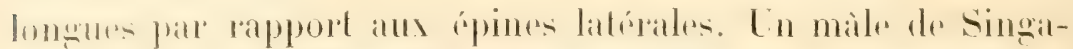

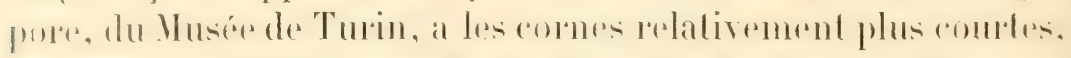

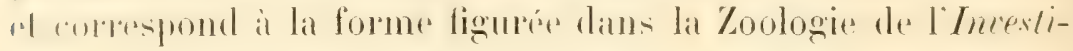

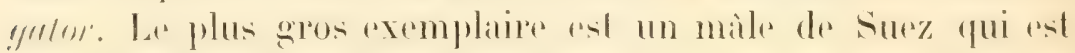
long de 66 millimètres et large de 41.

Signalé aussi à Beiloul (Cano).

\section{Genize GyCtax Dana.}

\section{Gyclax (Cyclomaia) suborbicularis Stm.}

Mithrux suborbicularis Stimpson, Proc. Ac. N. Se. Philadelphia, 18:37, p. 218. Cyclax spinicinctus Heller, S. B. Iliad. Wien, 43, 1861, p. 304, pl. 1, tig. 7-8. Cyclomia murguritatı A. Milne-Edwards, loc. cit., p. 236, pl. X, fig. 2-3. Cyclax (Cyclomaya) suborticularis Aleock, loc. cit., p. 22's (ubi syn.).

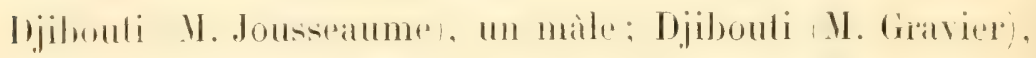
une femelle jeune; îles Musha (M. Gravier), un màle.

Le male de Djibouti a cing épines sur les bords latéraux;

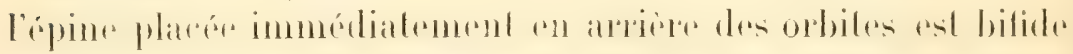
des la base a gauche, simple à droite; la femelle a six épines de chaque còté, la première bifide, la sixième très petite.

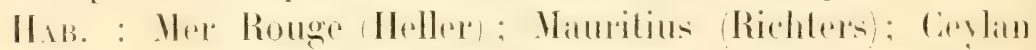
(Mïller); Inde (Mleock); Andamanes (Aleock); Maldives

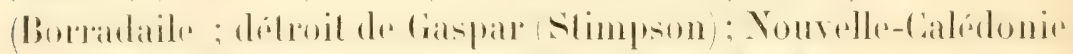

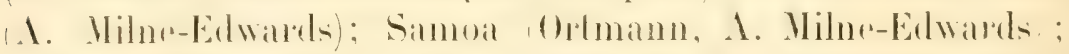
Rolumar Borrataile: iles Sandwide (1. Milno-Edwarlsi; détroil de Torrès (Calman).

\section{Genre STYY IABOGNATHUS von Martens. \\ Stylbognathus erythræus v. Mart.}

Yon Martens, Verh. Zool. Bot. Ges. Wien, 16, 1866, p. 379. - Paulson, loc. cit., pl. I, fig. 2 a-f. - Kossmann, loc eit., p. 13, pl. 1, 1ig. 1. - De Man, Not. Leyd. Mus., 1881, p. 93.

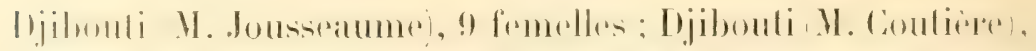
un mile el 2 femelles; mer Rouge (H. Jousseaume), une femelle: Obock (II. Jousseame), une femelle. 


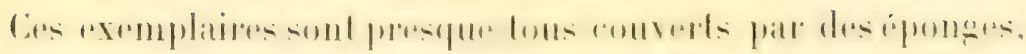

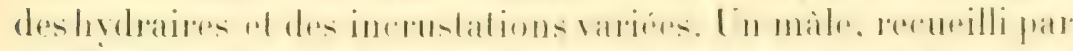

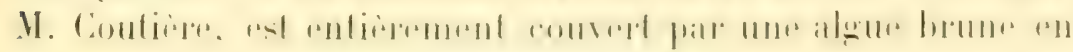
toulles ramififies.

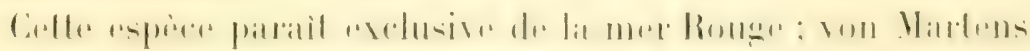
la signala de Ras Raïssi, De Man de Djodklah.

\section{GENRE S'l'ENOCIONOPS IAT.}

\section{Stenocionops curvirostris 1. M. Edw.}

A. Milne-Edwards, Ann. Soc. Ent. Hr. ('t), Y, 186å, p. 13\%, pl. I, lig. 1-1 e.Nobili, Bull. scient. Fr. Belg., XL, 1906, 1) 108.

Le type de cette espece vient de la mer Rouge. l'en ai vu

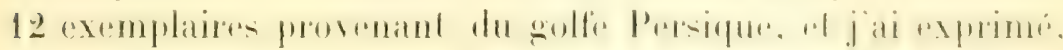
lor. cit., le doute que cette espèce soit identique it Sl. remicomis. D'apres von Wartens (Ine. chl., 1866), St. cemenomix se

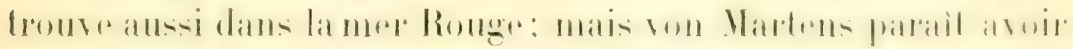
ignoré alors le S\% mervirostris décrit l'année précédente.

\section{GENRE PSEUDOMICIPPA HetLER.}

\section{Pseudomicippa nodosa Heller.}

Heller, loc. cit., p. 301, pl. 1, fig. 3-3. - Paulson, loc. cit., p. 9. - hossmann, loc. cit., p. 9.

Je n'en ai pas vu d'exemplailes.

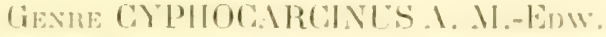

Cyphocarcinus minutus 1 . M.- Hlw.

Cyphocarcinus minutus A. Milne-Liwards, Nour. Arch. Jus., IV, 1868, 1). 73. pl. XIX, lig. 7-12. - Alcock, loc. cit., 1. 2.it. - Vobili, Bull. scient. Fr. Bely., XL, 1906, P. 109.

Ixion capreolus P'atulson, loc. cit., 1. 3, pl. II, ligr. 1.

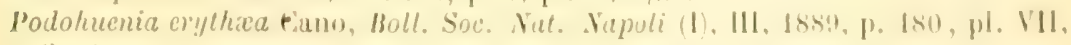
lig. : :.

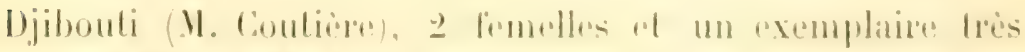
jeune mer Rouge (I. Ioustratume).

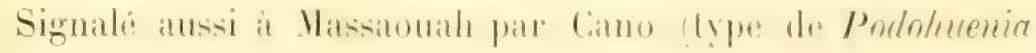
elylliliple 


\section{Genre Micippa Leach. \\ Micippa philyra (Herbst).}

Micippe platipes, Rüppell, loc. cit., p. 8, pl. I, lig. 4. - Heller, loc. cit., p. 299, pl. 1, tig. 2.

Micippe philyra et Paramicippe platipes II. Milne-Ldwards, loc, cit., I, p. 330. et 333 .

Micippe spatulifrons A. Nilne-Ldwards, loc. cit., p. 240, pl. XI, fig. 3.

Micippe philyra var. platipes Kossmann, loc. cit., p. 4 et 7, pl. III, fig. 3.

Wicippe philyra Alcock, loc. cit., 1. 249 (ubi syn.).

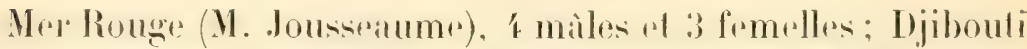
(M. Jousseanme, un male: golfe de: Tadjourah (M. Famot). une femelle; Djibouti (M. Coutière), un mâle.

Jareepte les idres de Aleoch que les nombereses formes de Miriplen is corues rostrales profondement bipartites doivent

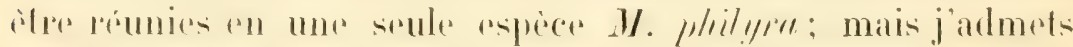

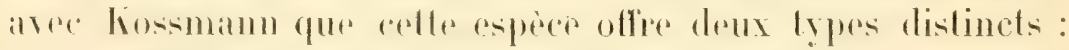

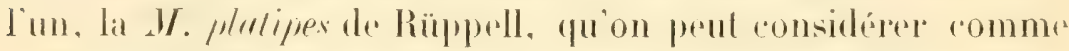
lat forme trpique. a bords lateraux sentement lubereules:

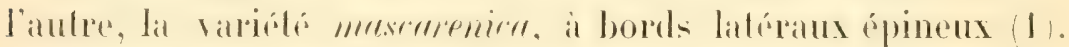
hossmann veut abahlie des différences ansi sur la forme du

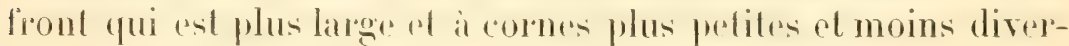

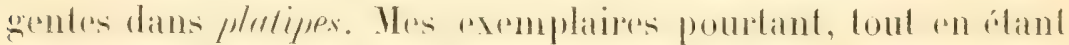

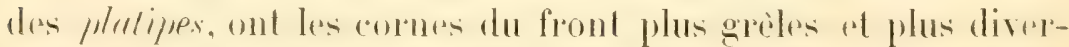

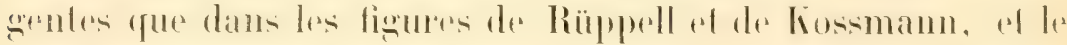

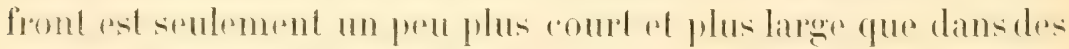
mascarenica du golfe Persique.

Micippa thalia (Herbst).

Ci. Aleock, loc. cit., p. 251 (ubi syn.).

Micippa miliaris Gerstïcker, Arch.f. Nat., 1856, ). 110. - Heller, los. cit., p. 298, pl. 1, fig. 1. - Kossmann, p. 4 et 8.

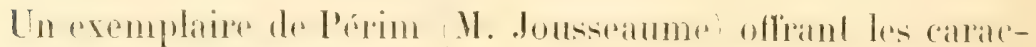
lieres de M. milinerix Gers.

(1) Alcock suppose que les diflérences entre la .1\%. philyra (platipes) el la M. mascarenica sont dues it un dimorphisme des mâles. Cette opinion n'est pas soutenable puisqu'on troure des mâles et des femelles des deux formes. 


\section{FAMILLE PARTHENOPIDA}

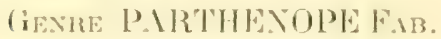

Parthenope horrida l'al).

Guérin, Iconog. R. Anim. Crust., pl. 111, fig, 1. - I1. Milne-Edwards, II. $n$. Cr., I, p. 360; Ill. Curier. It. Anim. Crust., pl. XXVI, lig. 2. - Aleock, luc.cit., p. 280 (uti syn.). - Stebbing, S. Afi. Crust. III, 1903, 11. 27.

Mer Rouge (V. Jousseaume), une femelle dont la caparace

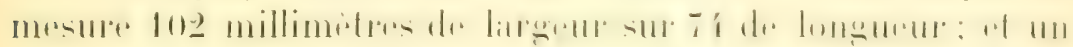

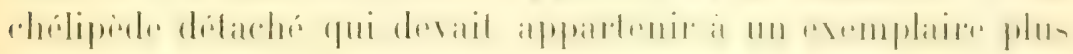
gros.

Djibouti, récifs du Pingouin el du Métére. Dragage 20 melres (Il. Gravier), une femelle avec aufs, large des 3̈3 millimetres el longue de 37 millimidres. Les lubercules des chélipedes sont moins développés que dans l'autre exemplaire plus gros.

\section{GENLE JAMBRLS LEACH. \\ Lambrus (Thyrolambrus) leprosus Nol). \\ P. IX, fier. T.}

Nobili, Bull. Ilus., 190.;, no 3, p. 399.

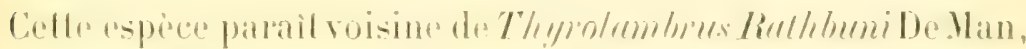
mais seon distingue pare la forme dibrente de la carapare qui

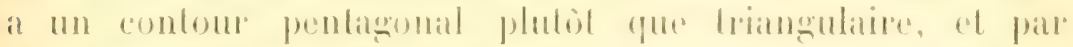
d'aubes nombereses particulatribis.

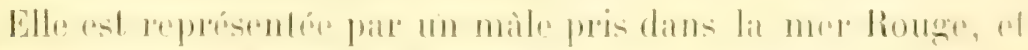
appantenant an Muse de Turin.

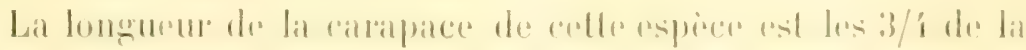

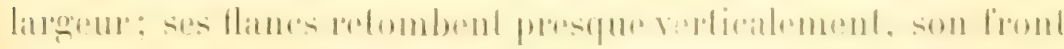

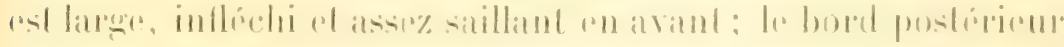

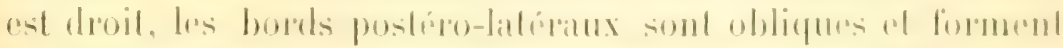

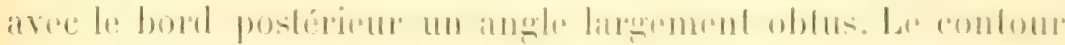

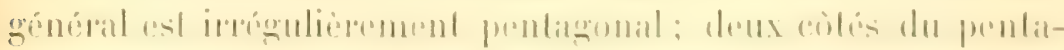

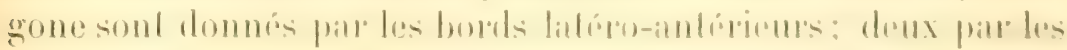

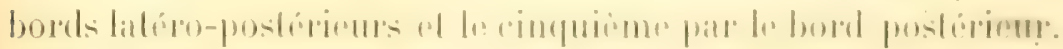

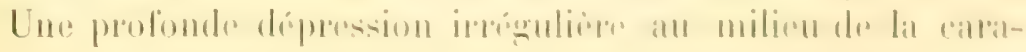


pace sipare la rigion gastrique de la rógion cordiale al des régions branchiales. La région gastrique est saillante, thans sal partie antéreure qui descend obliquement rers le firont elle offre une concavite, landis que sat partie postérieure ou mésogastrique est délimitée latéralement et en arriere en forme de gros lobe saillant at arrondi. La région sous-hépatiques savance en forme de tubereule arrondi bien net et bien séparé. La région hépatique n'est pas délimitée de la région gastrique ni des régions branchiales. Les flanes de la carapace, du front jusqu'it l'angle formé par les bords latéro-anterients avee les bords latéro-postérieurs, sont infléchis verticalement: ot l’on peut ainsi distinguer deux bords; l’un, le vai bord antéro-latéral qui n'est visible que partiellement en dessus. l'autre qui est la limite entre la partie dorsale et la partie latérale infléchie de la carapace. Le vai bord latéral est, sur la partie branchiale. divisé én 6-7 lobules très obscurs ot arrondis. Le hord de la partie dorsile offre un premier trait oblique en dehors qui rattarhe la région gastrique a la région branchiale le point de conjonction est marpué par un renflement luberculiforme le la région branchiale) et un deuxieme trait oblique en arriere et moins en dehors (plus rentrant) qui va jusqua au commenerment des bords postero-lateraux. L'angle formé it la rencontre est presque droit. Les bords postéro-latéraux sont minces; la surface inféro-latérale au-dessous d'eux est concave.

Le front est inflechi, concave en dessous et arrondi au bout; mesure a sa base rnter les yeux il est deux fois aussi large que long. Il est donc plus atroit que celui de Th. Rulhbuni De Man. La concavité du front se continue uniformément dans celle de la partie antérieure déclive de la région gastrique. La partie protogastrique se continue de còté sans démarcation dans les parties branchiales; la partie protogastrique est seulement plus haute que la branchiale, mais entre les deux il y an pont continu, et non des sillons séparants. La région branchiale est irrigulièment tuberculeuse et divisible avec difficulte en trois namulons déprimés. La région cordiale est bien distincte, mais elle st délimitée en arrière et sur les còtris plutòt par une dépression large et peu profonde que par un sillon net, atroit 
et profond, en fer de cheval comme dans Th. Ralhbuni ef

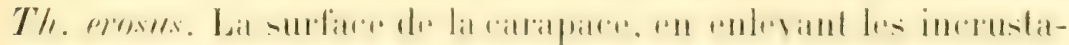

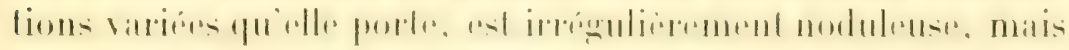

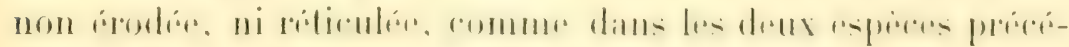
dentes. Les nodules sont petits.

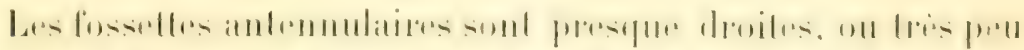

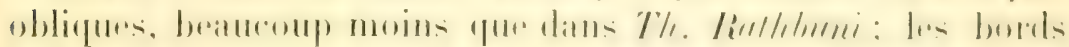

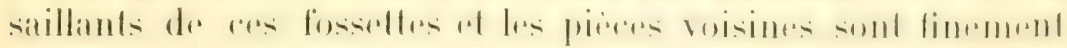

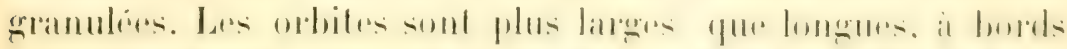

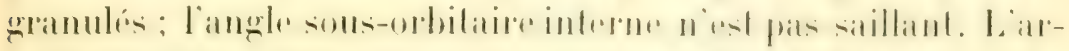

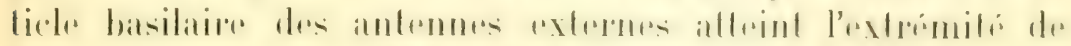

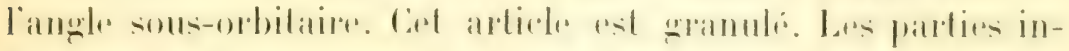

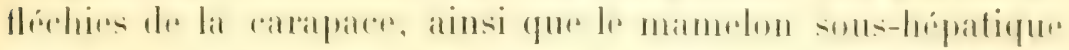

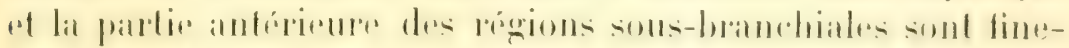

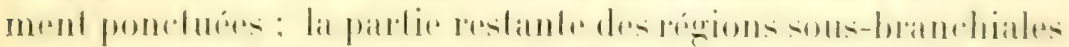

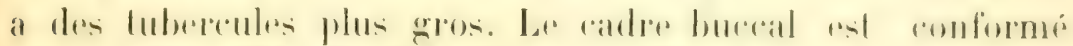

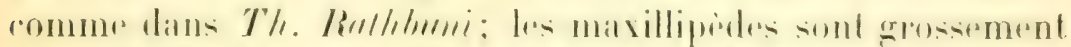

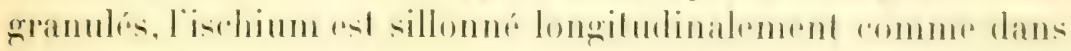
l'espèce nommée.

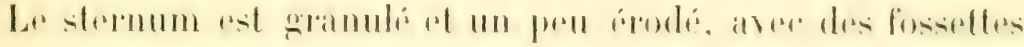

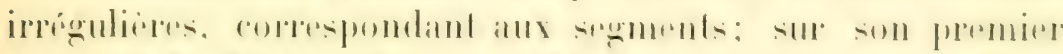

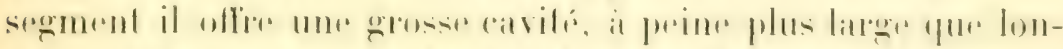

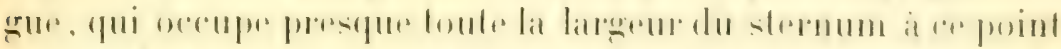

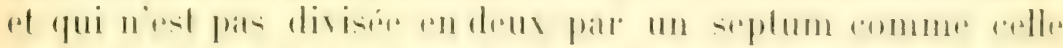
de T'. Rathbumi. L'abdomen est granuleux.

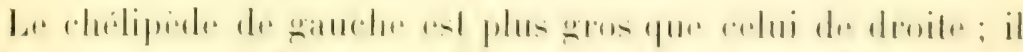

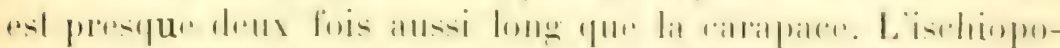

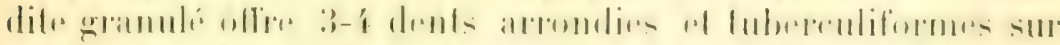

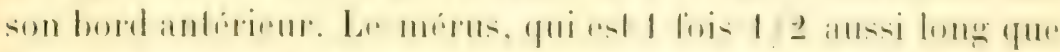

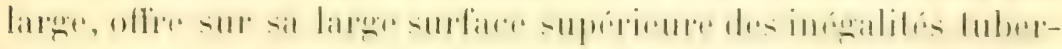

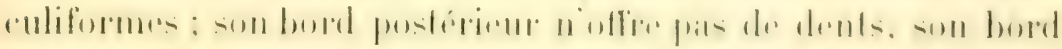

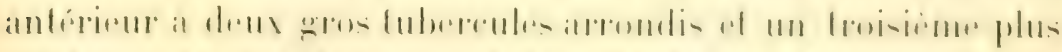

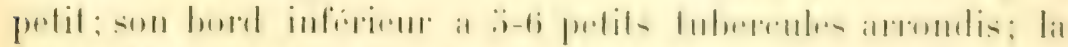

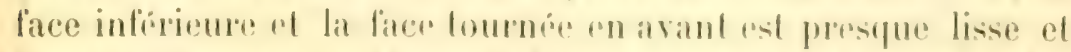

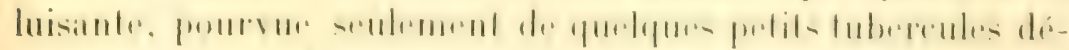

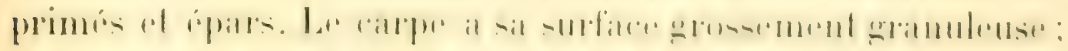

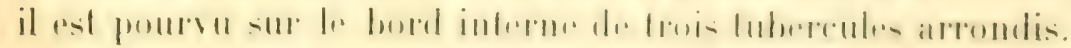

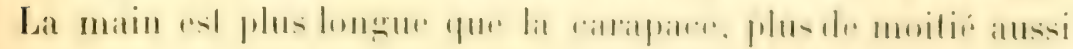


haute que longue a l'articulation du doigt; mais plus basse a larticulation "arpale. Son bord supero-externe est presfur lisse; sa face "xlerme a un gros fubereule déprime el granuleux pres de l'arliculation digitale ; an-desious de ce tubercule il y en a un autre plus putil, snivi par une langée transversale de lubereules de mime nalure. En desisous del desis de celtr rangée il y a dautres tubereules plus pertits, arrangés irrógulierement, mais avec fendance a se grouper. Complexivement la face extern. de la main ressemble beaucoup à celle de Th. Rorlhbumi. Le bord infóriene de la main of du doigt fixe forment une ligne droile. Le mai bord infrécer a quatre lubereules deprimés el comprimós allernés entre eux par des petits gramules. Le hord superieur de la main est très large; il forme prestue une seule surface avec la crite interne qui est décompée en trois gros lubrerules irréguliers. La face interne de la main est lisse el poredlanacée. Les doigts sont granulus; le doigt mobile st recourbé en bas et ne joint pas avec lo doigt fixe; il a deux petits tubercules sur sa surfare dorsale. Sa longueur en ligne droite de la base a la pointe est prestue les $2 / \ddot{\partial}$ de la longueur totale de la main, mais. par suite dre la rourbure, la distance entre l'arliculation w la pointe est à peine 1/4 de la longueur totale de la main.

Ces doigts ne sont pas dentés.

Le petit chéliperde a les mimes ornements, mais les lubercules et les saillies sont plus aigus et plus marqués; les doigts joignent bien, mais ne sont qu'irregulierement dentelés.

Les paltes ambulatoires sont plutot courtes; tous les articles sont l'égulièrement granulés al nodulenx; leurs méropodites offrent ruelques saillies dentiformes tres irrégulières, mais pas les dents cararteristiques disposécs en forme de filet gree

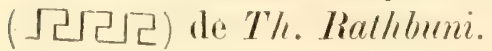

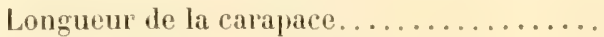

21,3 millim.

Largeur

$28, \ddot{3} \quad-$

Longueur du gros chélipède............

horizontale de la main.........

de la paume................

llauteur de la paume à l'articulation du

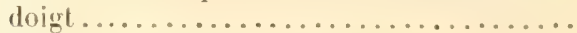

Hauteui de la paume à la base..........

i2

$2+$

18

14

11 


\section{Lambrus (Platylambrus) carinatus Eilw.}

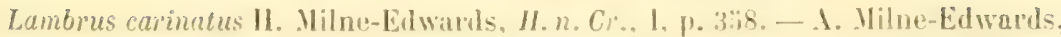

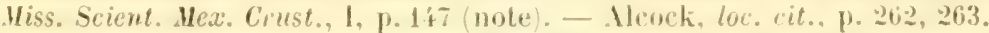

Lambrus serratus var. mossambicanus Bianconi, Mem. Accad. Bolognu, 1851, [1. 10:, pl. Xl, fig. :.

Djibouti (1). Coutiere), une femelle tres jeune dont la cara-

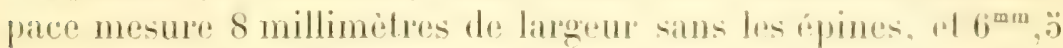
de longueur sius le rostre.

Ce jeune individu s'aceorde bien avec les deseriptions des

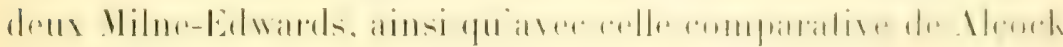
(je l'ai comparé avec un jeune $L$. grenson de Zamzibar), mais le rostre est aigu a la pointe et non obtus. Les hois prociminences sur la ligne médiane de la carapace sont bien développées, mais leur pointe est un peu arrondie. Il n'y a pas de ligne saillante en correspondance de l’ápine épibranchiale.

Le lobe infraorbitare est bilobé, mais le lobe interne n'est pas saillant.

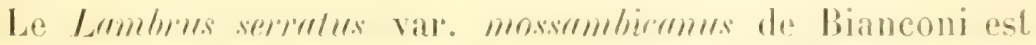

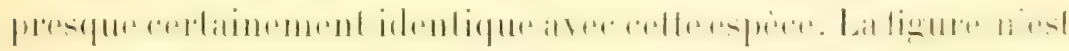
pas trop exacte, mais la description s'aceorde bien arec celles des deux Milne-Elwards el avee mon exemplaire.

11. D. Nilne-lidwards, Ior. ril., considere comme identique arec cette espece $u$ L. armulhus Bianconi. Je n'ai pas réussi it trouver une espece de ce nom dans la longue sére des Speri-

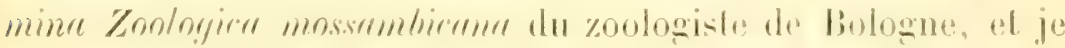
suppose qu'il y a lit mo lapsus rolmmi, ol quil stagil du L. mos-

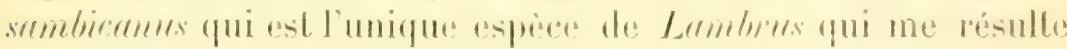
décrite par Bianconi.

\section{Lambrus Aulacolambrus pisoides $11.1 \%$.}

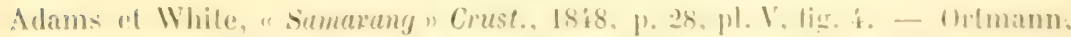

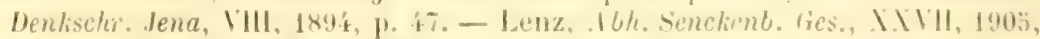

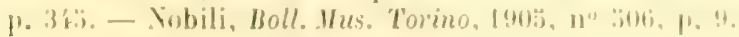
Aulacolombrus pisoides Paulson, loc, cit., 1'. !?.

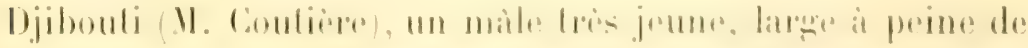

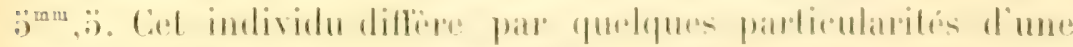

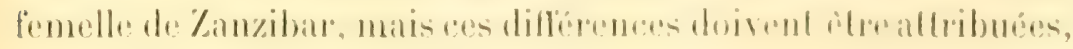


je crois, alu jeune igne de l'exemplaire. La carapace a un nombre plus petit de tubercules moruliformes; ces tubercules sont a peine discermables sur les chélipides, où on les voil en formation. Lal carapare a ses borkts phos courbés, et armés de sept tubercules aigus. Ces tubereules sont plus obtus dans la femelle de Zanzibar, ou ils sont aussi presque masqués par les gros tubercules moruliformes des régions branchiales; dans le màle de Ijibouli, ils sont plus visibles par le petil développement des pelits lubereules moruliformes. Le rostre est plus large et aussi moins saillant. Le bord extérieur de la main a six grosses épines au lieu de cinq.

Cette rare espece a déji be signalée dans la mer Rouge par laulson. Cie mème auteur fonda pour celle espece le genpe Aulurolumbrux en 183̈̈. Par une coüncidence étrange, le mème nom avec la mime signification systimatique fut employe trois années plus tard par M. 1. Milne-Edwards, evidemment sans connaître le genre de Paulson.

Has. : Mer Rouge (Paulson): Zanzibar (Ortmann, Lenz, Nobili); Philippines (Adam et White); Japon (Ortmann).

\section{Lambrus (Rhinolambrus) pelagicus Riipl).}

Lambrus pelagicus Rüppell, loc. cit:, p. 13. p1. IV, fig. 1. - H. Milne-Edwards, H.n.Cr., 1, p. 355. - De Han, Zool. Jahrb. Syst., VIII, 1895, 1. 494. - Alcock, loc. cit., p. 267 .

Lambrus affmis 1. Milne-Edwards, Nour. Irch. Mus., VIII, 1872. P. 261, pl. XIV, fig. 4.

Lambrus affinis var. heraldicus Paulson, loc, cit., p. 8, pl. III, fig. 4 -4 $c$.

Obock (M. Jousseaume), une femelle : Djibouti (M. Coutière), un màle: Périm (11. Jousseaume), 8 màles el 3 fermelles.

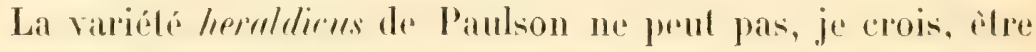
séparé de l'espere. Elle serait caraclérise surtout par le rostre denticuli, et par la portion anterieme de la carapace pourve de poils qui forment une toufle at la pointe du rostre: mais on lrouve fareilement tous les passigges dans une série un pen nombreuse d'individus. 


\section{Lambrus (Rhinolambrus montiger Nob).}

(I). XI, lig. 3 .

Nobili, loc. cit., p. 400.

\section{Djibouli (II. Coutiere), un mile.}

La carapace avee le rostre est i peine plus longue que large.

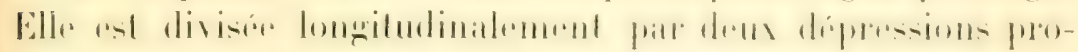

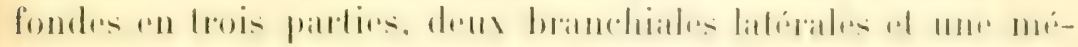

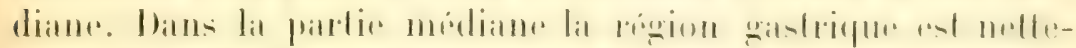

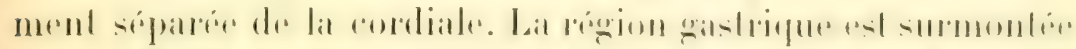

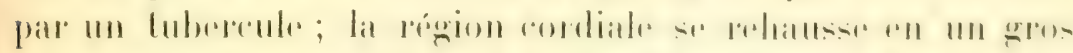

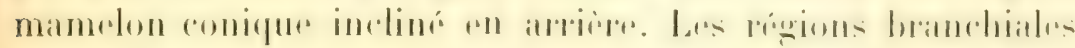

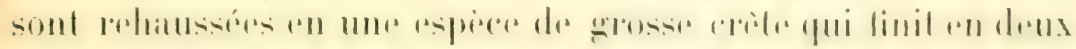

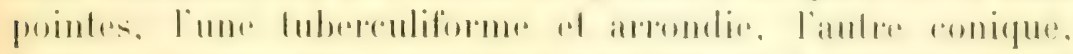

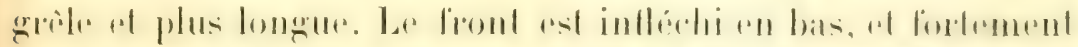

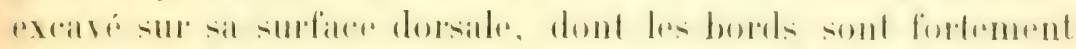

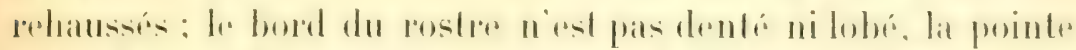

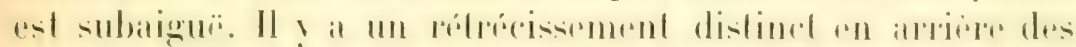

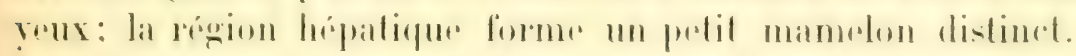

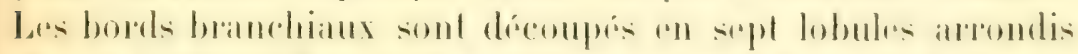

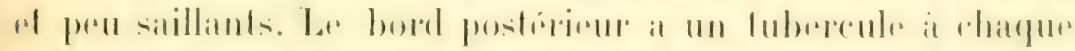

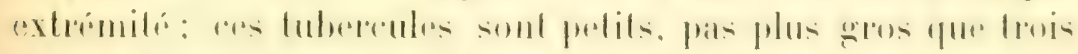

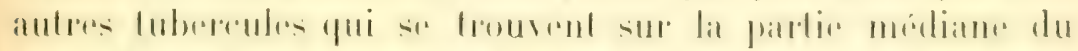

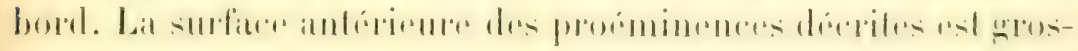

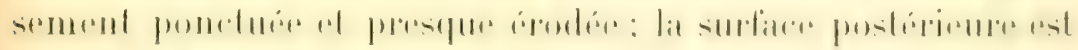
pourvare de gurelpues pelils luberecules.

Le sternum of liabdomen du miles sont irregulierement

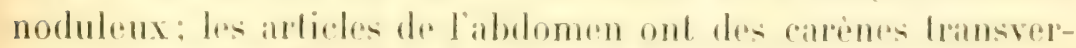

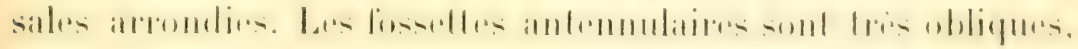
et commes divariguros par loinflechisioment du front. Les

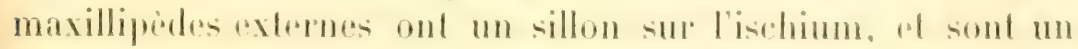
peug gramulents.

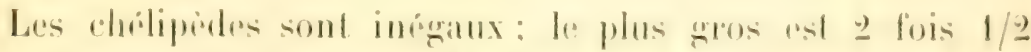

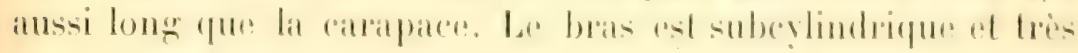

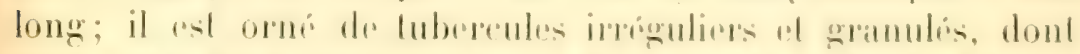

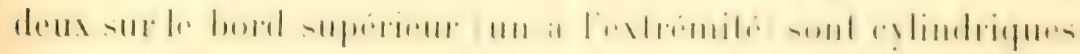

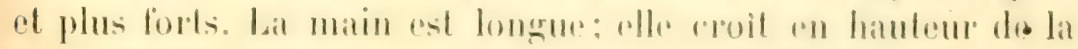

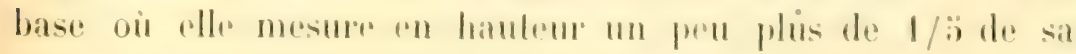


longuener a lanticulation digitale oil alle mesure "n hauleur 1/3 de sil longueur doigls compris. Le bord superiem ou

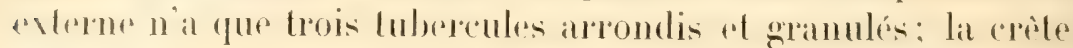
de la face suprepeme ou interne forme 3-'t lobes saillants et festonnés dans sa moilié distale, et offre ensuile une rangée der Lubercules granulés qui lendent a se transformer en lobes.

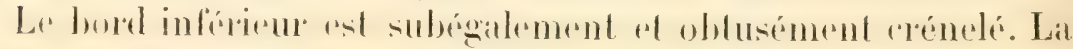

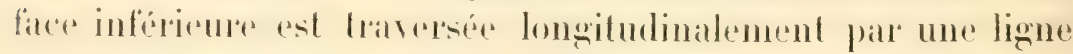
de potits luberenles granuleux. La surface dr tous les articles,

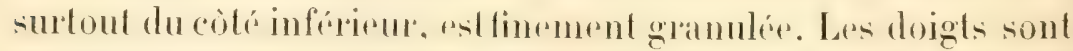

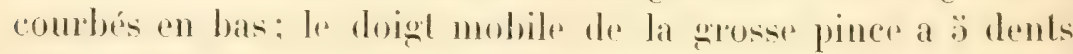

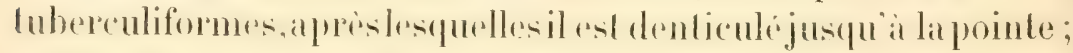
le doigt fixe a 3 dents : les doigh de la pelite pincesont armés plus faiblement.

Les mépopodites des palles ambulatoines ne soml pas dentés.

Longueur de la carapace............. 10 millim.

Largeur

$9.3-$

\section{Lambrus Pseudolambrus calappoides . W. Wh.}

Psendolambrus culappoides Paulson, loc. cit., P. 10, pl. III, lig. $3 a-b$. - Nobili, Bull. Scient. fr. Belgique, XL, 1906, P. 113.

Signalé dans la mere Rouge par l'aulson. Le nom Psendoleme

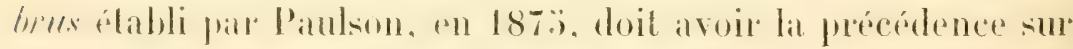
Parthenolambrus A. Milne-Edwark établi en 1878.

\section{GYGLOMETOPA}

\section{FINILLE PORTUNID E}

Genre POR'TUNUS Auct.

Portunus subcorrugatus A. Milne-Edwards.

A. Milne-Edwards, Arch. Mus., X, 1861, p. 402 , pl. XXXVI, fig. 2.

Mer Rouge (I. Jousseaume), un male.

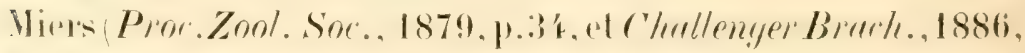

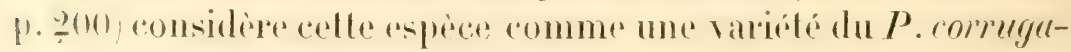

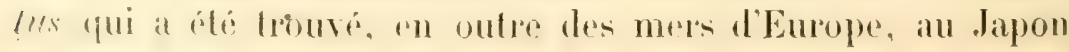




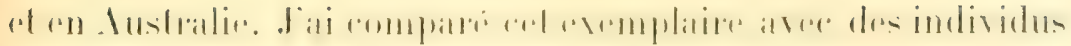

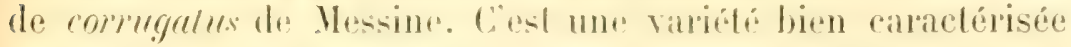

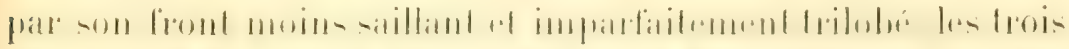

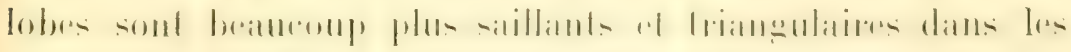
exemplaires de Messine of par les ligmes de la cartpace moins nombreuses mais phus saillantes.

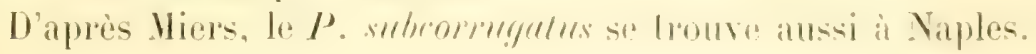

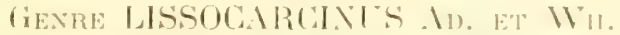

\section{Lissocarcinus orbicularis llanit.}

Dana, loc. cit., p. 288, pl. XYII, lig. 1. - A. Milne-Edwards, luc cit., p. \$18. - Richters, Decap. Maur., Seych., p. Lït. - Mier's, "llert" Crust., p. \$+1.

- Ortmann, Zool. Jahrt. Syst., III, 1893, p. 8\%. Alcock, J. As. Soc. Bengal, LIVIII, 1899, p. 20 (ubi syn.).- Borradaile, F. Gcoy. Maled. Laccad., 1, pt. 2, p. 200 .

Lissocarcinus pulihellus Müller, Verh. Nat. Ges. Busel, IIII, 1) 482, pl. V, fig. 6. - De Ilan, Ab. Senckenb. Ges., XXY, 1902, p. 6.1.

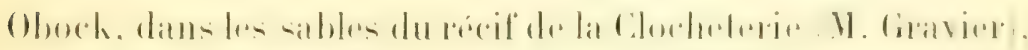
un mile large de 8 millimelres et long de $7^{\text {man }}, 3$.

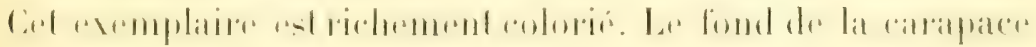
el des palles est blanchitre, avee de belles laches d'un brum

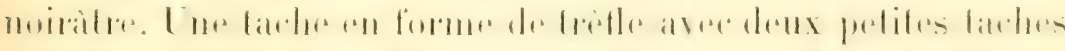
de colr, oceupe la parlie postérieme de la carapace. Sur la

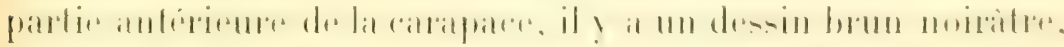

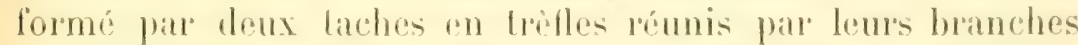
latérales. Une bitude de la mome couleur va de l'angle de l'or-

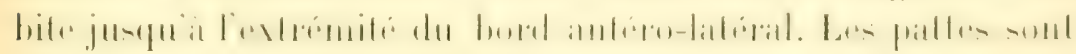
elégamment annelées de brun.

D'apees M. Borrataile, qui a observe ce clabe vivant aux iles Maldives, celle coloration est protertive et ressemble i

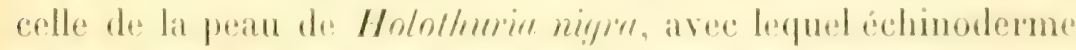
le crabu vil en symbiose ense cachant sous ou parmi les len-

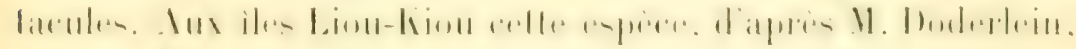
vil avee /lololhmrin "1/re.

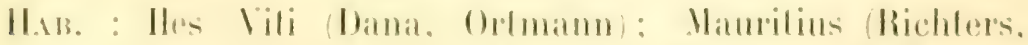

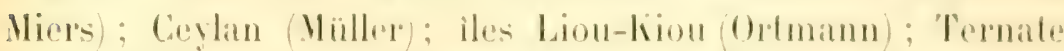

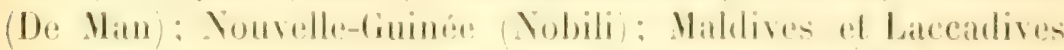

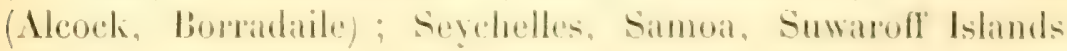
(Mier's). 
Genre Caphyra Guérin.

Dans mon itude Deropodi e Stommonpodi Eritrei del Wusen Zonlogirn dell I'nicersitu di Vupoli Annuario Mus. Zool. Tapoli, 1, n³ 3. 1901. 1. 11), jai donné un Lableau des espèces de Caphyro. Malheureusement, par suite de remaniements ypographiques faits apres la derniere revision des épreuves, une ligne a éte omise. Cielte ligne est d'importance capitale; sans elle mon tableau ne peut pas servir.

Le tableau doit donc être corrigé ainsi :

A. Zampe del quinto paio non arroresciate sul dorso del carapace.

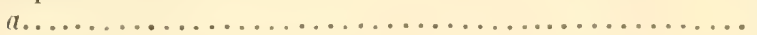

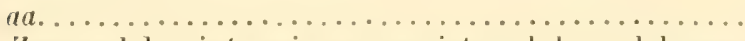

C. archeri Walk.

C. natutrix Zehnt.

A A. Zampe del quinto paio arrovesciate sul dorso del carapace.

$B$. Fronte, esclusi gli angoli, etc.

\section{Caphyra Monticellii Nob.}

Nobili, Ann. Hus. Napoli, 1, 1961, nº 3, p. 10.

Golfe de Tadjourah (II. Faurot), un mâle et une femelle.

Ces individus s'accordent avee ma description de la femelle typique du Musée de Naples, capturée parmi les plantes flottantes à 200 milles au nord de Massaouah. Les fines granulations qui caractérisent celte esperce sont bien visibles à la loupe, surtout dans lit partie antérieure du bouclier. Les deux lignes saillantes de la carapace, l’une sur la région gastrique, l'autre allant de la 4" dent d'un còte a celle de l'autre côté, sont bien marquées, mais plus dansle mile que dans la femelle.

La carapace de la femelle est bien convexe dans les deux directions, celle du màle beaucoup moins. Labdomen du mâle a les articles III-I soutés on une seule piece a bords convergents et un peu concaves. Le sixieme article est beaucoup plus large que long, ses bords latéraux sont combés. Le septieme article est triangulaire of it peu pris aussi long que large.

Longueur de la carapace.

Largeur
119 millim.

8,56 - 
Caphyra polita Heller.

Camptonyx politus Ileller, S. B. Akad. Wien., 43, 1861, 1, 3i9, 11. III, IV, lig. 26-32.

Caphyra polita 1. Milne-Edwards, Youv. Ireh. Mus., IX, 1873, 1\%.172. - Paulson, loc. cit., P. 151. - Yobili, loc. cit., p.11, 12.

Je n'ai pas vu d'exemplaires de cette espèce, signalée dans la mer Rouge par Ileller el l'aulson.

\section{Genre CilRUil Dish.}

\section{Carupa Iæviuscula Heller.}

Heller, Crust. Novara, p. 27, pL. III, fig. 2. - De Man, Not. Leyd. Mus., V, 1883, p. 1:2, et Arch. f. Wat., 1887, 1. 336. - Urtmann, Zool. Jehrb. Syst., VII, 1893, p. 68. - Alcock, loc. cit., p. 26 (ubi syn.).

Mer Rouge ot Obock (I. Jousseaume), 52 exemplaires;

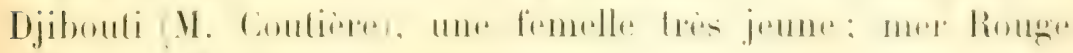
(Musée de Turin), \& individus.

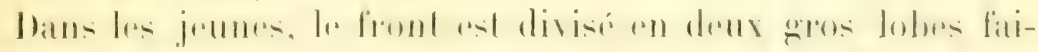

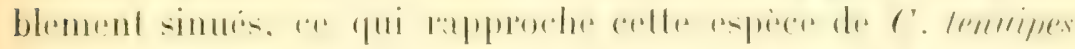

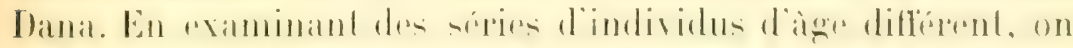

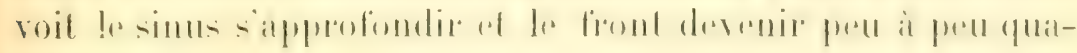
drilobé.

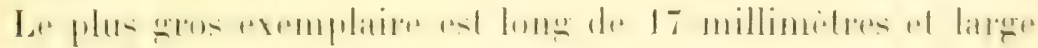
de 24 millimelres.

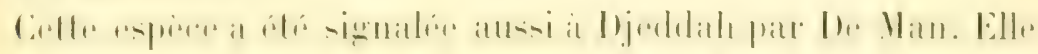

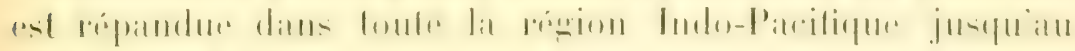
Japon et à '́ahili.

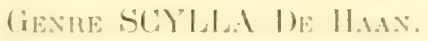

Scylla serrata forsh.

Cf. Aloock, loc. cil., p. $2 \pi$ (ubi syn.).

Mer Rouge (Husée de Turin), une prosite femelle: Assab (Musée de 'Turin), g gros miles.

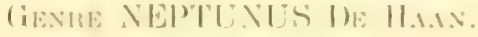

A. Mérognathe plus ou moins élargi ì son extremité antéto-externe. Camapace peu convexe. 
B. Derniere épine du bord latéro-antérieur beaucoup plus longue que les autres.

C. Angles postérieurs de la carapace arrondis. (Angle latéro-externe du mérognathe arrondi.) (Neptunus.)

c. Pas d'épines à l'extrémité postérieure du

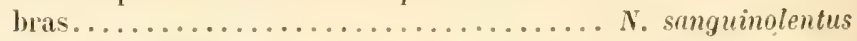
ce. Une épine à l'extrémité du bord posté- Herbs.

rieur du bras...................... pelagicus Linn.

CC. Angles postérieurs de la carapace carrés ou spiniformes (Hellenus).

b. Angles postérieurs carrés; carapace avec des groupes de granulations bien netles sur des saillies; front découpé en quatre dents, dont les deux médianes plus courtes que les

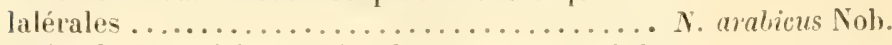

DD. Angles postérieurs de la carapace spiniformes.

d. Front tridenté, ì dents à peines marquées; deux épines sur le bord postérieur du bras, deux sur la main. Carapace très bosselée et granuleuse. Bords latéraux avec neuf dents. N. Alcocki Nob.

dd. Front f-denté, les dents médianes plus petites; une épine à l'extrémilé postérieure du hras, trois sur la main. Bords latéraux

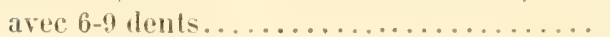

$B B$. Derniere épine des bords latéraux non ou un peu plus grosse que les autres Achelous).

(Derniere épine à peine plus grosse que les autres.

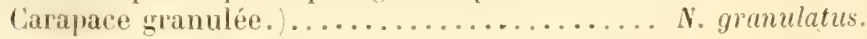

A. Mérognathe presque caré. Carapace convexe Pon-

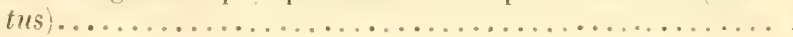

X. longispinosus Dana.

N. convexus (De Haan).

\section{Neptunus (Neptunus) pelagicus (Linn.).}

Cf. Alcock, loc. cit., p. 31, 3女 (ubi syn.).

Yombrems remplateres de la mor Rougre Mus. Turin : Mas-

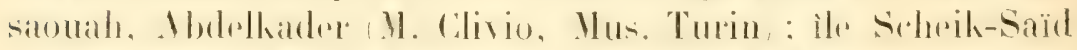

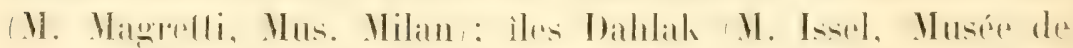

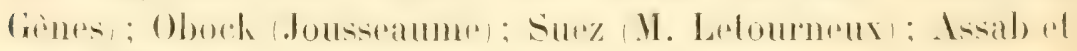
Aden (M. Ragazzi, Musée de Modène).

\section{Neptunus Neptunus) sanguinolentus (l,inn.).}

Cif. Alcock, loc. cit., p. 31-32. - Nobili, Ann. Itus. Napoli, I, 1901, nº 3, p. 9. Erythrée (Mus. Naples), 7 exemplaires jeunes. 
port a la rigion cordiale qui est saillante, soit par rapport au front qui est rehaussé. La carapace est couverte sur les parties saillantes de pelites gramulations, qui drviennent plus nombreuses ot plus grosses sur les bosselures des parties postérieures. De la grosse apine latérale de chaque coté part une grosse crète saillante et granulér, qui vient aboulir, après une courbe, près de la région cordiale. La région gastrique est obscurément partagér. La région cordiale et l'introstinale, qui ne sont pas séparées entre elles, sont divisées nettement en cing lobes tres saillants el granuleux, dont les deux anterieurs sont placés sur la mème ligne, et les trois autres sont disposés en arriere presque en demi-cercle. Ieux bourelets saillants et granulés s'itendent paralliement à ces régions de chaque còté, en commencant d'un point plací un peu en arriere de l'extrémité de la crète de l'épino latérale. ot śótendant jusqu’ì l'exfrémité postérieure de la carapace. a la base des angles latéropostérieurs. Les régions branchiales sont déprimées dans leur partie antérieure: dans la partie postérieme, en arrière de la crete de l'épine, elles sont dielives. Dans le large sillon qui sépare la région gastrifure de la région branchiale de chayue còté, il y a un petil lobule granulé.

Les angles postérieurs de la ratapace sont très aigus, spiniformes et bien saillants.

Les dents frontales sont en nombre de trois seulement, triangulaires, obluses et pen saillantes; celui du milien est légèrement plus court que lus deux lateraux. L'angle orbitaire interne est plus bas que les dents frontales. Les ortbites sont entièrement dorsales.

Les bords latéro-anterieurs forment une courbe a court rayou; ils sont armés de 9 dents alternativement grandes et petites; l'épine est courbée 'n arant, et plus de trois fois aussi longue que la huitième dent.

Les chélipèdes sont granulés. Le mérus offre deux épines à l'extrémite de son bord posterienr; sur son bord antérieur, il offre trois épines sur l'une des paftes el trois suivies d'une quatrieme plus petite sur l'aulre palle. Le carpe est gramuleux et orné de crètes; il a une épine assez courte sur le bord interne et trois sur sa face externe. La main est ornée de 


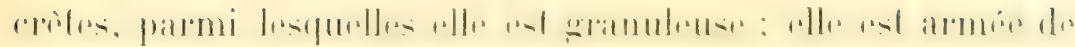

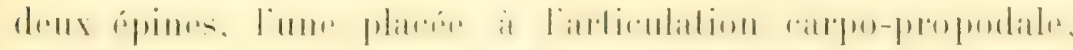
l'autre at l'arliculation proporlo-digitale.

Les pattes de la cinquieme paire n’ont pas d'épine sur le méropodile. Le dernier articte ast ovale, non rétréci vers la pointe.

Le sternum est finement gramuleux. Liabelomen du màle est triangulaire, étroil, arec le deuxieme el le troisieme article carénés, et avec les bords des articles suivants sinuenx.

L'unique exemplaire mesure:

Longueur de la carapace.............. $6 \frac{3}{3}$ millim.

Largeur de la carapace sous les épines.... $10^{\circ} \quad-$

\section{Neptunus (Achelous) granulatus (EW.).}

Lupea granulatu II. Milne-Edwards, H. n.Cr., I, p. fist.

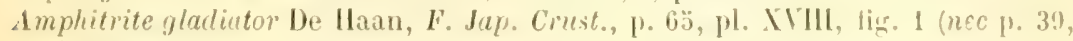
1). I, lig. 9).

Amphitrite specinsa Dana, loc. cit., p. 276, pl. XYII, fig. 1.

Achelous granulatus A. Milne-Elwards, loc. cit., p. 3ik.- Paulson, loc. cit., p. 38.

Neptumus (Achelous) grimulatus Miers, Challeng. Brach., p. 180. - Mcock, loc. cit., p. 32; tö (ulis syn.).

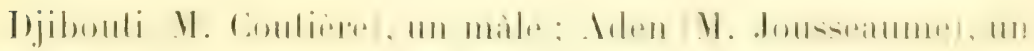
mâle.

Longueur de la carapace........ 13,;

Largeur $\quad-\quad \ldots \ldots \ldots$ 10,3 8 -

Signalé aussi a Builoul (Cino).

Neptunus (Pontus) convexus De llan.

Portunus (Pontus) concexus De IIan, loc, cil., I. !).

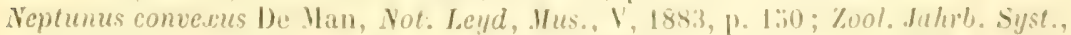

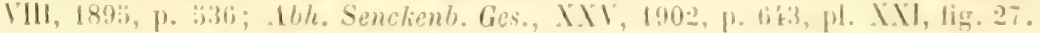
- Nobili, Am. Mus. Sapoli, 1, no 3, 1901, 1).9.

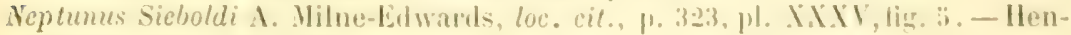
derson, loc. cil., 11.370.

Mer Rouge (D). Ragazzi, Musios de Modine), une lemelle large de 61 millimitres of longue de 3' millimitres.

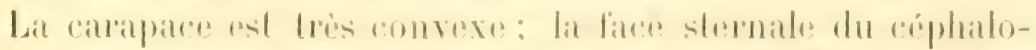

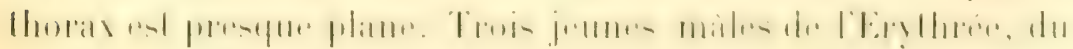
ANX. SC. NAT" zOOL. 
Muser dre Naples. dont jai parte ailleurs, blaient eonvexes sur la face stronale. Les crites des mains sont bien saillantes dans cette femelle; les parties entre les creles sont remplies de longs poils jaunitres, qui sont ausi abondants sur le rarpes sur le bras et sur la carapace.

Labdomen de la femelle ovigere est largement hiangulaire.

Haz. : Érthrén Vobili : Mauritius 1. Milne-Edwards: Ceylan Henderson); Mjeh, Malmahera. Termate (De Man); Moluques (De Haan).

Gente Chat B BDis De HaAn.

(Goniosoma A. Jilne-Edwards.)

A. Bords latéraux de la carapace armés de sept dents, dont la deuxième et la quatrième sont rudimentaires.......Ch. erythroductyla (Lam.).

AA. Bords latéraux de la carapace armés de six dents distinctes jusqu'à la base.

$B$. Sixième dent de la carapace égale aux autres. Plus que deux épines sur la main.

C. La première dent aiguë, la deuxième plus petite que les autres.

D. Carapace poilue avec une ligne transversale sur la région cordiale et une sur la région branchiale en arrière de la dernière dent......................... C. orientalis Dana.

DD). Carapace glabre, sans lignes transversales en arrière de la dernière dent............ C. Helleri A. Edw.

CC. La première dent aiguë, la deuxième égale aux autres. Une épine sur le bor'd postérieur du carpe de la cinquième patte............. C. merguiensis De Man.

CCC. La première dent tronquée (Main tuberculée, des lignes saillantes en arriere de la dernière

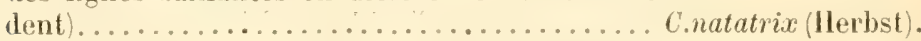

$B B$. La sixième dent plus longue que les autres. Deux

épines sur la main..................... Ch. anisodon De llaan.

AA A. La deuxième dent de la carapace presque entièrement fusionnée ạvec la première................. heterodon Nob.

Charybdis (Goniosoma) erythrodactyla (Lilli. .

Thalamita erythrodactyla II. Milne-Ewards, H. n. Cr., l, p. 46 .

Thalamira Teschoirei H. Milne-Edwards, Ann. Sc. Nat. (3), XYI, p. 200, tim. $5,6,7$.

Goniosoma erythrodactylum A. Milne-Edwards, Arch. Ifus., X, 1861, p. 369.- 


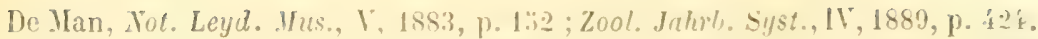
— Ortmann, Zool. Jukrb.Syst., VII, 1893. p. 81. - Ilenderson, loc. cit., p.37:i. - Tobili, Bull. scient. Fr. Bely., XL. 1906, p. 118, lig. 3.

Mer Rouge (Musée du Turin, a males at femelles.

Ces exemplaires different du gros spécimen de Youkahiva

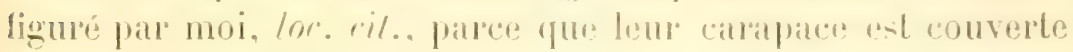
de poils couts, er qui n'es, probablement. quin caractere de jeunesse, parce quils sont beatcomp phus pelits. Les lignes lransversales sur la carapace sonl ansi beaucoup plus saillantes. Les dents trontales sont plus larges, plus rapprochées et honquées en arant, arec une disposition qui ressemble it celle de Thalomiln I)anis.

Le Lubercule sur le premier article du pedoncule des antennes externes est bien marequé. el dans quelques axemplates, il ast double el suivi par quelques gramulabions.

Har.: Djeddah (De Man): Ceylan (Itenderson); Maurilius (Adams et White): Célebes Thallwitz): Molupues Herklobs, A. Milne-Ledwards); Tahili De Man); iles Harquises 1. MilneEdwards, Ortmann): Honohulu (Lenz).

\section{Charybdis (Goniosoma) orientalis Dalla.}

Cr. Aleock, loc. cit., p. 63.

Une femelle de Oboek (II. dousseanmes.

Charybdis Goniosoma) Hellerii 1. M.-Ell:.

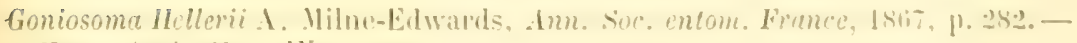
Nouv. Ark, Mus., IX, p. 1tiz.

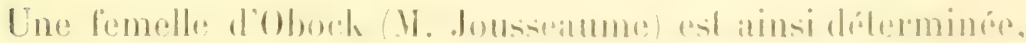

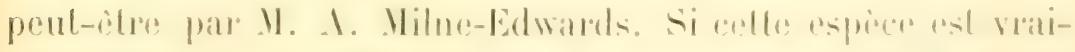

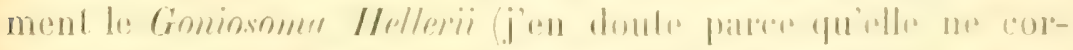

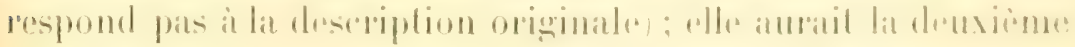

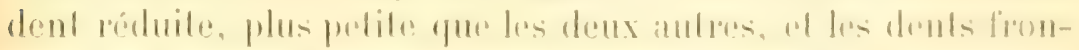

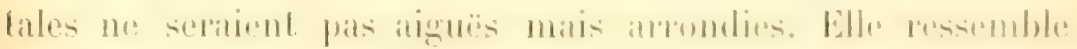

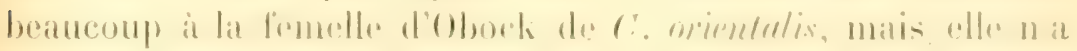

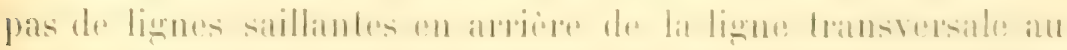

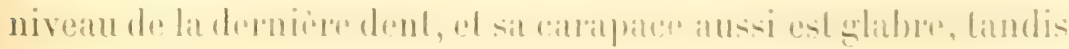


qu'elle ast poilue dans la femelle de nrientalix. Si cet exemplaire est rraiment le $G$. Hellerii, alor's $G$. mergniense ne peut aucumement itre identique aver Helleri, ansi que le soupconnait De Man, parce qu'il n'y a, dans l'exemplaire, aucune trace de l'épine sur lo carpe des pattes posterieures qui caractérise le merguiensis.

\section{Charybdis (Goniosoma) merguiensis the Man.}

Goniosoma merguiense De Ilan, J. Linn. Soc., XXIl, 1887-1888, p. 82, pl. V, fig. 3-4; Zool. Jahrb. Syst., 1895, VIII, p. 560.

Charybdis (Goniosoma) meryuiensis Alcock, loc. cit., p. ii.. - Nobili, Inn. Mus. civ. St. Nat. Genova, XL, p. 254 (1899) et 498 (1900); Bull. Mus. Torino, XVIII, $\mathrm{n}^{\circ} 455$, p. 31 .

Goniosoma Hellerii Henderson, loc. cit., p. 373.

Obock (II. Jousseaume), un màle large de 63 millimedres environ. Cet exemplaire est très poilu.

Charybdis (Goniosoma) sexdentata (Herl,st).

Thalamita sexdentata Rüppell, loc. cit., p. 4.

Celle espece a élé signalóe par hïppell dans la mer Rouge. Je n'en ai pas vu d'exemplaires.

Charybdis (Goniosoma) variegata (Fal..;.

Cano, loc. cit., p. 219.

Signalé à Assab par Cano.

Charybdis (Goniosoma) natatrix (Herbst).

Cf. Alcock, loc. cit., p. 61 .

Mer Rouge (M. Jousseaume), une femelle très jeune.

Charybdis (Goniosoma) heterodon Nob.

(Pl. VIII, fig. 4.)

Nubili, loc. cil., p. 401.

Colle espece, dont M. Jousicanme pecueillit une femelle it Obock, est caracterise par la deuxieme dent du bord latéro- 


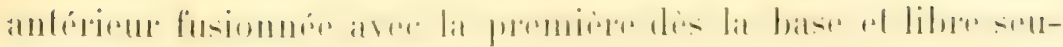
lement it la pointe, el par la forme du front.

La carapace est aplatie; sa longueur est les $2 / 3$ de sia largeur. La surface est glabre; finement poncture, ef fablement gratnulée près des dents. Les lignes granulenses en avant de la dernière sont normales et fables; il n'y a pas de lignes en arriere sur la région cordiale el sur los branchiales. Le front est divisé en 6 dents (exclus les anghles onbitanes internes) :

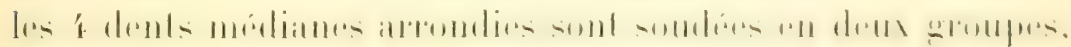
séparés entre eux par une fissure étroile: les dents externes sont un peu plus courtes ef triangulates. Le bord orbitaire supérieur n’a qu'une seule fissure: l'autre (l'externe) est close. La premiere dent est forte, el sur son bord externe, près de sa pointe est entaillée une pointe qui est celle de la deuxieme dent, entièrement soudée arec la premiere; cette espece na ainsi que ö dents libres de charque cote. La dent

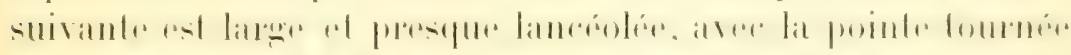

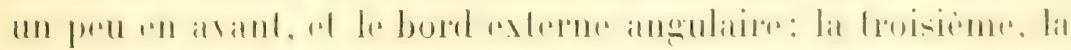

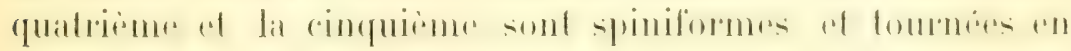
avant; la quatrieme est plus grèle que la troisieme et la cinquieme.

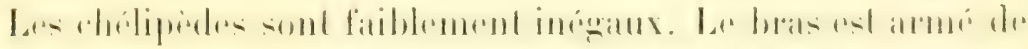

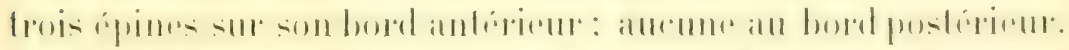

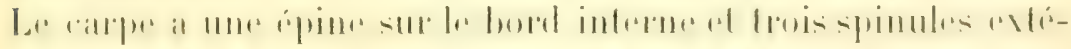

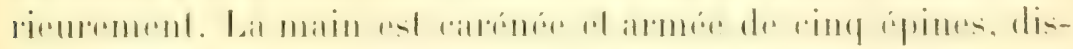
posées comme d'habilude: les creites sont lies fablement granuleuses, matis la surface entre les cretes est lisse ou presplue.

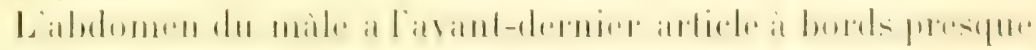

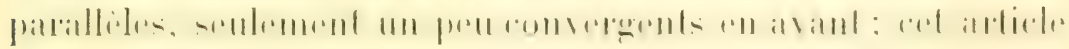
est atssi long que larere.

Les palles noul reen dre remarquable. Le mérus de la cinquieme pare n'est pats sillomne : il ast armé en dessous de l'épine habiluelle; les propodite est denticulé en dessolls.

Latgerur de la carapare. 27,3 millim.

Longuedur 18,0 


\section{Charybdis (Goniosoma) anisodon lle Haill.}

De Ilaan, F. Jap. Crust., p. 42.

Goniosoma anisodon A. Milne-Edwards, loc. cit., p. 381, pI. XXX, fig. \& ; Nouv. Arch. Mus., 1X, 1873, p. 167.

Mer Rouge (II. Jousseaume), un jeune mâle.

Har, : Oeian Indien (De Hatu): Chine (1. Milne-Edwards), Java (Hewlots, 1. Vilne-kdwards, De Han): Singapore Aleock, Tobilis: Philippines(Ortmann): Vourelle-Cálédonie (1. MilneEdwards?.

\section{Gexre ARCHIiS Paulson.}

Ce genre est ainsi caracterise dar l'aukon : Céphalothorax aplati, mais arec les principales regions convexes. Bord frontal anmé de 6 dents, les angles slss-orbitaires internes non compris. Bord antéreur presque droit: bords lateraus armés de 6 dents ! compris l’angle orbilate externes. dont lat dernioper est deux fois aussi longure que lavant-derniere et se prolonge en épine horizontale. l'ar ses dents frontales ce genre s'approche de Gomioxmmon. mais il s'en bloigme par ses antennes externes dont le deuxieme andicle est placer dans lorbite. Lus antenines internes sont obliques. Lindostome a des crètes. Les deux dernices articles des palles de la cinquième paire sont natatoires. Le sillon médian du sternum oecupe les trois derniers segments.

Ce gente ne me parait pas different du genpe Gonioneptumes Ortmann, 1893, que Alock considere justement comme un sous-genre de Charybdis.

\section{Archias sexdentatus Pauls.}

Paulson, loc. cit., p. 56, pl. VIll, fig. 3-3 b.

Obock (M. Jousseaume), un màle.

Cette rare espece n’a plus été retrourée depuis 1873. En 1902 Miss M. Rathbun derepivit un Arrhes sp. des iles Maldives.

La carapace de celte espero est tres ped convexe, les régions y sont bien délimitées el bosselées, aree les parties plus saillantes a les lignes transversales convertes de granulations arrondies. La surface de la carapace est poilue. 
Le front est divise en six lobes, dont les quatre mitoyens

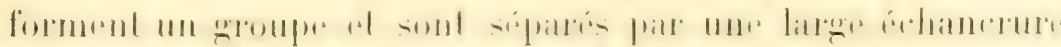

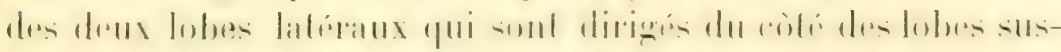

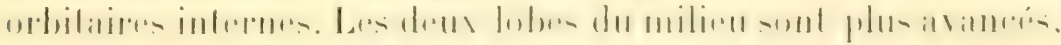

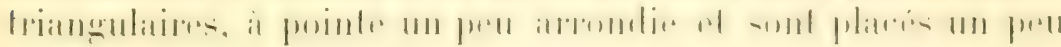
plus bas que les deux lobes voisins, qui sont peu avancés,

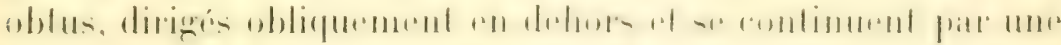

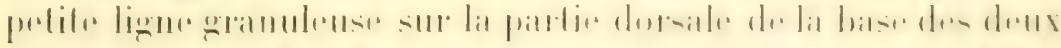

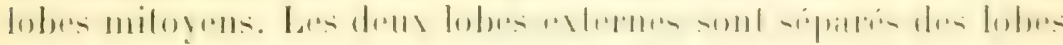

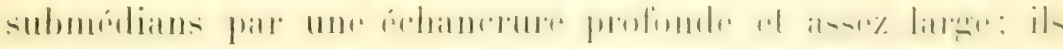

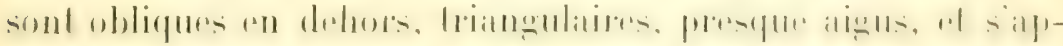

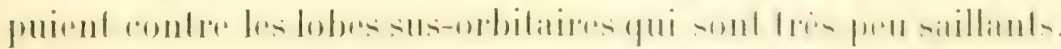

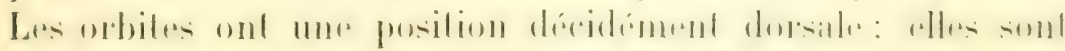

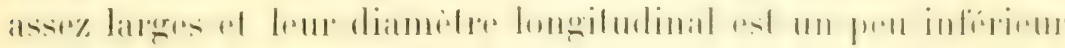
an diametre transversal.

Le bord superiem a deux fissures élroites; le bord inferrem a une échancrure large ef profonde: lo lobe sous-orbitare interne est arrondi et presque nul.

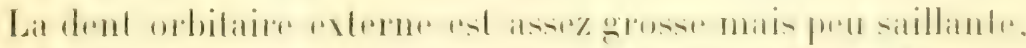

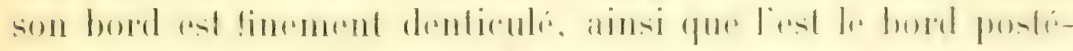

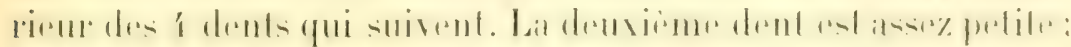

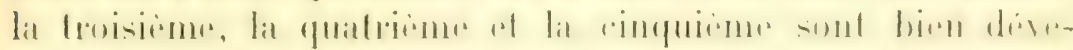

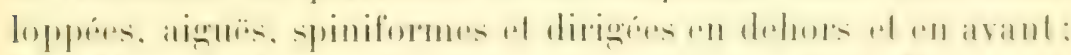

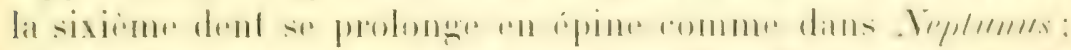

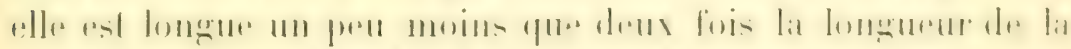

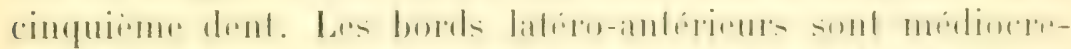

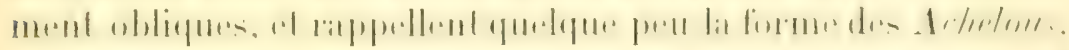

Le bord postérienre de la carapace ast phos large que de bord

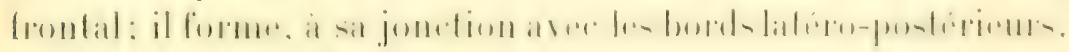
un angle presque droit, a pointe un peen arrondie. Les régions de la cartupace sont bien marguies: leurs sommets sont gratnuleux.

Lat région coldiale as nellement division an dens bobules. Il y a tune ligne transversile granuleuse lit granules anomdis,

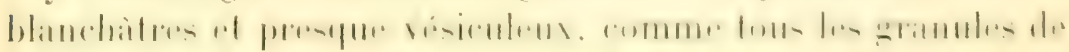

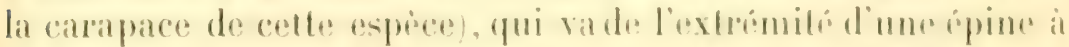

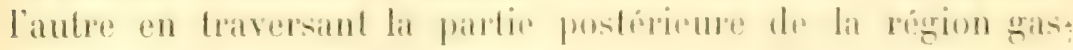
trique; ectle ligne est intertompur pare le sillon gastro-bran- 
chial. En arant de cefte ligne, sur le milieu de la région gastrique, il y a ume aubre ligne lransirrsale granulée, et, en avant de colle-ci, deux anteres pelites lignes. Les deux lobules de la région corliale ont aussi une ligne stillante, on rangée linéaire de gramulations; deux autres amas symétriques s'observent de chaque côté sur les régions branchiales.

Le mérognathe se prolonge en un lobe it son angle antéroexterne.

L'article basilaire des antemnes a une pelite crete faiblement granuleuse. Il entre dans lorbite el envoie 1 m prolongement vers le lobe sus-orbitaire interne; il l'atleint mais ne se fusionne pas avec; le fouct est placé entre ce lobe et le front, dans l'espace vide at an-dessous de la division entre le lobe externe du front et le lobe sus-orbitaire interne.

Le sternum est finement granuleux et ponctué.

Les chélipides sont a peine plus longs que la longurur de la carapace arec les epines. Le bras est finement granuleux sur sa surface interne et sa surface posterieure; ces granules deviennent squamiformes sur sal surface superieme, ainsi que sur le carpe. Le bord anterieur du bras est armé de trois épines, dont la premiere ou proximale est petite et les deux autres grosses et égales; le bord posterieur a une épine, le bord inférieur une épine aussi à l'extrémité. Le carpe a quatre épines; une du coté interne, longue el accire, atteignant presque la moilié de la paume, at trois du coté "xterne, avec de petites crites sillantes : la surface est granulée et poilue. La main est longute, comprimúe, of pas plus élroite que le bras. La paume est un peu plus courte que les doigts. Elle est armée de quatre eppines; sa surface est parcourue par cind cretes a granules squamiformes; l'espace entre les cretes est gramuleux et poilu. La face interne de la main a aussi deux cretes granuleuses: l'une au milieu, l'aulre prés du bord inférieur. Les doigts sont allongés, sillomnés, i pointe aigü; le doigt mobile a, a la base, une girosice dent lobiforme tommée en arrière.

Les pattes des deuxieme, troisieme dquatrieme pares sont grobles et allongées. Celles de la deuxième parre alleignent presque l'exlrémité des chélipedes. Les pathes de la cinquième 
paime ont une ípine est sillonné. Le doigt est ovale at allongé.

L'abdomen du nale es carené sur le deuxieme of le troi-

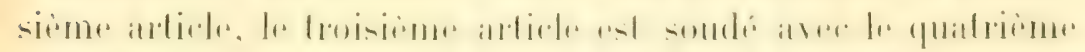

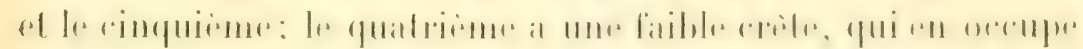

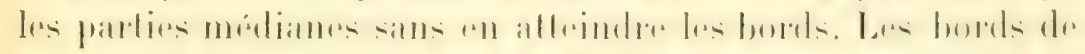

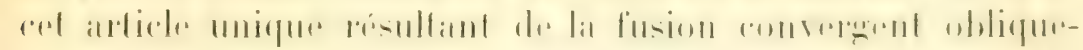
ment en avant, puis sont paralleles pour un certain trait. Le

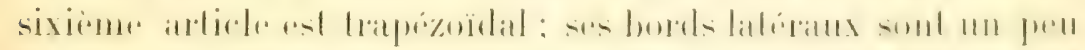

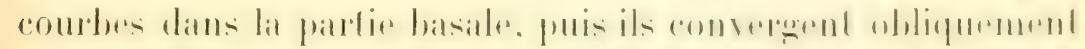

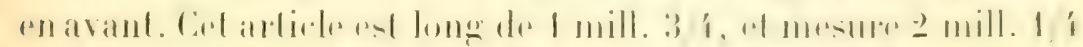

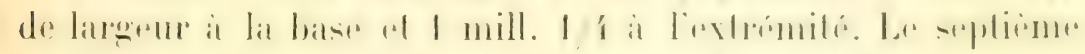

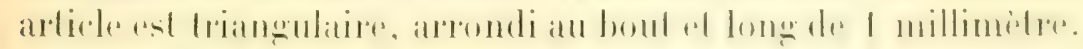

Largeur de la carapace avec les épines........ 16 millim.

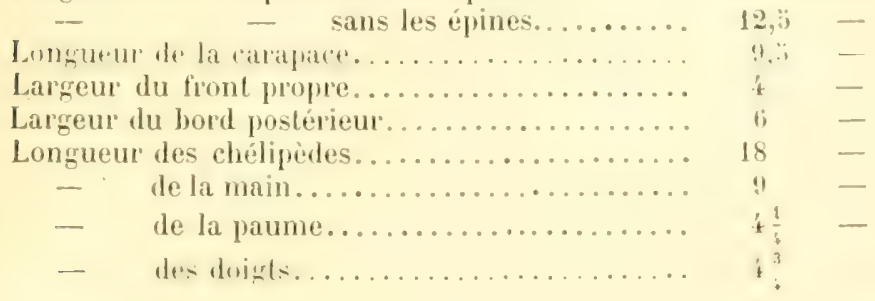

\section{GeNRE THALAMITA LAT.}

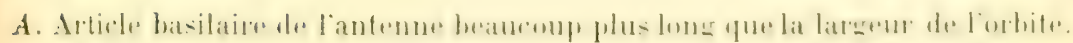

B. Front découpé en six lobes de largeur presque égale. (Lobes orbitaires internes exclus.

C. Quatrieme dent des bords latéro-anterieurs ígale ou presque égale atus autres.

D. Article basilaire des antennes porlant une créte granulée.

d. Rides transversales de la carapace peu saillantes. Mains it crètes fitibles....... Th. crenata Lat.

dd. Rides transversales de lit carapace trés fortes. Mains it mites rohustes........ Th. Danx Stm.

CC. Coualrieme dent plus petile que les autres.

e. Cirete de l'article basilaire des antenmes

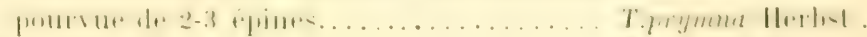

ee. Crite lisse..................... T. picta stm.

cee. Cicte tranulée................. T. Stimpson 1. I.lisw.

BB. Front découpé en deux lobes. (Angles orbilaires exclus.)

E. Lobes sus-orbitaires internes arpués, beancoup plus étroits que les lobes frontaux. 
$\boldsymbol{F}$. Lobes frontaux bien séparés; surface externe de la main avec des tubercules squamiformes et des crêtes............Th. sima Edw.

$F F$. Lobes frontaux non ou peu séparés; main presque lisse ou granulée seulement sur sa partie supérieure.

f. Dents latérales aiguës; la quatrième beaucoup plus petite, la cinquième plus proéminente............. T. Poissonii (Aud.).

ff. Les quatre premières dents latérales

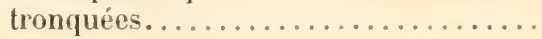

$E E$. Lobes sus-orbitaires internes droits ou presT. Chaptalii(Aud.). que; presque aussi larges que les lobes frontaux.

H. Crête de l'article basilaire de l'antenne lisse; quatrième dent rudimentaire...... T. integra Dana. HH. Crête de l'article basilaire de l'antenne granuleuse ou épineuse.

$h$. Crête granulée ou dentée.

Th. admeta var. Savignyi A. M.-Edw. hh. Crète pourvue de trois épines...... Th. admet $u$ var. in-

AA. Article basilaire de l'antenne égal à la largeur de l'ortermedia Borr. bite ou plus court.

G. Front quadrilobé. Une ligne saillante sur la région cordiale. Bords latéraux avec quatre dents sub-

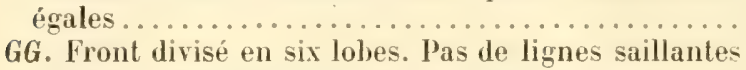
Th. De Mari Nob. sur la région coldiale. Quatre dents sur les bords latéraux, dont la troisième et la quatrième beaucoup

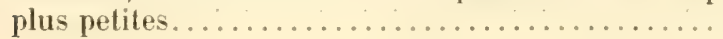

Th. bandusia Nob.

Thalamita crenata (Lat.).

H. Milne-Edwards, H. n. Cr., 1, p.461. - Rüppell, loc. cit., p. 6, pl. 1, fig. 2. - A. Milne-Edwards, loc. cit., p. 363. - Alcock, loc. cit., p. 73, 76 (ubi syn.).

Suez (II. Jousseaume), 4 màles, 3 femelles; mer Rougt (M. Jousseaumet. I mâle, 2 femelles; Obock (I. Jousseaume), I femelle; Massaouah (M. Tellini, Musée Turin), 3 mâles; Massaouah, Abdelkader (M. Clivio, Musée Turin), 2 màles, 1 femelle; Massaouah (M. Parenti, Muséc Modene), 1 màle; Massaouah (MM. Isiel el Beccari, Musée Gènes), 2 màles; Assab (M. Ragazzi, Musée Modene), I màle; Moka (M. Ragazzi. Musée Modène), 1 mâle.

Les jeunes sont, en génriral. un peu poilus, au moins dans les parties antérieures de la carapace, el les rides lransversales sont plus saillantes. 
saouah Tollini), pulite, mais bion developpée dans les deux femelles de Massaouah du Musée de Gènes.

La crete de l'article basilaire des antennes a 3-' épines libres dans les fomelles de Nassaonah et dans un male de mer houge ; 2 à gatuche ef 3 à droite dans la femelle de Oboch, et 3 ćpines phus ou moins soudées par la base dans la femelle de Matsiaouah (M. Tellini) et dans l'autre mâle de " mer Rouge ".

II. De Han a observi dans ses exemplaites de Jjoddah que les jeumes, moins larges que io millimiders, manquent de la crète granulense entre la crèle inférieme ol les épines de la main, qui apparait ensuite gradueflement e que l'on observe dans les individus larges de 6:3 millimedres. Plus tard, le meme auteur, en observant la présence de cefte creble dans les pxemplaires de l'océn Indien, conchait: The Herymi sperimens and those from the Red Sea are either carielies of one species or they belomy to different speries. In the laller rase I monpose to distinguish the Red Sea sperimems ax Th. pirlle, ele. Plus tard (\%ool. Jahrb., 189\%), il soulient encore la mime opinion, que les individus de la mer Rouge sont une variété distincte ou une nourelle espèce. Jo doute qu'on puisse ériger des différences spéciliques sur lo fait quin crolain caractere soit acquis un peu plus tòt ou un peu plus tard dans le développement par les exemplaires d'une localite, si les adultes arrivent tous a l'avoir. En tout cas, mes exumplaires n'autorisent pas cette séparation el prouvent que le caractire en question est seulement tres variable. Lusexemplaires de " mor Rouge ". Obock of Massaonah (I. Tellini) ont la crete bien développée, bien que l'exemptaire de Obock el l'un des miles de mer liouge n’aient que 29 et 2 ' millimelres de largeur. Les deux femelles de Massaouah du Musée de Gènes offrent a ce propos des différences entre elles. La femelle de 3il millimètres de largeur n’a qu'une trace de la crite sur la pince droite: sur la pince gauche, elle est indiquere par une rangive do granulations, tandis que l'autre femelle a la crite parfaitement formée sur les deux mains.

Le méropodite des patles de la cinquieme paire peut ètre sillomé ou lixse. Il est sillonmé dans l'une des femelles de Massionah, lisse dans l'autre. Il est lisse aussi dans la femelle 
de Obock el dans un mâle de " mer Rouge "; les sillons sont

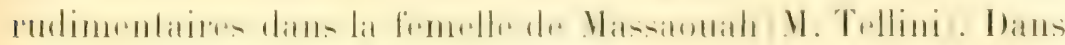

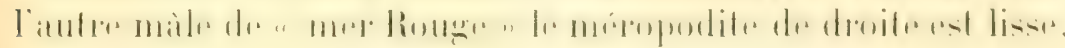
celui de ganche faiblement sillonné.

\section{Thalamita picta Stimpson.}

Stimpson, Proc. Icad. N. Sc. Philad., 18:38, [1. 39. - A. Milne-Edwards, loc.

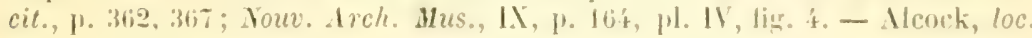
cit., p. 73,79 (ubi syn.).

Her Rouge (I. Jousseaume), une femelle.

Thalamita Stimpsoni 1. M.-Elw.

A. Milne-Edwards, loc.cit., p. 362, pl. XXXY, tig. 4. - Alcock, loc. cil., p. 79 (ubi syn.).

Suez (I. Jousseame), 3 exemplaires.

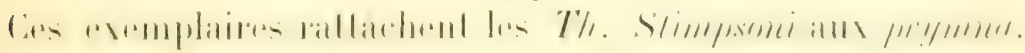
L'article basilaire de l'antenne offre en plus de la ligne de

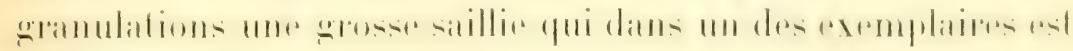
obtusément bidentée.

\section{Thalamita Chaptalii (Iud.).}

Portumes Chrotalii Audouin, Expl., P. 8:3. - Savigny, pl. II. fis. 1.

Thalamila Chaptulii H. Milne-Ldwards, H. H. CH., I, p. tho. - A. MilneEdwards, loc. cit., p. 360. - Cano, loc cit., p. 216. - Ncock, loc. cit., 1. 74,80 .

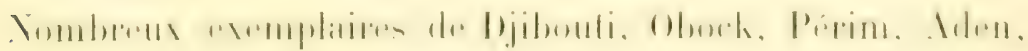
tous recueillis par $\mathrm{H}$. Jousseame.

Les quatre premieres dents sont toujours plus ou moins tronquées latératement; lat quatrieme ast un peu plus petite que les autres; la cinquirme est aiguë, non lronquée comme dans la ligure de Savigny.

\section{Thalamita Poissonil ( 1111 .).}

Portunus Poissonii Audouin, Exppl., p. 8i. - Savigny, ph. 11, lig. 3 (et:5?).

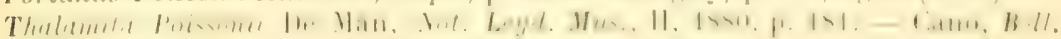

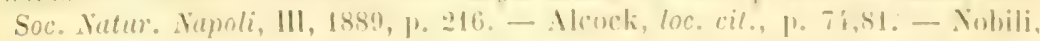

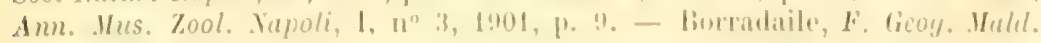
Luccul., 1, 1.:201. 


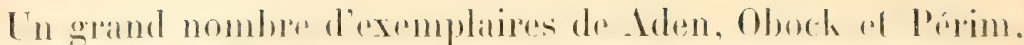

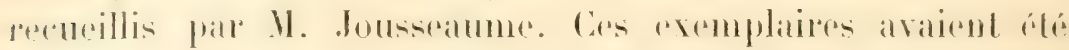
debemines dans les collections du Vuséum Th. ximm. Il n's a aurun doute que ces individus appartiennent at la forme ligurae

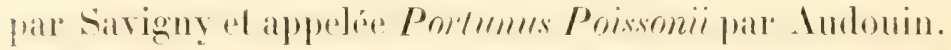

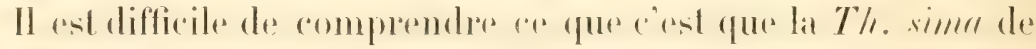
H. Vilne-Edwards. Cet andere ne domme ancume indicalion sur les caractères des mains, et sa description pourrait aussi sadapler a P. Poisonnii. Lat Th. simme de II. 1. Milne-Eolwards

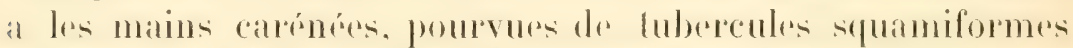

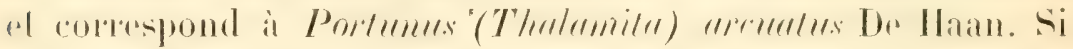

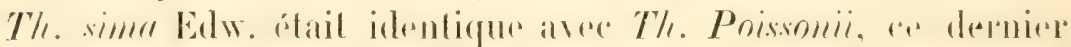
nom devrait avoir la précédence; et Th. sima A. H.-Edw. devrait s'appeler $T h$. arcuatu de Ilaan.

Les denticules du propodite de la ringuibume paire de palles ne sont pas un caractere distinctif entre celte espèce et

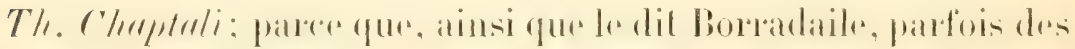

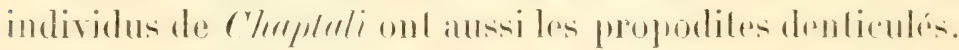

Aussi à Assab (Cano).

Thalamita sima (A. Milne-Erlwards).

A. Hilne-Edwards, loc. cit., p. 339. - Alcock, loc. cit., p. 7t, 81.

Lo jente exemplaire de delen dousseatume, parail appare-

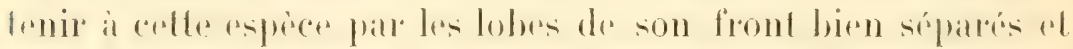

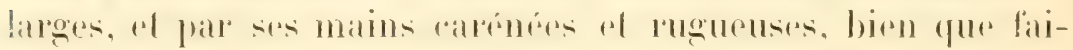
blement.

Thalamita admeta var. Savignyi (A. M.-Edw.).

Savigny, pl. IV, fig. 4 .

Portunis admete Audouin, Expl., p. 8'.

Th. Savignyi A. Milne-Edwatds, loc, cit., p. 33̈7; Nouv. Arch. Mus., IX, p. 163

- Kossmann, loc. cit., p. 49. - De Man, Not. Leyd. Mus., II, 1880, P. 180;

III, 1981, p. 99; Abh. Senckenb. Ges., XXV, 1902, p. 6ł:. - J. Lim. Soc.,

XXII, p. 73; Zool. Jahrb. Syst., VIII, 1895, p. 564. - Henderson, loc. cit., p. 372. - Alcock, loc. cit., p. 83.

Th. admeta Paulson, loc. cit., p. 58, pl. VIII, lig. 1-5 (pars).

Th. admete var. Savignyi Borradaile, loc. cit., p. 202.

Oboek et Púrim (II. Jousseaume), 8 exemplaires; mer Rouge M. Jousinaume, un male of une lomelle; lojibonli 
tout a fait dans un jeune exemplaire de Obock; elle est notablement plus petite qur les autres dans deux autres exemplaires de la mème localite, à peine un peu plus pelite dans deux autres individus de taille moyenne; olle est subégale a la cinquieme dans trois autres exemplaties assez gros de Obock aussi, dont le plus gros mesure $43 \times \geq 7$ millimètres.

d. La main niest pros partirulierement épurisue el sa surfare interne est purfois gramulée; los rếles gramulrives de la fare externe sont bien marquées aussi.

Quant it ce caractere, je dois observer que bien que les exemplaires d'ìge moyen et les femelles aient les mains plus allongées et moins grosses, la plupart des gros màles ont les mains épaisses, a doights plus courts qur la paume, ef mème, dans un exemplaire, obtus au bout, en offrant ainsi un caractere des admela.

On pourait faire les mèmes observations, pour l'autre différence des doigts plus droits, égaux a la paume ot non cannelés sur la face interne. Mes exemplaires offrent tous un ou deux sillons sur la surface interne.

\section{Thalamita admeta var. intermedia Borr.}

Borradaile, loc. cit., p. 203.

Th. Savignyi Nobili, Ann. Ius. Napoli, 1, no 3, 1901, P. 9.

Iles Musha (II. (iravier), une femelle mesurant 18 millimètres de largeur sur 11 de longueur.

Le front est saillant, la fissure médiane est itroite et profonde, les lobes sont faiblement sinues; la face interne des mains est lisse, l'externe offre cinf crêtes gramuleuses. Lal quatrième dent latérale est seulement un peu plus courte que la cinquième. L'article basilaire des antennes uxternes porte trois épines acérées, qui sonl caractéristiques pour cetle variété. L'exemplaire de l'Érythre du Musie de Naples, dont jai traité loc. cit., appartient aussi à cette variété.

Cette forme sapproche donc de Th. quadrilobrrla par les épines de l'article basilaire de ses antennes toul en se rallachant étroitement it Sactigmyi ot admele par son front. La quatrieme dent du bord hatéral parail ètre aussi celle de quadrilo- 


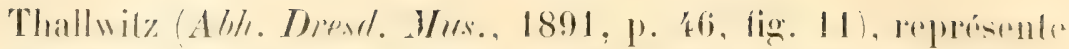
une firstre méliane profonde; la forme de We Van a, au eon-

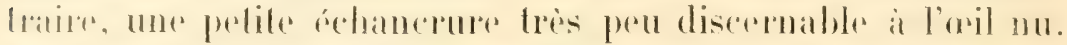
Les lobes exteriems, d'apres Thallwitz, sont dentiformes a!

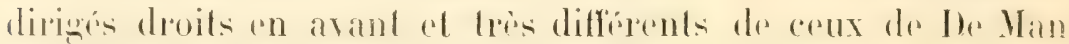
qui sont obliques et ì pointe obtuse et arrondie. Les quatre lobes sont sur la nerme ligne dans la forme de Thallwilz: landis que les drus módians sont plus saillants dans la forme de De Man, qui pour ce caractère s'approche de Woodmasoni el taprobranicr. Nème en supposant des inexatcludes dans la figure de Thallwitz, il me parait bien difterile quitle ne represente au moins avee approximalion les ratacteres do lesprece, el, en ce cas, elle serait bien diffirente.

2. Sur la partie postérieure de la carapace, il y a me ligme saliante lres marquér. plus large que le bord postérieur de la carapace, dont Thallwitz ne parle pas.

3. Les bords de la carapace sont armés de 4 dents

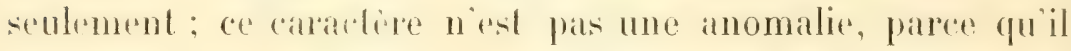
subereve dans la femedle de Atjeh de De Man et dans les deux atemplanes de Djibouli of de Obock. Lat quatriome dent qui tend id disparailte dans certaines esperes du groupe à article basilaire des antennes long, telles que admela, simer, Poixsmii, ete. disparait constamment dans certaines espreces du groupe a ardicle courl, telles que Th. Conperi Borr. Th. pilumnoüdes Borr., Th. Hanseni Alc. et Th. bandusia Nob.

4. La main des chélipèdes a cinq épines dans De Mumi, ef trois sentement daus mict" Thalw.

Je renvoie pour les autres caraclères de cetle espèce à la description et ì la figure de De Man.

Le plus gros exemplaire, celui de Obock, a les dimensions suivantes:

Largeur extrème de la carapace. . .

13 millim.

Longueur

Distance extraorbitaire

$8, \%-$

$10,5-$

H.13.: Aljeh. (De Man). 
face externe; elle est armée de quatre épines fortes et diune épine réduite.

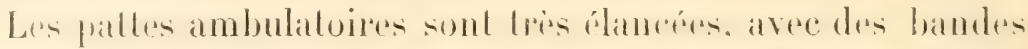

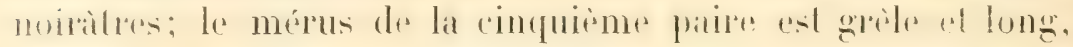

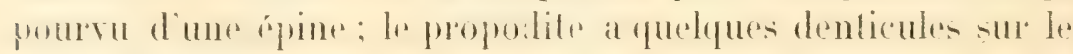
bord postérieur.

Largeur de la carapace............... $8 \frac{1}{\div}$ millim.

Longueur

Cetle espèce difrère de $T$ h. investigatoris par la forme et le

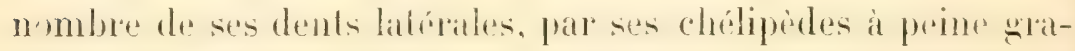
naleux, par sa main pourvue de côtes el de cinq épines; de T\%. Hanseni, alle dilfere nethement par son fronl divises en six lobes.

Gfare THALAMITOIDES A. M.-Enw.

Les deux espèces connues de ce genre se trouvent dans la mer houge. On peut facilement les reconnaître ainsi:

A. Bords latéro-antérieurs de la carapace armés de quatre dents. Th. quadridens A.

A. Bords latéro-antérieurs armés de trois dents. II.-Edw.

B. Article basilaire de l'antenne granulé

$B B$. Article basilaire de l'antenne pourvu de spinules dont 3-4 fortes. .

Th. tridens A. M.Edw.

Th. tridens var. spinigera Nob.

Thalamitoides quadridens A. M.-Eảw.

1. Milne-Edwards, Nouv. Arch. Mus., V, 1869, p. 149, pl. II, fig. 8-13. - De Ian, Arch.f. Nat., 1887, p. 332; Abt. Senckenb. Ges., XXY, 1902, p. 633.

Biler Rouge (Husée de Turin), un mâle. Cé exemplaire

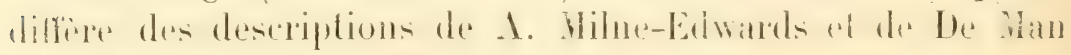

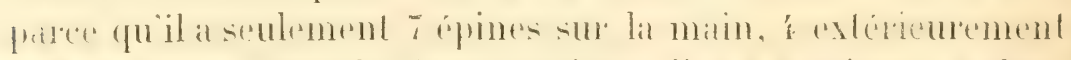

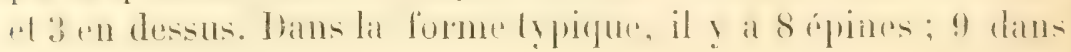
une variété de Upolu.

HAв. : Madagascar ( 1 . Milne-Edwards); Imboine el Ternate (De Man); Upolu ( . Milne-Edwards). 


\section{Thalamitoides tridens Vur. spinigera Nob.}

Nobili, loc. cit., p. 403.

Cf. Thalamitoides tridens A. Milne-Edwands, loc. cit., p. 149, pl. 11. lig. 1-7.-

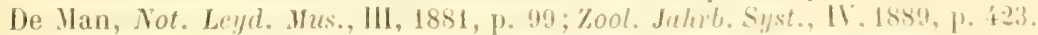
- Ortmann, itid., VI, 1) 86. - Nobili, Inn. Mus. Yipoli, 1, n' 3, 1901, P. 10 .

Hedrophthalmus thalamitoides Nauck, Zeitschr. Wiss. Zool., 1880, 1. :59, Lal. I, lig. 25.

Mer Rouge (Musée de Turin), 2 males, me lemelle: Obock (II. Jousseaume), 7 individus; une femello de localité inconnlle.

Ces exemplaires s'accordent avec les deux femelles de liile

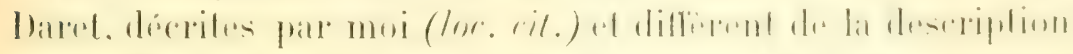

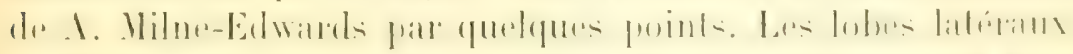

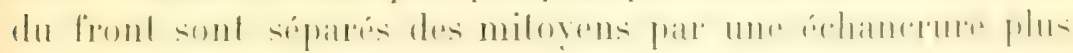
large; ils sont aussi plus arrondis. Les rides sur la rapapace

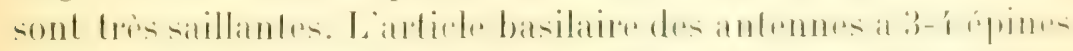
acérées el très fortes, visibles aussi d'en dessus entre le lobe orbitare ef le premier lobe frontal. Les mains ont 7 epines au lieu de 8, sauf dans un male de Obock qui a 7 épines sur une main el 8 sur l'autre.

La forme de l'abdomen d'un màle est de triangle étroil it

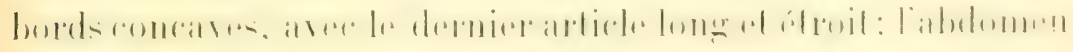

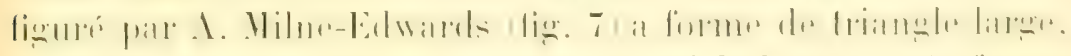
a bords convexes el avec le dernier article large. Cadte forme

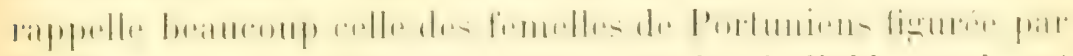
Pfefler el gui sont tris probablement des individus qui ont subi la castration parasitaire.

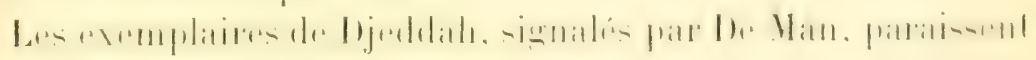
appartenir ansis it relle vilroté.

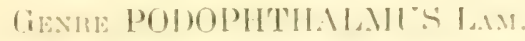

Podophthalmus vigil $[F .113$.$) .$

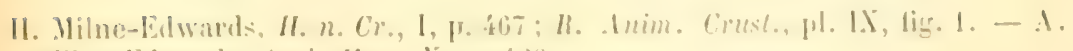
Milne-lidwarks, frek. Mus., I, p. izo.

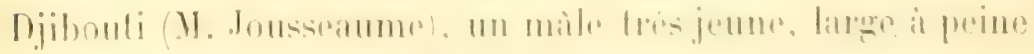
de 11 millimedres ed longe de 6 millimidres. 


\section{FIIILLE XANTHIDA.}

\section{Genre CitrpILIUS Jafach.}

Carpilius convexus (Forsk.) Ruppel.

Cancer convexus Forskal, Descr. Inim., p. 88.

Curplius convexus Rüppell, Besch. 2' Krabb. roth. Meer., p. 13, pl. III, fig. 2, p). I', lig. 6. - Vilne-Elwards, II. n. ('r., I, 1. 382, pl. XVI, lig. 9. - Dana, U. S. Expl. E.rp., I, p. L:!!, pl. III, lig. 5. - I. Milne-Edwards, Nune. Areh. Mus., I. 1865, 1. 21\%. - Ileller, s. B. .1ka1. Wien., XL1II, 1861, p. 319. Aleock, J. A. S. B., LXY, 1898, p. s0. - Vobili. A m. Mus. ('ir. Genova, XL, 1899 , p. 29.

Carpilius maculatus var. C. convexus Paulson, loc. cit., p. 22, pl. IV, tig. $5-5$. $c$.

Obock (II. Jousseaumej, nombreux individus; mer Rouge (Musée Bologne), ume femelle; Massaonah (IIM. Issel el Beccari, Musée Gènes).

La distribulion des laches rouges est variable. Dans les exemphares de Obock, chez qui la couleur est mieux conservere, il ? a ginfiralement une farhe annulaire rouge sur la région gastrique, aulour de laquelle se disposent des lignes rouges rayonnant et ramifiées.

\section{Genre Carpilodes A. M.-Edw.}

A. Carapace lisse ou pourvue de granulations non ou peu visibles à l'ail nu.

B. Granulations de la carapace extrêmement menues mème à la loupe. Lobes protogastriques non divisés longitudinalement................... lavis Edw.

$B B$. Granulations de la carapace bien distinctes à la loupe. Lobes protogastriques divisés en deux dans toute leur longueur...................... C. mber A. Edw.

A A. Carapace distinctement granulée à l'œil nu.

C. Lobes épigastriques fusionnés avec le plus interne des deux lobules de la région protogastrique. Pattes non sillonnées ni noduleuses.

$\nu$. Le sillon divisant la région protogastrique débouche dans le sillon circonscrivant la région mésogastrique. Lobe hépatique entier........ C. diodoreus Nob.

$D D$. Le sillon divisant la région protogastrique ne rejoint pas le sillon mésogastrique. Lobe hépatique divisé.

d. Carapace entièrement granuleuse........ C. rugatus A. Edw. $d d$. Carapace granulée seulement en avant el sur les côtés .................... v. vallantianus $\mathbf{A}$. Edw.

CC. Lobes épigastriques séparés du lobe protogastrique interne. Pattes sillonnées et noduleuses........................... rugipes Hell. 
Carpilodes lævis 1 . M.-Elw.

A. Milne-Edwards, Nouv. Areh. Mus., IX, 1873, p. 179, pl. V, lig. - De Man, Areh. f. Yat., 1887, 1. 2336.

Liomera Edwarlsi Kossmann, loc. cit., p. 2\%.

Carpilodes Edwardsi Nier's, Clulleng. Brach., 1886, p. 1333.

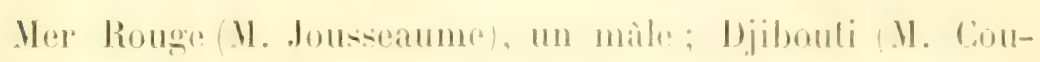

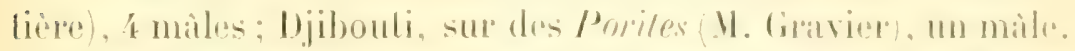

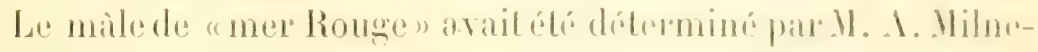
Edwards; les autres remplailes staceoldent hion avececelui-ci.

Linsi que U. de Man a fail observer, la carapace de celle.

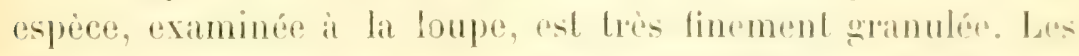

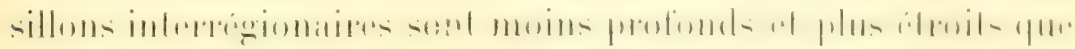

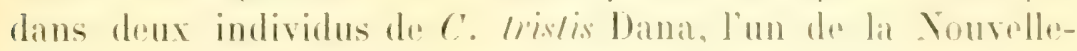

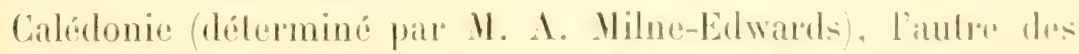
îlos Indamanes (díl. Meock). du Musée de Turin.

La région gastrique est bien délimitée, el un pelit sillon tres

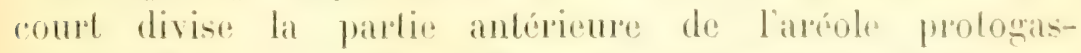
Lrigue 2) M (1). Les lobes I II sont nettement séparés dans un

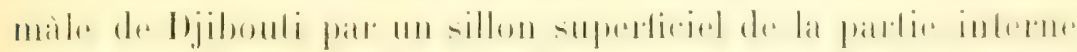
de l'aréole protogaslriege; le sillon est moins nel el parfois

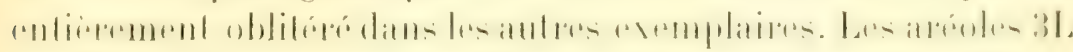
(1) 2L sont fusionnex ensemble ol cileonscoiles jat un sillon.

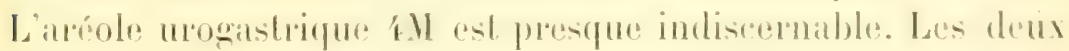

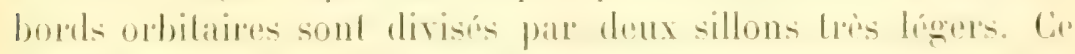
sont bien la les memes earatcleres que liossmann at donncis it sil

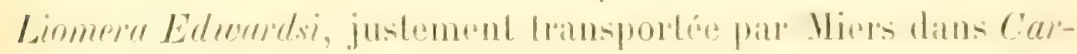

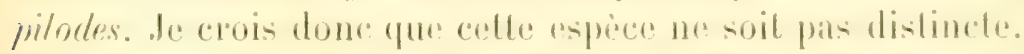

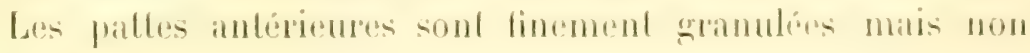

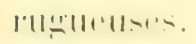

Carpilodes ruber $\Lambda ., 11 .-1311$.

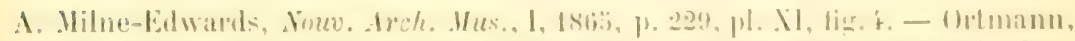

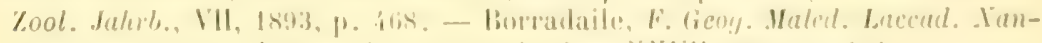

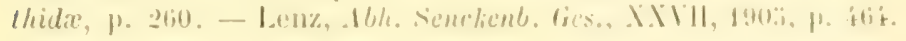

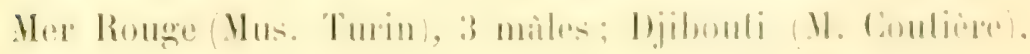
mu milt" jerunt?

(1) Dapres la terminolugie et lese désignations de Dana. 
Dans colle espece laréole protogastrique, ainsi que dans

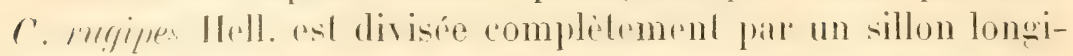
Iulinal qui rejoint en arrière le sillon bordant la région mésosalstrique, el l'aréle épigastrique sol neltement siparé du lobe prologastrique interne.

Les granulations qui courent la catapaces sont tres finces. hien que beancoup phus fortes que celles de lavix at lristix; olles sonl lròs régulieres, non sépares par les gros points of les petiles impressions qui domnent ì la carapace de mejpes son aspect rugueux. Ces granulations forment un revetement fin w mini, quion ne peut roir quà la loupe. Le front a un bord épais chez rugipes, plus mince chez miber.

La petile aréole triangulaire arondie qui s'observe dans rnglipes, entre le bond soureilier et le lobe externe de l'aréole protogastrique, est représentíc dans mber par une aire déprimir at granuleuse. L'aréole urogastrique rst séparée de li mésogastrique par un sillon entier. Les trois dernieres dents des bords latéro-anteriemsont triangulaires, aiguës, saillanter. non arrondies comme celles de rugipes. Les mains des pattes de la premier paire sont finement granulée a la loupe at pourvues de trois lignes saillantes longiludinales (invisibles it l'oril nu) el d'un sillon dans la partie supréreme de la fiace externe. Entre les lignes longitudinales, il y a des ponchutlioms assez nombreuses. Le "arpe a un sillon bifurqué incézilement. Les pattes ambulatoires sont finement granuledses. non sillonnées ni rugueuses.

Nouveau pour la mer Rouge. Habile : îles Sandwich (1. Milne-E(wards): Samoa (Orbmamn): Maldives (Borradaile).

\section{Carpilodes diodoreus (1) Nob.}

(Pl. X, fig. 9.)

Nobili, loc. cit., p. 403.

Djibouli, Obock el Périm (M. Jousseaume), 2 mâles et 6 femelles.

Celle espèce, par sa carapace granulée el par ses aréoles

(1) Atoốúpou vĩ̄sos nom de l'ile de Périm, dans Anonymi Periphs Maris Erythrxi. 


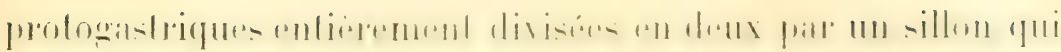

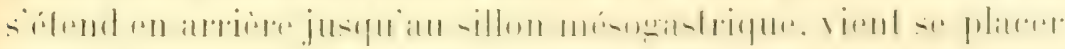
pres de $C$. ruber el $C$. mujpes, mais differe de ces especes par des nombrenx caracteres.

La carapace est notablement hombere of ses nombreuses

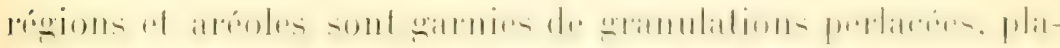
cées tout près les unes des autres of noffrant pas des poncluations ou des losseltes entre elles. Les sillons qui síparenl les arobles sont relativement larges et absolument lisses. I L aréoles ne sont mes saillantes par elles-mêmes, mais suivent la convexilé générale de li carapace.

L'aréole protogastrique de chaque còté ust dicisée drons lonle

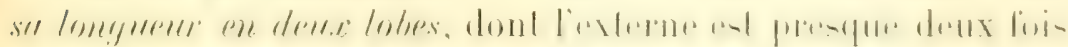

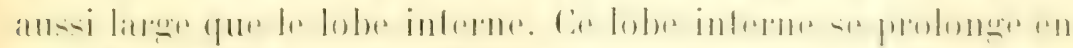

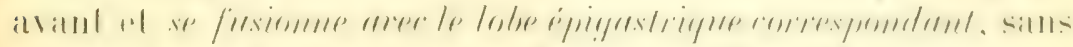

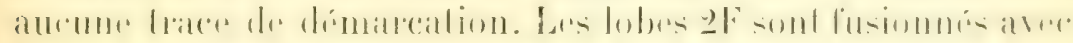

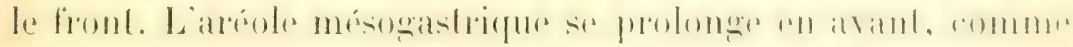

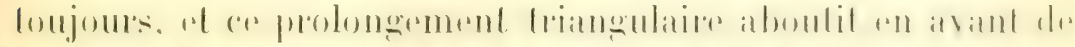

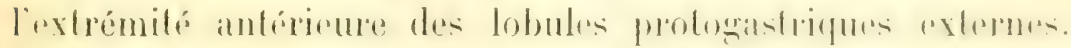

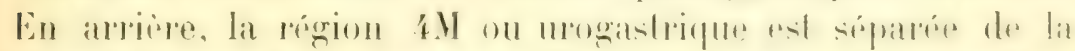

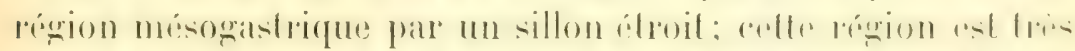

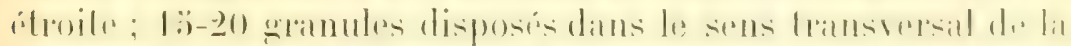

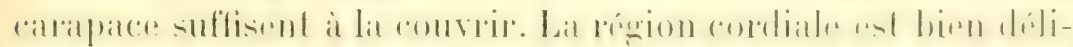

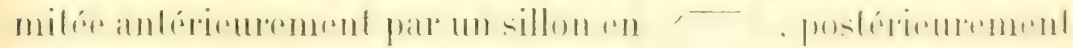

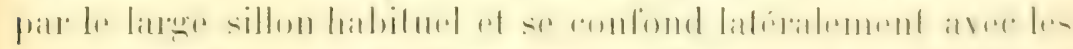

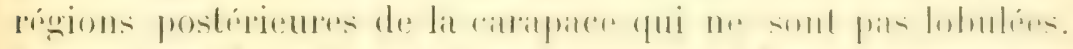

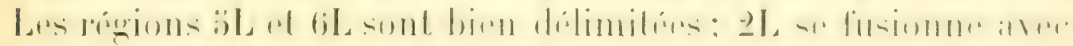

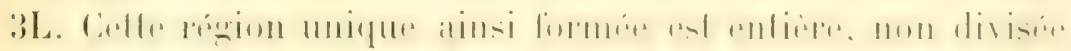
par aucun sillon.

Le front est infléchi; ses lobes sont arrondis, bien siparés

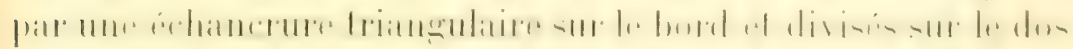
par le sillon frontal bien net qui s'unit en arribe atl sillon mesogastrique. Les lobes externes sont petils, oblus al adosien it l'orbite, mais séparés de celle-ci par un sillon. Lo bord supri-

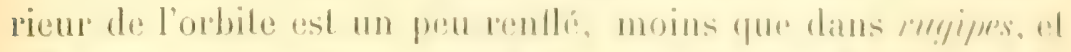

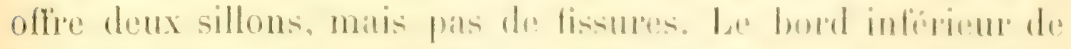
l'orbite ofire seul un sillon. I, sillon qui sipare le front de

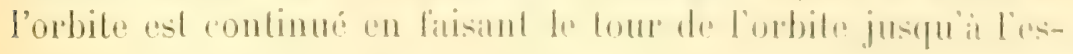


pace entre le premier lobe latérai el le deuxième. Entre le sillon postonbitate al le lobule protogastrique extrome, it la

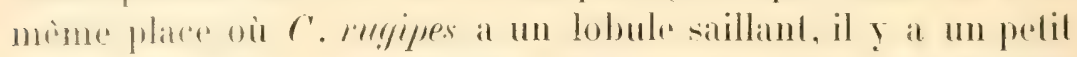

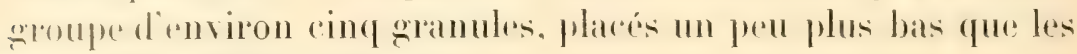
granulations des régions.

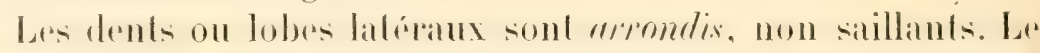
premiere lobe, résultant de ha fusion de Earre D, est plus loner

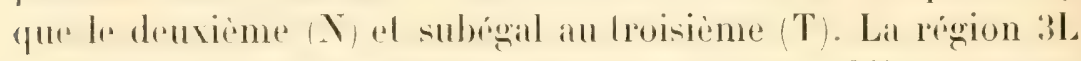
se fusionne avec T an formant ume soule arre oblique granuleuse. IR et $2 \mathrm{R}$ forment aussi une aire unique avec $\mathrm{S}$. Le

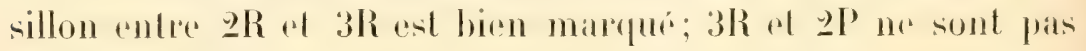
divisés.

Les granulations sont un peu plus gerosses sur les rigions placies en avant du sillon gastro-cordial, un peru plus petites, mais également nombreuses en arriere de re sillon. Sur le lobe protogastrique ratrue, on comple une mogenne de git granulations.

Les régions ptépgostomiques, sous-hépatiques, ele. ainsi que les maxillipedes el le sternum sont granuleus el ponelués. Labdomen dans les deux sexes est plus grossement ponclué que granuleux au milieu, gramuleux stor les còtés des deux premiers arlicles dans le mìle; un peu moins dans lit femelle.

Les chélipides sont aussi forlement granuleux que la cartpatee, matis non noduleux. Le carpe n'est pats sillommé. Ciel atlicle offre deux saillies coniques du coté interne, l'une placre pres de l'exlrémité de sa face supérieure, l'autre au-dessous de celle-ci, sur la face interne. Les doigls el lat paume sont subégaux.

La face externe de la paume est entierement rouverte de fortes granulations, dont quelques-unes plus saillantes lendent at se disposer en denx ou trois lignes longiludinales. Cidlesurfater noest pas rugueuse. Les doights sonl fortement carenés el sillonnós, el les carenes sont granuleuses on finemenl denticulés. La doigt mobile est fortement granuleus a sa bast. Les doigts ne joignent pas bien; ils sont excarés a la pointe, ot

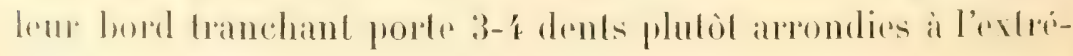
mité. Les doigts ont une couleur brun foncé. 
Les pattes ambulatoires ont des granulations, ainsi que la carapace el les chélipides. Ces ramulations occupent tous les arlicles, mème le daclylopodile.

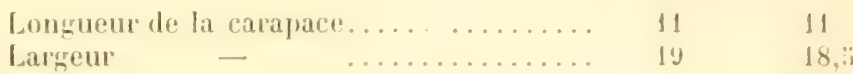

Chlomedius exigmes Targioni-Tozadli de Sumatra est ansi un Curpilodes, bien roisin de dindorens, qui a atusil la camapace couverte de fines granulations, les lobes protogastrigues divisés entierement et soudés avee les épigastriques. I'apros loxat-

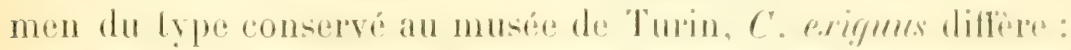

$1^{\circ}$ Par sa carapace beatroup phs introite. Le rapport entres la largeur el la longueur dans un màte de dindorens rst 1,72, dans le malle type de exigums il est de 1, 亿:

2" Le front est beaucoup plus large dans erifmes, mesurant

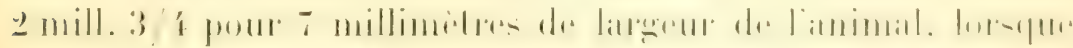

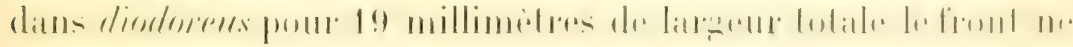
mestre que 3 mill. 1/4;

$3{ }^{\circ}$ La carapace est beaucoup moins bombée, el son depnier

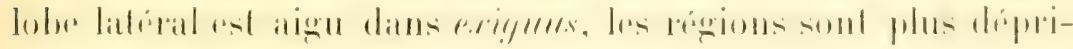
mées el les granules plus petits;

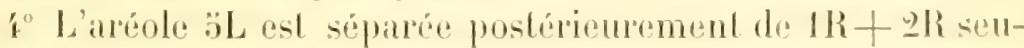
lement par un petil trait dans la partie antérienre dans erigmens ; elle est bien séparée dans diodnreus;

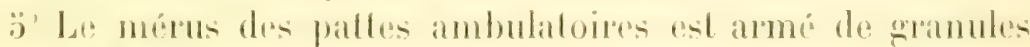

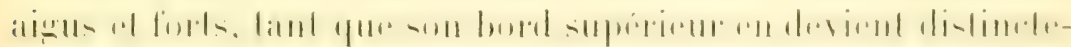
ment denticule dans exigums; les eranules du bord supérieur. bien que aigus, ne sont pas plus forts que les autres dans dindoreus.

$6^{\circ}$ Les deux derniers arlictes de l'abdomen du mile son subé-

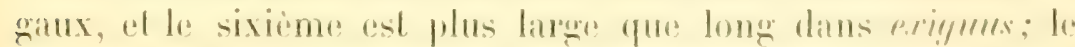
sixieme est plus long que le seplieme a ansi longer que latrose dans dindneres.

\section{Carpilodes rugatus (I.1Th.)}

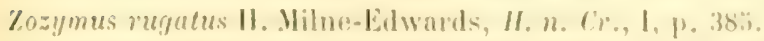

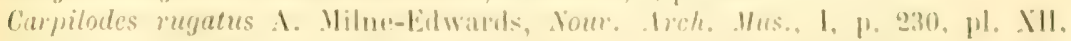

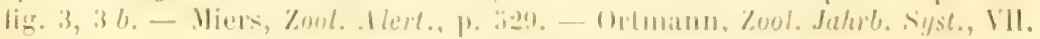
1893, 1. 468. - Nloock, loc. cil., P. 82, si (ubi s!mn.). 
Obock, a lintérieur des polypiess morts, sur la ligne des hauls fonds du récif de la Clochiterie, a mer basse, un mate Ch. Cravien); Djibouli ol Obock (H. Jousseaume), 3 mâles of 't femelles; mer Rouge (Musée de Turin), une femelle.

L'eremplaire recueilli par M. Gravier, qui ronserve ses coulems, est d'un pourpere violet lres beau. Les doigts des pinces unt la mème couleur violette dans la moitié proximale; ils sont blancs dans la moitié distale. Les doigts des pattes anbulatoires sont aussi violets dans la moilié basale, blance dans la distale; les ongles sont cornés. Les exemplaires du Dr. Jousseaume, qui ont séjourné dans l'alcool depuis 1897, ont une couleur rougeâtre.

Haв. : Her Rouge : Daedalus Shoal (Miers); Seychelles (Miers); Réunion (A. Milne-Edwards); Mauritius Richtersi: (Jcrin Indien et mer de Chine (1. Milne-Edwards); CocosIsland, Andamanes (Alcock): Nouvelle-Calédonie (A. MilneEdwards); Tahiti (Ortmann).

Garpilodes Vaillantianus A. M.-Edw.

A. Milne-Edwards, Nouv. Arch. Mus., I, 186ä, p. 231, pl. XI, fig. 3-36.

Signalé daus la mer Rouge, par M. A. Milne-Edwards.

\section{Carpilodes rugipes (Hell.).}

Actrodes rugipes Heller, S. B. Akad. Wien, XLllI, 1861, p. 330, pl. II, 1ig. 20. A ctæa rugipes Kossmann, loc. cit., p. 24.

Carpilodes rugipes A. Milne-Edwards, Nouv. Arch. Hus., I, p. 229, pl. XII, fig. 4. - Heller, "Novar( " Crust., p. 17. Paulson, loc. cit., p. 23, pl. IV, fig. 6. - Lenz, Abh. Senckenb. Ges., XXVII, 1905, p. 348.

Djibouti, Obock et Périm (M. Joussoaume): 2 miles at 3 femelles; mer Rouge (Mus. T'urin), un mâle.

Ces individus saceordent tres bien avece la description de Ifellere. Les lobules de la carapace sont tris saillants ot granuleux, mais les granules sont déprimés. Charque lobule offre ausisi des petites impressions el descoros points qui donnent aux lobules un aspect rugueux. Les aréoles épigastriques $1 . \mathrm{H}$ sont mellement séparées par un sillon profond du lobe interne des 2.1. Entre le bord sus-orbitaire ipais et le lobe raterne des 211. 


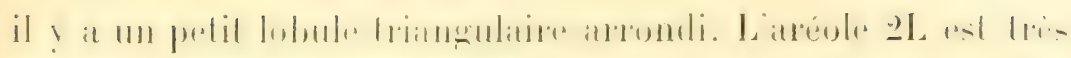
saillante, presque conique; la zone entre celle aréole el les

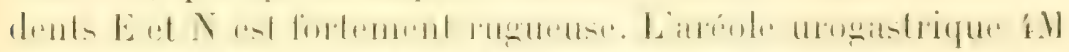

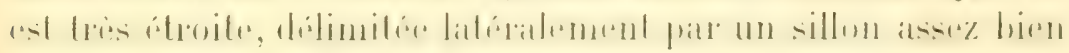
marqué, mais qui ne se conlinue pas au milieu. Les palles

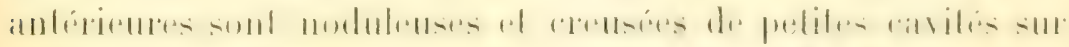
le carpe el sur le bord supérieur de ha main. Lal liace exterme

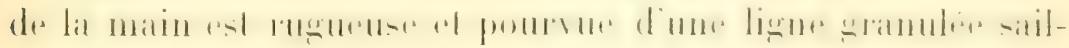

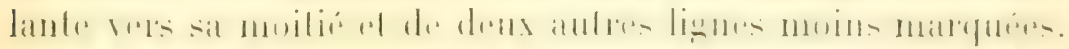

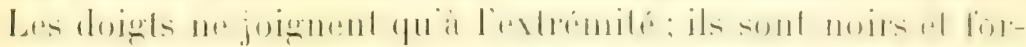

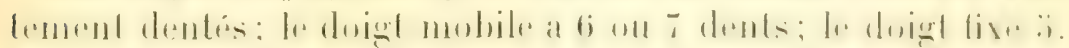
Les pattes ambulatoires sont fortement rugueuses.

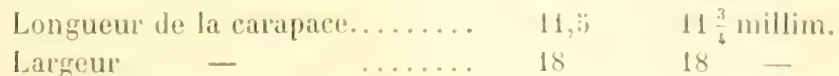

(iENIE IAOMERA DANA.

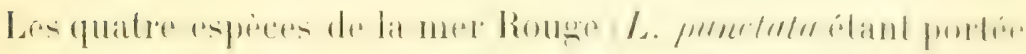
dans Linxantho) peuvent ètre distinguées ainsi :

A. Carapace non granuleuse.

13. Lobes des bords lateraux presque oblitérés. Hains pourvues de grosses granulations perlacées....... L. granosimanu A.

BB. Lobes des bords latéraux hien marqués. Pinces II.-EdW. non granuleuses. (Carapace très élargie; rapport entre la largeur et la longueur $=1,8$. Coulenr. rouge orangé; mains soment cerclées diun anneau (Iiii) ...............

A. Carapace granuleuse.

C. Carapace finement granuleuse. Lolses frontaux saillants et angulaires. I)oigt mobile des pinces court, fort el courbi........................... pubesecens Lidw.

CC. Carapace avee de grosses granulations. Lobes

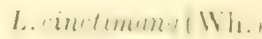
frontaux peu saillants et presque droits; doigts it peine plus coults pre la pamme, grèles, presigue: droits.............................. themistu (I) Man).

\section{Liomera cinctimana ( Withe)}

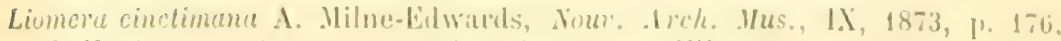

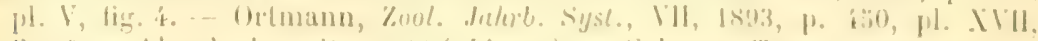
lig. 9. - Ncock, loc. cil., p. sis (uhisyn.). - Calman, Truns. Limn. Soc: (2). 1III, 1900, 1.. A.

Liomera lutu Danat, loc. cit., p. 161, j1. 111, lig. li. 
Mer Rouge (M. Joussaume), 2 femelles, ö mâles; Djibouti (M. Coulière), une femelle.

La coloration de aplle esperee est magnifique, matis aussi quelque peu rariable. Lal carapace et les palles sont d'un rouge?

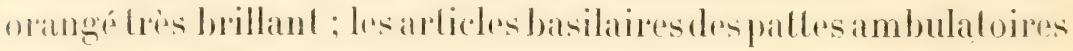
sont blancs: lextrémile des dachlopodites a aussi un amneatu blanc (en atcool). La femelle de Djibouti et un exemplanier lout jeune de " mer Rouge " ont la carapace blanchatre et les palles rouges. La bande noire qui cercele la main n'est pas tonjours constante : elle manque dans une femelle large de 3 millimedres, dans une autre de taille movenne, dans la femelle de lojibouti of dans le jemue exemplaioe deja menfiomé. Il est probable que cet ornement manque à la femelle. Seulement un màle large de 28 millimetres offie la bande sur les deux mains; dans les trois aulres rxemplaires it bande, olle at complete sur une seule pince, sur l'autre interrom 'le ou absente. Les pinces des femelles el du màle de 28 mill dedes sont igales. Dans deax autres moltes qui consedved des deux pinces, elles sont inégales: chez l'un la grosse pincerest a droitr. chez laule a gatuhe. Lat bande sol sur la pelite pince, chez le premier exemplaire, sur la grosse chez l'autre.

Le plus gros exemplaire $\left(O^{x}\right)$ est long de 19 millimetres et large de 35 millimètres.

Cielte espèce, répandue depuis l'Ifrique orientale jusqu in Polynésir, n’a pas encore été signalée dans la mer Rouge.

\section{Liomera pubescens var.}

Zozymus pubescens H. Milne-Eilwards, H. n. Cr., 1, 183', p. 38'.

Liomera pubescens A. Milne-Edwards, Nouv. Arch. Mus., 1, 186əे, p. 2.23, pl. XII, fig. 6-6a. - Ortmann, Zool. Jahrb. Syst., VII, p. 432.

Actrodes pubescens Miers, Proc. Zool. Soc, 1884, p. 10. - De Man, Not. Leyul. Mus., XIII, 1891, p. 4, pl. I, fig. 1.

Périm (M. Jousseaume), 3 mâles el \& femelles.

Ces individus different dutype de l'lle-de-France que M. Bouline a ell lobligeance de me communiquer. aimsi que des descriptions de A. Milne-Edwards et de De Man.

Les deux exemplaires plus gros $(a, b)$ et le type $(c)$ ont les dimensions suivantes : 


\begin{tabular}{|c|c|c|c|}
\hline & $a 0^{\circ}$ & 118 & $c 0^{\circ}$ \\
\hline aroeur de la carapace........ & $2 \%$ & 23 & 30 \\
\hline Longueur _ - & $1 ;, ; i$ & $1:$ & 18 \\
\hline
\end{tabular}

Les sillons de la rigion gastrique sont dislincts, moins que dans le type, et en cela anlant ces individus que le trpe diflerent de l'exemplaire dúcrit par Do Man, où ils mancpuaient, ol s'approchent mienx de la figure de 1. Milno-Edwards, où res

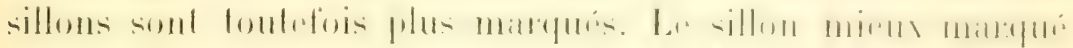

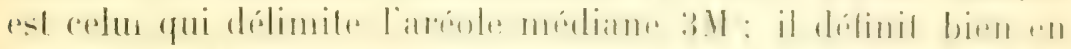

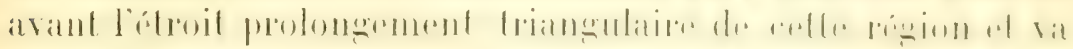
se joindre au sillon qui vient du front. Les sillons qui déli-

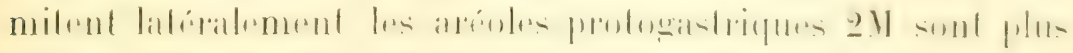
faibles, et ces areoles ne sont pas délimilées antérieurement.

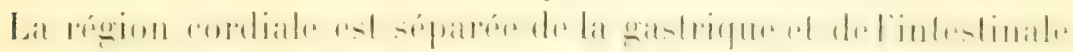

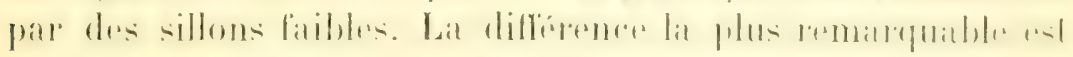
donnepp par les bords latéraux. Ceux-ci ne snnt prs dirisés du tout il n'y a amome trace des sillons qui devaient partager

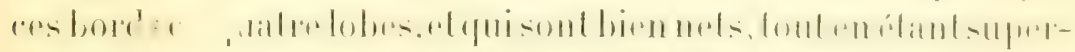

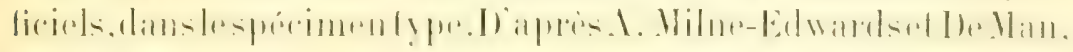

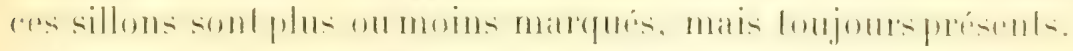

La carapace apparaît moins granuleuse que dans la figure.

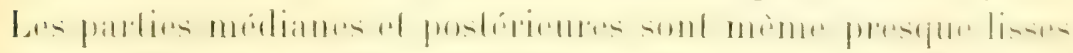

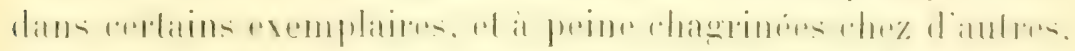
bien qu'it la loupe on les recomnaisse granulées. Les granulations deviennent plus nombreuses et plus grosies pres des bords latéraux et pres du front, el parmi les gramulations de taille usuelle, il y en a de nombreuses autres saillantes el subconiques. Sur ces borts latéro-antérieurs ces granulations deviennent coniques, aigues et forment une bordure it lat carapater composere d'environ 2.j granules coniques, dentiformes, di-

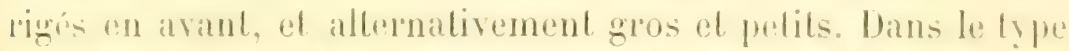
l1placées pros des borks deviennent aussi grosses que celles placees sur le loold ; les parties latérales el anterremes de la carapace portent de longe poils soyeux d'un jaume doré, qui

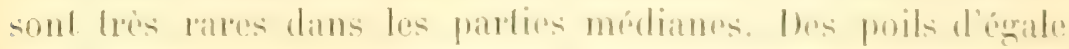

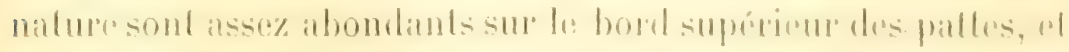
ansisur lo bord inférimer maismoins mombrems. 
Les pinces, dans le lype el dans les exemplaires de l'érim, birn que concordant arec la figure de De Han par la forme générale. "ul diflerent par les granulations plus régulièrement arrangées "n siries. La partie infériene de la surface externe de la main drpuis la hauteur du sillon du doigt lixe jusqu'au bord inféritur, est lisse et brillante dans les individus de Pórim, bien qu'elle apparaisse finement ponctuée it la loupe; dans le type cette partie est granulée.

La couleur des exemplaires de Périm est d'un rose très brillant. Je n'y vois aucune trace des laches blanches décrites par Miers et par De Man dans des exemplaires de Mauritius et des îles Fiji. Les doights sont foncés, el la coloration obscure du doigt fixe envahit un peu la main.

Le plus gros des màles a mème un anneau noirâtre qui cercele toute la main, comme dans L. cinctimana.

Cette rare espèce est nouvelle pour la mer Rouge. Elle n’a rté signalée qu à Mauritius, par Milne-Elwards et par Miers el aux îles Fiji par De Han et par Ortmann.

\section{Liomera themisto (De Man).}

Actxodes themisto De Man, Zool. Juhrb. Syst., IV, 1889, p. 417, pl. IX, fig. 3.

Mer Rougre (Musée de Turin), 2 mâles el 5 femelles.

Les dimensions de trois exemplaires sont:

\begin{tabular}{|c|c|c|c|}
\hline & o" & ? & \& \\
\hline Largeur de la carapace. & 22 & 24 & 17,5 \\
\hline Longueur - & 12 & 11,5 & 10,5 \\
\hline Distance extraorbitaire.......... & 11 & 11 & 9 \\
\hline 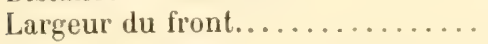 & 9,5 & 4,3 & $3, \dddot{2}$ \\
\hline
\end{tabular}

La carapace dans les exemplaires plus gros est proportionnellement plus large. Cétte espece a quelques affinilés aver L. pubescens, mais on peut lit dislinguer facilement pilr sil caratpace plus grossement granuleuse, plus poilue (en jugeant par let type de puliescens conservé à sec el par les exemplaires de la variété décrite) el par la forme de sés chripipedes. La région satrique est presque indéfinie, puisqu'dle n'est marquée qu" far la bifurcation du sillon postfrontal qui scetend en arriert a délimiter le prolongenent anterieur de la région masogit:- 


\section{Genre LiOXAN'THO Alcock.}

Tableau des espèces (d'après Alcock).

A. Chélipèdes inégaux; angle externe du front séparé du bord sus-orbitaire par une échancrure; divisions régionales de la carapace presque nulles............. Lumidus Alc.

$A A$. Chélipèdes égaux, angle externe du front fusionné avec le bord sus-orbitaire.

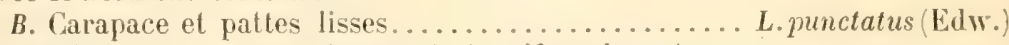

$B B$. Carapace et pattes finement et uniformément gra-

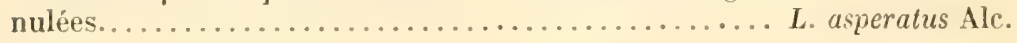

Lioxantho punctatus (Edw.).

Tantho punctatus H. Milne-Edwards, H.n. Cr., I, 1834, p. 396 . - A. MilneEdwards, Nouv. Arch., IX, 1873, p. 199, pl. VII, fig. 6. - De Man, Zool. Jahrb. Syst., IV, p. 420, et Not. Leyd. Mus., XII, 1890, ). 52, pl. III, lig. 1 .

Liomera maculata liaswell, Cat. A ustr. Crust., 1882, p. 47.

Liomera punctata Hiers, Zool. Alert., p. 528. - De Man, Arch. Nat., 188\%, 1. 238. - Ortmann, Zool. Jahrh. Syst., VII, 1893, p. 431.

Lioxantho punctatus Alcock, loc. cit., p. 91 (ubi syn.).

Djibouti et Obrock (II. Jousseaume), 2 males at 2 femelles: mer Rouge (Musée de Turin), 3 mâles et 4 femelles.

Les points roloriés sobservent surtout sur la carapace; ils sont rouge orangé, entourés par un pelit halo clair. Les paltes ambulatoires sont annelées d'orangé ou de rouge brun.

Les chélipèdes des femelles sont égaux, ceux des màles aussi śgaux ou parfois faiblement inégaux, ainsi que le dit Alcock et contrairement a l'exemplaire observé par De Man. Les doigh sont bruns, mais il y a toujours une bande claire a la base du doigt mobile, comme dans la figure de A. Milne-Edwards. La coloration noire du doigt fixe s'arribe a la batse de cet article chez la femelle te màle jermen, mais śélend sur la main dans le mâle adulte.

IIAв. : Mer Rouge : Daedalus Shoal (Miers); Seychelles (Miers); Mauritius (II. Milne-Edwards); Madagascar (Miers); Ceyalan (Miers); Maldives (Ortmann); Java (Je Man); Imboine (De

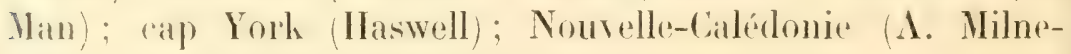
Edwards); Fiji et Samoa (Ortmann). 


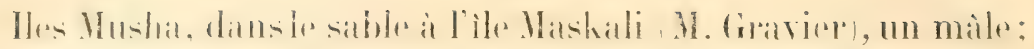

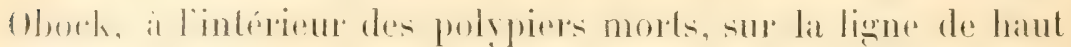

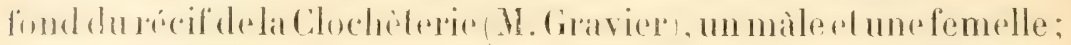
Obock, dragage dans les sables du récif de la Clochèterie

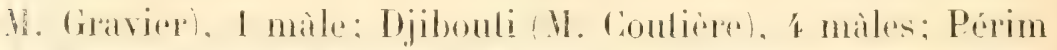

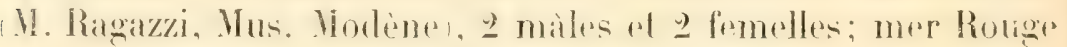
11. Jonseaume/. un male: une femelle de localite inconnue; Arlen (MH. Bonnier el Pérer), un jeune màle.

Cescrenplates atecordent asso bien avec la description etla lignar. Le front parait pourtant un peu différent. Il est conformé conme dans L. pumelutus, soit mellement bilobé et un peu infléchi en bas. Les divisions des bords latéro-antérieurs de la "anapace sont a peine indigues par un ou deux petits sillons lises ef superficiels quion voit entre les granulations qui revêfeut abondamment l's parties antéro-latéralesde la carapace. Un seul màte jeune d'block fait exception, parce qu'il offe deux sillons assez profonds, aboutissant à deux échancrures sur le bord de la carapace. qui est ainsi partagé en trois lobes nets.

Le doigt fixe des chélipedes est infléchi en bas. Les doigh ont la coloration foncén caractéristique de la plupart des Xanthides, et offent des series longiludinales de poncluations. Is -nnl un peu plus longs que le bord supérieur de la paume.

Les exemplaires bien conserves offrent deux colorations différentes. Le phus gros milu des iles Musha est d'un gris bleuatre uniforme: les autres individus ont les parlies supérieures des pattes d'un bean rouge orangé, of la carapace pourrue de plaques de la mème couleur sur fond jaunatre.

Le plus gros mâle a les dimensions suivantes :

Longueur de la carapace. .

Largeur

- du fronl

Longueur de la pince.

- de la main

des doigts.
18 millim.

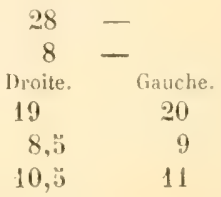

Ceile belle especes gui parait etre commune dans la partie mérilionale de la mer louger. n'est conmue que de Karachi of des Maldives. 
(IENRE ATERGIATS I)E HAN.

A. Carapace lisse.

B. Surface de la carapace non bosselée. Bords latéraux. obtus se contimuant sans domareation aver les hords" latéro-postérieurs....................... 1. reseus (Rüpp.).

$B B$. Surface de la carapace lonsselée. lionts litéru-antérieurs aigus et cristilormes, terminés en arrière par

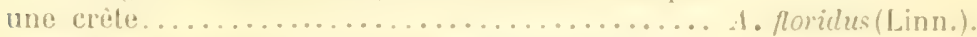
1. Surface de la carapace distinctement granulec...... A. granulutus Do: Han.

\section{Atergatis roseus Riipp.}

Carpilius roseus et C. maculutus Rüppoll, 1) 13, 1). III, fig. 3 et p. 15, I1. 1II, fig. 4 .

Atergatis roseus A. Milne-Liwards, Nour. Hrch. Wus., 1, 1863, p. 239. - hosmann, p. 19. - Paulson, [. 11, pl. 11, fig. 1-1 a (pmrs). - Alenck, p. 97. Atergatis lavigatus A. Milne-Ehwarts, loc. cit., p. 24, pl. XY, fig. 4. Atergatis scrobiculatus lleller, Sitzh. Had. Wien, ILIII, 1861, p. 310.

Djibouli (11. Jousseatume), fo jennes axemplatres, dont liun

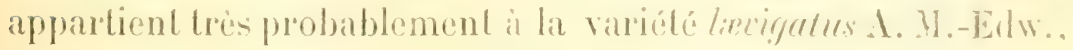
el un auber a la variebie albu liossm.

Obock (Il, Jousseamme), I fentelle lonerue de 13 millimelres, large de 80 millimetres, ol 2 jeunos moles, appretenanl it la valivé lievigatue 1. Edw.

Mer Rouge (M. Jousseanme), un jenne mile, aussi de la variétó laxirgalus.

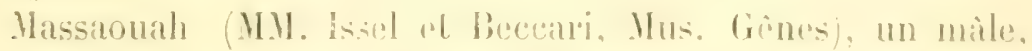

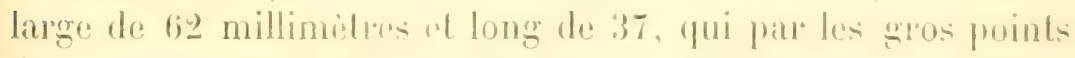

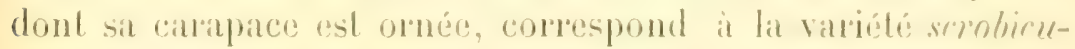

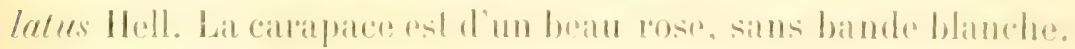

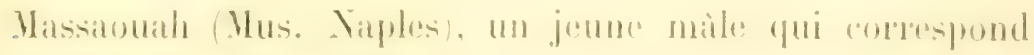

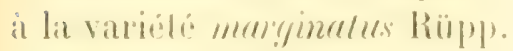

Atergatis floridus (Rumph. Limu.).

Cif. Alcock, loc cil., p. $98(u b i$ s!mu.).

Djibouti (II. Jousseanme), individus lo's jeunes.

Atergatis granulatus I) Min.

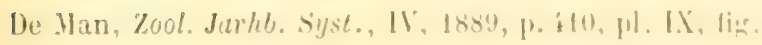


Obock et Périm (H. Jousseaume), 3 mâles; mer Rouge (Musée de Turin), ๖ mâles.

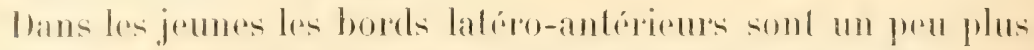

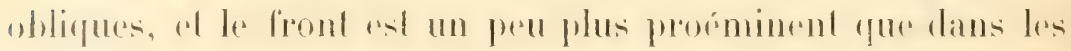
adultes. Les jeunes portent aussi une trace tres faible de la

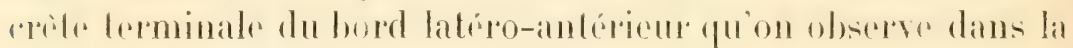
plupart des Atergalis.

Leseremplaters dobock at Périm onl les dimensions suivanles:

Largeur de la carapace....... $41 \quad 26,3 \quad 23$ millim.

Longueur $\quad-\quad \ldots \ldots \ldots$ 26 $16 \quad 16 \quad$

Distance extraorbilaire........ $17 \quad 11 \quad 10,5-$

Celle rare espèce n'esl commue que de Mauritius.

\section{GenRE LOPHACTAA A. M.-EDW.}

Tableau des espèces de la mer Ronge.

A. Carapace bien granuleuse.

B. Lohes prologastriques divisés longitudinalement.

C. Crète du hord supérieur de la main, forte, lisse sur les cotés et granulée en dessus. Granulations sur le lobe protogastrique externe en nombre de 16-25,

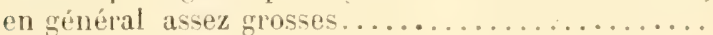

CC. Crète du bord supérieur de la main moins forte et

L. cristata A. II.Edw.

plutôt une ligne saillante granulée qu'une crète. Granulations de la carapace plus nombreuses et plus pelites; sur le lobe protogastrique externe, il y a

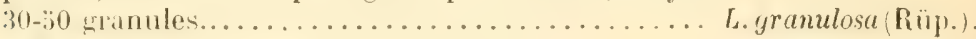
$B B$. Lobes protogastriques non divisés........... semigrunosu

(Hell.).

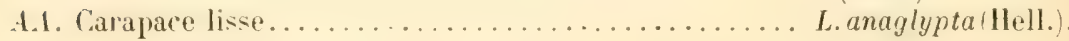

Lophactæa cristata A. M.-Edw.

A. Milne-Edwards, Nouv. Arch. Mus., 1, 1863, p. 246, pl. XVI, fig. 1. - De Man, Not. Leyd. Mus., III, p. 93, et Irch. f. Natury., p. 246. - Alcock, loc. cit., p. 100.

Mer Rouge Mus. Turin), 2 miles; un jeune màle trouvé parmi des gromelosu recueillis par M. Jousseaune a Djibouli,

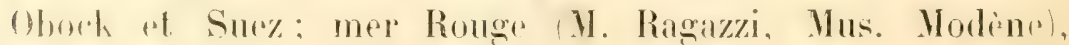
une femelle.

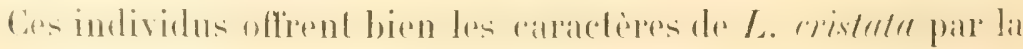




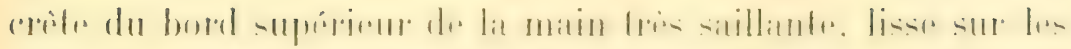
còtés el granulée sur le bord lranchant. Les deux mâles du

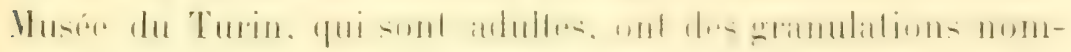
breuses et grosses sur lia fince externe ou postérieure de tous

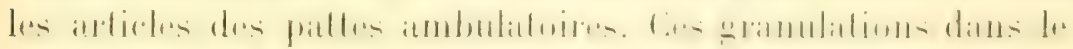

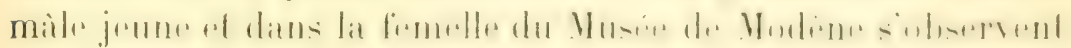
seulement sur le carpe ot le propodite.

Les granulations de la carapace sont plus grosses ol plus

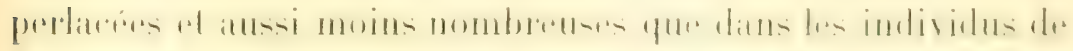
L. gramulosin. Le plus gros des mâles du Yusée de Turin a 10 granules sur l'un des lobes épigastriques, el environ 20

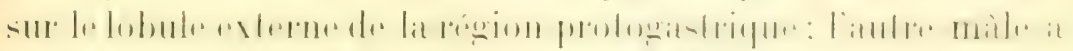

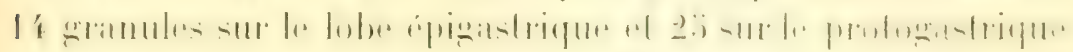

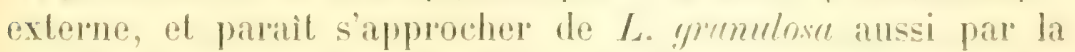
crete de la main moins lorte; le jeune mile n'a que 7 granules sur le lobe épigastrique el 16 sur le prologistrique externe. La femelle du Hosée de Modine a la crote de la main bien

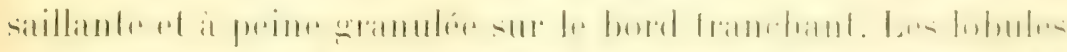

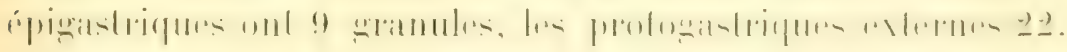

La carapace du plus gros màle est large de 32 millimetres ot longue de 22.

\section{Lophactæa granulosa (Riipp.).}

Xuntho granulosus Rüppell, p. 2't, pl. V, fig. 3.

Lophactxa granulosa A. Milne-Edwards, loc. cit., p. 237. - Hilgendorf, .I. B. Akad. Berlin, 1878, p. 78\%. - De Man. Not. Leycl. Mus., III, 1881, p. 9\%; : Arch.f. Nat., 1887, p. 246; 16h. Senclienh. Ges., XXV, 1902, p. 58:. - Acock, loc. cit., p. 100, 101 .

Djibouti, Suez el Obock (II. Jousseatume), 3 miles et 3 lemelles; îles Musha (.I. Cravier), une femelle: Oboch (W. Jousseatume), un gros mate; Djibouli (II. Coulicre), 2 femelles: Djibouti M. Gravier), une lemelle trouve à l'interieur d'un

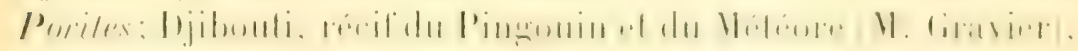
un mails.

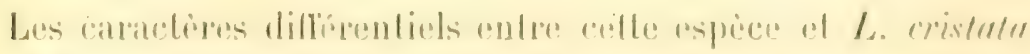

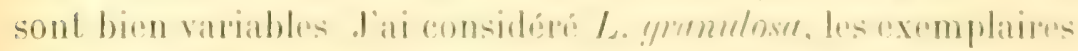

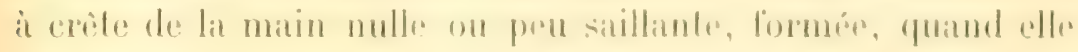
est présente, par une ligne de granulations plus errosises, el il 
carapace pourve de gramulations plus nombreuses et générulement plus petites. Le caractere négatif de labsence de cribl. -ur la main n est puint ahsolu, ainsi que Hilgendorf el De Man lavaient déja remarqué. Si je devais mime jugrio par les exem-

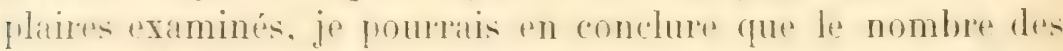
exemphares pontus de "rite est plus grand que le nombere de ceux qui en manquent. Inms la plupart des cas, il sagit d'une ligne de granulations saillantes. matis cette ligne pol si bien marquée, si isolée desantres gramulations. et souvent les reanules qui lat comprosent sont someles entere pux par la liatse. quäl net quane question de mots de lappeler ligne de granules sallant-

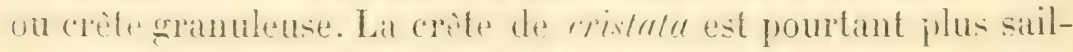
lante. of ses cotes sont lisies. Mais lexemplaire trouvé a Djihouti dans une Porites a une crite qui est presque autant saillante of lisst sur les fares, que "rlle de lat femelle de rristata du Musée de liodene. Ce mème individu a les granulations de lit

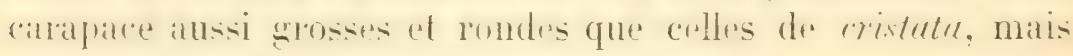
plus nombreuses.

La forme et les dimensions des granulations varient selon les individus: elles sont flus nombreuses dans gromulnsa. Lit femelle des iles. Mushat a 13 gramules sur les lobes épigastriques.

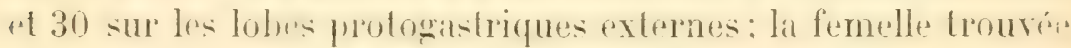
a Djibuti dans une Poritex, 20 granules sur les épigastriques

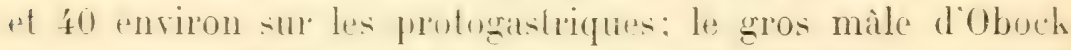
respectivement 23 a 30 . et une femelle de Djibouti, 20 et 40 environ.

Il n'est pas non plus un caracliere sùr celui suggéré par De Man, que les regions mésogastrique et cardiale sont presque

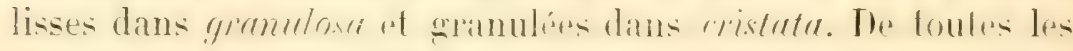
granulnsa examinfes trois sullement ont ces mèmes régions fresque lisses: dans les autres ans régions sont plus ou moins

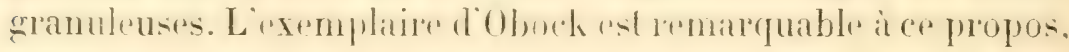
frarce que. pendant quil se manifeste une gromulose typique

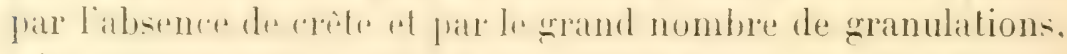
a les régions mécogatiolque of cardiale presque autant granulées que les autres rógions de la carapace.

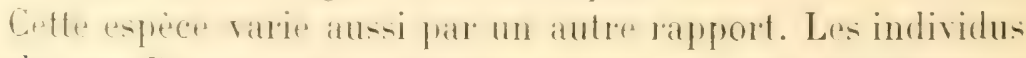

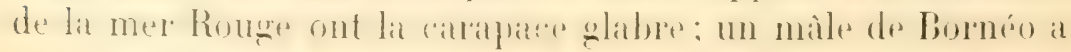




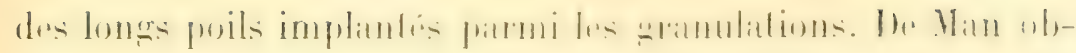
serva le mème fait entre un individu de l'ulo Edam. el un autre de Ternate.

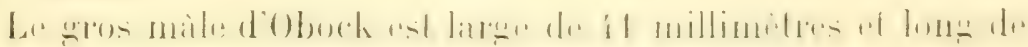
29 millimètres.

\section{Lophactæa semigranosa (Hell.).}

Atergatis semigranosus Heller, Sitzb. Akad. Wien, XLIll, 1861, p. 313.

Lophactxa semigranosa A. Milne-Edwards, Nouv. Arch., I, 1865, p. 2i8.lliers, "Alert ", 1884, p. 527. - Ortmann, Zool. Johrb. Syst., VII, 1893. p. 549. - De Man, Arch.f. Nat., 1887, p. 240 (en partic el ron les figures), et Abh. Senckenb. Ges., XXV, 1902, p. 582, pl. XXI, fig. 19.

Her Rouge (Musée de T'urin), 3 mâles el une femelle.

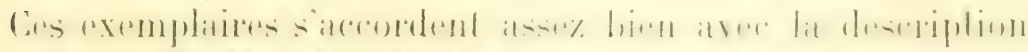

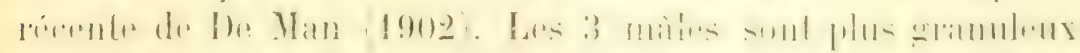

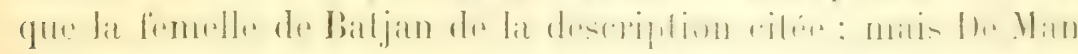
observe que les types de Heller de la mer houge sont aussi

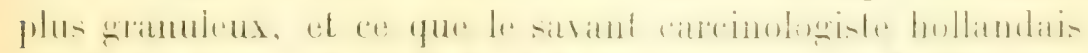

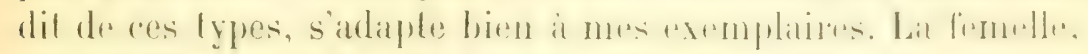
qui est plus pelite, est moins granuleuse que le mâle.

\begin{tabular}{|c|c|c|c|c|}
\hline & $\sigma^{*}$ & $0^{*}$ & 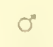 & \\
\hline Largeur de la carapace........ & 21,5 & 19,3 & 20 & 16 millim \\
\hline Longueur $\quad-$ & 15 & 13,5 & 14 & $10,3-$ \\
\hline
\end{tabular}

Hab.: Her houge, Tor (Heller); iles Mmirantes (Miers):

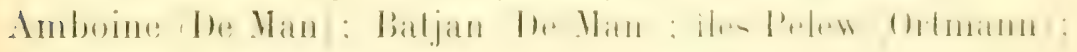
Maldives (Borradaile).

\section{Lophactæa anaglypta (Hell.).}

Atergatis anaglyptus Heller, loc. cit., p. 312, pl. 11, tig. 11-12.

Lophactza anaglypta A. Milne-Edwards, loc. cit., 1. 251. - De Man, Zool. Jahrb. Syst., IX, 1895, p. 498. - Nobili, Ann. Mus. Napoli, 1, n² 3, 1901, p. 12.

L. Helleri Kossmann, p. 21, pl. 1, fig. 2. - Nobili, luc. cil., 1. 13.

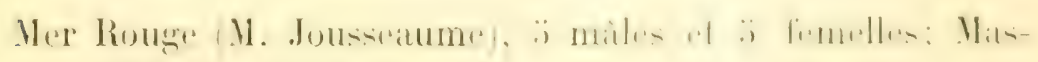
saouah (MN. Issel et Beceari, Mus. Gènes), une femelle.

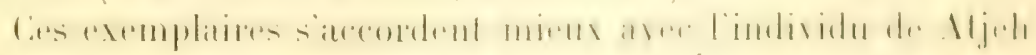
décrit par De Man qu'avec le mâle de l'Erylluée, décril par

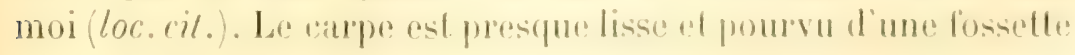


oblique; il est légierement irodé et pourvu de deux fossettes dans le mâle de l'Érthrée. Dans les hoos mâles plus gros, la couleur noire des doigls s'etend sur la paume; dans les femelles de dimensions presque ígales. al dans les màles plus jeunes la couleur noire s'arrête à la base des doigts. C"est li probablement une différence sexuelle qui se diveloppe avec l’àge.

En 1901 (loc. cit.), j’ai signale un exemplaire de L. Helleri, tout en exprimant lo doule que ces deux espèces ne pouvaient defe séparées. J'ai repris la question sur ces individus plus nombreux, et j’ai vu que les différences que j’avais réussi à trouver entre les deux individus du Musée de Naples, ne sont, ainsi que je supposais, qu'individuelles. Le front dans quelques exemplaires n'est pas fissuré; dans d'autres, il offre une fissure assez nette, dans d'autres une fissure a bord rapprochés et close. La main n’est pas proprement granulfe, mais souvent ses rugosilés sont un peu érodés, el alors la main apparaît légrement granuleuse. Il n’y a done lieu à séparer les deux espéces. Kossmann parait d'ailleurs avoir été trompé par la ligure de Ileller, puisque dans sa description, il ne fait aucune allusion à l'étroite affinité entre les deux formes.

Genre ATergatopsis A. M.-Edw. Atergatopsis Frauenfeldi (Heller).

Atergatis Frauenfeldi Heller, loc. cit., p. 311, pl. I, fig. 10.

Atergatopsis Frauenfeldi A. Milne-Edwards, lo: cit., p. 258. - Paulson, loc. cit., p. 133.

Djibouti (M. Coutière), 2 jeunes mâles.

La carapace est convexe et sa surface sit divisée par des sillons aussi superficiels que ceux de $A$. floridus en régions. Un sillon sipare les orbites d la carapace. Le sillon frontal est bien net et continué en arrière jusqu'i la régrion mésogastrique. Les lobes épigastriques el es postfrontaux sont à peine distinguables. L'aire protogastrique est divisée longitudinalement; les aréoles latérales sont discernables; la partie postérieure de la carapace n'est pas lobulée. Le front est infléchi en bats. large et formé par deux larges lobes à peine séparés all milieu, qui se rehaussent un peu de chaque colé à former les 
lobules externes. Les bords lidrimux, sans être en crête, sont saillants et minces. Ils sont découpes en quatre lobes, dont les deux premiers sont subégatux, le troisieme est plus long, le quatrieme est pelit, prestue dentiformr, mais obtus et peu saillant.

Les chélipedes sont égaux. La carpe el la main sont granulés, les gramules sur la partie inforleme at moyenne de lat main se

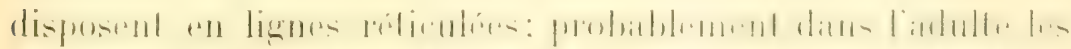

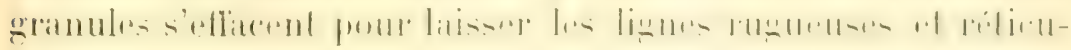
lées si fréquentes dans les Xanthions.

Le bord supérieur de la main na a pas de crête, comme dans

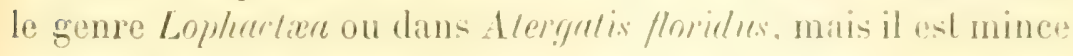
of tranchant, non arrondi. Les doigts, surtout lo doigh fixe, sont dentés el fortemenl cartenés.

Le bord superreur du méropodile des palles ambulaloires n'est pas caréné, mais il est arigu, a, examine aree une loupe? assez forte, il est finement denticule. Le bord inféro-externe esl saillant el échancré à l'extrémité. Le bord supérieur des aulres articles est aigu, non cirréné.

Caracteristipues de celte espece sont les ponchations nombreuses ef relativement grosses guion observe sur foute la carapace.

Le plus gros individu ast large de 7 millimelres.

Atergatopsis granulatus A. M.-EMW.

hossmann, loc. cit., p. :2:2.

Signalé par liossmann dans la mer Rouge.

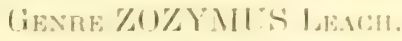

Zozymus æneus (linı.).

Dana, loc. cit., p. 192, pl. I, fig. 3. - Holler, loc cit., p. 326, - Alcock, loc. cit., p. 10't (ubi syn.).

Ateryatis (Zozymus) aneus laulson, loc. cil., p. 16, pl. 11, lit. 3-36.

Mer Rouge (Mus. Turin). Nombrenx exemplaires; mer Rouge

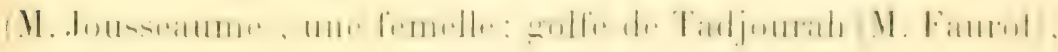
une femelle. 


\section{Genre ZOZYMODES Heller.}

\section{Zozymodes carinipes Hell.}

Zozymodes carinipes Heller, loc. cit., p. 327, pl. II, fig. 16-18.

Atrigatis carinipes Paulson, loc. cil., p. 18, pl. $1 \mathrm{~T}$, figr. 4-4 $b$.

:Leptodius (Yanthudius) cristatus Borradaile, Xanthidx IJaled. Laccad., p. 252. fig. 51 .

P'rim (Jousseaume), un mîlt: Obock (H. Jousseaume), 3 màles; Tyibonti of Aden (11. Jousseamme), 4 màles et une ferielle; Djibouti (N. Contiere), 2 mâls el une femelle.

Les exemplaires saceordent hien avee la description de Heller, mais non avec sa ligure qui est mavaise. Assez bonne est la figure de Paulson, bien (qu elle reproduise un trop̧ petit nombre de granules.

Les mains portent de nombreas poils sur la surface externe qui parfois masquent les granulations qui couvent la surface.

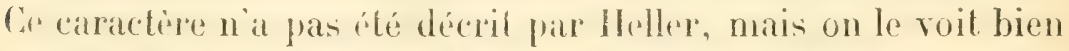
dans la figure d. Paulson. Le sillon sur la partie supérieure de la main est mienx marqué sur la pelite main que sur la grosse. Lar fice inférieure du céphaholhorax est couverte de poils très courts.

Je doute que Sumthodius ristatus Borrad. ne soit identiqur à cette espèce.

\section{Genre LOPHOZOZYMUS A. M.-EDw.}

\section{Lophozozymus pulchellus A. M.-Edw.}

A. Milne-Edwards, Arm. Soc. Ent. Fr. (4). VII, 1865, p. 273; Nouv. Arch., IX, 1873, p. 205, pl. 6, fig. 3. - Urtmann, Zoolog. Jahrb., VII, 1893, p. 458.

Mer Rouge (Nusée de Turin), un màle; mer Rouge (M. Jousseaume), une femelle.

La carapace est médiocrement convexe dans le sens longitudinal, presque plane en sens transversal dans les parties médianes; mais les parties antérieures des rógions branchiales sunt déclives parce qu'elles suivent la déclivité des parties antérieures de la rarapace. La surface est netlement lobulée, les lrigions y sont bien sfiparés. La longueur de la carapace est un per moins que les deux liers de sa lareen exprime entre les 


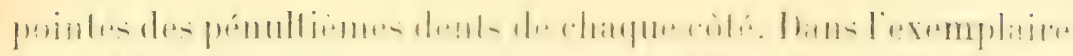
de la Touvelle-Calédonie décrit par 1. Milno-liswards, la lon-

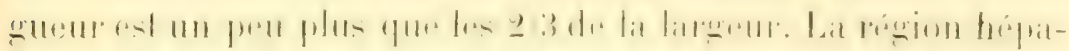

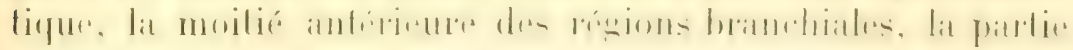

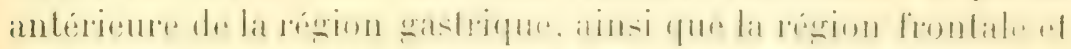

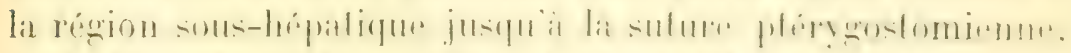

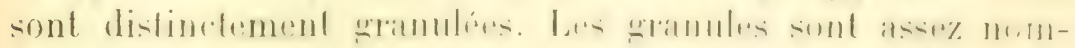

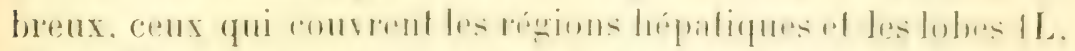

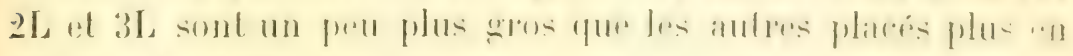
arrière. Le front mesure en largeur moins d'un tiers de la

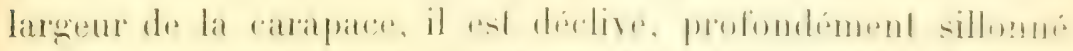

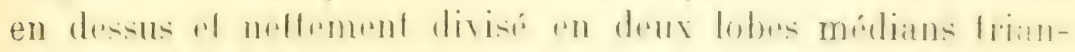

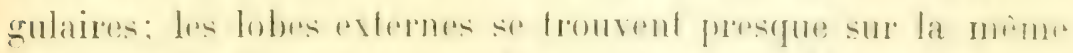

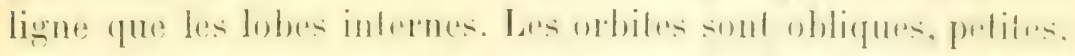

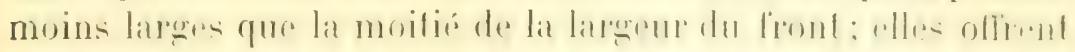

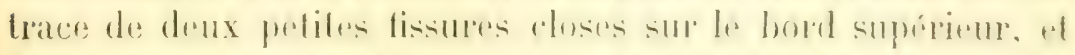

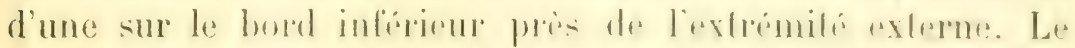

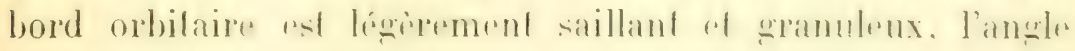
orbilaire externe est nul.

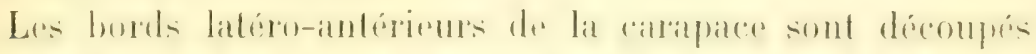

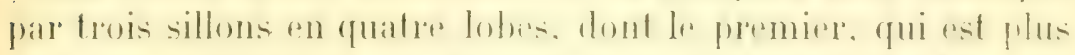

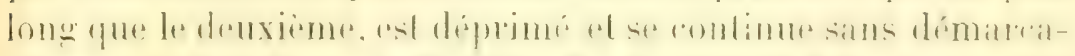

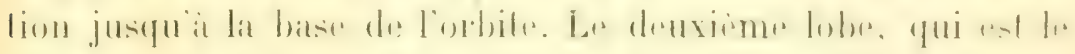
plus petil, est triangulaire, et, ì sa moilié, saillit en pointe

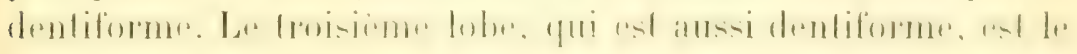
plus large de tous, et sa pointe cat portée plus en arrière de la

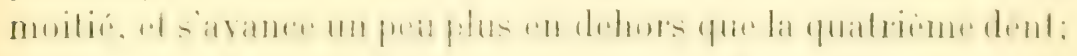
La plus prende largene do da carapace vient ainsi it se trouver entre les pointes des froisiemes dents de chaque cole. Le quatfrieme lobe ast distinctement dentilorme. Les prointes de la troisieme et de la quatrieme dent sont coniques of dirigres un peu en avant. Le bord latéral qui sur le premier lobe n'olire

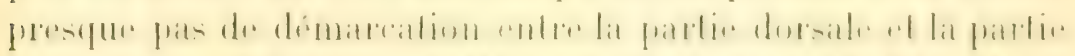

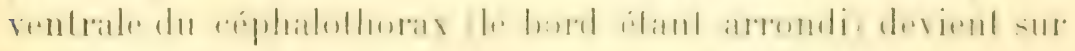
les trois autres lobes mince, aigu et lanchant.

La surface de la carapace offre deux lignes saillantes gramuleuses el interrompues: l'une oceupe la partie anterienre des

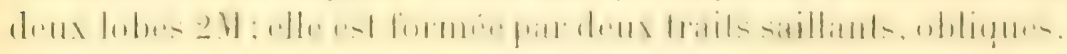


interrompus au milieu par le prolongement du lobe $3.1 \mathrm{M}$; latutre

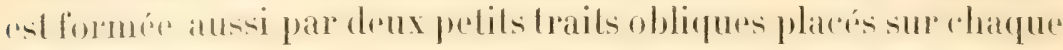
hobe :̈h, qui se continuent pare demx peetits traits obsolesernts sur la région gastrique.

Lus bords latero-postérims de la carapares sont tresobliques et plus longs que les bords latéro-antérieurs.

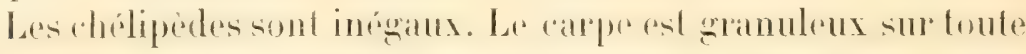
sil surfare "xferme el pres de l'ulremile il offre une pelite

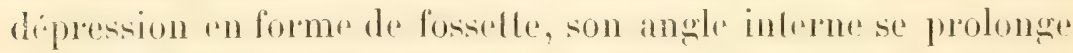
en forme de dent conique pen saillante. La main est aussi granuleuse ol rugueuse sur sa surfare exlerme : la paume est un pen plus longue que hes doigts, alle ast traverse par quelquelques lignes peen sillantes. Le doigt mobile est granulé a la base; il est comprimé af plutòt nince, finement sillonne; le lloigl fixe est plus fortement sillomné; il ast armé près de la base de deux grosises dents coniques disposées horizontalement.

Les pattes ambulatoires sont élégamment carénées.

Lat carapace (en alcool) a de larges plaques orangées.

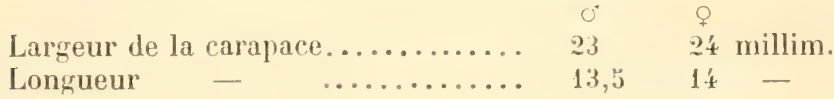

Nouveau pour la mor Rouge. Habite la Nouvelte-Calédonie et Samoa (1. Milno-Eolwards), at les îles Lion-hiou (O)tmann).

Genre EUXANTHUS Dana.

Euxanthus sculptilis Dana.

Ililgendorf, Decken's Reise Ost. Lfr., p. 106.

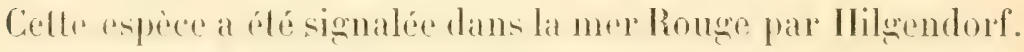
I la même place Hilgendorf a anssi un Euranthus frorgarius Melissa Strahl; mscr., Mus. Berol.), nom qui ne me résulte

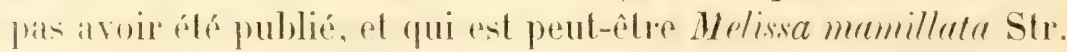
$=E u r$. melissa (Herbst); et un E. cavipes Strahl qui n'a pas non plus été publié el qui est peut-être Leptodius erripipes. 
GeNRE HYPOCOLPLS Rathisun.

(IIypocxelus Ilell., nom. maxoccup.)

\section{Hypocolpus sculptus (EdW.).}

Savigny, pl. Vl, fig. 2.

Cancer sculptus II. Hilne-Edwards, II. $n$. Cr., I, p. 37\%.

Hypocelus sculptus Heller, S. B. Acad. Hien, XLIII, 1861, p. 32.2. - A. Milne-

Edwards, Youv. Arch. Mus., 1, 1865, P. 295.-Kussmann. loc. cit., p. 29.

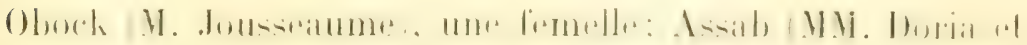
Beccuri, Mus. Gènes), une lemelle; Aden (M. Ragazzi, Mus. Modene), une femelle.

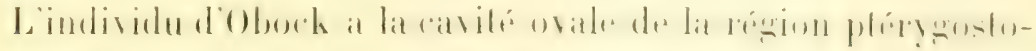
mienne moins profonde que les autres exemplaires.

Hab. : Her Rouge, Mamitius, Cochinchine, Japon.

\section{GeNre X\N'THO.}

\section{Xantho distinguendus De Haan.}

Cancer (Xantho) distinguendus De Haan, F. Japonica Crust., ए. ¿8. pl. XIII, lig. 7 .

Xantho distinguendus Ileller, S. B. Akad. Wien, XLIII, p.323. - Paulson, loc. cit., p. 32. - Alcock, loc. cit., p. 113 (ubi syn.).

Xantho macgilliwrayi Hiers, "Alert", p. 211, pl. XX, fig. c.

Medxus distinguendus De Man, Journ. Linn. Soc. London., XXII, p. 31.

Djibouli el Obock (H. Jousseaume), 8 males et 6 femeller. Liun des males porte une grosse saceuline.

\section{Xantho hirtipes lat.}

(if. I) Man, Milh. Humb, Ilus., XIll, 1896, p. 76, pl. I, fig. 1.

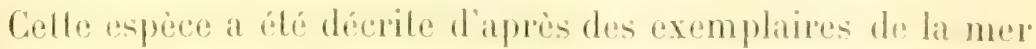

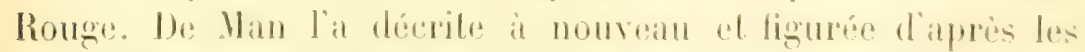

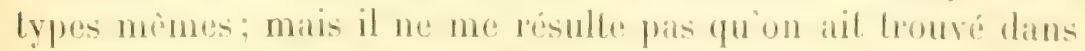
la mer houge d'aulres exemplatees apres les lypes.

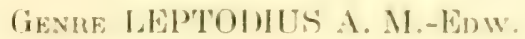

A. Carpe des paltes ambulatoires fortement bicarine; les cretes enclusent une cavité an atugn............... l. caripes 1)ana. A. Carpe des patles lisse.

B. Bords latéro-antérieurs de la carapace aver quatre dents. 
C. Carapace convexe. liégions à surface légèrement irrégulière..................... exaratus (Edw.).

CC. Carapace aplatie. Région à surface Lrè lisse. L. gracilis Dana.

BB. Bords latéro-antérieurs armés de cinq dents.

D. Front à lobes à peine concaves. Lobulation de

la carapace médiocrement accentuée........

L. sanguineus

(Edw.).

DD. Front à lobes profondement échancrés. Lobulation de

la carapace complète et à aréoles bien con-

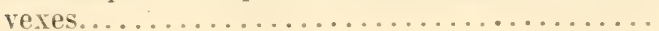

L. euglyptus Alc.

\section{Leptodius exaratus (Edw.).}

Savigny, pl. V, fig. 7.

Cancer inxqualis Audouin, Expl. P. C. Savigny.

Chlorodius exaratus H. Milne-Edwards, H.n.Cr., t. I, p. 402. - Atlas Cuvier, R. Anim. Crust., pl. XI, fig. 3.

Leptodius exaratus A. Milne-Edwards, Nouv. Arch. Mus., IV, p. 71. - Kossmann, loc. cit., p. 32, pl. II, fig. 1-6. - Alcock, loc. cit., p. 118 (ubi syn.).

Tantholividus De Haan, F. Jap. Crust., p. 48, pl. XIII, fig. 6.

Actæodes lividus Paulson, loc. cit., p. 26, pl. V, fig. 2-2 b.

Nombreux exemplaires de Massaouah (Nus. Turin, Gènes): Asrab (Mus. Modene); Obock, Djibouti, ete. (Musée de Paris).

L. erraratus est peut-être l'espèce lit plus commune de crabe dans la mer Rouge.

\section{Leptodius gracilis (Dana).}

Chlorodius gracilis Dana, p. 210, pl. XI, fig. 13.

Leptodius exaratus var. gracilis Miers, "Alert", p. 330. - Lenz, Abh. Senck. Ges., XXVII, p. 353.

Leptodius !racilis De Man, Arch. f. Nat., 1887, p. 287, pl. XI, fig. 2.

Her Rutge M. Jousieamme), une femelle, largeur 13 millimetres, longuen 9 millimetres. Ciet exemplaire differe de celui de l'île Noordwachter, dérit par De Man, par la carapace proportionnellement plus longue, par les chélipèdes a surface finement chagrine, et par les doigts moins fortement baillants et arqués.

Leptodius sanguineus (Edw.).

Chloradius sanguineus H. Milne-Edwards, H. n. Cr., 1, p. 402. - Dana, p. 207, pl. XI, fig. $11 a-d$.

Leptodius sanguineus A. Milne-Edwards, Nouv. Arch. Mus., IV, 1868, p. 71, et IX, 1873, p. 224. - De Man, Zool. Jahrb. Syst., VIII, 1895, p. 521, et 1 bh. 
Senckent. Ges., XIV. 1902, p. 602. Lenz. Abh. Senckenb. Gros., XXVI1, 190\%, p. $3 \% 2$.

?Chlorodius Erlwardsi Heller, S. B. .1kal. Hien, XIIII, p. 336.

fientho (Leptodius) sunyuineus Nicoch, loc. cit., p. 120 (uti sin.).

Mer Rouge (II. Jousseaume), i mites; Obock (II. Jousseaume), 3 males; Massaouah (I. Tellini, Mus. Thrin), un male.

Les exemplaires qui ont conservé les couleurs sont vivemenl leintés de rouge sur la carapace el sur les paltes. Le front est dans tous les exemplaires plus étroit que dans L. extromes. mais les lobes sont différemment échancris solon les premplaires, mais toujours plus protondément que dans exrnentux.

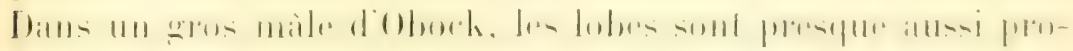
fondément échancerés que dans un cotype de L. eressimumus A. Edw. de la Vouvelle-Calédonie.

Acock considere le Ch. Edwerdsi IIell. conme identique it (20lle r.

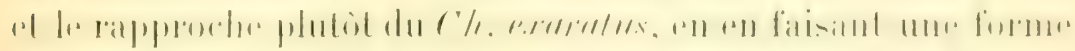
intermédiaire entre les Ch. exrratus el smonimens.

\section{Leptodius euglyptus Al.}

Xantho (Leptodius) euglyptus Alesch, loc. cit., p. 118, 1:21, et 1ll. Zool. Investiyator crust., pl. IXXVI, fir. 1 (1899).

Djibouti (11. Jousseanme), un mile el une femelle.

Ces exemplaires dillerent par quelques caracteres de la description el de la ligure d'Aleoch, mais ces diflobences ne me paraisient pas sortio de l'ordere habiluel des varialions des Leplodirs. Les dimensions des deux individus sont:

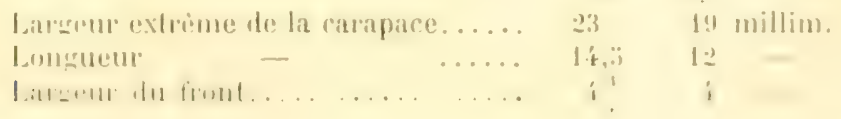

Dapres ces dimensions, on voil que la hargene du fronl asl

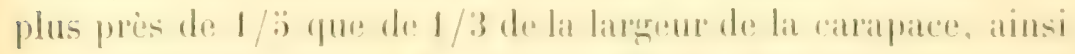

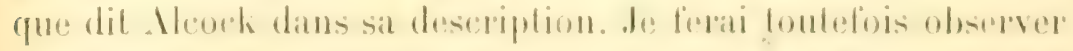
que dans lat ligure, le front proplex est un pen moins le 1/i de la largen de la carapater dimensions qui sébognenl ANX. SC. NAT. YOOL, ge siryin. IV, 11; 
moins des mirmmes. Par la largene proportionnelle du front

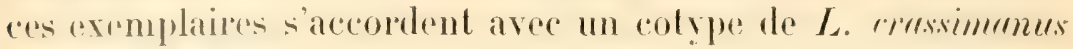
1. Ealw. La forme des lobes est aussi a pen pris la mème, paree que le bord des lobes internes est tress oblique, at les petils lobes externes sont triss saillants. Malgré ces affinitrs. les individus de l)jibouti ne penvent pas ide confondus avere L. ressimermux, parce que leur carapace est divisée rn un phus grand nombre de lobules, qui sont aussi beatucoup plus saillants. Les sillons interrégionnaires sont bien marqués, profonds el relativement alroits, surtout ceux qui siparent la région gastrique of renx qui la divisent en ses aróoles. L’aréolation de cette région est complite. L'aréole postfrontale (2F) est rapprochée do front. Les atréoles prémédiames 1.11 sont convexes el mal síparres des extramédianes 2J, qui sont divisies longitudinalement par un sillon: "resillon est la continuation de celui qui sipare les areoles of et la partio anterieme de I.II du borl sompilior. Ces mimes areoles 2 II sont encore divisées par des pelits sillons en divers lubereules saillants. Laréole 3.11 est noflement individualisér par un sillon bifurqué, continuation du sillon frontal. La petite aroble postmé-

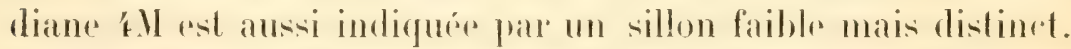

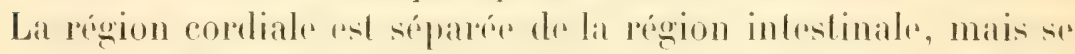
fond lateralement dans l’arole 3R. Les six arooles de lat région antéro-latirale sont bien séparress fa IL a la 22L sont pourlant presque fusiommins entre elles, matis divisés en deux gros lubereules: l'aréole öl porte trois bubercules. Les régions interslinales sont sipareses du bord postérieur de la carapace par un sillon bien marqué. Les cing dents antéro-laterales sont coniques, subobtuses et finement granulies. Entre la dent T el la dent $\mathrm{S}$, il y a un tubercule bien marqué qui est formé par 41 amas de granulations. Des granulations semblables, mais plus éparses, sobservent atusi ente les dents T at X. Le bord un peu renflé du front est finement gramulemx ansiof.

Les chélipèdes saccordent bien avec la figure. Les doigh sont excavís a la pointe, mais non dilates. Le propodite et le carpopodile des palles ambulatoires sont faiblement carénés al sillommón distinctement granuleux. Calte espece n'est conmur que de l'Inde (Mergui, Ceylan, iles Andamans). 


\title{
Leptodius cavipes (I)ana).
}

Chlorodius cnvipes Dana, p. $212, \mathrm{pl}$. Xll, ligr. 1 a-b. - Stimpson, Proc. Acad., N. Sc. Philad., 18:38, p. 34.

Leptodius cavipes De Man, Journ. L.S., XXII, 1887, p. 3i. - Alcock, loc. cit., P. 118, 122. - Lanchester, IProc. Kool. Soc., 1901, p. 3it0. - Lenz, Abh. Senckenb. lies., XXY11, 1905, P. 35 t.

Djibouti el Aden (I. Jousseaume), 2 males et 3 femelles:

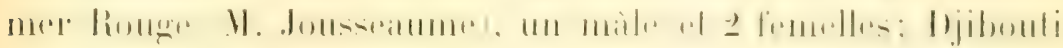
(II. Couliere), un mâle.

Les crêtes longitudinates sur les carpopodites sont bien

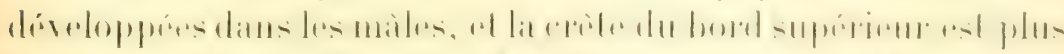

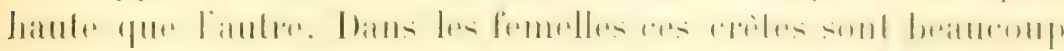

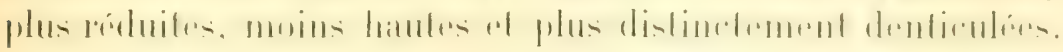
Mais un màle (mer Rouge) a les crôtes réduites el finement granulées el denticulées comme dans les femelles. C"est le

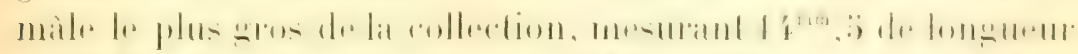

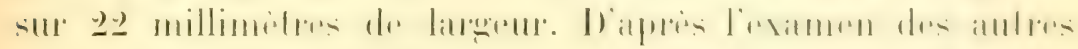
exemplaires, il me parait que les males plus jeunes ont les cretes plus fortes que les arlultes.

Linsi que Lanchester l'a observé, les rugosilís du carpe des

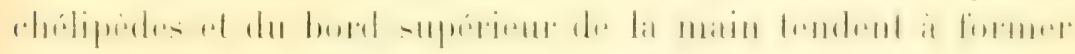
des fosselles.

Touvenu pour la mer Rouge. Mabite : iles Bonin (Slimpson).

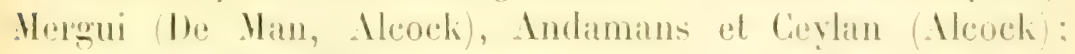
Penang (Lanchester); Zanzibar (Lenz).

\author{
(AENRE CYCIAOXNTIIOPS M. RATII, \\ (Cycloxanthus 1. Eilw, nom. procous.
}

Cycloxanthops lineatus ( I. M. - UlW.).

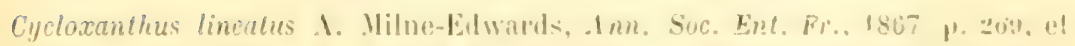

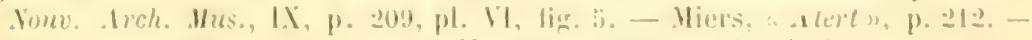
Henderson, Trums. Limn. Soc. (2), 1, 1893, p. 360. - Mlcock, loc. cit., p. 12\%. - Lenz, loc. cit., p. 3i9.

Iden (I. Jousseaume), un mite.

Largeur de la carapace. Longueur

Largeur du front.
19 millim.

13,5

(j) 
II y a meuf lignes ronges obliques de chaque coté de la carapace sur les paties laterates, el deux groupes de trois lignes longiludinales sur les parties postérieures de da carapare.

Yomera pour la mer Romge. Habite : Youvelle-Caledonie

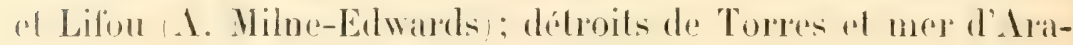
fura (Miers); Inde (Henderson); Zanzibar (Lenz).

\section{GenRe ETISUS FDW.}

\section{Etisus lævimanus Rand.}

Etisus lavimanus Randall, J. Acad. Nat. Sc. Philad., 1839, p. 11:. - Dana, loc. cit., p. 18., pl. Xl, fig. 1 a-b. - Kossmann, loc. cit., p. 30. - Alcock, loc. cit., p. 131 (uti sym.).

Etisus maculatus Heller, S. B. Acad. Wien, 43, P. 332.

Tijihouti (Y. Jousseatmes), un mile ef une lemelle: Obork

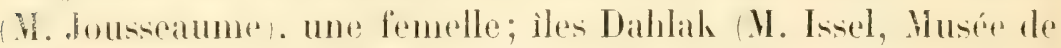
Gênes); mer Rouge (Mus. Turin), un màle et une femelle; Massaouah (Mus. Turin), un mâle.

La plupart de ces exemplaires offrent les petites taches brunes de $E$. maculatus.

\section{Genre E'TSODES DaNa.}

A. Des petites dents entre les dents du bord antéro-laté-

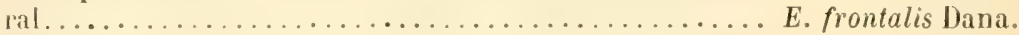

1. Pas de dents accessoires sur les bords antéro-latéга⿴囗十.

B. Longueur de la carapace égalant les trois quarts de la largeur. Surlace avec quelques lignes faiblement

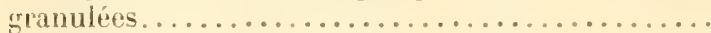

BB. Carapace plus allongée. Surface finement gra-

E. anaglyptus (Edw.).

E. electra (Herbsi).

Etisodes anaglyptus (Edw.)

Cancer anaglyptus II. Milne-Edwards, Atl. Cuvier, R. Anim. Crust., pl. XI, lig. 4.

Etisus anaglyptus H. Milne-Edwards, H. n. Cr., I, p. 411. - De Man, Nol. Leyd. Mus., XIII, 1891, p. 7.

Etisodes anaglyptus A. Milne-Edwards, Nouv. Arch. Mus., IX, p. 235. - Miers, "Alert ", p. 218. - Alcock, loc. cit., p. 133 (ubi syn.). - Lanchester, Proc. Zool. Soc, 1900, p. 739, pl. XLV, fig. כ̌. - Nobili, Ann. Mus. Napoli, I, n 3, 1901, p. 13. - De Man, 16h. Senckenb.Ges., XXY, 1902, p. 603̈. 


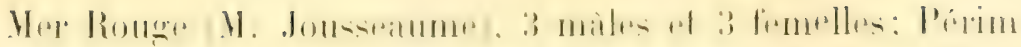
(Il. Jousseaume), un mile jeune; Massaouah (H. Issel, Mus. Gènes), 3 males; Erythré (Mus. Niples), 2 males.

Le plus gros male mesure lö millimetres de longuenr sur

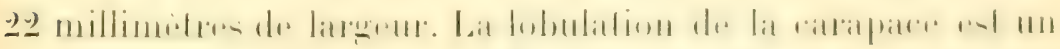
peu plus accentuee dans les femelles que dans les miles, et dans les jeunes plus que dans les adultes. Les lobules de lat

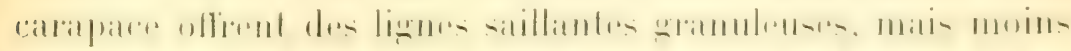
nombreuses et moins rógulieres que celles de E. elertill. Las

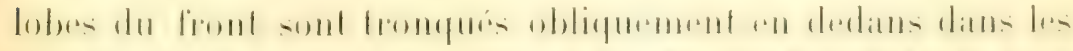

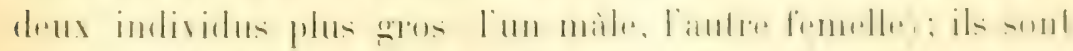

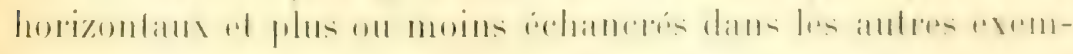
plaires. Les mains ont des lubercules disposés en sépies,

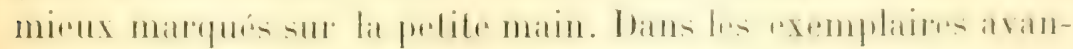

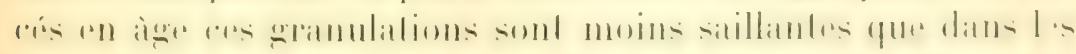

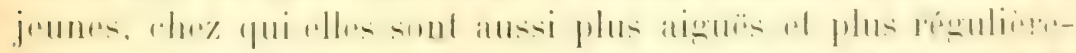

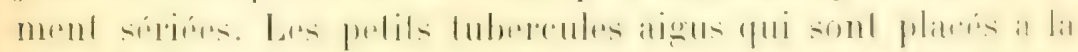

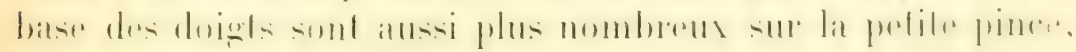
el plus aigus dans les jeunes.

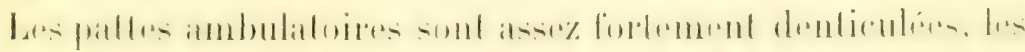

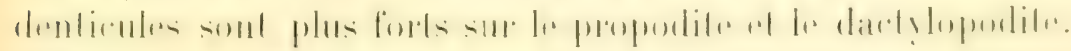

Dans les exemplaires mieux conservés la coloration rouge

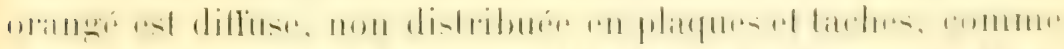

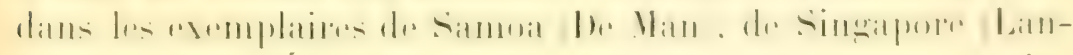
chester) et de l'Erylhree décrits par moi. La coloration noire du doigt fixe s'otend sur la patume; phos dans les jeunes qur dans les adultes.

Etisodes electra (Herbst).

Cancer electra Ilerbst, hrabb. Krebs., III, Is, p. 3i, pl. IJ, lig. 6.

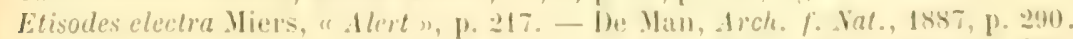
- Mlcock, loc. cil., 1) 1:33 (ubi syn.). - Calman, Trans. Linn. Soc. (2), I'lll, 1901, p. 7. - Borradaile, Tznth. maled. Laccad., p. 2033. - Nobili, Bull. scient. Fr. Bely., XL, 190ti, p. 1:20.

Etisodes sculptilis Ileller, S. B. Iliat. Wien, XIIII, 186i1, P. 233. - A. HilneEdwards, Nour. Arch. Mus., IX, 1873, p. 236, pl. IX, liz. 2.

Actaodes frontalis l'aulson, loc. cit., p. 27, pl. 1, liz., 3.

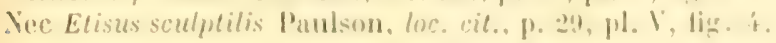

Her Rouge (11. Jousenume), 7 males et q femelles Djibouti (1). Coutiere), 19 males et une femelle; lojibouti (1) dous- 
seaume), ö males et unt femelle ; Assab (.VI. Issel et Buccertri, Mlus. (iènes), 2 màles et 2 femelles: Iden (II. Jousseaume), un màle; Obock (I. Jousseaume), 6 màles et 2 femelles.

Dans les jeunes la face externe de la main est loute couverte de petites granulations, qui dans les adultes se réduisent i la partie supérieure.

J'ai établi (lor. ril.) l'identilé de l'A rlipodes frontalis. Paulson arec celle espece: mais quant it la formo que Paulson appela Etisus sculptilis, je ne sais pas ce qu'elle est.

\section{Etisodes frontalis Dana.}

Etisodes frontalis Dana, loc. cit., p. 187, pl. IX, fig. 3. - De Man, Not. Leyd. Ius., XII, p. 8, pl. I, fig. 2. - Calman, Trans. Linn. Soc. (2), VIII, p. 7. ? Chlorodopsis frontalis Borradaile, Xanth, maled. Laccad., p. 261.

Djibouti (M. Jousseaume), une femelle; Ijjibouti (I. Coulière), 2 males et 2 femelles jeunes; mer Rouge (Nus. Turin), une femelle.

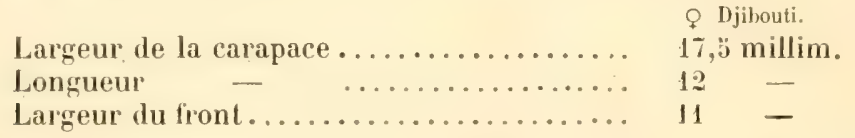

Le front a un double bord indistinctement et élégamment granule. Les a dents des bords latéro-antériems sont assez robustes; les deux premieres sont un peu obtuses, la troisième et la quatrieme sont spiniformes. Entre la deuxieme et la troisieme dent, il y a $2-3$ grinulations dentiformes. Une pelite dent semblable s'observe aussi entre la troisieme el la quatriemedent.

Les chélipèdes different un peu de la description de De Man. Ils offrent bien les mèmes ornements sur le carpe, le bord supérieur de la paume et des doigts, el aussi les mèmes lignes réticulées de petits granules: mais quelques-unes de ces granulations sont plus grosses et sont disposées en lignes longitudinales saillantes et plus ou moins bien marquées.

Cette rare espèce a été signalée dans la mer de Soulou (Lana), ¿ Upolu (De Man) et dans le détroit de Torres (Cal$\operatorname{man})$. 


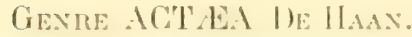

1. Doigts des chélipides tròs comprimés, Lanchants, cut-

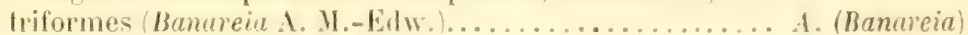

kraussi llell.

A A. Doigts des chélipèdes non ou médiocrement comprimés.

B. Pattes ambulatoires de forme ortinaile.

r. Catapatı lis-..................... bella (1)ana).

CC. Carapace couverte de petites granulations, ou de granules arrondis médiocres et épar's parmi les granulations petites.

D. Carapace beaucoup plus lare que longue (lon¿neur érale aux deux tier's de largeur ou moins). liords latéro-postérieurs fortement concaves.

$\boldsymbol{E}$. Doigts fortement excavés en cuillère. Carapace couverte d'un duvet noir coutt et tris dense..

LE. Doigts médiocrement excavés. Carapace portant des soies drues implantées parmi les poils.

DD. Longueur de la carapace plus que les deux tiers de la largeur.

F. Pattes lobulées comme la carapace.

G. Lobes de la carapace saillants et bien isolés. Sillons remplis d'un duvet bien visible.

!) Front très saillant, à échancrure médiane profonde; les lobes médians font un angle droit avee les externes. Bords latéro-antériems ilver rim lobes. . . . . . . . . . . . .

g!s. Front peu saillant, échancrure superdi(rielle; anzles des lobes medians larwement

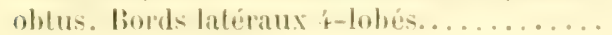

GG. Lohes de la carapace déprimés. Duvel des sillons presque invisible........................... FW. Pattes non lohulés, couredes de petits granules. Les granulations des lobes sont coniques, isolées, presque des tubercules........... 1. sabar Noh.

SCC. Carapace portant des tubercules. Patles tubereulées ou épineuses.

11. Catratee aver des tubercules isolés.

1. Hords latéro-antérieurs granulés mais non lubulin.

1I. Bords latín-interieurs lobulés.

L. Tubercules de la carapace peu nombreus, séparés par des sillons larges et lisses, des toulfes de poils longs sur la carapace ; front is lobes profondement echancres. Cramulations du bord supérieur des meropodites simplement coniques............... 1. nolulosa thih.

$L L$. Tubercules de la carapace déprimés et

1. tomentosa

(Edw.).

1. livsutissima Rüipl.).

1. rufopunctata

Edw.).

1. rufopunctatu var. retusu .iol.

1. speciosu (1)ana).

1. Helleri lidw. 
lisses; surface glabre; pattes avec de nombreux tubercules digitiformes............ polyacantha

(Hell.).

$L L L$. Tubercules de la carapace très nombreux, arrondis et subpédonculés; ceux des chélipèdes subsphériques et pédonculés; ceux des pattes clavés et fungiformes. Face inférieure du corps érodée.................. pisigera Nób.

HH. Carapace avec des tubercules confluents, formés de granules confluents.

$M$. Tubercules saillants, les granules qui les composent non entourés de ponctuation... A. granulata

(Aud.).

MM. Tubercules déprimés, les granules qui les composent entourés de ponctuations........ . calculosa (Edw.)

$B B$. Propodites et carpopodites des pattes ambulatoires carénés; les carènes laissent entre elles des cavités. A. fossulata (Gir.).

\section{Actæa (Banareia) Kraussi Hell.}

(P1. 10, fig. 4.)

Actac Kraussi Heller, S. B. Akad. Wien, V, 43, 1861, p. 316.

Nec Actæa Kraussi A. Milne-Edwards, Nouv. Arch. Mus., I, 1865, p. 260. - Nobili, Ann. Mus. civ. St. Net. Genova, XL, 1899, p. 258.

Voy. aussi Nobili, Bull. du Mus., 1905, n² 4, p. 235.

Djibouti (M. Coutière), un male ot une femelle très jeune; Djibouli (M. Jousseaume), une femelle avec aeufs: mer Rouge (Musée de Turin), un mâle el 2 femelles.

Cette espece est très caracteristiciue, mais elle n’a pas ité reconnue par $\Lambda$. Milne-Elwards. J'ai déja établi ailleurs / Inr. rit. que I’A. Krausvi I. M.-Edw. est une aulre espece, qui doit s'appeler A. Alphonsi Nob.

Cette espèce se reconnaît à sa carapace large, convexe, à sillons larges, peu profonds, glabres et tres nels, parmi lesquels les lobules nombreux de la carapace apparaissent comme des îlols granuleux et poilus.

Lit carapace est élargie, of uniformément arrondir en arc de cercle antérieurement; ses bords latéro-postérieurs sont concaves, un peu moins que dans A. hirstixima et tomentosa. Le front ne dépasse pas le contour général semi-circulaire de la partie antérieure de la carapace. Les bords latéro-antérieurs sont presque cristiformes, continus el à peine découpés en quatre lobes absolument non saillants. La carapace est uniformement convexe en sens longiludinal, et aussi, mais un peu moins. an sens transversal. Le rapport entre la largeur el la 
longueur de la carapace est de 1,3 dans la femelle et de 1,1 dans le màle. Le front est infléchi of divisé en quatre lobes,

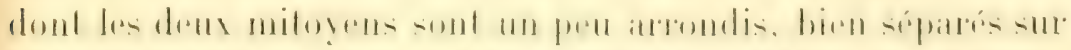

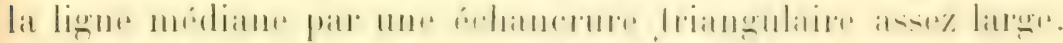

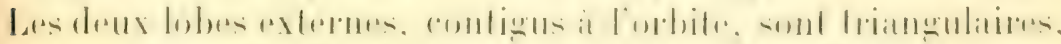

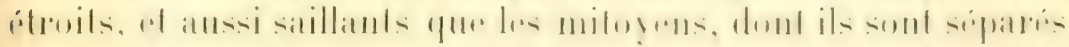
par un large simus arrondi. La surdace dorsale du front est

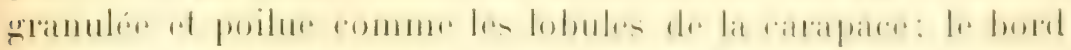

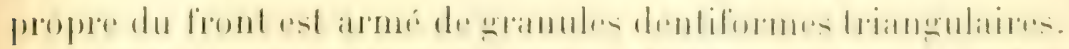

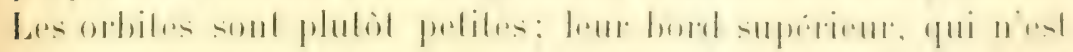
pas renflé ni accompagné par $11 n$ sillon post-orbilaire, esl

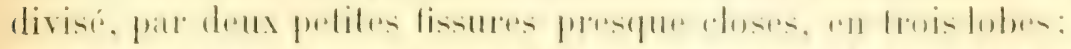

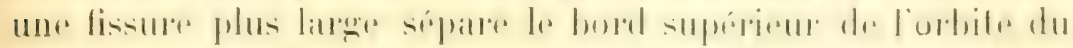

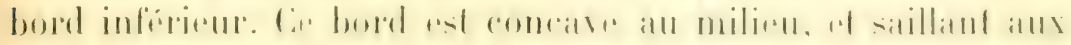
deux extrémités, sans former des vraies dents, atinsi qu'il paraitrait d'apres la description de Heller.

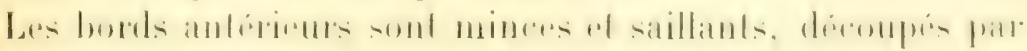

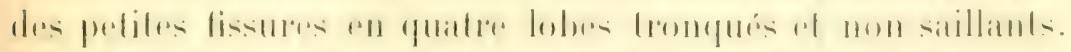

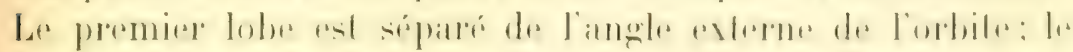

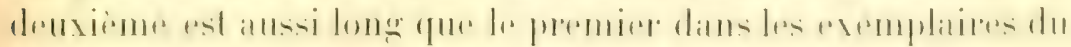

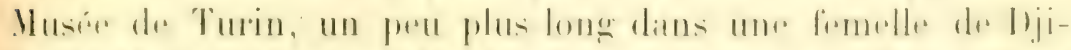

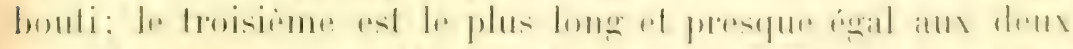

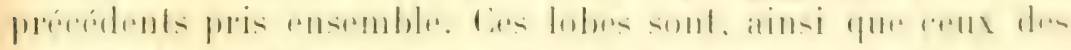

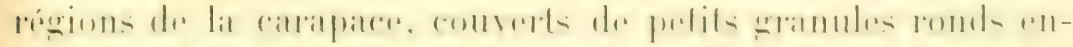

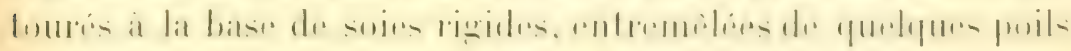

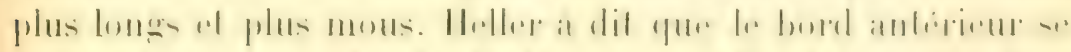

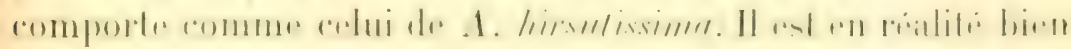

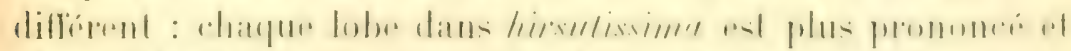

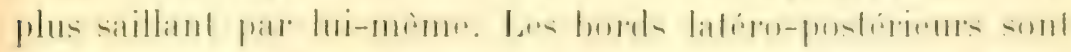
bien concaves, mais non marginés par un bord saillant, sauf dans le premier laist.

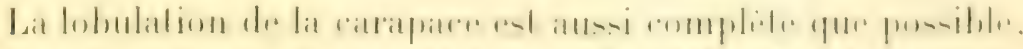

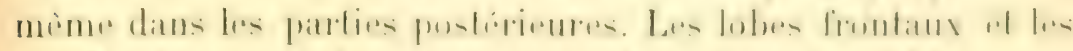

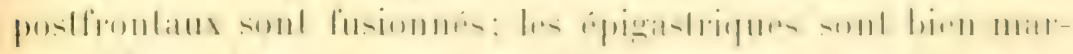

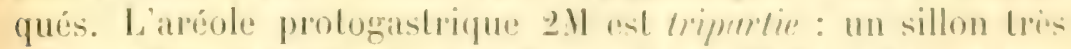

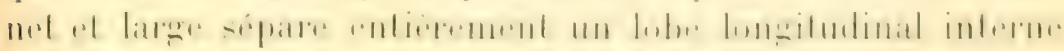
d'un lobe externe; celui-ci, yni ast plus large, est encore divisi

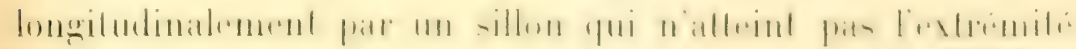


postérirure de l'aréole; ce lobe acquiert donc la forme diun $\mathbf{L}$. L. protongement anterieur de laire mésogatrique 3. II st détaché du corps de l'aire: l'aire propre est rncore divisie en deux parties; un sillon incompled sépare encore une pelite partie postérienre de ces deux moitiés. La région 4. II est divisie en yuatre tubercules. Les lobes latéraux sont lous sipares de ceux placés plus en arriere sont encore sublivises. öL et 6L sont partagés en petits lobules; les régions cordiale et intestinale 4t les régions postéro-latérales sont ausi fractionnesen lobules.

Le bord antérien du cadre buceal offre deux fissures bien neltes, mais moins larges que dans le spécimen ly pe de Banareia armala. Les maxillipedes externes sont poilus.

Les chélipiedes sont poilus, el le carpe ot la main sont poilus et granuleux ainsi que la carapace. Le mórus nofire pas de dents, mais seulement quelques petites granulations sur les bords parmi les longs poils qui les orment. Le carpe n a pas de sillier a l'angle interne: en dessus of en dehors, il offre les mimes granulations perlacées, entoures de soies, qu'on voit sur la cartpace. La main est grosse; chez les males la hauteur de la paume est égale a la longueur; la longueur de la paume est subegale a celle des doights chez les lemelles la main est plus petite. La main est lisse près de son bord inférieur, mais sur tout le reste de sa surface externe elle est hérissée de granules et poils identiques a ceux de la carapace, qui so disposent en rangées régulieres. La partie inférieure non granulée est quelque peu poncluée. Les doigts sont tres caractéristiques. Ils ne sont pas cylindriques el cannelés comme ceux des autres Actées, mais romprimés, tranchunts, cultriformes et lisses comme ceux de $B$. armulu. Chaque doigt offre 2-3 gros denticules arrondis près de sa base; ensuite les doigts sont lisses. Le doigt fixe est incliné un peu obliquement en bas, mais moins que dans $B$. armate. Les doigts ont une coloration brune; la pointe. qui est aiguä, ef une partie du bord tranchant sont blanes.

Les pattes ambulatoires sont gramulées et poilues aussi. Le sternum du male est granulí, celui de la femelle lisse.

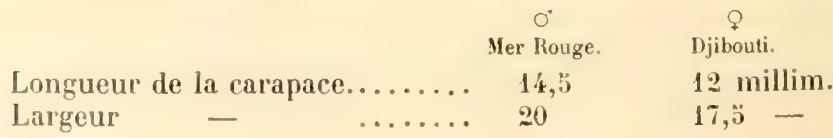


Par la forme de son bord antéro-lateral, par Jes régions

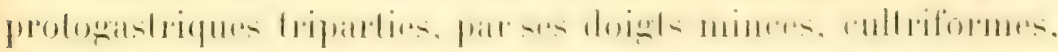

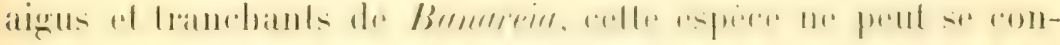

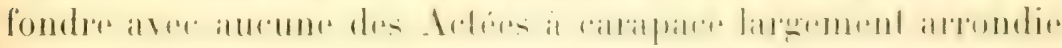
en avant el a bords postéreurement concaves. P'ar son bord

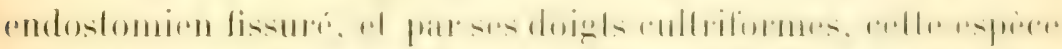

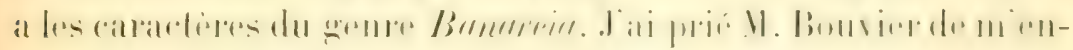
voyer le type de B. armaln $\Lambda$. M.-Edw. Cielte espece est lout a

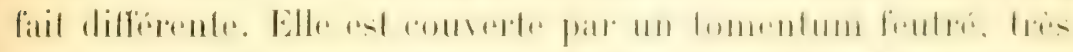

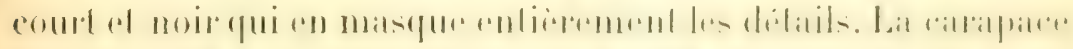

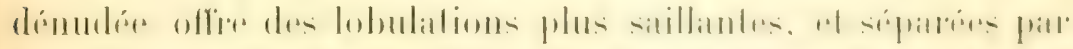

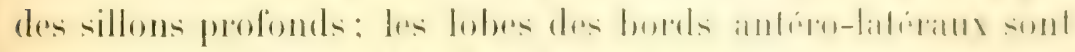

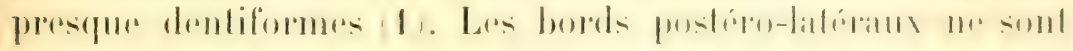

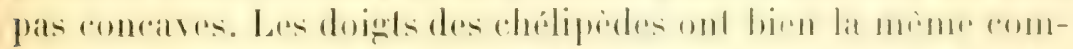

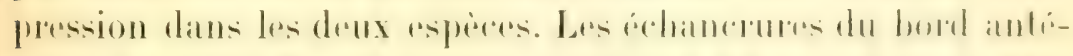

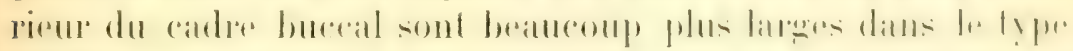
de $B$. armulne.

Je ne erois pas, d'ailleurs, que le genre Banarein, soit dis-

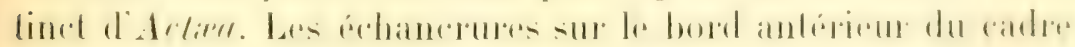
buceal s'observent souvent dans des Actées vaies, el si elles

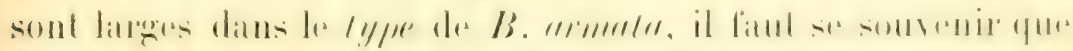

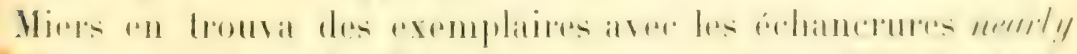

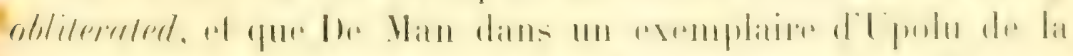
mème espece n'y trouva pas plus d'échancrures que dans un

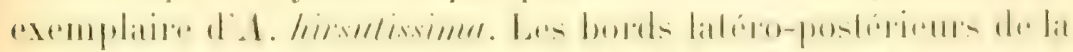

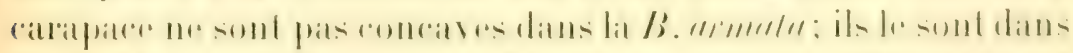

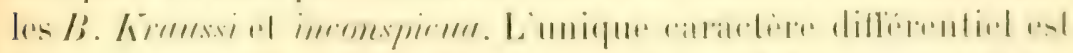

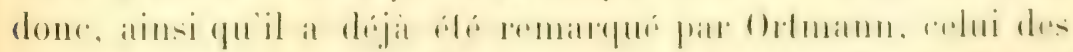
doigls; caractere qui ne me parait pas aroir it lui seul une

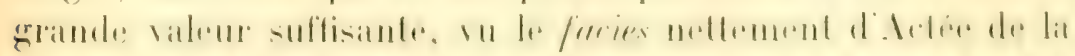

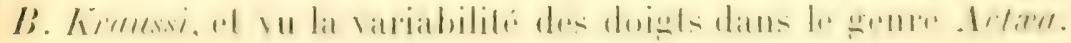

\section{Actrea bella (1)ana).}

Acticudes bellus l)ana, loc. cit., 1. 196, 11. X1, tig. 2. Actiea bella A. Milne-Edwards, loc. cit., p. 261. Carpilolles bellus Miers, Challeng. Brach., p. 13\%. - Cano, loc. cit.. 1. 199.

(1) La figure originale me parait rendre bien les conditions du type ot ne pas mériter les reproches de De Man. 
Une jeune femelle de Assab (exemplaire de Cano).

\section{Actæa tomentosa (Edw.).}

Zozymus tomentosus H. Milne-Edwards, $H . n$. Cr., t. I, p. 385, et Cuvier, Atl. R. Anim. Crust., pl. XI bis, fig. 2.

Actxodes tomentosus Dana, loc. cit., p. 197. - Heller, loc. cit., p. 328.

Actra tomentosa A. Milne-Edwards, Nouv. Arch. Mus., 1, p. 262. - Targioni-

Tozzetti, Crost. Brach. Anom. "Nagenta ", p. 35, pl. III, fig. 14, elc. - Ortmann, Zool. Jahrb. Syst., VII, 1893, p. 4:33. - Alcock, loc. cit., p. 140 (ubi syn.).

Her Rouge Musie de Turin); 2 males et une femelle; Aden (1I. Jousseaume), כ̈ exemplatres; Obock (M. Jousseaume), un mâle et 3 femelles; îles Musha (M. Gravier), un màle.

\section{Actæa hirsutissima (Rüpp.).}

Xantho hirsutissimus Rüppell, loc. cit., p. 26, pl. V, fig. 6.

Actxa hirsutissima De Haan, loc. cit., p. 18. - Dana, loc. cit., p. 164. - Heller, loc. cit., p. 314. - A. Milne-Edwards, loc. cit., p. 263. - Kossmann, loc. cit., p. 23. - Targioni Tozzetti, loc. cit., p. 37, pl. III, fig. 26. - Alcock, loc. cit., p. 141 (ubisyn.). - Doflein,Brach. Deutsch. Tief-See Exp., p. 102, pl. 32, fig. 1-2.

Her Rouge (I. Jousieaume), un màle; mer Rouge (Nusée de Turin), 3 males ot 3 trois femelles; Massaouah (MM. Issel et Beceari, Musée de Gènes), un maile; Obock (I. Jousseaume), 2 femelles.

Lat couleur des nombreuses soies drues de la carapace est Lantòt brun jaunatre clair: tantòt plus foncée; tantòt noirâtre.

\section{Actæa rufopunctata (Edw.).}

Xantho rufopunctatus H. Milne-Edwards, loc. cit., p. 389.

Actæe rufopunctata, A. Milne-Edwards, loc. cit., p. 268, pl. XVIII, fig. 1-1 a.Alcock, loc. cit., p. 142. - De Man, Abh. Senckenb. Ges., XXV, 1902, P. 697

Voy. aussi De Man, Not. Leyd. Ius., XIII, 1891, p. 2 (passim in descr. A. rugatx).

Arru (I. Jousseaume), 't miles at ö femelles; mer Rouge et Djibouli (I. Jousseaume), un màle; mer Rouge (II. Jousseaume), un mâle.

Cas exemplaires appartiennent it la forme typique décrite 


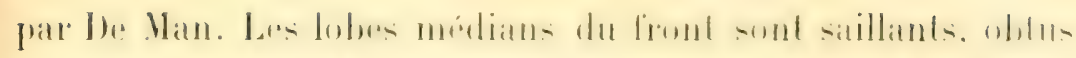

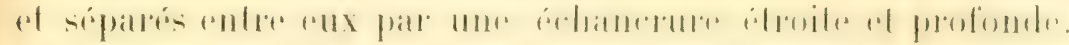
Le bord externe de ces lobes forme aver le bord interne des

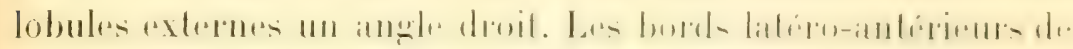
la carapace sont nettement découpés en cing lobes, dont le

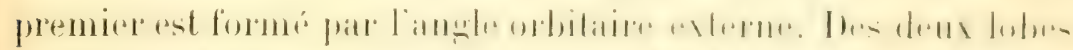

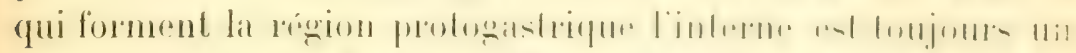

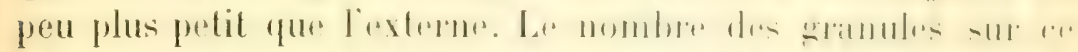

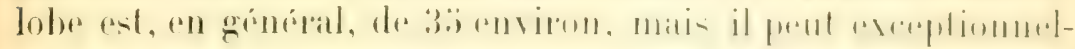
lement dépasser 40.

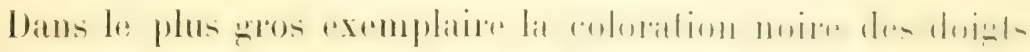
s'étend un peu sur la face externe et interne de la main ul sur presque tout le bord inférieur.

\section{Actæa rufopunctata var. retusa Nob.}

Actxe rufopunctata De Man, Not. Leyel. Ifus., II, 1880, 1). 172; ibid., 111, 1881, p. 96; H6h. Senckenb. Ges., XXY, 1902, p. 608.

Nobili, loc. cit., 1) 11.

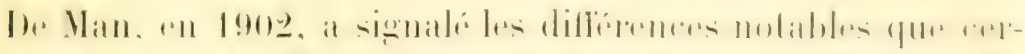

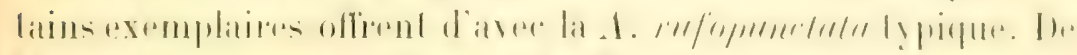

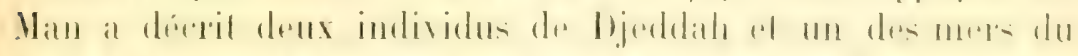

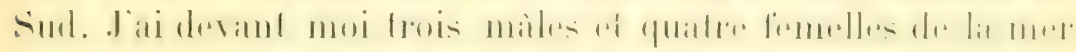

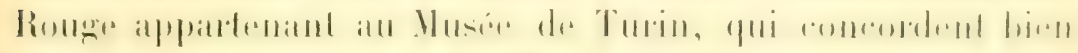
avec les exemplates de De Man, et different par les mimes caracteres des neul individus typipues de delen; ces carticteres sont:

1. Les lobes médians du front sont beancoup moins saillants; ils sont séparés entre oux par une exhancrure triangulate presque superficielle, non etroite at profonde commo

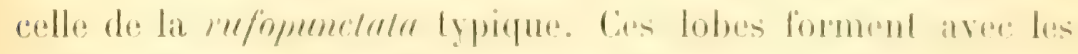
angles externes $u n$ angle latrement oblus, al parfois s'y rallatchent par une simple ligne concave.

2. Le premier lobe des bords latero-interiours as toujours

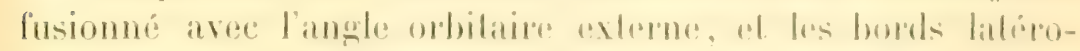
antérienrs résultent anosi divisis stollement en qualre lolues.

3. Les gramulations des lobules de la caraparer sont plus nombreuses el phas deprimes. Sur le lobe plerme do la rigion protogastripue, on comple on moyenne íi-is gramules. 
Ces ditlinences sont ronstantes. de crois que cette forme mérite d'être séparée, au moins comme variété.

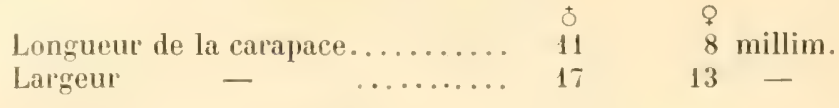

\section{Actæa speciosa (Dana).}

Actæodes speciosus Dana, loc. cit., p. 198, pl. XI, tig. 4. Actrodes nodipes Ileller, loc. cit., p. 329, pl. II, lig. 19. - De Man, Not. Loyd. Mus., II, 1880, p. 172.

Actæa speciosa Ottmann, Zool. Jahrb. Syst., VII, p. 4io. - Alcock, loc. cit., p. 143. - De Man, Lb/. Senclienb. Ges., XXV, 1902, p. 609. - Borradaile, F. Geog. mald. Laccad., I, p. 254 , lig. 42 c. - Lenz, Kool. Jahrb. Syst., XIY, $1901, p .460$.

Psaumis glabra Kossmann, loc. cit., p. 27, pl. I, fig. 4, pl. III, fig. 11.

Mer Rouge (Musere Turin), un maln: Djibouli M. Couliepes),

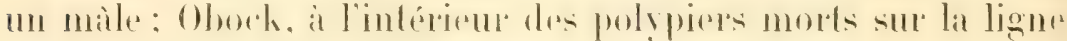
des hauts fonds du récif de la Clochèterie, à mer basse (M. Gravier), un màle; Arlen (M. Jousseaume), 2 mìles.

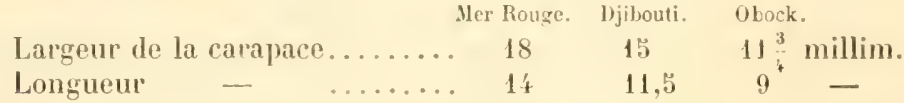

La longueu de la camapace est done un peu plus des 3/4

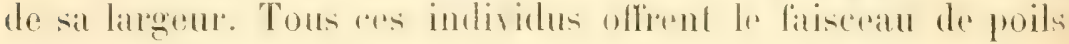
calacterisligue sur longle du dach lopodile des palles ambulat

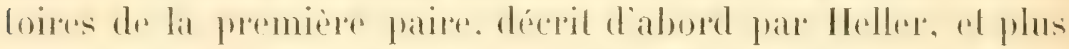

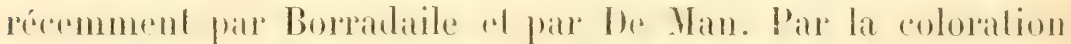
ils saceordent aussi arece la descriplion de Wr Man, mais les tatches ronges ne sont pas loujours distriburms sur les mèmes places.

Jai comparé solgmensement mes exemplaires avec la des-

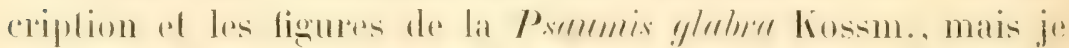

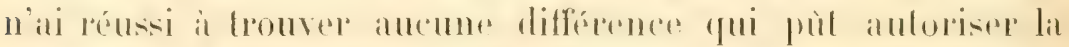

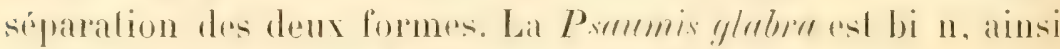
que Aleock s'en doutait, identique avec l'A. speciosa.

Actæa sabœa Nol.

(Pl. X, fig. 3.)

Nobili, loe. cit., p. 403.

Calle nourelle espece, représenté par mo ceptain nombre 
dans volveen toutes les granulations ont une lendance à se friduire, ol ainsi les granulations perlacés des lobules sont phes pelites el celles de la région mésogastrique of des parlies

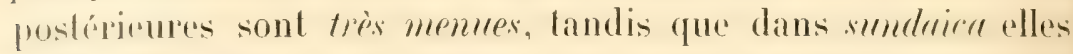
sont vésirnlenses el perlurres, bien que plus petitus que celles des parties lobuleses. Le nombre des granulations coniques sur les lobes parait ausid diflémont. Sur lo lobe externe de la région protogastrique, je compte 10-12 granules of quelpues autres

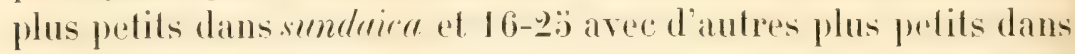
subeu. Dans sunduirr, l's arroles l' el le bord postérieur de la carapace ont des lignes de gromulalinns bien risibles, qui manquent absolument dans subeu, chez qui les granules de ces aréoles sont mime plus menus que ceux qui les précèdent. Les mèmes poils blouds aceompagnent les granulations dans les deux especes, mais ils pitraissent plus longs dans subrer. In duvet court se trouve entre les granules.

Les chélipedes sontsensiblement égatux dans les deux formes. Les gramulations sont plus faibles dans sabra, et celles de la partie inferieure de la face externe de la main sont arrangées en séries.

Les pattes ambulatoires sont ausi conformres igalement danslesteux especes, maislesgramulalionssontpedilesdanssuber.

La couleur est la même : rouge-pourpre.

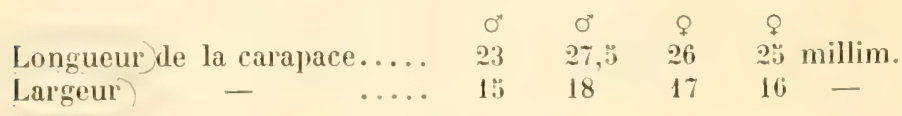

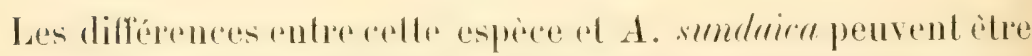
pésumées ainsi : bords latiraux nellement quadrilobés, lobulation bien marquée el a sillons inlerlobulaires larges: parlies postérieures de la carapace couvertes de granules tres menus et sans lignes saillantes pres du bord postérieur; granulations des lobes moins fortes et plus nombreuses.

\section{Actæa Hellerii A. M.-Edw.}

A. Milne-Edwards, Nouv. Arch. Mus., 1, 1865, p. 270, pl. XVII, fig. 3. - De Man, Not. Leyd. Mus., 111, p. 96 ; Arch. f. Nat., 1887, p. 261.

Mer Rouge (Musée de Turin), 8 mâles et 8 femelles. 
Les lobes mitoyens du front sont très avancés, mais arron-

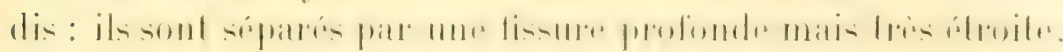

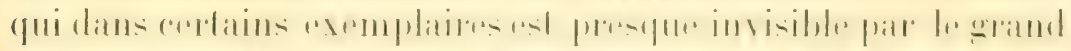

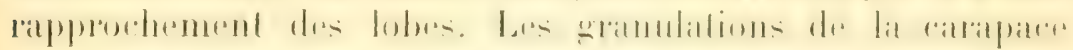

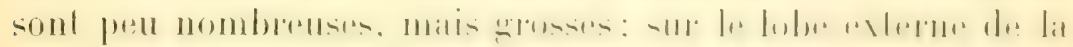

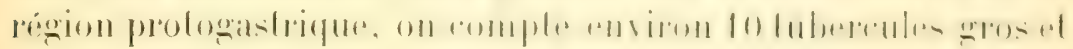
quelques autres pelits.

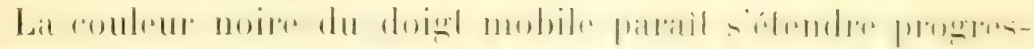

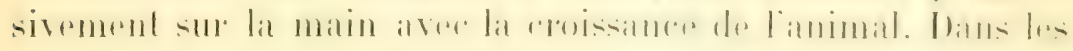
jeunes, la zone noire de la partie inférieure de la main est

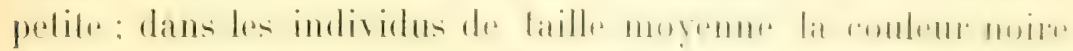

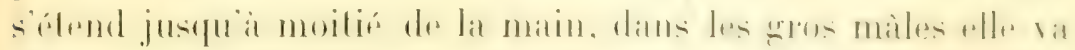
jusque près du bord postérieur.

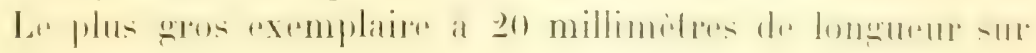
29 de largeur.

Cetle belle espèce n'est comnue que de Djeddah et d'Amboine, où elle a été signalée par De Man.

\section{Actæa nodulosa White.}

(l. X, fig. 2.)

Whith, Proc. Zool. Soc., 1847, p. 224. - Adams et White, "Samarang " Crust., 18'8. p. 39, pl. VIII, lig. 8. - A. Milne-Edwards, Vouv. Arch. Mus., 1, 186.;, p. 27T. - Alcock, loc. cit., P. 139, 1.t8 (ubi syn.).

Aren (II. Jousseaume), 2 f'emelles; Djibouti (II. Couliese). une fiomelle.

Ces exemplaires s'accordent mieux avec la description de Alcock qu'avec la ligure du Sammeme, parce que les lubercules sur le bord supérienr des ardictes des palles ambulattoires sont coniques el pointus, non claviformes comme dans la ligure.

La cartipace de la femelle plus grosse est large de 22 mu

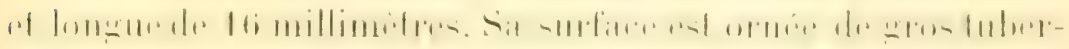
cules rouge orangé; les sillons interlobulates sonl lisses et larges. Les tubercules sont gros of an nombre redativemenl petil; sur le lobe externe de l'arre protogastrique, il y at

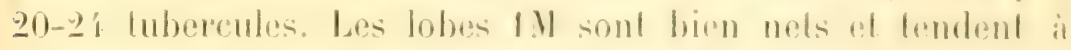
s'mir avec le lobe interne des 211. Les lobes postliontaux sonl distincts el granuleux. Tous les lobes antéro-latiraux ( $\left.\mathbf{L}_{2}\right)$ 
sont lien délimilés, mème l'aréole LL qui porte 16 lubercules. Les lobes postéro-latéraux ( $R$ ) ne sont pas séparés entre enx. Bien nettes sont les rigions intestinales 2I'; elles sont grossement fuberculées et sépracées par un sillon de la récrion cordiale of du bord postérieur granuleux. Liespace entre le bord postérieur de la carapace et une ligne imaginaire qui soit la contimuation du sillon gastro-cordial est aussi fuberculé, mais les fubercules sont moins gros que cenx qui couvrent les régions placées en avant de cette ligne. Liaréole mésogastrique 3.11 est tripartie, parce que deux sillons obligues se rencontrant en angle aigu relanchent le prolongement antírieur du rorps de l'aréole al divisent colui-ci en deux parties. All est aussi séparée de 3.II, alle est petile et pourvue de quatre tubercules.

Le front est bilobe ol chaque lobe st si profondément sinué que le liont apparail quadrilobé. Les deux lobes médians sont pourtant betucoup plus avancés; ils sont séparés par une frhancrue hrangulaire large. Le bord exterme de ces lobes est obligue; ils se rallachent alux lobes externes par un angle presque droil.

Les quatre dents du bord latéro-antérieur sont bien dislincles. plormes dem amas moreliforme de granulations w lubereules analogues a rens des lobes de la carapace. La pres-

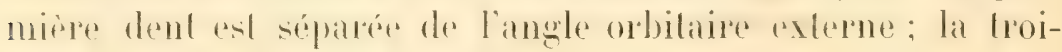
sième dent est la plus longue.

Lat carapace est glable dans les sillons of entre les lubereules. mais elle portre quelques loufles de longs poils brums disposés

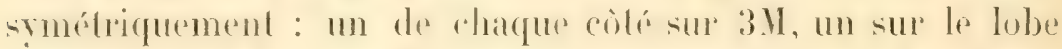

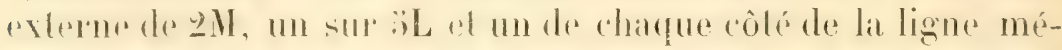
diane sur la région cordiale. Quelques petits groupes de poils s'observent aussi près des bords.

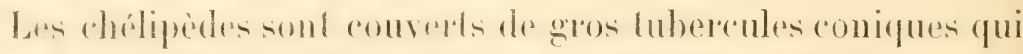
deviennent plus pelilo rers la partie infórieure de la main el qui lemdent a so disposer en rangées. Les doig̨ts sont sillonnés ol gramuleax pres de: la base: leur pointe n'est pas creusée en cuiller.

Lu palles ambulatoires sont fortement gramulese et leurs londs sont pourves de longs poils touffus. Les granulations de 


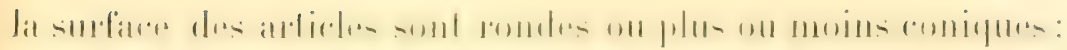

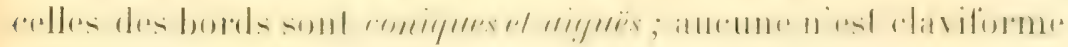
comme dans la figure du Strmerrireng.

Cetle espèce a éti trouvée dans le golle P'ersique, danst'Inde, it Maurilius el aux iles Andamans.

\section{Actæa polyacantha (Hell.)}

Chorodius polyacunthus Heller, S. B. Akad. Wien, XLIII, 1. 339, pl. III, fis. 21. Actea polyacentha Ortmann, Zool. Jaher. Syst., III, 1893, 1. 43:i.

Pilodius fragifer Paulson, loc. cit. (nec Adams et White).

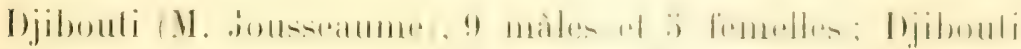
(II. Coulière), un màle; mer liouge (II. Jousseaume), une femelle; mer houge (Husée Turin), une femelle.

La position de cette espece est assez doutense. M. Heller la plaça dans Chlorodius, mais en ayant soin d'ajouter que sir position dans ce genre nur eine procisorische sei lirmen.

Ortmann, qui en vil un exemplaire de localité inconnue, lat plaça daus Arlex, près de A. acuntha. Nlcock a mème émis le donte que cette espece soit identique arec $A$. pernnii.

La position dans le genre Actarn me parail la plus probable, d'autant plus qu'elle a dans re genre un proche parent dans A. symosissime Bor's.

Le plus gros exemplaire, une lemelle, est long de 9, ö millimetres el large de 13 millimetres.

\section{Actæa pisigera Nol,.}

1'1. I. lice.1.

Aobili, loc. cil., fr. 40 '

Her Rouge (Musée de Turin), 4 individus.

Cielle espece est bien carateléisée par lat forme des lubercules arrondis el pédonculés qui ornent sit rabapatee at ses pattes.

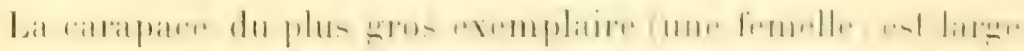

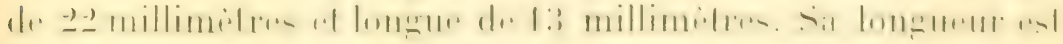

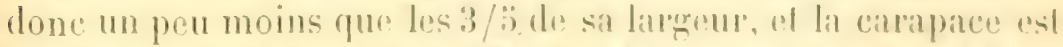

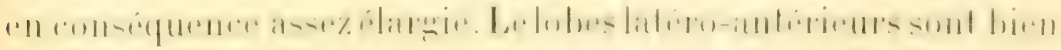

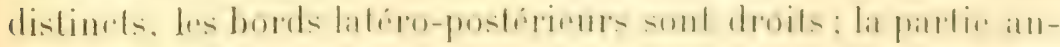


Léricure de la carapace décrit une couble a rayon assez larger. Toutr la surface de la carapace est couverte de gros lubereules pixifmmex, dont quelques-uns près des bords sont distinctement pédonculés. Cés Lubercules couvrent la surfice entiere de la carapare, mais, par suite de leur grosseur, le nombre en est petit; ainsi sur le lobe exlerme de la région prologastrique on ne comple que qualor\%e tubercules. Sur les régions en arriere de la dernière dent latérale les tubercules se font plus petits : sur la région cordiale ils tendent a se fusionner, en laissant entre eux de petites fossettes; ceile région apparait ainsi érodée. Sur le bord postérieur et sur les 2P ces granules sont disposés en rangée linéaire simple, interrompue au milieu sur 2P, et continue sur le bord postérieur.

Les gros tubercules de la partie antérieure de la carapace. examinés de près. apparaissent le plus souvent formés par d'autres granules at fubercules plus pelits. La lobulation de la carapace est complete dans la partie antérieure, mais par la grosien des granules les limites entre les lobes se laissent mal voir. Les aréoles de la région gastrique sont les plus nettes,

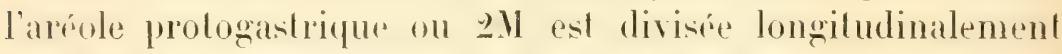
mair non completement; le lobe exterue est plus large que l'interne.

La front est divisi neltement sur la ligne médiane par une fisidre triangulaire ascer lange en deux lobes arrondis, qui sont beatucoup plus sallamts que les denx pedits lobes externes, aver lesfuels ils se rallachent par un angle aigu. Les orbites sont entoures ansi de gros tubercules pisiformes, qui sont aussi glos que lespare ontere chaque fissure; le bord orbilaire de cetle facon as entoure par 7 gros tuberentes. Les pédoncules oculares sont aussi luberculés. Lees lobes du bord anlirieur sont bien séparés, mais les deux premiers peu visibles par la erosedur des lubercules qui les comrent. Le premier lobe est sépaté de l'angle orbitaire "xterne: le premier el le deuxieme lobe sont arrondis, les deux suivants bien saillants.

Lat plus grande litrear de la carrapace est entre les lobes de la froisieme pare. Les bords latéro-postériems sont légèrement plus longes que les latéro-antérienr. Le bord postéreur est rectiligne. 
Les parties inférieures du céphalothorax sont tuberentées

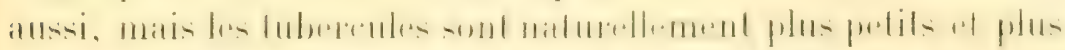

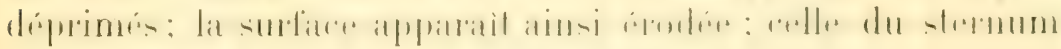
est grossement érodée.

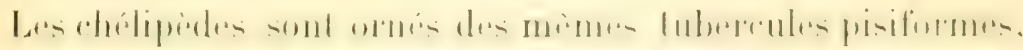

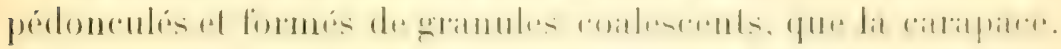
Le carpe a une saillie triangulaire interne. La main est ornée

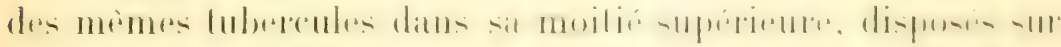

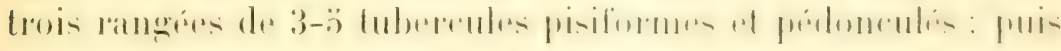

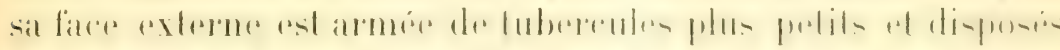

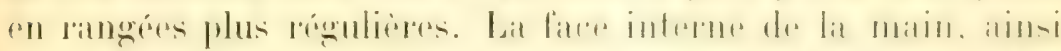

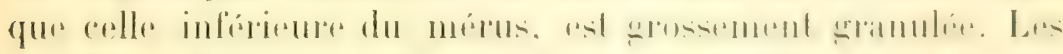

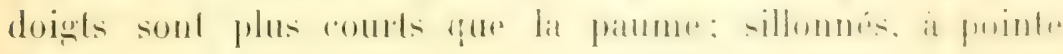
aiguë.

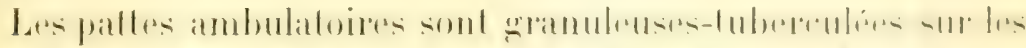

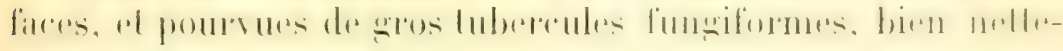
ment pédonculés sur le bord supérieur.

\section{Actæa granulata (Aud.).}

Savigny, pl. VI, fig. 2.

Cancer gramulatus Audouin, Expl., pl. 1. 87.

Cancer Sacignyi H. Milne-Ldwards, loc. cit., ]). 378.

Actæa granulata A. Milne-Edwards, loc. cit., P. 273. - Alcock, loc. cil. 1) 1301 (ubi syn.). - Nobili, Bull. seient. Fr. Bely., XL, 1906, 1. 127. - Stebling, South. 1fr. Crust., III, 190:, p. 30 .

Actaca Savignyi hossmamn, loc. cil., p. 23. - Ciano, loc. cil., p. 189.

Eucanthodes gramulutus Paulson, loc. cil., p. 30, pl. VI, fig. 3-3a.

Beifoul, profondeur 8 metres (M. Orsini, Musée 'Turini, une fenelle.

\section{Actæa calculosa (lidw.).}

Cincer calculosus 11. Milne-Liwards, loc. cit., p, 378.

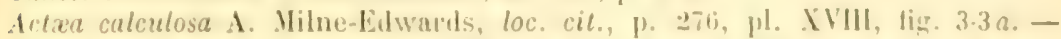
Calman, Trans. Linn. Soc. (2), VIII, p. 8. - Yobili, Ann. Mus. Zonl. Yapoli, $1, n^{\circ} 3, p \cdot 13$.

Euxanthus tuberculosus Miers, Zool. Alert, p. :20.i, pl. XIX, tig. A.

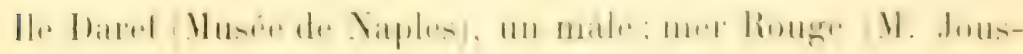
seaume), un màle et une lemelle.

Le màte de l'ìle Daret a été déji décrit par moi (lor. cil.). Les 
individus de "mer Rouge " ont les lubercules relativement déprimés ot formés parr des granules aplatis et confluents. médiocrement distincts les uns des autres et entourés de ponctuations assez nelles. Les pattes ambulatoires n'ont pas de tubercules spiniformes. Mais dans ces deux exemplaires le rapport entre la longueur el la largeur est plutoil de A. granulata:

\begin{tabular}{lccc} 
Longueur de la carapace........ & \multicolumn{1}{c}{} & 9 \\
Largeur & - & 12 & 12 millim.
\end{tabular}

Actæa fossulata Gir.

Cancer fossulatus Girard, Ann. Soc. Ent. Fr., 1859, p. 149, pl. 1V, fig. 2-2b. Actæa Schmardæ Heller, Sitz. Acad. IVien, XLIII, p. 318, pl. I, fig. 13. Actza fossulata A. Milne-Edwards, Nouv. Arch. Mus., 1, p. 279. - Alcock, loc. cit., p. 279. - Nobili, Ann. IIus. Zool. Napoli, I, no 3, 1901, p. 14. Psaumis fossulata Kossmann, loc. cit., p. 27, l. 1, fig. 3.

Mer Rouge et Ljibouti M. Jousseaume), 9 màles el 6 femelles. Massitouah MM. Issel et Beccari, Musée Gènesj, 7 màles et כ̃ femelles; ìle Scheik-ul-Ibu (Musie de Naples), 6 màles of 4 femelles; Djibouti, sur les Polypiers virants (H. Gravier). un mâle.

Ces exemplaires offrent une variabiliti remarquable quant aux fosselles de la carapare of des chélipedes. Parfois les fossettes sur le bord supérieur de la main sont si mal délimitées que cette partie de la main parait idre simplement érodée, parfois lesfosselles sont bien nettes, profondes, a fond lisse ef a bords granulés, disposéns soil parallelement a la petite du bord supéro-interne, soit en réseau.

\section{Gexte Chlorodtes (Edw.) A. M.-Edw. \\ Ghlorodius niger (Forsek.).}

Cancer niger Forskal, loc. cit., p. 89.

Chlorodius niger Rüppell, loc. cit., p. 20, pl. IV, fig. 7. - H. Milne-Edwards, H. n.Cr., I, p. 401. - Dana, loc. cit., p. 216, pl. XII, fig. 5 a-c. - Paulson,

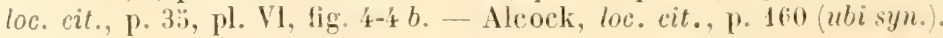

Très nombreux individus de Massaonah, Mden, Mssab, Djibouli, Obock, ele.

11. Cimarer a pris a bjibouti des exemplaires de celle espece virant par couples dans les Hirciniu. 


\section{Chlorodius bidendatus Nob.}

Chlorodius sp. Nobili, Amn. Mus. civ. Genova, XI, 1899, p. 258.

Chlorodius bidentatus Nobili, Ann. Hus. Zool. Nipoli, I, n 3, 1901, p. 1\%.

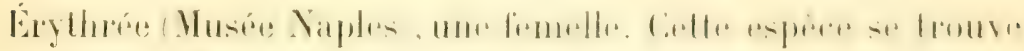
aussi dans la Nouvelle-fiuinée.

\section{Chlorodius lippus Nob.}

(P. X, fig. 8.)

Nobili, loc. cit., p. 405.

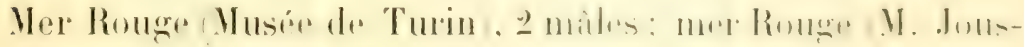
seaume), un màle, une femelle el un màle jeune.

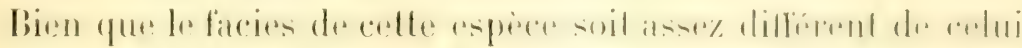

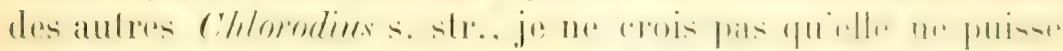

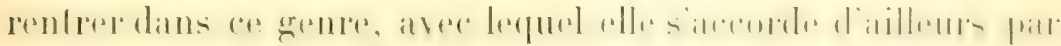

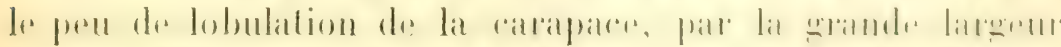

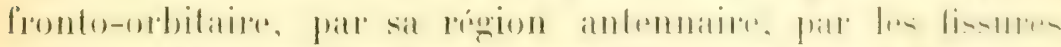

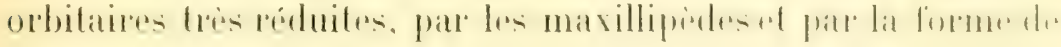
l'abdomen du mâle.

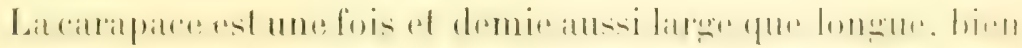
bombée, et suns truce de régions, si ce n’est le sillon en I qui

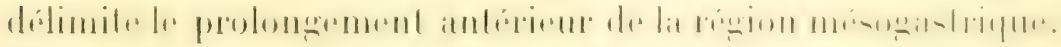

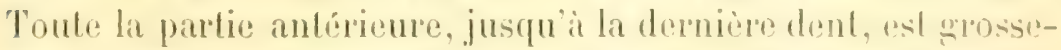
ment ponctuée; les ponctuations étant visibles it ail nu; lit carapace est d'ailleurs lisse el sans gramulations. Las parlius antéro-latérales nont aucune trace de lobulation, axceptí les lobes des bords. Lat distance entre les angles extermas des

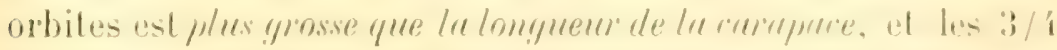

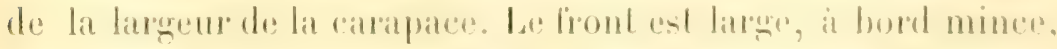
fablement achancer au milien of at angles exhermes oblus mais

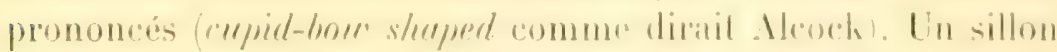

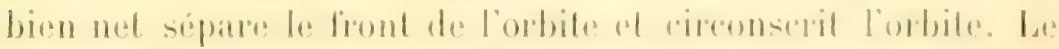

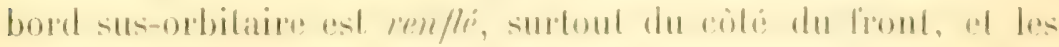
fissures y sont encore moins appatrentes que dans nigper alles

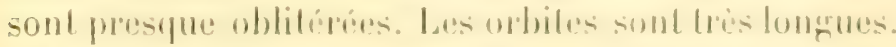

Le sternum est errosisement ponctur.

Les chélipedes sont subégitux. Les bords du bras sont tres 
finement granuleux, mais nont aucume saille. Le carpe est

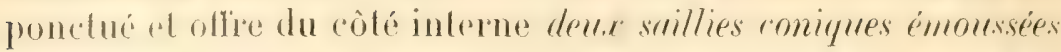
placres l'unc a l'angle interne, l'autre au-dessous de celle-ci. Les mains sont aussi lisses, ponctuées, et offrent sur la face externe un sillon parallèe d voisin au bord supérieur, qui, dans sa fartie antérieure. frud its'effacer avec l'àge. Les doights sont un peu plus longes que la paume, noirs, tres peu courbés, presque droits et excavés au boul du cooté interne. Le doigh mobile a 2 dents distinctes dans sa partic basalde; le doigh fixe "st grossement dente. Dans les jeunes les doigts sont faiblement sillonnis, dans les adultes les sillons deviennent des lignes de ponetuations superficielles.

Les paltes ambulatoires sont grêles el élanréex avec quelques longs cils rates sur les derniers articles. Les bords du méropodite sont fabblement granules; eeux des autres arlicles sont lisses. Les dactylopodiles, qui sont assez longs el grèles. poltent sur le bord inférieur et ì l'extrémité du bord supérieur des petites spinules cornées, comme celles de chl. burbrums Borr.

\section{Genre PHyMOdUS (Edw.).}

\section{Phymodius ungulatus (Edw.).}

Cf. Phymodius ungulatus Alcock, loc. cit., p. 162 (ubi lit.).

Nombreux "xemplaires d'obock M. Jousseaume); Djibouti (M. Coutière): Djibouti (M. Maindron); mer Rouge (Nus. Turin); Erythré Mus. Naples); Massaouah et Assab (MII. Jssel ot Beccari, Mus. Gênes).

\section{Phymodius monticulosus (Dana).}

Chlorodius monticulosus Dana, loc. cit., p. 206, pl. XI, fig. $9 a-f$.

Chlorodius obscurus Lucas. Voy. Astrolabe Zool., III, p. 26, pl. III, fig. 4.

Phymoclius obscurus A. Milne-Edwards, Nouv. Arch. Mus., IX, 1873, p. 220.

Phymodius monticulosus De Man, Zool. Jahrb. Syst., VII, p. 324. - Alcock, loc.

cit., p. 163 (ubi syn.). - Nobili, Annales Mus. Nat. Hung., 1905, 11, p. 490. Nec Yh. obscurus De Man, Not. Leyd. Mus., II, 1880, p. 174.

(Obock (11. Jousseaume), 3 malles; ces exemplaires concordent parfitiment arec la description d'Aleoch et avec la figure de Ph. obscurus Lucas. 


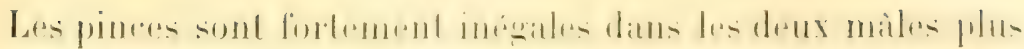

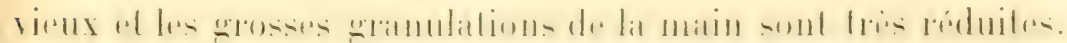

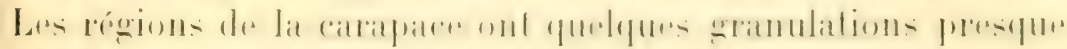
oblitérées.

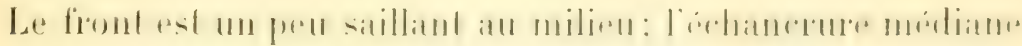
est très faible.

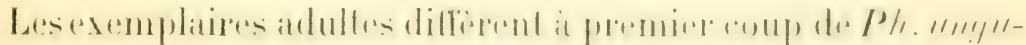

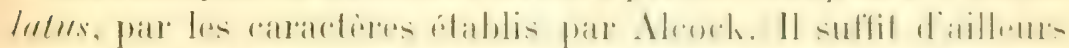

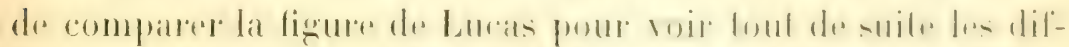

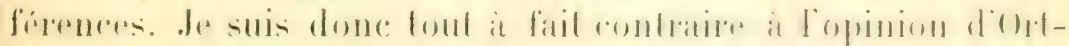

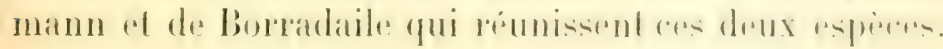

Longueur de la carapace........ $19 \quad 16$ millim.

Largeur $\quad-\quad \ldots \ldots \ldots \ldots 20 \quad 22 \quad-$

Phymodius sculptus A. M.-Ewd.

Chlorodius sculptus A. Milne-Edwards, Nouv. Arch. Mus., IX, p. 217, [1. VIII. lig. 4. - De Man, Yot. Leyd. Mus., III, 1881, p. 98, et Arch. f. Fatury., 1887, P. 279. - Ortmann, Zool. Juhrb. Sysl., VII, P. \&66. - Nobili, Ann. Mus, Lat. Hung., 190:, 11, p. 490.

Phymodius sculptus Alcock, loc. cit., p. 16; (ubi syn.).

Men Rouge (Musée Turin), 9 màles, une femelle.

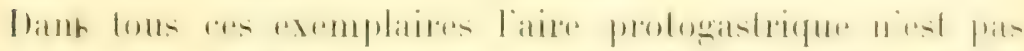

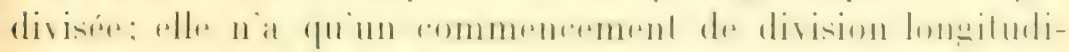

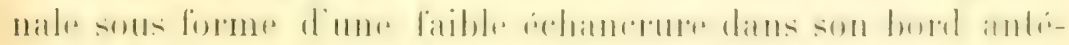
rieur. Lincision médiane du front est plus nette dans cette espece que dans les deux qui précedent. Les chélipedes sont

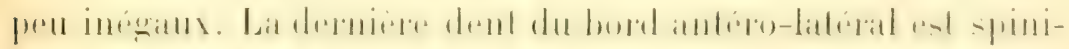
forme meme dans les adultes.

\section{Phymodius granulatus ('T'irn",-l'ozz.).}

Savigny, pl. $Y$, figr. 6 .

Pilodius granulutus T'argioni-Tozzelli, Crost. Brach. Anom. "Mayenta ", p. sio,

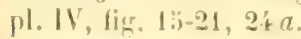

Hhymodius obscurus De Man, Fot. Leyd. Mus., II, 1880, \%. I7 (nec Ph. obscurus Luc.)

Phymodius grenulutus Nobili, Amn. Mus. Nat. Hun!., 190\%, II, 11. +10.

Djibouli (M. Mandron), un màte; mer Rouge (Mus. Turin). 2 màles; mer liouge (V. Jousseaume), un mile jeune: Dji- 
bouli . II. (navier), un male; Djibouti (M. Coulière), une femelle; Obock (M. Jousseaume), un mâle.

letle sipèce très intéressante et bien caractérisée avait été liguré par Savigny; mais Audouin ne lui donna aucun nom, el it la page 86 deson Expliculion des plunches, il se borna à en observer d'une façon sommaire les affinités et les diffirences d'arec plusieurs especes figures prar Ilerbst. Elle fut décrite en 1877, par le professeur Targioni-Tozzetli de Florence, d'après des exemplaires que le Musée de Florence avait reçus du général Clot-Bey, et qui provenaient probablement de la mer Rouge.

La description très précise de Targioni-Tozzetli sadapte parfaitement à mes exemplaires.

Ph. gromulatux se reconnait aisiment à sa carapace à lobulation bien nette, it ses lobules séparés par des sillons étroits el profonds, ¿̇ ses dents latérales plus átroites et moins arondies, dont la derniere est loujours spiniforme, et a son front nettement quadrilobé.

Les deux lobes moyens du front sont séparés par une échancrure nette; ils sont larges et arrondis; les deux lobes externes sont aigus, obliques, saillants et beaucoup plus distincts que ceux de loules les autres rsperes du mène genre. Les lobes de la carapace sont finement granulenx; les gramulations pres des còtés et dans la partie antériemp sont rondes, perlacées, et près du front of autour dro l'orbite se disposent en lignes.

Les chélipides ne different pas sensiblement de ceux de $P h$. zingulatus.

Les patles anbulatoires sont très poilues, a poils plus longs que ceux de ungulalus, et resisemblent beaucoup sous a pespect ì ceux de srulplus. I a pointe des doigts nest pas biunguiculée, parce que seulement l'onguicule corné lerminal est bien développé, l'autre est a peine plus gros que les denticules du bord inférieur.

Les jeunes ont les bords latero-antérieurs tout at fait spiniformes. Dans la femelle de Djibouli les eranulations sont beancoup plus fortes ef plus abondantes que dans les males.

La description que De Man a donnée d'un male de Djedilah allribur par lui a $P /$. olsemmes, sadaple de tout point a $P /$. granulatus. 


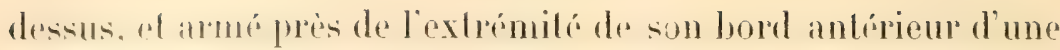
longue fiphe, son bord superient est denticule. Le carpe a atusi une ipine bien netle du còte interne, ol sa surface est orme de nombrenx petits luberenles arrondis. Les deux mains sont granuleuses, ou tuberculées sur toute leur surface "xterne; la partie infériener de la surface externe est finement granuleuse, la partie supérieure est tubereulers les tubercules du bord superieur, rus de profil, apparaissent mime dentiformes. Les doigts ne joignent pas sur la grosse pince des individus plus gros; ils joignent sur la petite pince; ils sont dentés et excavés au bout.

Les palles ambulatoires ont une longueur médiocre. Les méropodites sont finement denticulés sur les bords supérieurs: les carpopodites et les propodites aussi, mais plus fortement. Ces articles offrent aussi des longues soies. Les dactylopodites nont pas d'onguicule a l'extrémité, mais leur bord inférieur offre parfois des traces de denticulation.

La surface inférieure du corps est lisse.

Longueur de la carapace.

Largeur
4 millim.

$4,5 \quad-$

\section{Pilodius Martensi (Krauss).}

Menippe Martensi Krauss, Sudafr. Crust., 1843, p. 34, pl. III, fig. 1. - Hilgendorf, Decken's Reise Ostafi. Crust.

Trois exemplaires recueillis pat M. Jousseaune dans une localité non déterminée de la mer Rouge, me paraissent appartrnir à Menippe Marlenxi dont Hilgendorf signala la présence dans la mer houge. Hes exemplaires s'accordent parfaitement avec l'espece de lirauss. Toutefois cette espece n'a rien it faire avec une Menippe, tel que ce genre est entendu aujourd'hui. el ainsi que l'avait déja reconnu M. De Man. Elle a au conlaire une très grande affinité avec les deux espèces de Pilodiux décrites ici $(\mathbf{1})$.

(1) J'entends ce genre dans le sens récemment déterminé par M. De Man (16h. Senck. Ges., XXV, 1902, 1.619), non dans l'ancien sens de Dana qui comInenait aussi les Chorodopsis. Les trois esperees que j'ai vues ont tries peu de poils sur la carapace, et, par leur forme, tendent à se rapprocher de Chlorodius dont elles difrèrent surtout par leurs pattes ambulatoires dentées. 


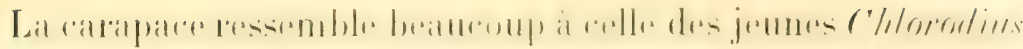

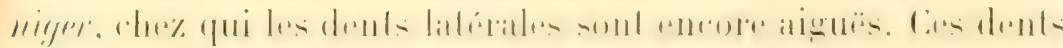
sont au nombre de 4 , dont la premiere el la quatrieme tres

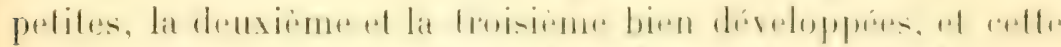

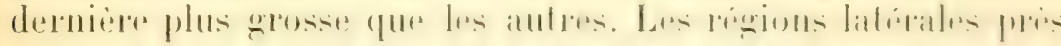

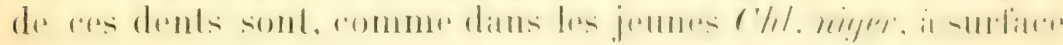

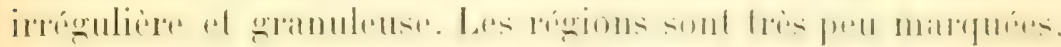
mais mieux distinctes que dans $P$. armiger.

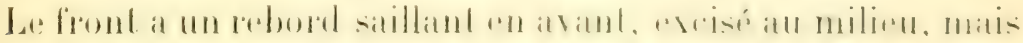

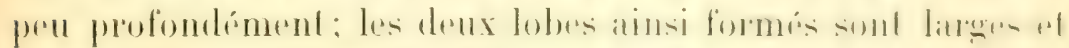
un peu obliques; les petits lobes ne sont pas séparés.

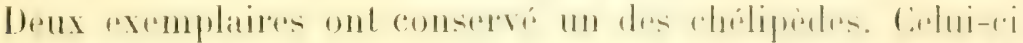

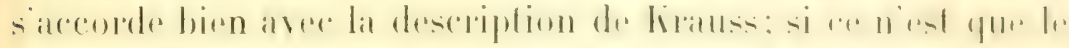

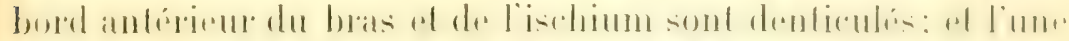

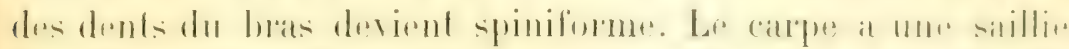

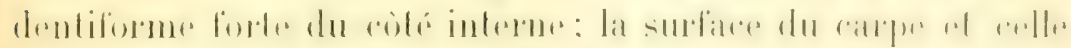
de la main sont très finement chagrinées.

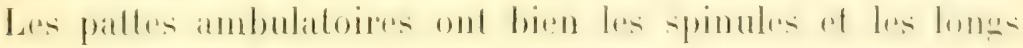

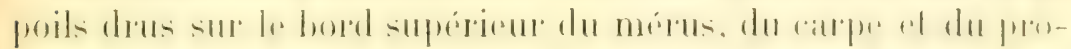
podite quion observe dans les antres especes de Pilodius. Le

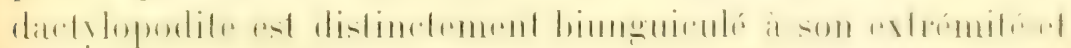
denliculé sur son bord inférieur.

Longueur de la carapace.............. 5 millim.

Largeur

\section{GiERE GHLOROIJOPSIS A. M.-EIMI.}

\section{Chlorodopsis areolata (E)}

Chlorodius areolatus 11. Milne-Edwards, H. n. Cr., I, p. 400.

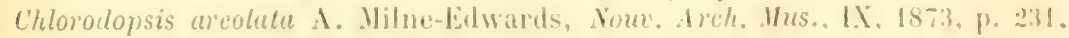
pl. VIII, fig. 8. - Miers, Zool. Alert, p. \$332. - De Man, Yot. Leygl. Mus.. XII, 1890, p. 34. - Alcock, loc. cit.. P. 1 lib (ubi s!ln.)

Mer houge (M. Jousseatume), 2 miles ul 2 femelles.

La carapace de celle spece a mose lobulation complite. Les lobes postfrontaux of les iphigistriques sont bien nots. Lai

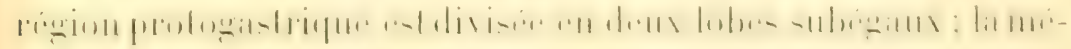

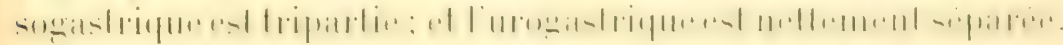

Les gramulations sonl atsog nombreuses; sur le lobe problogastrique externe on comple environ trente granules. 
Les granulations de la face externe de la main sont disposies en rangées longitudinales régulières. La coloration noire du doigh tixe s'étend sur la main dans les màles; elle s'arrète à la base du doigt dans les femelles. Celles-ci sont pourtant plus petites, et il est possible que la coloration s'étende avec l'ìge.

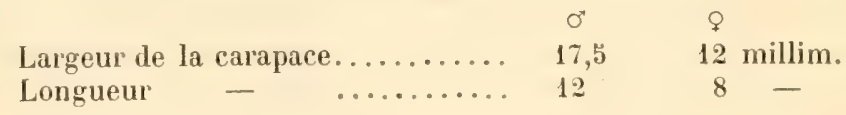

\section{Ghlorodopsis spinipes (Hell.).}

Pilodius spinipes Heller, loc. cit., p. 340, pl. IIl, fig. 22.

Etisus spinipes Paulson, loc. cit., P. 30, pl. VI, fig. 1.

Chlorodopsis spinipes A. Milne-Edwards, Nouv. Arch. Mus., IX, p. 230, pl. VIII, fig. 6. - De Man, Arch. f. Nat., 1887, p. 282. - Nobili, Ann. Mus. Napoli, 1, n 3,1901, p. 14.

? Chlorodopsis spinipes Alcock, loc. cit., p. 169.

?(hlorodepsis Wrood-Masoni Alcock, loc. cit., p. $170 ;$ Ill. Zool.Inecst., pl. XXXIII. lig. 7.

Mer Rouge (Nusée de Turin), 10 individus; Massaouah (II. Issel, Musée de Gènes), 3 mâles el une femelle; Érylhrér (Musée de Naples), a mâles: mer Rousen. Périm, Djibouti ef Obock M. Jousseaume), très nombrens individus: Djibouli (M. Couliere, M. Maindron, M. Cravier). quelques exemplaires.

Je serais porté, d'apres les descriptions de Aleock, at identifier ces exemplaires arec $C h l$. Ir nod-1Masnni, plutòt quarec Chl. spinipes.

L. front est presque toujours pourvu de denticules, au nombre de 3-ö par chaque lobe médian; ce qui serait, selon Acock, un caractere de Wond-Nasoni; dans spimipes, d'après Aleock, le front serait "ntier ou faiblement crénelé. Ce caracterest toutefois en contradiction avec la description the Heller, qui dil du front : mit s-4 feinen zähnchen besetzl. Si d'autre part quelques-uns des exemplaires ont le front presque entier, on trouve facilement tous les passages entre ceux-ci et les "xemplaires à front denticulé et mème spinuleux. La première fipine du bord antéro-latéral est variable en dimensions, souvent pertite, souvent plus développée. La deuxième dent est quelquefois simple, mais le plus souvent double. C'est la un 
comme d'autres exemplaires du golfe Persique vus par moi, ont ha coloration beaucoup plus chaire ed occupant seulement la moilié des doigts et sont atinsi intermédiaires entre la variété el la forme typique.

\section{Cymo quadrilobatus Miers.}

Mliers, Zool. Alert., p. 533. - Alcock, loc. cit., p. 175.

Djibouli (M. Jousseaume), un màle; mer Rouge (Musée de Turin), 2 mâles.

Genhe MenipPE De Haan.

Menippe Rumphii Fab.

Hilgendorf, Decken's Reise Ost. Afr., p. 107.

Indiquée par Hilgendorf dans la mer Rouge.

Genre PSEUdoziUs Dana.

Pseudozius caystrus Ad. et Wh.

Panopeus caystrus Adams et White, "Samarang " Crust., p. 42, pl. IX, fig. 2. Pseudozius planus Dana, loc, cit., p. 233, pl. XIII, fig. $6 a-h$.

Pseudozius caystrus Miers, Challeng. Bruch., p. 142. - De Man, Zool. Jahb. Syst., 17ll, p. 525. - Alcock, loc. cit., p. 181 (ubi syn.).

Astal) (Musée de Vodine), 1.1 màles, 6 femelles ; golfo de Tadjourah (M. Faurol), une femelle; Djibouti (II. Coutiere), 6 miles of 2 femelles; mer Rouge (II. Jousseaume), 60 expmplaires (II. Bouvier, in litt.).

Pseudozius sinensis A. M.-Edw.

A. Milne-Edwards, Ann. Soc. Ent. Fr., 1867, p. 278.

Aden (M. Jousseaume), 30 remplaires (M. Bouvier, in litl.).

Genre ozius Leach.

Ozius rugulosus Stm.

Cano, loc. cit., p. 204 .

Indiqué à Assab par Cano. 
Genre HETEROPILUMNUS DE MAN.

Heteropilumnus fimbriatus (Edw.).

pilumne fimbriatus II. Milne-Edwards, loc. cit., p. 416.

Ileteropilumnus fimbriatus De Ilan, Zool. Jahrb. Syst., VIII, 189:, P. 533. - Nobili, Ann. Jus. civ. Genova, XL, 1899, p. 260.

Nec P. fimbriatus llaswell.

Nec Cryptocoloma fimbriatum Mier's.

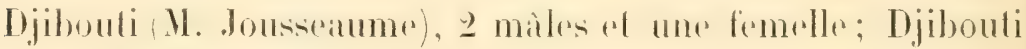
(M. Coutière), une femelle.

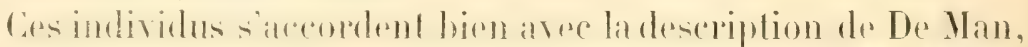
mais ils paraisisent stapplocher mienx des types de M. MilneEdwards parer que les patlies médiames ol posterientes de la

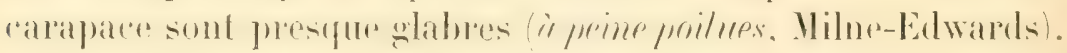

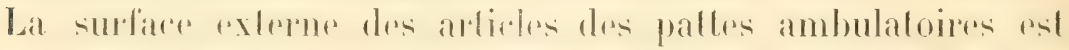

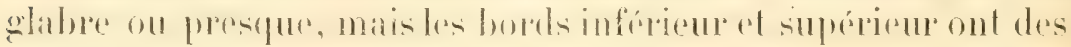
longs poils.

Las hois lobes du bord latéral varient quelque peru de forme.

Cette espèce n'a été trouvée que dans les parages de l'Australie.

\section{Gente PIIJMNUS latah.}

\section{Pilumnus trichophoroides De Man.}

De Man, Zool. Jahwb. Syst., VIII, p. 549 , pl. XIII, fig. 8. - Nobili, Bull. scient. Fr. Bely., XL, 1906, p. 13\%.

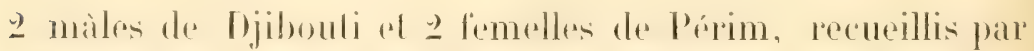
II. Jousseaume, ollerent les mèmes dithórences de la descripolion originale que los individus du galler Presigue décrils par

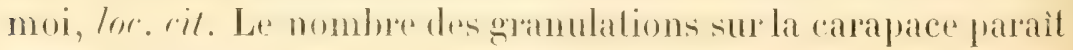
être variable.

\section{Pilumnus vespertilio Fab.}

Lif. Alcock, loc. cit., p. 192.

Pilumnus vespertilio var. Alcock, loc. cit., p. 193. - Nobili, Ann. IIus, zool. Napoli, 1, $\mathrm{n}^{0} 3,1901$, p. 15.

Ljibouli (M. Jousseaume), une femelle; Mjibouti (M. Gravier', une Pemelle; Suez (II. Jousseaume), 4 miles el 2 femeiles; mer Rouge (Nusée Turin), 2 mâles; Massaonah (MV. Clivio, 


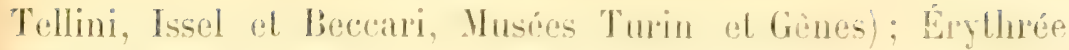
(Husée Naples).

De cette espece, très commune, on troure dans la mer houge autant la lorme typique que lar variate de Mleock.

\section{Pilumnus Forskalii Wiw.}

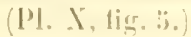

? Cancer incenus Forskial, loc. cit., [). (1).

Pilumnus Forskatii II. Milne-Edwards, loc. cit.. 1). 13. - Paulson, l c. cit., p. 36, pl. VII, fig. 1-1 $u$.

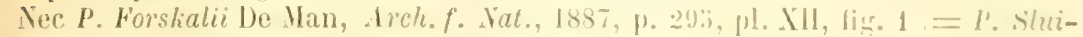
teri De Man).

Ver Rouge (Husée de Turin), un male; Djibonli (II. Jousseaume), un male; Djibouli (H. Couliere), une fornelle: (o)oces

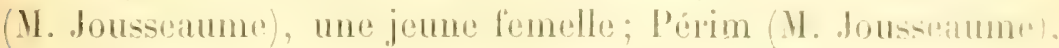
4 mates jelmes.

Cesexemplatres ont été comparés par moi an lype de MilneEdwards, conservé au Muséum, que .1. le probesseur E.-L. Bouvier a eu l'obligeance de m'envoyer. Je donne nne photogratphie du lype, qui vient aussi de la mer Rouge.

Ceelle espece, ainsi que nombrenx autres Pilummes, a lil carapace el les palles tries poilues. Les poils sont ales longues

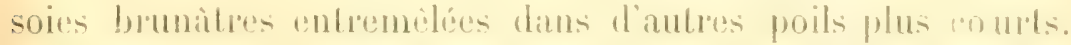
Les poils longs sont tris nombrems sur la partie anterinders du

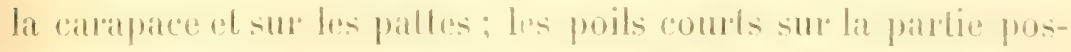
léreure. Aucune des soles longmes nest claviforme ni mollu comme celles de P'. expertilin.

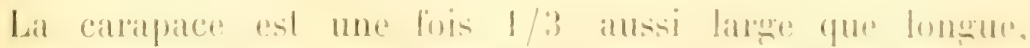

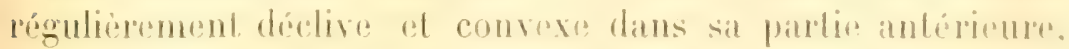
plane en andire. Lail stufiterest nellement divise en lobes patr des sillons peru prolonds, mais atsiry larges. Len lobes iprigats-

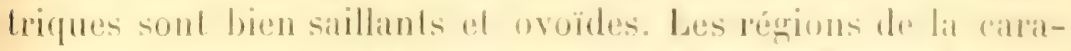
parer ont de-

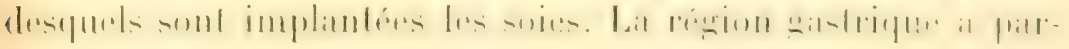

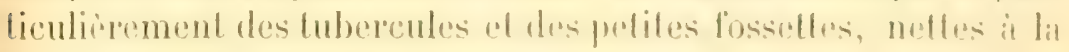
loupe. Vers les cotés les granules deviement atigus, pointus al blancs. Ces granules tendent it se grouper. Les rogrionspostéro- 
litérales sont aussi granuleuses, mais les gramules sont plus petils.

Le front est formé par deux larges lobes médians, bien séparés entre cux par une échancrure en V atsez étroite, ot nettement séprarés aussi des lobes externes spiniformes et dirigés un peu en dehor's. Ceux-ci sont bien séparés des lobes susorbitaires. Le bord des lobes médians est denticulé: les lobes externes sont granuleux, le bord frontal est pourvo de longs poils, suivis en arriere par une autre frange de poils implantés sur deux petites lignes granulées.

Le bord susorbitaire est finement crénelé; il offre deux fissures peu profondes mais asserz larges. Le bord inférieur est ausii crénelé, il est séparé par un petit sinus de l'angle orbitaire externe; le lobe interne est saillant et triangulaire.

Langle orbitaire est dentiforme et bien saillant; les trois dents épibranchiales sont aigurs et fortes, à pointespiniforme recouthere en avant, it entourées at la base et sur le bord postérieur de granules aigus. La dent sous-hépatique est bien nelle, entourée aussi de granulalions, dont souvent une est gronse et blanche comme la dent, al celle-ci apparait alors bidentée.

Les chélipèdes sont infígaux. Le mérus offre deux dents sur son bord supérieur: son bord antérieur, ainsi que relui de l'ischium, a quelques granulations aiguës.

Le carpe est fortement granulé; les granules sont coniques et relativement larges, ils se groupent en ilots poilus sur la surface glabre de l'article. La saillie interne est spiniforme. La face externe de la grosie main est entierement courerte de gros granules coniques, it pointe un peu inclince en avant, qui sont disposés en rangéres, dont deux sont sur le bord supérieur (avec une autre rangée irréguliere) ol ching sur la face externe. Le bord inférieur de la main a quelques granulations presqur oblitérées el des gros points. La disposition des granules sur la pelite main est à peu près la mime, mais les poils sont plus nombreux.

Lees pattes ambulatoires ont une longueur normale et sont très poilues. Sur le bord supérieur du mérus, du carpe et du propodite, mais surtout du carpe, il y a des granules aigus, 


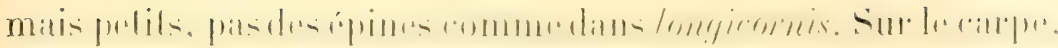
ces granules tendent it se disposer en trois rangées, dont la médiane est la plus forle.

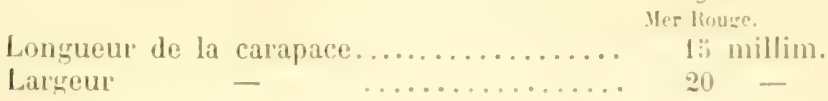

La femelle de Djibouli (II. Couliere) est peut-ritre un "remplaire anormal, paree que sa dent sous-hépalique est trés

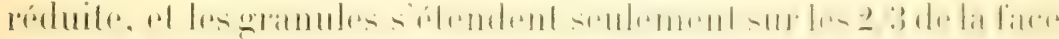

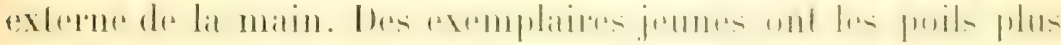
rares. Peut-être ils appartiennent a quelque variété.

Le Cancer incanus de Forshal a élé considéré parr M. Milne-

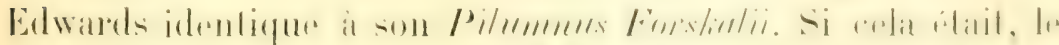

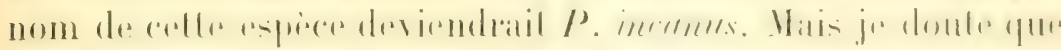
le $C$. inernus soil vraiment le $P$. Forstirlii. Bien que le caraclere lotus himsutus puisse convenil it celle espece (ainsi qu'a

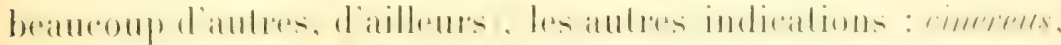

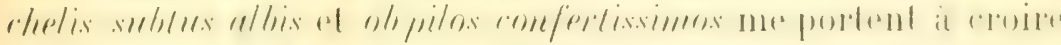

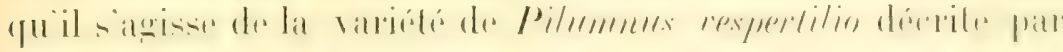

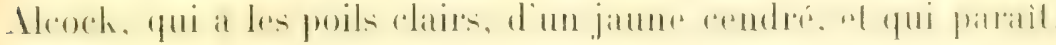

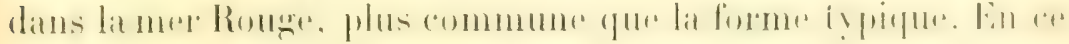
cas le nom inémus devail avoil la précédence sur revyersilin.

\section{Pilumnus Savignyi (Hell.).}

Savigny, pl. l', fig. 't.

Pilumnüs Savignyi Helled, Sitzb. Akad. Wien, 43, J. 345.- Xohili, Bull. scient. Fr. Belg., XL, 1906, p. 138.

Le lype de celle espece vient de la mer Rouge. Je n'en ai vu rue des exemplaires lu grolle l'ersigue.

\section{Pilumnus propinquus Nob.}

(1). IX, lig. 7.

Nobili, Bull. du Mus., 190:;, no 3, p. 163; Bull. scient. Fr. Belg., XL, I906, P. 1.0 .

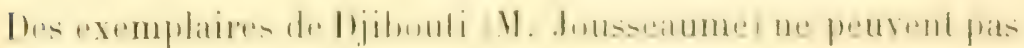


ithes siparos des lypes du golfe Persique par ancun carache lirri dir latrapace al des chéliprides; mais ils en different par fes mixopodiles des palles ambulatoires sans rpines. Les épines lembent d'ailleurs at se réduire el it disparaitre mime dans les exemplaires du golfe Persique.

Ta photographie de la planche IX est celle de deux individus du golfe Persique.

\section{Pilumnus hirsutus Stimp.}

Stimpson, Proc. Acad. n. Sc. Philud, !8:i8, p. 37. - Rathbun, Bull. Mus. Comp. Zool., XXXIX, p. 129.

Qnate exemplaires, dont l'un mesure 8 millim. 3/4 de largent sur 6 millimetres de longueur, s'accordent tres bien aree la description inedite de slimpson, publiée apres par Jliss Ralhbun, par la carapace poilue, mais moins que dans d'aulme especes, aver peu de poils plus longs, par les bords latero-intérieurs armés de qualre ópinestres aiguës y compris l'angle orbitaire exterme spiniformes, par l'absence de dent sous-hépalique, par la forme du front, par le bord inferieme des orbites denticulis ef par la grosise main samuleuse of poilues seulement sur la moitié de sa surface.

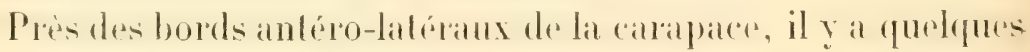

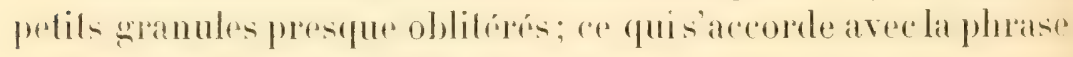
surface nearly smooth de la description de Stimpson.

\section{Pilumnus quadridentatus De Man.}

De Ulan, Zool. Jahrb. Syst., VIII, 189:, p. 337, pl. XlII, fig. 6.

Pilumnus seminudus De Man, J. Linn. Soc., XXII, p. 63 (nec Miers).

Djibouti (M. Coutière), 8 individus.

Les individus de Djibouli sonl lous plus jeunes que les ly pes de Pontianak, dont De Man a domné les dimensions. Ils diffèrent touledois des adultes fal les mimes diffépences que De Man a abablies pour ses exemplanires jemoes. Ces individus ont une largan moyenne de $6-7$ millimelres. Lal grosis main apparail, ¿ lis lonpe, granuleuse sur loule sa surface ; les gramules sont plus gros sur le bord supérieme. Lat surface anter les gramules 


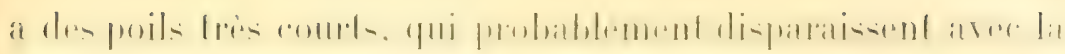
réduction de la granulation. Parmi les poils courts il y en a quelques-uns plus longs at elaviformes. Pres des bords antéro-

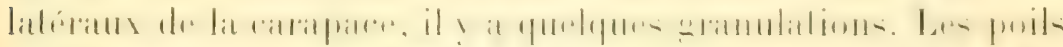
sur la carapace sont un peu plus nombreux fure daus les arlultes.

Celte espece est connue seulement de Mergui et Pontianak.

\section{Pilumnus lævimanus l Jinit.}

Dana, loc. cit., 1. 237, pl. Xlll, fig. 1. - A. Milne-Ldwards, Your. Arch. Mus., IX, p. 250, pl. X, fig. 4. - De Man, Arch. f. Wuturg., 1887, p. 301.

Mer Rouge (M. Jousseaume), 7 miles et 7 femulles.

\section{Pilumnus eudæmoneus (1) Nob.}

Nobili, loc. cit., P. $40 \%$.

Aden (H. Jousseaume), une femelle.

Djibouti (II. Jousseaume), un mite.

Cette espece appartient au groupe de P. nilidus 1.-M. Edw. et de P. gluberrimus Ilasw. el inermix Lasw., a carapace non lobulée et à dents latérales à peine marquées.

La carapace est large de $4^{\text {mm }}$, , el longue de $3^{\text {man }}, 3$ dans l'exemplaire de Aden et respectivement $9>6$ millimidres dans l'autre de Djibouli. Ello est convere d'avant en arriere el aussi de còté a còté, bien díclive dans la partie antérieure: le front n'est pas visible d'en haut. Lat surfice manque entierement de lobulation, si ce n'est le gros exemplate de bjibouti qui a des lobes épigastriques un peu prononcés. La region

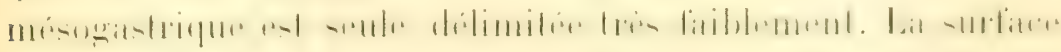
entière offre des gromules arrondis, petits, mais bien distincts. qui sont tres espacés entre eux, mais plus nombreux sur les colés de la carapace ou ils lorment aussi une petite ligne trans-

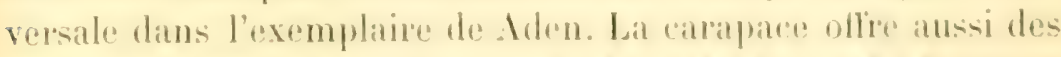

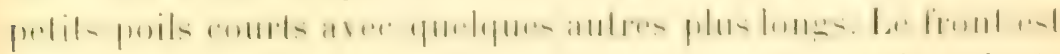
tres large (1 millimble et demi dans l'exrmplatere de Aden),

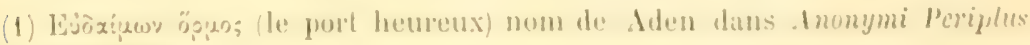
Maris Erythrio. 
inflechi, a bord droit, à peine sinué au milieu dans l'exenplaire de Aden, et nellement échancré dans celui de Djibouti plus vieux. Il est continué sans démareation avec le bord susorbitaire, et est sillonné en dessus. Lés bords latéro-antérieurs sont divisés en (quatr dents (y compris l'angle orbitaire), ¿t peine détachés du bord, presque égaux antre eux et finément granulés ou denticulés, Le bord supérieur des orbites n’a pas de fissures.

Les chélipèdes sont inégaux, le carpe est très granuleux; les deux mains sont couvertes sur leur face externe de granules nombreux et gros (relativement i la petitesse de l'exemplaire).

Ces granules sont disposés en rangíes qui courrent toule la surface externe de la main; parmi les granules, il y a des poils nombreux et très touffus.

Les pattes ambulatoires sont médiocres et poilues; le bord supérieur du mérus est denticulé.

Celte espece se reconnait aisément des trois autres nommées, par sa, carapace ayant des granules épars sur toute sa surface et par ses chélipèdes entierement granuleux of poilus.

\section{Pilumnus spongiosus Nob.}

(PL. X. fig. 6.)

Nobili, loc. cit., p. 406.

Ciette nouvelle espèce, représentée par 4 mâles et 3 femelles. recueillis par M. Jousseaume à Périm, paraît voisine de $P$. iermiculatu: Elw de la Nouvelle-Calédonie, mais en diffèr par plusieurs caractères.

Comme dans $P$. rermirulatus, la carapace est un peu déclive dans la partie fronto-orbitaire, mais trex aplatie pour le rest" de sa surface. Elle est divisée en régions; celles-ci sont aplaties mais distinctes. Les lobes épigastriques sont nets; les protogastriques sont séparés des mésogastriques el séparés aussi des lobes latéraux par un sillon assez profond. L'aréole urograstrique est séparée aussi. Les lobes latéraux antérieurs forment un bourrelet allongé al oblique, disposé parallèlement 


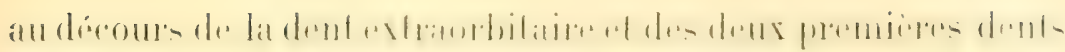
épibranchiales. Les régions latérales ont deux ou trois bour-

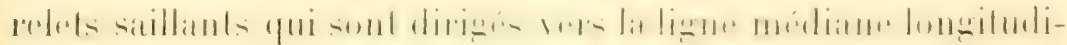

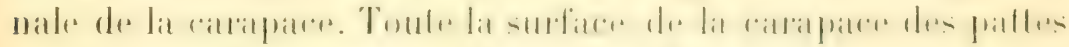

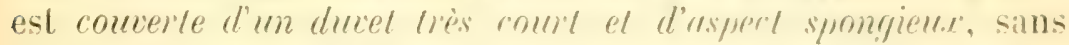
poils plus longs. Ce duvet suit exactement les saillies et les

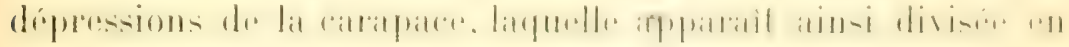
rides et figures el comme réliculée. Le rapport entre la longueur el la largeur de la carapace rust de 1: 1,33. Le front mesture a peine un quart de la Jargen de la carapace: il est formé par deux lobes un peu sinueux, síparés sur lat ligne médiane par une fissure en $V$ peu profonde, leur extrimité externe est aiguë, mais il n'y a pas de rrais lobes externes; les lobes sont toutelois séparés du bord sourcilier.

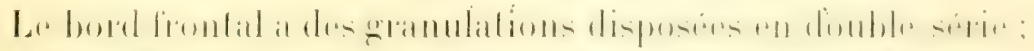

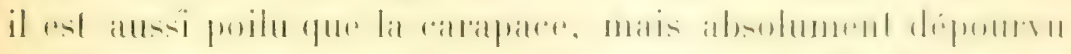
de la frange de longs poils de $P$. cermiculaks. Le bord orbilaire supérieur a deux fissures trimmulates; il est granulé. le

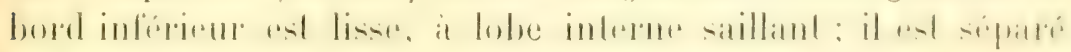

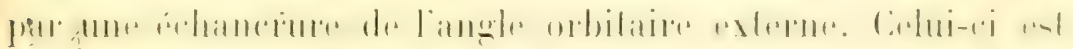
aigu mais non saillant.

Les bords latéro-antérieurs sont armés de quatre dents. sans compter la dent orbitare.

Lá premièe dent épibranchiale sist petite, Iriangulaire, aigue el séparee de l'ord)ite par un espace assez longe el concave, pourvu de 2-3 granules aigus; en dessous de cel espace, sur lar region sous-hépatique, entre lat dent et l'angrle orbitaire externe, il y a des gramulations subaigues of atsor grosses que l'on voit bien d'en haut en enlevant te duvel. La deuxieme dent est triangulaire, dirige en avant at end hohors;

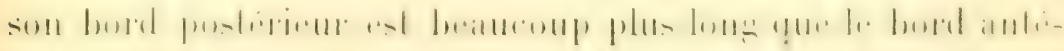
reur; lat froisieme dent est triangulate aussi, mais plus nellement dirigee an dehors; la plus grande largeur de la carratere se trouve alusi entre ces dents: le bord antiriente lo lomel posteriem de cedte dent no different pas sonsiblement en bon-

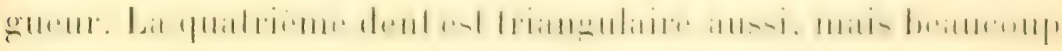

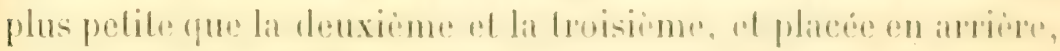
au commeneement des bords postero-litherax. 


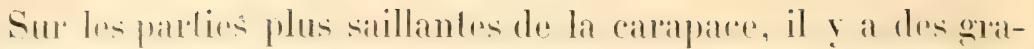

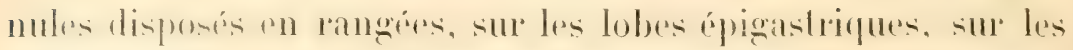
probogastriques, sur le bourrelel formi par les lobes lateraux et str les denx parties desrógions branchiales postérieures. Le long des bords postéro-latéraux il y a 6-7 de ces granules disposés "n rangée et espaces entre rux. Les granules sont visibles seulement en enlevant le duvet.

Les bords postéro-latéraux sont berminnup plus. Inngs: que les bords antéro-latéraux.

Les chélipèdes sont inégaux dans les deux sexps, dmeteux smi tomle leur surface ainsi (fut la rarapace, sans poils plus longs. Le bord superieur du bras n’a pas les deux dents de tant de Pilumnes, mais seulement quelques granules coniques; sur la face externe, cachés par le duvet, il y a des granules arrondis, espacés et peu nombreux.

Le bord anterieur de l'ischimm of du bras a des petits granules. Le carpe a la salille interne spiniforme et placée plutòt en arriere, sa surfiace offre des petits granules cachés sous le duvet. La main aussi est duveteuse sur toute sa surface; en enlevant le duvet, on voit des granulations petites sur sa partie supríreme el au long de l'arliculation avec le carpe; des granules plus gros forment deux lignes saillantes sur la face supréreure de la main. Partout ailleurs la surface de la main est lisse, mais ponctuée. Les doigls joignent bien et sont régulièrement dentés (4-30 dents); le doigt mobile esl subegal en longrterur au bord supérieur de la paume el plus court rew la longurem tolale de la paume; il est un peu granuleux et poilu a la base, sillonné longitudinalement. La petite pince est conformée comme la grosse pince, mais moins haute.

Les paltes ambulatoimes sont romprimeses of poilues comme lia carafrace: assez lomglus, mais moins que deux fois aussi longues que la carapace. Le mérus est lisse en dessus; le rappe of le propodile sont bicarenes an dessus; les doigts sont romprines at plus longs eque le propodite. Le bord postérieur An propodite ed du doigt offre une frange de poils un peu plus longs. ce qui ajoute it l'apparence presque nataloire des palles. 
Le sternum ast gránulé en sir partio antírieure el au long de la cavilé recerant l’abdomen: les granules sont ronds el perlacés.

L'avant-dernier article de l'abdomen du mate est plus long que le dernier.

Les dimensions de l'exemplatios plus gros (une femelle) sont:

Longueur de la carapace............ 13,5 millim.

largeur $\quad-\quad \ldots \ldots \ldots \ldots \ldots \ldots$ 18 18 -

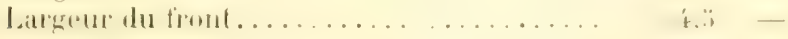

Trois autres espèces de la mer houge ont été décrites, que je n'ai pas vues.

\section{Pilumnus brachytrichus líssn.}

Kossmann, Zool. Eryelm, roth. Heer., 1, 1878, p. 39.

Cette espece devait etre revue d'apres le type, paree que la seule description originale n'est pas suffisante pour la liare reconnaitre avec simete.

\section{Pilumus Schrenkii Pauls.}

Paulson, loc. cit., 1). 2,2, pl. II, lig. $6-66$.

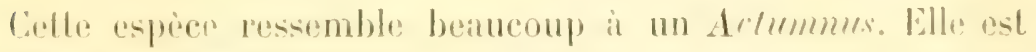
decrite ainsi par l'aulson:

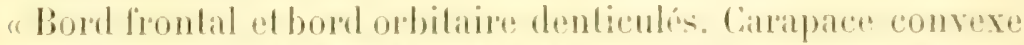

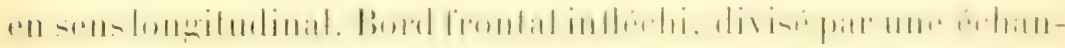
crube módiane en deux lobes un pen combris el denticulés. profondément séparés du borel orbilatere. Las donts próorbi-

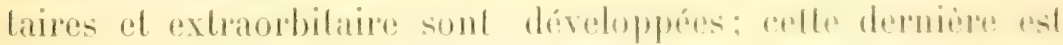

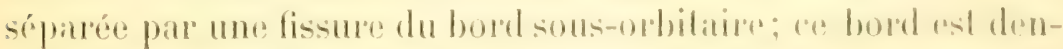

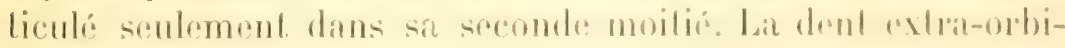

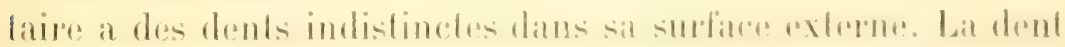

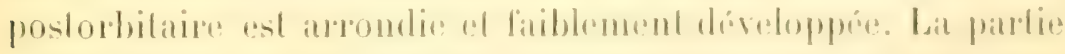

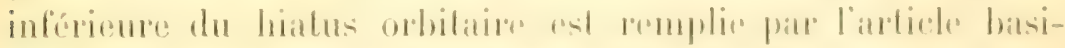

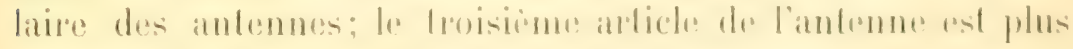

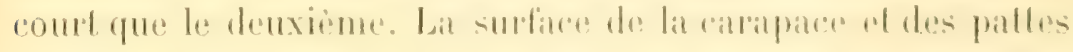
est duveleuse, aree des poils phus longs parmi le duved. Lai 


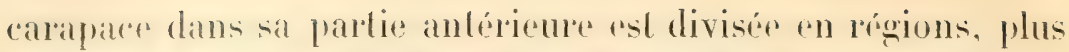
visibles en enlevant le duvel; sur la région hrepatique, il y a quatre tubercules, sur la région branchiale externe, du cóté gauche, il y a un autre tubercule (1).

"Les bords latéro-antérieurs ont 3 dents aiguës, dont la partie terminale est brillante, et séparé de la base large. Cihélipèdes subégaux : une dent aiguë sur le bord supéricur du mérus, le restant du bord lisse; bord antero-interne du carpe denté; quatre stries de denticules aigus sur la surface de cet article, dont l'une occupe le bord supérieur; surface externe consexe du propodus armée de tubercules aigus, décroissant vers la partie inférieure, seémoussant el śétendint aussi sur le doigt fixe; fubercules disposés sur la parlie supérieure, en partie en rangées obliques.....; doigls avec des dents obtuses; doigt mobile couvert de granules ì la base, sillomné sur la petite patte. Pattes ambulatoires comprimées, plus courtes of plus larges dans $P$. Forstialii. Un male large de $10^{\mathrm{mm}}, 8$, long de 8 millimètres. "

\section{Pilumnus asper Rüpp.}

Xantho asper Rüppell, fig. 8.

Cette espèce a été attribuée par Viers au genre Pilummus. $\Lambda$ près Rüppell, elle n'a plus été retrouvée.

\section{Genre ACTUMNUS Dana.}

\section{Actumnus globulus Hell.}

Heller, Sitzb. Akad. Wien, p. 341, pl. III, fig. 23. - A. Milne-Edwards, Nouv. Arch. Mus., 1, p. 286, pl. XVIII, fig. 4. - Ortmann, Denkschr. Jena, VIII, p. 52 .

Djibouti (I. Jousseaume), 't femelles; ile Dahlac (MM. Issel, at Beccari, Musée de Gènes), un mâle ; mer Rouge (I. Jousseaume), un màle; mer Rouge (Musée de Turin), un màle. Trouvé aussi à Tor (Heller) el à Assab (Cano).

(1) La figure en effet a un tubercule à gauche, et pas à droite. Les tubercules dans la figure sont coniques, pointus et blancs. (G. N.) 


\section{Actumnus Bonnieri Nob.}

Nobili, Bull. Mus., 190:;, no 163, p. 13; Bull. scient. Fr. Belg., XL, 1906, P. 132, pl. 32, tig. 11.

Une dizaine d'exemplaires recueillis par II. Jousseaume it Djibouti et is Aden.

J'ai comparé celte espèce avec $A$. selifer De Haan. Eille en diffère par ses régions très saillantes, neltement sóparées les unes des autres, fortement granuleuses, par son front el ses

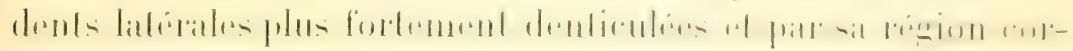
diale bipartie.

Deux exemplaires de Zanzibar de A. xelifer ont la carapace beaucoup plus poilue; les aréoles, bien que plus forles que dans A. Iomentoxus, ne sont pas ausid darement dílinies et sont

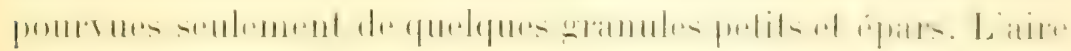
cordiale n'est pas divisée.

\section{Actumnus obesus Dana.}

Paulson, loc. cit., p. 4í, pl. I'll, fig. 2-2 a.

Celle espèce a été signalée dans la mer Rouge par Paulson. Cel aubur considere $A$. globuhus identique arec obesus, ce qui n'est pas juste.

(iENHE HETHROPANOPE STM.

\section{Heteropanope Vauquelini (Aud.).}

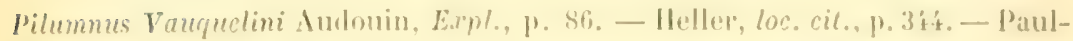
son, loc. cit., p. 43, pl. 11, lig. 7.

Heteropanope Vumguetini 1): Man, Zoul. Juherb. Syst., IV, 18s!, 1. i2.2, [1. II, liv. : :.

Mer Rouge el bjibouli (1). Joussenume), l't miles el

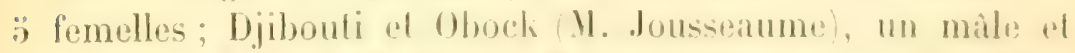
3 femelles: Beiloul (1) Orsini, Muse de Turin), une lemelle: mer Rouge Nuse de Turin), nombreux exemplaires.

Ces exemplaires sacenrent lous assez bien avec les descriplions de Heller or de De Man. Le bord inférieur de l'orbite dans tous les individus esterranulio ou crenelé comme dans 
la figure de Paulson. La distribution des poils sur la carapace est lres variable: somront la carapace est presque glabre, mais sombent ansi les lignes gramuleuses portent des longs poils peu nombreux.

\section{Heteropanope pharaonica Nob.}

(Pl. XI, lig. 1.)

Nol,ili, loc. cit., p. 407, fig. 2.

Une femelle recueille par M. Ragazzi dans une localití non indiquée de la mer Rouge, ol appartenant all Musée de Modiene.

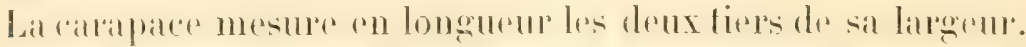

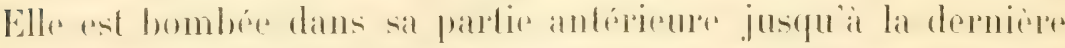

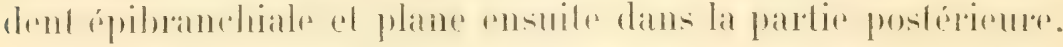

La surface est entièrement, glabre, et, examinée à la loupe,

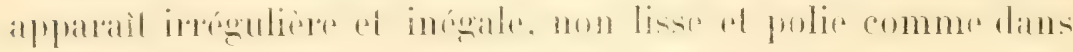

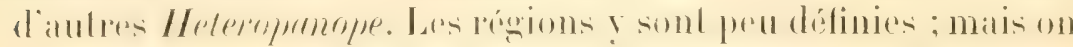

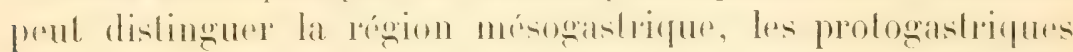
déclives en avant el mal séparées des lobes épigastriques qui sont pelits et assez distanls, et les régions $5 \mathrm{~L}$ et 6L. La

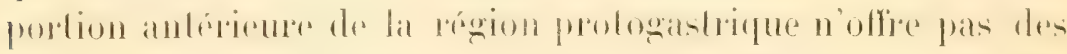
lignes nelles granuleuses, comme dans $H$. indica et afri-

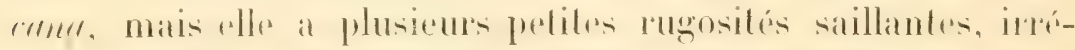

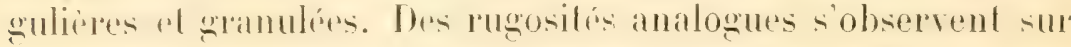
les régions antérieures latérales en arriere et en dedans des ders pormieres dents, of sur l'extrémité antérieure de öL qui est un peu rehausiée sur le plan des régions latérales. De grosses granulations s'observent parallèlement aux bords latléro-postéreurs; ces grosses gramulations, rxaminées à la loupe, apparaissent plutôt comme des rugosités granuleuses.

Le front est saillant, mince, hris pent declive: sa largeur est moins que 1/'́ de celle de la carapace. Léchancrure médiane est bien distincte mais petite, étroite, peu profonde, en forme dr I: les lobes médians, déterminés par cette fissure, sont plus âancís que les lobes latéraux; le bord frontal a un décours un peu oblique, et se rattache par une courbe aux lobes exterues qui sont arrondis. Le front est plus avancé que le lobe 
sus-orhitaine interne; les lobes externes du liront sont séparés par une échancrure bien nelte du lobe sus-orbilaire.

Les orbites mesurent 1/8 de lat largente de harapace; lene

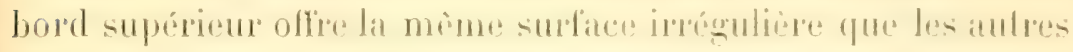

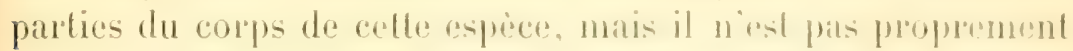

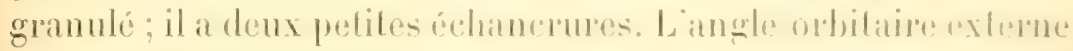
est peu saillant, it peine dentiforme. Lat distance cuthe les an-

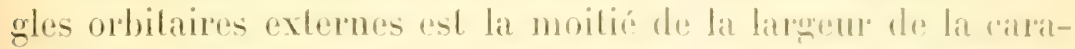
pace. Le bord inferieur de l'orbile est sippaté de l'angle orbilare externe par une exhancrure prolonde. Le lober sousorbilane externe est arrondi mais sillant, le lobe intorne es dentiforme el tres saillant, bien visible d'en desits.

Les bords latéro-antérieurs mesurent les 6/7 de lit longruent des bords latéro-postérieurs. Ils sont divisise en fi denls. Lal

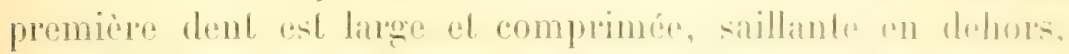
arrondie; sa portion antérieure qui se rallatche it l'angle de

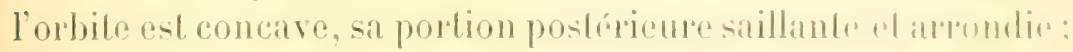
Ia longueur tolate de son bord anteriem est environ lo double de la longueur du bord postériem. La deusieme dent as atusi comprimée, à pointe obtuse; son bord antérieur, quiestenviron

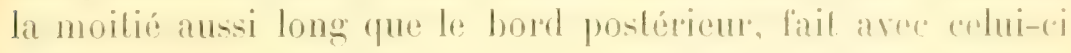

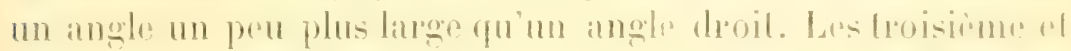

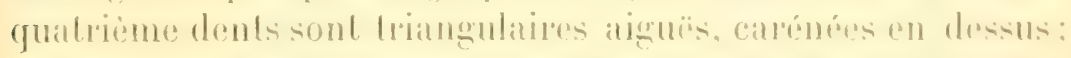
la troisieme dent savance plus que la quatrieme, al lat plus

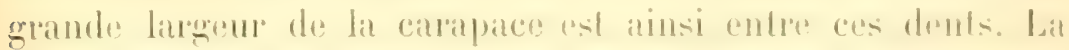

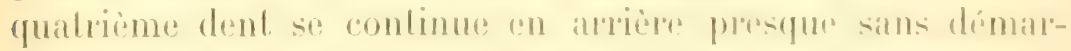
cation dans le bord postero-lateral de la carapalce. Toules ces dents sont impormliemment gramuleuses sur le bord, matis les granulations sont plutol des inregularites de lat surlatere que des

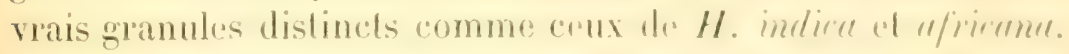
En dessous de la premieres dent, il y a sml la partire soushépalique un amas de gramulalions: las régions solls-hripalliques, plérygostomiques al sous-loranchiales sont gramulies.

Les crètes endostomiales sont bien nedtes el śefendent jusqu'au bord antérieur du cadre burcal.

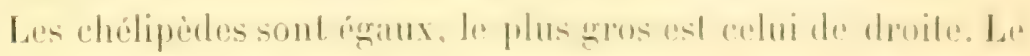
borel supérieur du brats de lat grosise pince estarme. un peu en arriere de son extrémite distale, d'ume dent aigui ef 1 men 
courbée en avant: le restant du bord est armé de fins gramules aigus. La face postérieure du bras est finement rugueuse, avec des poils très courts; sa face antérieure est granuleuse; le hord antérieur est granulé. La face supérieure du carpe est rugueuse, et parmi les rugosité fines, il y a aussi un. bon nombre de nodules et grosses rugosités. Son bord antírieur st aussi nodulé; sa saillie interne est forte et pourve d'une crête arrondie et obtuse en dessus.

La grosse main (dans la femelle) est un peu plus longue que la moitié de la largeur de la carapace, et sa hauteur est plus que moitié de la longueur. Le doig̨t mobile, mesuré en extension. est un peu plus long que le bord supérieur de la paume. La surface externe est médiocrement convexe; elle apparait à la loupe ponctuée, réticulée el granuleuse, arec trois còtes incompletes et lisses. Le bord supérieur a deux côtes irrógulierement et grossierement granuleuses, mais hien melles. Le doigt mobile est courbé, granulé aussibien que la paume ol sillonné en haut. Il offre une grosse dent arondie a si base, el quelques denti irrégulières après. Le doigt fixe est ausisillonné. Tout le corjos de l'animal est d'une couleur brune de rouille, et les doigts ont la mème couleur. La petite pince est conformée comme la croses : mais les deux crêtes du bord smperieur de la main sont plus fortement aceentures, par une dépression longitudinale qui oceupe la partie supéro-exteme de la paune; les doighsont plus longs et pourvus de plusieurs sillons.

Les patles ambulatoires sont élancres; l'avant-derniere paire mesure en longuem 1 fois 1/4 la laresem de la carapace. Ces paltes ont la surface ausis irreguliere fromme rouilles que la carapace of les chélipedes. Le carpe a dertx pedites cobles lisses at peu saillantes en desits: le propodus est lisse; les datclylopodites sont enveloppés dans des poils tomenteux, at sont plus longs que les proporlites. Bien qu'il y ail quelques poils longs it la base du méres, et sur le bord postérieur du propodus, ces poils ne forment pas des franges on des rangées comme dans les autres espèces du genre.

Le sternum est granulé.

Longueur de la carapace. 22 millim. 
Largeur du front. i millim.

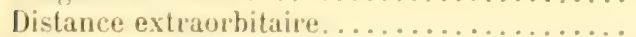

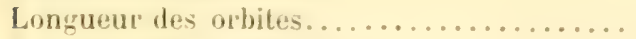

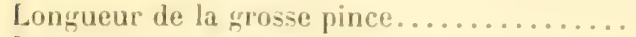

Longueur du doigt mobile...............

Hauteur de la grosse pince.

10

$4 \quad-$

$19-$

$10-$

\section{Gexre EURYCARGINUS A. M.-Enw.}

\section{Eurycarcinus natalensis (Krauss).}

Galene natalensis Krauss, Sudafr. Crust., 18ł3, p. 31, pl. I, fig. \&. - IIoflmann Crust. Echingd. Hadag., 187', p. 4. - Kossmann, loc. cit., p. 3\%.

Eurycarcinus Grandidieri A. Milne-Edwards, Ann. Soc, Ent. France, 1867. p. 277; Nouv. Areh. Mus., IV, 1868, p. 80, pl. XIX, tig. 13-16. - Aleock, Ioc. cit., p. 211.

Eurycarcinus natalensis Ililgendonf, M. B. Akad. Berlin, 1878, ए. 792. - Lenz et Richters, Abh. Senckenb. Ges., 1881, p. 2. Ortmann, Denksch. Jena, VIII. p. 49 .

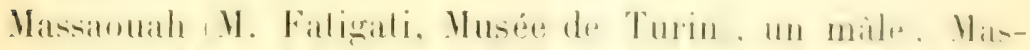

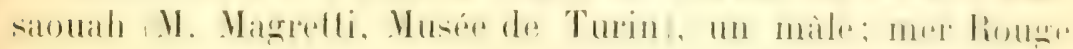
(II. Jousseaune), une femelle.

\section{Eurycarcinus orientalis 1. M.-Edw.}

(Pl. XI, fig. 5.)

A. Milne-Edwards, Ann. Soc. Ent. Fr., 1867, p. 27\%. - De Man, Not. Leygl. Ilus, XIV, p. 226.

Nec Eurycarcinus orientalis Alcock, loc. cit., 1. 210.

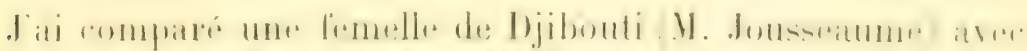

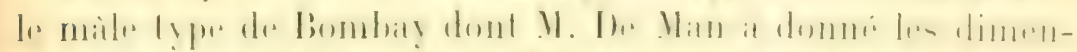

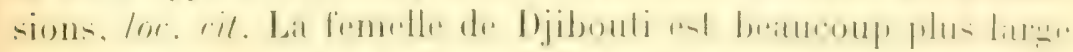

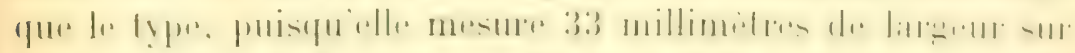
20 de longrueur. Le mitle a 26 mill. 22/3̈ de litrugrul sul 16

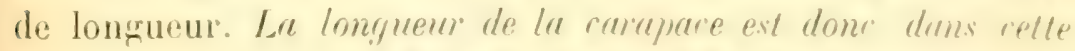

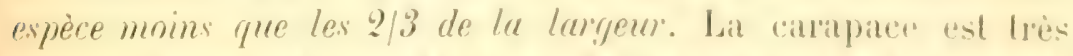

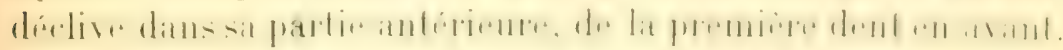

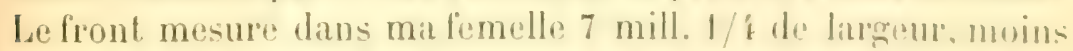

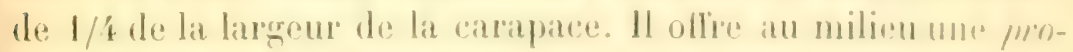

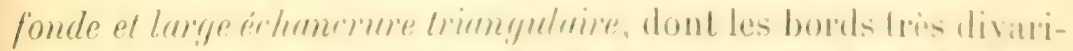

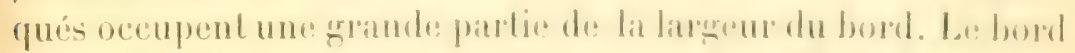

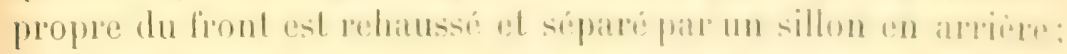

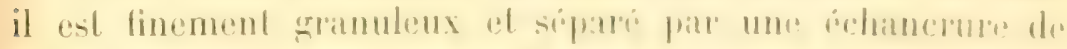

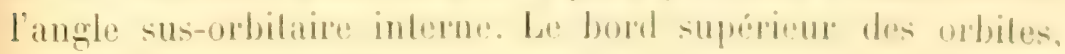

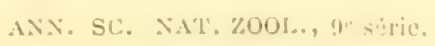


ainsi que les dents du bord latéro-antérieur, sont granulés ausi. Les bords latéro-antérieurs sont beaucoup plus courts qur les bords latéro-postérieurs; ils mesurent dans la femelle de Djibouti $9^{\mathrm{mm}}, 5$ de longurur (selon la rorde), les bords latéro-postérieurs mesurent $17^{\mathrm{mm}}$.̈. Le se trois premières dents sont tronquése; la deuxieme est un peu plus longue que la pre-

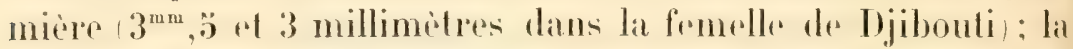
quatrieme dent as triangulaire ì pointe aigü, courbere en avant. Les parties de la carapace qui avoisinent ces dents sont granuleuses.

Il n'y a aucune trace de régions sur la carapace.

Les chelipedes sont inegaux dans les deux sexes: plus dams le mâle que dans la femelle.

Les trois derniers andicles des pattes ambulatoires ont des longs poils roux sur les bords.

\section{Eurycarcinus integrifrons De Man.}

(PI. XI, lig. 2.)

De IIan, Not. Leyd. Mus., 1, p. 55.

Eurycarcinus orientalis Alcock, loc. cit., p. 210 (nec A. Milne-Edwards).

Mer Rouge (Nusée de Turin), un mile: Mden (II. Jousseaume), 2 f'emelles.

Le front daus le male de "mer Rouge " du Yusée de Turin est entièrement droit, faiblement íchancré dans les 2 femelles de Alen, a qui concilie les deux descriptions de De Man el de Mleock. Par tous les autres carateleres ces trois exemplarires saccordent avee lesdescriptions cités. Il suftit de regarder les deux pholographies de la planche pour voir combien cotte espèce est différente de $\boldsymbol{E}$. orientralis.

La description de mientulis de Aleock convient par tout point it $E$. integrifions et non is orientalis.

La carapace est beaucoup plus atroile; elle mesure les deux liers ou nime plus de la largeur de la carapace, landis que dans $E$. mientalis elle est distinctement plus large, ce qui donur aux deux especes un aspect fres different. Le front ast moins que 1/4 de la largeur de la carapace: il est droit. arec

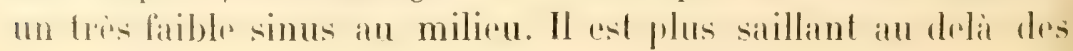




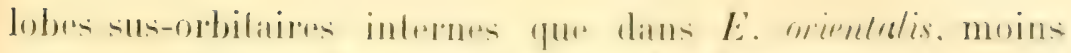

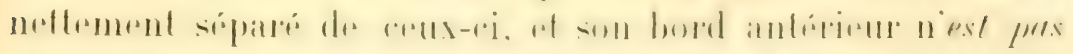
rehaussé ni séparé par un sillon de la surface du front.

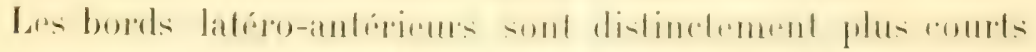

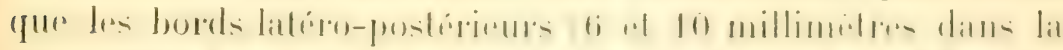

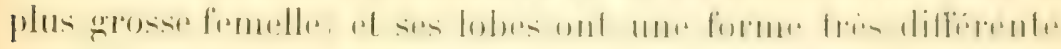
de celle de E. mienulix.

Les deux premiers sont arrondis, tronqués ef subeganux.

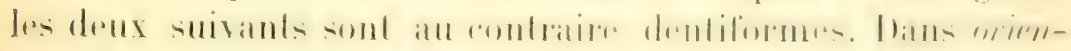

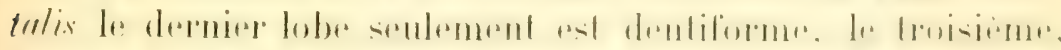

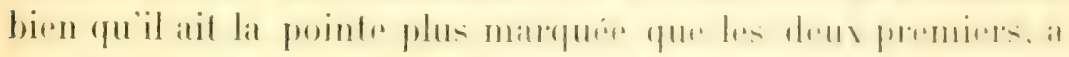
la mème forme que ceux-ci.

Les chélipedes sont inégaux, mime dans les femelles.

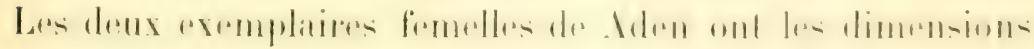
suivantes:

Longueur de la carapace......... $1: i \quad 13,3$ millim.

Largeur

……… 22

19,5

Lat localité du type est inconnue. Alcock retrouva cotte espece i Karachi, Bombay et aux iles Indamanes.

GHARE ERIPHIA I,IT.

Eriphia lævimana val, Smithi (Mlacl.).

Eviphia Smithi Macleay, Ann. S. Ifr., p. tio. - kinuss, loc. cil., p. 36, pl. II, fig. 3. - llotlmann, loc. cit., p. 6, pl. 1, fie. 1.

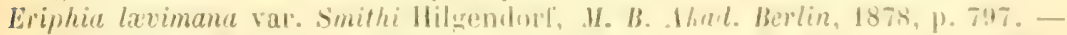
Miers, Inn. Uag. Nat. Hist. (ii), 1, 1880, p. 237. - Me Man, frch. F. Yat., 1887, 1. 327. - Mlock, loc. cil., 1. 216 (ubi syne). - Nobili, Moll, Mus. To-

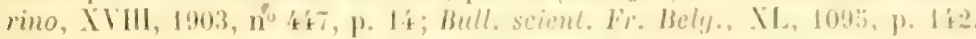

Obock (M. Mándron), 2 males; Mokia (M. Ragazzi, Musio de Vodene), un male ot une lemelle; Lssab) (II. Ratgazai. Musée de Hodino), 2 miths.

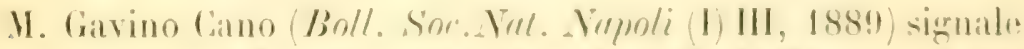
de

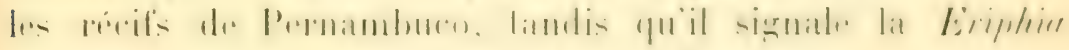
gnnaryme, espece americaine, a lssib)! Il y a peut-ebe lit une confusion d'exemplatios. 


\section{Eriphia scabricula Dana.}

Dana, loc. cit., p. 247, pl. XIV, fig. 5 a-b. - Alcock, loc. cit., p. 216.

Djibouti ou Obock (M. Jousseaume), un mâle tries jeune.

Un autre exemplaire de Djibouti (M. Coutiere), encore plus jeune, a la carapace et les pattes tres poilues, et trois dents seulement sur les bords de la carapace, qui sont reliées entre elles par de nombreux denticules.

\section{Genre TRAPEZIA Lat.}

Trapezia cymodoce (Herbst).

Cf. Ortmann, Zool. Jalub. Syst., X, 1897, p. 201. - Alcock, loc. cit., p. 219 (ubi syn.).

Trupezia ferruginea var. T. cœrulea Paulson, loc. cit., p. 46, pl. VII, fig. 4-4a.•

Djibouti (M. Jousseaume), 22 mâles et 16 femelles; Djibouti, vivant par couples a l'intérieur des cavités creusées dans les polypiers, surtout les Poriter (II. Gravier), quelques exemplaires; Djibouli (M. Coutiere), 10 màles at 8 femelles; Périm (M. Jousseaume), 1 ö mìles, 16 femelles; Massaouah (MM. Bonnier et P'érez), 13 màles el 9 femelles; Massaouah (MM. Issel el Beccari, Musée de Gènes), nombreux exemplaires; Massaouah (M. Vinciguerra, Musée de Turin); Assab (MV. Issel et Beceari, Husíc de Gènes), 3 màles et ä femelles; Érthrér (Nusée de Naples), 6 màles et 6 femelles; Obock (M. Jousseaume), 2 màles et 7 femelles; Mden (II. E. Simon), 7 màles et 3 femelles; Iden (M. Jousseaume), un mâle et une femelle; mer Rouge (M. Jousseaume), 6 mâles et כ̆ femelles; mer Rouge (Musée de Turin), 4 mâles et 3 femelles.

La $T$. fermginea var. $T$. romlea de Paulson est sans doute identique avec cette espèce, et correspond à $T$. crerulea Hell. La $T$. fermuinea var. $T$. cymodnce du mème aulrur n'est pas celte espèce; elle est probablement, a en juger par la synonymie, la $T$. fermigina var. dentata.

D'après les indications des étiquettes de II. Gravier la coul'ur de l'animal vivant est: "Cephalothorar bleu-violet, surfaces dorsales des appendices rouges. " 


\section{Trapezia ferruginea Lat.}

Cf. Ortmann, loc. cit., p. 202, 203. - Alcock, loc. cit., p. 220.

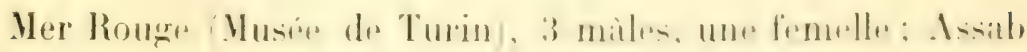

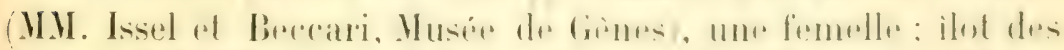

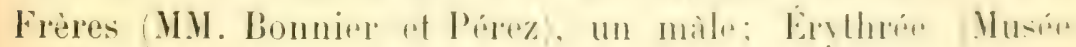
de Naples), un mâle.

\section{Trapezia guttata Rüpp.}

Rüppell, loc. cit., p. 27.- Heller, loc. cit., p. 331. - De Man, Not. Leyd. Mus, XII, 1890, p. 64. - Hiers, Challeng. Brach., p. 166, pl. Xll, fig. 1. - De Man, Abh. Senckenb. Ges., XXV, 1902, p. 640, pl. XXI, fig.2:.

T. ferruginea var. guttata Paulson, loc. cit., p. 47. -- Alcock, loc. cit., p. 220.

T. ferruginea guttata Ortmann, loc. cit., p. 203, 20\%.

Mer Rouge (Musée de Turin), 3 mâles el 2 femelles.

\section{Trapezia maculata (Mc: Leay).}

Dana, loc. cit., p. 256, pl. XV, fig. \& $a-d$. De Man, L'ch. $f$. Naturg., 1887, p. 318 , pl. XIII, fig. 2. - Alcock, loc. cit., p. 218, 221.

T. ferruginea maculata Ortmann, loc. cit., p. 203, 206.

T. ferruginea var. rufopunctata Paulson, p. 48, pl. YII, fig. 3 (nec T. rufopunctata Ilerbst, Ortmann).

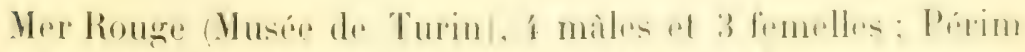

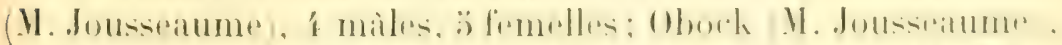

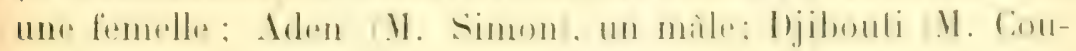
lière), un mâle, 4 femelles; illes Musha (II. Gravier), une

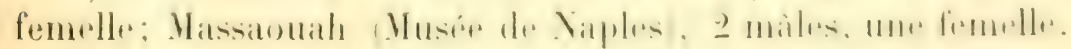

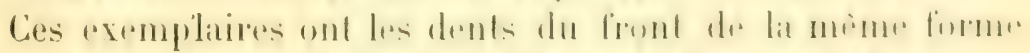

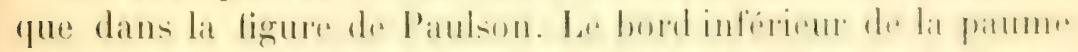

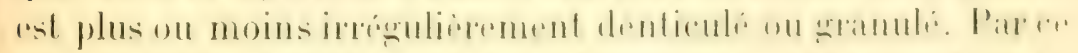

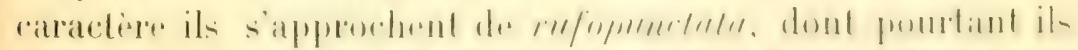

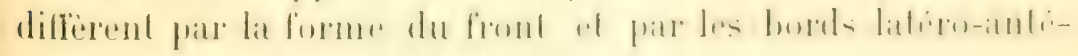
rieurs un peu divergents en arrière.

\section{Trapezia digitalis Lill.}

Ci. Ortmann, loc. cit., p. 203, 208. - Alcock, 10c. cit., p. 1 ib.

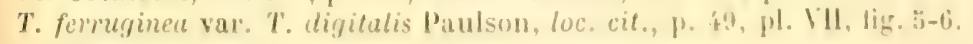

Mer Rouge (Musée de Thurin), ö males at 6 lemelles. 


\section{GenRE TETRAIJA DANA. \\ Tetralia glaberrima (Herbst).}

Cf. Ortmann, loc. cit., p. 209. - Alcoock, loc. cit., p. 224 (ubi syn.). - De

Man, Abh. Senckenb. Ges., XXV, 1902, p. 641, pl. XX'I, fig. 6.

Tetralia cavimana Paulson, loc. cit., p. 51, pl. VII et IX, fig. 1-1 d.

Tetralia cavimana var. cinctipes Paulson, loc. cit., p. 54, pl. VII, fig. 8.

Tetralıa heterodactyla Ilell., Paulson, loc. cit., p. 54.

Obock (I. Maindron), une femelle; Djibouti (M. Jousseaume), une femelle; mer Rouge (M. Jousseaume), a mâles et 2 femelles; Suez (II. Jousseaume), une femelle; Erythrée (Musée de Naples), 2 exemplaires; mer Rouge (Musée de Turin), 3 màles; Assab) (MM. Issel ot Becerai), 2 màles.

Gente QUADRELLA DANA.

Quadrella coronata var.

Nobili, Bull. scient. Fr. Belg., XL, 1906, p. 143.

Entre les îles Hanich et la cote d'Mrabie (MM. Bonnier et Pérez), כ̆ mâles et une femelle.

Genre LyBJA Rathi.

(Melia Lat., nom. præoccup.)

Lybia denticulata Nob.

(Pl. VIII, lig. 6.)

Nobili, loc. cit., p. 408.

Mer Rouge (M. Jousseaume), un mâle el une femelle.

Cette espece differe nettement de L. lessellala (dont j’ai comparé des exemplaires de Mldabra) par la forme de sa carapace dont les bords lateraux sont denticulés et par la longueur de ses pattes ambulatoires.

La carapace est un peu plus large que longue, et sa forme est lout à fait différente de celle d L. lessellala. Le's bords latéroantérieurs sont plus dirergents en arriere et en dehors, subégaux en longueur aux bords postéro-latéraux qui convergent en arrière.

Les bords latéro-antérieurs dans tessellata sont distinctement plus courts que les bords latéro-postérieurs; et i la jonction 
des bords il y a une dent bien distincte ef forle. Cette dent manque dans $L$. denticulalu, mais les bords sont finement denticulís.

La surface de la carapace es peu irreguliere; lat portion an-

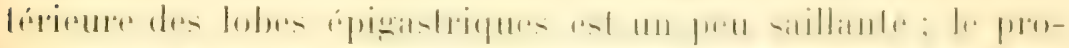

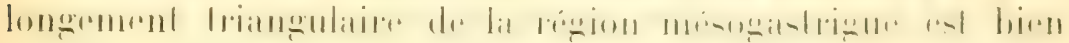
visible. l'ar-ci, par-lit la carapace a encore de faibles traces de

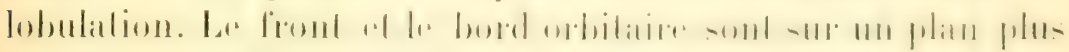
bas que le restant de la carapace qui est bombe. I front os avancé, plat, mais près de son exlrémité il sönfléchil verlicatlement en bas: son bord anteriem inflichi savance au miliou

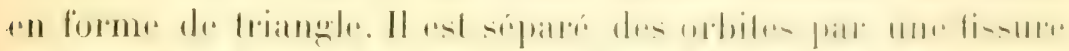

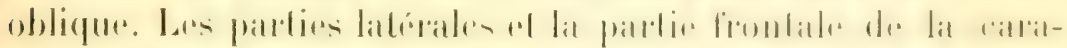

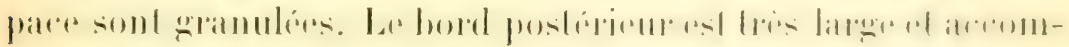
pagné par un sillon.

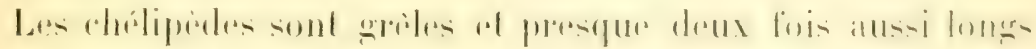

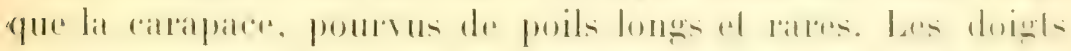
sont un peu phus courts que la carapace. al different de ceux

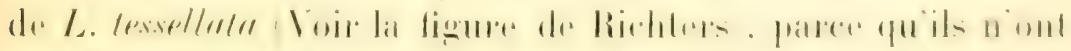

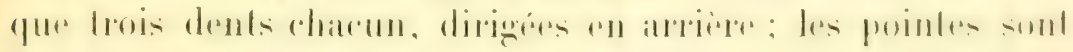
forlement recourbées en dedans.

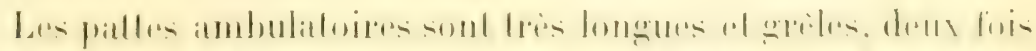

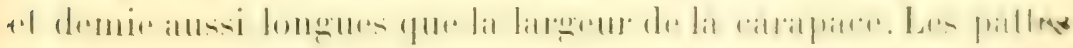

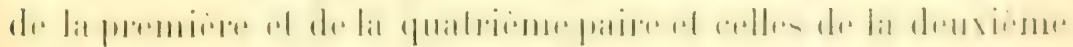
ef de la troisieme paire sont rigales.

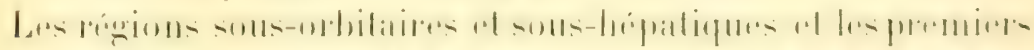

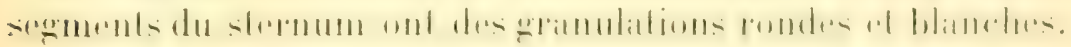

Longueur de la carapace............ 10 millim.

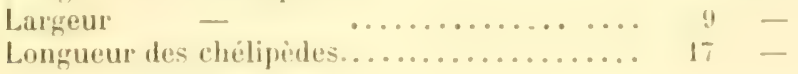

La femelle a entre les doighto des masses charmues lopp diti:riorées pour pouvone "n reconnailme lat nature, mais qui sonl

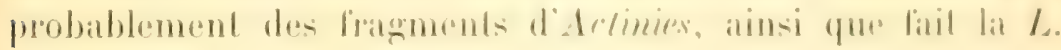
lesw/lılı.

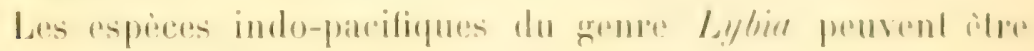
dispostes ainsi :

A. Bords latéro-antérieurs non deroupres an aros lohes

B. Une dent a la jonction des hords pusteriems ave: 
les bords latéro-antérieurs. Bords latéro-antérieurs plus courts que les latéro-postérieurs. Front peu saillant. Doigts des chélipèdes avec de nombreuses dents. Pattes de longueur médiocre............ L. tessellata Lat.

$B B$. Bords latéraux finement denticulés. Front saillant. Bords latéro-antérieurs subégaux aux latéropostérieurs. Doigts des chélipèdes avec trois dents. Pattes deux fois et demie aussi longues que la largeur de la carapace...................... denticulata Nob.

AA. Bords antéro-latéraux grossement lobulés.

C. Carapace aussi longue que large, à surface divisée en tubercules nombreux. Pattes ambulatoires de la première paire plus grèles et plus courtes que celles de la quatrième...................................

$C C$. Carapace plus large que longue, à surface moins lobulée ; pattes de la première paire égales à celles de la quatrième........................... pugil Alc.

\section{CATOMETOPA}

Famille GONOPLACIDA.

Genre EUGRATE De HaAn.

Eucrate crenata De Haan.

Je Haan, F. Jap. Crust., p. 51, pl. XV. tig. 1. - Ortmann, Zool. Jahrb. Syst., 1894, p. 688, pl. XXIII, fig. 4. - Alcock, J. As. Soc. Bengal., LXIX, 1900, p. 300. - Nobili, Boll. Mus. Torino, XVlll, 1903, no 455, p. 35; Bull. scient. Fr. Belg., XL, 1906, p. 145 .

Pilumnoplax sulcatifrons Targioni Tozzetti, loc. cit., p. 102, pl. VII, fig. 2.

Ver Rouge (M. Jousseaume), un male et une femelle: Aden (M. Jousseaume), une femelle jeune.

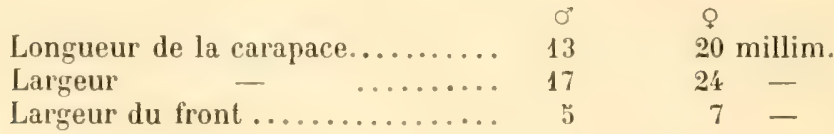

Les taches rouges sont bien marquées dans le màle; elles manquent dans la femelle. La jeune femelle de Aden offre les mêmes différences que les jeunes exemplaires du golfe Persique, décrits par moi (loc. cit.).

H.ıв. : Japon (De Haan, Targioni, Ortmann); Hong-Kong (Alcock); Singapore (Nobili); Inde, Indamans (Acoch); golfe Persique (Nobili). 
Eucrate crenata var, dentata (Stm.) Alc.

Alcock, loc. cit., p. 301 .

?Heteroplax dentatus Stimpson, Proc. Acad. S. Sc. Philad., 1858, p. 9'.

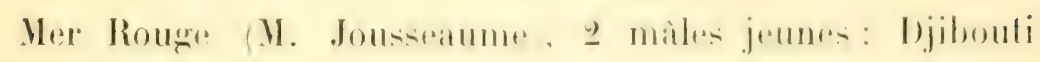
(II. Coutière), 3 jeunes màles.

Lat denxieme et la quatriome dent laterale sont petites lit premiere el la troisiemesont heaturoup plus saillantes. Labhatncrure frontale est parfois nulle, parfois a peine marquare. La

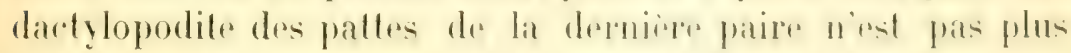

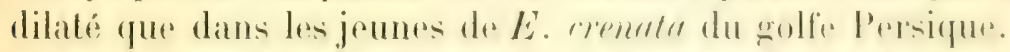

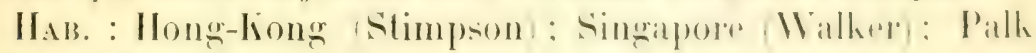
Strait (Aleock).

Genre LibYS'T'ES A. M.-Enw.

Libystes nitidus A. M.-Edw.

A. Milne-Edwards, Ann. Soc. Ent. Fr., 1867, p. 285 ; Nouv. Arch. Mus., IV, 1868, p. 83, pl. XXVI, fig. 5-7.

Une femelle de Djibouti (II. Jousseaume).

l'ai comparé eet exemplaire au type de Zanzibar, pare qüil offrait des différenes remarefuables d'arece la figure: mats.

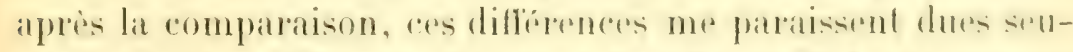

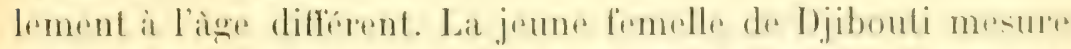

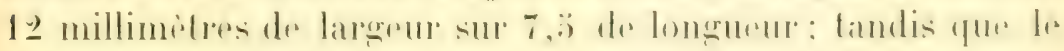

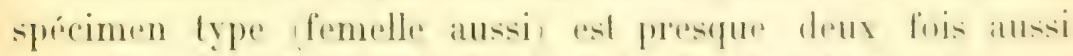

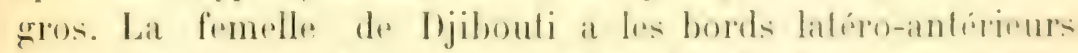
gramuleux denticulés: quelques-uns des denticules sont phos saillants, sans toulefois alleindre les dimensions des dents de

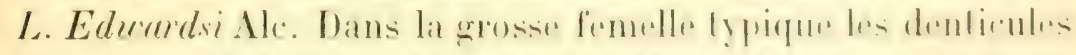

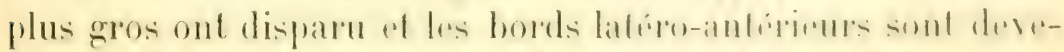

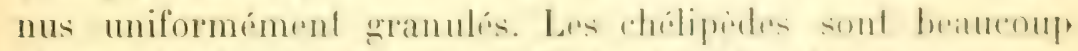

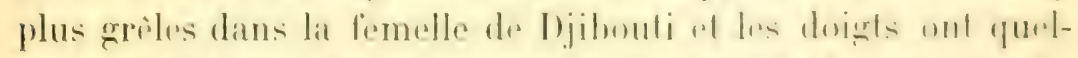

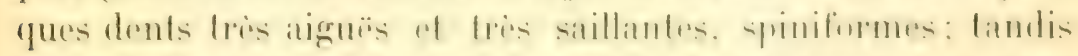

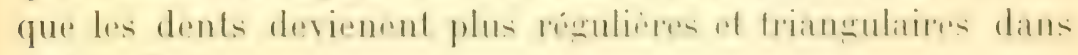
la femelle type.

Les dactylopodites de la cinquieme paire ont la forme

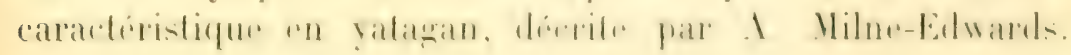




\section{GenRe PARANOTONYX Nob.}

\section{Paranotonyx curtipes Nob.}

(PI. VIII, fig. T.)

Nobili, loc. eit., p. 408.

Mer Rouge (M. Jousseaume), une femelle.

Je cois necessaire d'atablir une nouvelle roupe gémérique pour colle forme qui est voisine de Solonyer el de C'eraloplate, mais qui ne peut rentrer dans aucun de ces genres. Les caraclères du genre peuvent ètre établis ainsi :

La carapace ressemble a celle de Ceralnplar : elle est tris élargie, bombée, dóclive en avant, plus large en avant quen arriere et les régions n'y sont pas dislinctes. Le's angles latéroantérieurs sont arrondis. La carapace est marginée tout autour par une crete saillante. Le bord fronto-orbitaire mesure les 2/3 de la largen de la carapace; le front est un peu arqué en avant. Les bords postérieurs sont convergents.

Les pédoncules oculaires soml molniles. Las antemuless se replient obliquement dans des fosseltes propres; le fouet des antennes est placé dans l'orbite.

L'épistome est birn formé; le radre bureral se rétréril en aremt, l'ouverture de la bouche est entierement fermér par les maxillipedes qui ont lo mérus dilalé, liès longye el anssi lony quel'isrhium. Le "palpe " des maxillipedes est fort, son dernier arlicle est pourvu de longs poils.

La forme de ses maxillipioles dislingue ce genre de Ceratnplax el de Notony $x$.

Le petit exemplaire est large $6^{\mathrm{mm}}, \ddot{\partial}$ et long un peu plus de 4 millimètres. La carapace est plus large en avant qu'en arière, parce que les bords latéraux convergent en arrière. La surface en est finement poncture et peul-itre poilue (dans les individus bien conservés), trés déclive en avant ou le bord du front n'est pas visible d'en haul. Il n'y a aucune lare de régions. Le front mesure environ 1/3 de la largeur de lil carapace, il est légèrement aryué en avant, mais non fichan"ré; ses bords latéraux sont obliques. Les orbites sont petites "l sans fissures. Le front, les orbites, les bords latéraux de la cirrapace el mème le bord postérieur sont marginés par un 


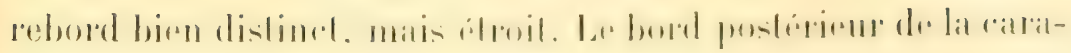
pace mesure les $2 / 3$ de la largenr totale.

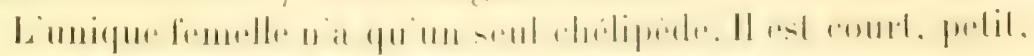

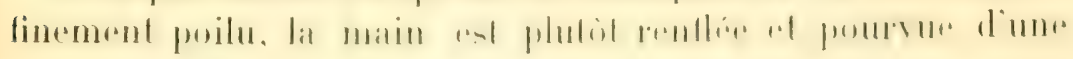
crête assez forte on dehor's pres du bord inférieur.

Les paltes ambulatoires sont courles it peine un peu plus longues que la latroen de la carapace; alles sont finement poilues.

Les doigh sont un peu crochus at courts; sur les pattes de la troisieme el de la quatrieme paire ils mesurent it peine la moilie de la longueur des propodiles. Dans les Volomyer of Ceratoplax les pattes sont beaucoup plus longues.

\section{GENRE XENOPHTHALMOIES RICHT.}

Xenophthalmodes Mobii Richt.

De Man, Not. Leyd. Mus., XII, 1890, J. 68, pl. II, fig. ̈̈.

Signalé à Djeddah par M. De Man.

\section{FIMILE PINNOTERIIIE.}

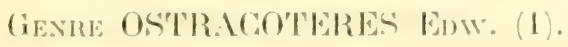

A. Dactylopodite de la dernime paire de palles crochu comme celui des autres palles.

B. Carapace plus ou moins arrondie. Carpe et propudus des maxillipides externes asse\% loners........ O. triduenx liüpl.

BB. Carapace presque quadrangulaire. Carpe et propodites des maxillipedes tres courts............ o. affinis Edw.

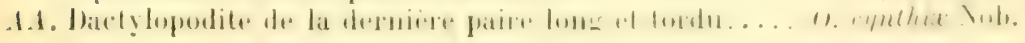

\section{Ostracoteres tridacna Riipp.}

Savigny, pl. IIl, tig. 1.

Pinnotheres veterum Audoin, Expl. (nec Bose

Pimotheres tridacna Rüpjell, Inc. cil., p. 2.2. pl. Y' lig. .․

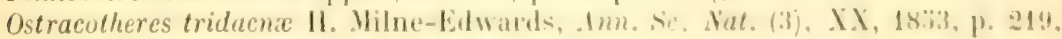
pl. 11, fig. 10. - Paulson, loc. cit., 1. 70, - Kossmann, loc. ril. 11. 62, Adensamer, Ann. K. K. Hofmus, II ien, XII, Is!7, P. 10!. Ostracotheres Savignyi II. Milne-Ldwarils, loc. cil., p. 21!?. ? Ostracotheres Savigmyi Adensamer, loc. cil., p. 108.

(1) Puisque le nom Ostracoteres a la mime origine que Pinnoteres, an doil l'écribe de la meme faron aree $t$ et non th. 
Suez (M. Vaillant), 10 màles et 13 femelles. Massaoualr (MII. Issel et Beccari, Musée de Gènes), un mâle et une femulle; Obock (M. Jousseaume), 4 màles et 7 femelles; mer Rouge (M. Jousseaume), 3 mâles et 2 femelles.

II. Adensamer (Inr. rit.) signale une O. Sacignyi de la mer Rouge prise dans les Ascilies et une O. tridarnx puis les Tridacnes.

Cet auteur considère donc les deux espèces comme distinctes, mais malheureusement il n'a pas donné ses raisons. Peut-ètre s'agit-il d'une autre espèce. Je erois avec liossmann et Paulson. que l'O. Sacignyi n'est pas differente de tridarnx. M. MilneEdwards parait avoir fondé son espèce sur la figure de Savigny, qui représente les poils du bord supérieur des pattes plus forts qu'ils ne sont habituellement.

\section{Ostracoteres affinis Edw.}

11. Milne-Edwards, Ann. Sc.nat. (3), XX, 1833, p. 220, pl. Xl, fig. 11 .

Cette espèce est bien caractéristique, et ne paraît pas avoir éte revue, après la première description de 1853 .

II. Jousseaume en recueillit une femelle à Djıbouti.

La carapace est presque quadrangulaire, plus large que longue.

Dans la femelle de Djibonti, la largeur de la carapace est de 8 millimètres, sa longueur est de 6 millimètres. La largeur est done 1,33 fois la largeur, tandis que ce rapport est de 1.11 dans O. spondyli Nob., de 1,07 dans tridarne et les deux dimensions sont subégales dans $O$. cynthix. Les téguments sont aussi notablement plus minces que dans les autres espéces. Le borl antérieur de la carapace forme une courbe régulière. Les bords latéraux sont droits. Le bord postérieur st droit aussi. La carapace et les patles sont légèrement duveteuses.

Las maxillipèdes externes sont poilus et correspondent à la figure de: Milne-Edwards par la forme du "palpe ". Le propodite court est arrondi en avant au lien d'ètre tronqué oblifurement comme dans les autres especes. Le fouet de l'exopode est formé par un seul article et par une partie flagelliforme. 


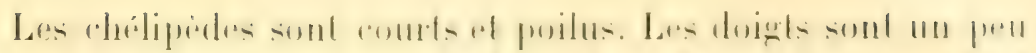

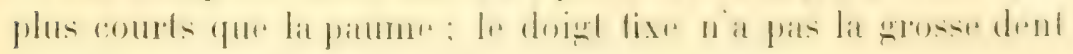
de 0 . tridacnie.

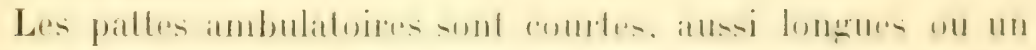

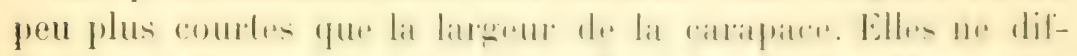

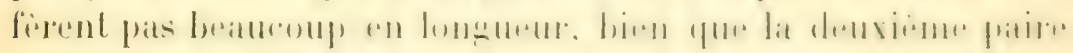

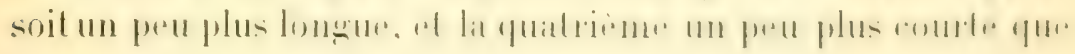
les autres.

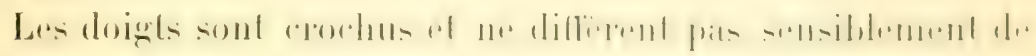
ceux des autres especes, excepte 0 . cynthice.

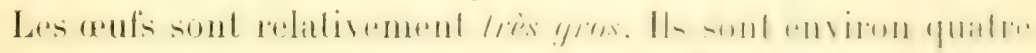
fois aussi gros que cenx de (). spondyli Yob., ou ils ont les dimensions normales des autres P’innoléridés.

Celle espèce n'est connue que de l'Ile de Firance.

\section{Ostracoteres cynthiæ Nob.}

Nobili, loc. cii., 1). 409.

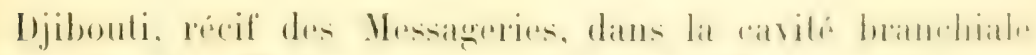
d'une Cynlhia (II. Gravier), un mile et une fenelle.

Cette espèce se reconnait facilement par ses doigls de la dernière paire aplatis.

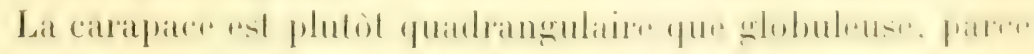

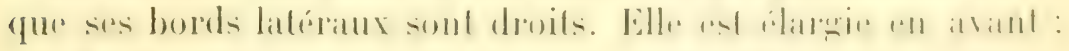
son bord antéricur se rattache par une courbe aux bords lateraux. Lat surfice est unie. sins trace de lobulation, el très bombée, le front est bien détaché du bord antérieur de la rarapace, laminaire et avancé, légèrement saillant au milieu, et un peu concave sur sa surfice dorsale médiane, rehaussé de cóté. Ises yeux sont noirs.

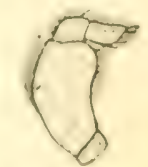

Fiz. 9. - Mavillipinde externe di:

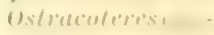
thiir Nish.

La largeur de la carapatee est subergale it sil fomgurme? compris le front. Les parties declives lithro-posterieures de lit carapace sont poilues.

L'ischio-mérognathe des maxillipreder extermes a le: bord postéreur convexe, l'anteriente concatre, un pren angulaire it l'extrémite. Le propodus "st allongr. loonque obliquement au 
bout. Lat fitce externe des maxillipedes est poilue, les bords sont ciliés.

Les chélipèdes sont assez gros et légèrement duveteux. La main a un contour hriangulaire; elle est forte, avec les doigts gros el robustes, poilus, subégaux a la longueur de la paume, ou un peu plus longs, at garnis de dents irregulières.

Les pattes ambulatoires sont grèles, à peine plus longues que la largeur du rorps, légerement poilues, mais portant des franges de poils sur le carpe et sur le propodite. Les doigts des trois premieres paires sont allonges. gribles, cilies, un peu recourbés a la pointe; cenx de la quatrième ou dernire paire sont longs, aplatis, poilus et à bords sinueux, dans la lemelle.

Le male est plus petil que la femelle, sia carapace est plus arrondie: ses chólipèdes sont plus gros. at ses doigts de la cinquirme paire de paltes sont peu différents des autes.

La femelle mesure environ 7 millimetres de longueur et de largeur.

\section{Gente PINNOTERES Iat.}

1. I)actylognathite unguiforme ou slyliforme greble), insire sur le bord inférieur du prognathite.

B. Doigts des pattes ambulatoires tous d'égale longueur. (Dactylus des maxillipèdes dépassant l'extrémité du propodus, carapace quadrangulaire.)..... P. pectinicola

Bürg.

BB. Doigts des pattes de longueur ditlérente.

C. Doigts des pattes de la 3 e et de la $4^{\mathrm{e}}$ paire plus longs que ceux de la $1^{\text {re }}$ et de la $2^{\mathrm{e}}$ paire.

D. Doigts de la $3^{\text {e }}$ paire plus longs que ceux de la

$4^{\mathrm{e}}$ paire. (Carapace élargie, arrondie en avant.) P. purpureus Alc.

$D D$. Doigts de la $3^{\text {e }}$ paire égaux ou plus courts que ceux de la s $^{\mathrm{e}}$ paire.

E. Carapace au moins un quart plus large que longue.

$e$. Extrémité du prognathite arrondie. Doigls de la $1^{\text {re }}$ et de la $2^{\mathrm{e}}$ paire très courts; doigts de la 3 paire de longueur médiocre...... P. lutescens Nob.

$e e$. Extrémité du prognathite tronquée ou étroite. Doigts de la $1^{\mathrm{re}}$ et de la $2^{\mathrm{e}}$ paire de longueur normale.

F. Carapace assez convexe, lisse; bord postérieur peu concave. Doigts des pattes de la $3^{\mathrm{e}}$ paire plus longs ou égaux à ceux de la te $^{\mathrm{e}}$ paire..... P. pernicola Bürg.

$F F$. Carapace aplatie avec des dépressions larges et irrégulières. Bord postérieur profondément 
coneave. Doigts do la ze paire plus courts que

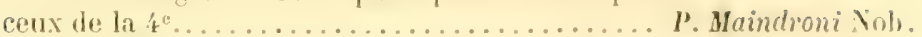

EE. Carapace $1 /: i$ plus lare que longue. front saillant. Joigts de la dernime paire styliformes, grêles, loners et pilus.................... Bomadailei Wob.

C C. Doigls de la 3" pair" aussi lones que ceux de la fr" el de la 2 " paire. Doigls de la p" paire trís longt..... P’. Coulderei Cob. A. Dactylognathite spatuliforme et gros. Carapace cor-

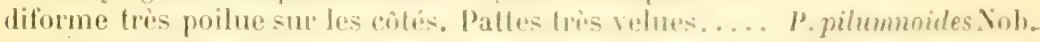

\section{Pinnoteres pectinicola Bïrs.}

Bürger, Kool. Jaterb. Syst., IIII, 189:5, p. 36:, pl. IX, lig. 1 et pl. X, lig. 1.

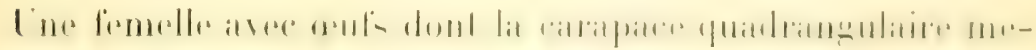

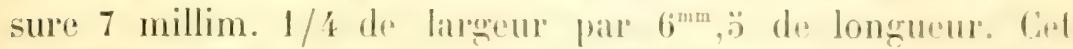

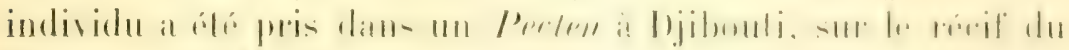
Météore, par M. Cih. Gravier.

Cet exemplate s'aceorde de tout point avec la description

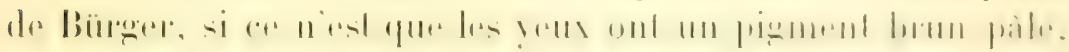
tandis que le pigment manque dans le lype de perfinirnle.

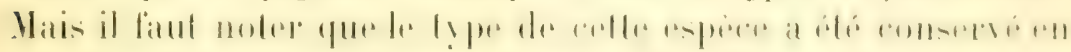

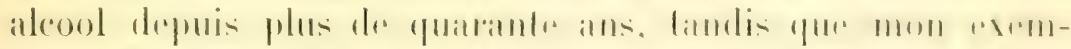

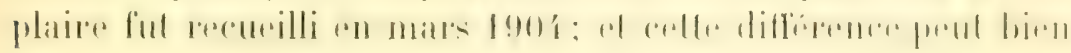
etre due a l'aclion décolorante de l'aleool.

\section{Pinnoteres purpureus $\mathrm{Alc}$.}

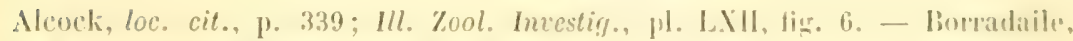
H. Geog. Muld. Lacend., vol. 11, p. \$31.

Djibouti (11. Jousceanme), une fomelle un pen plus petite

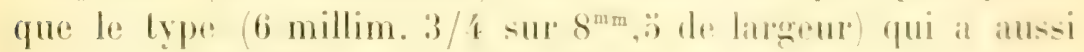
la portion frontate un peu plus saillante, mais qui s'areorde:

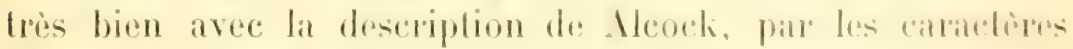
de ses gnathostegites, de ses chélipedes ples palles.

Mer Rouge (No Jousseatume), une liemelle.

Cette esperee n'est connue que des iles Indimans of des Naldives.

\section{Pinnoteres pernicola Biir...}

Bürger, loc. cil., p. 37\%, pl. IX, lig. 17; pl. 1. lig. 16. - Vohili, Imn. Mus. Cin. Genova, XI, 1899, 1. 26ir,

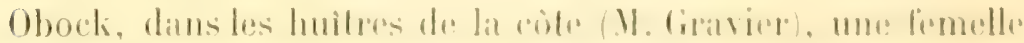

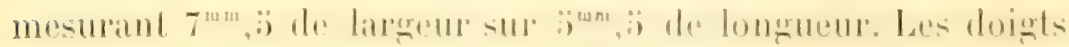


de la troisième paire sont, comme dans un des exemplaires de Bürger, aussi longs que ceux de la quatrième.

Ciette espèce n'est comnue que des Philippines et de la Nouvelle-Guinée.

\section{Pinnoteres lutescens Nob.}

Nobili, loc. cit., p. 409.

Djibouti (M. Coutière), 4 femelles.

Cette espèce est extrèmement voisine de $P$. modiolicola Bürg., i laquelle elle ressemble parfaitement dans le facies général. Par lobligeance de M. le professeur E. Ehlers, jai pu comparer le type unique de P. modinlirola conservé au Musée de Göttingen, et j’ai trouvé entre les deux espèces les différences suivantes:

1. La carapace est plus large. Dans la plus grosse femelle

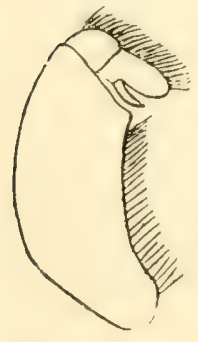

Fig. 10. - Maxillipede exteme de Pinnoteres lutescens Nob. elle mesure 10 millimètres de largeur sur 7 de longueur. Dans modiolicola, la carapace est large d'environ 8 millimètres, et longue d'environ 7 millimètres. Elle est aussi proportionnellement plus convexe dans modiolicola et sa surface est unie; elle est aplatie dans lutescens et sa surface offre aussi des dépressions irrégulieres, moins fortes pourtant que celles du P. Mrindroni, décrite après.

2. Le bord postérieur de la carapace est parfaitement rectiligne dans modiolicola, faiblement concave dans lutescens.

3. Les chélipèdes sont plus grèles et plus allongés dans lutescens et les proportions sont différentes. La paume est a peine 1 fois 1/2 aussi longue que les doigts, elle est presque 2 fois aussi longue que les doigts dans modiolirola); elle us aussi presque 2 fois aussi longue que haute dans luterens, tandis qu'elle est 1 fois $1 / 2$ aussi longue que haute dans modintirolu. La face externe est beaucoup phus couvexe dans modintirnlr, ef elle se rétrécit moins prés de la hase de la paume; le bord supérieur de la paume est donc plus oblique 
dans heserens. Les doigh de modiolieolu nont pas des poils sur l. bord tranchant: ils ont quelques poils seulemene sur le bold

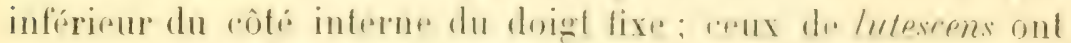
des petites foutfies de poils a l'extremite du bord lramelant et la face interne et une frange de poils bien nette.

4. Les doigts de la premiere el de la denxiome parip sont

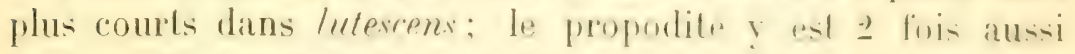

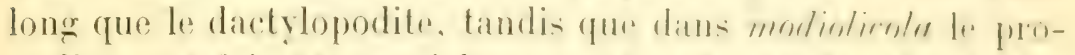
podite est 1 fois 1 a ausi long que les doigts. Les doigts des

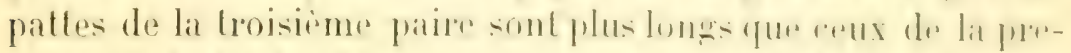

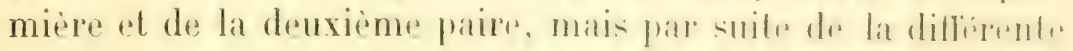
longueur des doights de la premiere et de la deuxieme patre

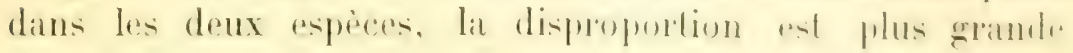
dans lutescens.

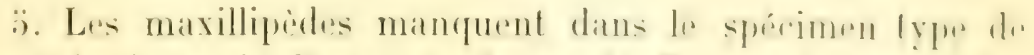

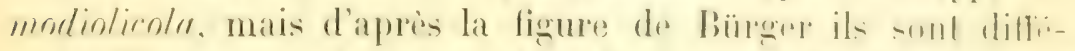

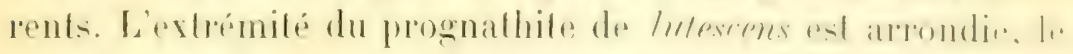

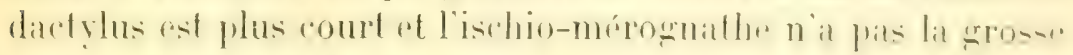
saillie antérieure de modiolicola.

\section{Pinnoteres Coutieri Nob.}

Nobili, l. cit. p. 409.

Cette petite espèce, représentée par une femelle recurillit: par II. Coutiere, it Djibonti, est aussi voisine de modiolicola Bürg. En comparant avec le type de modinticola, je note les différences suivantes:

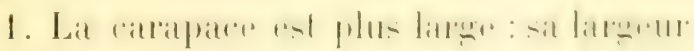
est de $8^{\text {mm }}, 5$ et sa longueur de $6^{\text {mm }}, 3$.

2. Le palpe des hectognathes est plus gros el le propodus plus allongé.

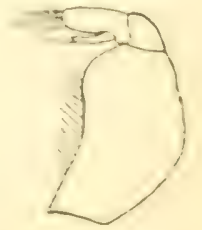

Fig. 10. - Maxillipide externe de pire noteress Contieri.

3. La portion palmaire de la main es seulement un peu plus longue que les doigls; elle est I fois $1 / 2$ aussi longue que haule.

1. Les dactylopodites de la troisieme paries sont aussi lones que cenx de la premiere of de la deuxieme: dans modinlienle. ils sont plus tongs. Les doights de lat quatrieme paire sont

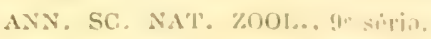
$1 \mathrm{~V}, 20$ 
longs, st liformes, poilus, et 2 fois ansi longs que ceux de la troisième paire.

P. arcophilus. Bürg. qui est aussi voisine de cette espèce, diffère par les mèmes caractères.

\section{Pinnoteres Borradailei nov. nom.}

Pinnoteres tenfipipes Borradaile, loc. cit., p. 431, fig. 113. (Nec P. tenuipes Bürg.).

P. Rouxi Paulson, loc. cit., p. 70, pl. IX, fig. 2. (Nec H. Milne-Edwards.)

Mer Rouge V. Jonswatume), dins les Pinna, 7 femelles: Périm (II. Jousseaume), „j femelles; Djibouli (II. Couliere), une femelle.

Ces exemplaires saceorlent avec la description de Borradaile, ainsi fuarec relle de l'aulson, mais la carapace dans les gros axemplaires pamil bre un peu plus flargie. Dans 2 femelles la carapace est longue de 11 et $11^{\mathrm{mm}}, \tilde{5}$, et large de 13 of $1: 3^{\mathrm{mm}}, 0$. Le daclylus hiren diveloppé alteint l'extrémité du frognalhitr on mème la dépasse légèrement. Le hord infriver de la main us cilió du colrinterne, ainsi que le sont les doigts sur le bord préhensile.

Paulson a bien figure celle espece sous le nom de P. Rouri Celte identificalion ne me parail pas exacte. Nous ne connaissons que la ligure des hectognalhes de cette espece; mais ceux-ci sont conformés différemment.

Le nom tenmipes aynt dógit éte employi par Bürger j’ai proposé pour cette espèce celui de Borradailei.

\section{Pinnoteres Maindroni Nob.}

(Pl. VIII, fig. 8.)

Nobili, l. cit. p. 410.

Obock (M. Maindron), une femelle.

L'espèce est voisine de $P$. consors Bürg., et a, comme celle espèce, la carapace à contour polygonal, et doigts des pattes des deux dernières paires plus longs que ceux des deux premières paires.

La carapace a une forme subtrapézoïdale avec le bord 
antérieur très larege, it angles Jatérax arrondis mais asse\% étroits. Les bords latéraux sont obliques et divergents en

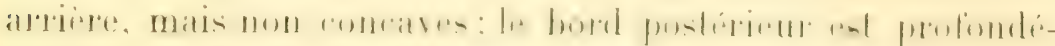
ment sinué au milisu. La carapace est entirement glabre. aplatie, el mème crensée de dépressions irrégulieres el trè

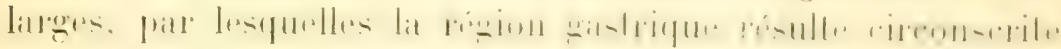
en relief.

Le front et les yeux ne sont pas risibles den lanul: le fronl est droil, mais son bord antérienr manque. el, vu d'en bas, il apparail tribbé parce que les trois prolongements sous-frontaux se raltachent directement au front. Les yeux sont pelits et bruncitres. Les maxilliperdes externes ont le "palpe "assez gros, aveu le propodus allongé, pourvu d'une faible saillie a son extrémité, mais autrement coupé

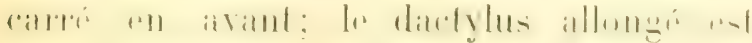
étroil, arrondi à l'extrémité el dépasse légèrement l'extrémité du propodus.

Les chélipèdes sont grèles el inermes: I

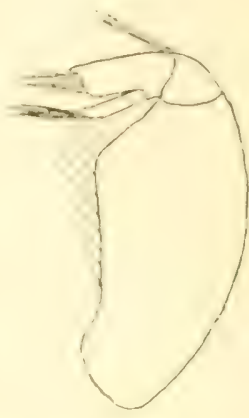

Fig. 11. - .laxilliped. externe d, loinnoleves.MaindioniNoh. mérus est grềle et allongé, le carpu court.

La main est étroite, allongée; la paume est un pru moins que 2 fois aussi longue que les doigls. Autant les chélipedes que les autres paltes sont dépourvus de poils.

Les paltes ambulatoires sont greiles, phutól courtus, ou de longuenr médiocere. Les pattes de la trobieme paire sont les plus longues. Les paltes de la promione de da deuxiemes

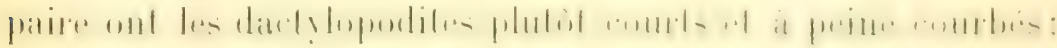

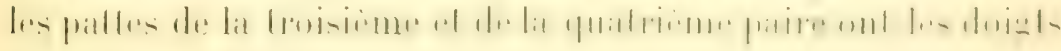
longs; ceux de la hooisieme paire sont un pen plus courts que ceux de la qualrieme.

Longueur de la carapace ............

Largent

\section{Pinnoteres pilumnoides Nob.}

Tobili, I. cit. 1. \&10.

Djibouli (H. Coutione), une lemelle.

Celle espèce est voisine, par son lacies, de P'. Irupeziformis 
Nauck et P. villoxisimus Dofl. La carapace est longue de 9 millimètres et large de $10^{\mathrm{mm}} .5$; elle est moins élargie que dans $P$. trapeziformis. La surface supérieure de la carapace se rétrécit fortement en arrière; le contour de la carapace est ainsi cordiforme, et le bord postérieur est large seulement de " miflimètres, soit à peine les $2 / 5$ de la largeur de la carapace.

La surface dorsale de la carapace a sur les côtés des long̣s

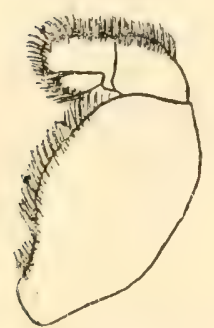

Figr. 12. - Maxillipide cxterne de Pinnoteres pilumnoücle Nob. poils feutrés, analogues à ceux qui couvrent les pattes et les parties latérales infléchies. De chaque côté de la partie postérieure de la région gastrique et de la cordiale il y a une faible dépression. La partie antérieure de la carapace s'infléchit beaucoup; le front est distinct et a la forme d'un lobe assez large, bilobé et sillonné; latéralement, il est bien séfaré des orbites qu'il devance. La partie antérieure de la carapace est très faiblement fcourbée, fles bords latéraux sont presque droits dans le premier trait, puis convergent en arrière. Les yeux sont noirs.

Les pinces sont tris poilues sur la surface; la paume est un peu plus longue que les doigts : ceux-ci sont gros, robustes et joignent bien: ils sont bien excavés an bout. La face interne de la main est plus poilue que la face externe.

Les patles ambulatoires sont rourtes et trapues, d'égale ongueur, excepté la dernière paire; elles sont courertes de nombreux poils tres longs et touffus, et sont terminées par des doigts courts et crochus, d'égale longueur.

Le carpe des hectognathes ast plus gros que le propodus; cet article est arrondi en avanl. Le dacty̧lus est arrondi en avant, spatuliforme, tres gros at phus courl que le propodus. Les hectognathes sont très poilus.

L'abdomen de la femelle est conformé différemment que dans les autres P'innotires parce que ses premiers articles ne sont pas visibles d'en dessus.

l'ar la forme de ses gnathopodes celte espece s'approche de P. trapeziformis Nauch. Mais dans trapeziformis, la cara- 


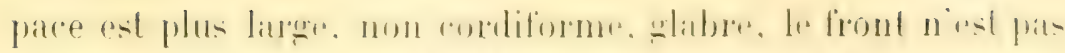

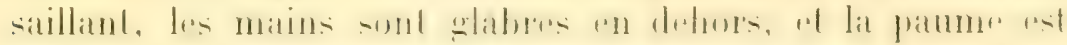
2 fois aussi longue que les doigts, etc. (Ci". Bürger, loc. rit.. p. 380, pl. IX, lig. 26, el pl. X, fig. 23).

$P$. villosissimus, récemment décrit par Doflein (Brach. Deutsche Tiefsee Exp., p. 123̈, pl. XXXVII, fig. 6-7), ressemble beaucoup par son facies it celte espece, mais ses

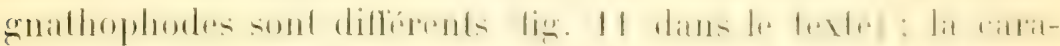

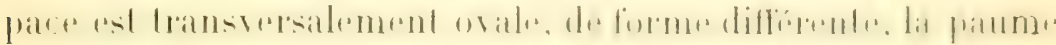

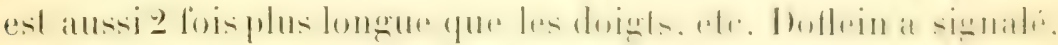
i propos de son espèce, le fail intéressant qu'elle a de très

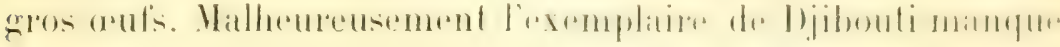
d'oufs.

\section{GENRE DURCKHEIMIA I)E MIAN (RUpr?ms.).}

Durckheimia carinipes Je Jan.

De IIn, Zool. Jahrb. Syst., IV, 1899, p. It2, jl. X, fig. 12.

Gette espece est connue seuloment par l'unique axemplatire, lype du Musée de Francfort-sur-Mein.

\section{FAIILLE OCYPODIDA.}

(IENTE OC)POI) I F.113.

A. Ligne stridulante de la grisse main absente.

0. cordimena

A. Ligne stridulante bien déreloppée. l)esm.

B. Ligne stridulante formée de tubercules ot de petites rides. Angles orbitaires externes aigus dans les adultes.

C. Ligne stridulante formine pat it)-iol rides et tubercules. Propodites des pallos ambulatoires de

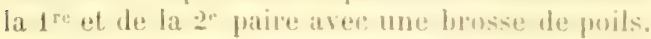
Angles orbitaires aigus mais peu saillants.... O. ceratophlhame l'ill.

CC. Ligne stridulante forme de 7 ij rides et lubercules. limsses sur la premiere paire de paltes seulement. Angles orditaires aigus et tres sail.

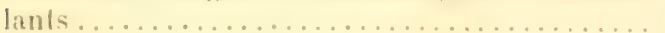

BB. Ligne stridulante formee soulement de rides (bioJinusemumri Noh. 100). Angres orhilaires arrondis dans les adultes

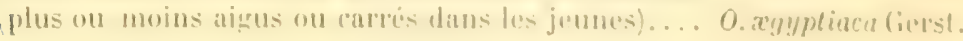




\section{Ocypoda cordimana Desm.}

Cf. Ortmann, Zool. Jahrb. Syst., X, 1897, p, 360, 362 (ubi syn.). - Alcock loc. cit., p. 345,349 .

Massaouah (M. Clivio, Musée de Turin), un màle large de உ't millimètres et long de $20^{\mathrm{mm}}, \mathrm{b} ;$ Obock (M. Jousseaume), une femelle longue de $17^{\text {m }}$, כ̆ et large de 21 millimètres.

\section{Ocypoda ceratophthalma Pall.}

Cette espèce fut signalér par II. Milne-Edwards en Égrpte, et par von Martens à liosseir. Je n'en ai pas vu d'exemplaires. adultes de la mer Rouge; un jeune exemplaire de l'île Daret du Iusée dr Naples, appartient probablement à celle espece.

\section{Ocypoda Jousseaumei Nob.}

Nobili, Bull. Wus., 1905, n 4, p. 233, fig. 2.

Obock (M. Jousseaume), un mâle.

\section{Ocypoda ægyptiaca Gerst.}

Gerstäcker, Arch. f. Nat., 1856, p.134. - Heller, luc. cit., p. 361. - Hotlmann, loc.cit., p. 14. - Mier's, Ann. Mag., Nat. Hist. (5), II, 1898, p. 409.De Man, Not. Leyd. Mus., III, 1881, p. 247. - Miers, Ann. Mag. Nat. Hist.(5), X, 1882, p. 381, pl XVII, fig. 3. - Ortmann, loc. cit., p. 360, 366. - Nobili, Ann. Mus. Zool. Napoli, 1, no 3, 1901, p. 16; Bull. scient. Fr. Belg., 1906, 1). 152 .

O. ceratophthalma Kossmann, loc. cit., p. 55 (nec Pallas).

O.cerathophtalmu var. ceratophthutmi-xyyptic a Paulson, loc. cit., p. 64.

Abdelhaderprès de Massaouah (M.Clivio, MusséTurin), - màles et 3 femelles; Érthrée (11. Tellini, Vusée Turin), 2 màles et une femelle; Scheïk-Sä̈d, près de Massaouah (M. Magretti), 2 femelles; Massaouah (11. Issel el Beccari, Musée Gènes), 3 màles el a femelles; Melen (11. Doria, Musée Gènes), une femelle; Ras Garibal-Assab (M. Ragazzi, Musée Modène), 3 màles; Suez (11. Jousseaume), 3 mâles; Obock: (M. Maindron), כ̌ indisidus jeunes: ile Musha sur le sable (M. Gravier), un male: golfe de Tadjourah (M. Faurot), 2 mâles jeunes. 


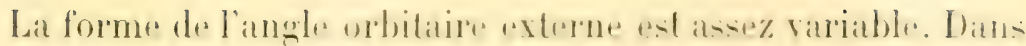

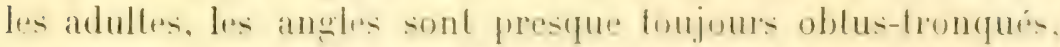
romme dans lat ligure de Mlars. matis parfois les angles sonl

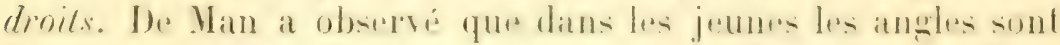
droils. En jugeanl dopros mes exemplatires. les individus dige

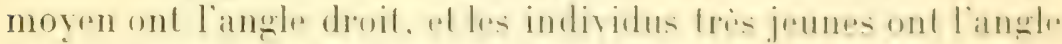

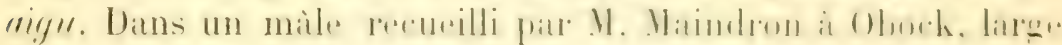

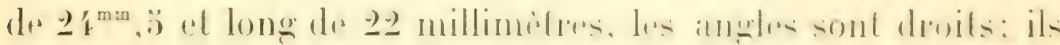
sont aigus dans un male dre lat mime localiti. latere de la mil-

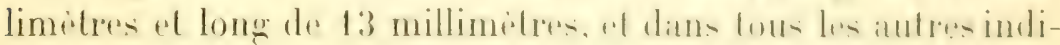
vidus plus petits.

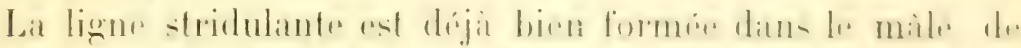
2年"

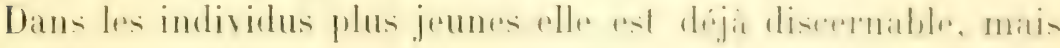
moins marquée.

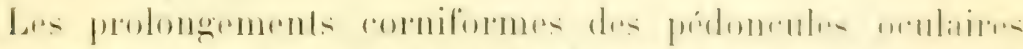

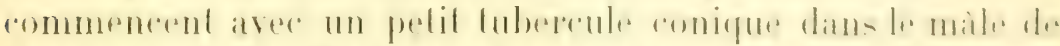

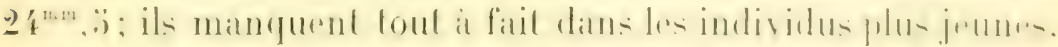

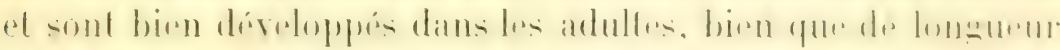

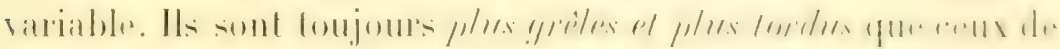
reratophthalmut.

Un exemplaire femelle recueilli is Suez par M. Jonswaume

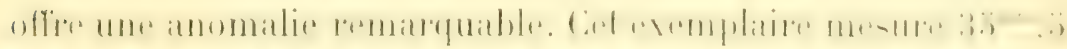

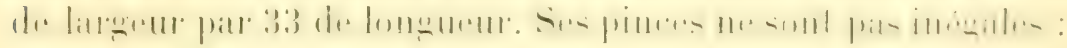
l'une grosse el pourve de ligne stridulante, l'aulre pretite el

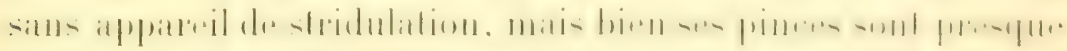
égales et ressemblant it la petite punce des exemplatires normaux, el dépourvues de ligne stridulante. l'eut-eitres y at-l-il li un cas de régénérution hypolypingue de la pincor.

\section{GiEHE ULiL LEACH.}

\section{'ielesimus Lat.}

A. Front large entre la base des yenx, mesurant de un cimpuieme is un sivieme de la largeur de la earapace.

B. Deux crètes granulées sur le bral interne de la main, l'une pris de l'artirulation des doights, l'antre oblique dans la moitié postirieure.

U. anmulipe's (Lat.).

BB. Une seule crète gramulée prés de l'aticulation des

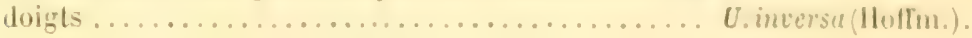


$A$ A. Front mesurant moins d'un sixième de la largeur de la carapace.

C. Front mesurant environ un dixième de la largeur de la carapace. Crêtes granuleuses de la main indistinctes. Doigts peu comprimés................

CC. Front mesurant environ un quinzième de la largeur de la carapace. Crêtes granuleuses de la main proéminentes. Doigts presque laminaires.

D. Doigts cultriformes.

U. tetragonon

(Herbst).

$D D$. Doigl fixe excavé en W.

U. marionis

(Desm.).

U. marionis var. nitida Dana.

\section{Uca annulipes (Lat.).}

Gelasimus annulipes Lat. - Milne-Edwards, H. n. Cr., II, p. 55, pl. XVIII, lig. 10-13, et Ann. Sc. Yut. (3), XYIII, 1852, p. 149, pl. IV, tig. כ. - Kingsley, Proc. Acad. N. Sc. Philad., 1880, p. 148, pl. X, fig. 22. - De Man, J. Linn. Soc., XXII, 1887, p. 118, pl. VIIl, fig. 5-7. - Alcock, loc. cit., p. 354.

Gelasimus annulipes var. albimana Kossmann, loc. cit., p. 53.

L'a annulipes Ortmann, Zoal. Jahtb. Syst., X, p. 33̈4. - Nobili, Boll. Mus. Torino, XVI, 1901, no 397 , p. 13, fig. A-B.

Djibouti (M. Jousseanme), un màle; Oboch (M. Jousseaume), 2 màles; Périm (M. Jousseaume), un mâle; Assab, Ras Garibal (M. Ragazzi, Nusée de Modène), 3 mâles.

Ces exemplaires appartiennent a la forme de la partie occidentale de la région Indo-Pacifique (Cf. YoвıLs, loc. cit.), soil à la forme qui a la dent subapicale du doigt fixe petite, ce qui donne à l'extrémité de ce doigt un aspect tronqué. Celte forme est la mème que le Gelasimus perplexus Edw.

\section{Uea inversa (Hoffm.).}

Gelasimus inversus Ilofrmann, Crust. Echinod. IIadag. Pollen et Vandam, Rech. Faune Madag.), p. 19, pl. IV, fig. 23-26 (1874). - De Man, Not. Leyd. Mus., XIII, 1891, p. 21, 24, pl. IV, fig. 12. - Alcock, loc. cit., p. 352-355.

Uca inversa Ortmann, loc. cit., p. 351. - Walker et Scott, Crust. Abd-el-Kuri, p. 216. - Nobili, Bull. scient. Fr. Belg., XL, 1906, p. 151.

Abdelkader et environs de Massaouah (M. Clivio, Musée de Turin), nombreux exemplaires; Assab (I. Ragazzi, Musée de Gênes), 3 mâless; mer Rouge (Musée Turin), 2 màles; Arafali M. Ragazzi, Musée Nodène), a màles et une femelle; Obock (.I. Maindron), un màle; Djiloouti (II. Maindron), un màle; Ujibouti (M. du Bourg de Bozas), un màle; Obock, dans la vase 


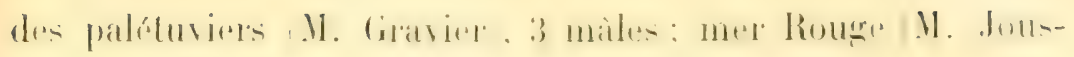
seaume), 2 mâles.

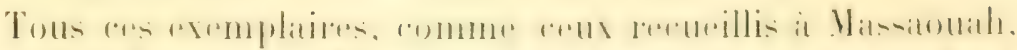

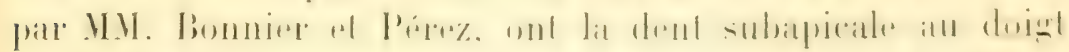
mobile, et appartiennent i la forme typique. Celte forme

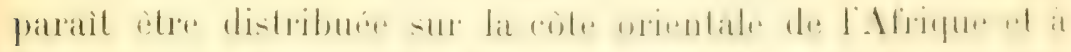

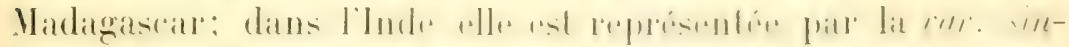

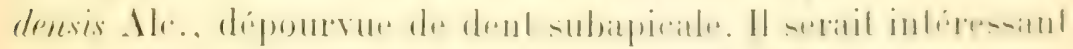
de connaître quelle forme se trouve it $\mathbf{l y d}$ d-el-huri.

Longueur de la carapace....... $\quad$ i $\quad 10$ millim.

\begin{tabular}{|c|c|c|c|}
\hline Largeur & ......... & 12 & 19 \\
\hline Longueur & - de la pince ............ & 18 & 28 \\
\hline 一 & de la paume.......... & 6 & 8 \\
\hline - & du doigt mobile........ & 12 & 20 \\
\hline Hauteur d & le la paume............. & 6 & 10 \\
\hline
\end{tabular}

Uca tetragonon (Herbst).

Cancer tetragonon Herbst, I, II, p. 2:37, pl. XX, fir. 110.

Gelasimus tetragonus Rüppel, loc. cit., p. 2:, pl. V, fih. ". - Milne-Edwards H. n. Cr., II, p. 52; Ann. Sc.nat. (3), XVIII. p. 147, pl. III, lig. 9. - De Man,

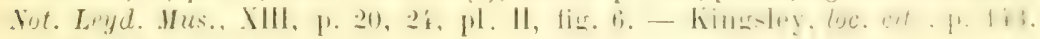
pl. IX, fig. 11 .

Gelusimus tetragonon Alcock, loc. cit., p. 3333, 357 (ubi syn.).

Uca tetragona Ortmann, loc. cit., p. 34t8.

Uca letragonon Nobili, Bull. scient. Fr. Belg., 1906, p. 151.

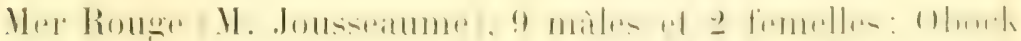

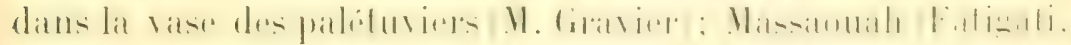

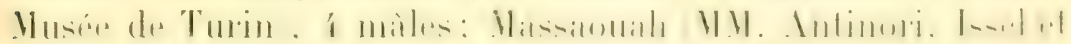

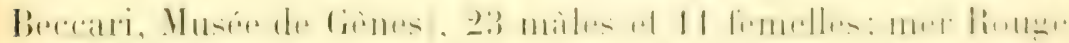

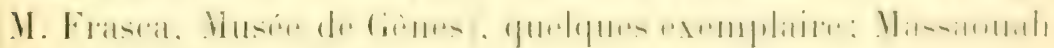
(II. Clivio, Musée de T'urin), 3 miles.

Une des femelles recuillies par 11 . Jonsecaume at de grande

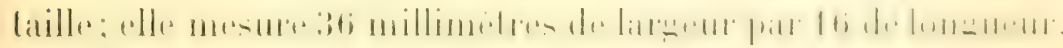
Dans ces exemplaires, on observe le mime lail que javalis

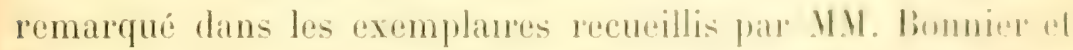
Pérez, soil que les femelles ont les méropodites des palles ambulatores granuleux sur la surdace externe of crandir sur le bord superieur. Lat coloration est lat mime que celle derrite jar moi (Inr. ril.)

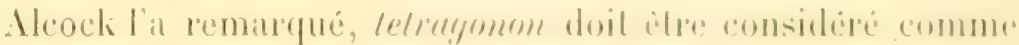
substandil of non eomme un aljerelif. 


\section{Uca Marionis (Desm.).}

Gelusimus Marionis Desmarest, Cons. Crust., p. 124, pl. XIIl, fig. 1. - MilneEdwards, Ann. Sc. Nat. (3), XVIII, p. 145, pl. III, fig. 5 (nec H. n. Cr., II, p. 53). - Kingsley, loc. cit., p. 141, pl. IX, fig. 8. - Alcock, loc. cit., p. 353, 359. - De Man, Abh. Senckenb. Ges., XXV, 1902, p. 477.

Djibouli, dans le sable vaseux (1I. Gravier), un mâle qui a les dimensions suivantes :

Longueur de la carapace............ 8,5 millim.

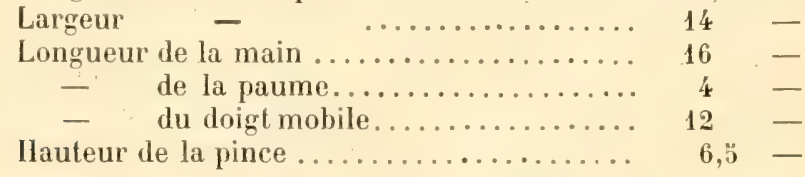

La forme du doigt fixe de la grosse pince s'approche de la ligure de Vilne-Edwards, mais le bord tranchant est plus droit, et moins recourbé en $\mathrm{S}$. Cette pince est exactement égale à relle d'un jeune $U$. Duxwmieri de Samarinda (Bornéo) du Musée de Turin. La dent du bras el la dent du carpe sont très réduiles. Le doigt mobile est profondément sillonné sur sa face externe. Des deux crêtes de la face interne de la main celle qui est parallèle à l'articulation du doigt est presque oblitérée; l'autre est très saillante. H. Milne-Edwards a employé deux fois te nom de Gelaximus Mariomis pour deux especes différentes. La première fois, dans l'Hixtoire nromelle des Crustarér, il désigne sous ce nom l'espece qu'il appellera plus tard Gelasimus perplexus, et qui "st identique avec la forme occidentale de $U$. ammlipes, la deuxieme fois, il désigne rraiment l'espèce de Desmarest.

Uca Marionis var. nitida Dana.

Gelasimus vocans H. Milne-Edwards, Ann. Sc. Nat. (3), XVIII, p. 14́5, pl. III, lig. 4 (nec H.n. Cr., II, p. 54). - De Man, Not. Leyd. Mus., XIII, 1891, p. 23, pl. II, fig. ${ }^{\circ}$.

Gelasimus nitidus Dana, loc. cit., p. 316, pl. XIX, fig. 5.

G. Marionis var. nitidus Alcock, loc. cit., p. 333, 360 .

Djibouti, dans le sable vaseux en face de la Résidence (11. Gravier), ? mâles; Obock (M. Jousseaume), un màle jeune. Les deux màles de Djibouti sont adultes el ont les dimensions suivantes : 
II. Alcuch a décrit me espèce d'Aden, D. affinix, qui, très probablement, est identique avec $D$. sulcala. Dotillu affinis diffère de sulcala par trois points : $1^{\circ}$ absence de l'épine sur la face inférieure du bras; $\underline{\beth}^{\circ}$ présence d'un tympanum sur la face supérieure du méropodite des pattes de la dernière paire: 30 doights des chélipèdes pas aussi longs que la paume.

Nes exemplaires (excepté deux qui ont les doights subégaux a la paume) ont tous les doigts plus longs que la paume : caraclire de sulfata. Mais pour les deux autres caracteres, ils sont bien variables et s'approchent souvent d'affinis plus que de sulralu. Ainsi sur 20 exemplaires examinés, fi nont pus d'épines. anl bras et 1 t ont des tympans bien formés sur les dernières pattes. De plus, aucun des exemplaires noffre la combinaison simultanée des caractères de la présence de l'épine et de l'absence dutympan, ce qui ferait d'eux des $D$. sulcuta, mais ces caractères rarient d'un individu a l'autre, paree qu on trouve des individus avece épine et tympan, et d'autres sans épines et sans tympan. Deux seuls exemplaires offrent les caracteres d'affinis. liss autres ont les caractères de sulcata el d'affinir mélangés. Jo crois donc que ces deux espèces sont fondées sur des caraclieres tres variables et que affinis ne peut pas itre dislincte.

Les individus extrèmement jeunes n'ont que des traces trè faibles de sillons, nont pas des lympans aux paltes, et ont des aires membraneuses sur le slermum, qui pourtant ne forment pas des tympans comme dans le D. fenestrutu. Dans les individus d'àge moyen les sillons ont les bords plus tranchés que dans les adultes où ils tendent à s'blargir et à avoir les bords moins nets.

\section{Gente PARACLISTOSTOMA Dé MAN.}

\section{Paraclistostomá Leachii (Aud.).}

Savigny, pl. II, fig. 1 .

Macrophthalmus Leachii Audouin, Expl.

Cleistostoma Leachii H. Nilne-Edwards, H. n. Cr., II, p. 68. - De Man, Mitth. Hamb. Hus., XIII, 1896, p. 90.

Cleistostoma Leachii var. penicillata Paulson, loc. cit., p. 67, pl. VIl, fig. 6-6 b.

Ner Rouge (II. Jousseaume), 2 males, 3 femelles: mel Rouge (Musée Turin), 3 mâles, 3 f'emelles.

P'aulson a décrit une variéti de cette espece, quil caracté- 
Les paltes ambulatoires ont une épine à l'extrémité des méropodites, forte et aiguë, qui n’est pas représentée dans la ligure de Milne-Edwards, mais qui a été mentionnée dans sa jremière deseription de 1848. Le bord supérieur du méropodite est finement granulé-denticulé; l'extrémité du bord inféricur est finement, presque imperceptiblement, denticulée.

Les paltes ont des bandes annulaires incomplètes de couleur noire, les pédoncules oculaires sont annelés de la mème façon.

\section{Macrophthalmus brevis (Herbst.}

Cancer brevis Herbst, pl. LX, fig. 4.

Macrophthalmus brevis Hilgendorf, Decken's Reise Ost.-Afr., p. 86, pl. IIl, lig. ‘. - De Man, Not. Leyd. Mus., II, p. 70.

M. Grandidieri A. Milne-Edwards, Ann. Soc. Ent. Fr., 1867, p. 285 ; Nouv. Arch. Mus., IV, 1868, p. 84, pl. XX, fig. 8-11. - Lenz, Abh. Senckenb. Ges., XXVII, 1905, p. 365.

Her Rouge et Périm (M. Jousseaume), 9 màles et ő femelles.

Ces exemplairessaccordent tres bien arec la description et la ligure de llilgendorf de $\boldsymbol{M}$. brevis (que ce mème auteur signala aussi dans la mer Rouge), de mòme qu'ils concordent arec les descriptions de M. Gromdidieri, données par A. Milne-Edwards et par Lenz, ainsi qu’avee un spécimen-lype examiné de Zanzihar. Je crois done que les deux espèces doivent ìre réunies.

La doigt mobile du màle est dépourvu de grosses dents sur le bord tranchant; le doigt fixe a un lobe oblique. La cride oblique de la partie inférieure de la main manque dans la femelle, mais le bord inférieur de la main devient caréné et tranchant. Le doigt fixe des pinces dans les exemplaires dìge moyen est presque droit avec le bord inférieur de la main; dans les gros exemplaires il est fortement infléchi.

Le gros mâle mesure :

Largeur de la carapace.................

Longueur

23 millim.

Longueur de la main...................... 11 .

\section{Macrophthalmus depressus Rüppell.}

Rüppell, loc. cit., p. 17. pl. IV. fig. 6.- H. Milne-Edwards, H. n. Cr., II, p. 66 ; Ann. Sc. Nat. (3), XVIII, p. 159. - Heller, loc. cit., p. 362. - De llan, Not. Leyd. Mus., III, p. 255 ; Arch.f. Naturg., 1887, pl. XV, fig. 3. - Paulson, loc. 
Ilot des Frères (MN. Bonnier et Pérez), un mâle et une femelle.

\section{Grapsus strigosus (Herbst).}

Gf. Alcock, loc. cit., p. 393 (ubi syn.).

Golfe d'Akabah (M. Arconati, Musée de Turin), quelques exemplaires; mer Rouge (I. Jousseaume), un jeune mâle; Assab (M. Ragazzi, Musée de Modène), quelques exemplaires.

Genre GeOgrapsus Stm.

Geograpsus Grayi (Ew d.).

Signalé dans la mer Rouge par Hilgendorf sous le nom de G. rubidus Stm.

\section{Genre MeTOPOGRapsus Edw. \\ Metopograpsus messor (Forsk.).}

Cf. Alcock, loc. cit., p. 387 (ubi. syn.).

Mer Rouge (Musée de Turin), 6 exemplaires; golfe d'Akabah M. Arenati, Musée de Turin), (j exemplaires; Suez (II. Des(champls. Musée de Turin), 7 môles et 2 femelles; Massaouah II. Clivio, Husée de Turin), 2 màles; mer Rouge (M. Jousseame), כ̈ mâles et 2 femelles; Suez (M. Létoụneux), 3 màles.

\section{Genre BRACHynotus De HaAn.}

\section{Brachynotus harpax Hilg.}

Hilgendorf, Sitzb. Ges. Nat. Fr. Berlin, 1892, n 4, p. 38. - De Man, Zool. Jahrb. Syst., IX, 1895, p. 124, pl. XXIX, fig. 26.

Iden, 12 màles et 6 femelles (Types de Hilyendor'f); Djibouti (II. Coutière), un màle et une femelle; Djibouti (M. Jousseaume), une femelle: Pórim (I. Jousseaume), 2 femelles; mer Rouge (II. Jousseaume), 2 mâles et une femelle.

Tous ces exemplaires, y compris les types de Hilgendorf, $\therefore$ accordent parfaitement avec la description de De Man. Les dents des bords latéraux sont plus aigueis el plus détachées dans 


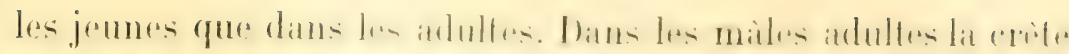

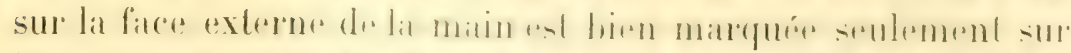

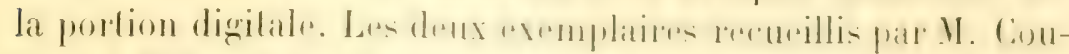

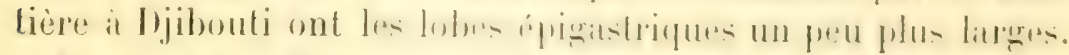

HaB. : Aden (Hilgendorf'), Aljeh (De Man).

\section{Genre Planes Beth. \\ Planes minutus (Linn.).}

Cf. Nautilograpsus minutus H. Milne-Edwards, Ann. Sc. Nat. 3, XX, p. 17. Kingsley, Proc. Acad. Philudelphiu, 1880, P. 202. - A. Milne-Edwards of Bouvier, Crust. Hirond., 189', p. 49.

Planes minutus Stebbing, S. Afr. Crust., 111, 190\%, p. ‘ 3.

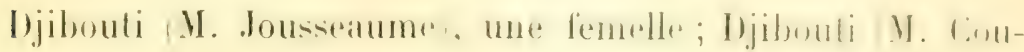
tière) un mâle et une femelle.

Gentre PSELdograpses Eaw.

Pseudograpsus erytræus Kossm.

Kóssmann, loc. cit., p. 61, pl. I, fig. : a, et pl. 1II, lig. 1'-15.

Heterogrupsus erythræus Kingsley, loc. cit., p.

Mer Rouge. (I. Jousseaume), 8 individus.

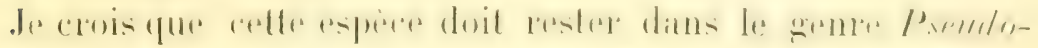

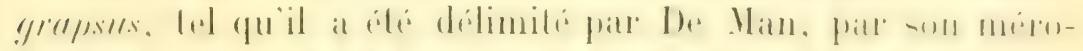

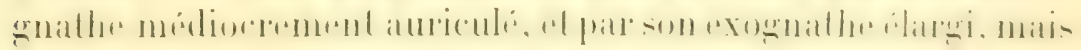
moins large que la moitié de l’ischiognathe.

(ime GYGLOGRAPSUS EDW.

Cyclograpsus lophopus Nol.

(PI. XI, fig. 4.)

Sobili, loc. cit, p. \&11.

Djibouti (M. Coutière), 6 femelles.

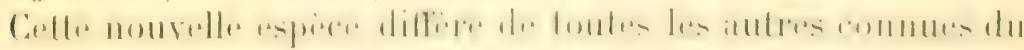

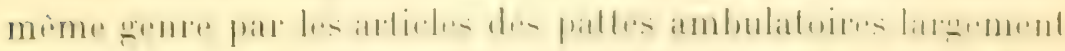
dilatés et carénés; les pattes ressemblant aninsi it celles d'un Atergalis.

La carapace a la forme caracteristique des Cyclograpses

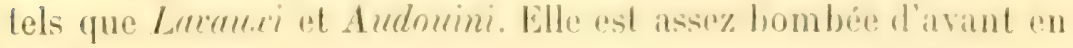
ANA. SC. NAT. ZOOL... !r série. 
arrière, surtout en avant, ot aussi en sens transversal. Les bords latéro-antérieurs ne snnt pas découpés en dents, ni interrompus mar des fissures. Ils sont minces, caréniformes et tranchants; finement granulés à la loupe. La surface entière du corps et des pattes aussi est finement poilue.

Le front est moins large que la moitié de la largeur de la carapace, avancé, lamelleux, à bord antérieur bien visible d'en haut et un peu romere en avant; ses angles externes sont aigus; il se rattache au bord orbitare supérieur par une ligne concave. Le bord orbitaire supripieur est dirigé un peu en dehors et en avant; l'angle orbitaire externe est peu marqué.

Il ny a aucune division de régions sur la carapace; les lobules épigastriques sont à peine marqués; le sillon en $\mathrm{H}$ est à peine risible à la loupe, parce quil est tris faible et superficiel.

Les pédoncules oculaires sont gros et priformes, aplatis supérieurement ot renflés on lessous. His remplissent exaclement l'orbite. La crète sus-orbitaire est granulée mais non découpée en lobes.

Lé chélipedes sont égaux dans la femelle. Le mérus n’a pas de saillies ni de denticules particulierement développés. Le carpe est faiblement gramulé en dessus : il a une faible saillie obtuse du cote interne. La face externe de la main est bombée. granulée; les granules sont plus serrés sur la face supépieuro qui est bordér par une mince crobe granuleuse. Les doigh sont finement dentés sur le bord tranchant; le doigt mobile a ö-6 dents spiniformes disposées en rangée longitudinale sur son bord supérieur.

Les pattes ambulatoires ont les méropodites carénés en dessus, avec une dent subterminale; carénés chargis en dessous; la largeur du mérus de la troisième paire est les 9/3 de la longueur de l'arlicle. Les carpes et les propodites sont courts; les carpes sont fortement lricarénés. Les doights sont faiblement poilus et plus courts que les propodites. Les pattes de la première paire sont phus grèles que cilles de la dernière paire.

Longueur de la carapace. $11,3 \mathrm{millim}$, 
Largeur du front.

Distance extraorbitaire

Largeur du bord postérieur.
$5,3 \quad-$

9

$6, ; \quad-$

\section{Gevre SESARMA SAY.}

Sesarma (Sesarma) Jousseaumei Nol.

(Pl. VIII, fig. 9.)

Nobili, loc. cit., p. 411.

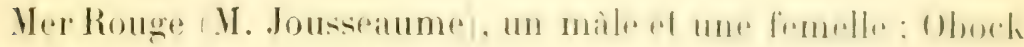
(M. Jousseaume), un mâle.

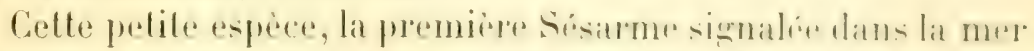

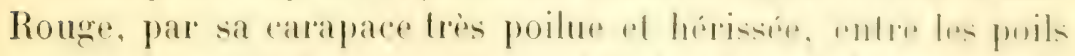

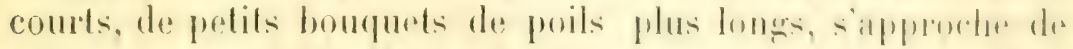
S. pontianarensis De Man et de S. lanalum Alcock.

Lat carapare es carrée, a bords lateratux paralledes, qui con-

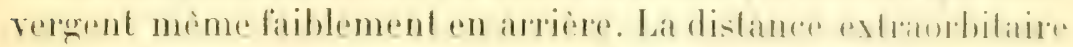

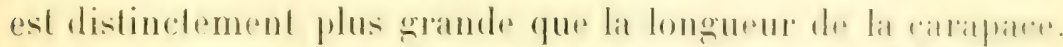

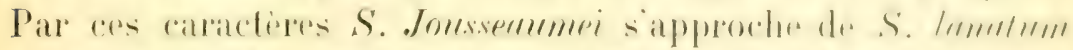

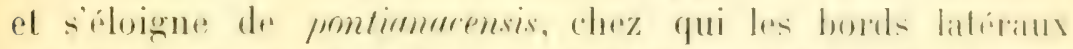

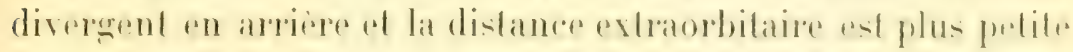
que la longueur de la carapace.

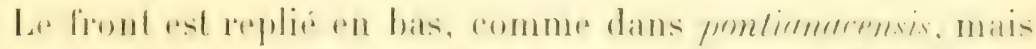

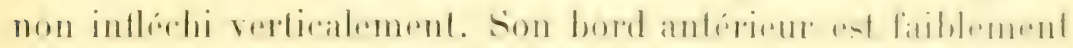

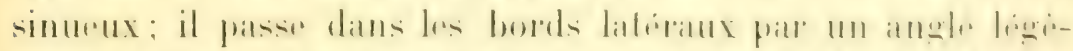

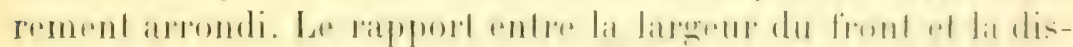
tance extraorbitaire est de 5 : 9 .

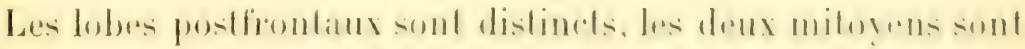

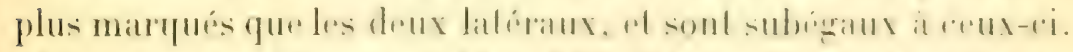

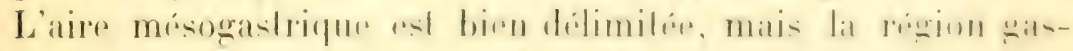
trique n’est pas délimitée sur les cotés.

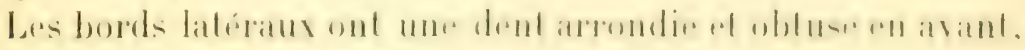
mais assez bien délachée des bords.

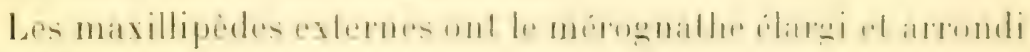
du còté externe.

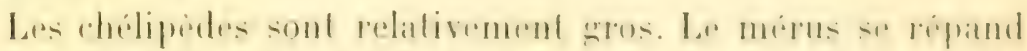

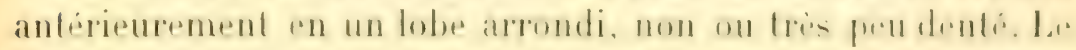

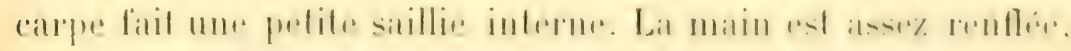
sa fice externe est couverle par une larege platgue do poils lres 
louffus. Toute la surface externe est couverte de poils, mais ceux de la main sont plus longs et plus nombreux. Le bord inférieur de la main se continue en ligne droite avec celui du doigt fixe. Le doigt mobile n'a pas d'ornements particuliers; il est plus long que le bord supérieur de la paume, el à peu près égal à la longueur totale de la paume. Les doigts sont baillants. La face interne de la main est lisse.

Les pattes ambulatoires sont entierement couvertes par les mimes poils qu'on voit sur la carapace of les chélipèdes. Ces pattes sont plus courtes que dans pontianacensis. Les méropodites sont une fois et demie aussi longs que larges, leur bord postérieur est élargi, tronqué en avant et denticulé comme celui de pontianacensis. Le méropodite de la derniere paire est denticule sur lout son bord postéricur. Les propodites sont it peine une fois et demie aussi longs que les dactylopodites, tandis qu'ils le sont trois fois dans pontianacensis.

\begin{tabular}{|c|c|c|}
\hline & $\sigma$ & \& \\
\hline Longueur de la carapace... & 4,5 & 4 millim \\
\hline Distance extraorbitaire......... & $5 !$ & $4,5-$ \\
\hline argeur du front... & $2 \frac{3}{4}$ & $2,5-$ \\
\hline
\end{tabular}

S. Irnatum Alcock diffère par ses bords latéraux trideniés, par ses méropodites entiers, etc.

\section{PLAGUSIIN $\bar{E}$}

Genre PLAGUSIA Lat.

Plagusia squamosa (Herbst).

Signalée par Heller dans la mer Rouge.

Genre PerGnon Gistel.

(Acanthopus De Haan. Liolophus Miers, nom. præocc.).

\section{Percnon planissimus (Herbst).}

Cf. Liolophus planissimus Alcock, loc. cit. (ubi syn.).

Percnon planissimus Rathbun, Proc. U. S. Nat. Hus., X.XII, 1900, p. 281.Nobili, Ann. Mus. Nat. Hung., 1905, II, p. 501.

Mer Rouge (M. Jousseaume), nombreux exemplaires. 


\section{FAIILE PALICIDAE \\ Gevre Palicus Pint.}

\section{Palicus Jukesii White.}

Cymopolia Jukesii White, 1847. - Miers. Voy. Erebus and Terror. Zool. Crust., P. 3, pl. III, fig. \& $a-c(1875)$; Rep). Challeng. Brach., p. $335(1886)$.

Pulicus Jukesii Bouvier, Bull. Soc. Philom. Paris (8), IX, 1. 1-2. - Calman, Trans. Linn. Soc. (2), Vll1, 1900, p. 29, pl. 1, fig. 9-13. - Alcock, loc. cit., [1. 15̆1. Cymopolia carinipes Paulson, loc. cit., p. $73, \mathrm{p}$ I. IX, lig. f-it a.

Her Rouge (Musée de Turin), deux exemplitires.

\section{INCERTAE SEDIS}

\section{FAMILLE HAPALOCARCINIDAE}

Gentre CRYP'l'OGHYRUS HeLt.

\section{Cryptochyrus coralliodytes Hell.}

Heller, loc. cit., p. 366, pl. II, fig. 33-39 (1861).

Lithoscaptus paradoxus A. Milne-Edwards. Maillard, lle Rénion. Crust, p. 10-12(1863).

Lithoscaptus paradoxus Paulson, loc, cit., p. 72.

Les types de ce genre inrerlic sedix provenaient de la mer Rouge. Je n'en ai pas vu d'exemplaires de cette provenance; bien que j’en aie examine de nombreux recurills aux îles Gambier par M. Seurat. Paulson considere Lilhos-

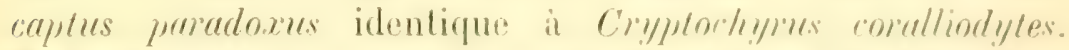

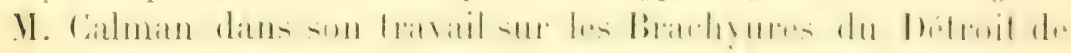
Torres, a clairement exposí les caracteres do I/upuloraminus marsupialix Stimp., of chabli ses affinités avec Crymlorhyrtex.

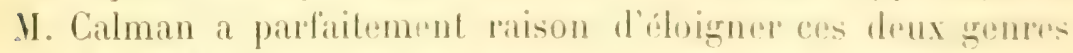

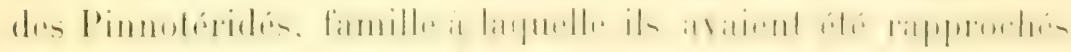
par Stimpson el l'aulson. Leme allinilé aroes les fluninider. soutenue par $\mathbf{\Lambda}$. Milne-lidwats ne me parail pas clario.

La présence de /I. mmosmpralis Stm. dims la mor Rouge n'usl pas certaine, patree (gue los galles sur les coraux décrites pall

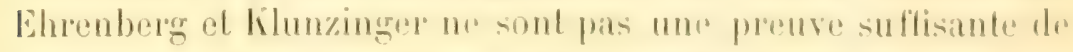
sat présence dans cette latum, mais il est fres problable qu'il siy irouve atussi. 


\title{
STOMATOPODA
}

\author{
Genre GONODAC'TYLUS Lat. (I). \\ (Incl. Protosquilla Brooks.)
}

Gonodactylus tripinosus var. pulchellus Miers.

Gonodactylus trispinosus White (nomen nudum). - Dana, U. S. Expl. Exp.. I, 1852, p. 623. - Miers, Ann. Mag. Nat. Hist. (5), V, 1880, p. 121, pl. Ill, fig. 10.

Gonodactylus trispinosus var. pulchellus Miers, loc. cit., p. 122. - Ortmann Denkschr. Jena, VIII, 1894, p. 61.

Protosquilla trispinosa Brooks, Rep. Challeng. Stomat. (XVl), 1886, p. 71. Henderson, Trans. Linn. Soc. (2), V, 1893, p. 45o. - Borradaile, Proc. zool. Soc., 1898, p. 34, pl. V, fig. 1.

Protosquilla trispinosa var. pulchella De Man, Abh. Senckenb. Ges., XXV, 1902, p. 920 .

Aden (M.Jousseaume), un màle long de 32 millimètres.

Les deux tubercules spinuleux latéraux du telson sont étroits, allongés et en forme d'amande. Les rugosités au milieu du cinquième segment abdominal manquent. Le telson est movable, non soudé avec le sixième segment.

Il y a des larges bandes transversales noires comme dans les Lysiosquilla; deux bandes sont sur la carapace.

\section{Gonodactylus spinosissimus (Pfeff.).}

Gonodactylus spinosissimus Pfeffer, Mitth. Naturch. Hus. Hamburg., VI, 1888, (1889), 4º, p. 35. - Bigelow, Proc. U. S. Nat. Mus., XVII, 1894, p. 493.

Mer Rouge (M. Jousseaume), une femelle; Obock (M. Jous seaume), une femelle.

D'après Pfeffer, il y a seulement deux crètes sur le cinquìme segment de l'abdomen. Dans ces exemplaires les deux crètes sont parfaitement distinctes, mais la crète marginale du segment est aussi double, et il y a donc quatre cretes et quatre dépressions. Sur le sixième segment il y a quatre tubercules manillaires, hérissés de spinules aciculaires rayonnantes; les

(1) Vaccepte entièrement les idées de IIansen (Isop. Cum. Stomat. Plankton Exp., 1895) que Protosquilla n'est pas séparable de Gonodactylus. 
deux médians sont plus apprimés al platers un pell plus an arrière des deux latéraux. An Jonge de: larticulation do rin-

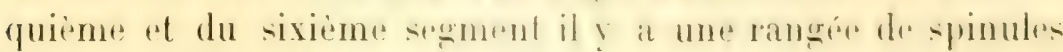
très menues non décrites par Pfefler.

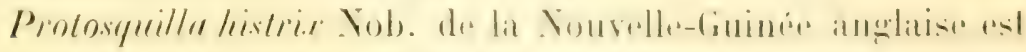
voisine de celte espece : mais elle en dillepe par son ringuieme

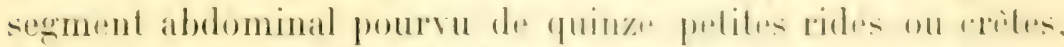
déterminant entre elles des fositelles. pal soll sixieme soment

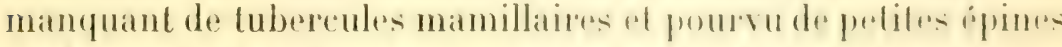
de forme très différente, soil non ardoulaters, mats entuter, plus grosses el courbees, disposies uniformément an rangras.

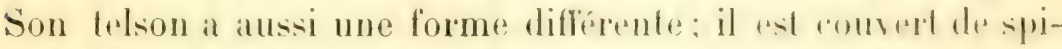
nules de mème forme que celles du sixieme serment atusid ditns les espaces entre les tubercules, tandis que dans li. spmoxisy-

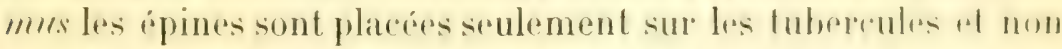

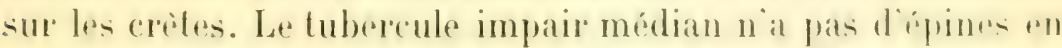
dessus, mais sendement tout autome de la base. Liangle litteroantérieur de la carapace est arrondi, non dentiforme; il t's

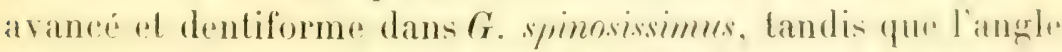
postérieur du cinquieme stogment de l'abdomen al normate-

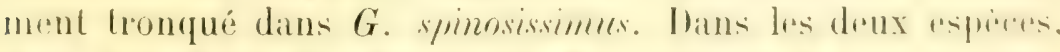
ainsi que dams $P$. Brorlili lle Van, les you sonl triangulaires.

\begin{tabular}{|c|c|c|c|}
\hline Longueur totale................. & $2 t$ & 30 & millim \\
\hline de la carapace.......... & 6 & 8 & - \\
\hline du bord antérieur de la & & & \\
\hline rasapare............ & i & : & - \\
\hline Lageut de la carapace............ & ;) & 6 & \\
\hline Longueur de l'ueil............... & 3 & 3 & - \\
\hline$\ldots \ldots \ldots \ldots \ldots$ & 1,8 & $1 \stackrel{3}{6}$ & - \\
\hline 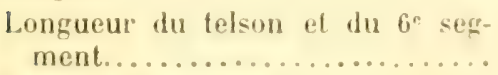 & $6,3 i$ & 7,3 & - \\
\hline Lareesur du telson. . . . . . . . . & $\because$ & ;.: & - \\
\hline
\end{tabular}

HAn. : 'anzibar.

\section{Gonodactylus chiragra (Fab.).}

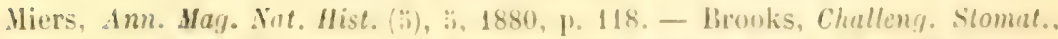
186, p. 156, ph. XV, tig. \&. - Je Man, Kool. Jahrb. Sy/st., X, 1898. 1. 694.

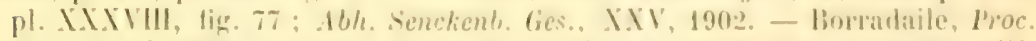

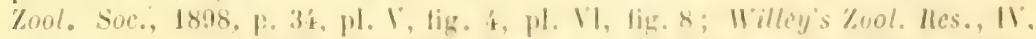
1899, p. 401. - Nohili, Ann. Mus. Tapoli, 1, n³, 1901, 1. 16; Bull. scient. 
Fr. Belg., 1906, XL, p. 15\% - Lanchester, Stomat. Mald. Laccad., 1902, p. 4'5, p1. XXIII (pars). - Jurich, Stomat. Deutsch. Tiefsee Exp., 1904, p. 375, pl. XXVI, fig. 4-4a, 5-5a. - Paulson, loc. cit., p. 127.

Gonodactylus falcatus Kossmann, loc. cit.

11. Djibouti (M.Coutière), une femelle longue de 7 's millimetres, a l'ancre a l'extrémilé de la crète médiane, mais les crêtes sont plus rentlées et l'ancre est plus courte que dans un exemplaire de Sarawak du Musée de Turin, qui correspond bien à la forme figurée par De Man. Dans un mâle de 65 millimètres la cròte médiane est encore plus renflée, presque comme dans la var. tumidı Lanchester, el l'ancre est à peine discernable, ainsi que dans trois autres màles longs respectivement dè 58 , 47. 40,3 millimètres et dans une femelle longue de 38 millimètres.

Dans ces exempiaires le telson est proportionnellement plus. court, la dent latérale moins marquée ; ils tendent donc à rattacher la var. tumidus Lanch. aver la var. incipiens Lanch. qui correspond a la forme typique selon De Man ou à la var. A. de Borradaile.

b. Obock (M. Jousseaume), 13 exemplaires qui rentrent dans la forme pourvue d'anre de longueur différente. L'ancre est bien distincte dans 12 exemplaime $(70,0 \% q)$ entre et 60 millimètres; les branches de l'ancre sont plus ou moins réunies à la carène et plus ou moins longurs. Dans un màlı long de 61 millimetres les cornes de l'ancre sont soudées avec la carène qui est assez renflée. Dans ce mème màle les angles externes de la plaque rostrale, ainsi que dans un autre malte ut dans ö femelles sont arrondis, tandis qu'ils sont plus ou moins spiniformes dans les autres six exemplaires màles. Ces derniers s'approchent donc de la var. acutirostrix De Man, mais ils ont l'ancre bien développée. Ils s'accordent avec les exemplaires d'Amboine décrits parmoi (Ann. Mus. Nryoli, Inr. rit.).

$\therefore$ Ner Rouge, Aden, Obock et Périm (M. Jousseaume), 10 màles et 11 femelles.

Sur ces exemplaires on peut faire les mèmes observations que sur ceux qui précèdent. L'ancre est bien formée dans is miles et 10 femelles; elle est soudée avec la carène médiane dans deux gros"exemplaires, un màle de 66 millimètres et unt 


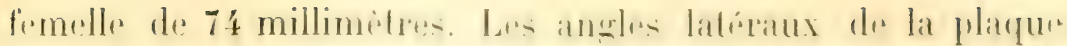

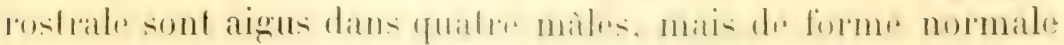

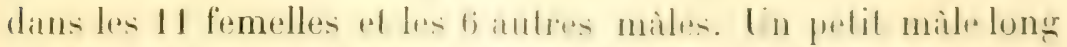

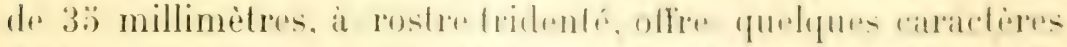

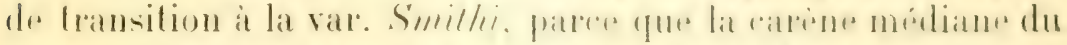

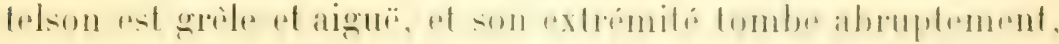

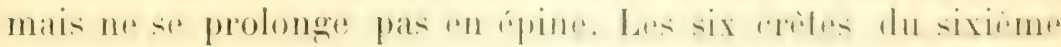

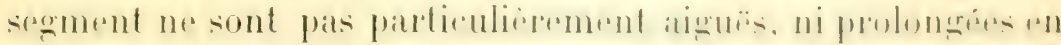
épine.

Tous aes exemplaires de la mer Rouge differment des antres

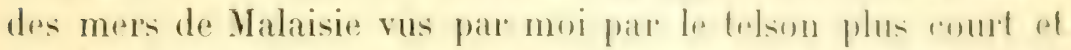
par les rertes plus renflées of par lo corpes dius les gros individus, plus trapu.

Gomodertylus pletysoma Woodmason, en jugenal d'atpres lat

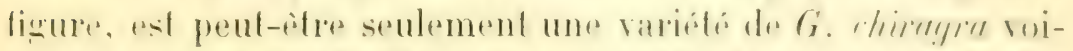

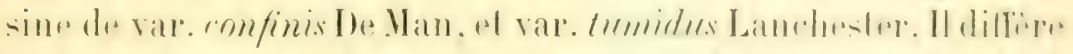

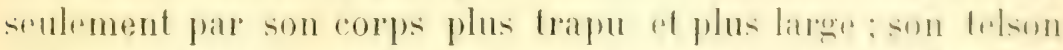

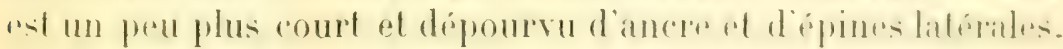

\section{Gonodactylus chiragra var. Smithi (Poc.).}

Gonodactylus Smithi Pocock, A. M. N. H. (6), 11, 1893, p. 47, pl. X.X B, fig. 1. Cionodactylus chiragra var. Smithi Borradaile, Proc. zool. Soc., 1898, p. 3'. pl. I'. lig. 2, et Willey's Zool. Res., IV, P. 402. - Lanchester, loc. cit., p. '17, pl. XXIII, fig. 't-3.

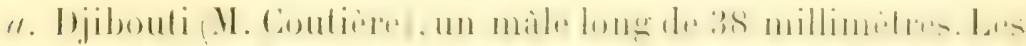

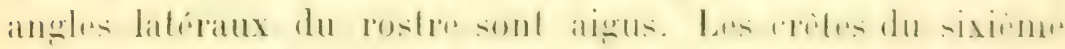

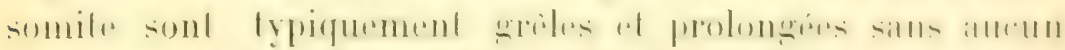

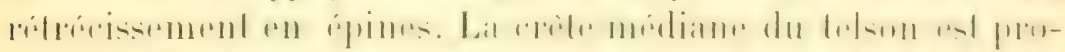

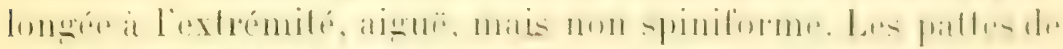

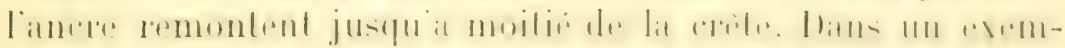
plaire tres jeune long de $1^{\text {mon }}, 0$ les pattes de l'ancre se prolongent encore plus.

b. Obock (11. Jonsseaume), 7 femelles. Ainsi que dans

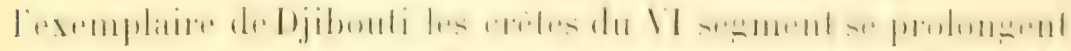

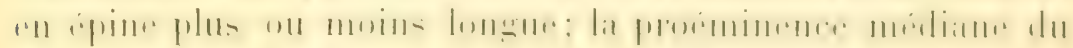
telson est plus ou moins spiniforme it lextremité, mais son bord extreme rentre obliquement, jamatis il nest arrondi on rectiligne, comme dans la forme ancrese de $G$. chirugre. 
c. Mre Rouge, Aden, Périm et Obock (M. Jousseaume), 6 mâles et 10 femelles.

Le rostre a dans tous les angles latéraux aigus.

d. Mer Rouge (Musée de Turin), une grosse femelle longue de 53 millimètres.

La crète médiane du telson est très grèle, distinctement prolongée en épine; les pattes de l'ancre dépassent la moitié de la crète. Cet exemplaire correspond à la var. Smithi l de Lanchester.

Has.: Mer d'Arafura (Pocock); Rotuma, Funafuti, Loyalty Islands (Borradaile); Maldives (Lanchester); golfe Persique (Nobili).

\section{Gonodactylus De Mani Hend.}

Gonodactylus n. sp. ? De Mlan, Arch. f. Naturg., 1887, p. 574, pl. XXIl, fig. 7. Gonodactylus De Mlanii Henderson, Trans. Linn. Soc. (2), V, pl. XL, fig. 23, 24.

- Nobili, Boll. Mus. Torino, XX, no 506, p. 11 ; Bull. scient. Fr. Belg., 1906, $\mathrm{XL}, \mathrm{p.} 158$.

Gonodactylus spinosus Lenz, Abh. Senckenb. Ges., p. 387, pl. XLIII, fig. 12 (nec Bigelow).

Obock (M. Jousseaume), 6 [mâles et 4 femelles; Djibouti (M. Coutière), 8 màles et 9 femelles; mer Rouge (M. Jousseaume), 2 mâles et 2 femelles; Aden (M. Jousseaume), un màle et 2 femelles; Djibouti (II. Jousseaume), 2 femelles; illes Musha, dans le sable du récif, dragage à 15 mètres (M. Gravier), un mâle; Djibouti, récifs du Pingouin et du Vétéore (II. Gravier), \& exemplaires; baie de Djibouti sur les Stylophora (M. Gravier); baie de Djibouti, dans les canaux de Hirrinia, et dans les sables à Balanoglossus (M. Gravier).

Har.: Pulo Edam (De Man); Rameswaram (Henderson); golfe Persique (Nobili); Zanzibar (Lenz, Nobili).

\section{Gonodactylus spinosus Bigelow.}

IBigelow, John Hopkins Univ. Circ., 106, p. 101, 1893; Proc. U. S. Nat. Mus., XVII, 1894, p. 493.

(ionodactylus chiragra var. spinosus Lanchester, loc. cit., p. 4ö, pl. XXIll, fig. 14 .

Djibouli (I. Coutière), un màle et 2 femelles; Djibouti, récil' du l'ingouin et du Météore (M. Gravier), un màle. 


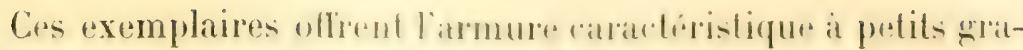
nules aigus et spinuliformes smll toml le telson. el concordent pourla plupart des caracteres a we les deseriplions de Bigedow.

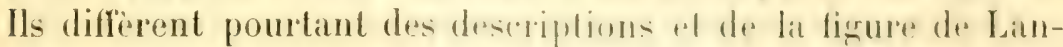

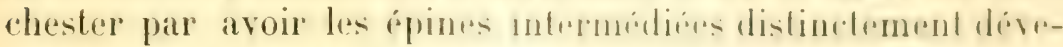

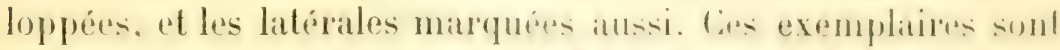

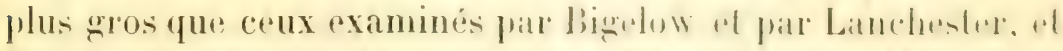
bigcelow mentionne dans ses exemplitipes lun smenll teelle on

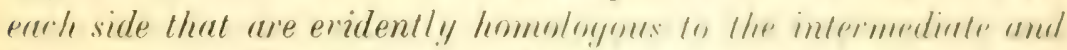

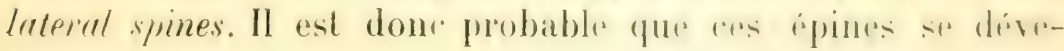
loppent avec l'âge.

Cetle espece si mon identification stexted est atu fond un G. De Mani à armure épineuse très développée.

Jes trois exemplates sont longs de 2t. 2-2 at 33 millimetres. IIAB. : Mauritius Bigelow); Maldives Borradaile).

\section{Gonodactylus brevisquamatus Pauls.}

Paulson, loc. cit., p. 127, pl. XXI, fig. 3-3g.

Cofte espece tris rare, non plus retrourece apros l'aulson. al

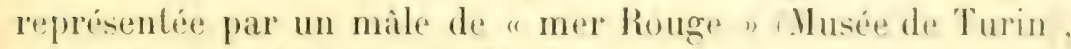
el une femelle de Djibouti (II. Jousseaume).

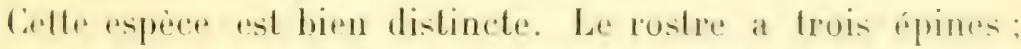
lépine médiane dépasse la moitio des pédoncules ocularios

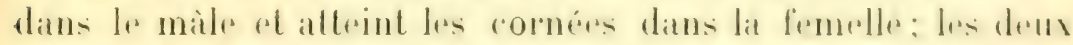

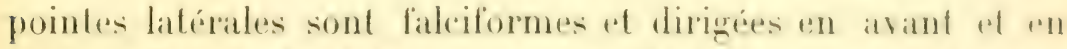

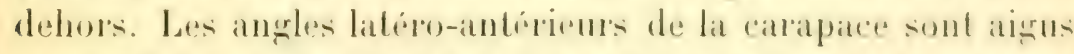

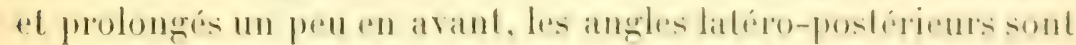

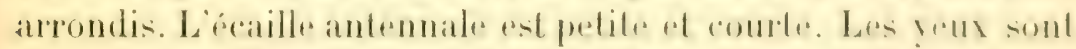

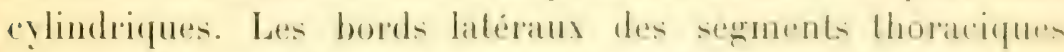
découverts sont tronqués.

La caractéristique de cette espece est la lorme du sixieme

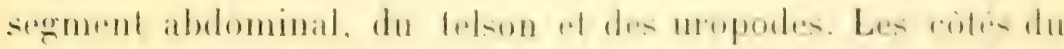

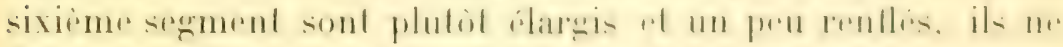

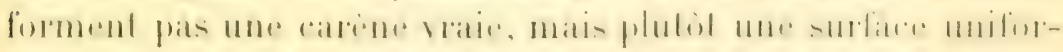
mément surélevée, séparée par un sillon de lat critr voisins.

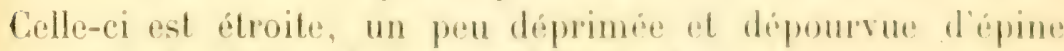

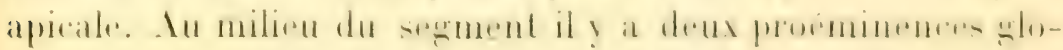


bulenses, lisses, sans épines, disposées parallèlement dans le mile. et formant entre elles un angle dans la femelle de Djibouti (et dans la figure de Paulson).

Le telson est court; il n'a pas d'épines latérales (ses bords laléraux sont courbés uniformément); à son extrémité il a quatre épines ; les submédianes aiguës et étroites, séparées par un sinus arrondi, environ deux fois aussi longues que les deux externes (en mesurant le bord interne); celles-ci sont un peu plus larges et moins aiguës. Il n'y a pas de dents entre ces épines, les submédianes sont entièrement lisses, non denticulées: sur le bord interne. Le telson offre trois proéminences, la médiane très grosse, arrondie-obovée, plus saillante, parfaitement lisse, les deux latérales allongées et moins saillantes. La crète qui passe sur la dent externe est assez renflée et lisse.

L'article basilaire des uropodes est bifurqué en dessous 'n deux épines, dont l'interne est beaucoup plus grèle que l'externe. La branche externe des uropodes est lisse dans sa plus grande partie, et pourvue seulement de deur épines falciformes a son extrémité (trois dans la figure de Paulson). Le segment distal est cilié sur le bord et couvert de poils sur toute la surface; l'endopode est aussi poilu, il a forme de $f \rho r$ de lance, mais il est un peu tordu.

Les mesures du mâle sont :

Longueur totale.

Largeur du telson.
23 millim.

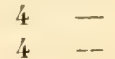

G. De Mani a les crètes dı telson disposées de la mimr. fiacon, mais il differe nettement de bresisquemalux, en plus que par ses spinules, par la présence de la dent latérale, par les épines terminales du telson denticulées, par l'exopode des uropodes garni d'épines mobiles, par son rostre unispineux, elc.

G. expinosus Borr. qui manque d'épines latérales at qui a une disposition analogue des crètes et des proéminences lisses, a les épines intermédiées réduites, rostre unispineux, uropodes pourvus d'épines mobiles, etc. 


\section{Gonodactylus glaber Brooks.}

Gonodactylus glabrous Brooks, Challeng. Stomat., 1). 62, pl. XIt, fig. 8; pl. XV, fig. 7 et 9. - Borradaile, Hroc. Zool. Soc., 1898, p. 45̌ - - Kohili. Ann. Mus. civ. Genova, XL, 1899, J) 276. - Bigelow, loc. cil., p. 493. - De Man, Abh. Senckenb. Ges., XXY, 1902, p. 913, jl. XXVII, fig. 6\%. - Jurich. loc. cit., p. 376.

Gonodactylus glaber, Henderson, loc. cil., p. 43.4. - Nobili, Am. Mus. Napoli. 1, no 3, 1901, p. 17; Boll. Wus. Torino, XX, no 506, 1905, p. 11.

Gonodaclylns graphurus, Nobili, Amn. Mus. Yapoli, I, 1901, no 3, I. 16.

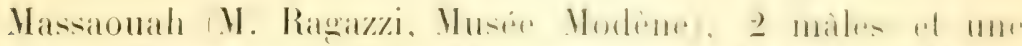

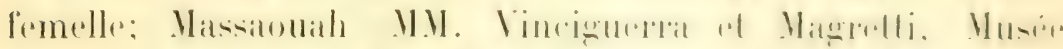

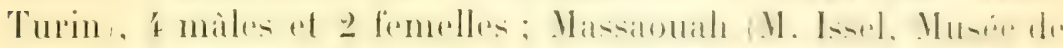

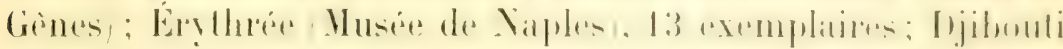
(11. Couliere), 6 màles et 4 femelles; Obock, Iden el Sue\%

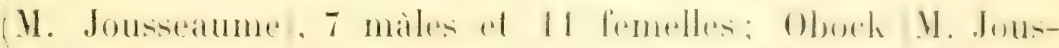

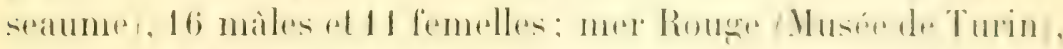

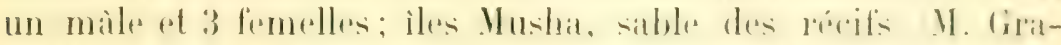

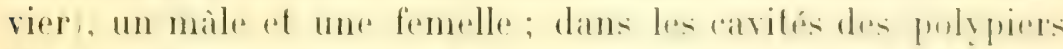

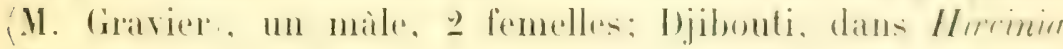
(II. Gravier), un mile.

Tous ces 97 exemplaires, ainsi que deux autres de

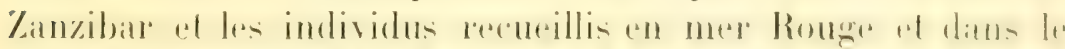

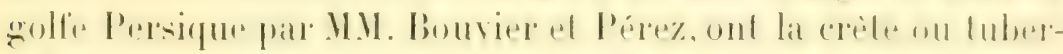

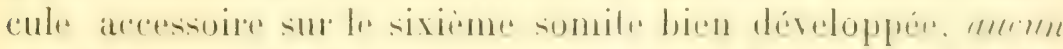

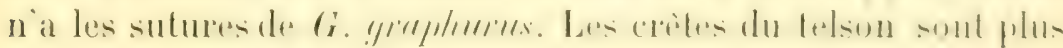
ou moins renflées ou plus ou moins aiguës.

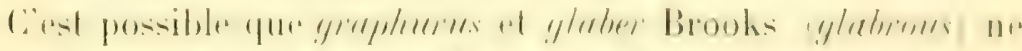

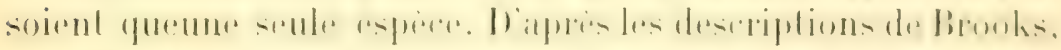

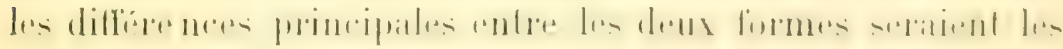
suivantes :

G. graphurus lliers.

1. Des sutures sur le dos des cinq premiers somites de l'abdomen.

2. Une crète ou luhercule accessoiro entre les six crites du sixieme segr- 1 ment abdominal.

3. Carenes du telson renflées.

t. Le basipodote des mopopes a deux épines; la lamelle de l'exopode est plus longue que moitie du deuxieme article. (i. glabrous lirooks.

1. Pas de sutures sur le dos des cinu premiers articles.

2. Pas de crite aropsobre sur le sixiome segment abdominal.

3. Carines du telson zribles, nun renHies

f. Le? basipodite des uroporles a une serule eprose; la lamelle de l'exopode est plus courte que moitie du denxieme articte. 
Lo truisième caractère peut être tout de suite écarté parce que Henderson (loc. rit.) nous informe que dans les types de grayhurs. les carènes sont encore plus grèles que dans glabrous, et j’ai en 1901, donné le nom de G. graphurus Broolisi au G. graplmurus décrit par Brooks, qui parait ètre une forme beaucoup plus rare que l'autre à carines grêles ou médiocrement renflées.

Restent les deux autres caractères. Mes exemplaires par l'absence de sutures tergales seraient des glaber, par la présence du lubercule accessoire seraient des grophurus. Leurs uropodes en font aussi des glaber.

Lequel des caractères a plus de valeur? Dans le musée de Gènes, il y a de nombreux exemplaires de Massaouah, déterminés glaber par Hansen, qui ont le tubercule très distinct, et n'ont pas de sutures. M. Lanchester observa des exemplaires en conditions égales, igalement déterminés par le mème carcinologiste. I. Hansen donne done plus d'importance ¿a la présence des sutures qu’a celle du tubercule. La concordance aussi des caractères des uropodes et la forme de l'endopode de la premièe pairre de pléopodes me portent à peésent à considérer ces excmplaires comme des glaher, bien qu'ils aient le tubercule de graphurus.

Le Cancer falcrutus de Forskal, par ses rarinis longitudinalibus, conceris, jonne murronatis, mumero quinque est certainement identique avec grophurus ou avec glaber, non avec chirayra, ainsi que des auteurs (Kossmann entre autres) ont supposć. Mais à laquelle des deux formes correspond l'espèce de Forskal, c'est ce qu'on ne peut pas déduire de sa descriplion.

La couleur paraìt ètre très variable. D’après les notes qui accompagnent les exemplaires de M. Gravier, elle était gris bleutre avec des taches sombres dans certains exemplaires des iles Musha; et bleu ardoise, avec les appendices bleu clair dans le mâle capture dans Hirrinir, à Djibouti.

Genre GORONIDA Brooks.

Coronida trachura ( $\mathrm{v}$. Martens).

Gonodactylus trachurus von Martens, Sit:b. Ges. Naturf. Fr. Berlin, 1881, p. 83. - Miers, Proc. Zool. Soc., 1884, p. 16, pl. 1, fig. 3. 
mer Rouge. Les différences observées par Broolis entre ses individus de Honolulu et d'autres de Saint-Thomas, sont donc individuelles, et celle espece est sujetle a varier dans une mème localité.

Un jeune exemplaire, long de 17 millimetres, recueilli par M. Coutière à Djibouti, et un autre des îles Musha, recueilli par V. Gravier à 20 mètres de profondeur, sont dans le stade monodartylu. Le doigt est apparemment entier, mais, examiné au microscope, on y voit, sous les téguments, les épines déjà formées.

Un jeune exemplaire de Djibouti offre une belle coloration avec deux bandes longitudinales violettes et les bords des segments clairs pointillés de violet.

\section{Pseudosquilla pilænsis De Man.}

De Man, Journ. Linn. Soc., XXII, 1887-88, p. 296. - Bigelow, loc. cit., p. 499.

Djibouti ou Obock (II. Jousseaume), une femelle.

Cet exemplaire est en manvais état et manque de telson. Je l'attribue également à $P$. piliensis par la forme de son corps qui ressemble a une Lyyinsquillu, par ses yeux aplatis, dilatés. avec les cornées bilobées, pour aroir seulement trois épines (en plus de celle terminale) sur le dernier article des pattes ravisseuses, et par la forme du prolongement distal du basipodite des uropodes. Celui-ci pourtant a six épines d'un còté et sept de l'autre, tandis que dans le spécimen type, il y a seulement cinq épines.

Cette espèce n'a été trouvée qu’à Elphinstone Island dans l'archipel de Mergui.

\section{Pseudosquilla megalophthalma Bigelow.}

Pseudosquilla megalophtalma Bigelow, loc. cit., p. 500.

Djibouti et Obock (11. Jousseaume), 2 mâles et 3 femelles. Ces exemplaires different de la description de Bigelow, par les caractères suivants :

$1^{\circ}$ Les segments abdominaux II el III n'ont pas d'épinc is leur angle postérieur. 


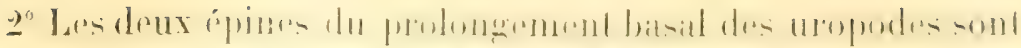

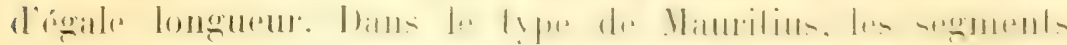

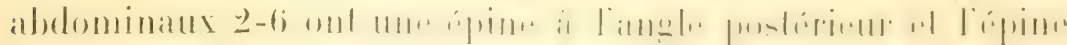

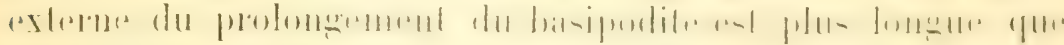
l'épine interne. de ne crois pas toutelois que ces diflérences aient beaucoup d'importance, paree (jue dans $P_{x}$. rilinll on observe les memes variations.

La couleur est caractéristique. Sur la carapace il y a une

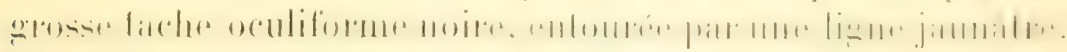
Les angles postérieurs du cinquiène el du sixiemes segnent de l'abdomen sont aussi colorés de noir aver une ligne jaunatre. Le dos du doigt des paltes ravissenses, el les épines el les crètes du telson ont une couleur violellr.

Le plus gros individu mesure:

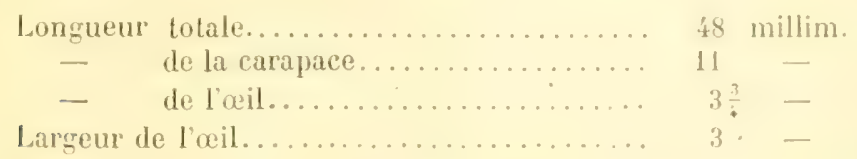

Cielte espece n'est connue que par un seul exemplaire de l'ile Maturice.

\section{GENRE LYSIOSQUILLA DANA.}

\section{Lysiosquilla multifasciata Wood-.lison.}

Wood-Mason, Fig. Uescr. $9 \mathrm{sp}$. Squillidx, 1895. - Nolvili, Boll. Mus. Torinu. XVIII, 1903, no $4 \div 7$, p. 30

Obock (11. Gravier), dans les sables at bulanoyloswn. Trois individus.

Ces exemplaires sont rehativement jeunes, par rapport it une femelle de samarinda (Borméo), do latquelle ils diberent

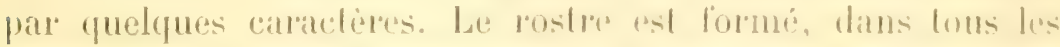

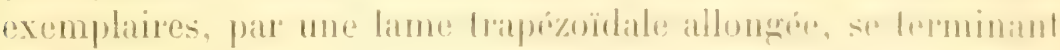

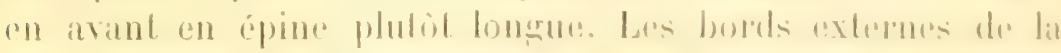

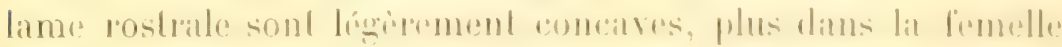

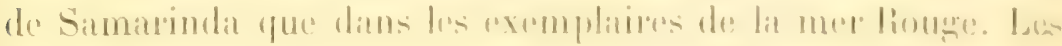

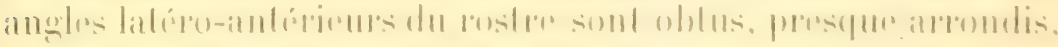

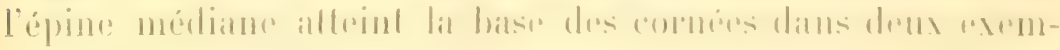

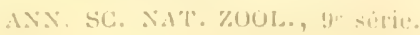


plaires et dans la femelle de Bornéo, el seulement la moitio du pédoncule dans l'autre individu.

Les repines mobiles de l'anneau antennaire dépassent la moilić du pédoncule oculaire. L'écaille des antennes externes ust elliptique.

Les angles latéro-antérieurs de la carapace sont obtus, ainsi que les angles posterieurs. La carapace s'élargit tn arrière. Les pleurons des segments thoracipues découverts sont arrondis-tronqués. Le sixieme segment de l’abdomen se prolonge en épine à ses angles postérieurs.

Le telson a deux épines marginales de chaque còtí, et deux épines submédianes qui sont plus courtes que les ćpines mobiles insérées en dessous. Entre la première épine marginale, qui est la plus longue, et la deuxieme, il y a une apine courte; 4 épines sont disposées entre la deuxième marginale el la subnédiane qui n'est pas plus longue que les épines intercalaires. Entre les 2 épines submédianes, il ! a 8 spinules dans le màle ('́ dans l'une des femelles de Oboch et dans celle de Samarinda, 6 dans l'auler femelle de Obocki). Deux des 8 spinules du màle sont plus rourtes et insérées plus haut.

Sur la surface dorsale du telson, on observe ö épines disposées en arc de cercle.

Le basipodite des mopodes a une épine en dessus, ef se prolonge en dessous dans les 2 épines habituelles, dont l'interne est plus longue que l'externe; l'exopodite offre 6 épines mobiles:

Le carpe des pattes ravisseuses a une spinule en dessus; la main a 4 épines mobiles; le doigt a 6 épines (y compris l'apicale) denticulées, la première très courte, la deuxième plus courte que la troisième subégale à la cinquième, ou un peu plus courte, la quatrieme longue. Dans la femelle de samarinda, il n'y a que 5 épines pou chaque doigh, dans le lype de Bombay il y a 5 épines d'un côté et 6 de l'autre.

La coloration est celle des autres Lysiosquilla, par bandes transversales noires, dont trois sur la carapace.

Les plus gros exemplaires mesurent: 


$\begin{array}{ccc}0 & c \\ \text { Obock. } & \text { sumanda. } \\ 31 & 33 & \text { millim. } \\ 6 & 10 & - \\ 3 & \because 3 & -\end{array}$

Celle espèce n'est comnue que de bombar al de Samarinda.

\section{Lysiosquilla vicina Nol.}

Nobili, Bull. du Mus, 190', no :, p. 228 (Diagn. prélim.).

Obock, dans les sables à Balanorlossus (I. Ciravier), un mâle.

Cel exemplaire manque matheureusement des palles latrisseuses. Celle espèce est voisine de Lyysiostuilla ligrina. Tol). de Bornéo (1).

Le rostre est une lame rectangulaire prolongée en avanl en 3épines, les deux externes correspondant aux angles antorieurs du rostre. L'épine médiane atteint à peine la base de la portion chilinisée des pédoncules oculares.

Le bord postérieur du sixième segment abdominal, du côte

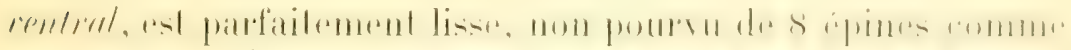

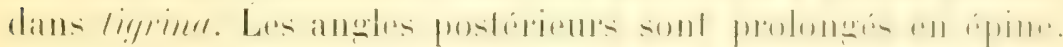

Le telson a 8 épines sur tout son bord, soit 3 latérales de chaque còté el 2 submédianes. La première ápine latérale. placée près de la base, est la plus longue; la deuxieme el lia troisieme sont subégales, les 2 submédianes sont un peu

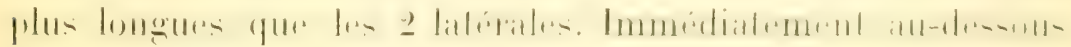
des 2 épines submédianes il y a 2 spinules mobiles. aussi longues que les 2 submédianes et cachees par alles. Entre les 2 épines submédianes, sur le bord inférieur du tulson, sont implantées 12 spinules, dont les 2 médianss sonl un peu plus courtes elplacées un peu plus haut que les aluher. Il y a ume épine sutre la première el la denxième épine mareginale. unes

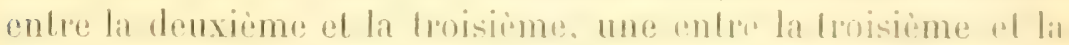
submádiane de chaque colf. las ipheses sont implantées un

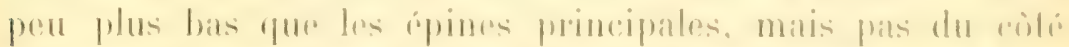

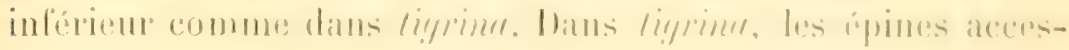

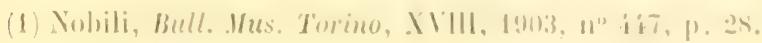


soires sont plus nombreuses; il y en a : une en arrière et au-

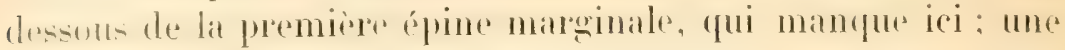

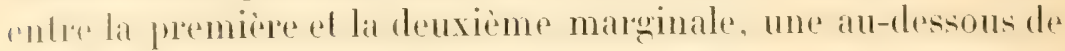
lit deuxieme marginale qui manqur aussi icd, unr au-dessous de la troisième marginale (qui manque à tigrina).

Sur le dos du telson il y a כ̌ épines disposées en arc de cercle, comme dans multifasciala el vicina.

La L. vicina diffère done de la I. ligrina par son rostre tris-

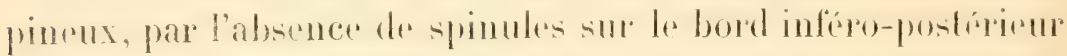
du sixieme segment abdominal ef par un plus pedit nombres

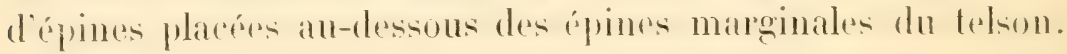

Longueur tota

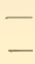

totale

de la carapace...............

du lelson (sans épines)......
27 millim.

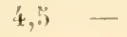

$2 \quad-$

Genre SQUILLA FAB.

Squilla massauensis Kossm.

Kossmann, Zool. Ergebn. roth. Meer., II, 1880, p. 99.

Massaouah (M. Fatigati, Musée de Turin), 7 mâles et

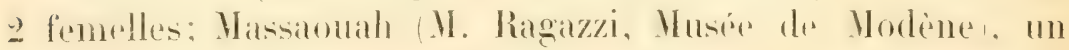
màke: Jijibouti. dans une llatpue an milien du sable raseux, a l'ouest de la Résidence (M. Gravier'), un mâle.

Bigelow (loc cil.) place avec doute cette espèce parmi les synonymes de $S$. nepa. Elle est, par contre, plus voisine de

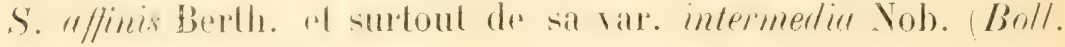
Mus. Torino, XVIII, 1903, no 454, p. 38, 39).

Les yeux sont gros, obliques sur le pédoncule, et disposís de faron que le sillon médian des cornces prolongé passe str 1: bord interne du pridoncule, at ainsi une moilie de l'arl est

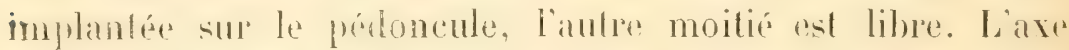
pédonculaire est plus court que laxe coméen. Le rostere as lígèrement plus long que large a la base, et se rétrécit en avant; il a seulement les petites crêtes latérales.

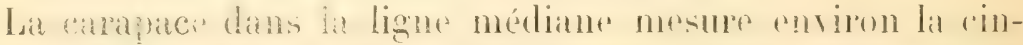

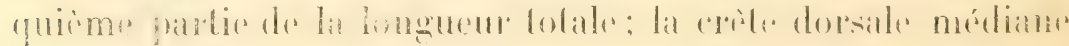
est terminée en arrière par une épine. Antérienrement celte crête se bifurque à son extrémité par une partic assez courle; 


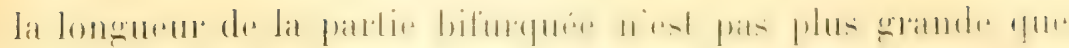
celle de S. affinis. Les angles latéro-intérieurs de la carapace

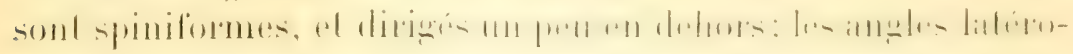
postérieurs sont arrondis.

Le premier segment thoracique déconvert n’a pas d'uphes ventrales, mais il offre deux processus latériuxcomme dans nepr el affinis, dont l'antérieur est plus gros que le proususus

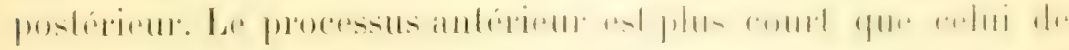
S. affinis. Le deuxième segment a deux processus latúnux. dont le premier est aigu el courbé, presque spiniforme, non large et plus ou moins tronqué comme celui de S. nepun el

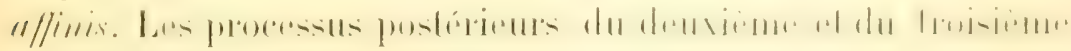
somite découverts sont aigus.

L'abdomen a 8 carènes longiludinales. La carène margi-

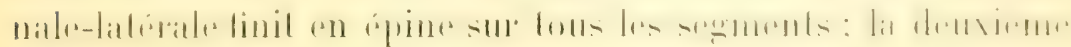

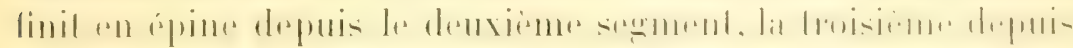
le troisième; les deux dorsales médianes sont épinensés sur

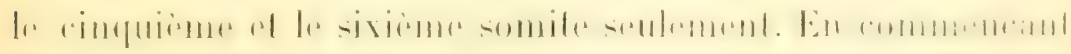
du deuxième segment, on observe entre la deuxième ol lit troisieme crète latérale deux tubercules bien distinets, renfléx el de forme irrégulière, entre lesquels il y a une petitu losselta. Dans nepre et affinis il y a seulement la petite fossctfe: lus tubereules manquent entierement. Les cretes sur le sixime segment sont plus renflées que dans nepun el "flinis.

Le telson offre les meilleures diflérences. Sur le dos de cer segment, on observe la mome crète longitudinale a las mèmes lignes de fossettes de ehargue cobte de la creite. al les mèmes rangées radiantes de points creux comme dans nepu. "ffinis el lant d'autres espreces. Mais dans nepu el aflinis la surface du telson est aplatie, les fossettes el les points sont imprimés simplement. Salis aucune seuplure loul aulour. Dans mossmensis, an contraire, la suldace du lalson est sculptie avec des luberentes et des bords penflis qui aceompagnent les dépressions. Le long de la rangée de fosidelles

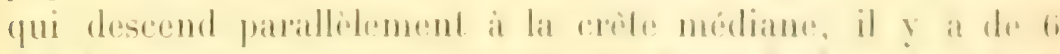

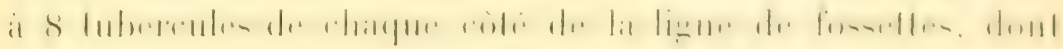

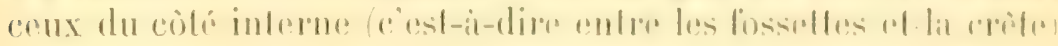
sont plus lorls que ceux du coté externe, qui se lusionnent un 


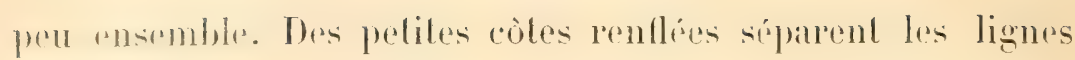
de points.

Sur le bord postérieur il y a 3 denticules submédians, 6 intermédiés et 2 latéraux.

L'épine interne du prolongement basal des mopodes est la plus longue; l'exopodite a 9 épines mobiles.

Le doigh des paltes ravisselses est sinué en dehors, moins que dans nepun, et plubol romme dans mat S. "ffinis var. intermedir. Le doigl a 6 ípines. Le propodus a $2-3$ épines mobiles. Le carpe at seulpturé, el a 3 dents sur son bord superieur.

Longueur totale du corps sans rostre...... 134 millim.

- de la carapace................ 28

- de l'axe pédonculaire............

- $\quad-$ cornéal.................

Longueur du rostre.

Liexemplaire do Djibouli, que j’ai vu frais, était de couleur pris verdatre; les eretes des segments abdominaux et les épines du sixieme segment of du lelson sont rouges. Les uropodes sont bleu foncé.

\section{ADDENDA}

Pour compléter le catalogue des décapodes de la mex Rouge, il faut ajouter aussi les deux espèces suivantes:

Parathoë rotundata (Miers), signalée par Miers, à Suez.

Carcinus maenas (Linn), signalé dans la mer Rouge par Alcock. 


\section{APPENOIE}

Ion travail était sous presse guand parut un travail de Klunzinerer, Die Spitz-und Spitzmundhrubben (Oxyphyncha und Orystomata) des roten Meeres. Stultgart, 1906. Je ne pourais plus citer ce travail dans le texte du mien; je rais donc en donner ici un résumé, avee quelques observalions critiques.

Klunzinger décrit ringt-cing espèces de Oxy'hingues:

Camposcia relusa lat. (figure), Koseir.

"Simocarcinus camelus Kilz. n. sp). (ligures), El 'Tor'.

Trigonothir pyramidatus (Ilell.) (ligures).

Mencetus monoceros (Latr.), Koseir.

Acanthonyx elongatus (Wh.), Viers. Koseir, Suez. A. consolnimu Paulson nec

A. II.-Edw. est considéré par Kilunzinger identique ì A. clongutus.

stenocionops curvirostris A. M.-Ldw. (d'après A., Milne-Edwatds).

Stilbognathus erytheres v. Mart., lioseir.

* Herlistia corniculata Klz., n. sp., Koseir.

- Herbstia contiguicornis Klz., n. sp.

"Hyastenus Brockii De Man., Koseir.

Schizophrys aspera Edw., Koseir.

Cyclax suborbicularis Stimps. (figures), Koseir.

Micippe platipes Rupp. (figures), Koseir.

Hicippe thatia var. miliaris Gerst. (d'apres Gerstacker).

'Peudomicippe nodosa Heller, Koseir.

Tylocarcinus styx Ilerbst, Koseir.

Ixion capreolus Paulson. Klunzinger n'a pas vu celle espèce; il donne la traduction de la description de Paulson. J'ai déjà émis l'idée Bull. Sc. Fr. Bely., XL, 1906, p. 110) que celte espèce est identique à C'yphocurcinus minutus 1. M.-Edw., el qu'un autre synonyme en est Podohuenia erythra Cano, espice non citée dans le travail de kilunzinger.

Parathö̈ rotundata Miers (figures), Koseir.

Lambrus (Rhinolambrus) pelagicus Rüpp. (d'apres 1. Milne-Edwards el liüppell).

Lainbrus (Aulacolambrus) pisoides Ad. et Wh. ('après l'aulson).

Lambrus (Pscudolambrus) calappoides Ail. et Wh. d'apres Mlock, Adams et White el Paulson).

" Heterocrypta petrosa Klz. (ligures), n. sp., Koseir.

Parthenope horrida lab., lioseir.

- Parthenope acuta Klz. (figures), Koseir. Culle aspece fondésur un exemplaire tres jeune me pratait roisine mais distincte de Thyrolumbrus leprosus Tol. . 190:i,

- Liomedon pentagoms lilzer. Celte espre de Kosuje me parail identique is Eumedtur convictor Bouvier el seurat, lono", llouve par II. Seurat atux iles

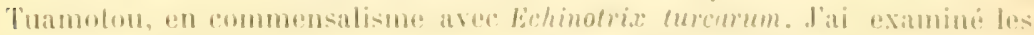

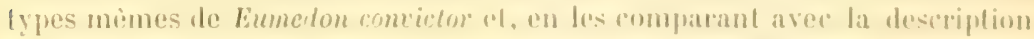

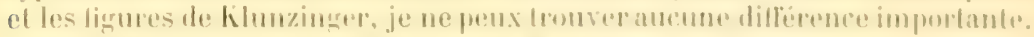

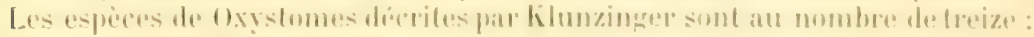

Caluppa hepatica Linn., hiseres.

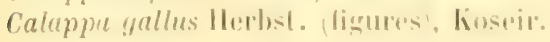


Matuta Banksii Leach. (figures).

Matuta victor Fab. (d'après les auteurs).

Leucosiu fuscomaculata Miers, Koseir. L. urania var. signate Paulson est justement considérée par Klunzinger identique à $L$. fuscomaculata; mais la substilution nécessaibe du nom signat 1875 , a fuscomacrelate 1879) ni est pas faite par l'auteur.

Philyru plutychira De Ilaan (figures), Koseir. Lïdentité de Myra variegatu Rinppell avec cette espié est reconnue. Mais si l'on armet l'identité complite de l'espèce de Rüppell (voir ce travail p. 169), le nom de Rüppell a la priorité sur celui de De Haan.

Myra fugax Fab.

Ebalia gramulata Rüpp., Koseir.

Ebalia orientalis Kossm. (d'après Kossmann).

Cullidatylus Kessterii Paulson. Klunzinger n'a pas vo celle espece, qüil décrit d'après Paulson, tout en doutant que sa position dans Callidactylus soit inexacte. J'ai établi (p.163) que cette espèce est identique avec Jyra darnleyensis Haswell, 1879.

Oreophorus horridus Rüpp. (d'après Rüppell).

Cryptocnemus tuberosus Klzg. (figures). Cette espèce est presque cerlainement identipue a Nursia mubifera. Les figures ef la description de Klunzinger s'adaptent très bien à mes exemplaires (Cf. p. 1ə̋3).

Cymopoliu carinipes Paulson. Citée d’après Paulson. Calman a déjì étahli eus 1900, l'identité de cette espèce avec Pulicus Jukesi Wh.

Le travail de Klunzinger ajoute done septeperes de oxyrhynques marqués par ") à celles de mon catalogue, ce qui porte le nombre des espèces de la mer

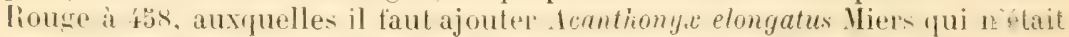
pas compris dans la liste par familles donnée à la page 5 de ce travail, et les deux ajoutées à la page 342 ; ce qui porte le nombre des espèces sigualées dans la mer Rouge à 461 . 


\section{EXPLICATION DES P'LANCHES}

\section{PIATIIE I}

Fig. 1. - Metapenæus cognatus Nob. liostre, $x\{$.

Fig. 2. - Vetapenaus Stebbingi Nob. Rostre, $\times$; ; $2 a$; petasma, $\times\{; 2 b$, thélycum, X4.

Fig. 3. - Metapenxus consobrimus Nob. Rostre d'un mile; $3 a$, pélasma, $x$ i: $3 b$, thélycum, $\times \dot{4}$.

Fig. 4. - Hetapenxus Vaillanti Nob. Pétasma, $\times 1: ;$; tique).

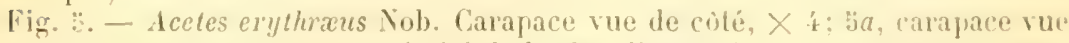
d'en dessus, $X 4 ; \ddot{b}$, extrémité de la deuxieme paire de pérépqodes, $X: 30:$ : màle, $\times 1$ l'; préhension du màle, $\times 150$.

\section{PLANCHE II}

Fig. 1. - Lucifer Hanseni Nob. Femelle, $\times 1$; 1 a, sixieme articlu de l'abelomen et telson du mâle, $\times 1$; ; $1 b$, telson du mâle vu de côté, $\times: 0 ; 1 c$, émission du spermatophore dans un mâle, $\times 1: 0$.

Fig. 2. - Virbius jactans Nob. Rostre, $\times 30 ; 2 b$, extrémilé d'une patte, $\times 50$.

Fig. 3. - Latreutes mucronatus var. mullidens Yob. $\times 1$ t.

Fig. 't. - Hippolysmata vittata Slm. Liostre, $X$ i.

Fig. 3. - Hippolysmata multiscissa Noh. Rostre, $X$ \&.

Fig. 6. - Periclimenes soror Wob. Rostre, $x$ i.

Fig. 7. - Coralliocaris superbe 1)ana. Anomalie du lostre, $X$ \&.

Fig. 8. - Coralliocaris rhodope Nolb. X' ; Sa, extrémité d'un péréopode.

\section{PANCIIE III}

Fir. 1. - Paschocaris paschalis (Itell.). Mandibule, $X$ l't, el délails des parties incisives et molaires, $X \$ 0 ; 1 a$, deuxime maxillipede, $X 11 ; 1$, premier maxillipede, $\times 1$; 16 , premiere maxille, $\times 1$; $1 d$, deuxime maxille. Y $1 \mathrm{i}$

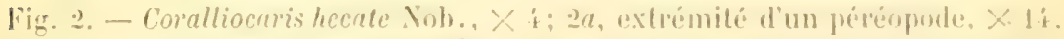

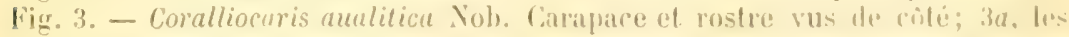
mèmes vas d'en haul; 36, chélipiede; 3c, doigh d'un péréopode. X 1't.

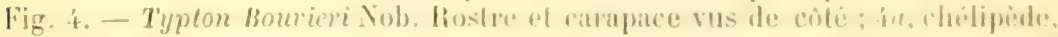

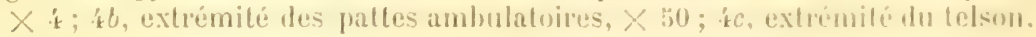
$\times: 30$.

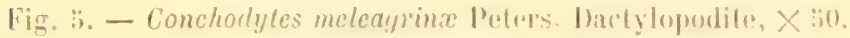

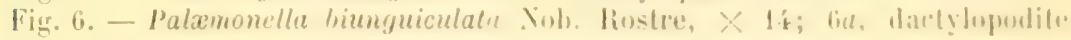
$\times: 0$.

\section{PLATIII: II}

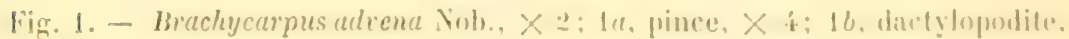
$\times: 0$.

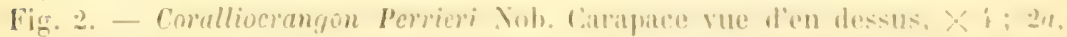
mandibule, $\times 50 ; 20$, premiere maxille, $\times 1$; 20 , deuxieme matle, $\times 1$; 
$2 d$, premier maxillipède, $\times 14 ; 2 c$, deuxième maxillipède, $\times 14 ; 2 f$, extrémité du troisième maxillipède, $\times 14 ; 2 g$, premier péréopode, $\times 4 ; 2 h$, denxième péréopode, $\times 14 ; 2 i$, dactylopodite des pattes ambulatoires, $\times 50$.

Fig. 3. - Processa Coutierei Nob. Rostre et antennes vus d'en haut, $\times 14$; les mêmes vus de côté, $\times 14$.

\section{PLANCIIL V}

Fig. 1. - Nikoides Danx Pauls. Extrémité de la carapace et du rostre, $\times 4 ; 1 a$, mandibule, $\times 14 ; 1 b$, première maxille, $\times 14 ; 1 c$, deuxième maxille, $\times 14$; $1 d$, premier maxillipède, $\times 14 ; 1 e$, deuxième maxillipède, $X 14 ; 1 f$, patte simple de la première paire, $\times 7$.

Fig. 2. - Callianassa Calmani Nob. Ischium, mérus et carpe du gros chélipède, $\times 4 ; 2$, main du gros chélipède, $X$ 千;2b, petit chélipède, $X\{$.

\section{PLANCHE VI}

Fig. 1. - Axiopsis æthiopica Nob. Carapace, $\times 4 ; 1$, pédoncule des antennes, $\times 14 ; 1 b$, gros chélipède, $\times 4 ; 1 c$, petit chélipède, $\times 4 ; 1 d$, extrémité d'une patte de la troisième paire, $X 14 ; 1$, telson et uropode, $X 4$.

lig. 2. - Callianassa Jousseaumei Nob. Grosse pince, $X 2$; $2 a$, petite pince, $\times 2$; $2 b$, telson et uropodes, $\times 4 ; 2 c$, maxillipède externe, $\times 4 ; 2 l$, extrémité de la troisieme paire de pattes, $X$ 4.

lig. 3. - Callianassa Bouvieri Nob. Grosse pince, $\times 4$; $3 a$, extrémité de la Iroisieme paire de pattes, $\times 14 ; 3 b$, telson et uropodes, $\times 4$.

Fig. 4. - Callicmassa Gravieri Nob. Grosse pince, $\times 4$; 4 , pelite pince, $\times 4$; $4 b$, extrémité de la troisième paire de pattes, $\times 14 ; 4 c$, maxillipèdes externes, $\times 14 ; 4 d$, telson et uropodes, $\times 4$.

\section{PLANCIIE VII}

Fig. 1. - Callianassa Coutierei Nob.1, ischium et mérus du gros chélipède, $\times 4 ; 1 a$, telson et uropodes, $\times 4 ; 1 b$, extrémité des pattes de la troisième paire, $\times 4$.

Fig. 2. - Callianassa Rosæ Nol. Rostre, $\times 4 ; 2 a$, mérus du gros chélipède, $\times 4 ; 2 b$, telson, $\times 2 ; 2 c$, extrémité des pattes de la troisième paire, $\times 4$; $2 d$, doigts de la grosse pince.

Fig. 3. - Galathea ægyptiuca Pauls. Chélipède, $\times 4$.

\section{PLANCHE VIII}

Fijg. 1. - Galathea hrumilis Nob. Carapace, $\times 14 ; 1 a$, chélipède, $\times 14$.

Fig. 2. - Petrolisthes leptocheles Ilell., $\times 2$.

lig. 3. - Polyonyx pedalis Nob., X 2.

Fing. 4. - Charybdis heterodon Nob., X 2 .

Fig. 3. - Pilodius armiger Nob., $\times 4$.

lig. 6. - Libya denticulata Nob., $\times 4 ; 6 a$, pince, $\times 4$.

Fig. 7. - Paranotonyx curtipes Nob., $\times 4 ; 7 a$, maxillipède, $\times 14$.

lig. 8. - Pinnoleres Maindroni Nob., $\times 4$.

Fig. 9. - Sesarma Jousseaumei Nob. Maxillipède, $\times 14$.

\section{PLANCIIE IX}

Jig. 1. - Ebalia granulata Rüpp. Type.

Fig. 2. - Ebalic abdominalis Nol.

Vig. 3. - Ebalia lacerlosa Nob. 
Hi... i. - Vursia Joussearmei Nob.

Pis. ::. - Cryptodromia grunulata Kossm.

Fi... A. - Nucia tuberculosa A. M.-Edw.

Fis. :. - Lambrus leprosus Nob.

\section{PLANCHE Y}

Fiı. 1. - Actra pisigera Nob.

Fï. 2. - Actra nodulosa Ad. et Wh.

lï. 3. - Actaca sabxa Nob.

I.i... 4. - Actere Kraussi IIell.

Pi... 5. - Pihmmes Forskalii Eis. Type.

Pi... 6. - Pilumnus spongiosus Tob.

Fï. 7. - Pilumnus propinquus Xob. (Individus types du golfe ['ersigue.

lïr. 8. - Chlorodius lippus Nob.

Fin. 9. - Curpilodes diodoreus Nob.

\section{PLANCIIE XI}

lï. 1. - Heteropunope pluraonica Nob.

Fin. 2. - Eurycarcinus integrifrons De Man.

Vis. 3. - Lambrus montiger Nob.

Fin. 4. - Cyclograpsus lophopus Wob.

Pï. :3. - Euryearcinus orientalis 1. II.-EdW. 


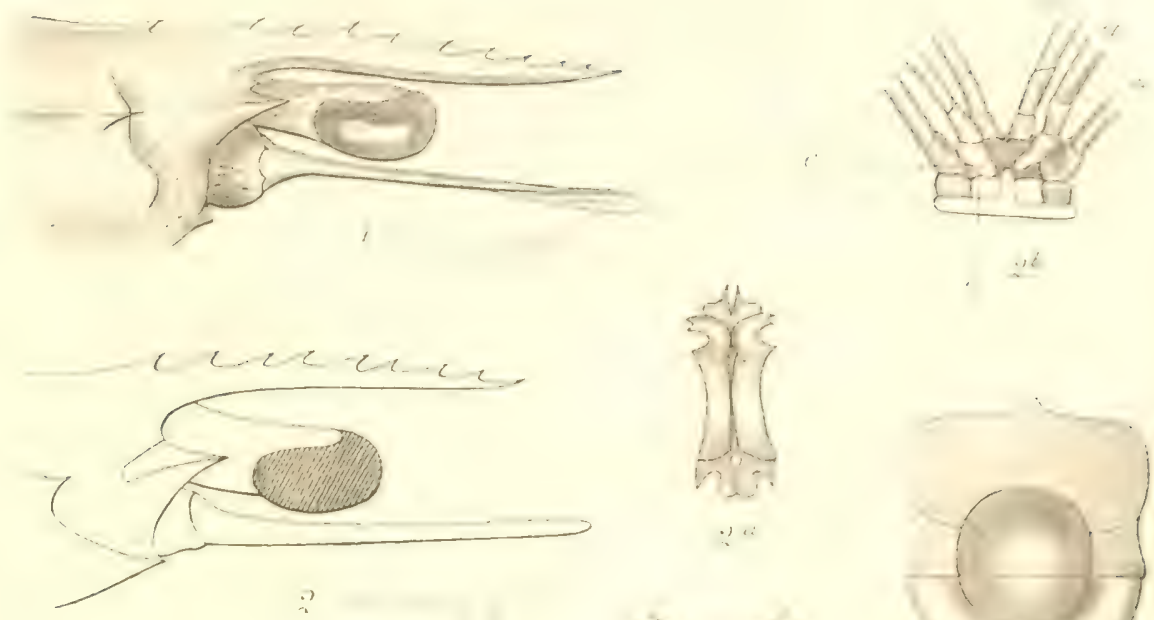

$\because \cdots$

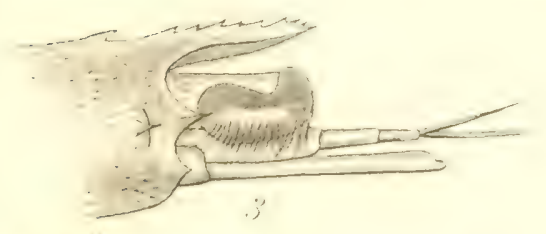

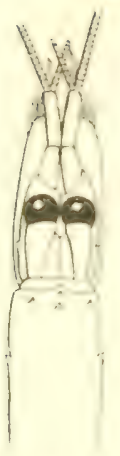

$\because \cdots$
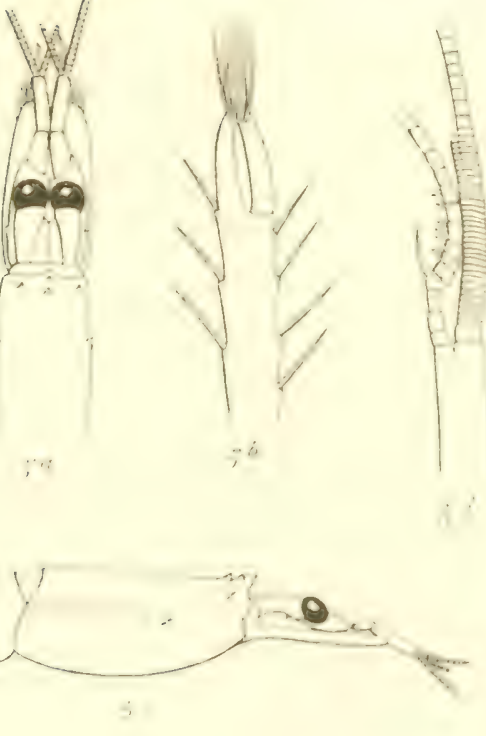

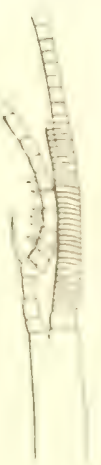

in

.$j \cdot 1$

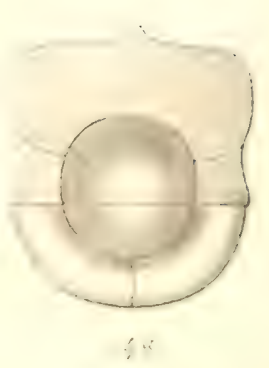

ib

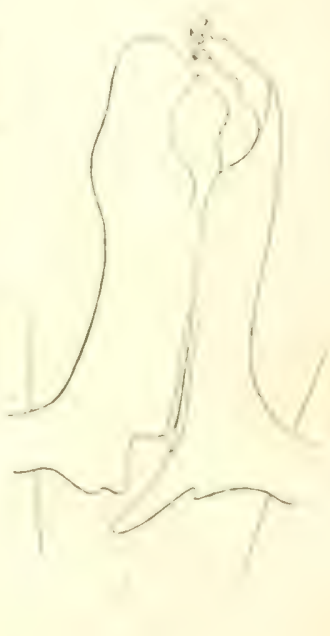

;

$\therefore 812 \div$

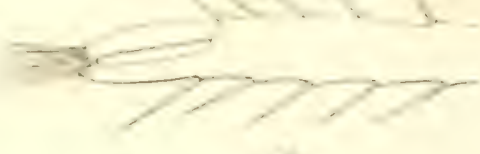

$(\because, \because o b), \because e l$

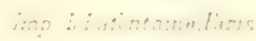

$\therefore \because \cdots, \div \div \div$ 

Anndes Se not. 9\% Serie.

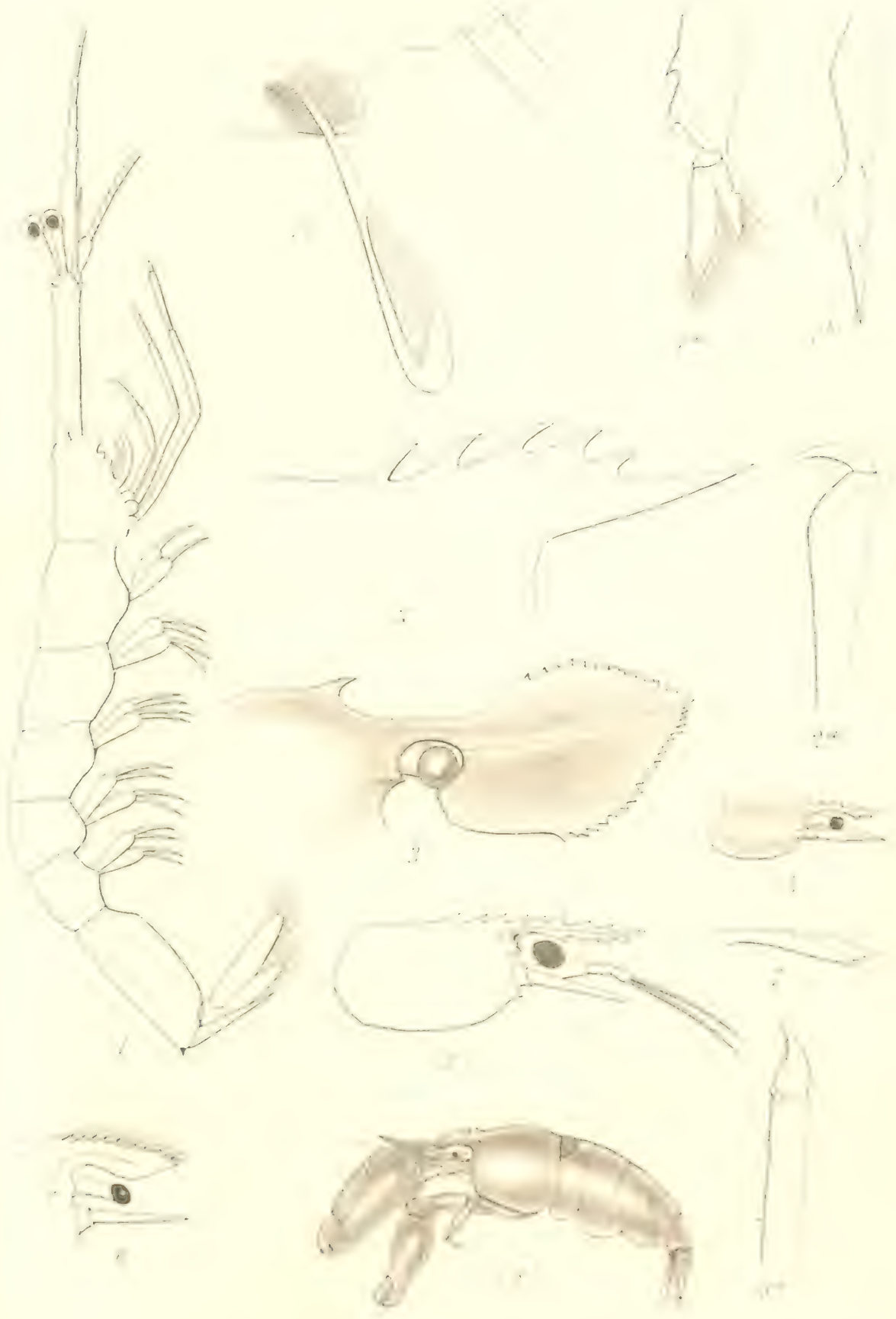




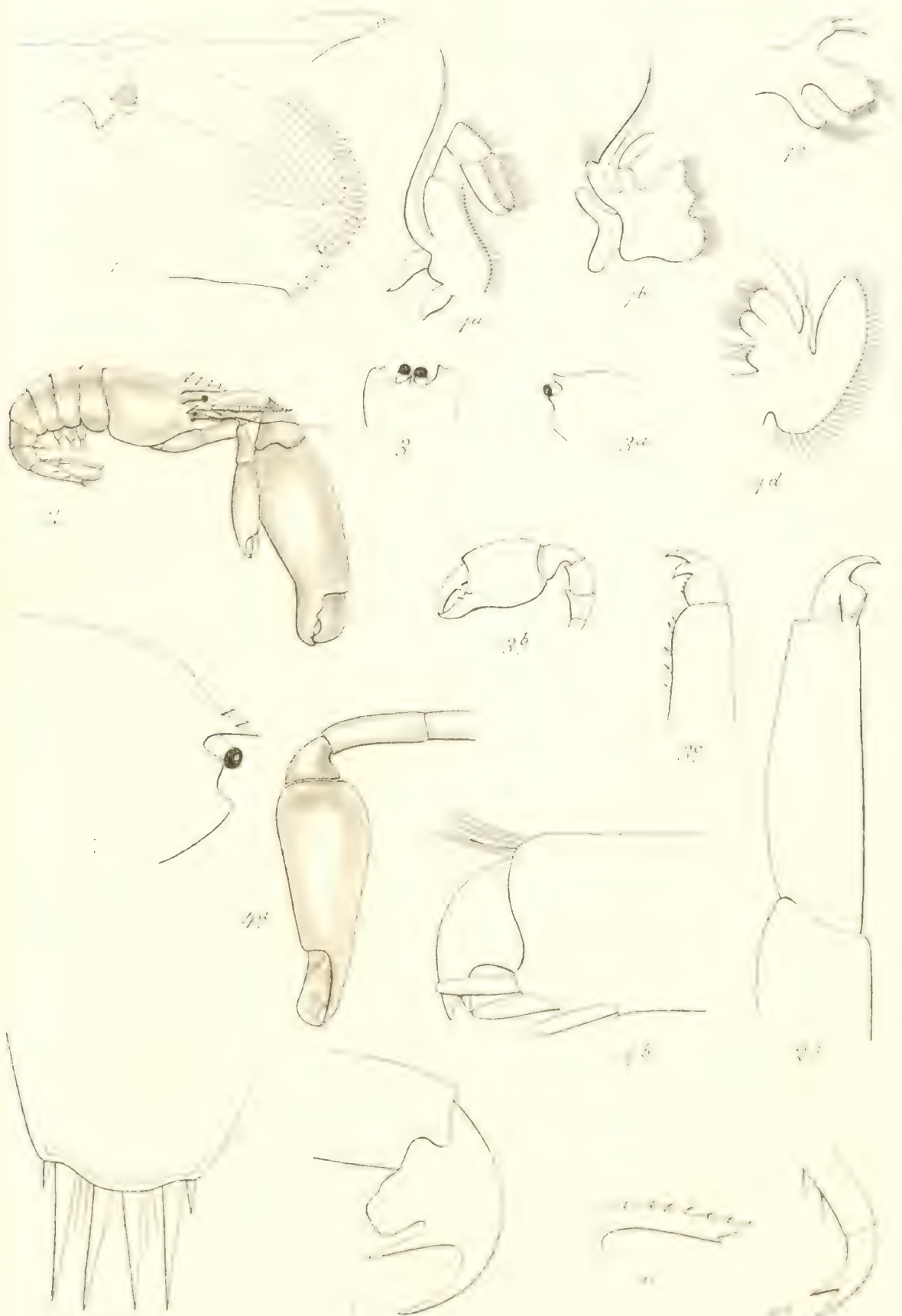

C.Nobitian
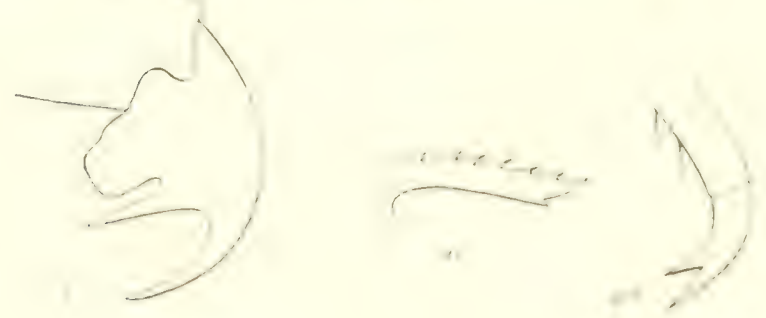


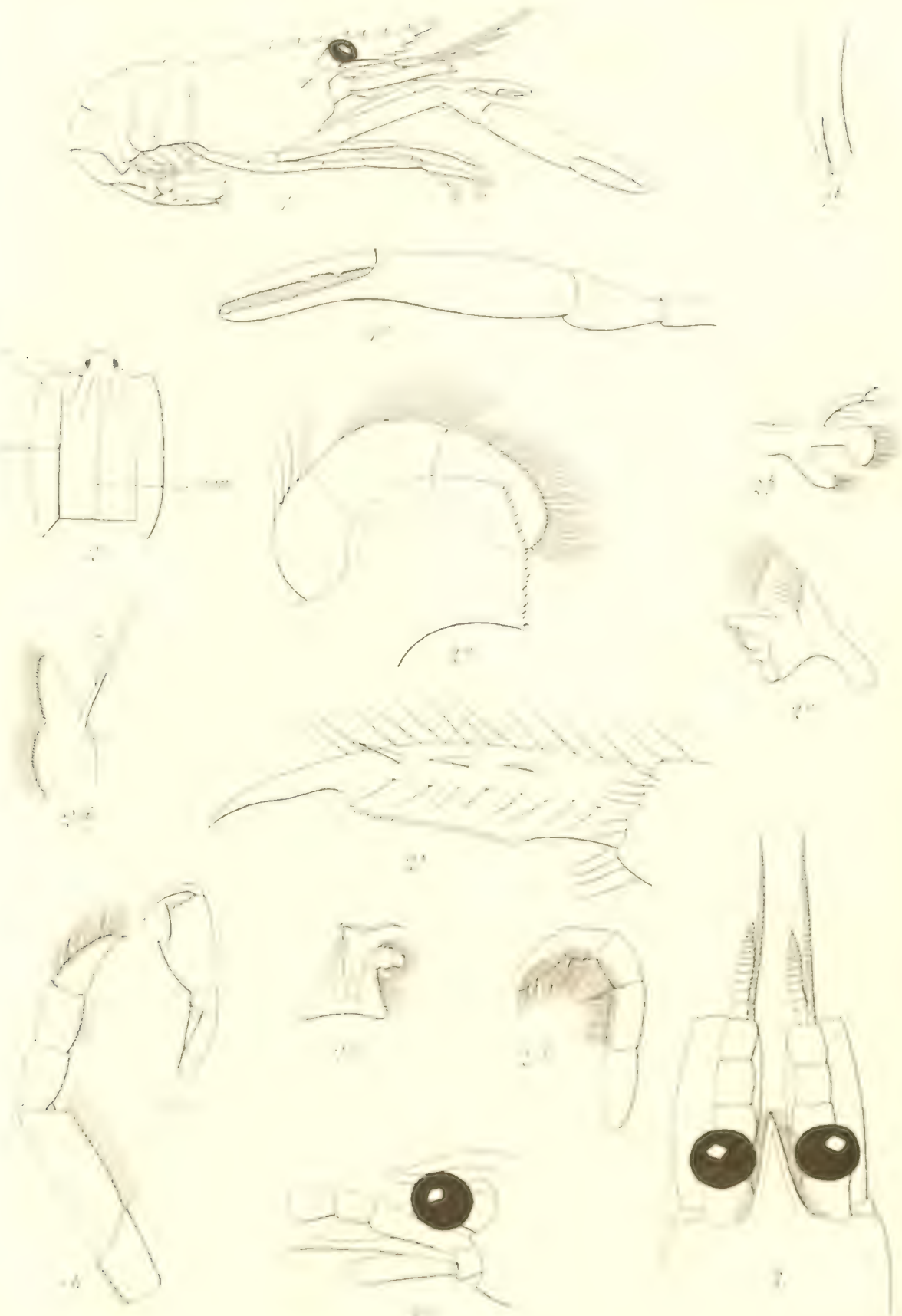


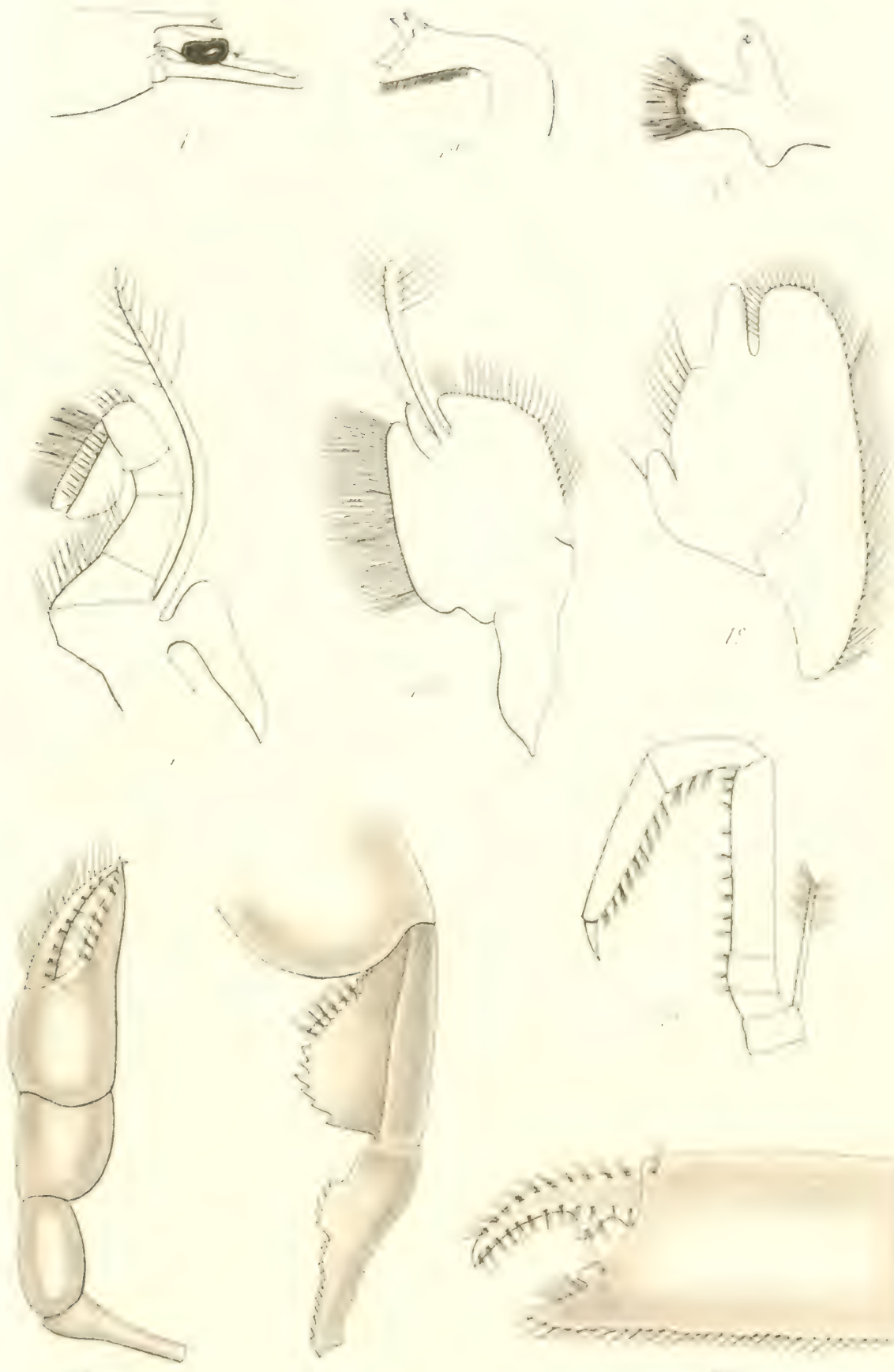

,
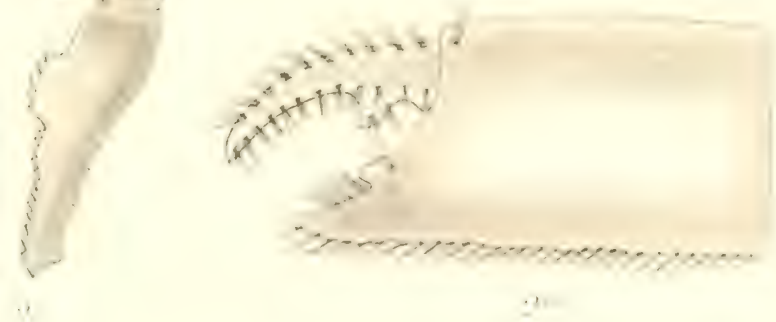


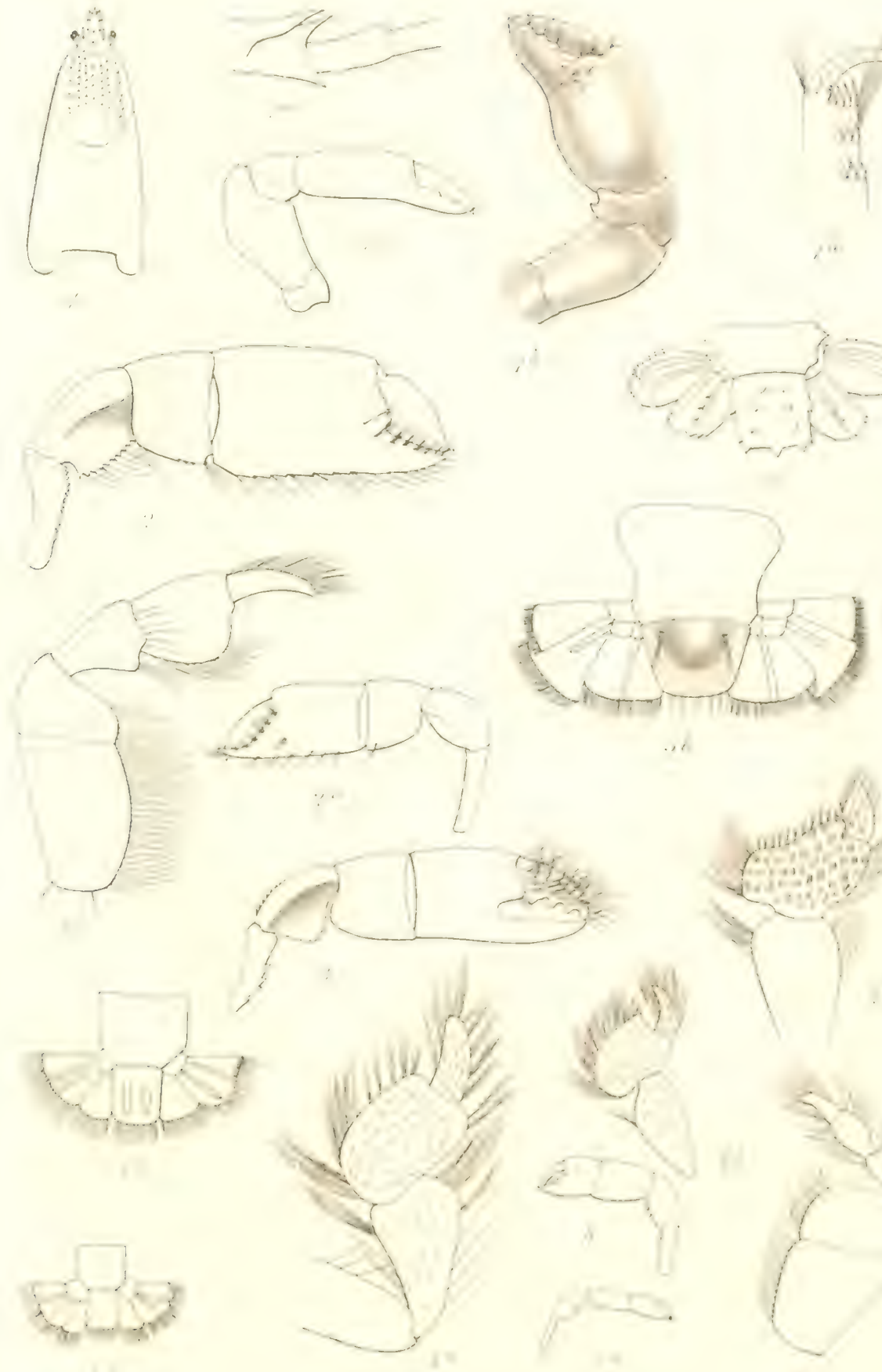
$x \rightarrow y=3, y=0$

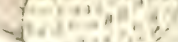
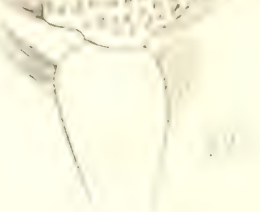

Ann des fonat ge Simio:

Zool. TH: P\%. T.
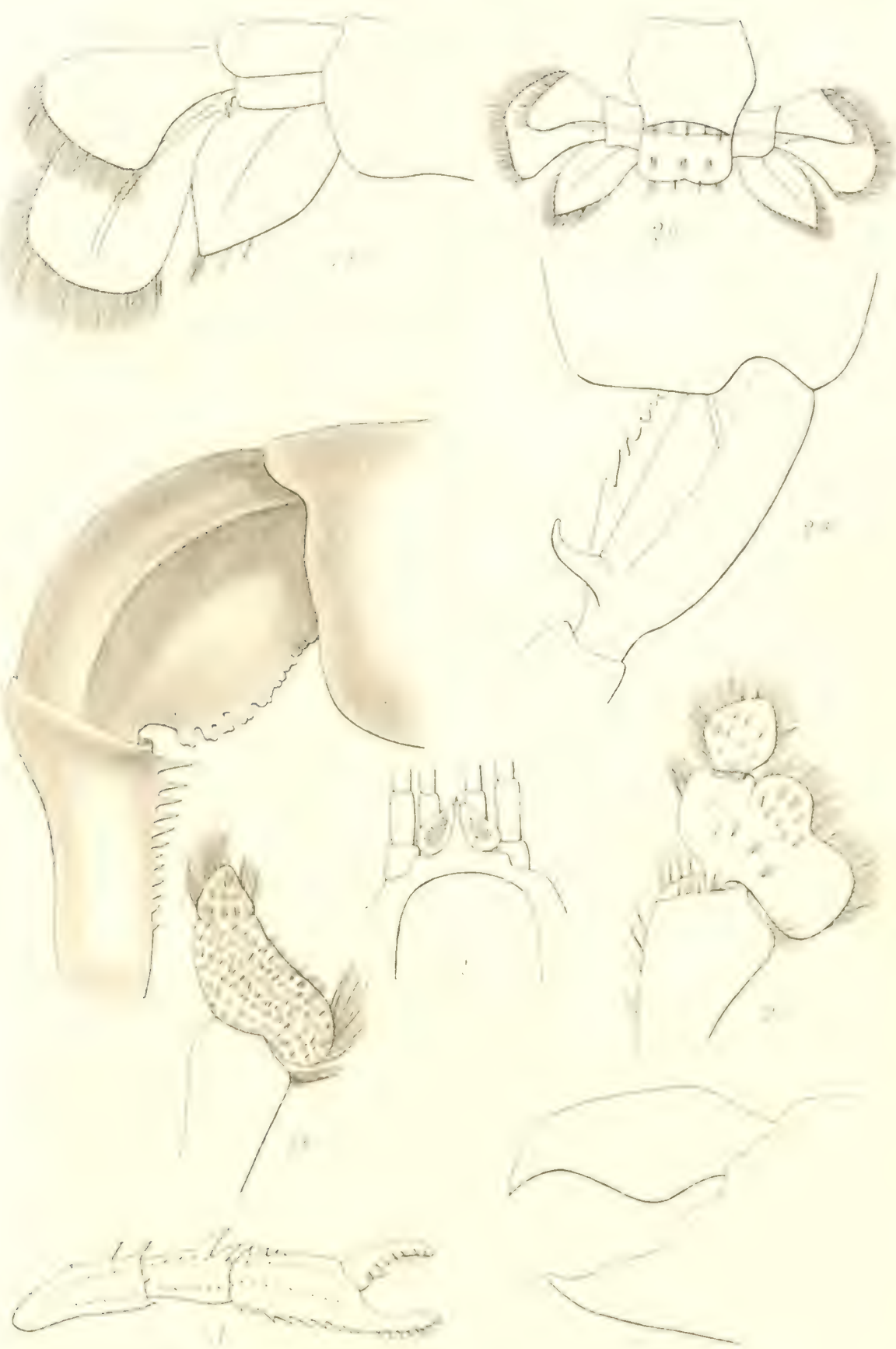


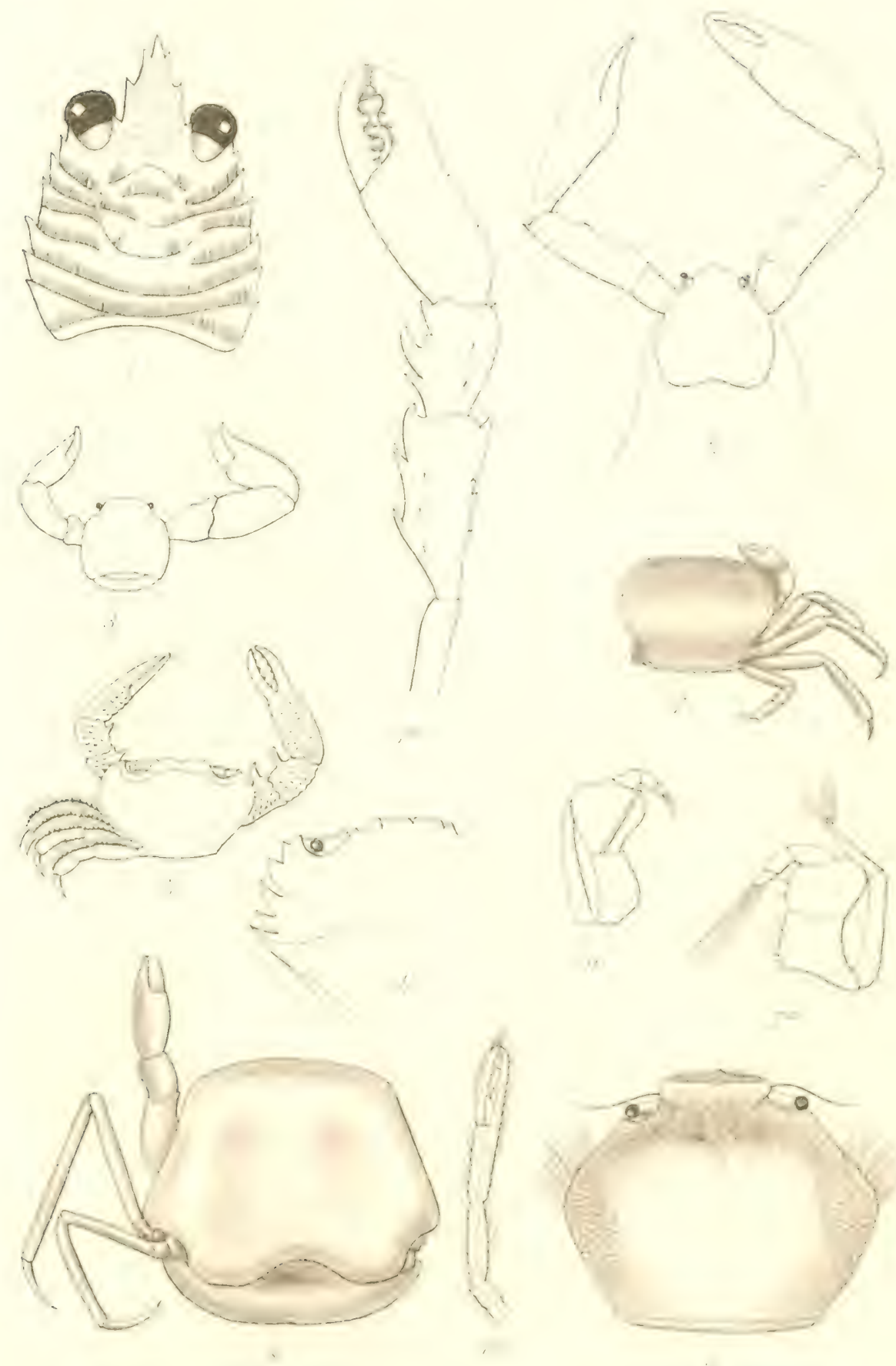


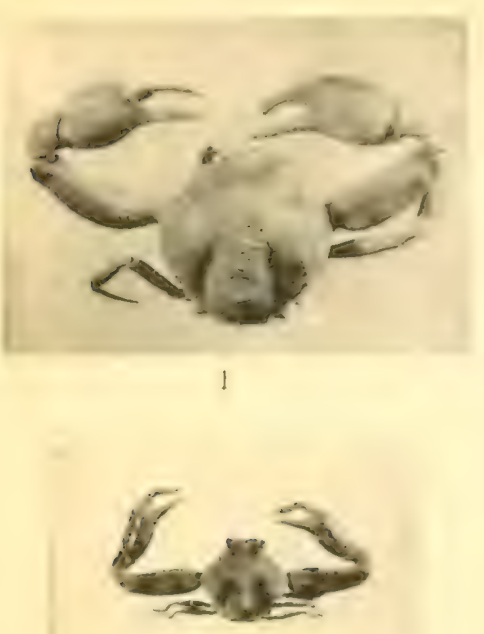

ir

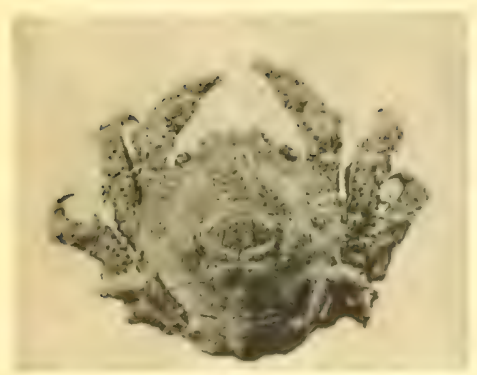

5
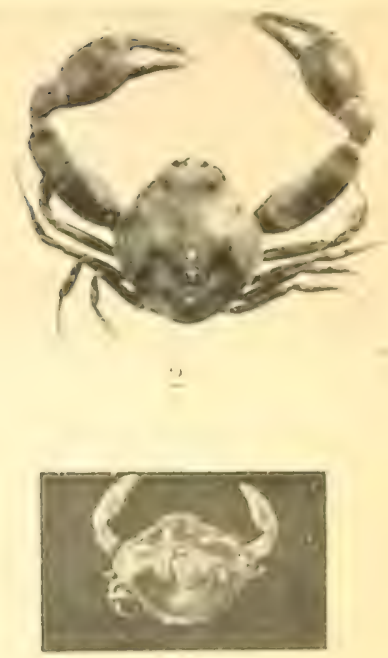

1

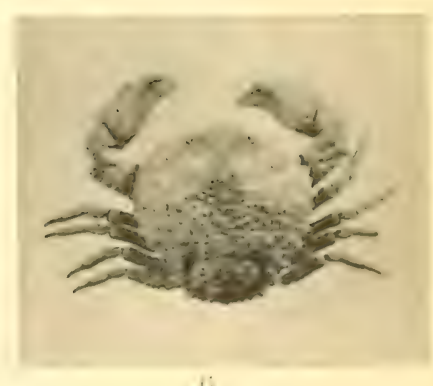

is

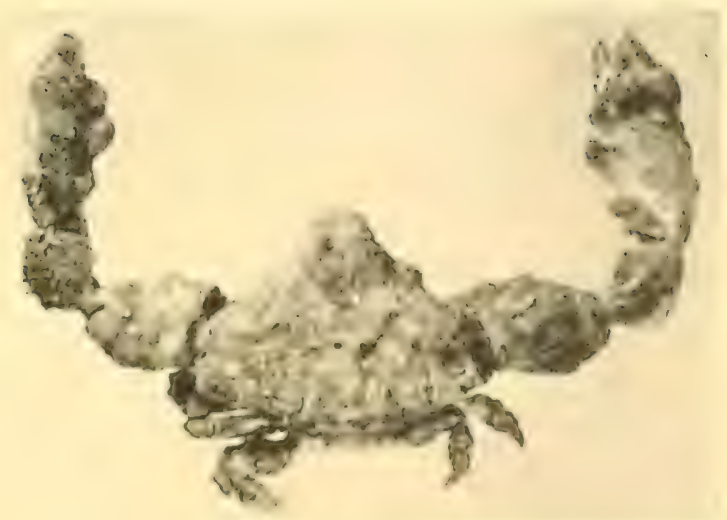





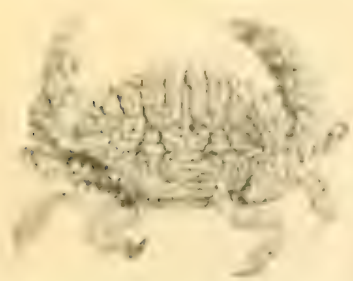

1

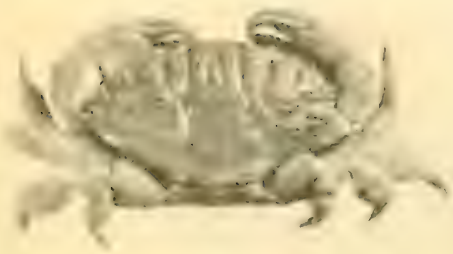

$\because$

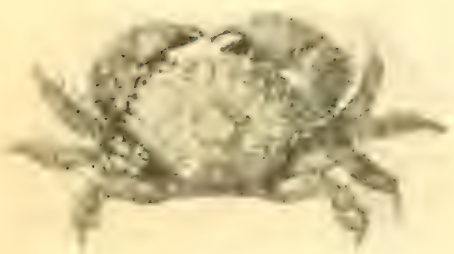

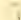

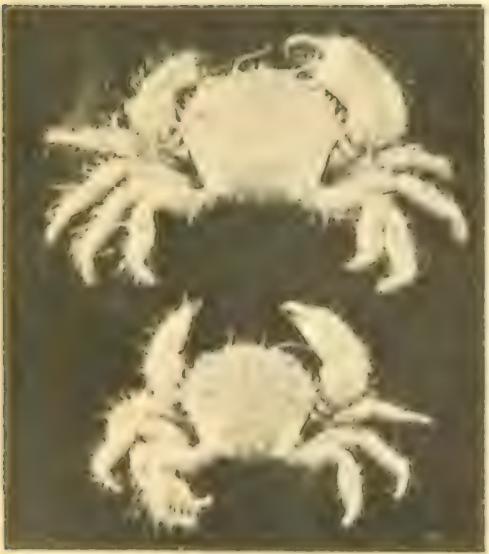

;
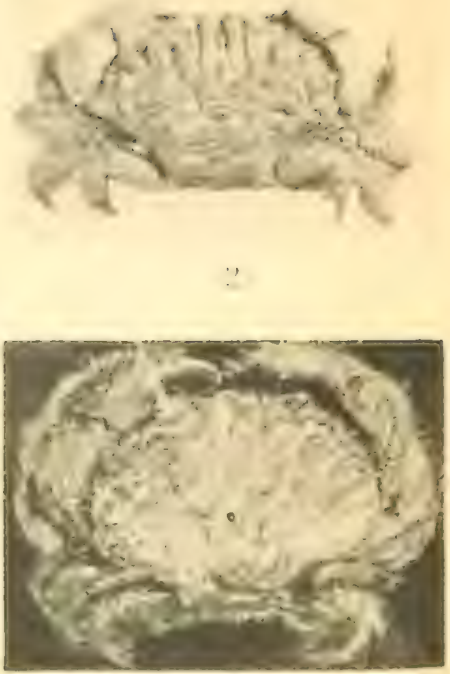

!

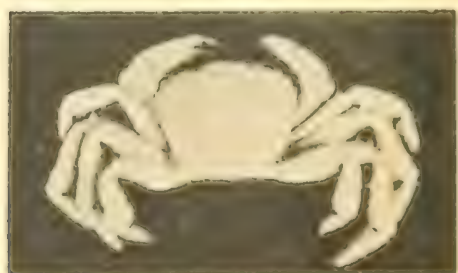

i.
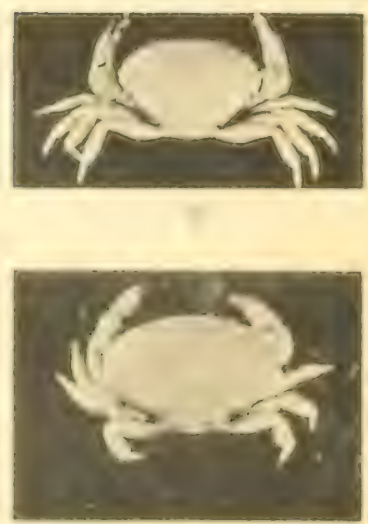

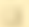




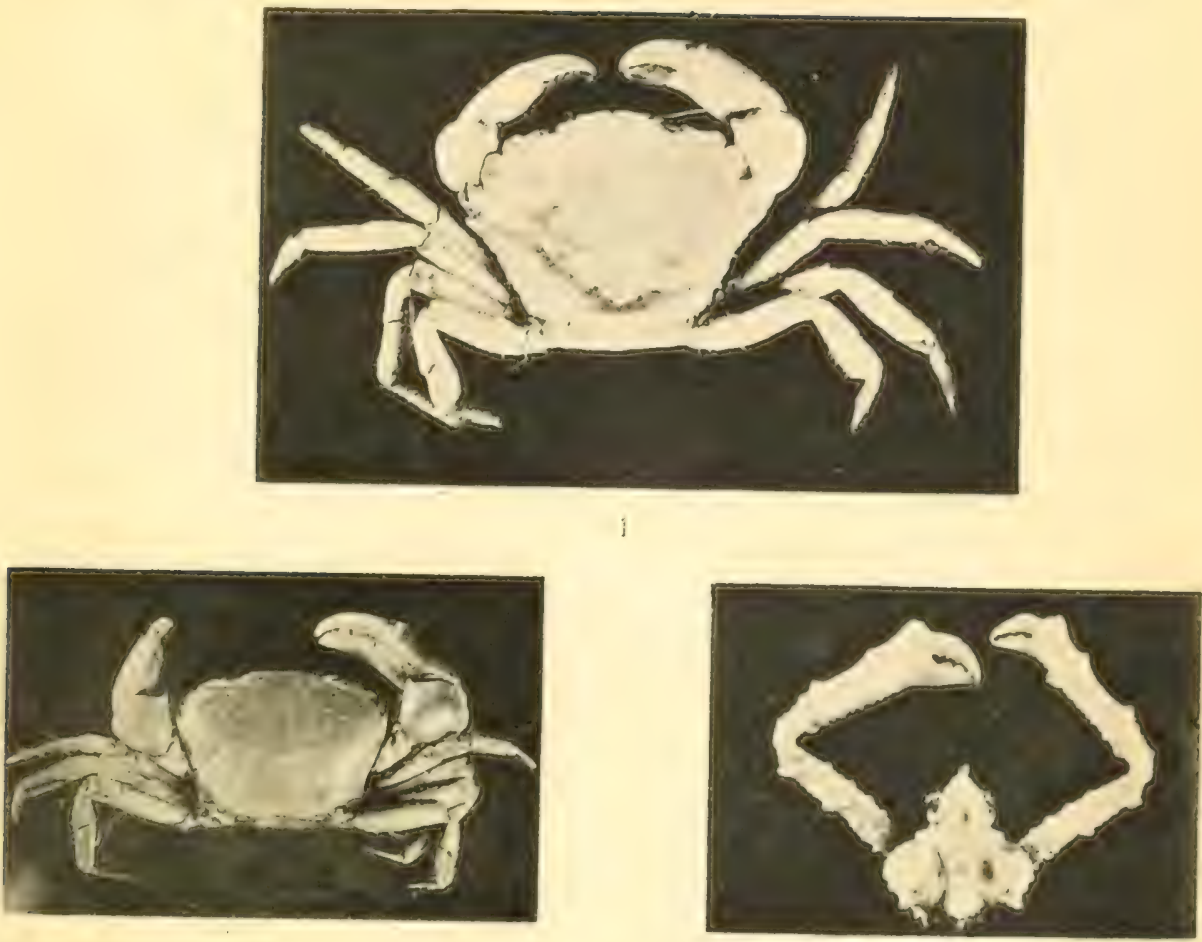

2
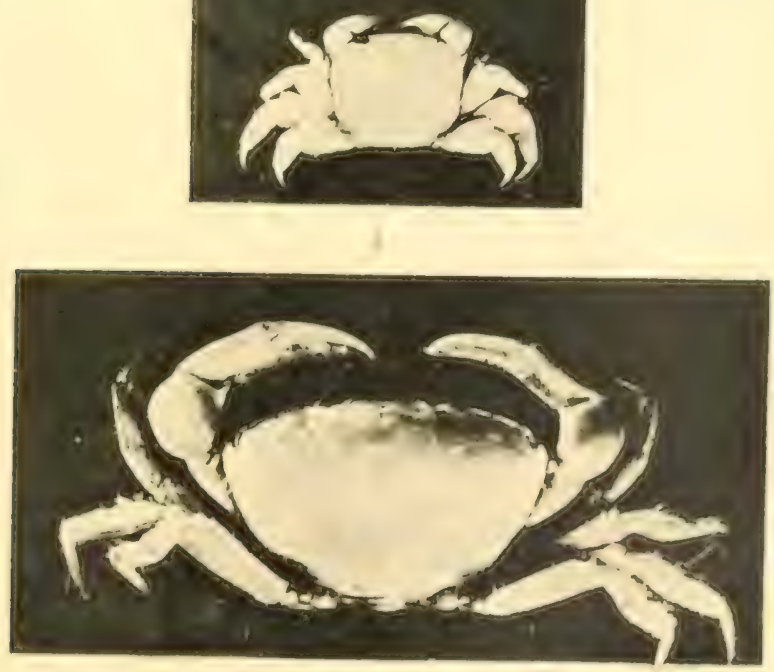



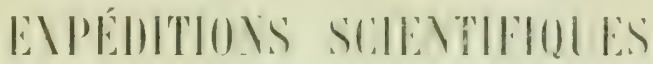

\section{TRAVAILLEUR ET I)U TMLISMMAN}

\author{
PENDANT LES ANTEES 1880, 1881, I88? LI 186:"
}

Ouvrage publié sous les auspices du Ministère le l'Instruction publique, sous la direction de $\mathbf{H}$. A. Mic.e-Édwandos, membre de l'Iustitut, président de la commission des dragages sous-marins, professeur-administruteur du Iluséum d’histoire naturelle :

Poissons, par M. L. VaIrLast, professeur-administrateur du Mluscum d'histoire uaturelle, membre de la Commission des dragages sous-narins. I fort volume

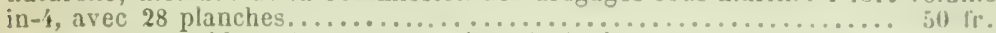

Brachiopodes, par M. P. Frscien, membre de la Commission des dragages sonzmarius et D.-P. OEHLent, membre de la Société géologique de France. 1 vol.

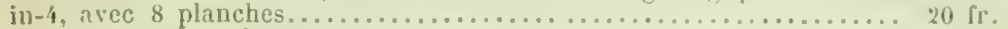

Echinodermes, par Edm. Peraner, professeur-administratcur au Jusćum d'histoirc naturelle. I vol. in- $\mathbf{6}$, avec planches noires et en conleurs.......... $50 \mathrm{fr}$.

Mollusques teslacés, par Arnould Locint. Tome 1, 1 fort volume, gr. in-i arre

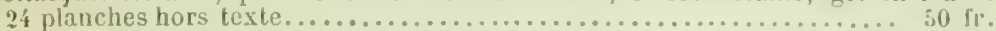

Tome 11. I vol. gr. in-'t avec nombreuses planches hors texte (sous presie).

Crustacés décapodes. Première partie: Brachyures et Anomoures, par A. MIr.xi:Enwans, et E.-L. Bovvien, professeur au Iluséum. 1 vol. in-8 avec 32 plan-

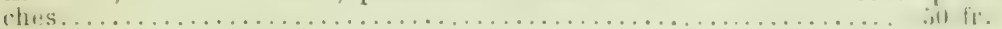
L'ouvrage comprendra en outre: Introduction. - Bryozodires, Annelides, Eponges, rrolozonives.

\section{La Géographie}

\section{BULLETIN \\ II: 1.1 \\ Société de Géographie}

PUBLTE TOUS I, ES MUIS P.I

LE Barror HULOT', Secrétaire général de la socicité

E.1

11. Cimanes RABOT, Secrétaire de la Rédaclion

ABONNEMENT ANNUEL : PAIUS : $24 \mathrm{fr}$. - DEMARTMENT: $26 \mathrm{fr}$.

Etmangen: $28 \mathrm{fr}$. - Prix du muméro: $2 \mathrm{fr} .50$.

Chaque unméro, du format grand in-8, composé de so pages at accoupragné de cartes et de gravures nombreuses, couprend des mémoires, une chronique, une

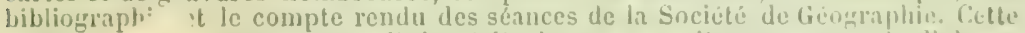
publical: pas un recueil de récits de voyages pittoresques, mais d'observalinseignements seientitiques.

¿digée par des spécialistes pour charque partie dı munle fait te plus bref délai, toutes les nouvelles recues des voyaneurs en société de Géographice et présente un résumi des renseignemente .r les publications étrangéres : elle constitue, en un mot, uแ résumé du rent géographique pour charque mois. 


\section{A LA MEME IIIBRAIRIE}

Traité de Zoologie, par M. Elmond Perrier, membre de l'hustitut, direcleur du Muséum d'bistoire nalurelle. 2 vol, gr. in-8.

Fascicule I. - Zoologie genérale, avec 458 figures dans. le texte...... 12 fr.

Fascicule 1I. - Prolozoaires el Pllyfozoaires, avec $243 \mathrm{fig}$. dans le texte. $10 \mathrm{fr}$.

Fascicule 11. - Arthropodes, avec 278 gravures............... $8 \mathrm{fr}$.

Fascicule IV. - Vers, Mollusques, avec 566 gravures.............. 16 fr.

Fascicule V. - Amphioxus, Verlélıres, avec 97 gravures........... $6 \mathbf{6 r}$.

FAscicule VI. - Vertébrés (sous presse).

Les Colonies animales et la formation des Organismes, par .I. Eilmond Perriek, in 'mbre de l'Instilut, professeur an Mluséum d'histoire naturelle. 2e édition, avec 2 planches hors texte ef 158 figures dans le texte. 1 fort vol. $\mathrm{gr}$.

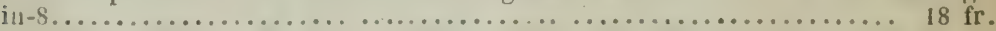

Les Enchainements du Monde animal daus les temps géologiques, par $\mathrm{M}$. Albert Gacbrix, de l'Institut, professeur au Muséum d'histoire naturelle.

Fossiles primaires. 1 vol. gr. in-8 avec 285 figures dans le texte..... 10 fr. fossiles seconilaires. 1 vol. gr. in-S arec $30 \mathrm{i}$ firtires dans le texte.... $10 \mathrm{fr}$. Mammifires tertiaires. 1 vol. gr. in-8 arec 312 figures dans le texte.. $10 \mathrm{fr}$.

Essâs de Paléontologie philosophiogue, par A. Gaudry, membre de l'Institut de France et de la Société royale de Londres, professeur au Muséum d'histoire naturelle. 1 vol. grand in-8 avec 204 gravures dans le texte.......... $8 \mathrm{fr}$.

Traité de Géologie, par M. A. oe LApiparent; membre de l'Institut, professeur à l'école libre des liautes Etudes. Guvrage couronué par l'Institut. 4o èdition, entierement refoudue et considéräblement augmentece. 3 rol. grand in- 8 , xiv1912 pages avec 850 gravures dans le texte................... 35 fr.

Cours de Minéralogie, par I1. A. de LAPranevt, 3e édition très augmentée. 1 vol. grand in-8 de 650 pages avec 619 gravures dans le texte et une plauche chro-

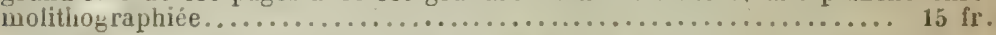

Traité de Botanique, par VAN TrEGHEM, membre de l'Institut, professeur au Muséum d'histoire naturelle. Denxième édition entièrement refoudne et corrigée. 2 vol. grand in -8 avec 1,213 gravires dans le texte.......... $30 \mathrm{fr}$.

Nouvelles Archives du FIuséum d'histoire naturelle, publices par III. les professeurs-administrateur: de cet-établissement:

11 parait chaque anuée 1 vol. gr. in-t, publié en 2 fascicules avec planches en noir et en couleurs. Chaque volume séparément................... $40 \mathrm{fr}$.

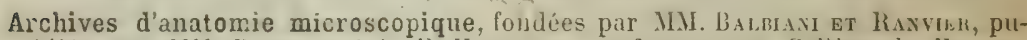
bliees par MII. Ranvien et L.-1:. IIenkegur, professeurs au Cullege de France. Les Archives d'anatomie microscopique forment par an 't 1. Iscicules in-8, d'environ 150 pages, paraissant à des époques indéterminécs avec planches en noir et en couleur. Paris, Dép. et Union postale....................... $50 \mathrm{fr}$.

Guides du Touriste, du Naturaliste et de l'Archéologue, publiés sous la direction de M. Marcellin Boule:

Chaque volume, cartonné toile ánglaise .................. 4 fr. 50

Le Cantal, par MM. Marcellin Boule et Louis. Fairges. 1 vol. avec 85 dessins et photographies et 2 cartes en conleurs.

La Lozère, par MM. Ernest Cono et Gústave Cond, avec la collaboration de M. Armand VIRE. 1 vol. avec 87 dessins et photographies et 4 cartes en couleurs.

Le Puy-de-Dôme et Vichy, par Mil. Marcellin Boule, Ph. Glangeadd, Rouchon, A. Verniére. 1 vol. avec 109 deșins et photographies et 8 cartes en couleurs.

La Haute-Savoie, par M. Marc Le Roux, 1 vol, avec dessins et photographies et 4 cartes en couleurs (sous presse). 

















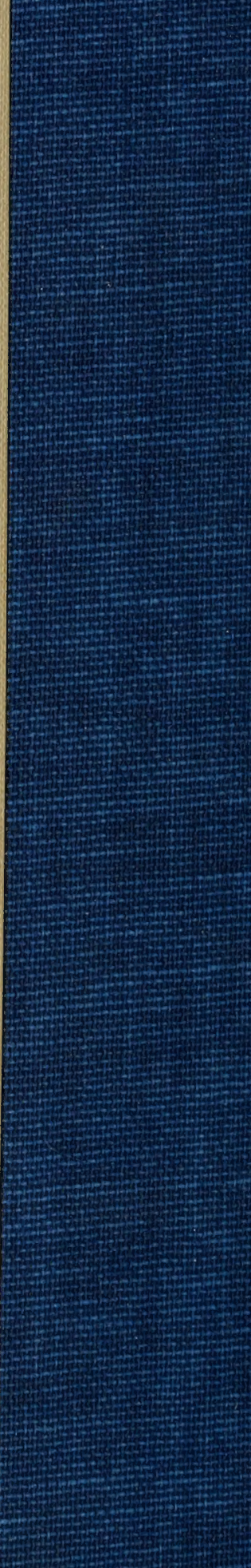

